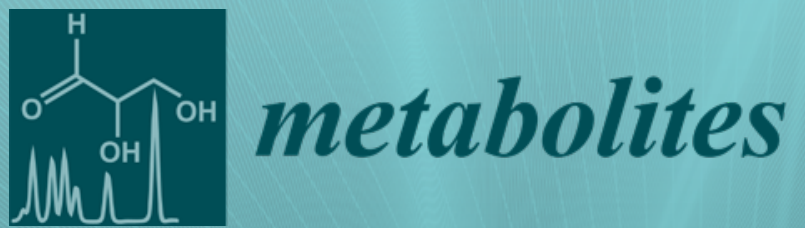

\title{
Metabolomic \\ Applications in \\ Animal Science
}

Edited by

Shozo Tomonaga

Printed Edition of the Special Issue Published in Metabolites 
Metabolomic Applications in Animal Science 



\section{Metabolomic Applications in Animal Science}

Editor

Shozo Tomonaga

MDPI • Basel $\bullet$ Beijing $\bullet$ Wuhan $\bullet$ Barcelona $\bullet$ Belgrade $\bullet$ Manchester $\bullet$ Tokyo $\bullet$ Cluj $\bullet$ Tianjin 
Editor

Shozo Tomonaga

Kyoto University

Japan

\section{Editorial Office}

MDPI

St. Alban-Anlage 66

4052 Basel, Switzerland

This is a reprint of articles from the Special Issue published online in the open access journal Metabolites (ISSN 2218-1989) (available at: https://www.mdpi.com/journal/metabolites/special_ issues/Animal_Sci).

For citation purposes, cite each article independently as indicated on the article page online and as indicated below:

LastName, A.A.; LastName, B.B.; LastName, C.C. Article Title. Journal Name Year, Article Number, Page Range.

ISBN 978-3-03943-647-7 (Hbk)

ISBN 978-3-03943-648-4 (PDF)

(C) 2020 by the authors. Articles in this book are Open Access and distributed under the Creative Commons Attribution (CC BY) license, which allows users to download, copy and build upon published articles, as long as the author and publisher are properly credited, which ensures maximum dissemination and a wider impact of our publications.

The book as a whole is distributed by MDPI under the terms and conditions of the Creative Commons license CC BY-NC-ND. 


\section{Contents}

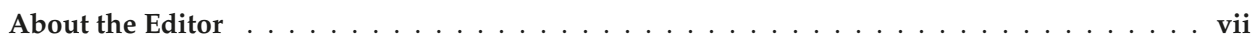

Preface to "Metabolomic Applications in Animal Science" . . . . . . . . . . . ix

Masaaki Taniguchi, Aisaku Arakawa, Motohide Nishio, Toshihiro Okamura, Chika Ohnishi, Kouen Kadowaki, Kimiko Kohira, Fumika Homma, Kazunori Matsumoto and Kazuo Ishii Differential Metabolomics Profiles Identified by CE-TOFMS between High and Low Intramuscular Fat Amount in Fattening Pigs

Reprinted from: Metabolites 2020, 10, 322, doi:10.3390/metabo10080322 . . . . . . . . . . . 1

Shoko Sawano, Keishi Oza, Tetsuya Murakami, Mako Nakamura, Ryuichi Tatsumi and Wataru Mizunoya

Effect of Gender, Rearing, and Cooking on the Metabolomic Profile of Porcine Muscles

Reprinted from: Metabolites 2020, 10, 10, doi:10.3390/metabo10010010 . . . . . . . . . . . . . .

Xiao Wang and Haja N. Kadarmideen

Metabolite Genome-Wide Association Study (mGWAS) and Gene-Metabolite Interaction Network Analysis Reveal Potential Biomarkers for Feed Efficiency in Pigs

Reprinted from: Metabolites 2020, 10, 201, doi:10.3390/metabo10050201 . . . . . . . . . . . . .

Priyanka Banerjee, Victor Adriano Okstoft Carmelo and Haja N. Kadarmideen

Integrative Analysis of Metabolomic and Transcriptomic Profiles Uncovers Biological Pathways of Feed Efficiency in Pigs

Reprinted from: Metabolites 2020, 10, 275, doi:10.3390/metabo10070275 . . . . . . . . . . . . 49

Kamil Stastny, Kristina Putecova, Lenka Leva, Milan Franek, Petr Dvorak and Martin Faldyna

Profiling of Metabolomic Changes in Plasma and Urine of Pigs Caused by Illegal

Administration of Testosterone Esters

Reprinted from: Metabolites 2020, 10, 307, doi:10.3390/metabo10080307 . . . . . . . . . . . . 67

Tomoya Yamada, Mituru Kamiya and Mikito Higuchi

Gas Chromatography-Mass Spectrometry-Based Metabolomic Analysis of Wagyu and

Holstein Beef

Reprinted from: Metabolites 2020, 10, 95, doi:10.3390/metabo10030095 . . . . . . . . . . . . . . .

Timothy D. W. Luke, Jennie E. Pryce, Aaron C. Elkins, William J. Wales and

Simone J. Rochfort

Use of Large and Diverse Datasets for ${ }^{1} \mathrm{H}$ NMR Serum Metabolic Profiling of Early Lactation Dairy Cows

Reprinted from: Metabolites 2020, 10, 180, doi:10.3390/metabo10050180

Timothy D. W. Luke, Jennie E. Pryce, William J. Wales and Simone J. Rochfort

A Tale of Two Biomarkers: Untargeted ${ }^{1} \mathrm{H}$ NMR Metabolomic Fingerprinting of BHBA and NEFA in Early Lactation Dairy Cows

Reprinted from: Metabolites 2020, 10, 247, doi:10.3390/metabo10060247 . . . . . . . . . . . 11

Sajid Latif, Paul A. Weston, Russell A. Barrow, Saliya Gurusinghe, John W. Piltz and Leslie A. Weston

Metabolic Profiling Provides Unique Insights to Accumulation and Biosynthesis of Key Secondary Metabolites in Annual Pasture Legumes of Mediterranean Origin Reprinted from: Metabolites 2020, 10, 267, doi:10.3390/metabo10070267 . . . . . . . . . . . . 135 
Saki Shimamoto, Kiriko Nakamura, Shozo Tomonaga, Satoru Furukawa, Akira Ohtsuka and Daichi Ijiri

Effects of Cyclic High Ambient Temperature and Dietary Supplementation of Orotic Acid, a Pyrimidine Precursor, on Plasma and Muscle Metabolites in Broiler Chickens

Reprinted from: Metabolites 2020, 10, 189, doi:10.3390/metabo10050189 . . . . . . . . . . . . . 155

Tatsuhiko Goto, Hiroki Mori, Shunsuke Shiota and Shozo Tomonaga

Metabolomics Approach Reveals the Effects of Breed and Feed on the Composition of Chicken Eggs

Reprinted from: Metabolites 2019, 9, 224, doi:10.3390/metabo9100224 . . . . . . . . . . . . . . . . 171

Marilena Bazzano, Luca Laghi, Chenglin Zhu, Enrica Lotito, Stefano Sgariglia,

Beniamino Tesei and Fulvio Laus

Exercise Induced Changes in Salivary and Serum Metabolome in Trained Standardbred, Assessed by ${ }^{1} \mathrm{H}-\mathrm{NMR}$

Reprinted from: Metabolites 2020, 10, 298, doi:10.3390/metabo10070298 . . . . . . . . . . . . . 183

Chenglin Zhu, Sabrina Fasoli, Gloria Isani and Luca Laghi

First Insights into the Urinary Metabolome of Captive Giraffes by Proton Nuclear Magnetic

Resonance Spectroscopy

Reprinted from: Metabolites 2020, 10, 157, doi:10.3390/metabo10040157 . . . . . . . . . . . . . 197

Satoko Naoe, Hiroshi Tsugawa, Mikiko Takahashi, Kazutaka Ikeda and Makoto Arita

Characterization of Lipid Profiles after Dietary Intake of Polyunsaturated Fatty Acids Using Integrated Untargeted and Targeted Lipidomics

Reprinted from: Metabolites 2019, 9, 241, doi:10.3390/metabo9100241 . . . . . . . . . . . . . . 211

Susumu Muroya, Shuji Ueda, Tomohiko Komatsu, Takuya Miyakawa and Per Ertbjerg MEATabolomics: Muscle and Meat Metabolomics in Domestic Animals

Reprinted from: Metabolites 2020, 10, 188, doi:10.3390/metabo10050188 


\section{About the Editor}

Shozo Tomonaga earned his Ph.D. in Agriculture from Kyushu University in March 2008. He was a JSPS Postdoctoral Fellow from April 2008 to October 2009. From October 2009, he was an assistant professor in Kyushu University. In April 2012, he moved to Kyoto University as an assistant professor. He is a scientist in Animal Science. He has investigated the dietary effects of imidazole dipeptides, especially carnosine. He is interested in stress and studied nutritional metabolism during stressful conditions. He has challenged applications of metabolomics in Animal Science by GC-MS and LC-MS. 



\section{Preface to "Metabolomic Applications in Animal Science"}

Metabolomics has been a useful method for various study fields. However, its application in animal science does not seem to be sufficient. Metabolomics will be useful for various studies in animal science: Animal genetics and breeding, animal physiology, animal nutrition, animal products (milk, meat, eggs, and their by-products) and their processing, livestock environment, animal biotechnology, animal behavior, and animal welfare. More application examples and protocols for animal science will promote more motivation to use metabolomics effectively in the study field.

Therefore, in this Special Issue, we introduced some research and review articles for "Metabolomic Applications in Anmal Science". The main methods used were mass spectrometry or nuclear magnetic resonance spectroscopy. Not only a non-targeted, but also a targeted, analysis of metabolites is shown. The topics include dietary and pharmacological interventions and protocols for metabolomic experiments.

Shozo Tomonaga Editor 



\title{
Differential Metabolomics Profiles Identified by CE-TOFMS between High and Low Intramuscular Fat Amount in Fattening Pigs
}

\author{
Masaaki Taniguchi ${ }^{1, *}$, Aisaku Arakawa ${ }^{1}$, Motohide Nishio ${ }^{1}$, Toshihiro Okamura ${ }^{1}$, \\ Chika Ohnishi ${ }^{2}$, Kouen Kadowaki ${ }^{3}$, Kimiko Kohira ${ }^{4}$, Fumika Homma ${ }^{4}$, Kazunori Matsumoto ${ }^{4}$ \\ and Kazuo Ishii ${ }^{1}$ \\ 1 Institute of Livestock and Grassland Science, National Agriculture and Food Research Organization, \\ Tsukuba Ibaraki 305-0901, Japan; aisaku@affrc.go.jp (A.A.); mtnishio@affrc.go.jp (M.N.); \\ okamut@affrc.go.jp (T.O.); kazishi@affrc.go.jp (K.I.) \\ 2 Miyazaki Station, National Livestock Breeding Center, Kobayashi Miyazaki 886-0004, Japan; \\ c0ohnisi@nlbc.go.jp \\ 3 Ibaraki Station, National Livestock Breeding Center, Chikusei Ibaraki 308-0112, Japan; k0kadowk@nlbc.go.jp \\ 4 National Livestock Breeding Center, Nishigo Fukushima 961-8511, Japan; k0kohira@nlbc.go.jp (K.K.); \\ f0honma@nlbc.go.jp (F.H.); k0matsumoto@nlbc.go.jp (K.M.) \\ * Correspondence: masaakit@affrc.go.jp; Tel.: +81(0)29-8388627
}

Received: 25 June 2020; Accepted: 5 August 2020; Published: 7 August 2020

\begin{abstract}
The amount of intramuscular fat (IMF) present in the loin eye area is one of the most important characteristics of high-quality pork. IMF measurements are currently impractical without a labor-intensive process. Metabolomic profiling could be used as an IMF indicator to avoid this process; however, no studies have investigated their use during the fattening period of pigs. This study examined the metabolite profiles in the plasma of two groups of pigs derived from the same Duroc genetic line and fed the same diet. Five plasma samples were collected from each individual the day before slaughter. Capillary electrophoresis-time of flight mass spectrometry (CE-TOFMS) was used to analyze the purified plasma from each sample. Principle component analysis (PCA) and partial least squares (PLS) were used to find the semi-quantitative values of the compounds. The results indicate that branched-chain amino acids are significantly associated with high IMF content, while amino acids are associated with low IMF content. These differences were validated using the quantification analyses by high-performance liquid chromatograph, which supported our results. These results suggest that the concentration of branched-chain amino acids in plasma could be an indicative biomarker for the IMF content in the loin eye area.
\end{abstract}

Keywords: biomarker; CE-TOFMS; intramuscular fat; meat quality; metabolomics; porcine

\section{Introduction}

Pork consumers depend primarily on meat quality when making purchasing decisions. Intramuscular fat (IMF) content in the loin eye muscle, also known as marbling, is a particularly valuable trait of high-quality pork and is associated with the meat's flavor. IMF content may vary by breed types, muscle types, genders, ages, feeding conditions, and final slaughter weight [1-3]. The heritability of IMF content in Duroc breed pigs has been estimated to be moderate to high ( 0.39 to 0.69 ) [4-11]. This has led to improvements in the phenotypic value of livestock in recent decades, despite that IMF is recognized as a polygenic trait $[1,12]$.

Pigs grown on energy restricted diets accumulate significantly more IMF content when their gene expression profiles in relation to protein, glycogen and lipid turnover are altered [13]. Dietary regulation, particularly of lysine, has also resulted high amounts of IMF in pork [14]. A better 
understanding of the molecular and biological mechanisms underlying adipocytes development within muscle tissue could help to create effective nutritional controls during the fattening period of livestock, however few studies have been conducted to date.

Metabolomics are of particular interest in animal production as postmortem aging of meat can be predicted using time course analysis of capillary electrophoresis-time of flight mass spectrometer (CE-TOFMS) metabolomics. Such findings can contribute to the improvement of pork meat quality checks prior to slaughter [15]. More recently, Muroya et al. [16] proposed a novel concept, "MEATabolomics", which combines the muscle biology and meat metabolomics of domestic animals. Additionally, metabolomics data can be defined as intermediate phenotypes, as metabolites are found between the genome level and the external phenotype level, such as growth rate, fat deposition and other economic traits. Metabolomics could lead to "next-generation phenotyping" approaches that improve the prediction of breeding values and selection schemes [17]. Furthermore, Suravajhala et al. [18] reported that high-throughput omics technologies have contributed to our understanding of complex biological phenomena, disease resistance, and holistic production improvements. Hence, metabolomics data may have a potential to improve prediction accuracy of genomic selection and to enhance the impact of breeding schemes for traits related to animal production by incorporating multiple layers of the high-throughput omics approach. In this context, development of non-invasive measurement technologies to obtain the metabolomics data is key for precise evaluation of target traits.

The objective of this study was to analyze and compare metabolite profiles in high and low IMF content pigs, in order to identify metabolites that could be used as IMF content indicators in pigs. To achieve this goal, we used a CE-TOFMS as our primary metabolomics technology due to its highly-sensitive, broad-range detection ability. We used the CE-TOFMS system to screen for candidate metabolites via a semi-quantitative method, then used an absolute quantification method to identify which candidate metabolites are associated with IMF content. We concluded that branched-chain amino acids (BCAA), glycine (Gly), anserine, and carnosine could be used as potential non-invasive biomarkers to estimate IMF content in the loin eye muscle of pigs before slaughter.

\section{Results}

\subsection{Phenotypic Characteristics}

Pigs were separated into low and high IMF groups at two locations of the National Livestock Breeding Center (NLBC), based on IMF (\%) content in the loin eye muscle. The mean proportion of IMF content in pigs at the Miyazaki (MIY) station were $3.1 \%$ and $8.7 \%$, respectively. The mean proportion of IMF content in pigs at the Ibaraki station (IBR) were $2.9 \%$ and $5.4 \%$, respectively (Table 1). The differences were highly significant at both stations. Moisture (\%) measured in the muscle tissues of low IMF pigs were significantly higher than in the high IMF pigs at both stations. At the MIY station, protein $(\%)$ and loin eye area $\left(\mathrm{cm}^{2}\right)$ in low IMF pigs were significantly $8 \%$ and $11 \%$ higher than in high IMF pigs, respectively. At the IBR station, cooking loss (\%) was significantly $15 \%$ higher than those of high IMF pigs. 
Table 1. Comparison of growth performance and carcass characteristics between high and low intramuscular fat (IMF) groups at two stations.

\begin{tabular}{|c|c|c|c|c|c|c|}
\hline \multirow{2}{*}{$\begin{array}{l}\text { Growth Performance and } \\
\text { Carcass Characteristics }\end{array}$} & \multicolumn{3}{|c|}{ Miyazaki (MIY) Station } & \multicolumn{3}{|c|}{ Ibaraki (IBR) Station } \\
\hline & L-IMF MIY & H-IMF MIY & $p^{* 1}$ & L-IMF IBR & H-IMF IBR & $p^{* 1}$ \\
\hline IMF (\%) & $3.101 \pm 0.255$ & $8.699 \pm 1.441$ & $p<0.001$ & $2.868 \pm 0.550$ & $5.447 \pm 0.797$ & $p<0.001$ \\
\hline Days old at slaughter & $149.2 \pm 9.99$ & $152.2 \pm 13.64$ & N.S. & $154.4 \pm 8.357$ & $169.8 \pm 6.997$ & $p<0.05$ \\
\hline Moisture (\%) & $73.96 \pm 0.339$ & $69.98 \pm 0.966$ & $p<0.001$ & $73.81 \pm 0.978$ & $71.48 \pm 0.444$ & $p<0.01$ \\
\hline Crude protein (\%) & $21.82 \pm 0.173$ & $20.23 \pm 0.917$ & $p<0.01$ & $22.35 \pm 0.149$ & $22.02 \pm 0.350$ & N.S. \\
\hline Cooking loss (\%) & $24.68 \pm 1.548$ & $26.52 \pm 1.823$ & N.S. & $28.04 \pm 1.966$ & $24.38 \pm 2.269$ & $p<0.05$ \\
\hline WHC $(\%) * 2$ & $68.30 \pm 5.489$ & $72.30 \pm 4.511$ & N.S. & $68.14 \pm 5.824$ & $68.94 \pm 4.199$ & N.S. \\
\hline WBSF $(\mathrm{kg}) * 3$ & $2.392 \pm 0.338$ & $2.048 \pm 0.489$ & N.S. & $2.963 \pm 0.550$ & $2.838 \pm 0.478$ & N.S. \\
\hline Tenderness $\left(\mathrm{kg} / \mathrm{cm}^{2}\right)$ & $38.09 \pm 5.586$ & $34.81 \pm 2.010$ & N.S. & $46.45 \pm 6.442$ & $44.82 \pm 6.710$ & N.S. \\
\hline Pliability & $1.497 \pm 0.0918$ & $1.375 \pm 0.0609$ & N.S. & $1.527 \pm 0.0442$ & $1.494 \pm 0.0679$ & N.S. \\
\hline Toughness $\left(\mathrm{kg} / \mathrm{cm}^{2} \times \mathrm{cm}\right)$ & $8.163 \pm 1.474$ & $8.054 \pm 1.082$ & N.S. & $10.27 \pm 1.900$ & $10.15 \pm 1.888$ & N.S. \\
\hline Brittleness & $1.631 \pm 0.0750$ & $1.662 \pm 0.0864$ & N.S. & $1.568 \pm 0.0880$ & $1.632 \pm 0.110$ & N.S. \\
\hline Average daily gain (g/day) & $1141.3 \pm 62.70$ & $1035.9 \pm 188.8$ & N.S. & $1006.7 \pm 53.91$ & $1008.8 \pm 46.73$ & N.S. \\
\hline Loin eye area $\left(\mathrm{cm}^{2}\right)$ & $35.06 \pm 1.318$ & $31.45 \pm 2.059$ & $p<0.05$ & $35.10 \pm 3.369$ & $34.29 \pm 3.219$ & N.S. \\
\hline Back fat thickness $(\mathrm{cm})$ & $2.597 \pm 0.547$ & $3.376 \pm 0.548$ & N.S. & $2.044 \pm 0.568$ & $2.476 \pm 0.661$ & N.S. \\
\hline Statue height $(\mathrm{cm})$ & $63.16 \pm 1.895$ & $61.22 \pm 0.722$ & N.S. & $65.48 \pm 1.001$ & $64.16 \pm 2.330$ & N.S. \\
\hline Body length (cm) & $107.6 \pm 3.855$ & $105.28 \pm 2.285$ & N.S. & $89.7 \pm 39.35$ & $106.5 \pm 5.040$ & N.S. \\
\hline
\end{tabular}

${ }^{* 1}$ Statistical significance is defined when $p<0.05$. N.S. denotes no significance $(p>0.05) .{ }^{* 2}$ WHC: Water-holding capacity $(\%)$ by centrifugation at $10,000 \times g .{ }^{* 3}$ WBSF: Warner-Bratzler Shear Force value $(\mathrm{Kg})$ measured after cooling of the carcass.

\subsection{Screening of Differential Metabolomics Profiles with CE-TOFMS}

The total number of metabolites detected in the MIY samples were 201 (144 cations and 57 anions), which were used as the semi-quantitative values (relative area based on the internal standard). Likewise, the IBR metabolites detected were 152 (104 cations and 48 anions). The mean detection rates of metabolites, which are defined as the proportion of metabolites identified among either 201 (MIY) or 152 (IBR), were $82.0 \%$ and $88.6 \%$, respectively. The mean detection rate of metabolites at IBR was greater than at MIY, despite the fact that more metabolites were detected at MIY than IBR.

Principal component analysis (PCA) was carried out based on the semi-quantitative values (relative area) of all detected metabolites. At MIY, the high IMF group tended to be in the PC1 positive and PC2 negative regions, while the low IMF group showed higher variability (Figure S1 PCA (A)). The high IMF group at IBR was found in the PC3 and PC4 positive regions, but high and low IMF groups were neither separated by PC1 nor PC2 (Figure S1 PCA (B and C)).

Partial least squares (PLS) was then used to identify possible relationships between IMF content and metabolite profiles at MIY and IBR (Figure 1. Tables S1 and S2). Since the PLS was performed between two groups (low or high IMF), we only considered PLS1 separation. All high IMF pigs and low IMF pigs were clearly in the positive and negative PLS1 scores. Therefore, we considered that positive loading values were associated with high IMF and negative loading values were associated with low IMF (Table 2). Statistically significant metabolites with positive loading values at MIY were leucine (Leu), O-acetylhomoserine/2-aminoadipic acid, 1-methylnicotinamide, choline, and phosphorylcholine, while all 10 metabolites detected with negative loading values were significant. Statistically significant metabolites with positive loading values at IBR were urea and gluconic acid, while those of the negative loading values were threonine (Thr) and diethanolamine. At both stations a BCAA, valine (Val) was identified from the positive loading metabolites. At MIY, Leu and isoleucine (Ile) were also identified from the positive loading metabolites. However, as we observed in the PCA analysis, BCAAs (Leu, Ile, and Val), tryptophan (Trp) and amino acid metabolites ( $\mathrm{O}$-acetylhomoserine/2-aminoadipic acid, $\mathrm{N}, \mathrm{N}$-dimethylglycine) were also detected in the list of positive PLS1 loading metabolites at MIY. Negative PLS1 loading metabolites at MIY included amino acids (Gly and beta-alanine ( $\beta$-Ala)), amino acid metabolites ( $N$-acetylornithine, $N$-acetyllisyne, 5 -oxoproline, 
N5-ethylglutamine), peptides (anserine-divalent, carnosine) and organic acids (creatine, cis-aconitic acid). At IBR, urea, 3-hydroxybuyric acid, nicotinamide, mucic acid and homocitrulline were commonly observed in the top positive loading metabolites on PCA3 and PCA4, While negative PLS1 loading metabolites consisted of several amino acids (Thr, Asn, Arg, Lys, methionine (Met), and Tyr) and amino acid metabolites (5-hydroxylysine and hydroxyproline).
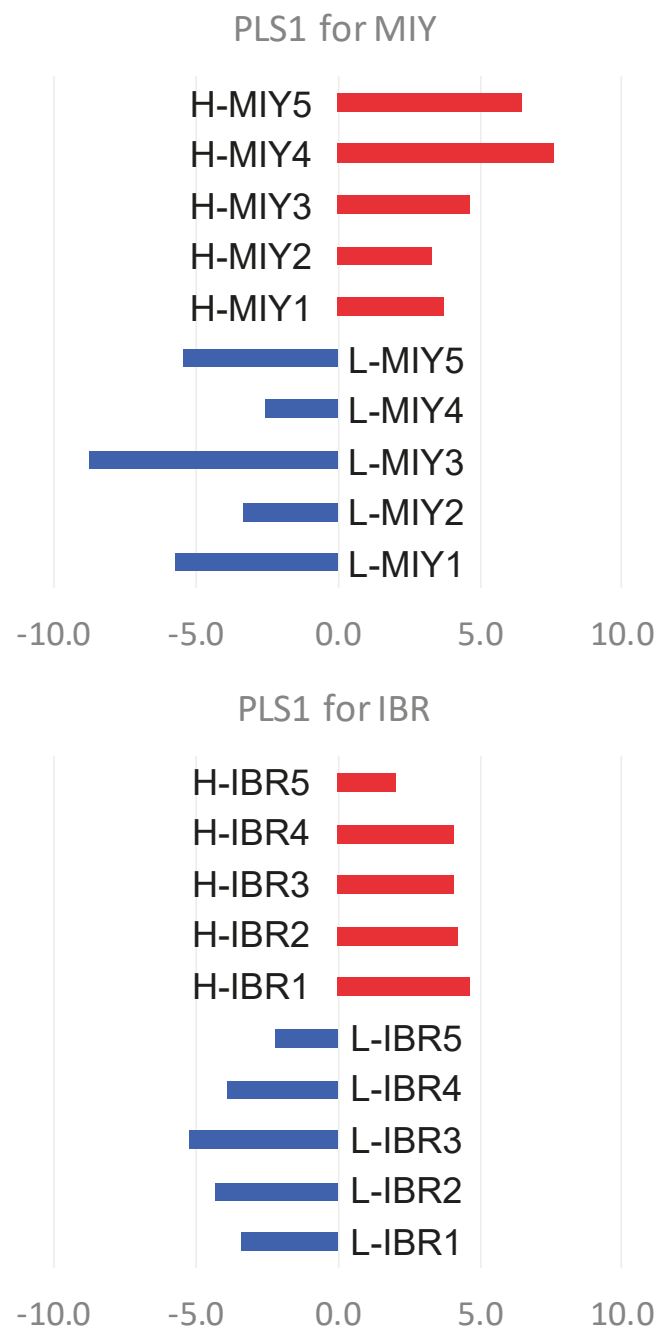

Figure 1. Partial least squares (PLS) analysis by the metabolites detected from pig plasma samples showing the difference in the intramuscular fat contents at the two stations. Blue and red bars indicate low and high IMF pigs, respectively. Top and bottom panes show the results of PLS in MIY and IBR stations, respectively. 
Table 2. Top 10 metabolites with positive and negative high loading values in partial least square at two stations.

\begin{tabular}{|c|c|c|c|c|c|}
\hline \multicolumn{3}{|c|}{ MIY Station } & \multicolumn{3}{|c|}{ IBR Station } \\
\hline \multirow{2}{*}{ Metabolites } & \multicolumn{2}{|c|}{ PLS1 } & \multirow{2}{*}{ Metabolites } & \multicolumn{2}{|c|}{ PLS1 } \\
\hline & $R * 1$ & $p$ & & $R * 1$ & $p$ \\
\hline \multicolumn{6}{|c|}{ Positive loading } \\
\hline Leu & 0.731 & $1.6 \times 10^{-2}$ & Urea & 0.729 & $1.7 \times 10^{-2}$ \\
\hline $\begin{array}{l}\text { O-acetylhomoserine } \\
\text { 2-aminoadipic acid }\end{array}$ & 0.727 & $1.7 \times 10^{-2}$ & Gluconic acid & 0.654 & $4.0 \times 10^{-2}$ \\
\hline 1-Methylnicotinamide & 0.711 & $2.1 \times 10^{-2}$ & 3-hydroxybutyric acid & 0.544 & $1.0 \times 10^{-1}$ \\
\hline Choline & 0.645 & $4.4 \times 10^{-2}$ & Isethionic acid & 0.505 & $1.4 \times 10^{-1}$ \\
\hline Phosphorylcholine & 0.637 & $4.8 \times 10^{-2}$ & Nicotinamide & 0.494 & $1.5 \times 10^{-1}$ \\
\hline Ile & 0.609 & $6.1 \times 10^{-2}$ & Val & 0.493 & $1.5 \times 10^{-1}$ \\
\hline Creatine & 0.603 & $6.5 \times 10^{-2}$ & Taurine & 0.488 & $1.5 \times 10^{-1}$ \\
\hline Val & 0.582 & $7.8 \times 10^{-2}$ & Mucic acid & 0.476 & $1.6 \times 10^{-1}$ \\
\hline$N, N$-dimethylglycine & 0.569 & $8.6 \times 10^{-2}$ & Homocitrulline & 0.475 & $1.7 \times 10^{-1}$ \\
\hline $\operatorname{Trp}$ & 0.470 & $1.7 \times 10^{-1}$ & Sarcosine & 0.452 & $1.9 \times 10^{-1}$ \\
\hline \multicolumn{6}{|c|}{ Negative loading } \\
\hline Gly & -0.861 & $1.4 \times 10^{-3}$ & Thr & -0.716 & $2.0 \times 10^{-2}$ \\
\hline Anserine_divalent & -0.840 & $2.3 \times 10^{-3}$ & Diethanolamine & -0.705 & $2.3 \times 10^{-2}$ \\
\hline$N$-acetylornithine & -0.834 & $2.7 \times 10^{-3}$ & Thymidine & -0.611 & $6.0 \times 10^{-2}$ \\
\hline$N$-acetyllysine & -0.796 & $5.9 \times 10^{-3}$ & 5-hydroxylysine & -0.589 & $7.3 \times 10^{-2}$ \\
\hline 5-oxoproline & -0.753 & $1.2 \times 10^{-2}$ & Asn & -0.566 & $8.8 \times 10^{-2}$ \\
\hline Carnosine & -0.741 & $1.4 \times 10^{-2}$ & Arg & -0.533 & $1.1 \times 10^{-1}$ \\
\hline Creatinine & -0.707 & $2.2 \times 10^{-2}$ & Lys & -0.521 & $1.2 \times 10^{-1}$ \\
\hline$N^{5}$-ethylglutamine & -0.705 & $2.3 \times 10^{-2}$ & Hydroxyproline & -0.514 & $1.3 \times 10^{-1}$ \\
\hline cis-Aconitic acid & -0.685 & $2.9 \times 10^{-2}$ & Met & -0.505 & $1.4 \times 10^{-1}$ \\
\hline$\beta$-Ala & -0.683 & $2.9 \times 10^{-2}$ & Tyr & -0.459 & $1.8 \times 10^{-1}$ \\
\hline
\end{tabular}

$* 1 R$ indicates correlation coefficient between PLS score and each metabolite levels. Since $O$-acetylhomoserine/ 2-aminoadipic acid were detected within the identical single peaks having the consistent $\mathrm{m} / \mathrm{z}$ (molecular mass/electric charge of ions) and MT/RT (migration time/retention time) by the CE-TOFMS system employed in this study, the values in the table indicated the combination of two compounds: The $m / z$ and MT/RT for $O$-acetylhomoserine/2-aminoadipic acid is 162.076 and 12.10 , respectively.

\subsection{Integrated Analysis of Metabolomics Data Using Absolute Quantification}

With the CE-TOFMS metabolomics analysis system, the concentration in micro molar (mM) of 110 metabolites was calculated using a standard curve method. We then focused on the differences in these selectively quantified metabolites between the low and high IMF groups at the two stations. Consequently, the total number of metabolites detected in any of the eight samples from MIY and IBR was $59 \mathrm{mM}$. The mean number of cations detected at MIY was 38.7, and the mean number of anions at MIY was 34.6. At IBR, cations were 13.7, while anions were 11.9. The mean detection ratios of quantified metabolites at MIY were $88.8 \%$ and $78.9 \%$ at IBR. This indicates that there were a greater number of quantified metabolites identified at MIY than IBR. For the 59 quantified metabolites, we analyzed relationships between IMF content and the metabolites.

We used PLS to further determine which metabolites are definitively associated with IMF content. The PLS score plot showed that the low and high IMF groups at both stations were more separated by PLS2 than PLS1 or PLS3 (Figure 2), while the two stations themselves were clearly separated by PLS1 (Figure 2). We then extracted the top five metabolites with both positively and negatively high loading of PLS2 (Table 3, Table S3). Positively loading PLS2 metabolites included the BCAAs Leu, Ile, and Val, and creatine, which were found to be significant metabolites with relatively moderate correlation coefficient (R). Comparison of these BCAAs and creatine concentrations at MIY indicated 
that the high IMF group displayed a significantly higher amount or tended to show greater amounts of the metabolites than those in the low IMF group. Basically, these same metabolites, BCAAs and creatine in the high IMF group at IBR also showed a greater amount than those of low IMF, although the difference was not as large as at MIY.
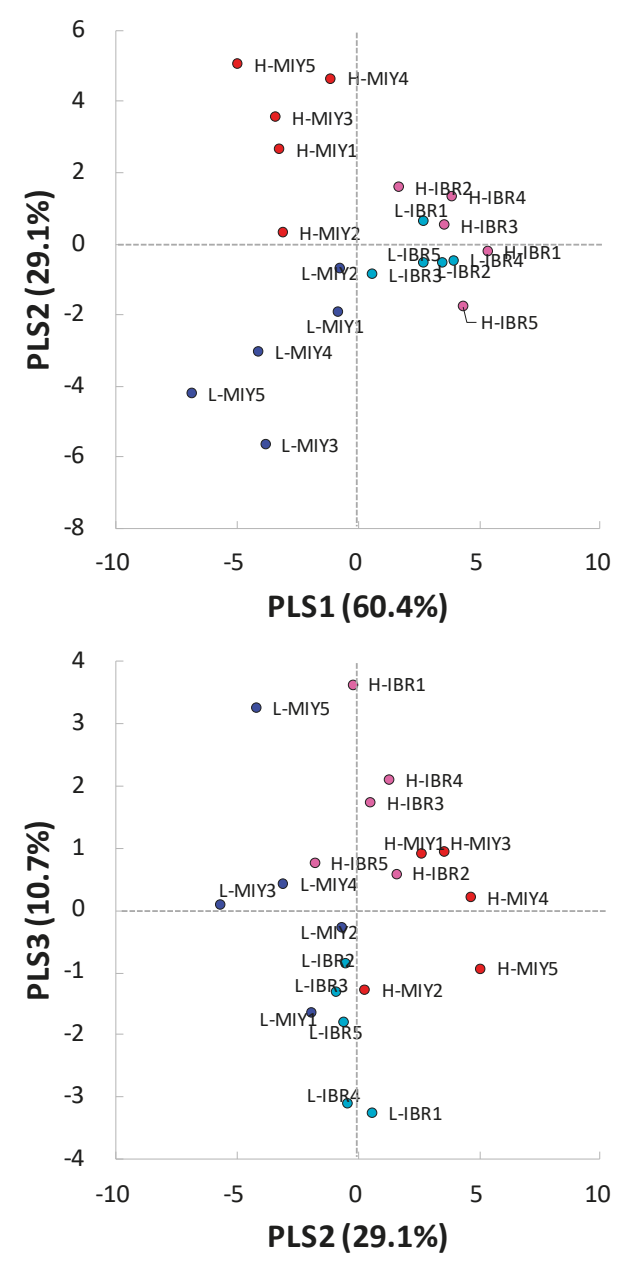

Figure 2. Integrated analyses of partial least squares by the metabolites detected from pig plasma samples showing the difference in the intramuscular fat contents at the two stations. Blue and red dots indicate low and high IMF pigs from MIY station, respectively. Cyan and magenta dots indicate low and high IMF pigs from IBR station, respectively. Top and bottom panes show the results of PLS analyses with PLS1 and 2 and with PLS2 and 3, respectively.

All metabolites from the negatively loading PLS2 metabolites were determined significant with slightly higher correlation coefficients as compared to those in the positively loading metabolites. Similar to the positive loading metabolites, the differences in the negatively loading PLS2 metabolites concentrations between the high and low IMF groups at MIY were clearer and more significant than those at IBR. Consequently, all metabolites identified by PLS2 factor loadings were commonly found in the list of metabolites which were identified with the one-factor PLS analysis at MIY and IBR (Table 2). 


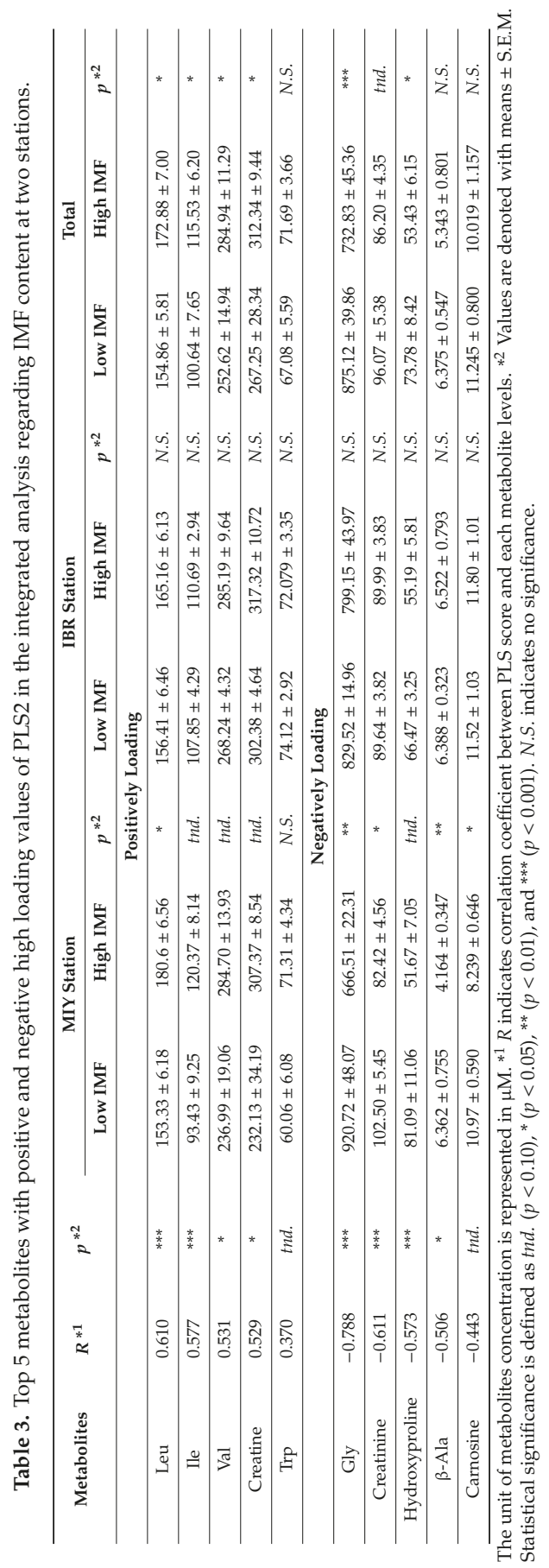




\subsection{Comparison of Amino Acids and Related Metabolites}

Since several amino acids and their related metabolites were identified as the differential metabolites between IMF groups, we next focused our analysis on those metabolites derived from BCAAs such as 3-methyl-2-oxovaleric acid (2K3MVA)/4-methyl-2-oxovaleric acid (2-oxoleucine), 2-oxoisovaleric acid (2-KIV) (Figure 3). The combined amount of Ile and Leu in the high IMF group at MIY was significantly higher than in the low IMF group. The amount of Ile and Leu at IBR, and Val at both stations showed a similar tendency, although the difference was not significant. Likewise, the amount of 2K3MVA/2-Oxoleucine and 2-KIV in the high IMF groups at both stations were greater than in the low IMF groups. There was no significant difference in the total free amino acid contents between the low and high IMF groups. The mean concentrations and standard deviations were $3428 \pm 240(\mu \mathrm{M})$ and $3258 \pm 182(\mu \mathrm{M})$, respectively.
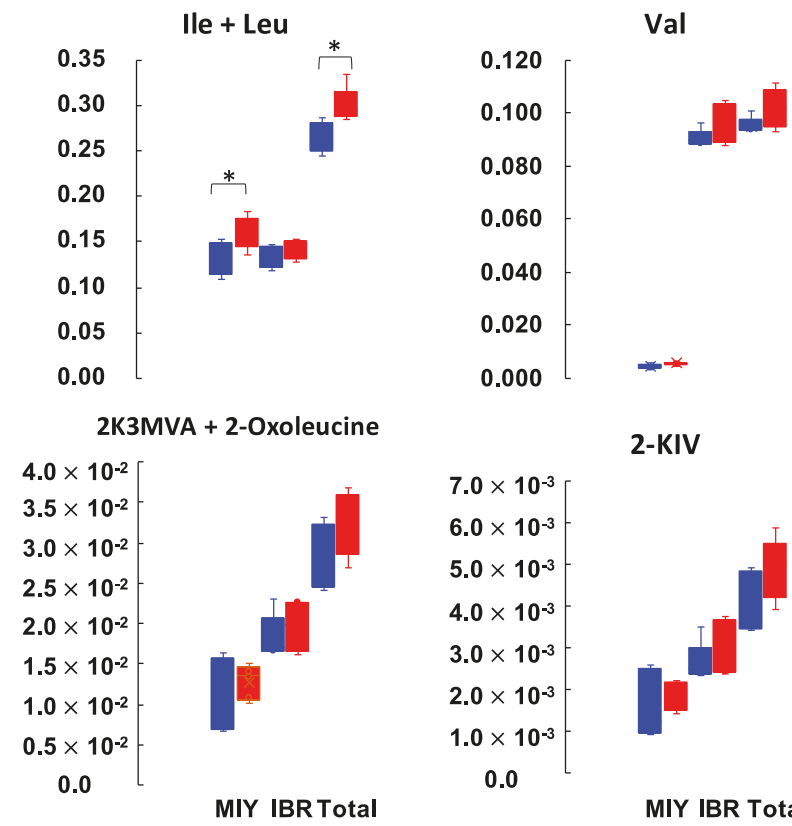

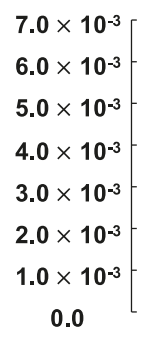

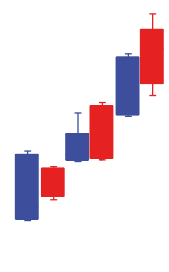

MIY IBR Total

Figure 3. Comparisons of branched-chain amino acids and their degraded metabolites detected from pig plasma samples showing the difference in the intramuscular fat contents at the two stations. Blue and red bars indicate low and high IMF pigs, respectively. Total shows the sum of MIY and IBR. Y-axis indicate the relative area of each metabolites normalized by the internal standard. 3-methyl-2-oxovaleric acid (2K3MVA) and 4-methyl-2-oxovaleric acid (2-oxoleucine), which are the degraded metabolites of Ile and Leu, respectively, could not be separately detected by the capillary electrophoresis-time of flight mass spectrometer (CE-TOFMS). Statistical significance is defined as * $(p<0.05)$.

\subsection{Amino Acid Content Analysis in the Muscle Tissue of Pig Carcass}

Per our CE-TOFMS metabolomics results, we concluded that BCAAs were associated with high IMF content in loin eye muscle. We examined the free amino acid contents of 20 amino acids with taurine, carnosine and anserine (Asn) in the muscle tissues of pig carcasses in order to better understand the mechanism by which high IMF content had either resulted from or was caused by high BCAAs in plasma (Table 4).

At MIY, high IMF pigs had significantly higher concentrations of Asn in loin eye muscle tissue than low IMF pigs, while Gly, carnosine and Asn in high IMF pigs were significantly lower than low 
IMF. The results of Gly and Carnosine matched the results shown in Tables 2 and 3. At IBR, low IMF pigs had greater concentrations of Asp, His, Met, Trp, phenylalanine (Phe), Ile, and Leu in loin eye muscle tissues than those of high IMF pigs. The BCAA contents of loin eye muscles in low IMF pigs were significantly higher than those of high IMF pigs. This result is quite opposite from the results obtained from the plasma metabolomics. As seen in the plasma metabolomics, the total free amino acid content of loin eye muscle tissues displayed no difference between the low and high IMF groups.

\section{Discussion}

This study sought to identify the metabolites associated with IMF content in pork loin eye muscle using CE-TOFMS metabolomics data detected in pig plasma before slaughter. We selected five castrated boars with low IMF content, and five castrated boars with high IMF content in the loin eye muscle from two NLBC stations in Japan (IBR and MIY), for a total of 20 pigs. The difference in IMF content in the high IMF pigs was 2.8 times greater than the low IMF pigs at MIY. At IBR, the difference in IMF content in high IMF pigs was 1.9 times greater than the low IMF pigs. In contrast the moisture content (\%), crude protein content $(\%)$, and loin eye area $\left(\mathrm{cm}^{2}\right)$ in both high IMF groups were either significantly less or tended to be less than those of the low IMF groups. Most of the growth-related traits and the eating-quality traits displayed no differences between the groups. The metabolites differentially identified between the IMF content levels were therefore considered to be primarily associated with IMF content, and secondly with moisture content. Possible partial relationships may have occurred between the candidate metabolites for IMF content, crude protein content, loin eye area or cooking loss (Table 1).

Plasma samples from each pig were profiled using semi-quantification results derived from CE-TOFMS metabolomics. As indicated in the PCA analysis results, large variations were observed, particularly in the low IMF groups at both stations, although the high IMF groups had relatively close PCAs plots. No nutritional controls or genetic factors were used to generate these differences. Since no obvious separation was observed in the IMF content differences from either station, it can also be concluded that none of these factors were involved in samples obtained during this study and it is natural to see such vague separations (Figure S1).

Next, we conducted the PLS analysis. Our results clearly depicted variations in the candidate metabolites in the first PLS1 screening; more specifically, that high IMF groups had positive PLS1 values, while low IMF groups had negative PLS1 values (Figure 1). The metabolites with high positive loading values of PLS1 consistently included BCAAs and amino acid metabolites. This led us to conclude that BCAAs are potential biomarkers for IMF content in pork loin eye muscles. Metabolites with negative loading values of PLS1 included several kinds of amino acids and amino acid metabolites, which suggests that an increased amount of amino acids and amino acid metabolites in plasma may represent lower IMF content (i.e., leaner meat) (Table 2).

We used the quantification results from the CE-TOFMS to validate these findings. We applied the integrated PLS analysis using quantification data from the four groups (Figure 2). Although the PLS1 accounted for $60.4 \%$ of the total IMF variability, it appeared to obviously discriminate station differences, which was not the original purpose of this study. The PLS2 showed the comprehensible separation between the IMF content groups. Even though the number of metabolites quantified in the integrated analysis was 59 out of 201 at MIY and 152 at IBR during the screening analysis, BCAAs were commonly extracted from the list of metabolites with high loading PLS2 values (Table 3).

According to the correlation coefficients estimated in Tables 2 and 3, metabolites with higher $R$ values showed clear differences between the high and low IMF groups. Additionally, metabolite concentration differences between the two IMF groups at MIY were more obvious than those at IBR. Nevertheless, the concentration of metabolites in the high IMF groups was consistently greater at both stations, with the opposite holding true for metabolites with negative loading PLS2 values.

Since BCAAs were indicated to correspond to IMF content, we examined the potential BCAA-related metabolites closer (namely 2K3MVA, 2-oxoleucine, and 2-KIV) (Figure 3). Since 2K3MVA and 2-oxoleucine could not be separately identified by the CE-TOFMS system, additional amounts of 
2K3MVA and 2-oxoleucine and their original BCAAs (Ile and Leu) were applied (Figure 3). The relative amount of metabolites degraded from BCAAs (2K3MVA, 2-oxoleucine, and 2-KIV) indicated a tendency similar to the original BCAAs. Even if the differences were not statistically significant, all of these metabolites follow the patterns of "low in low IMF", and "high in high IMF". One explanation for this may be that the baseline amount of BCAAs in high IMF pig plasma is greater than in low IMF pigs, which means that the amount of metabolized BCAAs might follow the same pattern. If this is the case, then it is quite important to analyze the amino acid content in tissues that metabolize amino acids.

Post slaughter amino acid analysis was conducted on the loin eye muscle tissues (Table 4). Results of this analysis were inconsistent with the metabolomic plasma analysis, that is, the amino acid tissue analysis showed that high IMF pigs at MIY had significantly lower amounts of Gly, Car, and Ans than low IMF pigs. This suggests that the amount of Gly, Car, and Ans (divalent) detected in the plasma of high IMF pigs might be correlated with the intake of those metabolites into muscle tissues. The amino acid analysis at IBR indicated that the amount of Asp and Met in the low IMF group were significantly greater than those in the high IMF group, which matched our plasma screening results. It is interesting that the amount of BCAA in the muscle tissues of the low IMF group were significantly greater than in the high IMF group. Additionally, the BCAA differences at MIY were not as obvious as in the plasma samples (N.S.), although the same trend was observed in both the plasma and the muscle tissues. These results suggest that the amount of BCAA in plasma is positively associated with high IMF content in the loin eye muscle.

Table 4. Analysis of amino acid contents in the loin eye muscle tissues from two stations.

\begin{tabular}{|c|c|c|c|c|c|c|}
\hline \multirow{2}{*}{ Amino Acid $* 1$} & \multicolumn{3}{|c|}{ MIY Station } & \multicolumn{3}{|c|}{ IBR Station } \\
\hline & Low IMF & High IMF & $p^{* 2}$ & Low IMF & High IMF & $p^{* 2}$ \\
\hline Asp & $9.6 \times 10^{-2} \pm 8.1 \times 10^{-3}$ & $9.5 \times 10^{-2} \pm 2.1 \times 10^{-2}$ & N.S. & $8.1 \times 10^{-2} \pm 3.3 \times 10^{-2}$ & $3.2 \times 10^{-2} \pm 1.0 \times 10^{-2}$ & $*$ \\
\hline Glu & $5.5 \times 10^{-1} \pm 1.0 \times 10^{-1}$ & $6.2 \times 10^{-1} \pm 1.2 \times 10^{-1}$ & N.S. & $6.8 \times 10^{-1} \pm 1.5 \times 10^{-1}$ & $5.7 \times 10^{-1} \pm 9.3 \times 10^{-2}$ & N.S. \\
\hline Asn & $1.6 \times 10^{-1} \pm 2.8 \times 10^{-2}$ & $2.3 \times 10^{-1} \pm 5.0 \times 10^{-2}$ & * & $2.6 \times 10^{-1} \pm 6.9 \times 10^{-2}$ & $2.4 \times 10^{-1} \pm 1.7 \times 10^{-2}$ & N.S. \\
\hline Ser & $4.9 \times 10^{-1} \pm 9.1 \times 10^{-2}$ & $4.8 \times 10^{-1} \pm 9.9 \times 10^{-2}$ & N.S. & $5.5 \times 10^{-1} \pm 1.2 \times 10^{-1}$ & $4.5 \times 10^{-1} \pm 5.1 \times 10^{-2}$ & N.S. \\
\hline Gln & $1.658 \pm 0.250$ & $1.490 \pm 0.144$ & N.S. & $1.485 \pm 0.438$ & $9.9 \times 10^{-1} \pm 1.4 \times 10^{-1}$ & tnd. \\
\hline His & $1.7 \times 10^{-1} \pm 2.0 \times 10^{-2}$ & $1.7 \times 10^{-1} \pm 2.6 \times 10^{-2}$ & N.S. & $1.9 \times 10^{-1} \pm 2.3 \times 10^{-2}$ & $1.5 \times 10^{-1} \pm 2.3 \times 10^{-2}$ & * \\
\hline Gly & $1.62 \pm 0.26$ & $1.213 \pm 0.0966$ & * & $1.852 \pm 0.498$ & $1.789 \pm 0.481$ & N.S. \\
\hline Thr & $3.4 \times 10^{-1} \pm 5.9 \times 10^{-2}$ & $3.6 \times 10^{-1} \pm 6.2 \times 10^{-2}$ & N.S. & $3.9 \times 10^{-1} \pm 7.5 \times 10^{-2}$ & $3.3 \times 10^{-1} \pm 3.1 \times 10^{-2}$ & N.S. \\
\hline$\beta$-Ala & $6.7 \times 10^{-1} \pm 2.3 \times 10^{-1}$ & $4.4 \times 10^{-1} \pm 1.0 \times 10^{-1}$ & N.S. & $5.1 \times 10^{-1} \pm 8.3 \times 10^{-2}$ & $5.6 \times 10^{-1} \pm 1.6 \times 10^{-1}$ & N.S. \\
\hline Arg & $3.6 \times 10^{-1} \pm 5.9 \times 10^{-2}$ & $3.5 \times 10^{-1} \pm 6.6 \times 10^{-2}$ & N.S. & $4.0 \times 10^{-1} \pm 8.3 \times 10^{-2}$ & $2.9 \times 10^{-1} \pm 3.5 \times 10^{-2}$ & thd. \\
\hline Ala & $2.143 \pm 0.325$ & $2.329 \pm 0.397$ & N.S. & $2.643 \pm 0.472$ & $2.170 \pm 0.293$ & N.S. \\
\hline Tau & $2.341 \pm 0.441$ & $2.16 \pm 0.289$ & N.S. & $2.404 \pm 0.772$ & $1.829 \pm 0.920$ & N.S. \\
\hline Car & $26.41 \pm 1.56$ & $20.99 \pm 2.35$ & *** & $28.95 \pm 1.65$ & $27.60 \pm 2.20$ & N.S. \\
\hline Ans & $8.5 \times 10^{-1} \pm 6.0 \times 10^{-2}$ & $6.6 \times 10^{-1} \pm 8.4 \times 10^{-2}$ & ** & $7.1 \times 10^{-1} \pm 5.7 \times 10^{-2}$ & $7.3 \times 10^{-1} \pm 1.2 \times 10^{-1}$ & N.S. \\
\hline Tyr & $2.8 \times 10^{-1} \pm 4.7 \times 10^{-2}$ & $2.7 \times 10^{-1} \pm 4.8 \times 10^{-2}$ & N.S. & $3.0 \times 10^{-1} \pm 5.8 \times 10^{-2}$ & $2.5 \times 10^{-1} \pm 2.3 \times 10^{-2}$ & N.S. \\
\hline Val & $3.9 \times 10^{-1} \pm 6.1 \times 10^{-2}$ & $4.8 \times 10^{-1} \pm 5.7 \times 10^{-2}$ & tnd. & $5.3 \times 10^{-1} \pm 8.0 \times 10^{-2}$ & $4.4 \times 10^{-1} \pm 5.6 \times 10^{-2}$ & N.S. \\
\hline Met & $2.5 \times 10^{-1} \pm 4.1 \times 10^{-2}$ & $2.7 \times 10^{-1} \pm 4.6 \times 10^{-2}$ & N.S. & $3.0 \times 10^{-1} \pm 3.4 \times 10^{-2}$ & $2.3 \times 10^{-1} \pm 2.1 \times 10^{-2}$ & $* *$ \\
\hline $\operatorname{Trp}$ & $8.8 \times 10^{-2} \pm 7.8 \times 10^{-3}$ & $9.9 \times 10^{-2} \pm 5.9 \times 10^{-3}$ & tnd. & $1.2 \times 10^{-1} \pm 9.8 \times 10^{-3}$ & $1.0 \times 10^{-1} \pm 5.2 \times 10^{-3}$ & $* *$ \\
\hline Phe & $3.3 \times 10^{-1} \pm 4.4 \times 10^{-2}$ & $3.5 \times 10^{-1} \pm 4.1 \times 10^{-2}$ & N.S. & $3.9 \times 10^{-1} \pm 4.0 \times 10^{-2}$ & $3.4 \times 10^{-1} \pm 1.2 \times 10^{-2}$ & $*$ \\
\hline Ile & $3.1 \times 10^{-1} \pm 5.7 \times 10^{-2}$ & $3.4 \times 10^{-1} \pm 5.1 \times 10^{-2}$ & N.S. & $3.8 \times 10^{-1} \pm 4.9 \times 10^{-2}$ & $3.1 \times 10^{-1} \pm 2.5 \times 10^{-2}$ & * \\
\hline Leu & $7.0 \times 10^{-1} \pm 1.2 \times 10^{-1}$ & $6.3 \times 10^{-1} \pm 1.0 \times 10^{-2}$ & N.S. & $7.3 \times 10^{-1} \pm 1.1 \times 10^{-1}$ & $5.5 \times 10^{-1} \pm 6.2 \times 10^{-2}$ & $*$ \\
\hline Lys & $4.4 \times 10^{-1} \pm 8.1 \times 10^{-2}$ & $4.2 \times 10^{-1} \pm 8.1 \times 10^{-2}$ & N.S. & $4.5 \times 10^{-1} \pm 8.1 \times 10^{-2}$ & $3.5 \times 10^{-1} \pm 3.5 \times 10^{-2}$ & tnd. \\
\hline Hyp & $3.142 \pm 0.253$ & $2.897 \pm 1.630$ & N.S. & $2.099 \pm 0.198$ & $2.054 \pm 0.0751$ & N.S. \\
\hline Pro & $9.6 \times 10^{-1} \pm 8.9 \times 10^{-2}$ & $2.790 \pm 3.31$ & N.S. & $5.4 \times 10^{-1} \pm 1.04 \times 10^{-1}$ & $3.7 \times 10^{-1} \pm 1.3 \times 10^{-1}$ & tnd. \\
\hline $\mathrm{FAA}^{* 3}$ & $14.42 \pm 0.732$ & $15.67 \pm 3.60$ & N.S. & $15.26 \pm 2.76$ & $12.40 \pm 1.87$ & N.S. \\
\hline
\end{tabular}

The unit of amino acid concentration is represented in $\mu \mathrm{mol} / \mathrm{g} .{ }^{* 1}$ This list includes analysis result of amino acids together with Taurine, Carnosine and Anserine. ${ }^{* 2}$ Statistical significance is defined as $t n d .(p<0.10),{ }^{*}(p<0.05)$, $* *(p<0.01)$. N.S. indicates no significance. ${ }^{* 3}$ FAA denotes total amount of free amino acids.

Recently, it was demonstrated that surplus dietary Ile, one of BCAAs, increased the IMF content in skeletal muscle via the upregulation of lipogenic genes by stimulating lipogenesis in skeletal muscle tissue of finishing pigs [19]. In the previous study, Luo et al. clearly showed the effect of extra Ile in the 
diet on lipogenesis in the muscle tissue, serum cholesterol levels, and fatty acid composition without affecting growth performance. However, it is not possible to further discuss whether Ile (BCAA) intake into the muscle cells affected lipid synthesis due to the lack of Ile level in muscle tissue [19]. In contrast, it has been well known that BCAA are effective amino acids to regulate protein synthesis because of a greater increase in protein synthesis than degradation [20-22].

Therefore, one possible explanation for this is that the availability of BCAA and/or the intake of BCAA in the muscle tissue of low IMF pigs are more effective than in high IMF pigs, which results in the pig building more muscle mass. It is possible, therefore, that the availability of BCAA in the muscle tissue of high IMF pigs is relatively low, which would mean that some kind of molecular mechanism(s) could be triggered that could enhance the preadipocytes near the muscle cells.

The Duroc pigs used for this study were derived from the one previously established to improve IMF content at NLBC. The heritability of IMF in the Duroc genetic line established at NLBC has been previously determined to be 0.52 [23]. Although there were certain differences in the metabolite profiles between MIY and IBR by the PLS1 (Figure 2), these observed differences may be due to environmental factors only, since the genetic Duroc line and the feeding regimes were nearly identical at each location.

Previously, Katsumata et al. [14] reported that a low lysine diet fed to finishing pigs increased IMF content, although the authors recognized that the influence of restrictive amino acid nutrition on IMF content needs to be researched further. Restricted amino acid diets may increase stress sensitivity in pigs, since it has been indicated to do so in other animals [14,24,25]. In the case of beef marbling improvements, vitamin A restriction in the diet of beef steers increased IMF content due to hyperplasia of the adipocytes, although subcutaneous fat depth was not affected [26,27]. This method of feeding beef cattle diets with low vitamin A is well-known and used worldwide. In addition to the vitamin A restriction, it is suggested that a genotype of alcohol dehydrogenase 1C (ADH1C) has an interactive effect with vitamin A on IMF content, because of the differential transcriptional regulation that potentially occurs in this genotype [28]. It is plausible that nutritional control combined with certain genetic backgrounds may increase or decrease the IMF content in meat animals. However, there is still an inevitable phenotypic variation in IMF content, as is the nature of quantitative traits. Therefore, a non-invasive method, such as plasma metabolomics by CE-TOFMS, could be of benefit to determine the physiological status of meat producing animals, since it can measure a broader range of compounds than other metabolomics systems.

In conclusion, in this study we found that pigs with high IMF content in the loin eye muscle also had an increased amount of BCAA in their blood plasma. We also found that pigs with low IMF content in the loin eye muscle had an increased amount of Gly, Ans, and Car in their blood plasma. We suggest, therefore, that BCAA, Gly, Ans, and Car are potential biomarkers that could be used to estimate IMF in the loin eye muscle before slaughter. Further study of these biomarkers is required, since their amounts were not always consistently demonstrated in this study.

\section{Materials and Methods}

\subsection{Animals}

This study used castrated boars from the Duroc breed, which has been genetically bred for high IMF content as reported in the previous study [23]. All pigs used in this study were fed identical diets based on the Japan Nutrition Standards (NILGS 2016) and were raised at two locations of the NLBC (IBR and MIY) in Japan.

At each location, five high IMF content pigs and five low IMF content pigs were chosen from among the herd, for a total of 20 animals. Other than IMF content, growth performance and carcass characteristics of pigs were compared (Table 1). Aside from the fact that low IMF pigs took longer to gain slaughter weight, there were no significant phenotypic variables between any of the four IMF groups. 
Blood samples were collected in a vacuum blood collection tube with EDTA/2Na from the jugular vein of each pig after fasting overnight. After blood samples were collected, the pigs were slaughtered at the abattoir located at each station. The blood collection tube was kept on ice and centrifuged with $3000 \times g$ at $4{ }^{\circ} \mathrm{C}$ for $10 \mathrm{~min}$. Plasma was removed from the blood collection tube and placed into a new sample tube, then snap frozen, and stored in $-80^{\circ} \mathrm{C}$ freezer until use. All procedures involving animals were performed in accordance with the National Livestock Breeding Center's guidelines for care and use of laboratory animals.

\subsection{Semi-Quantitative Metabolomics (Basic Scan) by Capillary Electrophoresis-Time of Flight Mass Spectrometry (CE-TOFMS)}

Preprocessing was initiated by adding $50 \mu \mathrm{L}$ pig plasma to $450 \mu \mathrm{L}$ methanol containing $50 \mu \mathrm{M}$ Internal Standard Solution 1 (H3304-1002, Human Metabolome Technologies (HMT), Tsuruoka, Yamagata, Japan) followed by mixing. To the mixture, $500 \mu \mathrm{L}$ chloroform and $200 \mu \mathrm{L}$ ultrapure water were added and then mixed. After centrifugation at $2300 \times g$, in $4{ }^{\circ} \mathrm{C}$ for $5 \mathrm{~min}, 400 \mu \mathrm{L}$ supernatant of the mixture was removed to ultrafiltration using UltraFree MC PLHCC (HMT) and centrifuged at $9100 \times \mathrm{g}$ at $4{ }^{\circ} \mathrm{C}$ for $120 \mathrm{~min}$. The filtrate was once frozen-dried and dissolved in $50 \mu \mathrm{L}$ ultrapure water just before applying to Agilent CE-TOFMS system (Agilent Technologies, Santa Clara, CA, USA). Metabolome analysis was performed by Basic Scan package of HMT using the CE-TOFMS based on the methods described previously [29,30]. Briefly, CE-TOFMS analysis was carried out using an Agilent CE capillary electrophoresis system equipped with an Agilent 6210 time-of-flight mass spectrometer (Agilent Technologies, Santa Clara, CA, USA). CE-TOFMS was operated using Agilent G2201AA ChemStation software version B.03.01 (Agilent Technologies, Santa Clara, CA, USA), compounds were detected using the cation or anion modes. Briefly, the compounds were electrophoresed through fused silica capillaries ( $50 \mu \mathrm{M} \times 80 \mathrm{~cm}$, Agilent Technologies, Santa Clara, CA, USA) with $30 \mathrm{kV}$ under 50 mbar and $10 \mathrm{~s}$ pressure injections. The electrophoresed compounds were ionized with either ESI Positive or Negative MS ionization. The scan range for the compounds was between 50 and 1000 $\mathrm{m} / \mathrm{z}$. Detected peaks from the CE-TOFMS system were processed by MasterHands ver2.17.1.11 [31]. Signal peaks corresponding to isotopomers, adduct ions, and other product ions of known metabolites were excluded, and remaining peaks were annotated according to the HMT metabolite database library, which includes 900 metabolites, based on their $\mathrm{m} / \mathrm{z}$ values with the MTs determined by TOFMS. Areas of the annotated peaks were then normalized based on internal standard levels and sample amounts to obtain relative levels of each metabolite.

\subsection{Absolute Quantification of 110 Target Metabolites for CE-TOFMS Analysis}

Based on the same preprocessing and the analysis system, primary 110 metabolites involved in the pathways of glycolytic/gluconeogenesis, TCA cycle, pentose phosphate, lipid metabolites, and nucleic acid metabolites were absolutely quantified based on one-point calibrations using their respective standard compounds.

\subsection{Measurement of Free Amino Acids, Peptides, and Carcass Traits in Muscle Tissues}

The procedure used to analyze the free amino acids and peptides was carried out using the exact same method described in [32]. Briefly, $0.10 \mathrm{~g}$ samples of loin eye muscle tissues were drawn from minced raw meat (about $15 \mathrm{~g}$ in total). Samples were homogenized with $4.24 \mathrm{~mL}$ of ultrapure water, $4 \mathrm{~mL}$ of $\mathrm{N}$-hexane and $0.16 \mathrm{~mL}$ of internal standard solution mixed with norvaline $(5 \mathrm{nmol} / \mu \mathrm{L})$ in ultrapure water, and then centrifuged at $1750 \times g$ for $5 \mathrm{~min}$. The underlayer was mixed with $4 \mathrm{~mL}$ of $\mathrm{N}$-hexane and centrifuged at $1750 \times \mathrm{g}$ for $5 \mathrm{~min}$. The resultant underlayer was then mixed with $3.6 \mathrm{~mL}$ of acetonitrile and centrifuged at $1750 \times \mathrm{g}$ for $10 \mathrm{~min}$. The resulting supernatant was filtered through a $0.45-\mu \mathrm{m}$ microfilter (Millex-LH; Merck Millipore, Billerica, MA, USA), and the filtrate was then mixed with $45 \%$ acetonitrile solution and analyzed for free amino acids and peptides using an Agilent 1260 infinity high performance liquid chromatograph equipped with an Agilent 1260 Binary 
Pump, 1260 HiP Degasser, 1260 HiP ALS autosampler, 1290 thermostat, 1260 Thermostatted column compartment control module, 1260 diode array detector and a Poroshell 120 EC-C18 column $(3.0 \times 100$ $\mathrm{mm}, 2.7 \mu \mathrm{m}$; Agilent). The eluents used were: (i) $20 \mathrm{mmol} / \mathrm{L}$ disodium hydrogen phosphate (pH 7.6); and (ii) acetonitrile/methanol/water $(5: 5: 1, v / v / v)$. Amino acids and peptides were identified through the comparison of their retention times with those of established standards. The concentrations of each were calculated using internal and external standard solutions and expressed as $\mu \mathrm{mol}$ per $\mathrm{g}$ of meat and $\mu$ mol per $1 \%$ of moisture in meat, respectively. The internal standard solutions were used to account for matter lost during analysis, and the external standard solutions $(1,10,50$, and $100 \mathrm{pmol} / \mu \mathrm{L})$ were used to plot a calibration curve for each amino acid and peptide. Carcass traits were analyzed according to the methods described previously [32,33]. Moisture content was determined in duplicates by drying $2 \mathrm{~g}$ samples of meat drawn from the minced raw meat (approximately $30 \mathrm{~g}$ in total) for $24 \mathrm{~h}$ at $105^{\circ} \mathrm{C}$. IMF content was determined by Soxhlet extraction of the dried samples with diethyl ether for $16 \mathrm{~h}$. Crude protein content was determined using $1 \mathrm{~g}$ samples of meat drawn from the minced raw meat (approximately $30 \mathrm{~g}$ in total) by the Kjeldahl method using a nitrogen distillation titration device (2400 Kjeltec auto sampler system, FOSS, Hillerod, Denmark). Cooking loss was determined by the weight difference of meat samples (approximately $50 \mathrm{~g}$ ) before and after heating at $70^{\circ} \mathrm{C}$ for $1 \mathrm{~h}$ in a water bath. After cooking loss measurement, the meat sample was then used for WBSF measurement with crosshead speed of $200 \mathrm{~mm} / \mathrm{min}$ using Instron (model 5542; Instron, University Avenue Norwood, MA, USA). WHC was determined using meat samples (approximately $0.5 \mathrm{~g}$ ) drawn from the minced raw meat which were centrifuged by $10,000 \times g$ for $30 \mathrm{~min}$. Determination of tenderness, pliability, toughness, and brittleness were performed using meat samples prepared for the same as the WBSF using Tensipresser (model TTP-50BX II, Takemoto Electric Inc., Tokyo, Japan).

\subsection{Data Analysis}

Metabolomics data acquired by the CE-TOFMS system were normalized and the semi-quantitative and absolute quantification data were then analyzed by PCA and PLS [34] using MATLAB (The MathWorks, Natick, MA, USA) and R programs [35] developed by HMT Co. Ltd. Differences between high and low IMF groups were examined by Welch's $t$-test. Significant difference was defined by $p<0.05$.

Supplementary Materials: The following are available online at http://www.mdpi.com/2218-1989/10/8/322/s1, Figure S1. Principal component analysis by the metabolites detected from pig plasma samples showing the difference in the intramuscular fat contents at the two stations. Blue and red dots indicate low and high IMF pigs, respectively. (A) The PCA performed with PC1 and PC2 for pigs from MIY station; (B) The PCA performed with PC1 and PC2 for pigs from IBR station; (C) The PCA performed with PC3 and PC4 for pigs from IBR station.

Author Contributions: Conceptualization, M.T. and K.I.; Methodology, M.T., C.O., K.K. (Kouen Kadowaki), K.K. (Kimiko Kohira), F.H., and K.M.; Validation, M.T., A.A., M.N. and T.O.; Formal Analysis, M.T. and A.A.; Investigation, M.T.; Resources, C.O. and K.K. (Kouen Kadowaki); Data Curation, A.A., M.N. and T.O.; Writing-Original Draft Preparation, M.T.; Writing—Review \& Editing, M.T., A.A., M.N., T.O., C.O., K.K. (Kouen Kadowaki), K.K. (Kimiko Kohira), F.H., K.M., and K.I.; Visualization, M.T.; Supervision, M.T.; Project Administration, K.I.; Funding Acquisition, K.I. All authors have read and agreed to the published version of the manuscript.

Funding: This research was supported by a grant from the Project of the Bio-oriented Technology Research Advancement Institution, NARO (the special scheme project on advanced research and development for next-generation technology).

Acknowledgments: We thank Shozo Tomonaga for his constructive comments.

Conflicts of Interest: The authors declare no conflict of interest. The funders had no role in the design of the study; in the collection, analyses, or interpretation of data; in the writing of the manuscript, or in the decision to publish the results.

\section{References}

1. Hocquette, J.F.; Gondret, F.; Baéza, E.; Médale, F.; Jurie, C.; Pethick, D.W. Intramuscular fat content in meat-producing animals: Development, genetic and nutritional control, and identification of putative markers. Animal 2010, 4, 303-319. [CrossRef] [PubMed] 
2. Apple, J.K. Nutritional effects on pork quality in swine production. In National Swine Nutrition Guide; Factsheet No. PIG 12-02-02; U.S. Pork Center of Excellence: Clive, IA, USA, 2015.

3. Schwab, C.R.; Baas, T.J.; Stalder, K.J. Results from six generations of selection for intramuscular fat in Duroc swine using real-time ultrasound. II. Genetic parameters and trends. J. Anim. Sci. 2010, 88, 69-79. [CrossRef] [PubMed]

4. Fernández, A.; de Pedro, E.; Núñez, N.; Silió, L.; García-Casco, J.; Rodríguez, C. Genetic parameters for meat and fat quality and carcass composition traits in Iberian pigs. Meat Sci. 2003, 64, 405-410. [CrossRef]

5. Won, S.; Jung, J.; Park, E.; Kim, H. Identification of genes related to intramuscular fat content of pigs using genome-wide association study. Asian Aust. J. Anim. Sci. 2018, 31, 157-162. [CrossRef]

6. Davoli, R.; Catillo, G.; Serra, A.; Zappaterra, M.; Zambonelli, P.; Meo Zilio, D.; Steri, R.; Mele, M.; Buttazzoni, L.; Russo, V. Genetic parameters of backfat fatty acids and carcass traits in Large White pigs. Animal 2019, 13, 924-932. [CrossRef]

7. Hovenier, R.; Kanis, E.; van Asseldonk, T.H.; Westerink, N.G. Breeding for pig meat quality in halothane negative populations-A review. Pig News Inf. 1993, 14, 17N-25N.

8. Sellier, P. Genetics of meat and carcass traits. In Genetics of the Pig; Rothchild, M.F., Ruvinsky, A., Eds.; CAB International: Wallingford, Oxon, UK, 1998; pp. 463-510.

9. Newcom, D.W.; Baas, T.J.; Schwab, C.R.; Stalder, K.J. Genetic and phenotypic relationships between individual subcutaneous backfat layers and percentage of longissimus intramuscular fat in Duroc swine. J. Anim. Sci. 2005, 83, 316-323. [CrossRef]

10. Suzuki, K.; Irie, M.; Kadowaki, H.; Shibata, T.; Kumagai, M.; Nishida, A. Genetic parameter estimates of meat quality traits in Duroc pigs selected for average daily gain, longissimus muscle area, backfat thickness, and intramuscular fat content. J. Anim. Sci. 2005, 83, 2058-2065. [CrossRef]

11. Solanes, F.X.; Reixach, J.; Tor, M.; Tibau, J.; Estany, J. Genetic correlations and expected response for intramuscular fat content in a Duroc pig line. Livest. Prod. Sci. 2009, 123, 63-69. [CrossRef]

12. Cesar, A.S.M.; Regitano, L.C.A.; Koltes, J.E.; Fritz-Waters, E.R.; Lanna, D.P.D.; Gasparin, G.; Mourao, G.B.; Oliveira, P.S.N.; Reecy, J.M.; Coutinho, L.L. Putative Regulatory Factors Associated with Intramuscular Fat Content. PLoS ONE 2015, 10, e0128350. [CrossRef]

13. Da Costa, N.; McGillivray, C.; Bai, Q.; Wood, J.D.; Evans, G.; Chang, K.C. Restriction of dietary energy and protein induces molecular changes in young porcine skeletal muscles. J. Nutr. 2004, 134, 2191-2199. [CrossRef] [PubMed]

14. Katsumata, M. Promotion of intramuscular fat accumulation in porcine muscle by nutritional regulation. Anim. Sci. J. 2011, 82, 17-25. [CrossRef] [PubMed]

15. Muroya, S.; Oe, M.; Nakajima, I.; Ojima, K.; Chikuni, K. CE-TOF MS-based metabolomic profiling revealed characteristic metabolic pathways in postmortem porcine fast and slow type muscles. Meat Sci. 2014, 98, 726-735. [CrossRef] [PubMed]

16. Muroya, S.; Ueda, S.; Komatsu, T.; Miyakawa, T.; Ertbjerg, P. MEATabolomics: Muscle and meat metabolomics in domestic animals. Metabolites 2020, 10, 188. [CrossRef]

17. Fontanesi, L. Metabolomics and livestock genomics: Insights into a phenotyping frontier andits applications in animal breeding. Anim. Front. 2016, 6, 73-79. [CrossRef]

18. Suravajhala, P.; Kogelman, L.J.A.; Kadarmideen, H.N. Multi-omic data integration and analysis using systems genomics approaches: Methods and applications in animal production, health and welfare. Genet. Sel. Evol. 2016, 48, 38. [CrossRef]

19. Luo, Y.; Zhang, X.; Zhu, Z.; Jiao, N.; Qiu, K.; Yin, J. Surplus dietary isoleucine intake enhanced monounsaturated fatty acid synthesis and fat accumulation in skeletal muscle of finishing pigs. J. Anim. Sci. Biotech. 2018, 9, 88. [CrossRef]

20. Zhang, S.; Chu, L.; Qiao, S.; Mao, X.; Zeng, X. Effects of dietary leucine supplementation in low crude protein diets on performance, nitrogen balance, whole-body protein turnover, carcass characteristics and meat quality of finishing pigs. Anim. Sci. J. 2016, 87, 911-920. [CrossRef]

21. Zheng, L.; Wei, H.; He, P.; Zhao, S.; Xiang, Q.; Pang, J.; Peng, J. Effects of supplementation of branched-chain amino acids to reduced-protein diet on skeletal muscle protein synthesis and degradation in the fed and fasted states in a piglet model. Nutrients 2017, 9, 17. [CrossRef]

22. Wang, Y.; Zhou, J.; Wang, G.; Cai, S.; Zeng, X.; Qiao, S. Advances in low-protein diets for swine. J. Anim. Sci. Biotech. 2018, 9, 60. [CrossRef] 
23. Ishii, K.; Arata, S.; Ohnishi, C. Estimates of genetic parameters for meat quality and carcass traits in Duroc pigs. In Proceedings of the World Congress on Genetics Applied to Livestock Production, Auckland, New Zealand, 8 February 2018. Abstract Number 408.

24. Smriga, M.; Kameishi, M.; Uneyama, H.; Torii, K. DietaryL-lysine deficiency increases stress-induced anxiety and fecal excretion in rats. J. Nutr. 2002, 132, 3744-3746. [CrossRef] [PubMed]

25. Srinongkote, S.; Smrige, M.; Toride, Y. Diet supplied with L-lysine and L-arginine during chronic stress of high stock density normalizes growth of broilers. Anim. Sci. J. 2004, 75, 339-343. [CrossRef]

26. Harris, C.L.; Wang, B.; Deavila, J.M.; Bushboom, J.R.; Maquivar, M.; Parish, S.M.; McCann, B.; Nelson, M.L.; $\mathrm{Du}, \mathrm{M}$. Vitamin A administration at birth promotes calf growth and intramuscular fat development in Angus beef cattle. J. Anim. Sci. Biotechnol. 2018, 9, 55. [CrossRef] [PubMed]

27. Kruk, Z.A.; Bottema, M.J.; Reyes-Veliz, L.; Forder, R.E.A.; Pitchford, W.S.; Bottema, C.D.K. Vitamin A and Marbling Attributes: Intramuscular Fat Hyperplasia Effects in Cattle. Meat Sci. 2018, 137, 139-146. [CrossRef]

28. Ward, A.K.; McKinnon, J.J.; Hendrick, S.; Buchanan, F.C. The impact of vitamin A restriction and ADH1C genotype on marbling in feedlot steers. J. Anim. Sci. 2015, 90, 2476-2483. [CrossRef]

29. Ohashi, Y.; Hirayama, A.; Ishikawa, T.; Nakamura, S.; Shimizu, K.; Soga, T. Depiction of metabolome changes in histidine-starved Escherichia coli by CE-TOFMS. Mol. Biosyst. 2008, 4, 135-147. [CrossRef]

30. Ooga, T.; Sato, H.; Nagashima, A.; Sasaki, K.; Tomita, M.; Soga, T.; Ohashi, Y. Metabolomic anatomy of an animal model revealing homeostatic imbalances in dyslipidaemia. Mol. Biosyst. 2011, 7, 1217-1223. [CrossRef]

31. Sugimoto, M.; Goto, H.; Otomo, K.; Ito, M.; Onuma, H.; Suzuki, A.; Sugawara, M.; Abe, S.; Tomita, M.; Soga, T. Metabolomic profiles and sensory attributes of edamame under various storage duration and temperature conditions. J. Agric. Food Chem. 2010, 58, 8418-8425. [CrossRef]

32. Sakuma, H.; Saito, K.; Kohira, K.; Ohhashi, F.; Shoji, N.; Uemoto, Y. Estimates of genetic parameters for chemical traits of meat quality in Japanese black cattle. Anim. Sci. J. 2017, 88, 203-212. [CrossRef]

33. Okumura, T.; Saito, K.; Sakuma, H.; Nade, T.; Nakayama, S.; Fujita, K.; Kawamura, T. Intramuscular fat deposition in principal muscles from twenty-four to thirty months of age using identical twins of Japanese Black steers. J. Anim. Sci. 2007, 85, 1902-1907. [CrossRef]

34. Yamamoto, H. PLS-ROG: Partial least squares with rank order of groups. J. Chemom. 2017, 33 , e2883. [CrossRef]

35. R Core Team. R: A Language and Environment for Statistical Computing; R Foundation for Statistical Computing: Vienna, Austria, 2019; Available online: https://www.R-project.org/ (accessed on 29 May 2020).

(C) 2020 by the authors. Licensee MDPI, Basel, Switzerland. This article is an open access article distributed under the terms and conditions of the Creative Commons Attribution (CC BY) license (http://creativecommons.org/licenses/by/4.0/). 



\title{
Effect of Gender, Rearing, and Cooking on the Metabolomic Profile of Porcine Muscles
}

\author{
Shoko Sawano ${ }^{1,2}$, Keishi Oza ${ }^{2}$, Tetsuya Murakami ${ }^{3}$, Mako Nakamura ${ }^{2}$, Ryuichi Tatsumi ${ }^{2}$ and \\ Wataru Mizunoya ${ }^{2,4, *}$ \\ 1 Department of Food and Life Science, School of Life and Environmental Science, Azabu University, \\ Sagamihara 252-5201, Japan \\ 2 Department of Bioresource Sciences, Faculty of Agriculture, Kyushu University, Fukuoka 819-0395, Japan \\ 3 Fukuoka Agriculture and Forestry Research Center, Chikushino 818-0004, Japan \\ 4 Department of Animal Science and Biotechnology, School of Veterinary Medicine, Azabu University, \\ Sagamihara 252-5201, Japan \\ * Correspondence: mizunoya.wataru.k91@kyoto-u.jp; Tel.: +81-42-769-1704
}

Received: 3 December 2019; Accepted: 20 December 2019; Published: 22 December 2019

\begin{abstract}
To clarify the relationship between the fiber type composition and meat quality, we performed metabolomic analysis using porcine longissimus dorsi (LD) muscles. In the LD of pigs raised outdoors, the expression of myosin heavy chain (MyHC)1 (slow-twitch fiber marker protein) was significantly increased compared with that of $\mathrm{MyHC1}$ in pigs raised in an indoor pen, suggesting that rearing outdoors could be considered as an exercise treatment. These LD samples were subjected to metabolomic analysis for examining the profile of most primary and secondary metabolites. We found that the sex of the animal and exercise stimulation had a strong influence on the metabolomic profile in the porcine skeletal muscles, and this difference in the metabolomic profile is likely in part due to the changes in the muscle fiber type. We also examined the effects of cooking $\left(70{ }^{\circ} \mathrm{C}\right.$ for $\left.1 \mathrm{~h}\right)$. The effect of exercise on the metabolomic profile was also maintained in the cooked muscle tissues. Cooking treatment resulted in an increase in some of the metabolite levels while decreasing in some other metabolite levels. Thus, our study could indicate the effect of the sex of the animal, exercise stimulus, and cooking on the metabolomic profile of pork meat.
\end{abstract}

Keywords: pork; meat; skeletal muscle; fiber type; cooking

\section{Introduction}

Free amino acids stimulate taste and can modify the palatability of foods depending on the concentrations at which they are present in the foods [1-4]. To date, free amino acids are considered as the most important taste components of meat. In addition to free amino acids, purine nucleotides, such as inosine monophosphate (IMP), are also thought to be important components of taste in meat, thereby considerably potentiating the taste responses of free amino acids [5]. The presence of IMP suggests the existence of taste modifiers, as well as the tastants in food and the complexity in the induction of the taste stimuli. Although these free amino acids and purine nucleotides are undoubtedly important components of meat taste, we think there are more substances that affect meat taste and flavor. For example, taurine was not thought to be a taste substance because it is tasteless; however, taurine has been reported to show a positive correlation with umami intensity and is thought to impart full-bodied taste in meat taste or flavor by an unknown mechanism [6]. Thus, metabolomic substances as well as taurine could have the potential to affect meat taste and flavor. One-by-one analysis of each substance contained in meat will take a long time, but it is possible to depict the whole metabolite profiling of meat as the combination and association of these metabolites form the overall taste and flavor. 
Metabolomics in food analysis is the study of the metabolites present in the foods to describe and predict the properties of the food palatability and taste. Analytical methods have been developed to elucidate the profile of the components responsible for the overall food taste and flavor. For example, metabolomic analysis is performed for the metabolite profiling of strawberries and for identifying fruit attributes influencing hedonics and sensory perception based on consumer ratings [7]. A few metabolomic applications have been reported in meat. Metabolomic analysis has been performed to clarify the sensory characteristics and flavor of beef [8,9], the effect of finishing forage on beef [10], the effect of storage condition of ground beef [11], and the key metabolites during the postmortem aging of beef [12] or pork [13]. The approach to find a novel biomarker that indicates meat quality in relation to the water-holding capacity has also been reported [14]. However, more analyses need to be performed to clarify the relationship between various meat characteristics and metabolites.

Skeletal muscle tissues are composed of slow-twitch (type 1) and fast-twitch (type 2) muscle fibers. Metabolically, slow-twitch fibers have abundant mitochondria and myoglobin and rely on oxidative metabolism, whereas fast-twitch fibers have less mitochondria and myoglobin and mainly rely on the glycolytic pathway. In addition to these metabolic traits, the muscle fiber composition affects various meat properties, such as the color, $\mathrm{pH}$, water-holding capacity, tenderness, and nutritional value of meat [15]. In our previous study, we confirmed that there was a strong positive correlation between myosin heavy chain (MyHC)1 (slow-twitch fiber marker protein) composition and total free amino acid concentrations in bovine muscles, thereby suggesting that a high content of slow-twitch fibers contributes to the intense flavor of meat derived from amino acids [16]. In fact, in a tasting panel evaluation of lamb, which is a red meat rich in slow-twitch fibers, the lamb meat was classed as having a more intense flavor than white meat, which is assumed to be rich in fast-twitch fibers [17].

Thus, we thought that meat rich in slow-twitch fibers may have a different composition of metabolites from that in the fast-twitch predominant meat and this composition may not consist of only free amino acids. The aim of this study was to provide an insight into the profiling of pork meat metabolites under two conditions known to affect muscle fiber types: sex of the animal and exercise treatment. In humans, the proportion of slow-twitch fibers in women has been reported to be higher than that in men [18-20]. This greater proportion of the slow-twitch fibers in women could partially explain their higher oxidative capacity. The adaptive benefits of exercise training are commonly attributed to the fast-to-slow fiber type transition and increased mitochondrial energetic capacity [21]. Moreover, we also examined the alterations in the metabolite profiling induced by the cooking of pork meat. Although the sample size in this study was limited, the obtained results were remarkable, and these findings will contribute to the progress in the analysis of taste and flavor of meat in the future.

\section{Results and Discussion}

First, we measured the muscle fiber type compositions in the longissimus dorsi (LD) muscle tissue samples from barrows (castrated males) and gilts (females), which were raised indoors (sedentary) and outdoors (exercising animals). MyHC1 (slow-twitch fiber marker) and MyHC2 (fast-twitch fiber marker) isoform compositions were measured by SDS-PAGE. The percentage of $\mathrm{MyHC1}$ isoforms in the barrows and gilts was similar but it was slightly higher in the gilts (18.0\% vs. $22.3 \%$ in sedentary pigs, $30.6 \%$ vs. $33.4 \%$ in exercising pigs) (Figure 1). We found that the exercise treatment increased the slow-type MyHC1 composition significantly in the outdoor animal group of the barrow and gilt compared to that in the sedentary group of the barrow and gilt $(20.2 \pm 2.2 \%$ vs. $32.0 \pm 1.4 \%$, $p<0.05$ by $t$-test, $n=2$ for each group). It is well-known that prolonged exercise training could induce fast-to-slow fiber type transition. In this study, the pigs reared outdoors exercised voluntarily. Voluntary wheel running has been widely used as a model of non-interventional exercise training that induces adaptive changes in the skeletal muscle fiber type from fast-twitch to slow-twitch fibers. More specifically, the fiber type transition among fast-twitch subtypes (types $2 \mathrm{~A}, 2 \mathrm{X}$, and $2 \mathrm{~B}$ ) is commonly induced by endurance exercise; shift from type $2 \mathrm{~B} / 2 \mathrm{X}$ toward type $2 \mathrm{X} / 2 \mathrm{~A}$. It is reported that voluntary 
wheel running induced fiber type transition of $2 \mathrm{~B} / 2 \mathrm{X}$-to-2A in mice [22] or 2B-to-2A/2X in rats [23]. However, a shift from type 2-to-type 1 fibers may occur in limited muscle tissues under longer duration, higher volume endurance type events. For example, voluntary wheel running for four weeks induced type 2-to-type 1 fiber type conversion in rat plantaris muscle but not in soleus muscle [24]. Similarly, type 2-to-type 1 fiber type conversion in pigs also occurred during voluntary exercise although the muscle tissue was LD in our experiment. In this study, the proportion of slow-twitch fibers was slightly higher in gilts, which is in accordance with that reported previously in humans, although the effects of castration on the porcine muscle fiber type have still not been elucidated.

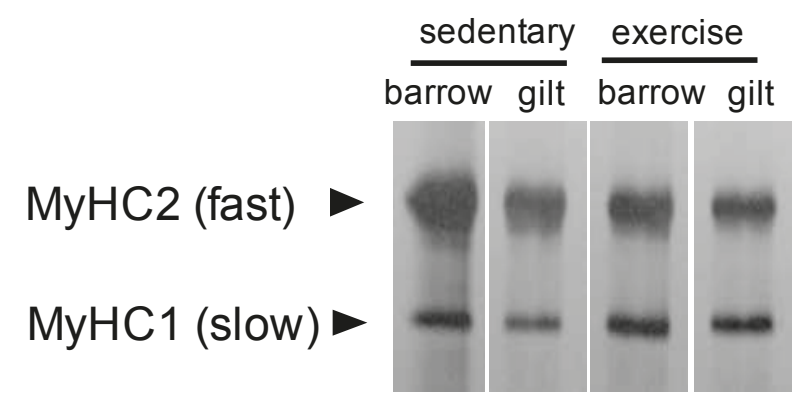

Figure 1. Separation of myosin heavy chain (MyHC) isoforms of the porcine longissimus dorsi (LD) muscles from sedentary or exercising barrows and gilts by using SDS-PAGE.

In this study, we performed global CE-TOFMS analysis to target metabolites involved in primary and secondary metabolism, such as sugars, amino acids, nucleotides, and other ionic metabolites in porcine LD muscles of sedentary barrow and gilt, exercising barrow and gilt, and cooked gilts. In this analysis, 130 peaks were detected. The analyzed metabolite profile of the LD muscles, i.e., pork loin, showed notable changes in each experimental condition: sex, exercise, and cooking. A principle component analysis (PCA) was performed to visualize the condition-related effects on the metabolites in LD muscles (Figure 2). Overall, the PCA demonstrated clear clustering in sex and cooking conditions. The peaks of the exercising barrow and gilt showed a slight separation from each other but they were still clustered together. Surprisingly, the separation was profound between the sedentary barrow and gilt, suggesting that the exercise stimulus decreased the sex differences seen in sedentary animals. Exercise is known to increase oxidative capacity. Hence, the alteration of oxidative capacity might be attenuated in gilts because females have higher oxidative capacity intrinsically.

The hierarchical clustering analysis (HCA) of the metabolites in the LD muscles showed a remarkably different pattern in the different experimental conditions (Figure 3). The results of the HCA classification were very similar to those of the PCA analysis. The sedentary barrow sample showed a different metabolite pattern from that of the sedentary gilt muscles or the exercising barrow muscle. 


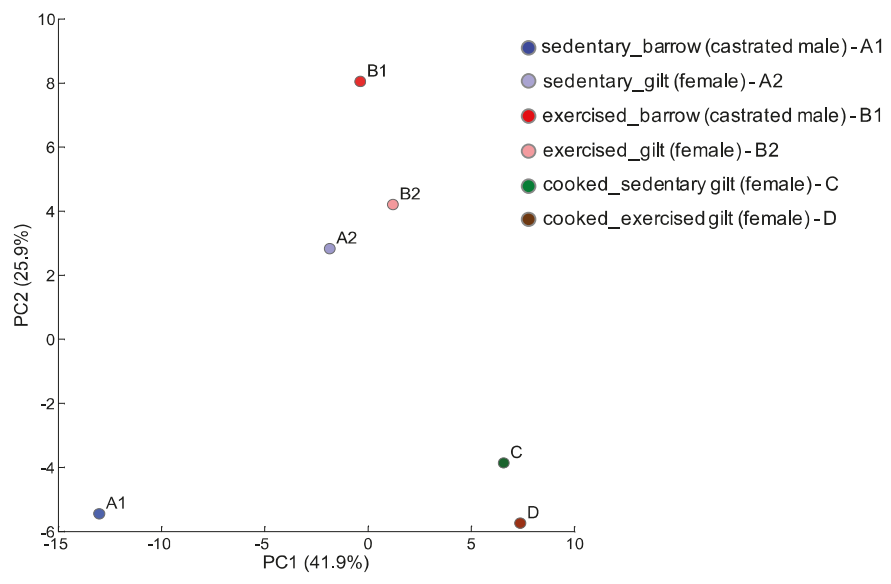

Figure 2. Principal component analysis (PCA) of the porcine longissimus dorsi (LD) muscle metabolomic profiles of sedentary or exercising barrows and gilts. We also analyzed cooked gilt LD muscles.

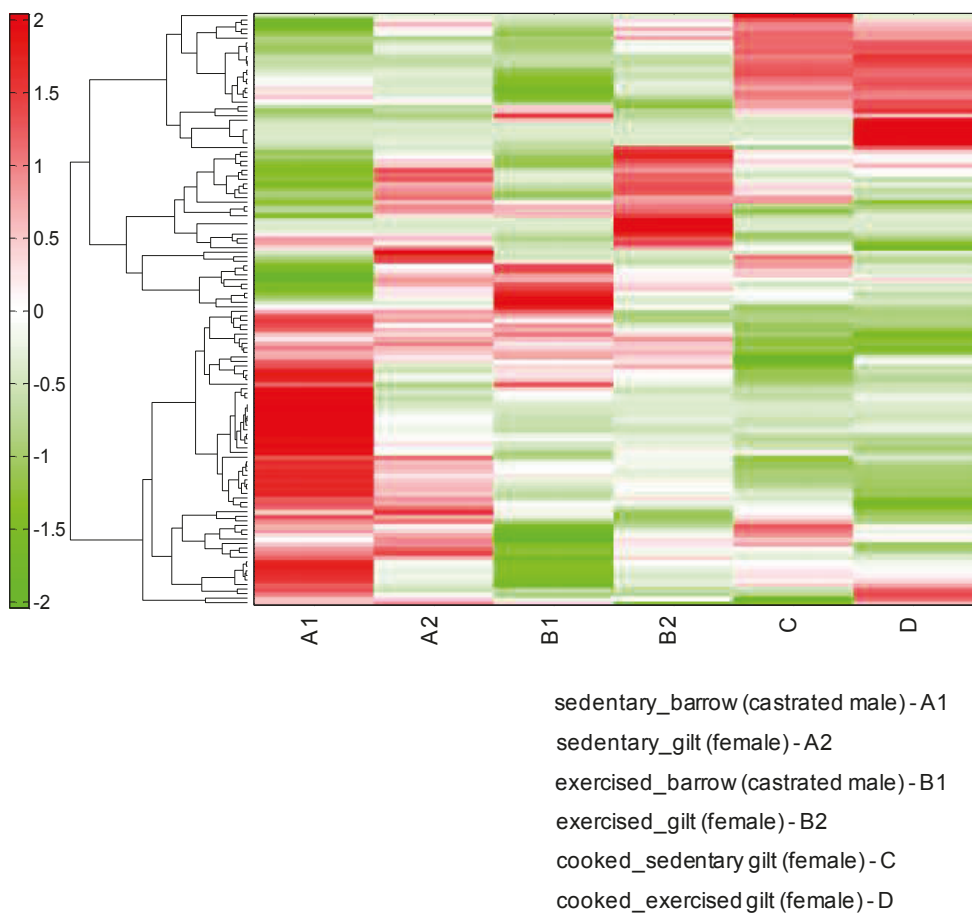

Figure 3. Hierarchical clustering analysis (HCA) of the porcine longissimus dorsi (LD) muscle metabolomic profiles of sedentary or exercising barrows and gilts. We also analyzed cooked gilt LD muscles.

Table 1 shows the changes in the metabolite ratios under the three different conditions. There was a notable increase in the sugars of fructose-6-phosphate and glucose-6-phosphate in the meat of the sedentary gilt compared to that in the meat of the sedentary barrow. Interestingly, the levels of these two sugars were also markedly increased by the exercise stimulus. The ratio of the gilt/barrow and 
exercise/sedentary seems to have common metabolic features. Since both conditions are known to show higher oxidative metabolism, the substrates in the glycolytic pathways might be spared. The exercise did not evoke marked alterations in the metabolites in gilts, while the metabolites in barrows showed various differences due to exercise. These findings indicate that there may be sex differences in the response to exercise.

Table 1. Metabolite ratios in the three different conditions. Numbers in the red boxes are a ratio of $>2$ and those in blue boxes are a ratio of $<0.5$. The intensity of the color corresponds to the magnitude of the ratio $<0.1$ or $>10$.

\begin{tabular}{|c|c|c|c|c|c|c|}
\hline \multirow{2}{*}{$\begin{array}{l}\text { Compound } \\
\text { Name }\end{array}$} & \multicolumn{2}{|c|}{ Gilt/Barrow } & \multicolumn{2}{|c|}{ Exercise/Sedentary } & \multicolumn{2}{|c|}{ Cooked/Uncooked } \\
\hline & Sedentary & Exercise & Barrow & Gilt & Sedentary & Exercise \\
\hline $\begin{array}{l}\text { 1-Methylhistidine, } \\
\text { 3-Methylhistidine }\end{array}$ & 0.59 & 1.09 & 0.80 & 1.46 & 0.64 & 0.64 \\
\hline 2-(Creatinine-3-yl) propionic acid & N.D. & N.D. & N.D. & N.D. & N.D. & N/A \\
\hline $\begin{array}{l}\text { 2-Aminoisobutyric acid, } \\
\text { 2-Aminobutyric acid }\end{array}$ & 0.78 & 1.10 & 0.58 & 0.81 & 0.65 & 0.73 \\
\hline 2-Hydroxybutyric acid & 1.10 & zero & zero & zero & 0.79 & N.D. \\
\hline 2-Hydroxyvaleric acid & 2.38 & 0.67 & 1.76 & 0.49 & 0.87 & 0.96 \\
\hline 3-Hydroxybutyric acid & 1.28 & 1.90 & 0.92 & 1.37 & 1.00 & 0.73 \\
\hline 3-Methyladenine & N/A & 1.25 & N/A & 1.05 & zero & zero \\
\hline 4-Methylpyrazole & 1.18 & zero & 0.89 & zero & zero & N/A \\
\hline 5'-Deoxy-5'-methylthioadenosine & N.D. & N.D. & N.D. & N.D. & N.D. & N/A \\
\hline 5-Oxoproline & 0.71 & 1.10 & 0.42 & 0.65 & 0.67 & 2.39 \\
\hline Adenosine & N.D. & N.D. & N/A & N.D. & N.D. & N/A \\
\hline ADMA & 0.96 & 1.22 & 0.63 & 0.81 & zero & 1.67 \\
\hline ADP & 0.93 & zero & 3.11 & zero & 3.96 & N/A \\
\hline ADP-ribose & N/A & 0.66 & N/A & 0.84 & 0.40 & 0.53 \\
\hline Ala & 0.73 & 0.84 & 0.73 & 0.84 & 0.70 & 0.82 \\
\hline AMP & 0.93 & 0.10 & 14.49 & 1.63 & 7.84 & 5.90 \\
\hline Anserine_divalent & 1.03 & 1.60 & 0.83 & 1.29 & 0.72 & 0.63 \\
\hline Arg & 0.84 & 1.42 & 0.60 & 1.01 & 1.28 & 1.38 \\
\hline Argininosuccinic acid & zero & N.D. & zero & N.D. & N.D. & N.D. \\
\hline Asn & 0.72 & 1.39 & 0.51 & 0.99 & 1.09 & 1.15 \\
\hline Asp & 0.45 & 0.93 & 0.58 & 1.20 & 1.05 & 1.07 \\
\hline ATP & N.D. & N.D. & N.D. & N.D. & N/A & N/A \\
\hline Betaine & 1.33 & 1.44 & 0.96 & 1.04 & 0.90 & 0.76 \\
\hline Butyrylcarnitine & 0.36 & 1.49 & 0.19 & 0.79 & 0.67 & 0.74 \\
\hline Carnitine & 0.94 & 1.24 & 0.67 & 0.88 & 0.72 & 0.63 \\
\hline Carnosine & 1.33 & 1.26 & 1.01 & 0.96 & 0.96 & 0.84 \\
\hline Choline & 0.43 & 0.87 & 0.35 & 0.70 & 0.40 & 0.48 \\
\hline Citrulline & 0.71 & 1.26 & 0.43 & 0.76 & 0.74 & 0.65 \\
\hline Creatine & 1.06 & 1.13 & 0.91 & 0.98 & 0.91 & 0.78 \\
\hline Creatinine & 1.33 & 1.34 & 0.82 & 0.82 & 1.25 & 3.68 \\
\hline Cys & 4.65 & 1.53 & 3.22 & 1.06 & 1.47 & 1.02 \\
\hline Cysteine glutathione disulfide & 0.21 & N/A & zero & 0.56 & zero & 0.55 \\
\hline Cystine & 0.06 & N.D. & zero & zero & zero & N.D. \\
\hline Cytidine & 1.05 & 1.12 & 0.78 & 0.84 & 0.83 & 0.96 \\
\hline $\begin{array}{l}\text { Daminozide } \\
\text { Ala-Ala }\end{array}$ & N/A & N/A & N.D. & 1.14 & 1.90 & 1.42 \\
\hline Diethanolamine & 1.06 & zero & 1.24 & zero & zero & N.D. \\
\hline Dyphylline & 2.69 & 0.83 & 2.88 & 0.89 & 0.99 & 0.90 \\
\hline Ethanolamine & 0.91 & 1.01 & 0.87 & 0.97 & 0.56 & 0.50 \\
\hline Ethanolamine phosphate & 0.80 & 0.84 & 1.04 & 1.09 & zero & 0.68 \\
\hline
\end{tabular}


Table 1. Cont.

\begin{tabular}{|c|c|c|c|c|c|c|}
\hline \multirow{2}{*}{$\begin{array}{l}\text { Compound } \\
\text { Name }\end{array}$} & \multicolumn{2}{|c|}{ Gilt/Barrow } & \multicolumn{2}{|c|}{ Exercise/Sedentary } & \multicolumn{2}{|c|}{ Cooked/Uncooked } \\
\hline & Sedentary & Exercise & Barrow & Gilt & Sedentary & Exercise \\
\hline Fructose 6-phosphate & 36.20 & 0.42 & 69.38 & 0.80 & 1.55 & 0.98 \\
\hline GABA & 1.13 & 1.16 & 0.76 & 0.78 & zero & zero \\
\hline Gln & 0.87 & 0.77 & 0.88 & 0.78 & 0.50 & 0.66 \\
\hline Glu & 0.62 & 1.79 & 0.36 & 1.04 & 1.06 & 0.94 \\
\hline Glu-Glu & 2.20 & 1.90 & 1.38 & 1.19 & 1.27 & 1.00 \\
\hline Gluconic acid & 2.48 & 2.10 & 1.35 & 1.14 & 0.88 & 0.49 \\
\hline Gluconolactone & N/A & 1.50 & N/A & 1.15 & 1.14 & zero \\
\hline Glucose 1-phosphate & N/A & 0.51 & N/A & 0.85 & 1.15 & 0.72 \\
\hline Glucose 6-phosphate & 43.39 & 0.39 & 83.94 & 0.76 & 0.81 & 0.54 \\
\hline Glutathione (GSH) & 0.97 & 0.89 & 1.09 & 1.00 & 0.78 & 0.65 \\
\hline Glutathione (GSSG)_divalent & 0.25 & 0.81 & 0.21 & 0.68 & 0.17 & 0.26 \\
\hline Gly & 0.83 & 1.14 & 0.63 & 0.87 & 0.75 & 0.75 \\
\hline Gly-Asp & zero & N.D. & zero & N.D. & N.D. & N/A \\
\hline Gly-Gly & N.D. & $\mathrm{N} / \mathrm{A}$ & N.D. & N/A & N.D. & zero \\
\hline Gly-Leu & N.D. & N.D. & N.D. & N.D. & N/A & N.D. \\
\hline Glyceric acid & N/A & 2.59 & N/A & 0.81 & 0.40 & 0.65 \\
\hline Glycerol & 1.34 & 0.63 & 1.87 & 0.88 & 0.94 & 0.95 \\
\hline Glycerol 3-phosphate & 0.97 & 1.24 & 0.57 & 0.73 & 0.51 & 0.93 \\
\hline Glycerophosphocholine & 1.00 & 0.64 & 1.58 & 1.01 & 0.61 & 0.71 \\
\hline GMP & 1.12 & 1.30 & 1.05 & 1.22 & 1.06 & 0.89 \\
\hline Guanine & 0.45 & 1.03 & 0.35 & 0.80 & 0.74 & 1.13 \\
\hline Guanosine & 0.91 & 0.84 & 0.76 & 0.70 & 0.93 & 1.04 \\
\hline His & 0.73 & 1.15 & 0.62 & 0.99 & 1.06 & 0.89 \\
\hline His-Glu & N.D. & N.D. & N.D. & N.D. & N.D. & N/A \\
\hline Homocarnosine & 1.41 & 1.28 & 1.18 & 1.07 & 0.94 & 0.81 \\
\hline Hydroxyproline & 0.79 & 0.96 & 0.62 & 0.76 & 0.48 & 0.70 \\
\hline Hypotaurine & 0.43 & 0.70 & 0.66 & 1.06 & 0.46 & 0.60 \\
\hline Hypoxanthine & 0.46 & 1.15 & 0.33 & 0.83 & 1.00 & 1.23 \\
\hline Ile & 0.84 & 1.24 & 0.60 & 0.88 & 1.31 & 1.47 \\
\hline IMP & 1.50 & 1.63 & 1.03 & 1.12 & 0.94 & 0.93 \\
\hline Inosine & 1.24 & 1.02 & 1.03 & 0.85 & 0.97 & 0.98 \\
\hline $\begin{array}{l}\text { Isobutyric acid } \\
\text { Butyric acid }\end{array}$ & 0.84 & N/A & zero & 1.46 & zero & zero \\
\hline Isoglutamic acid & 0.71 & zero & 0.41 & zero & zero & N.D. \\
\hline Lactic acid & 1.43 & 1.23 & 1.14 & 0.98 & 0.82 & 0.77 \\
\hline Leu & 0.86 & 1.43 & 0.59 & 0.99 & 1.38 & 1.39 \\
\hline Lys & 0.74 & 1.38 & 0.53 & 0.99 & 1.18 & 1.26 \\
\hline Malic acid & 0.46 & 1.63 & 0.34 & 1.20 & 0.76 & 0.41 \\
\hline Malonylcarnitine & N/A & N.D. & N.D. & zero & zero & N.D. \\
\hline Met & 1.07 & 1.62 & 0.63 & 0.96 & 1.81 & 1.83 \\
\hline Methionine sulfoxide & N.D. & N.D. & N.D. & N.D. & N/A & N/A \\
\hline $\begin{array}{l}\text { myo-Inositol 1-phosphate } \\
\text { myo-Inositol 3-phosphate }\end{array}$ & N.D. & N/A & N.D. & N.D. & N/A & 1.00 \\
\hline$N$-Acetyllysine & 1.81 & 1.57 & 1.20 & 1.04 & 1.95 & 1.88 \\
\hline$N$-Acetylneuraminic acid & N.D. & N.D. & N.D. & N.D. & N/A & N/A \\
\hline $\mathrm{N}$-Acetylornithine & 1.43 & 2.00 & 0.86 & 1.20 & 2.11 & 1.82 \\
\hline$N$-Methylalanine & 0.76 & 1.22 & 0.56 & 0.89 & 0.57 & 0.60 \\
\hline$N^{5}$-Ethylglutamine & 0.44 & 0.94 & 0.65 & 1.39 & 0.72 & 0.75 \\
\hline$N^{6}{ }_{,} N^{6}, N^{6}$-Trimethyllysine & 1.80 & 1.21 & 1.92 & 1.29 & 0.74 & 0.65 \\
\hline$N^{6}$-Methyllysine & 0.66 & 0.73 & 1.04 & 1.15 & 1.00 & 0.95 \\
\hline
\end{tabular}


Table 1. Cont.

\begin{tabular}{|c|c|c|c|c|c|c|}
\hline \multirow{2}{*}{$\begin{array}{l}\text { Compound } \\
\text { Name }\end{array}$} & \multicolumn{2}{|c|}{ Gilt/Barrow } & \multicolumn{2}{|c|}{ Exercise/Sedentary } & \multicolumn{2}{|c|}{ Cooked/Uncooked } \\
\hline & Sedentary & Exercise & Barrow & Gilt & Sedentary & Exercise \\
\hline $\mathrm{NADH}$ & 0.89 & 2.43 & 0.28 & 0.76 & 1.21 & 1.02 \\
\hline Nicotinamide & 2.27 & 0.89 & 2.16 & 0.85 & 0.79 & 1.05 \\
\hline O-Acetylcarnitine & 0.83 & 1.72 & 0.32 & 0.67 & 1.43 & 1.51 \\
\hline $\begin{array}{l}\text { O-Acetylhomoserine } \\
\text { 2-Aminoadipic acid }\end{array}$ & 0.73 & 0.88 & 0.57 & 0.69 & 0.50 & 0.69 \\
\hline Ornithine & 0.89 & 2.10 & 0.40 & 0.94 & 0.76 & 0.79 \\
\hline Pantothenic acid & 0.71 & 1.20 & 0.57 & 0.96 & zero & 0.81 \\
\hline Phe & 1.01 & 1.27 & 0.71 & 0.90 & 1.54 & 1.62 \\
\hline Phosphorylcholine & 0.36 & 0.77 & 0.45 & 0.98 & 0.81 & 1.09 \\
\hline Pro & 0.68 & 1.05 & 0.53 & 0.82 & 0.75 & 0.84 \\
\hline Putrescine & 0.91 & 0.99 & 1.00 & 1.09 & zero & zero \\
\hline Ribulose 5-phosphate & 1.40 & 1.34 & 1.07 & 1.03 & 1.43 & 1.45 \\
\hline S-Adenosylhomocysteine & 1.07 & 0.84 & 1.26 & 1.00 & 1.16 & 1.35 \\
\hline S-Adenosylmethionine & 1.45 & 1.12 & 1.38 & 1.06 & 0.59 & 0.67 \\
\hline S-Methylcysteine & 0.90 & 1.43 & 0.79 & 1.25 & 0.87 & 0.57 \\
\hline Saccharopine & N/A & 0.70 & N/A & 1.17 & 0.75 & 0.60 \\
\hline Sedoheptulose 7-phosphate & zero & zero & 0.40 & N.D. & N.D. & N.D. \\
\hline Ser & 0.68 & 1.34 & 0.51 & 1.01 & 1.14 & 1.09 \\
\hline Spermidine & 0.82 & 1.01 & 0.69 & 0.85 & 0.66 & 0.86 \\
\hline Spermine & 2.92 & 1.35 & 1.82 & 0.84 & 1.08 & 1.14 \\
\hline Stachydrine & 1.52 & N/A & zero & 0.78 & 0.83 & 0.91 \\
\hline Succinic acid & 0.67 & 0.62 & 0.89 & 0.82 & 0.54 & 0.76 \\
\hline Taurine & 0.50 & 0.89 & 0.61 & 1.08 & 0.51 & 0.67 \\
\hline Terephthalic acid & N.D. & N.D. & N.D. & N.D. & N.D. & N/A \\
\hline Thiamine & 1.08 & 1.72 & 0.52 & 0.84 & 1.04 & 1.00 \\
\hline Thiamine phosphate & 1.31 & 1.17 & 1.21 & 1.09 & zero & zero \\
\hline Thr & 0.69 & 1.35 & 0.50 & 0.98 & 1.07 & 1.03 \\
\hline $\begin{array}{l}\text { Thr-Asp } \\
\text { Ser-Glu }\end{array}$ & 1.10 & N.D. & zero & zero & 1.52 & N/A \\
\hline Trigonelline & 1.08 & 1.58 & 0.59 & 0.86 & 0.98 & 0.70 \\
\hline Trp & 0.92 & 1.24 & 0.74 & 0.99 & 1.32 & 1.36 \\
\hline Tyr & 0.92 & 1.60 & 0.60 & 1.05 & 1.50 & 1.55 \\
\hline $\begin{array}{l}\text { UDP-glucose } \\
\text { UDP-galactose }\end{array}$ & N.D. & zero & N.D. & N.D. & N.D. & N.D. \\
\hline $\begin{array}{l}\text { UDP- } N \text {-acetylgalactosamine } \\
\text { UDP- } N \text {-acetylglucosamine }\end{array}$ & N.D. & N/A & N.D. & N/A & N.D. & zero \\
\hline UMP & 1.77 & 1.97 & 1.11 & 1.24 & 0.99 & 0.74 \\
\hline Urea & 0.99 & 0.91 & 0.73 & 0.67 & 0.76 & 0.76 \\
\hline Uridine & 1.14 & 0.85 & 0.97 & 0.73 & 0.83 & 0.99 \\
\hline Val & 0.74 & 1.24 & 0.55 & 0.92 & 1.09 & 1.14 \\
\hline$\beta$-Ala & 0.89 & 0.96 & 0.76 & 0.82 & 0.82 & 0.64 \\
\hline$\beta$-Ala-Lys & 1.98 & 1.44 & 1.41 & 1.02 & 0.66 & 0.74 \\
\hline$\gamma$-Butyrobetaine & 1.06 & 1.44 & 0.61 & 0.83 & 0.81 & 0.76 \\
\hline$\gamma$-Glu-Cys & N.D. & N/A & N.D. & N/A & N.D. & zero \\
\hline
\end{tabular}

N.D. denotes it could not be detected in both conditions. N/A denotes it could not be calculated due to "the division by zero". The "zero" denotes its numerator was not detected.

As expected, the cooked LD muscles contained a completely different profile of the substances compared with that in the respective uncooked muscles. Even after the cooking treatment, there were still clear differences between the sedentary and exercised samples. In fact, the differences in the profile of the metabolites in the uncooked muscles was maintained even after heating at $70{ }^{\circ} \mathrm{C}$ for $1 \mathrm{~h}$. Cooking treatment increased the levels of $\mathrm{N}$-acetylornithine, ribulose 5-phosphate, $\mathrm{N}$-acetyl lysine, $\mathrm{N}$-acetylneuraminic acid, ATP, methionine sulfoxide, methionine, phenylalanine, tryptophan, and tyrosine compared with those in the four uncooked samples. In particular, ATP, N-acetylneuraminic 
acid, and methionine sulfoxide were detected only in the cooked samples, thereby suggesting that these substances are unique in cooked meat. It was interesting to note that ATP emerged after cooking because ATP is thought to be an unstable molecule. Conversely, the cooking treatment decreased the levels of diethanolamine, isoglutamic acid, succinic acid, glutamine, reduced glutathione, thiamine phosphate, gamma-aminobutyric acid, ethanolamine, putrescine, and ethanolamine phosphate compared with those in the four uncooked samples. In particular, thiamine phosphate, gamma-aminobutyric acid, and putrescine were not detected in the cooked samples, thereby suggesting that these substances were degraded or metabolized by cooking.

The levels of volatile aroma compounds were not measured in this study because the adopted CE-TOFMS is not optimized for the identification of these compounds. Flavor is an important factor for determining the palatability of food and the taste formed by the cooking of food. For example, the sugars in the meat are likely the precursors of the flavor components produced by the Maillard reaction, which is one of the most important pathways occurring primarily between the amino acids and the reducing monosaccharides for flavor formation in cooked foods [25]. Meinert et al. reported that glucose and glucose-6-phosphate increased the flavor-related volatile components more than ribose and ribose 5-phosphate in minced pork [26]. In our analysis, lipids were not targeted, although they are responsible for the volatile components of the meat flavor after cooking [27]. It should be emphasized that a comprehensive analysis of the volatile aroma compounds responsible for the flavor of meat would contribute to the understanding of whole meat palatability in the future.

The sex of the animal and exercise stimulation influence the type of metabolites contained in porcine skeletal muscles. The metabolic profile of sedentary gilt was already similar to exercised barrow even though MyHC1 expressions were increased in both the gilt and barrow. We thought that the regulation of metabolism and that of $\mathrm{MyHC}$ expression are independent although these two factors are closely related. In fact, adaptations in muscle metabolic capacity to prolonged exercise training can occur without fiber type alterations [28]. Thus, we assumed the change in the metabolic profile was partly attributed to the changes in the muscle fiber type, and partly attributed to other factors such as upregulation of metabolic enzyme expression. The effect of exercise is also maintained in the cooked muscle tissues. It is necessary to verify whether the changes observed in this study were not just individual differences. In the future, we will try to elucidate the relationship between metabolite profiles and taste (flavor) of meat by integrating sensory evaluation and metabolome analysis.

\section{Materials and Methods}

\subsection{Meat Sample}

The pork loins of Large White were obtained from a local meat shop. The pigs were reared in a farm located in the Fukuoka Agriculture and Forestry Research Center (Chikushino, Japan). The pigs were slaughtered in an approved slaughterhouse and dressed according to Japanese standard commercial procedures, then distributed to the meat shop. We chose the entire pork loins from one barrow and one gilt raised indoors in a $1.9 \mathrm{~m} \times 3.5 \mathrm{~m}\left(6.7 \mathrm{~m}^{2}\right)$ pen or raised outdoors where they were allowed to graze over a total area of $369 \mathrm{~m}^{2}(12.3 \mathrm{~m} \times 30 \mathrm{~m})$ during daytime for $6 \mathrm{~h}(9: 30$ to 15:30) for 32 days in finishing period. From the entire pork loins chilled for $120 \mathrm{~h}$ since slaughter (normal storage period in Japan), we excised the LD muscles. The excised middle LD muscle blocks were vacuum packed and frozen at $-30{ }^{\circ} \mathrm{C}$ until preparation. We used the single sample for each treatment $(n=1)$.

\subsection{Sample Preparation}

Muscle samples were thawed overnight at $4{ }^{\circ} \mathrm{C}$. The small portion of the middle LD muscle tissue was ground to powder with a mortar and pestle, cooled with liquid nitrogen, and stored at $-20^{\circ} \mathrm{C}$ for metabolomic analysis or at $-80{ }^{\circ} \mathrm{C}$ for protein assay. For obtaining cooked samples, the small block (about $4 \mathrm{~g}$ ) of thawed LD was vacuum packed and heated in a water bath at $70{ }^{\circ} \mathrm{C}$ for $1 \mathrm{~h}$ and cooled under running tap water for $30 \mathrm{~min}$. The cooked muscle tissue was snap frozen in liquid nitrogen and 
ground to powder with a mortar and pestle, cooled with liquid nitrogen, and stored at $-20{ }^{\circ} \mathrm{C}$ until metabolomic analysis.

\subsection{MyHC Isoform Content Determination}

A motor-driven small pestle was used to homogenize each thawed uncooked sample of the muscle ( $50 \mathrm{mg}$ ) in an SDS solution (10\% SDS, $40 \mathrm{mM}$ DTT, $5 \mathrm{mM}$ EDTA, and $0.1 \mathrm{M}$ Tris-HCl buffer (pH 8.0)) on ice. The SDS solution contained the Protease Inhibitor Cocktail for Use with Mammalian Cell and Tissue Extracts (Nacalai Tesque, Inc., Kyoto, Japan) in a 1:100 ratio. The sample homogenates were heated in boiling water for $3 \mathrm{~min}$. The total protein concentrations were assayed using the Pierce BCA Protein Assay Kit (Thermo Fisher Scientific, Waltham, MA, USA), with bovine serum albumin as the standard. The samples were diluted in 2× sample buffer (100 mM DTT, 4.0\% SDS, $0.16 \mathrm{M}$ Tris- $\mathrm{HCl}$ (pH 6.8), $43 \%$ glycerol, and $0.2 \%$ bromophenol blue) and $\mathrm{dH}_{2} \mathrm{O}$ to give final protein concentrations of $20 \mathrm{ng} / \mu \mathrm{L}$ in $1 \times$ sample buffer. These protein samples were subjected to high-resolution SDS-polyacrylamide gel electrophoresis for assessing the $\mathrm{MyHC}$ isoform composition, as described in detail previously [29]. The gel contained 8\% acrylamide (acrylamide/bisacrylamide ratio $=99: 1$ ) and 35\% (v/v) glycerol. After loading the samples (100 ng protein), electrophoresis was performed at a constant voltage of $140 \mathrm{~V}$ for $22 \mathrm{~h}$ at $4{ }^{\circ} \mathrm{C}$. The gels were stained with Silver Stain Kanto III (Kanto Chemical Co. Inc., Tokyo, Japan) and dried. The bands were captured on an imager (Fusion SL-4, Vilber Lourmat), and the relative contents of the MyHC isoforms were quantified by densitometry using the ImageJ 1.34s software (Rasband W, National Institutes of Health, Bethesda, MD, USA). MyHC isoforms were identified according to their different migration rates $(\mathrm{MyHC} 1>2)$.

\subsection{Sample Pretreatment for Metabolome Analysis}

Metabolome measurements were performed through a facility service at Human Metabolome Technologies (HMT) Inc., Tsuruoka, Japan. Briefly, approximately $30 \mathrm{mg}$ of frozen uncooked or cooked LD samples was plunged into $1200 \mu \mathrm{L}$ of $50 \%$ acetonitrile/Milli-Q water containing internal standards (Solution ID: 304-1002, Human Metabolome Technologies, Inc., Tsuruoka, Japan) at $0{ }^{\circ} \mathrm{C}$ to inactivate the enzymes. The tissue was homogenized 4 times at $1500 \mathrm{rpm}$ for $120 \mathrm{~s}$ by using a tissue homogenizer and then the homogenate was centrifuged at $2300 \times g$ at $4{ }^{\circ} \mathrm{C}$ for $5 \mathrm{~min}$. Subsequently, $400 \mu \mathrm{L}$ of the upper aqueous layer was centrifugally filtered through a Millipore 5-kDa cutoff filter at $9100 \times g$ at $4{ }^{\circ} \mathrm{C}$ for $120 \mathrm{~min}$ to remove the proteins. The filtrate was centrifugally concentrated and re-suspended in $50 \mu \mathrm{L}$ of Milli-Q water for CE-MS analysis.

\subsection{CE-TOFMS Analysis}

CE-TOFMS was performed using an Agilent CE Capillary Electrophoresis System equipped with an Agilent 6210 Time of Flight mass spectrometer, Agilent 1100 isocratic HPLC pump, Agilent G1603A CE-MS adapter kit, and Agilent G1607A CE-ESI-MS sprayer kit (Agilent Technologies, Waldbronn, Germany). The systems were controlled by the Agilent G2201AA ChemStation software version B.03.01 for CE (Agilent Technologies, Waldbronn, Germany). The metabolites were analyzed using a fused silica capillary $(50 \mu \mathrm{m}$ i.d. $\times 80 \mathrm{~cm}$ total length), with a commercial electrophoresis buffer (Solution ID: H3301-1001 for cation analysis and H3302-1021 for anion analysis, HMT) as the electrolyte. The sample was injected at a pressure of 50 mbar for $10 \mathrm{~s}$ (approximately $10 \mathrm{~nL}$ ) for the cation analysis and $25 \mathrm{~s}$ (approximately $25 \mathrm{~nL}$ ) for the anion analysis. Spectrometry was performed by scanning from $\mathrm{m} / \mathrm{z} 50$ to 1000. Other conditions were as described previously [30].

\subsection{Data Analysis}

Peaks were extracted using the automatic integration software MasterHands (Keio University, Tsuruoka, Japan) in order to obtain peak information, including $m / z$, migration time for CE-TOFMS measurement (MT), and peak area [31]. Signal peaks corresponding to isotopomers, adduct ions, and other product ions of known metabolites were excluded, and the remaining peaks were annotated 
with putative metabolites from the HMT metabolite database based on their MTs and $m / z$ values determined by TOFMS. The tolerance range for the peak annotation was configured at $\pm 0.5 \mathrm{~min}$ for MT and $\pm 10 \mathrm{ppm}$ for $\mathrm{m} / \mathrm{z}$. In addition, peak areas were normalized against those of the internal standards and then the resultant relative area values were further normalized by the sample amount. HCA and PCA were performed using HMT's proprietary software, PeakStat and SampleStat, respectively. The detected metabolites were plotted on metabolic pathway maps by using the VANTED (Visualization and Analysis of Networks containing Experimental Data) software [32].

\subsection{Statistics}

Results of MyHC analysis are expressed as means \pm SE. We used a two-tailed $t$-test calculated by Excel 2016 for Mac (Microsoft), and significance was set at $p<0.05$.

Author Contributions: Conceptualization, S.S. and W.M.; methodology, K.O., T.M., S.S., and W.M.; validation, M.N., R.T., and W.M.; writing-original draft preparation, S.S. and W.M.; writing-review and editing, S.S. and W.M.; visualization, S.S.; supervision, W.M.; project administration, W.M.; funding acquisition, S.S., R.T., and W.M. All authors have read and agreed to the published version of the manuscript.

Funding: This research was funded by a research grant from the Mishima Kaiun Memorial Foundation to S.S. This research was funded by a research grant from the Ito Foundation and a research grant from the Kyushu University Interdisciplinary Programs in Education and Projects in Research Development (P\&P) Grant to W.M. This research was also supported by the Japan Society for the Promotion of Science (JSPS) KAKENHI Grant Numbers JP19H03109 to W.M.; JP17H03908 to W.M. and R.T.; JP17K07807 to W.M. and S.S.

Acknowledgments: We would like to show our gratitude to Shojiro Kasa (Fukuoka Agriculture and Forestry Research Center) for providing information about the produced pigs.

Conflicts of Interest: The authors declare no conflict of interest.

\section{References}

1. Schiffman, S.S.; Hornack, K.; Reilly, D. Increased taste thresholds of amino acids with age. Am. J. Clin. Nutr. 1979, 32, 1622-1627. [CrossRef] [PubMed]

2. Schiffman, S.S.; Sennewald, K.; Gagnon, J. Comparison of taste qualities and thresholds of D-and L-amino acids. Physiol. Behav. 1981, 27, 51-59. [CrossRef]

3. Nishimura, T.; Ra Rhue, M.; Okitani, A.; Kato, H. Components contributing to the improvement of meat taste during storage. Agric. Biol. Chem. 1988, 52, 2323-2330.

4. Kato, H.; Rhue, M.R.; Nishimura, T. Role of free amino acids and peptides in food taste. In Flavor Chemistry: Trends and Developments, Proceedings of ACS Symposium Series; Teranishi, R., Buttery, R.G., Shalnidi, F., Eds.; American Chemical Society: Washington, DC, USA, 1989; Volume 388, pp. 158-174.

5. Nelson, G.; Chandrashekar, J.; Hoon, M.A.; Feng, L.; Zhao, G.; Ryba, N.J.; Zuker, C.S. An amino-acid taste receptor. Nature 2002, 416, 199. [CrossRef]

6. Suzuki, K.; Shioura, H.; Yokota, S.; Katoh, K.; Roh, S.G.; Iida, F.; Komatsu, T.; Syoji, N.; Sakuma, H.; Yamada, S. Search for an index for the taste of Japanese Black cattle beef by panel testing and chemical composition analysis. Anim. Sci. J. 2017, 88, 421-432. [CrossRef]

7. Schwieterman, M.L.; Colquhoun, T.A.; Jaworski, E.A.; Bartoshuk, L.M.; Gilbert, J.L.; Tieman, D.M.; Odabasi, A.Z.; Moskowitz, H.R.; Folta, K.M.; Klee, H.J. Strawberry flavor: Diverse chemical compositions, a seasonal influence, and effects on sensory perception. PLOS ONE 2014, 9, e88446. [CrossRef]

8. Jiang, T.; Bratcher, C.L. Differentiation of commercial ground beef products and correlation between metabolites and sensory attributes: A metabolomic approach. Food Res. Int. 2016, 90, 298-306. [CrossRef]

9. Lee, S.M.; Kwon, G.Y.; Kim, K.-O.; Kim, Y.-S. Metabolomic approach for determination of key volatile compounds related to beef flavor in glutathione-Maillard reaction products. Anal. Chim. Acta 2011, 703, 204-211. [CrossRef]

10. Carrillo, J.A.; He, Y.; Li, Y.; Liu, J.; Erdman, R.A.; Sonstegard, T.S.; Song, J. Integrated metabolomic and transcriptome analyses reveal finishing forage affects metabolic pathways related to beef quality and animal welfare. Sci. Rep. 2016, 6, 25948. [CrossRef] [PubMed] 
11. Argyri, A.A.; Mallouchos, A.; Panagou, E.Z.; Nychas, G.-J.E. The dynamics of the HS/SPME-GC/MS as a tool to assess the spoilage of minced beef stored under different packaging and temperature conditions. Int. J. Food Microbiol. 2015, 193, 51-58. [CrossRef] [PubMed]

12. Muroya, S.; Oe, M.; Ojima, K.; Watanabe, A. Metabolomic approach to key metabolites characterizing postmortem aged loin muscle of Japanese Black (Wagyu) cattle. Asian Australas. J. Anim. Sci. 2019, 32, 1172-1185. [CrossRef] [PubMed]

13. Muroya, S.; Oe, M.; Nakajima, I.; Ojima, K.; Chikuni, K. CE-TOF MS-based metabolomic profiling revealed characteristic metabolic pathways in postmortem porcine fast and slow type muscles. Meat Sci. 2014, 98, 726-735. [CrossRef] [PubMed]

14. Welzenbach, J.; Neuhoff, C.; Looft, C.; Schellander, K.; Tholen, E.; Große-Brinkhaus, C. Different statistical approaches to investigate porcine muscle metabolome profiles to highlight new biomarkers for pork quality assessment. PLoS ONE 2016, 11, e0149758. [CrossRef] [PubMed]

15. Choi, Y.M.; Kim, B.C. Muscle fiber characteristics, myofibrillar protein isoforms, and meat quality. Livest. Sci. 2009, 122, 105-118. [CrossRef]

16. Mashima, D.; Oka, Y.; Gotoh, T.; Tomonaga, S.; Sawano, S.; Nakamura, M.; Tatsumi, R.; Mizunoya, W. Correlation between skeletal muscle fiber type and free amino acid levels in Japanese Black steers. Anim. Sci. J. 2019, 90, 604-609. [CrossRef]

17. Valin, C.; Touraille, C.; Vigneron, P.; Ashmore, C. Prediction of lamb meat quality traits based on muscle biopsy fibre typing. Meat Sci. 1982, 6, 257-263. [CrossRef]

18. Brooke, M.H.; Engel, W.K. The histographic analysis of human muscle biopsies with regard to fiber types: I. Adult male and female. Neurology 1969, 19, 221-233. [CrossRef]

19. Simoneau, J.A.; Bouchard, C. Human variation in skeletal muscle fiber-type proportion and enzyme activities. Am. J. Physiol. 1989, 257, E567-E572. [CrossRef]

20. Staron, R.S.; Hagerman, F.C.; Hikida, R.S.; Murray, T.F.; Hostler, D.P.; Crill, M.T.; Ragg, K.E.; Toma, K. Fiber type composition of the vastus lateralis muscle of young men and women. J. Histochem. Cytochem. 2000, 48, 623-629. [CrossRef]

21. Holloszy, J.O.; Booth, F.W. Biochemical adaptations to endurance exercise in muscle. Annu. Rev. Physiol. 1976, 38, 273-291. [CrossRef]

22. Allen, D.L.; Harrison, B.C.; Maass, A.; Bell, M.L.; Byrnes, W.C.; Leinwand, L.A. Cardiac and skeletal muscle adaptations to voluntary wheel running in the mouse. J. Appl. Physiol. 2001, 90, 1900-1908. [CrossRef] [PubMed]

23. Kriketos, A.; Pan, D.; Sutton, J.; Hoh, J.; Baur, L.; Cooney, G.; Jenkins, A.; Storlien, L. Relationships between muscle membrane lipids, fiber type, and enzyme activities in sedentary and exercised rats. Am. J. Physiol. Regul. Integr. Comp. Physiol. 1995, 269, R1154-R1162. [CrossRef] [PubMed]

24. Bigard, A.X.; Sanchez, H.; Birot, O.; Serrurier, B. Myosin heavy chain composition of skeletal muscles in young rats growing under hypobaric hypoxia conditions. J. Appl. Physiol. 2000, 88, 479-486. [CrossRef] [PubMed]

25. Mottram, D.S. Flavour formation in meat and meat products: A review. Food Chem. 1998, 62, 415-424. [CrossRef]

26. Meinert, L.; Tikk, K.; Tikk, M.; Brockhoff, P.B.; Bredie, W.L.; Bjergegaard, C.; Aaslyng, M.D. Flavour development in pork. Influence of flavour precursor concentrations in longissimus dorsi from pigs with different raw meat qualities. Meat Sci. 2009, 81, 255-262. [CrossRef] [PubMed]

27. Tikk, K.; Haugen, J.-E.; Andersen, H.J.; Aaslyng, M.D. Monitoring of warmed-over flavour in pork using the electronic nose-correlation to sensory attributes and secondary lipid oxidation products. Meat Sci. 2008, 80, 1254-1263. [CrossRef]

28. Van der Zwaard, S.; Brocherie, F.; Kom, B.L.; Millet, G.P.; Deldicque, L.; van der Laarse, W.J.; Girard, O.; Jaspers, R.T. Adaptations in muscle oxidative capacity, fiber size, and oxygen supply capacity after repeated-sprint training in hypoxia combined with chronic hypoxic exposure. J. Appl. Physiol. 2018, 124, 1403-1412. [CrossRef]

29. Mizunoya, W.; Wakamatsu, J.; Tatsumi, R.; Ikeuchi, Y. Protocol for high-resolution separation of rodent myosin heavy chain isoforms in a mini-gel electrophoresis system. Anal. Biochem. 2008, 377, 111-113. [CrossRef] 
30. Soga, T.; Ohashi, Y.; Ueno, Y.; Naraoka, H.; Tomita, M.; Nishioka, T. Quantitative metabolome analysis using capillary electrophoresis mass spectrometry. J. Proteome Res. 2003, 2, 488-494. [CrossRef]

31. Sugimoto, M.; Wong, D.T.; Hirayama, A.; Soga, T.; Tomita, M. Capillary electrophoresis mass spectrometry-based saliva metabolomics identified oral, breast and pancreatic cancer-specific profiles. Metabolomics 2010, 6, 78-95. [CrossRef]

32. Junker, B.H.; Klukas, C.; Schreiber, F. VANTED: A system for advanced data analysis and visualization in the context of biological networks. BMC Bioinform. 2006, 7, 109.

(C) 2019 by the authors. Licensee MDPI, Basel, Switzerland. This article is an open access article distributed under the terms and conditions of the Creative Commons Attribution (CC BY) license (http://creativecommons.org/licenses/by/4.0/). 
Metabolite Genome-Wide Association Study (mGWAS) and Gene-Metabolite Interaction Network Analysis Reveal Potential Biomarkers for Feed Efficiency in Pigs

\author{
Xiao Wang and Haja N. Kadarmideen * \\ Quantitative Genomics, Bioinformatics and Computational Biology Group, Department of Applied Mathematics \\ and Computer Science, Technical University of Denmark, Richard Petersens Plads, Building 324, \\ 2800 Kongens Lyngby, Denmark; xiwa@dtu.dk \\ * Correspondence: hajak@dtu.dk
}

Received: 1 April 2020; Accepted: 11 May 2020; Published: 15 May 2020

\begin{abstract}
Metabolites represent the ultimate response of biological systems, so metabolomics is considered the link between genotypes and phenotypes. Feed efficiency is one of the most important phenotypes in sustainable pig production and is the main breeding goal trait. We utilized metabolic and genomic datasets from a total of 108 pigs from our own previously published studies that involved 59 Duroc and 49 Landrace pigs with data on feed efficiency (residual feed intake (RFI)), genotype (PorcineSNP80 BeadChip) data, and metabolomic data (45 final metabolite datasets derived from LC-MS system). Utilizing these datasets, our main aim was to identify genetic variants (single-nucleotide polymorphisms (SNPs)) that affect 45 different metabolite concentrations in plasma collected at the start and end of the performance testing of pigs categorized as high or low in their feed efficiency (based on RFI values). Genome-wide significant genetic variants could be then used as potential genetic or biomarkers in breeding programs for feed efficiency. The other objective was to reveal the biochemical mechanisms underlying genetic variation for pigs' feed efficiency. In order to achieve these objectives, we firstly conducted a metabolite genome-wide association study (mGWAS) based on mixed linear models and found 152 genome-wide significant SNPs ( $p$-value $<1.06 \times 10^{-6}$ ) in association with 17 metabolites that included 90 significant SNPs annotated to 52 genes. On chromosome one alone, 51 significant SNPs associated with isovalerylcarnitine and propionylcarnitine were found to be in strong linkage disequilibrium (LD). SNPs in strong LD annotated to FBXL4, and CCNC consisted of two haplotype blocks where three SNPs (ALGA0004000, ALGA0004041, and ALGA0004042) were in the intron regions of FBXL4 and CCNC. The interaction network revealed that CCNC and FBXL4 were linked by the hub gene N6AMT1 that was associated with isovalerylcarnitine and propionylcarnitine. Moreover, three metabolites (i.e., isovalerylcarnitine, propionylcarnitine, and pyruvic acid) were clustered in one group based on the low-high RFI pigs. This study performed a comprehensive metabolite-based genome-wide association study (GWAS) analysis for pigs with differences in feed efficiency and provided significant metabolites for which there is significant genetic variation as well as biological interaction networks. The identified metabolite genetic variants, genes, and networks in high versus low feed efficient pigs could be considered as potential genetic or biomarkers for feed efficiency.
\end{abstract}

Keywords: feed efficiency; biomarkers; SNPs; GWAS; metabolomics; RFI; pigs; pathways

\title{
1. Introduction
}

Large populations are generally essential for genome-wide association study (GWAS) to obtain sufficient statistical power for the identification of genetic polymorphisms [1]. However, 
some intermediate phenotypes like metabolites could potentially avoid this problem, as they are directly involved in metabolite conversion modification [2,3]. As the end products of cellular regulatory processes, metabolites represent the ultimate response of biological systems associated with genetic changes, so metabolomics is considered the link between genotypes and phenotypes [4]. Metabolomics refers to the measurements of all endogenous metabolites, intermediates, and products of metabolism and has been applied to measure the dynamic metabolic responses in pigs $[5,6]$ and dairy cows $[7,8]$. Additionally, metabolites could provide details of physiological state, so genetic variant-associated metabolites are expected to display larger effect sizes [9]. Gieger et al. (2008) firstly used metabolite concentrations as quantitative traits in association with genotypes and found their available applications in GWAS [9]. Do et al. (2014) [10] conducted GWAS using residual feed intake (RFI) phenotypes to identify single-nucleotide polymorphisms (SNPs) that explain significant variation in feed efficiency for pigs. Our previous study found two metabolites (i.e., $\alpha$-ketoglutarate and succinic acid) in a RFI-related network of dairy cows which could represent biochemical mechanisms underlying variation for phenotypes of feed efficiency [8].

In this study, we aimed to identify genetic variants (SNP markers) affecting concentrations of metabolites and to reveal the biochemical mechanisms underlying genetic variation for pigs' feed efficiency. Our study is based on two of our previously published papers and datasets used therein [6,11]. Briefly, the experiment consisted of 59 Duroc and 49 Landrace pigs with data on feed efficiency (RFI), genotype (PorcineSNP80 BeadChip) data, and metabolomic data (45 final metabolite datasets derived from liquid chromatography-mass spectrometry (LC-MS) system). While our previous studies only looked at metabolome-phenotype associations [6], we report an integrated systems genomics approach to identify quantitative trait loci (QTLs) or SNPs affecting metabolite concentration via metabolite GWAS methods (mGWAS), where each metabolite is itself a phenotype. To the best of our knowledge, this is the first study to link the genomics with metabolomics to identify significant genetic variants associated with known metabolites that differ in pigs with different levels of feed efficiency. Main aims of our study are as follows:

1. Find significant SNP markers associated with all the metabolites in the metabolomics dataset using mGWAS method and then reveal the biochemical mechanisms underlying genetic variation for porcine feed efficiency using 108 Danish pigs in low and high RFI conditions, genotyped by 68K PorcineSNP80 BeadChip array.

2. Annotate identified significant SNP markers to porcine genes.

3. Annotate metabolites and identify enriched metabolic pathways and gene-metabolite networks to find the potential biomarkers that were strongly associated with feed efficiency.

\section{Results}

\subsection{First Component Score and Significant Metabolic Pathways of 45 Metabolites}

The partial least squares-discriminant analysis (PLS-DA) results revealed that the first component score (component 1 ) explained more than $75 \%$ variation of all 45 metabolites (Figure $1 \mathrm{~A}$ ). It showed that metabolite values of Duroc were higher than those of Landrace, the same as metabolites from second sampling time higher than those from first sampling time. In addition, the Duroc and Landrace pigs were clearly stratified, especially using the metabolite values between Duroc from first sampling time and Landrace from second sampling time (Figure 1A). The most significant metabolic pathways were the aminoacyl-tRNA biosynthesis; following by the arginine biosynthesis; the arginine and proline metabolism; and the alanine, aspartate, and glutamate metabolism (Figure 1B). As the pathway impact of the aminoacyl-tRNA biosynthesis was zero, we discarded this significant pathway and only used the metabolites enriched in the other three significant pathways for GWAS (Table 1). Thus, the metabolite means for 5 compounds in the arginine biosynthesis (arginine, aspartic acid, citrulline, glutamic acid, and ornithine); 5 compounds in the arginine and proline metabolism (arginine, glutamic acid, ornithine, proline, and pyruvic acid); and 4 compounds in the alanine, aspartate, and glutamate metabolism 
(alanine, aspartic acid, glutamic acid, and pyruvic acid) metabolites were calculated and shown in Table 1.

A

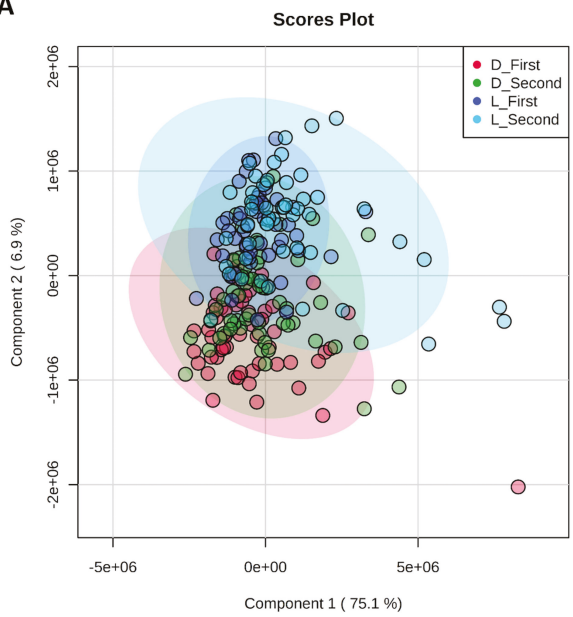

B

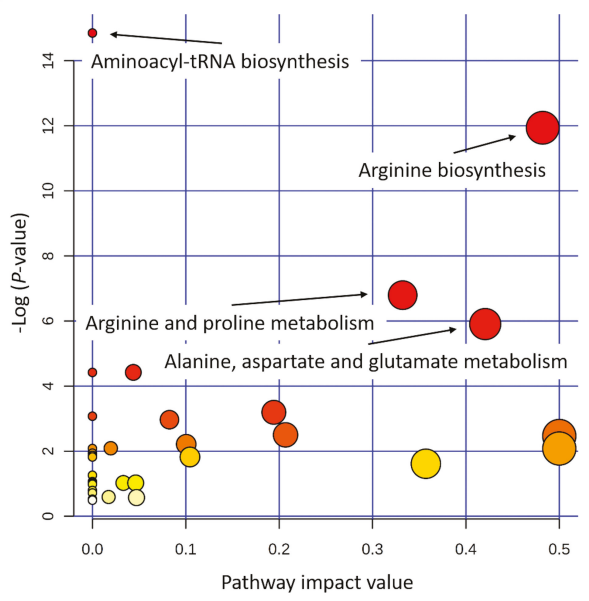

Figure 1. (A) Partial least squares-discriminant analysis (PLS-DA) of 45 metabolites. Note: D/L with first/second indicates the sampling time of Duroc/Landrace pigs. (B) Metabolic pathways for 45 metabolites using Homo sapiens as the library. Note: The size and color of the circles for each pathway indicate the matched metabolite ratio and the $-\log (p$-value), respectively.

\subsection{Genome-Wide Significant SNPs and Gene Annotation}

Metabolite based GWAS for first, second, and combined two sampling times revealed 152 genome-wide significant SNPs (Supplementary Table S1) in association with 17 metabolites (Supplementary Table S2). Unfortunately, no significant SNP was detected in association with first component scores $\left(p\right.$-values $\left.\geq 2.78 \times 10^{-6}\right)$ and metabolites enriched in the significant metabolic pathways $\left(p\right.$-values $\geq 1.74 \times 10^{-4}$ ); thus, GWAS results of these two scenarios were not listed. Manhattan plots of genome-wide association for isovalerylcarnitine and propionylcarnitine are shown in Figure 2, and Manhattan plots for the other 43 metabolites are shown in the Supplementary Figure S1. Along the whole genome, significant SNPs associated with isovalerylcarnitine and propionylcarnitine from the second sampling time were mainly located on the chromosome one (Figure 2). The overlapped significant SNPs associated with more than two different metabolites were shown in Table 2, where 57 significant SNPs on genome level were associated with isovalerylcarnitine and propionylcarnitine from the second sampling time. In addition, another 3 metabolites (1-hexadecyl-sn-glycero-3-phosphocholine, 1-myristoyl-sn-glycero-3-phosphocholine, lysoPC (16:0)) were also significantly associated with 10 SNPs (Table 2). 
Metabolites 2020, 10, 201

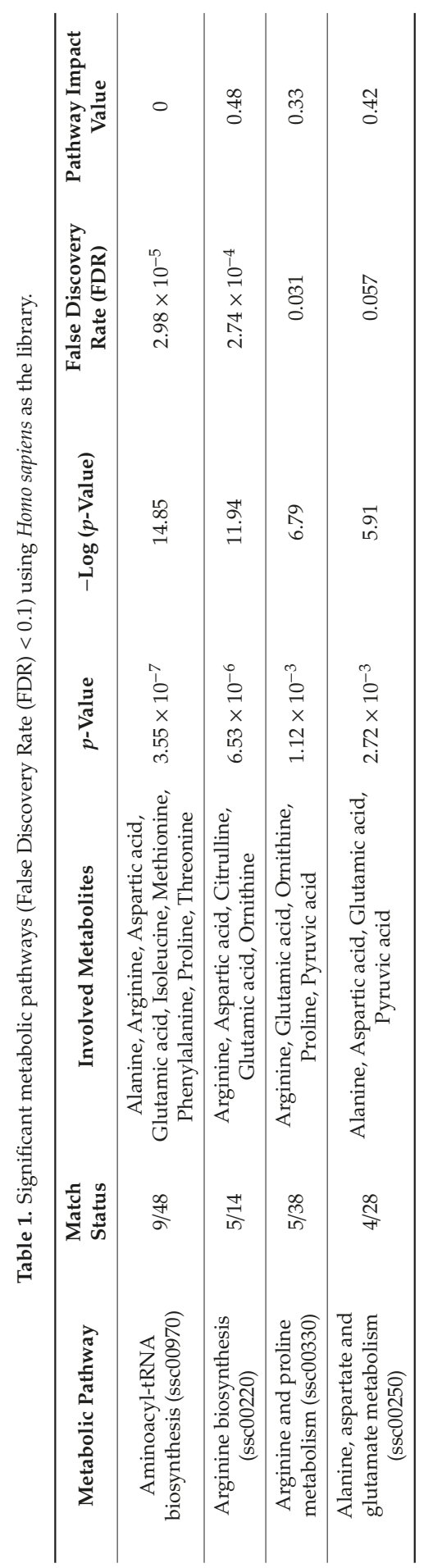



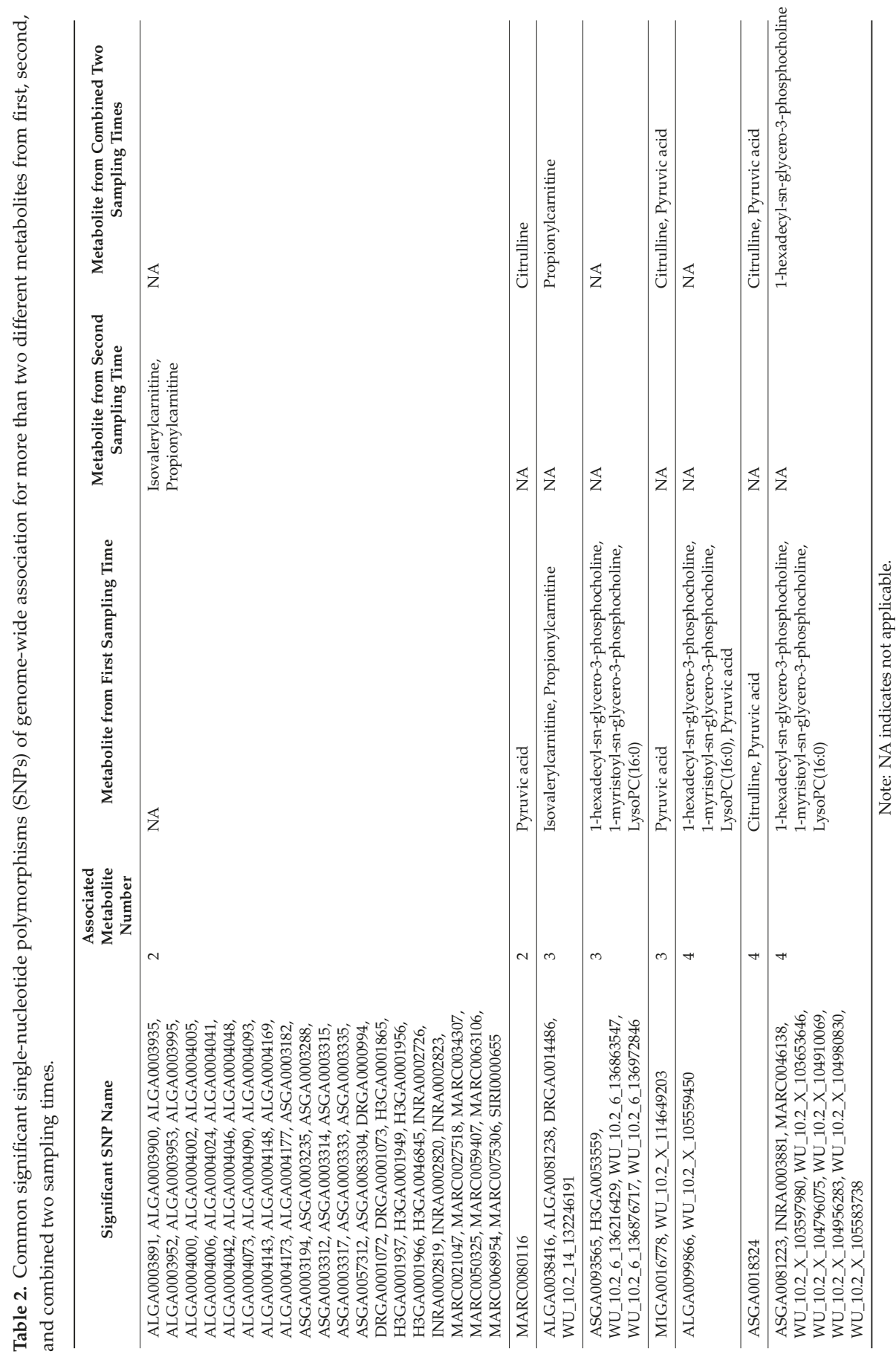
A

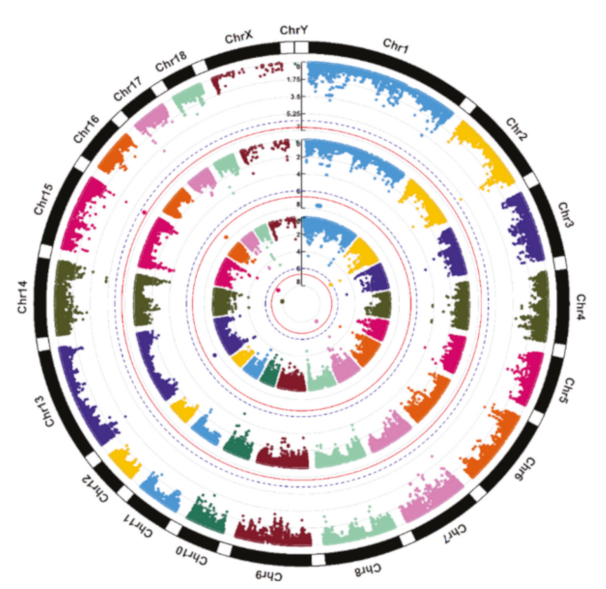

B Propionylcarnitine

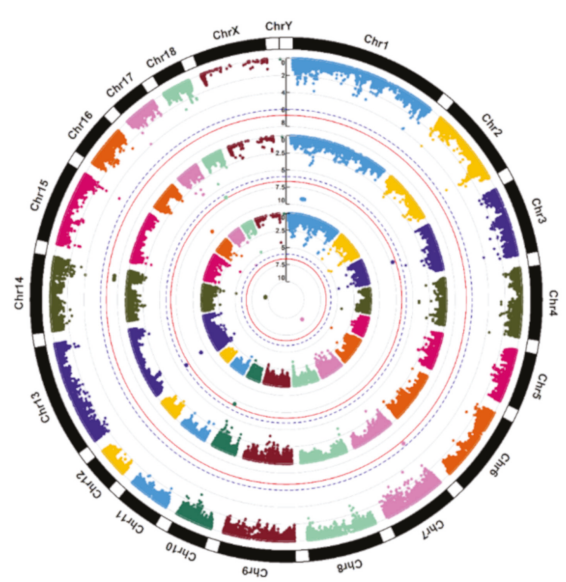

Figure 2. Manhattan plots of genome-wide association for (A) isovalerylcarnitine and (B) propionylcarnitine. Note: $Y$-axis indicates the $\log _{10}(p$-value). Blue dotted and red solid lines indicate the genome-wide threshold of 0.05 and 0.01 after Bonferroni multiple testing, respectively. The three tracks indicate the metabolites from first sampling time, second sampling time, and combined two sampling times from outside to inside.

After annotation of significant SNPs to the neighboring genes and gene components (Sscrofa10.2/susScr3), we found that 90 significant SNPs were within a 500-kb window of 52 neighboring genes (Supplementary Table S1) and that 6 significant SNPs were directly located in the gene components of 5 genes (Table 3). For example, if we only consider the SNPs on chromosome one, we found 29 significant SNPs were near 9 genes (Supplementary Table S1), whereas ALGA0004000, ALGA0004041, and ALGA0004042 were located in the introns of FBXL4 and CCNC (Table 3). These results show that these genes may be involved in regulating abundance of the metabolites that are significantly different between high and low RFI pigs. Between using porcine RefSeq database of Sscrofa10.2/susScr3 and Sscrofa11.1/susScr11, the results of significant SNPs annotated to the genes overlapped greatly, but SNPs had different distances to the annotated genes between two versions (Supplementary Table S1). In Table 3, we found that the annotations of ALGA0004042 and ALGA0061605 to CCNC and MTRF1 were changed from 9th intron and 5th intron to 8th intron and 9th intron, respectively, when we used the Sscrofa11.1/susScr11 database. 
Metabolites 2020, 10, 201

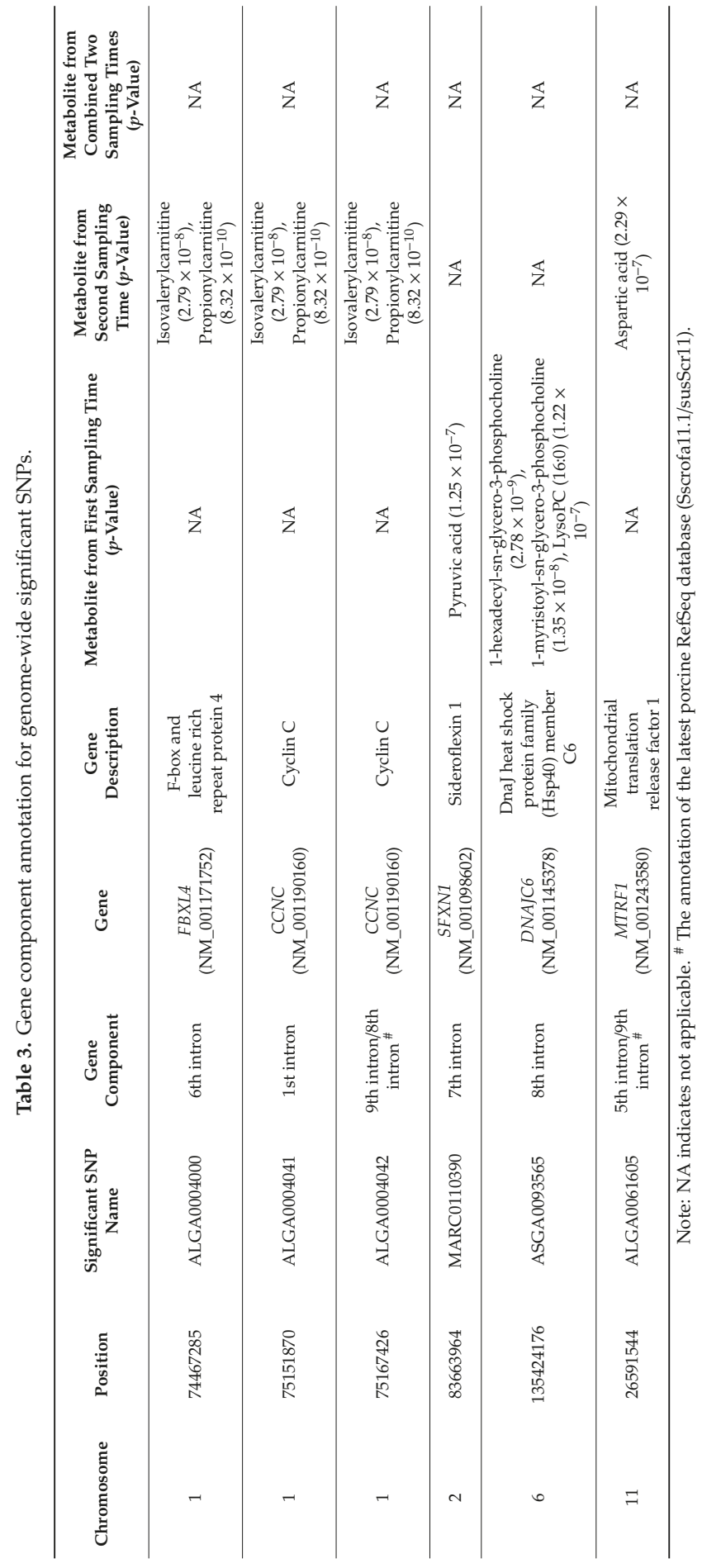


The linkage disequilibrium (LD) pattern for all significant SNPs is shown in the Supplementary Figure S2. From the LD results for 58 significant SNPs on chromosome one, we found that 51 significant SNPs associated with isovalerylcarnitine $\left(p\right.$-value $\left.=2.79 \times 10^{-8}\right)$ and propionylcarnitine $\left(p\right.$-value $\left.=8.32 \times 10^{-10}\right)$ from second sampling time were in strong LD (Figure 3). Among the 58 significant SNPs, five of them were not in LD with the other 53 significant SNPs (Supplementary Figure S2), so they were excluded in the haplotype visualization in the Figure 3. In detail, SNPs annotated to LOC780435 (NM_001078684), FHL5 (NM_001243314), FBXL4 (NM_001171752), CCNC (NM_001190160)/MCHR2 (NM_001044609), and SIM1 (NM_001172585) were in block 2, block 4, block 6, block 8, and block 9/10, respectively. Furthermore, ALGA0004000 in the 6th intron of FBXL4 was in LD of block 6, together with another five SNPs (INRA0002726, MARC0075306, ALGA0003995, ALGA0004002, and ALGA0004005) that were located in the intergenic regions of FBXL4. Especially, three SNPs in strong LD consisted of block 8 with two SNPs (ALGA0004041 and ALGA0004042) located in the second and ninth intron of CCNC (Figure 3, Table 3, and Supplementary Table S1). The number of significant SNPs in strong LDs of the other chromosomes was less than the significant SNP number on chromosome one (Supplementary Figure S2). Notably, MARC0110390 in the 7th intron region of SFXN1 (NM_001098602) on chromosome two and ALGA0061605 in the 5th intron region of MTRF1 (NM_001243580) on chromosome eleven were not in the LD with other SNPs. However, ASGA0093565 in the 8th intron region of DNAJC6 (NM_001145378) was in strong LD with WU_10.2_6_135312468 that was annotated to LEPROT (NM_001145388) (Supplementary Table S1 and Supplementary Figure S2).

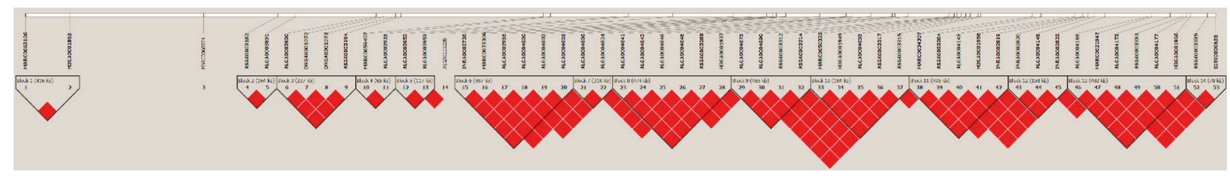

Figure 3. Linkage disequilibrium (LD) pattern for 53 significant SNPs on chromosome one. Note: the solid line triangle indicates LD. One square indicates LD level $\left(\mathrm{r}^{2}\right)$ between two SNPs, and the squares are colored by the $\mathrm{D}^{\prime} \&$ LLOR standard scheme. D' \& LLOR standard scheme is that red indicates LLOR $>2, \mathrm{D}^{\prime}=1$; pink indicates $\operatorname{LLOR}>2, \mathrm{D}<1$; blue indicates $\operatorname{LLOR}<2, \mathrm{D}^{\prime}=1$; and white indicates $\mathrm{LLOR}<2, \mathrm{D}^{\prime}<1$. LLOR is the logarithm of likelihood odds ratio and the reliable index to measure $\mathrm{D}^{\prime}$.

\subsection{Gene and Metabolite Interaction Network}

The most significantly enriched gene-based pathways were the human papillomavirus infection (ssc05165) with five genes (i.e., CCND2, CTNNB1, JAK1, LAMC1, and NFKB1), followed by the PI3K-Akt signaling pathway (ssc04151) with five genes (i.e., CCND2, F2R, JAK1, LAMC1, and NFKB1) and the hepatitis $\mathrm{C}(\mathrm{ssc05160)}$ with four genes (i.e., CLDN8, CTNNB1, JAK1, and NFKB1) (Figure 4A). Based on the gene-gene interaction network analysis, CCNC was in good connection with CDK8, CDK3, and N6AMT1 whereas N6AMT1 was linked to FBXL4 (Figure 4B). Unfortunately, no gene-metabolite interaction network was found in this study. After the clustering of the SNP-related gene component-associated metabolites (Table 3), we found that aspartic acid, 1-hexadecyl-sn-glycero-3-phosphocholine, 1-myristoyl-sn-glycero-3-phosphocholine, and lysoPC(16:0) were clustered in the lower cluster while the upper cluster included the metabolites of isovalerylcarnitine, propionylcarnitine, and pyruvic acid (Figure 4C). Results show that metabolites from Duroc pigs have higher values in the upper cluster than those from lower cluster, but the metabolite values of Landrace pigs are higher in the lower cluster (Figure 4C). Afterwards, we investigated the metabolite values of aspartic acid, isovalerylcarnitine, propionylcarnitine, and pyruvic acid for which the associated significant SNPs were in the introns of MTRF1, FBXL4/CCNC, SFXN1 (Table 3). Generally, propionylcarnitine from the low RFI group had higher values while other three metabolite values in the high RFI group seemed higher, but they are not significantly different between low and high RFI groups ( $p$-value $>0.05$ ) (Figure $4 \mathrm{D}$ ). 
A

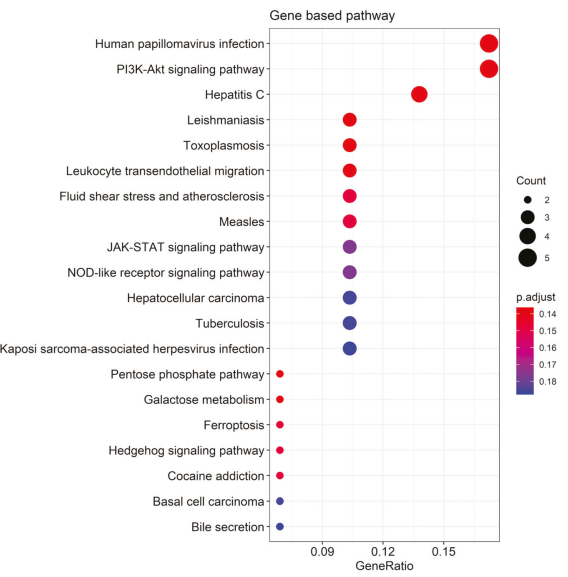

C

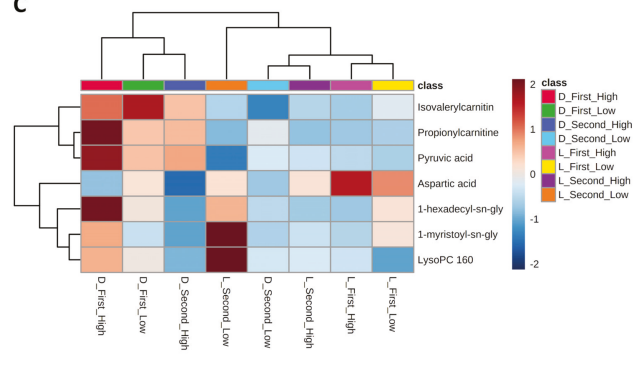

B

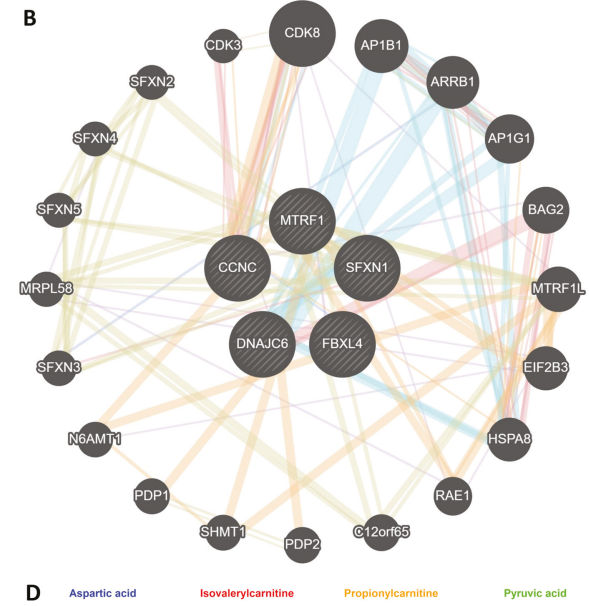

D

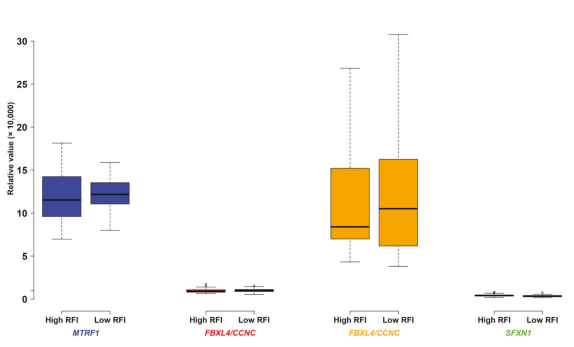

Figure 4. Gene pathway, metabolite cluster, and the interaction network: (A) Pathway for significant SNP-related genes. (B) Network for the genes in which significant SNPs were annotated to gene components. (C) Heatmap cluster for the metabolites that were associated with significant SNPs annotated to gene components. (D) Metabolites (i.e., aspartic acid, isovalerylcarnitine, propionylcarnitine, and pyruvic acid) values in high and low residual feed intake (RFI) pigs associated with the genes in which significant SNPs annotate to gene components. Note: The high RFI pigs and low RFI pigs were from left and right parts of all the pigs $(n=108)$ with one SD of actual RFI values.

\section{Discussion}

\subsection{Metabolites in the PLS-DA and Metabolic Pathways of Pigs}

The previous study reported that different breed types performed differently in RFI variation [8], so RFI-related metabolomics could be breed specific. Therefore, different breeds tend to exhibit different metabolite abundance values, for example, in studies involving the colostrum of pigs [12,13], the milk and plasma of cattle [8,14], the yolk and albumen of chickens [15,16], the plasma of dogs [17], and the fruit metabolite content of tomatoes [18]. In pigs, the heritability and genetic correlation of production traits of Duroc, Landrace, and Yorkshire pigs vary. Duroc pigs showed lower heritability of feed efficiency but greater performance of growth traits $[19,20]$. The metabolomics of this study showed that metabolite values vary between two pig breeds and between the sampling times (Figures 1A and 4C), as the metabolite profiles would change according to the breeds and time points [6]. Metabolites of Duroc from first sampling time and Landrace from second sampling time were apparently stratified, probably because metabolite values of these two groups and their metabolite profiles were different. However, metabolites of Duroc from second sampling time and Landrace from first sampling time were very close, probably because metabolite values of these two groups and their metabolite profiles 
were very similar (Figure 1A). Hence, the breed differences between Duroc and Landrace pigs were driven both by genetic and metabolic factors.

The arginine biosynthesis pathway (ssc00220), where arginine, aspartic acid, citrulline, glutamic acid, and ornithine were significantly enriched in our study (Table 1), plays a crucial role in amino acid metabolism, particularly in the synthesis of citrulline and proline in pigs [21]. By linking arginine, glutamate, and proline in a bidirectional way, the arginine and proline metabolism pathway (ssc00330) biosynthesizes arginine and proline by glutamate. It is observed that proline metabolism is associated with metastasis formation of breast cancer [22]. In dairy cattle, the alanine, aspartate, and glutamate metabolism (ssc00250) identified in the gene-based pathways of our study (Table 1) is the potential metabolic biomarker between the low and high feed efficient conditions [8].

\subsection{Genome-Wide Significant SNP-Related Genes Associated with Metabolites}

The previous GWAS for Duroc pigs identified two pleiotropic QTLs on chromosome one and seven for feed efficiency [20]. Do et al. (2014) [10] revealed 19 significant SNPs located on several chromosomes (e.g., one, three, seven, eight, nine, ten, fourteen, and fifteen) that were highly associated with feed efficiency in Yorkshire pigs. In addition, other studies also found significant SNPs associated with RFI on other chromosomes, for example, SNPs on chromosome five in the growing Piétrain-Large White pigs [23], on chromosome two in a crossed populations [24], on chromosome six in Large White pigs [25], etc. [26,27].

In this study, significant SNPs were mainly located on chromosome one (58/152), but the associated metabolites only mapped to 1-hexadecyl-sn-glycero-3-phosphocholine (1.7\%), 1-myristoyl-sn-glycero-3-phosphocholine $(1.7 \%)$, isovalerylcarnitine $(47.0 \%)$, isoleucyl proline $(0.9 \%)$, propionylcarnitine $(47.0 \%)$, and lysoPC(16:0) $(1.7 \%)$. Obviously, isovalerylcarnitine and propionylcarnitine primarily derived from amino acid catabolism were the major metabolites that associated with nine significant SNP-related genes (i.e., CCNC, FBXL4, FHL5, LOC780435, MAT2B, MCHR2, PNISR, RRAGD, and SIM1) on chromosome one (Supplementary Table S1). A previous study indicated that the amount of isovalerylcarnitine could decrease in the plasma and liver tissues but greatly increased in the muscle tissue, as a byproduct of leucine catabolism [28]. The isovalerylcarnitine compound was reported to be found in high amounts in the colostrum and milk of sows [29]. As a key role in the mitochondrial fatty acid transport and high-energy phosphate exchange, propionylcarnitine could improve cardiac dysfunction by reducing myocardial ischaemia [30].

\subsection{Gene and Metabolite Interaction Network}

Based on the gene interaction node N6AMT1, one gene-gene interaction was found to connect CCNC with FBXL4 (Figure 4B), in which significant SNPs were annotated to gene components and associated with isovalerylcarnitine and propionylcarnitine (Table 3). As the members of $C D K 8$ mediator complex that can regulate $\beta$-catenin-driven transcription, $C C N C$ encodes the cell cycle regulatory protein cyclin $C$ and results in the protein dysfunction due to the mutations of CCNC [31,32]. CCNC is also believed to increase the quiescent cells to maintain CD34 expression after knocking down CCNC expression in human cord blood [33], while the amplification of CCNC was in a relationship with the unfavorable prognosis [34]. FBXL4 is considered to participate in oxidative phosphorylation, mitochondrial dynamics, cell migration, prostate cancer metastasis, circadian GABAergic cyclic alteration, etc. [35-39]. The association results in pigs found that blood and immune traits were associated with the SNPs of FBXL4 [40]. The node N6AMT1 is responsible for DNA 6mA methylation modification as the first glutamine-specific protein methyltransferase characterized in mammals; thus, glutamine could be regulated by the genes that promote porcine growth performance [41,42].

\subsection{Associations Linking SNP Genotypes, Metabolites, and RFI}

To investigate the direct association between SNP genotypes and RFIs, we also conducted GWAS for RFI (i.e., where the GWAS model included RFI as phenotype and SNPs as fixed effect/explanatory 
variable) in the mixed linear model. Unfortunately but as expected due to small sample size, the results showed that no genome-wide significant associations were found between SNPs and RFI values $\left(p\right.$-values $\left.\geq 2.09 \times 10^{-4}\right)$. However, the top SNP was DRGA0008061 $\left(p\right.$-values $\left.=2.09 \times 10^{-4}\right)$, and we found five genes (ANGPTL2, AUTS2, GRIFIN, PTRH1, and SIRT5) in which the top ten SNPs were annotated (Supplementary Table S3). In our previous studies, Banerjee et al. (2020) [11] also revealed that DRGA0008061 was one of the top significant SNPs associated with RFI after genome-wide epistatic interaction network analysis for feed efficiency using the same genotypes and pig populations as used in our current study. Meanwhile, Carmelo et al. (2020) [6] used Kolmogorov-Smirnov test to identify the significant metabolites associated with feed efficiency traits at two time points in Duroc and Landrace pigs. They found that 1-hexadecyl-sn-glycero-3-phosphocholine, 1-myristoyl-sn-glycero-3-phosphocholine, isovalerylcarnitine, lysoPC(16:0), and phosphocholine were significantly ( $p$-value $<0.05$ ) associated with RFI and other feed efficiency traits [6]. By matching the results from Carmelo et al. (2020) [6] with our results, we found that these five metabolites were also our main significant SNP-associated findings in GWAS (Table 3). Therefore, the triangular association of genotypes (SNP), metabolomics (metabolite), and feed efficiency (RFI) is established via our mGWAS (SNPs affecting metabolites) and GWAS (SNPs affecting RFI) and is linked with the previous studies [6,11].

\section{Materials and Methods}

\subsection{Animals, Metabolites, and Genotypes}

A total of 108 pigs were involved in this study including 59 Duroc and 49 Landrace pigs that were part of our own previous published studies [6,11]. The detailed description of the animal experiment and phenotype, metabolite, and genotypes data collection are available from our previously published studies, and all data used in this study were derived from our datasets that were already made public. Metabolite data [6] were accessed using MetaboLights accession ID MTBLS1384 with a link: https://www.ebi.ac.uk/metabolights/MTBLS1384. Genotype data [11] were accessed from National Center for Biotechnology Information (NCBI) GEO accession number: GSE144064 with the following link: https://www.ncbi.nlm.nih.gov/geo/query/acc.cgi?acc=GSE144064. The genotype data was sequenced using GeneSeek-Neogen PorcineSNP80 BeadChip containing 68,528 loci based on the version Sscrofa10.2/susScr3 [11].

As in Carmelo et al. (2020) [6], all the pigs were purebred uncastrated males derived from sixteen-sire families in four generations and fed on the same diets. They had RFI values calculated for each pig as the difference between the observed daily feed intake (DFI) and the predicted daily feed intake (pDFI) [6] following the method of Nguyen et al. (2001) [43]. Nguyen et al. (2001) [43] firstly corrected the DFI for batch and sex and their interaction effects (i.e., fixed effects) and then estimated the pDFI from different regression models including growth rate and back fat after adjustments for above fixed effects; hence, Carmelo et al. (2020) [6] could compute RFIs in the same way by correcting fixed effects in their study. Finally, our study directly used RFIs together with other phenotypes by accessing the public dataset with a link: https://www.ebi.ac.uk/metabolights/MTBLS1384. The range of actual RFI values of Duroc were from -10 to 14 , while Landrace's RFI value range was from -14 to 17 (Figure 5). The previous study conducted the metabolite-trait association analysis for RFI, so it was suggested that fatness or other factors should be adjusted in the calculation of RFI to achieve more accurate association results in their study [6]. As similar means of RFI for Duroc and Landrace pigs were observed in Figure 5 of our study, we assumed that fatness was adjusted in the calculation of RFI, but we cannot determine it. In this study, we selectively chose the extreme left and extreme right tails of distribution of feed efficiency (i.e., actual RFI values) distribution of all the pigs $(n=108)$ with one standard deviation (SD) from the mean $[11,44]$ of actual RFI values. Then, they were defined as high RFI pigs (RFI $\leq-5.23, n=11$ ) and low RFI pigs ( $R F I \geq 5.23, n=16$ ), respectively (Figure 5). The overview of the analysis workflow is shown in Figure 6 and included five scenarios of phenotypes 
in the GWAS analysis based on different transformations of metabolites. The five types of phenotypes were (1) the metabolites from first sampling time, (2) the metabolites from second sampling time, (3) the metabolites from combined two sampling times (i.e., metabolite values from first and second sampling times were combined as an integrated dataset, where each pig had two metabolic values for one metabolite, but genotypes were same for the metabolite values between first and the second sampling times from the same pig), (4) the first component score (component 1) from partial least squares-discriminant analysis (PLS-DA), and (5) the metabolites enriched in the significant metabolic pathways (Figure 6).

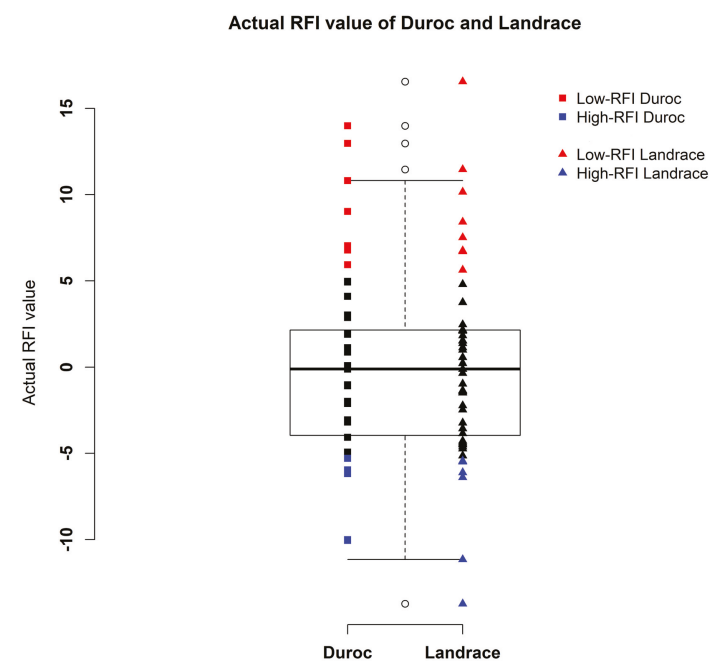

Figure 5. Distribution of actual RFI values of Duroc $(n=59)$ and Landrace $(n=49)$ pigs.

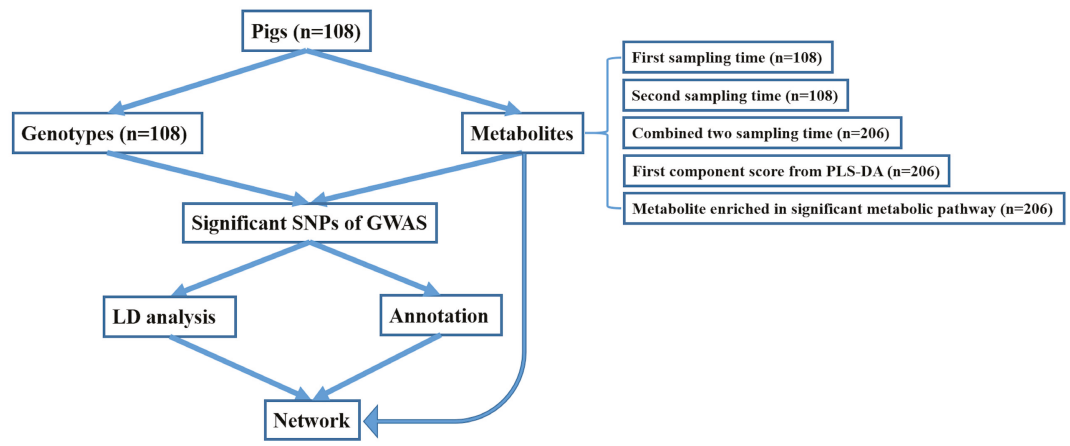

Figure 6. Overall analysis workflow.

Metabolite data was downloaded by accessing MetaboLights accession ID MTBLS1384 with a link, https://www.ebi.ac.uk/metabolights/MTBLS1384, and were collected in two sampling times (i.e., the first sampling time was at the age when pig weighted approximately $28 \mathrm{~kg}$, and the second sampling time was 45 days after the first sampling time) for each pig by the previous study [6]. Finally, 45 metabolites were used in this study (Figure 7) including 16 annotated metabolites (i.e., 1-hexadecyl-sn-glycero-3-phosphocholine, 1-myristoyl-sn-glycero-3-phosphocholine, 
(3-Carboxypropyl)trimethylammonium, 5-methyl-5,6-dihydrouracils, acetaminophen, acetylcarnitine, benzoic acid, cotinine, creatinine, indoleacrylic acid, isoleucyl proline, isovalerylcarnitine, leucyl methionine, lysoPC(16:0), manNAc, and propionylcarnitine) and 29 identified metabolites (i.e., 4-aminobenzoic acid, alanine, arginine, aspartic acid, carnitine, citrulline, cytidine, disaccharide, glutamic acid, guanine, guanosine, hypoxanthine, inosine, isoleucine, lactic acid, methionine, monosaccharide, nicotine amide, ornithine, phenylalanine, proline, pyruvic acid, riboflavine, sorbitol, thiamine, threonine, thymidine, uridine, and xanthine).

\section{Statistical description of metabolites}

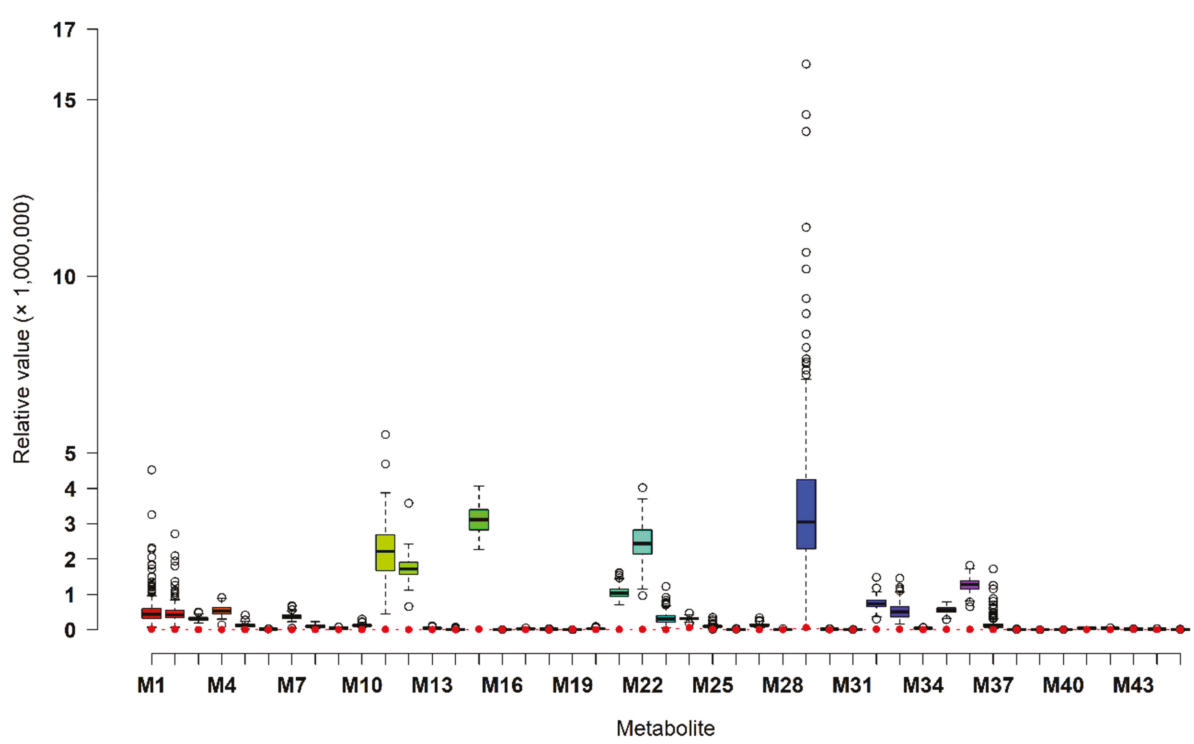

Figure 7. Statistical description of 45 metabolites from combined two sampling times. Note: The red solid circle indicates the limit of detection (LOD) relative value of each metabolite. LOD refers to the lowest value of a metabolite that the LC-MS method can detect. M1 to M45 indicate the metabolites of 1-hexadecyl-sn-glycero-3-phosphocholine, 1-myristoyl-sn-glycero-3-phosphocholine, (3-Carboxypropyl)trimethylammonium, 4-aminobenzoic acid, 5-methyl-5,6-dihydrouracils, acetaminophen, acetylcarnitine, alanine, arginine, aspartic acid, benzoic acid, carnitine, citrulline, cotinine, creatinine, cytidine, disaccharide, glutamic acid, guanine, guanosine, hypoxanthine, indoleacrylic acid, inosine, isoleucine, isoleucyl proline, isovalerylcarnitine, lactic acid, leucyl methionine, lysoPC(16:0), manNAc, methionine, monosaccharide, nicotine amide, ornithine, phenylalanine, proline, propionylcarnitine, pyruvic acid, riboflavine, sorbitol, thiamine, threonine, thymidine, uridine, and xanthine, respectively.

The genotype data was downloaded from NCBI GEO database with accession number: GSE144064 with a link, https://www.ncbi.nlm.nih.gov/geo/query/acc.cgi?acc=GSE144064, that was issued by the previous study [11]. After removing the markers with duplicated SNP positions (i.e., coordinates) ( $\mathrm{n}=274)$, unannotated SNP positions $(\mathrm{n}=2618)$, and no genotypes $(\mathrm{n}=3903)$ from GeneSeek-Neogen PorcineSNP80 BeadChip (68,516 SNP markers here), 61,721 SNP markers remained. Afterwards, we performed the imputation for missing markers using pedigree (i.e., all the pigs were derived from sixteen-sire families in four generations) by FImpute software (version 3) [45], as the closer relatives usually share longer haplotypes; therefore, pedigree information could contribute towards the FImpute software, achieving more accurate imputation $[45,46]$. Quality control (QC) for the imputed genotypes 
was conducted based on the criteria of Hardy-Weinberg equilibrium (HWE $>10^{-7}$ ) and minor allele frequency (MAF $\geq 0.001$ ) by PLINK software (version 1.9) [47].

In this study, we also combined the metabolite values from the first sampling time and the second sampling time as an integrated dataset, so each pig had two values in one metabolite. However, the genotypes for two metabolite values were the same if one metabolite value was from the first sampling time of one pig while the other metabolite value was from the second sampling time of the same pig. In other words, each pig had two different metabolite values but the same genotypes; thus, $\mathrm{QC}$ results of the integrated dataset $(n=206)$ were different from the unintegrated dataset $(n=108)$, especially for HWE but not for MAF. Finally, the genotypes for the first sampling time and the second sampling time retained 47,109 SNP markers after removing unqualified 9337 ( $\mathrm{HWE} \leq 10^{-7}$ ) and 5275 (MAF < 0.001) markers, while the genotypes for the combined two sampling times retained 42,393 SNP markers after removing unqualified 14,053 ( $\mathrm{HWE} \leq 10^{-7}$ ) and 5275 (MAF < 0.001) markers.

\subsection{Partial Least Squares-Discriminant Analysis and Metabolic Pathway Enrichment for 45 Metabolites}

The partial least squares-discriminant analysis (PLS-DA) and metabolic pathway analysis for the 45 metabolites were performed by MetaboAnalyst software (version 4.0) [48] using Homo sapiens as the library. Fishers' exact test and relative betweenness centrality were used for the overrepresented analysis and the pathway impact value calculation (i.e., sum of importance measures of the matched metabolites divided by the sum of the importance measures of all the metabolites), respectively [49]. The first component scores (component 1) and metabolites enriched in the significant metabolic pathways after false discovery rate (FDR) correction of multiple hypothesis testing (FDR $<0.1$ ) were selected as phenotypes of the transformed metabolites for GWAS. The mean calculated for the metabolites enriched in each significant metabolic pathway was considered as transformed metabolite values; thus, each pathway had one transformed metabolite value (i.e., the mean).

\subsection{Mixed Linear Model Based Association Analysis}

In this study, we considered other environmental factors (e.g., age) the same among all the pigs, so we only used breed and RFI as the fixed effects to directly link metabolites with genotypes. GWAS for 45 single metabolites and transformed metabolites (i.e., component 1 and enriched metabolites) was conducted by mixed linear model based association analysis in GCTA software (version 1.93.0) [50]. The mixed linear model is as follows:

$$
\mathrm{y}=\mathrm{X} b+g+e,
$$

where $y$ is the vector of phenotypes (i.e., metabolites from the first, second, and combined two sampling times and the transformed metabolites); $b$ is the vector of fixed effects including intercept, breed effects (i.e., Duroc and Landrace pigs), RFI effects (i.e., actual RFI values included as covariates), and SNP effects (i.e., candidate SNPs included as covariates) to be tested; $X$ is the design matrix for fixed effects that includes SNP genotype indicators (i.e., 0,1 , or 2); $g$ is the vector of polygenic effects as random effects that are the accumulated effects of all SNPs; and $e$ is the vector of residual effects. The polygenic and residual variances are $\operatorname{Var}[g]=G \sigma_{g}^{2}$ and $\operatorname{Var}[e]=I \sigma_{e}^{2}$, where $G$ and $I$ are the genetic relationship matrix (GRM) calculated using all SNPs and identity matrix, respectively.

\subsection{Significant SNPs and Their Annotated Genes}

The significant SNPs for GWAS were defined when the $p$-values were less than the threshold after Bonferroni correction for multiple hypothesis testing on genome level. The threshold for metabolites from the first and second sampling times was $1.06 \times 10^{-6}$ (i.e., $0.05 / 47109$ ), while the threshold for combined two sampling times was $1.18 \times 10^{-6}$ (i.e., $0.05 / 42393$ ). Then, the significant SNPs were annotated to the genes and gene components (i.e., promoters, exons, and introns) of porcine RefSeq database (Sscrofa10.2/susScr3) downloaded from University of California Santa Cruz (UCSC) genome browser (https://genome.ucsc.edu/cgi-bin/hgTables), where a window of $500 \mathrm{~kb}$ was used for the 
annotation of intergenic regions to neighboring genes. In addition, we used the reference SNP (rsfSNP) ID (i.e., specific rs number) of significant SNPs to annotate them to the genes and gene components of latest porcine RefSeq database (Sscrofa11.1/susScr11).

Linkage disequilibrium (LD) analysis to display the potential haplotypes for the significant SNPs was performed using Haploview software (version 4.2) [51]. SNPs with a distance larger than $500 \mathrm{~kb}$ were ignored in the pairwise comparisons for LD analysis.

\subsection{Gene-Based Pathway Enrichment Analysis and Gene-Metabolite Interaction Network}

We used R package KEGG.db (version 3.2.3) of Sus scrofa species to annotate SNP-related genes in the gene-based pathway enrichments. Based on the adjusted $p$-values (p.adjust) $<0.2$ under FDR control, the gene-based pathways were finally realized by R package clusterprofiler (version 3.12.0) [52]. The gene-gene interaction networks were created by GeneMANIA server [53,54] with default settings using Homo sapiens as the library. Then, the gene-metabolite networks for interactions between SNP-related genes and phenotype-related metabolites were created by MetaboAnalyst tool [55] with default settings using the same library of Homo sapiens. Significant SNP-associated metabolites based on the low-high RFI pigs were hierarchically clustered by Ward's method in Euclidean distance [56]. Then, a heat map for averaged metabolite clustering was visualized by MetaboAnalyst tool [48].

\section{Conclusions}

We utilized metabolic and genomic datasets from a total of 108 pigs that were made available for this study from our own previously published studies $[6,11]$ in publicly available data repositories. These studies involved 59 Duroc and 49 Landrace pigs and consisted of data on feed efficiency (RFI), genotype (PorcineSNP80 BeadChip) data, and metabolomic data (45 final metabolite datasets derived from LC-MS system). Utilizing these datasets, our main aim was to identify genetic variants (SNPs) that affect 45 different metabolite concentrations in plasma collected at the start and end of the performance testing of pigs categorized as high or low in their feed efficiency, as measured by RFI values. Genome-wide significant genetic variants could be then used as potential genetic or biomarkers in breeding programs for feed efficiency. In order to achieve this main objective, we performed GWAS in the mixed linear model-based association analysis and found 152 genome-wide significant snps $\left(p\right.$-value $\left.<1.06 \times 10^{-6}\right)$ in association with 17 metabolites that included 90 significant SNPs annotated to 52 genes. On chromosome one alone, we found SNPs in strong LD that could be annotated to FBXL4 and CCNC; it consisted of two haplotype blocks, where three SNPs (ALGA0004000, ALGA0004041, and ALGA0004042) were in the intron regions of FBXL4 and CCNC. The interaction network analyses revealed that $C C N C$ and FBXL4 were linked to each other by N6AMT1 gene and were associated with compounds isovalerylcarnitine and propionylcarnitine. The identified genetic variants and genes affecting important metabolites in high versus low feed efficient pigs could be considered as potential genetic or biomarkers, but we recommend that these results are validated in much higher sample size.

Supplementary Materials: The following are available online at http://www.mdpi.com/2218-1989/10/5/201/s1, Supplementary Table S1. All the significant SNPs of genome-wide association with chromosome, position, and p-value information for metabolites from first, second, and combined two sampling times. Supplementary Table S2. All the metabolites in association with significant SNPs from first, second, and combined two sampling times. Supplementary Table S3. Top ten SNPs associated with residual feed intake (RFI). Supplementary Figure S1. Manhattan plots of genome-wide association for 43 metabolites. Supplementary Figure S2. Linkage disequilibrium (LD) pattern for all significant SNPs.

Author Contributions: H.N.K. was a grant holder and lead PI for the FeedOMICS project who conceived and designed the experiments and made the related datasets available in public repositories. X.W. analyzed the data under the supervision of H.N.K., X.W. and H.N.K. wrote the first draft of this paper, which was improved by H.N.K. All authors have read and agree to the published version of the manuscript.

Funding: Xiao Wang was funded by the FeedOMICS research project, headed by Haja Kadarmideen at DTU Denmark. FeedOMICS project was funded by the Independent Research Fund Denmark (DFF)—Technology and Production (FTP) grant (grant number: 4184-00268B). 
Acknowledgments: Authors thank open access platforms MetaboLights and NCBI-GEO from which we downloaded the datasets for research reported in this study and cited under the section "availability of data and materials". The authors thank Claus Thorn Ekstrøm from Faculty of Health and Medical Sciences, University of Copenhagen for his advice on statistical modelling.

Conflicts of Interest: The authors declare that there are no conflict of interest.

Availability of Data and Materials: All datasets used in this paper are from public repositories. Metabolite data were accessed using MetaboLights accession ID MTBLS1384 with a link: https:/www.ebi.ac.uk/metabolights/ MTBLS1384. Genotype data were accessed from NCBI GEO accession number GSE144064 with the following link: https://www.ncbi.nlm.nih.gov/geo/query/acc.cgi?acc=GSE144064). The details of these datasets can be found in Carmelo et al. (2020) [6] and Banerjee et al. (2020) [11].

$\begin{array}{ll}\text { Abbreviations } \\ \text { Component 1 } & \text { First component score } \\ \text { DFI } & \text { Daily feed intake } \\ \text { FC } & \text { Fold change } \\ \text { FDR } & \text { False discovery rate } \\ \text { LC-MS } & \text { Liquid chromatography-mass spectrometry } \\ \text { GRM } & \text { Genetic relationship matrix } \\ \text { GWAS } & \text { Genome-wide association study } \\ \text { HWE } & \text { Hardy-Weinberg equilibrium } \\ \text { LD } & \text { Linkage disequilibrium } \\ \text { LOD } & \text { Limit of detection } \\ \text { LLOR } & \text { Logarithm of likelihood odd ratio } \\ \text { MAF } & \text { Minor allele frequency } \\ \text { mGWAS } & \text { Metabolite GWAS } \\ \text { NCBI } & \text { National Center for Biotechnology Information } \\ \text { pDFI } & \text { Predicted daily feed intake } \\ \text { PLS-DA } & \text { Partial least squares-discriminant analysis } \\ \text { QC } & \text { Quality control } \\ \text { QTL } & \text { Quantitative trait locus } \\ \text { RFI } & \text { Residual feed intake } \\ \text { SNP } & \text { Single nucleotide polymorphism } \\ \text { UCSC } & \text { University of California Santa Cruz }\end{array}$

\section{References}

1. Wellcome Trust Case Control Consortium. Genome-wide association study of 14,000 cases of seven common diseases and 3,000 shared controls. Nature 2007, 447, 661-678. [CrossRef] [PubMed]

2. Suravajhala, P.; Kogelman, L.J.; Kadarmideen, H.N. Multi-omic data integration and analysis using systems genomics approaches: Methods and applications in animal production, health and welfare. Genet. Sel. Evol. 2016, 48, 38. [CrossRef] [PubMed]

3. Beebe, K.; Kennedy, A.D. Sharpening Precision Medicine by a Thorough Interrogation of Metabolic Individuality. Comput. Struct. Biotechnol. J. 2016, 14, 97-105. [CrossRef] [PubMed]

4. Fiehn, O. Metabolomics-The link between genotypes and phenotypes. Plant Mol. Boil. 2002, 48, 155-171. [CrossRef]

5. Liu, H.; Chen, Y.; Ming, D.; Wang, J.; Li, Z.; Ma, X.; Wang, J.; Van Milgen, J.; Wang, F. Integrative analysis of indirect calorimetry and metabolomics profiling reveals alterations in energy metabolism between fed and fasted pigs. J. Anim. Sci. Biotechnol. 2018, 9, 41. [CrossRef]

6. Carmelo, V.A.O.; Banerjee, P.; Diniz, W.J.D.S.; Kadarmideen, H.N. Metabolomic networks and pathways associated with feed efficiency and related-traits in Duroc and Landrace pigs. Sci. Rep. 2020, 10, 1-14. [CrossRef]

7. Kenéz, Á.; Dänicke, S.; Rolle-Kampczyk, U.; Von Bergen, M.; Huber, K. A metabolomics approach to characterize phenotypes of metabolic transition from late pregnancy to early lactation in dairy cows. Metabolomics 2016, 12. [CrossRef] 
8. Wang, X.; Kadarmideen, H.N. Metabolomics Analyses in High-Low Feed Efficient Dairy Cows Reveal Novel Biochemical Mechanisms and Predictive Biomarkers. Metabolites 2019, 9, 151. [CrossRef]

9. Gieger, C.; Geistlinger, L.; Altmaier, E.; De Angelis, M.H.; Kronenberg, F.; Meitinger, T.; Mewes, H.-W.; Wichmann, H.-E.; Weinberger, K.M.; Adamski, J.; et al. Genetics Meets Metabolomics: A Genome-Wide Association Study of Metabolite Profiles in Human Serum. PLoS Genet. 2008, 4, e1000282. [CrossRef]

10. Do, D.N.; Strathe, A.B.; Ostersen, T.; Pant, S.; Kadarmideen, H.N. Genome-wide association and pathway analysis of feed efficiency in pigs reveal candidate genes and pathways for residual feed intake. Front. Genet. 2014, 5. [CrossRef]

11. Banerjee, P.; Adriano, V.; Carmelo, O.; Kadarmideen, H.N. Genome-Wide Epistatic Interaction Networks Affecting Feed Efficiency in Duroc and Landrace Pigs. Front Genet. 2020, 11, 121. [CrossRef] [PubMed]

12. Helke, K.L.; Nelson, K.N.; Sargeant, A.M.; Jacob, B.; McKeag, S.; Haruna, J.; Vemireddi, V.; Greeley, M.; Brocksmith, D.; Navratil, N.; et al. Pigs in Toxicology: Breed Differences in Metabolism and Background Findings. Toxicol. Pathol. 2016, 44, 575-590. [CrossRef] [PubMed]

13. Picone, G.; Zappaterra, M.; Luise, D.; Trimigno, A.; Capozzi, F.; Motta, V.; Davoli, R.; Costa, L.N.; Bosi, P.; Trevisi, P. Metabolomics characterization of colostrum in three sow breeds and its influences on piglets' survival and litter growth rates. J. Anim. Sci. Biotechnol. 2018, 9, 23. [CrossRef] [PubMed]

14. Sundekilde, U.; Frederiksen, P.D.; Clausen, M.R.; Larsen, L.B.; Bertram, H. Relationship between the Metabolite Profile and Technological Properties of Bovine Milk from Two Dairy Breeds Elucidated by NMR-Based Metabolomics. J. Agric. Food Chem. 2011, 59, 7360-7367. [CrossRef]

15. Goto, T.; Mori, H.; Shiota, S.; Tomonaga, S. Metabolomics Approach Reveals the Effects of Breed and Feed on the Composition of Chicken Eggs. Metabolites 2019, 9, 224. [CrossRef]

16. Yin, J.D.; Shang, X.G.; Li, D.F.; Wang, F.L.; Guan, Y.F.; Wang, Z.Y. Effects of Dietary Conjugated Linoleic Acid on the Fatty Acid Profile and Cholesterol Content of Egg Yolks from Different Breeds of Layers. Poult. Sci. 2008, 87, 284-290. [CrossRef]

17. Middleton, R.P.; Lacroix, S.; Scott-Boyer, M.-P.; Dordevic, N.; Kennedy, A.D.; Slusky, A.; Carayol, J.; Petzinger-Germain, C.; Beloshapka, A.; Kaput, J. Metabolic Differences between Dogs of Different Body Sizes. J. Nutr. Metab. 2017, 2017, 1-11. [CrossRef]

18. Zhu, G.; Wang, S.; Huang, Z.; Zhang, S.; Liao, Q.; Zhang, C.-Z.; Lin, T.; Qin, M.; Peng, M.; Yang, C.; et al. Rewiring of the Fruit Metabolome in Tomato Breeding. Cell 2018, 172, 249-261.e12. [CrossRef]

19. Do, D.N.; Strathe, A.B.; Jensen, J.; Mark, T.; Kadarmideen, H.N. Genetic parameters for different measures of feed efficiency and related traits in boars of three pig breeds. J. Anim. Sci. 2013, 91, 4069-4079. [CrossRef]

20. Ding, R.; Yang, M.; Wang, X.; Quan, J.; Zhuang, Z.; Zhou, S.; Li, S.; Xu, Z.; Zheng, E.; Cai, G.; et al. Genetic Architecture of Feeding Behavior and Feed Efficiency in a Duroc Pig Population. Front. Genet. 2018, 9, 220. [CrossRef]

21. Dekaney, C.M.; Wu, G.; Jaeger, L.A. Gene expression and activity of enzymes in the arginine biosynthetic pathway in porcine fetal small intestine. Pediatr. Res. 2003, 53, 274-280. [CrossRef] [PubMed]

22. Elia, I.; Broekaert, D.; Christen, S.; Boon, R.; Radaelli, E.; Orth, M.F.; Verfaillie, C.M.; Grunewald, T.G.P.; Fendt, S.-M. Proline metabolism supports metastasis formation and could be inhibited to selectively target metastasizing cancer cells. Nat. Commun. 2017, 8, 15267. [CrossRef] [PubMed]

23. Gilbert, H.; Riquet, J.; Gruand, J.; Billon, Y.; Fève, K.; Sellier, P.; Noblet, J.; Bidanel, J.-P. Detecting QTL for feed intake traits and other performance traits in growing pigs in a Piétrain-Large White backcross. Animal 2010, 4, 1308-1318. [CrossRef]

24. Shirali, M.; Duthie, C.-A.; Doeschl-Wilson, A.; Knap, P.W.; Kanis, E.; Van Arendonk, J.; Roehe, R. Novel insight into the genomic architecture of feed and nitrogen efficiency measured by residual energy intake and nitrogen excretion in growing pigs. BMC Genet. 2013, 14, 121. [CrossRef] [PubMed]

25. Sanchez, M.P.; Tribout, T.; Iannuccelli, N.; Bouffaud, M.; Servin, B.; Tenghe, A.; Déhais, P.; Muller, N.; Del Schneider, M.P.; Mercat, M.-J.; et al. A genome-wide association study of production traits in a commercial population of Large White pigs: Evidence of haplotypes affecting meat quality. Genet. Sel. Evol. 2014, 46, 12. [CrossRef]

26. Onteru, S.K.; Gorbach, D.M.; Young, J.M.; Garrick, D.J.; Dekkers, J.C.M.; Rothschild, M.F. Whole Genome Association Studies of Residual Feed Intake and Related Traits in the Pig. PLoS ONE 2013, 8, e61756. [CrossRef] 
27. Do, D.N.; Ostersen, T.; Strathe, A.B.; Mark, T.; Jensen, J.; Kadarmideen, H.N. Genome-wide association and systems genetic analyses of residual feed intake, daily feed consumption, backfat and weight gain in pigs. BMC Genet. 2014, 15, 27. [CrossRef]

28. Makowski, L.; Noland, R.C.; Koves, T.R.; Xing, W.; Ilkayeva, O.R.; Muehlbauer, M.J.; Stevens, R.D.; Muoio, D.M. Metabolic profiling of PPAR $\alpha-/-$ mice reveals defects in carnitine and amino acid homeostasis that are partially reversed by oral carnitine supplementation. FASEB J. 2009, 23, 586-604. [CrossRef]

29. Kerner, J.; Froseth, J.A.; Miller, E.R.; Bieber, L.L. A study of the acylcarnitine content of sows' colostrum, milk and newborn piglet tissues: Demonstration of high amounts of isovaleryl-carnitine in colostrum and milk. J. Nutr. 1984, 114, 854-861. [CrossRef]

30. Bartels, G.L.; Holwerda, K.J.; Kruijssen, D.A.C.M.; Remme, W.J. Anti-ischaemic efficacy of L-propionylcarnitine-A promising novel metabolic approach to ischaemia? Eur. Heart J. 1996, 17, 414-420. [CrossRef]

31. Lew, D.J.; Dulić, V.; Reed, S.I. Isolation of three novel human cyclins by rescue of G1 cyclin (cln) function in yeast. Cell 1991, 66, 1197-1206. [CrossRef]

32. Arai, E.; Sakamoto, H.; Ichikawa, H.; Totsuka, H.; Chiku, S.; Gotoh, M.; Mori, T.; Nakatani, T.; Ohnami, S.; Nakagawa, T.; et al. Multilayer-omics analysis of renal cell carcinoma, including the whole exome, methylome and transcriptome. Int. J. Cancer 2014, 135, 1330-1342. [CrossRef]

33. Miyata, Y.; Liu, Y.; Jankovic, V.; Sashida, G.; Lee, J.M.; Shieh, J.H.; Naoe, T.; Moore, M.; Nimer, S.D. Cyclin C regulates human hematopoietic stem/progenitor sell quiescence. Stem Cells 2010, 28, 308-317. [CrossRef]

34. Bondi, J.; Husdal, A.; Bukholm, G.; Nesland, J.M.; Bakka, A.; Bukholm, I.R.K. Expression and gene amplification of primary (A, B1, D1, D3, and E) and secondary (C and H) cyclins in colon adenocarcinomas and correlation with patient outcome. J. Clin. Pathol. 2005, 58, 509-514. [CrossRef]

35. El-Hattab, A.W.; Dai, H.; Almannai, M.; Wang, J.; Faqeih, E.A.; Al Asmari, A.; Saleh, M.A.M.; Elamin, M.A.O.; Alfadhel, M.; Alkuraya, F.S.; et al. Molecular and clinical spectra of FBXL4 deficiency. Hum. Mutat. 2017, 38, 1649-1659. [CrossRef] [PubMed]

36. Ballout, R.A.; Al Alam, C.; Bonnen, P.E.; Huemer, M.; El-Hattab, A.W.; Shbarou, R. FBXL4-Related Mitochondrial DNA Depletion Syndrome 13 (MTDPS13): A Case Report With a Comprehensive Mutation Review. Front. Genet. 2019, 10, 39. [CrossRef]

37. Bonnen, P.E.; Yarham, J.W.; Besse, A.; Wu, P.; Faqeih, E.A.; Al-Asmari, A.M.; Saleh, M.A.M.; Eyaid, W.; Hadeel, A.; He, L.; et al. Mutations in FBXL4 cause mitochondrial encephalopathy and a disorder of mitochondrial DNA maintenance. Am. J. Hum. Genet. 2013, 93, 471-481. [CrossRef] [PubMed]

38. Stankiewicz, E.; Mao, X.; Mangham, D.C.; Xu, L.; Yeste-Velasco, M.; Fisher, G.; North, B.; Chaplin, T.; Young, B.; Wang, Y.; et al. Identification of FBXL4 as a Metastasis Associated Gene in Prostate Cancer. Sci. Rep. 2017, 7, 5124. [CrossRef] [PubMed]

39. Li, Q.; Li, Y.; Wang, X.; Qi, J.; Jin, X.; Tong, H.; Zhou, Z.; Zhang, Z.C.; Han, J. Fbxl4 Serves as a Clock Output Molecule that Regulates Sleep through Promotion of Rhythmic Degradation of the GABAA Receptor. Curr. Biol. 2017, 27, 3616-3625.e5. [CrossRef]

40. Li, Y.; Yang, S.L.; Tang, Z.L.; Cui, W.T.; Mu, Y.L.; Chu, M.X.; Zhao, S.H.; Wu, Z.F.; Li, K.; Peng, K.M. Expression and SNP association analysis of porcine FBXL4 gene. Mol. Biol. Rep. 2009, 37, 579-585. [CrossRef]

41. A Cabrera, R.; Usry, J.L.; Arellano, C.; Nogueira, E.T.; Kutschenko, M.; Moeser, A.; Odle, J. Effects of creep feeding and supplemental glutamine or glutamine plus glutamate (Aminogut) on pre- and post-weaning growth performance and intestinal health of piglets. J. Anim. Sci. Biotechnol. 2013, 4, 29. [CrossRef] [PubMed]

42. Hsu, C.B.; Lee, J.W.; Huang, H.J.; Wang, C.H.; Lee, T.T.; Yen, H.T.; Yu, B. Effects of Supplemental Glutamine on Growth Performance, Plasma Parameters and LPS-induced Immune Response of Weaned Barrows after Castration. Asian Australas. J. Anim. Sci. 2012, 25, 674-681. [CrossRef] [PubMed]

43. Nguyen, N.H.; McPhee, C.P.; Daniels, L.J.; Wade, C.M. Selection for growth rate in pigs on restricted feeding: Genetic parameters and correlated responses in residual feed intake. Proc. Assoc. Advmt. Anim. Breed. Genet. 2001, 14, 219-222.

44. Wang, X.; Ma, P.; Liu, J.-F.; Zhang, Q.; Zhang, Y.; Ding, X.; Jiang, L.; Wang, Y.; Zhang, Y.; Sun, D.; et al. Genome-wide association study in Chinese Holstein cows reveal two candidate genes for somatic cell score as an indicator for mastitis susceptibility. BMC Genet. 2015, 16, 111. [CrossRef]

45. Sargolzaei, M.; Chesnais, J.; Schenkel, F. A new approach for efficient genotype imputation using information from relatives. BMC Genom. 2014, 15, 478. [CrossRef] 
46. Wang, X.; Su, G.; Hao, D.; Lund, M.S.; Kadarmideen, H.N. Comparisons of improved genomic predictions generated by different imputation methods for genotyping by sequencing data in livestock populations. J. Anim. Sci. Biotechnol. 2020, 11, 3-12. [CrossRef]

47. Purcell, S.M.; Neale, B.; Todd-Brown, K.; Thomas, L.; Ferreira, M.A.; Bender, D.; Maller, J.; Sklar, P.; De Bakker, P.I.W.; Daly, M.J.; et al. PLINK: A Tool Set for Whole-Genome Association and Population-Based Linkage Analyses. Am. J. Hum. Genet. 2007, 81, 559-575. [CrossRef]

48. Xia, J.; Wishart, D.S. MetPA: A web-based metabolomics tool for pathway analysis and visualization. Bioinformatics 2010, 26, 2342-2344. [CrossRef]

49. Xia, J.; Wishart, D.S. Web-based inference of biological patterns, functions and pathways from metabolomic data using MetaboAnalyst. Nat. Protoc. 2011, 6, 743-760. [CrossRef]

50. Yang, J.; Lee, S.H.; Goddard, M.E.; Visscher, P.M. GCTA: A Tool for Genome-wide Complex Trait Analysis. Expanding the Spectrum of BAF-Related Disorders: De Novo Variants in SMARCC2 Cause a Syndrome with Intellectual Disability and Developmental Delay. Am. J. Hum. Genet. 2011, 88, 76-82. [CrossRef]

51. Barrett, J.C.; Fry, B.; Maller, J.; Daly, M.J. Haploview: Analysis and visualization of LD and haplotype maps. Bioinformatics 2005, 21, 263-265. [CrossRef] [PubMed]

52. Yu, G.; Wang, L.-G.; Han, Y.; He, Q.-Y. clusterProfiler: An R Package for Comparing Biological Themes Among Gene Clusters. OMICS A J. Integr. Boil. 2012, 16, 284-287. [CrossRef] [PubMed]

53. Mostafavi, S.; Ray, D.; Warde-Farley, D.; Grouios, C.; Morris, Q. GeneMANIA: A real-time multiple association network integration algorithm for predicting gene function. Genome Biol. 2008, 9, S4. [CrossRef] [PubMed]

54. Warde-Farley, D.; Donaldson, S.L.; Comes, O.; Zuberi, K.; Badrawi, R.; Chao, P.; Franz, M.; Grouios, C.; Kazi, F.; Lopes, C.T.; et al. The GeneMANIA prediction server: Biological network integration for gene prioritization and predicting gene function. Nucleic Acids Res. 2010, 38, W214-W220. [CrossRef]

55. Chong, J.; Soufan, O.; Li, C.; Caraus, I.; Li, S.; Bourque, G.; Wishart, D.S.; Xia, J. MetaboAnalyst 4.0: Towards more transparent and integrative metabolomics analysis. Nucleic Acids Res. 2018, 46, W486-W494. [CrossRef]

56. Ward, J.H., Jr. Hierarchical Grouping to Optimize an Objective Function. J. Am. Stat. Assoc. 1963, 58, $236-244$. [CrossRef]

(C) 2020 by the authors. Licensee MDPI, Basel, Switzerland. This article is an open access article distributed under the terms and conditions of the Creative Commons Attribution (CC BY) license (http://creativecommons.org/licenses/by/4.0/). 



\title{
Article \\ Integrative Analysis of Metabolomic and Transcriptomic Profiles Uncovers Biological Pathways of Feed Efficiency in Pigs
}

\author{
Priyanka Banerjee, Victor Adriano Okstoft Carmelo and Haja N. Kadarmideen * \\ Quantitative Genomics, Bioinformatics and Computational Biology Group, Department of Applied Mathematics \\ and Computer Science, Technical University of Denmark, 2800 Kongens Lyngby, Denmark; \\ priyankabnrj@gmail.com (P.B.); vaocar@dtu.dk (V.A.O.C.) \\ * Correspondence: hajak@dtu.dk; Tel.: +45-45255223
}

Received: 21 May 2020; Accepted: 4 July 2020; Published: 6 July 2020

\begin{abstract}
Feed efficiency (FE) is an economically important trait. Thus, reliable predictors would help to reduce the production cost and provide sustainability to the pig industry. We carried out metabolome-transcriptome integration analysis on 40 purebred Duroc and Landrace uncastrated male pigs to identify potential gene-metabolite interactions and explore the molecular mechanisms underlying FE. To this end, we applied untargeted metabolomics and RNA-seq approaches to the same animals. After data quality control, we used a linear model approach to integrate the data and find significant differently correlated gene-metabolite pairs separately for the breeds (Duroc and Landrace) and FE groups (low and high FE) followed by a pathway over-representation analysis. We identified 21 and 12 significant gene-metabolite pairs for each group. The valine-leucine-isoleucine biosynthesis/degradation and arginine-proline metabolism pathways were associated with unique metabolites. The unique genes obtained from significant metabolite-gene pairs were associated with sphingolipid catabolism, multicellular organismal process, cGMP, and purine metabolic processes. While some of the genes and metabolites identified were known for their association with FE, others are novel and provide new avenues for further research. Further validation of genes, metabolites, and gene-metabolite interactions in larger cohorts will elucidate the regulatory mechanisms and pathways underlying FE.
\end{abstract}

Keywords: feed efficiency; linear model; metabolomics; pigs; transcriptomics

\section{Introduction}

Feed represents about $60-70 \%$ of total pork production costs in modern pig production. Thus, to decrease costs and increase profitability, it is pivotal to identify feed efficient (FE) animals [1]. However, due to the polygenic architecture of FE, individual pigs in a herd exhibit considerable variation in FE despite belonging to similar genetic background and environment [2]. Considering this variation, different methods have been proposed and widely used to measure the FE, including feed conversion ratio (FCR) and residual feed intake (RFI) [1,3]. FCR is the ratio of feed intake (FI) per unit body weight gain and is affected by many factors such as breed, sex, diet, and environmental conditions [4,5]. Pigs with low FCR are considered high FE and vice-versa. RFI estimates the difference between actual and expected FI predicted on production traits as average daily gain (ADG) [6]. Since FCR considers both FI and weight gain, and FCR is also one of the critical predictors of FE, it suggests that feed efficient pigs may possess different physiological-biochemical profiles compared to the inefficient ones [2].

Based on the advances in omics technologies, several approaches have been adopted to shed light on the genetic mechanisms underlying FE in pigs. Among these omics technologies, transcriptomics 
and metabolomics have provided tools to elucidate the molecular basis of FE. While transcriptomics allows us to have a transcriptional snapshot of genes underpinning the phenotype under investigation, metabolomics bridges the gap between genotype and phenotype. Recently, an increasing number of metabolomic studies have reported the role of metabolites in economically important traits [7], such as meat quality [8,9], pre-slaughter stress [10], and FE [3]. Likewise, several transcriptomic studies have pointed out candidate genes underpinning FE and other related-traits such as immune response, growth, and metabolism in pigs [11-13].

Recently, we have investigated RNA-seq data on the 41 Danish production pigs that underwent feed efficiency and performance testing trials to identify differentially expressed genes and gene networks and reported 13 genes as potential biomarkers for feed efficiency [14]. Despite the new insights into key genes and molecular mechanisms reported in these studies, these approaches rely solely on data from a single biological layer. It has been shown that the integration of transcriptomics datasets with genomic and other omics datasets (systems genomics) increases the power to detect causal and regulatory factors and molecular pathways underlying complex phenotypes or diseases in animals $[15,16]$.

To gain further insights into biochemical aspects of complex traits, data integration analysis has emerged as a fruitful tool, unveiling potential biomarkers via integration of metabolomics and transcriptomics [17]. By the development of analytical technologies for data integration, the assessment of system-wide changes of transcript levels as surrogate measurements of metabolic rearrangements can be widely assessed. Metabolite-transcript co-responses using combined profiling can yield vital information on the complex biological regulation of the trait. Transcriptome-metabolome integration is a powerful combination as the metabolome is affected by the phenotypic measurements to which the global measures of transcriptome can be anchored [18]. Therefore, herein, we integrated data from metabolome-transcriptome approaches to unveil the unique gene-metabolite pairs. To this end, we adopted a two-step framework, as follows: (1) we first employed the numerical integration of gene-metabolite levels to identify gene-metabolite interaction pairs separately for the breeds (Duroc and Landrace) and FE groups (low and high FE) using IntLIM R-package; (2) next, a knowledge-based integration approach based on pathway over-representation analysis was used to reveal the underlying pathways in each group (breed-specific and FE-specific). To the best of our knowledge, this is the first study of its kind to ever combine high throughput metabolomics data with RNA sequence based gene expression data in pigs to unravel the missing links between genes and metabolites and to shed light on the molecular basis that characterizes the specific differences based on breed and feed efficiency.

\section{Results}

\subsection{Descriptive Statistics and Linear Model Analysis for genes and Metabolites}

The data on 749 metabolites and 25,880 genes from 40 samples were generated using untargeted metabolomics and transcriptomic approaches, respectively. We utilized data of 405 annotated metabolites (see methodology) for further analysis. For the transcriptomic data generated on 25,880 genes, we analyzed the data for each of the two groups (breed-specific and FE-specific), as described in the methodology. The genes with a gene count $<1$ were removed, resulting in 20,233 genes for both the groups. The gene count data for each group (breed-specific and FE-specific) was normalized, and the linear model was fitted into the data as given in the methodology. The genes were also screened for their chromosomal information from the Ensembl Sus scrofa database. After normalization, removal of values $<0$ and obtaining the gene chromosomal location information, 17,726 (breed-specific), and 17,697 (FE-specific) genes were retained in each group. As a quality control for IntLIM, we filtered out genes with the lowest $5 \%$ of the variation, which gave 16,839 genes (breed-specific) and 16,812 genes (FE-specific) that were subjected to IntLIM analysis. A schematic representation of the study design and analysis steps are given in Figure 1. We performed the PCA analysis (Figure S1) on the filtered metabolome-transcriptome data, which included 405 metabolites, and 16,839 genes (breed-specific), 
and 16,812 genes (FE-specific). The results of PCA analysis for the metabolites-genes based on breed and FE groups are shown in Figure S1.

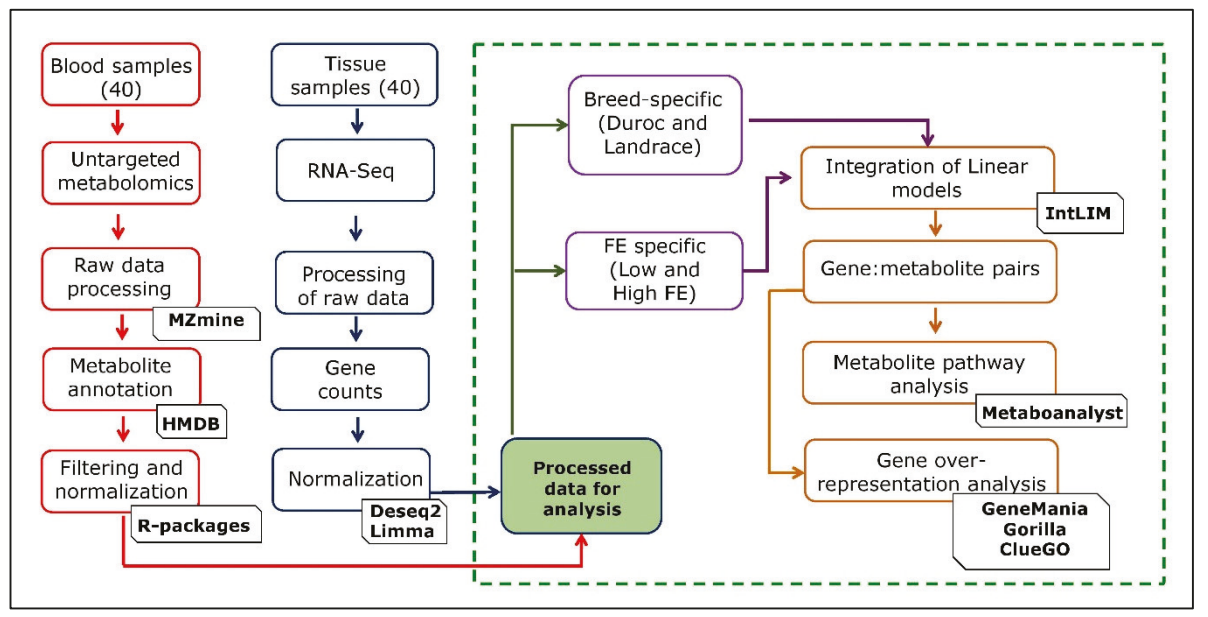

Figure 1. Schematic representation of the study design and analysis steps.

\subsection{Gene Metabolite Interaction of Breed-Specific and FE-Specific Groups}

From the IntLIM analysis, we identified gene-metabolite pairs that have a strong association with respect to the breed (Duroc and Landrace) and FE (low and high FE) groups. For the breed-specific groups, all possible combinations of gene-metabolite pairs $(6,819,795$ model runs) were evaluated, using Duroc and Landrace as the breed-group. Based on this approach, we identified 21 gene-metabolite associations (false discovery rate-FDR adjusted $p$-value $\leq 0.1$ and correlation difference effect size $>0.1$ ) (Table 1). Clustering these pairs by the direction of association (positive and negative correlations) within each breed group revealed two major clusters (Figure 2a) in each breed. First, the Duroc correlated/Landrace anti-correlated cluster consists of seven gene-metabolite pairs (three unique metabolites and five unique genes) with a high positive correlation in Duroc and low or negative correlation in Landrace (Figure 2a). Second, the Duroc anti-correlated/Landrace correlated cluster consists of 14 gene-metabolite pairs (10 unique metabolites and nine unique genes) with relatively high negative correlations in Duroc and positive correlations in Landrace. The two top-ranked gene-metabolite pairs (ranked in descending order of absolute value of Spearman correlation difference between Duroc and Landrace) in the Duroc correlated and anti-correlated clusters were ENSSSCG00000028124 (SNRPN)—Rhodamine B (Figure 2b) and ENSSSCG00000000401 (GLS2) - cystathionine ketimine (Figure 2c) respectively. SNRPN and Rhodamine B are positively correlated in Duroc ( $\mathrm{r}=0.7$ ) but negatively correlated in Landrace $(\mathrm{r}=-0.5)$ (Figure $2 \mathrm{~b})$. GLS2 and cystathionine ketimine are negatively correlated in Duroc $(r=-0.9)$, but positively correlated in Landrace $(\mathrm{r}=0.2)$ (Figure 2c).

Similarly, we used IntLIM for the FE-specific group and evaluated all possible combinations of gene-metabolite pairs (6,808,860 models), with low and high FE as a binary phenotype. With this approach, we identified 12 FE-specific gene-metabolite correlations (FDR adjusted interaction $p$-value $\leq 0.1$, and a Spearman correlation difference $>0.1$ ) involving eight unique gene and metabolites each (Table 2). The heat map with gene-metabolite Spearman correlation for low and high FE group showed a clear separation between the two groups (Figure 3a). The high FE-correlated cluster of 12 gene-metabolite pairs (eight unique genes and metabolites with high correlations in high-FE groups) were negatively correlated with the low-FE group. The two gene-metabolite pairs (ranked in descending order of absolute value Spearman correlation difference between high and low FE group) 
in high-FE correlated clusters were ENSSSCG00000025106 (THNSL2)—pyrocatechol (Figure 3b) and ENSSSCG00000036609 (TBXT)—ketoleucine (Figure 3c), respectively. Both pairs showed a positive correlation in high-FE group $(\mathrm{r}=0.6, \mathrm{r}=0.5)$ while showed a negative correlation in the low-FE group $(\mathrm{r}=-0.7, \mathrm{r}=-0.3)$ (Figure $3 \mathrm{~b}, \mathrm{c})$.

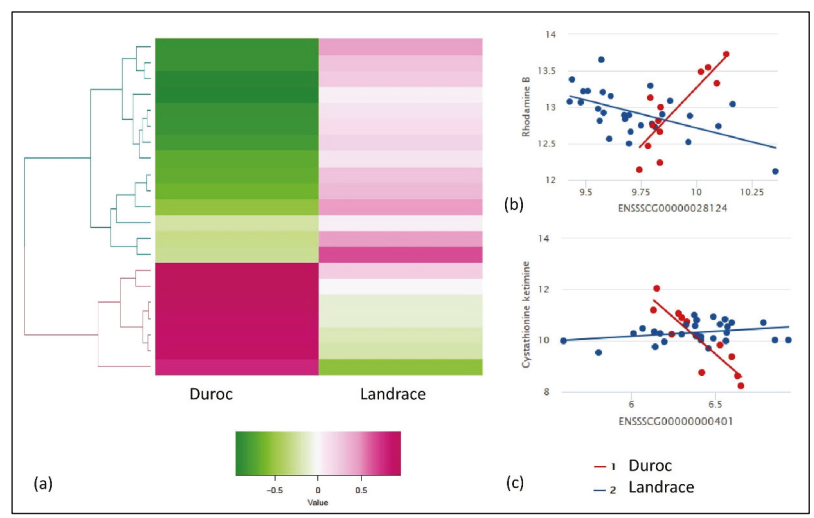

Figure 2. Results of IntLIM applied to breed-specific groups. (a) Clustering of 21 identified gene-metabolite pairs (FDR adjusted $p$-value of interaction coefficient $<0.1$, Spearman correlation difference $>0.1$ in Duroc and Landrace breeds, (b) Gene-metabolite difference in ENSSSCG00000028124 $(S N R P N)$-rhodamine B (FDR adjusted $p$-value $=0.1$, Duroc Spearman correlation $=0.7$, Landrace Spearman correlation $=-0.5)$, (c) Gene-metabolite difference in ENSSSCG00000000401 (GLS2)—cystathionine ketimine (FDR adjusted $p$-value $=0.01$, Duroc Spearman correlation $=-0.9$, Landrace Spearman correlation $=0.2$ ).

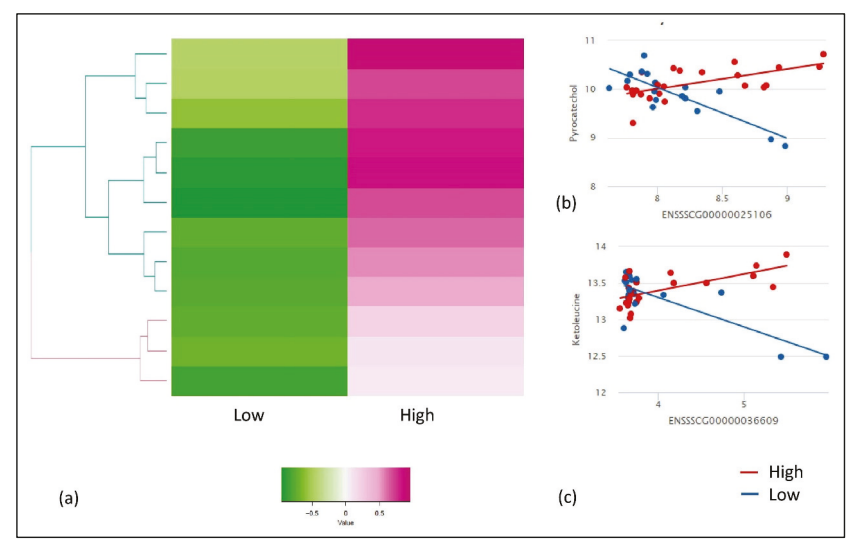

Figure 3. Results of IntLIM applied to FE-specific groups. (a) Clustering of 12 identified gene-metabolite pairs (FDR adjusted $p$-value of interaction coefficient $<0.1$, Spearman correlation difference $>0.1$ in high and low FE groups, (b) Gene-metabolite difference in ENSSSCG00000025106 (THNSL2) -Pyrocatechol (FDR adjusted $p$-value $=0.06$, High-FE Spearman correlation $=0.6$, Low-FE Spearman correlation $=$ -0.7), (c) Gene-metabolite difference in ENSSSCG00000036609 (TBXT)—ketoleucine (FDR adjusted $p$-value $=0.08$, High-FE Spearman correlation $=0.5$, Low-FE Spearman correlation $=-0.3$ ) 


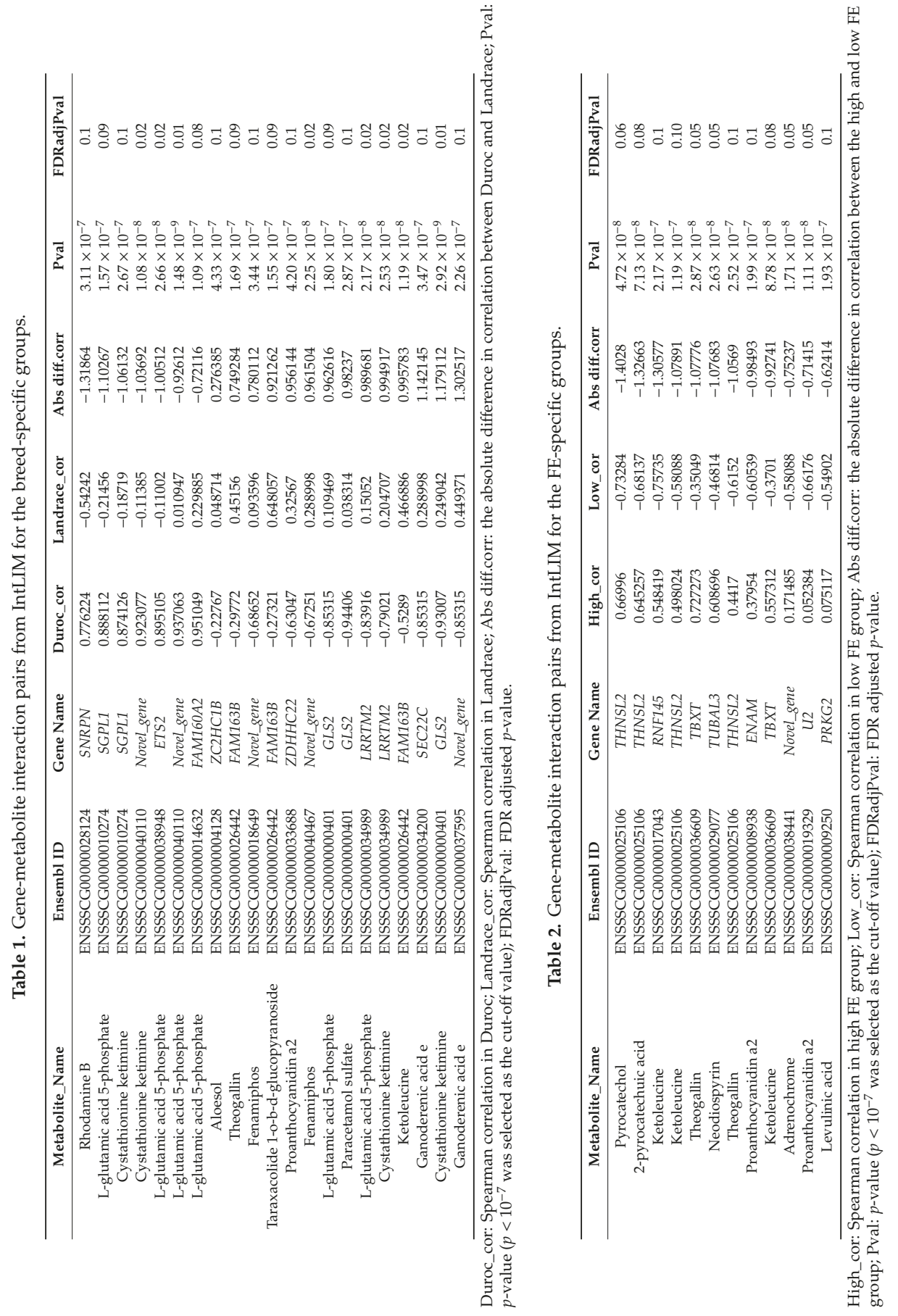




\subsection{Pathway and Gene Ontology Over-Representation Analysis}

We identified the pathways associated with the unique metabolites in each cluster identified from breed-specific (21) and FE-specific (12) interactions. The three unique metabolites from Duroc correlated/Landrace anti-correlated clusters were associated with arginine and proline metabolism $(p$-value $=0.02)$. Furthermore, the ten unique metabolites from Duroc anti-correlated/Landrace correlated cluster were associated with valine-leucine-isoleucine biosynthesis $(p$-value $=0.01)$ and valine-leucine-isoleucine degradation $(p$-value $=0.07)$ along with arginine and proline metabolism $(p$-value $=0.07)$. The eight unique metabolites from high-FE correlated/low-FE anti-correlated cluster were associated with valine-leucine-isoleucine biosynthesis $(p$-value $=0.01)$ and degradation $(p$-value $=0.07)$. The pathways associated with the metabolites in breed-specific and FE-specific clusters for unique metabolites are given in Supplementary Table S1a.

Unique and mappable genes from each group (breed-specific-each cluster, and FE-specific) were screened by using GeneMania to generate a composite functional association network that includes all the evidence of co-functionality. From the breed-specific group, unique genes (4 genes) from Duroc correlated/Landrace anti-correlated cluster (Table 1) mapped to 20 genes based on co-functionality from GeneMania (Table S1b). The gene-ontology enrichment analysis of the identified 24 genes (unique genes from Table 1 and co-functional genes from GeneMania) revealed enrichment of the regulation of hemopoiesis, response to thyroid hormone, and the sphingolipid catabolic process (Table S1c). These genes were enriched for the sphingolipid metabolism KEGG pathway (adjusted $p$-value corrected with Bonferroni step down $\leq 0.05)$ (Supplementary Table S1d). Unique and mappable genes (6 genes) identified from Duroc anti-correlated/Landrace correlated cluster (Table 1) were co-functional with 20 genes based on GeneMania (Table S1b). The gene ontology enrichment analysis of these 26 genes (unique genes from Table 1 and co-functional genes from GeneMania) revealed the ER to Golgi vesicle-mediated transport and membrane fusion (Table S1c) as an enriched biological process. Butanoate metabolism, alanine-aspartate-glutamate metabolism, and valine-leucine-isoleucine degradation were significantly enriched KEGG pathways from the Duroc anti-correlated/Landrace correlated cluster (Supplementary Table S1d). Similarly, from the FE-specific group, unique mapped genes (7 genes) from high-FE correlated/low-FE anti-correlated clusters (Table 2) were co-functional with 20 genes identified from GeneMania (Table S1b). These genes were involved with the cGMP metabolic process, purine nucleotide biosynthesis, and phosphorus-oxygen lyase activity pathways (Table S1c). The top significant KEGG pathway enriched was the cGMP-PKG signaling pathway (Supplementary Table S1d).

\section{Discussion}

FE is an important quantitative trait, which quantifies the efficiency of nutrient conversion from the feed to a tissue that is of nutritional and economic significance [19]. Understanding the molecular mechanisms underlying FE will be advantageous in the efficient selection of pigs and benefit the pig producers. In the Danish pig industry, Duroc is the most popularly used terminal sires in combination with crossbred Landrace X Yorkshire breeds [20], so the selection pressures for FE in Duroc is higher as compared to Landrace. Thus, in the current study, we attempted to identify the gene-metabolite interactions specific to each breed. FE is a complex trait influenced by environmental and health factors and involves many organs. Skeletal muscle, being the largest organ in the body, is an essential location for the metabolism of carbohydrates and lipids [21,22]. It plays a significant role in the utilization and storage of energy acquired from the feed. Thus, understanding the difference in the regulatory processes from a divergent FE group will add a layer of knowledge to the understanding of biological mechanisms involved with this complex trait.

A plethora of metabolome and transcriptome studies for FE in pigs are reported [3,9,10,12]. However, to the best of our knowledge, markers from the integration of metabolome and transcriptome in Duroc and Landrace pigs has not been done before. Herein, we unveiled the gene-metabolite relationships that are phenotype dependent. This approach highlighted molecular functions and 
pathways that are strongly evidenced by the integration study. Evaluating phenotype-specific relationships between metabolites and genes assists us to elucidate novel co-regulation patterns that would not be identified by single approaches. In the current study, we integrated untargeted metabolomic and transcriptomic data. We used a numerical data integration approach that employed the integration of a linear model (IntLIM package) to predict metabolite levels from gene-expression in a phenotype dependent manner [23].

We attempted to identify the breed-specific and FE-specific gene-metabolite pairs in the current study. The PCA analysis showed a difference in the expression of genes in Duroc and Landrace. However, PCA for the FE group did not exhibit significant clusters between groups, which may be due to the small sample size evaluated here. With our current metabolome-transcriptome analysis, we identified 21 gene-metabolite breed-specific pairs and 12 gene-metabolite FE-specific pairs.

\subsection{Breed-Specific Pathway Analysis}

In the breed-specific analysis, two clusters were identified between Duroc and Landrace breeds. The Duroc correlated/Landrace anti-correlated cluster associated L-glutamic acid 5-phosphate metabolite with the FAM160A2, ENSSSCG00000040110, ETS2, and SGPL1 genes; cystathionine ketimine metabolite with ENSSSCG00000040110 and SGPL1 genes and Rhodamine B with the SNRPN genes. The arginine and proline metabolism pathways were associated with the unique metabolites from this cluster. The gene ontology enrichment analysis of the unique genes identified from this cluster with the co-functional genes found enrichment for the multicellular organismal process, sphingolipid catabolic process, regulation of hemostasis, and coagulation pathways. Sphingolipid metabolism associated with the SGPL1 and GBA genes was identified as the top KEGG pathway. SGPL1 (sphingosine-1-phosphate) catalyzes the final step of the sphingolipid pathway by irreversibly converting sphingosine-1-phosphate $(S 1 P)$ to its by-products [24], thereby regulating S1P. S1P plays a role as a muscle trophic factor by activating quiescent muscle stem cells (satellite cells) for efficient skeletal muscle repair and regeneration [25]. The role of FE on skeletal muscle mass was well established from previous studies wherein the improvement of muscle properties and an increase in muscle mass is attributed by selection for low RFI in pigs [26]. S1P is also reported to trigger glutamate secretion and potentiates depolarization-evoked glutamate secretion [27]. Glutamic acid has been found to play a crucial role in FE as it improves the FE of weaned piglets [28]. This supports the results in the current study where SGPL1 was associated with L-glutamic acid 5-phosphate as a significant gene-metabolite pair. A brief overview of the role of SGPL1 in sphingolipid-metabolism regulating FE is given in Figure 4. Therefore, further investigation of SGPL1-L-glutamic acid 5-phosphate gene-metabolite pair, which was positively correlated with Duroc while negatively correlated with Landrace concerning FE traits, could be a major avenue for breed-specific research and its effect on FE and meat quality traits.

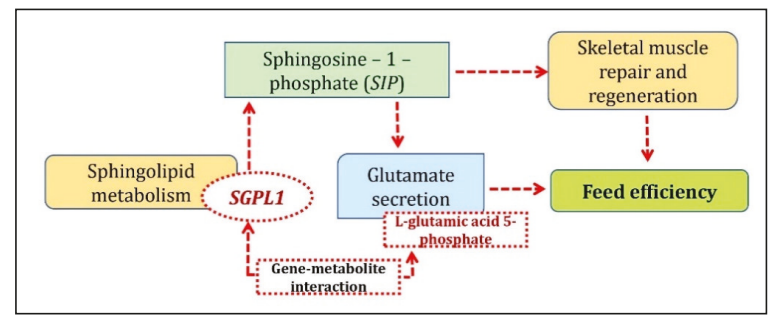

Figure 4. Biological mechanism showing the involvement of SGPL1-L-glutamic acid 5-phosphate gene-metabolite pair underlying sphingolipid metabolism identified from Duroc correlated/Landrace anti-correlated cluster.

We also identified the $S N R P N$ gene and Rhodamine B relation in the Duroc correlated cluster. A previous study showed the SNRPN gene (small nuclear ribonucleoprotein polypeptide N) was 
ubiquitously expressed in pigs [29]. Small nuclear ribonucleoproteins and heterogeneous small nuclear riboproteins play roles in nucleolar ribosomal RNA (rRNA) and messenger RNA (mRNA) synthesis in conjunction with spliceosome activity responsible for cleaving on introns from the pre mRNA molecule [30]. Furthermore, in a study of FE in broiler chickens, a high FE phenotype exhibited enrichment of ribosome assembly including small nuclear ribonucleoprotein, as well as nuclear transport and protein translation processes than low FE phenotype [31].

The Duroc anti-correlated/Landrace correlated cluster identified aloesol-ZC2HC1B, Proanthocyanidin a2-ZDHHC22; fenamiphos metabolite with ENSSSCG00000040467, ENSSSCG00000018649 gene; ganoderenic acid e metabolite with ENSSSCG00000037595 and SEC22C genes; FAM163B gene with taraxacolide 1-o-b-d-glucopyranoside, theogallin, and ketoleucine metabolites; LRRTM2 gene with cystathionine ketimine and L-glutamic acid 5-phosphate metabolites and GLS2 gene with L-glutamic acid 5-phosphate, cystathionine ketimine, and paracetamol sulfate metabolites. One of the significant pathways in Landrace correlated cluster was valine, leucine, and isoleucine degradation which included the $A B A T$ and $A C A D S$ genes (co-functional genes) identified in the current study. Valine, leucine, and isoleucine are branched-chain amino acids (BCAA) and have a crucial role in protein synthesis and energy production [32]. The degradation of BCAA can be glucogenic (valine), ketogenic (leucine and isoleucine), or both (isoleucine). The end products from their degradation, succinyl-CoA and/or acetyl-CoA can enter the tricarboxylic acid (TCA) cycle for energy generation and gluconeogenesis or may act as precursors for lipogenesis and ketone body production through acetyl-CoA and acetoacetate [33]. Glucose metabolism and the TCA pathway in the skeletal muscle is a key pathway regulating FE traits in pigs [34]. In an interesting proteomic study involving glucose metabolism and the TCA cycle reported earlier, the proteins catalyzing the conversion of glucose to pyruvate and oxaloacetate were up-regulated in high-FE pigs while those involved in the conversion of pyruvate to lactate or acetyl-CoA were down-regulated in high-FE pigs [34]. This resulted in inhibition of the TCA cycle in high-FE pigs due to the down-regulation of key catalytic proteins [34]. Thus, the pathway identified with BCAA in the current study may cause differences in FE concerning specific breed as evident from the indirect link with TCA and FE (Figure 5). The BCAA also affects protein synthesis, as reported earlier in a study with reduced degradation of rat skeletal muscle proteins [35]. Additionally, leucine activates mTOR signaling, one of the central regulators of cell growth and metabolism along with an increase in fatty acid oxidation. With all these supporting facts of BCAA regulating cellular metabolism, protein, and fatty acid degradation, which are also key factors influencing FE, the role of the valine-leucine-isoleucine pathway in FE cannot be overlooked. Furthermore, this pathway has been found over-represented in a GWAS study for RFI in beef cattle [36] and pigs [3].

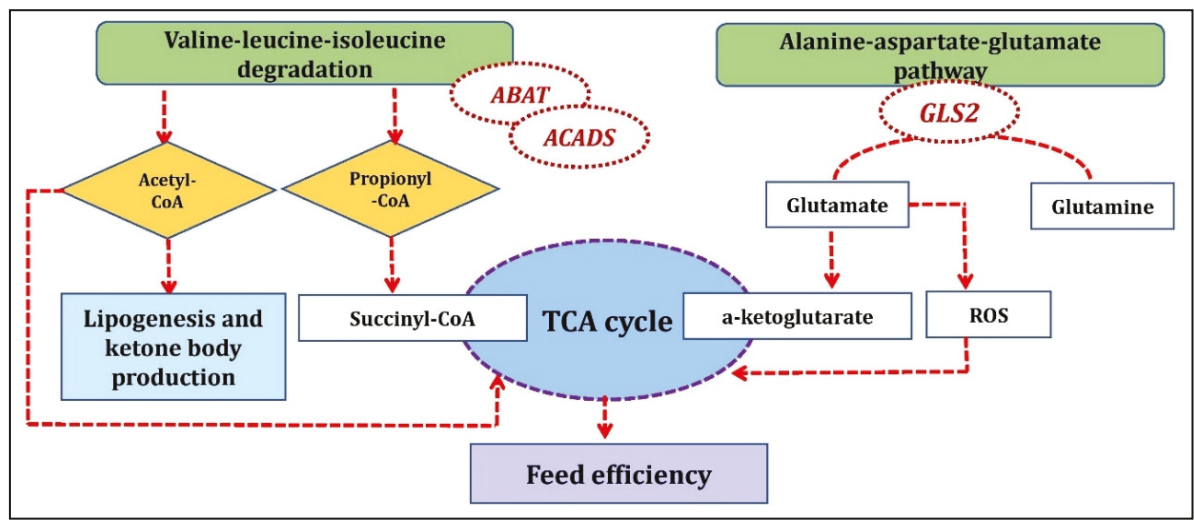

Figure 5. Biological mechanism showing the involvement of genes identified from Duroc anti-correlated/Landrace correlated clusters underlying feed efficiency. 
We identified the alanine-aspartate-glutamate metabolism KEGG pathway involving the GLS2 and $A B A T$ genes within the Duroc anti-correlated/Landrace correlated cluster. GLS2 is a mitochondrial glutaminase that catalyzes glutamine to glutamate which is further converted to a-ketoglutarate, an important substrate for the citric acid cycle to produce ATP in mitochondria. Glutamate is also a precursor of reduced glutathione (GSH), an important antioxidant molecule, and a scavenger for ROS (Reactive oxygen species) [37]. ROS are regulated by FE related traits as reported from the previous studies, higher levels of ROS production and oxidized mitochondrial proteins have been found in the muscle of low FE chickens [38] while in pigs, ROS production in mitochondria was higher in semitendinosus muscle of less efficient pigs selected for high RFI compared to high efficient pigs (low RFI) [39]. GLS2 interacted with L-glutamic acid 5-phosphate and cystathionine ketimine metabolites as identified from Duroc anti-correlated/Landrace correlated clusters (Figure 5).

The unique metabolites identified in the breed-specific clusters were also previously reported in another study for the metabolomic analysis of FE related traits in Duroc and Landrace [3]. The breed-specific unique metabolites such as aloesol and ketoleucine affected FE in Duroc [3]. In contrast, rhodamine B, taraxacolide 1-o-b-d-glucopyranoside, and ganoderenic acid were underlying testing daily gain (TDG) in Duroc [3]. Theogallin and ketoleucine were involved with TDG and daily gain (DG) in Duroc and Landrace and RFI in Duroc [3]. L-glutamic acid 5-phosphate, cystathionine ketimine, and paracetamol sulfate were associated with FE and RFI in Landrace [3]. It is worth highlighting the interaction of metabolites L-glutamic acid 5-phosphate and cystathionine ketimine identified in this study. While these metabolites interact with the SGPL1 gene as identified in the Duroc correlated cluster, on the contrary, they interact with the GLS2 gene in the Landrace correlated cluster. Both SGPL1 and GLS2 were in the top significant pathways in their respective cluster. Therefore, these gene-metabolite interactions which are highly specific to breed differences open up the avenues for further research to extrapolate differences in FE related traits concerning breeds.

\section{2. cGMP-PKG Pathway Involved with FE-Specific Analysis}

In the FE-specific analysis, we found 12 significant gene-metabolite pairs. The gene-metabolite pairs in high-FE correlated/low-FE anti-correlated cluster were TBXT gene with theogallin and ketoleucine metabolites; THNSL2 gene with pyrocatechol, 2-pyrocatechuic acid, ketoleucine, and theogallin metabolites; TUBAL3 gene with neodispyrin metabolite, RNF145 gene with ketoleucine metabolite, ENAM gene and U2 snRNA with proanthocyanidin a2 metabolite, ENSSSCG00000038441 gene with adrenochrome metabolite, and PRKG2 gene with levulinic acid metabolite. The pathway analysis with the unique metabolites identified from high-FE correlated/low-FE anti-correlated clusters was over-represented for valine-leucine-isoleucine biosynthesis and degradation pathway. The unique mapped genes and co-functional genes were enriched for the following biological processes: lyase activity, cGMP metabolic process, phosphorus-oxygen lyase activity, and cyclic purine nucleotide metabolic process. cGMP-PKG, purine metabolism, and renin secretion were the KEGG pathways identified in this cluster. The cGMP pathways were also identified in the studies reported earlier with FE related traits with pigs [40] and beef cattle [41]. The PRKG2 gene, one of the main predictors for cGMP pathways and also identified in this study, encodes the serine/threonine-protein, which binds to inhibits the activation of several receptor tyrosine kinases and is a regulator of the intestinal secretion, bone growth, and renin secretion (https://www.ncbi.nlm.nih.gov/gene/5593). PRKG2 encodes for CGKII (guanosine 3,5-cyclic monophosphate (cGMP)-dependent protein kinase II) and is abundantly expressed in intestinal epithelium. CGKII relays signaling through a membrane-associated, cGMP-producing enzyme, guanylyl cyclase (GC). The catalytic activity of this receptor-enzyme is triggered by two locally produced ligands, the peptides guanylin and uroguanylin [42]. The GC is activated by nitric oxide (NO) and catalyzes the conversion of intracellular guanosine- $5^{\prime}$-triphosphate (GTP) to cyclic guanosine-3', $5^{\prime}$-monophosphate (cGMP). This enzyme has two forms: a membrane protein and a soluble form with specific kinetic properties and tissue distributions. The soluble GC (sGC) form is a heterodimeric protein consisting of $\alpha\left(\alpha_{1}\right.$ and $\left.\alpha_{2},\right)$ and $\beta\left(\beta_{1}\right.$ and $\left.\beta_{2}\right)$ subunits encoded by distinct 
genes [43]. An alpha subunit of guanylyl cyclase, GUCY1A2 and a beta subunit GUCY1B3 was identified as co-functional genes in the current study and were involved with cGMP, phosphorus metabolic process, nitrogen metabolic process pathways as identified in the current study. Uroguanylin is a gastrointestinal hormone primarily involved in fluid and electrolyte handling. It has recently been reported that prouroguanylin, secreted postprandially, is converted to uroguanylin in the brain and activates the receptor guanylate cyclase-C (GC-C) to reduce food intake in mice [44]. Reduced FI is a characteristic feature for the selection of the pigs known for high FE [1]. The overview of the mechanism involving the cGMP-PKG pathway and its role in FE is given in Figure 6.

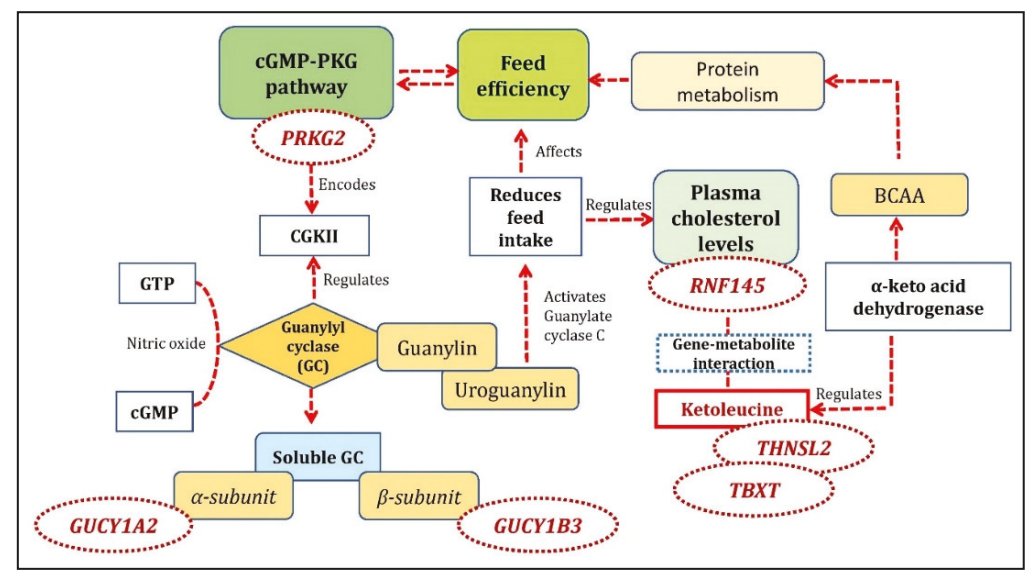

Figure 6. An overview of gene-metabolite pairs affecting the cGMP-PKG pathway involved with feed efficiency.

A positive correlation between FI and plasma cholesterol levels is previously established in pigs [45]. RNF145, which was positively correlated in the high-FE cluster participates in key signaling pathways of cholesterol homeostasis [46]. RNF145 expression is induced in response to LXR activation and high-cholesterol diet feeding [46]. Transduction of RNF145 into mouse liver inhibits the expression of genes involved in cholesterol biosynthesis and reduces plasma cholesterol levels. On the other hand, its inactivation increases cholesterol levels both in the liver and plasma [46]. In this study, RNF145 was identified with ketoleucine as a significant gene-metabolite pair in this cluster (Figure 6). Ketoleucine is an abnormal metabolite that arises from the incomplete breakdown of branched-chain amino acids (https://hmdb.ca/metabolites/HMDB0000695). Ketoleucine is regulated by branched-chain $\alpha$-keto acid dehydrogenase. Studies reported that the branched-chain $\alpha$-keto acid dehydrogenase catalyzes the irreversible oxidative decarboxylation of all three branched-chain keto acids (BCKA) derived from branched-chain amino acids (BCAA), i.e., $\alpha$-ketoisocaproate (ketoleucine) [47]. They demonstrated changes in BCKA activity that showed a significant alteration in BCAA and protein metabolism during starvation in rats [47]. BCAA also plays a crucial role in FE by regulating energy homeostasis in addition to lipid and protein metabolism as reported in pigs [48].

Apart from RNF145, gene-metabolite interaction of ketoleucine with TBXT and THNSL2 was also identified in this study. THNSL2 was reported among the top 40 significantly differentially expressed genes of characterized proteins between high- and low-ADG steers from a liver transcriptome profiling of beef cattle [49]. This gene has also been associated with abdominal and visceral fat in humans based on GWAS [50]. An interaction of proanthocyanidin a2 with ENSSSCG00000019329 (U2 snRNA) was also identified with low but positive correlation with high-FE cluster while a negative correlation with the low-FE cluster. U2 spliceosomal snRNAs are the molecules found in the major spliceosomal machinery of all eukaryotic organisms and affect their gene expression [51]. U2 snRNA plays a central role in the splicing of mRNA precursors by regulating a dynamic set of 
RNA-RNA base-pairing interactions [52]. From the previously reported studies, the role of precursor mRNA in gene expression has been established as it removes the intronic sequence from immature RNA, leading to a production of mature mRNA that might differ in function [53]. Regulation of pre-mRNA splicing by nutrients modulates the carbohydrate and lipid metabolism [53]. U2 interacts with proanthocyanidin a2 in the current study. Proanthocyanidin a2 is an antioxidant and has a broad spectrum of biologic properties against oxidative stress [54]. Proanthocyanidin significantly increased the activity of antioxidant enzymes such as superoxide dismutase, glutathione peroxidase, and catalase [54]. The role of antioxidant activity with FE was reported earlier in beef cattle as low feed efficient steers had greater superoxide dismutase and glutathione peroxidase activity than the high feed efficient ones, potentially using a greater proportion of energy [55]. Thus, as evident from all these facts, the potential role of proanthocyanidin a2-U2 interaction in high-FE pigs identified in the current study might be interesting to explore.

The gene-metabolite pairs identified in the present study over-represented some pathways that have been reported to have a role in FE related traits. Some of the genes identified are novel and were not included in the pathway analysis. Since these gene-metabolite pairs selected have a highly significant correlation with respect to each study group, a detailed study of these genes and metabolites are needed to better understand their role in FE related-traits. Further studies on the identified gene-metabolite pairs may assist in the discovery of biomarkers as these significant pairs identified directly reflect the phenotype as revealed by the candidate gene-expression with the downstream metabolite differences in pigs with low and high FE groups.

\section{Materials and Methods}

\subsection{Data Resource and Phenotype Generation}

The pigs used in this experiment were raised at the pig testing station "Bøgildgård" operated by SEGES within Landbrug and Fødevarer (L\&F: Danish Agriculture and Food Council). Pigs were ad libitum fed and free water supplied. The authors of this study were not responsible for animal husbandry, diet, and care as the testing station is a facility within the Danish breeding program run by SEGES. The initial bodyweight of the pigs before the testing period was approximately $7 \mathrm{~kg}$, followed by a 5 -week acclimatization phase. For the phenotypic traits, we calculated FCR and RFI, as reported in our previous study [40]. We considered the same classification of animals in this study as efficient and inefficient (low and high FCR, respectively), as previously reported [40]. The classification was done by selecting pigs that were one standard deviation above or below the mean FCR for each breed as previously reported.

\subsection{Gene Expression Profile, Metabolite Profile, and Data Analyses}

For transcriptome analysis, we collected psoas major muscle from 40 purebred uncastrated male pigs from two breeds comprising of 12 Danbred Duroc and 28 Danbred Landrace. The tissue samples were preserved in RNAlater (Ambion, Austin, TX, USA) immediately post-slaughter and stored at $-25{ }^{\circ} \mathrm{C}$ until subsequent analysis. Total RNA isolation and sequencing were carried out by BGI Genomics. Paired-end sequencing (100 bp) was performed on the BGISEQ-500 platform after Oligo dT library preparation. Read quality control, mapping, and gene counts were reported elsewhere [14]. Lowly expressed genes were filtered out, and the gene counts normalization was carried out by applying the variance stabilizing transformation (VST) function from DeSeq2 [56].

To identify significant gene-metabolite pairs, we analyzed the data considering two approaches, i.e., breed-specific (Duroc-Landrace) and FE-specific (low-high FE groups). Thus, we fitted a linear model for adjusting the read counts with the covariates using the Limma R package [57]. For adjusting the data to identify breed-specific differences, we adopted the following model:

$$
y_{i j k l}=\mu+F_{i}+R_{j}+A_{k}+P_{l}+\varepsilon_{i j k l}
$$


where $y_{i j k l}$ : is the normalized read counts; $\mu$ : is the intercept; $F_{i}$ : is the fixed effect of the FE group (two levels, high and low); $R_{j}$ : is the covariate for the RIN values; $A_{k}$ : is the covariate for the animal's slaughter age, in days; $P_{l}$ : is the fixed effect of the pen ( 8 levels); $\varepsilon_{i j k l}$ : is the random residual effect associated with each observation.

To identify differences between high and low FE groups, the breed effect was added in the linear model, as follows:

$$
y_{i j k l}=\mu+B_{i}+R_{j}+A_{k}+P_{l}+\varepsilon_{i j k l}
$$

where $y_{i j k l}$ : is the normalized read counts; $\mu$ : is the intercept; $B_{i}$ : is the fixed effect of the breed (two levels, Duroc and Landrace); $R_{j}$ : is the covariate for the RIN values; $A_{k}$ : is the covariate for the animal's slaughter age, in days; $P_{l}$ : is the fixed effect of the pen (eight levels); $\varepsilon_{i j k l}$ : is the random residual effect associated with each observation.

Regarding the identification of the metabolites, we used an untargeted metabolomic approach, as reported elsewhere [3]. In summary, $5 \mathrm{~mL}$ of blood samples at two-time points were collected from jugular venipuncture of each non-fasted pig into the EDTA tubes and immediately placed on ice. The plasma samples extracted from 109 animals (59 Duroc and 50 Landrace) were subjected to metabolomics analysis, as described in a previous study [3]. The metabolite data from this study were accessed using MetaboLights accession ID MTBLS1384 with a link: https://www.ebi.ac.uk/metabolights/ MTBLS1384. Due to the need for paired data to carry the integrative analysis, only those samples with both metabolite and RNA-Seq data were used herein. The metabolite data from time-point two in 40 pigs were log-normalized before fitting into a linear model. Only those with the relative standard deviation $>0.15$ were used based on the raw counts. As proposed for the RNA-seq, we adjusted the log-normalized metabolite data considering both approaches. First, the following model was employed for the breed-specific analysis:

$$
m_{i j k l}=\mu+F_{i}+D_{j}+A_{k}+P_{l}+\varepsilon_{i j k l}
$$

where $m_{i j k l}$ : is the is the log-normalized concentration of each metabolite $(n=749) ; \mu$ : is the intercept; $F_{i}$ : is the fixed effect of the FE group (two levels, high and low); $D_{j}$ : is the fixed effect of the batch for metabolomic analysis (two levels); $A_{k}$ : is the covariate for the sampling age, in days; $P_{l}$ : is the fixed effect of the pen (8 levels); $\varepsilon_{i j k l}$ : is the random residual effect associated with each observation.

For the FE-specific group approach, we fitted the data as follows:

$$
m_{i j k l}=\mu+B_{i}+D_{j}+A_{k}+P_{l}+\varepsilon_{i j k l}
$$

where $m_{i j k l}$ : is the is the log-normalized concentration of each metabolite $(n=749) ; \mu$ : is the intercept; $B_{i}$ : is the fixed effect of the breed (two levels, Duroc and Landrace); $D_{j}$ : is the fixed effect of the batch for metabolomic analysis (two levels); $A_{k}$ : is the covariate for the sampling age, in days; $P_{l}$ : is the fixed effect of the pen (8 levels); $\varepsilon_{i j k l}$ : is the random residual effect associated with each observation.

The metabolites were annotated with HMDB (Human metabolome database) based on library search of the masses in HMDB with a mass uncertainty of $0.005 \mathrm{Da}$ or $5 \mathrm{ppm}$. Those metabolites that did not correspond to HMDB entries were left unannotated and removed from the analysis.

\subsection{Integration of Transcriptomic and Metabolomic Data Based on the Linear Model}

To uncover the complex relationship between metabolites and genes, we adopted a linear model framework using the IntLIM (Integration of Linear model) R-package (version 0.1.0) (https://github.com/ mathelab/IntLIM) [23]. The IntLIM approach allows us to integrate the metabolomic-transcriptomic data considering a case-control design. Thus, as initially proposed, we compared the breeds (Duroc vs. Landrace) and the FE groups (low and high FCR animals). As a quality control step from IntLIM, we filtered out genes with the lowest $5 \%$ of the variation. Gene and metabolite exploratory analyses were performed by applying Principal Component Analysis to identify breed- and FE-specific clusters. 
The linear model for data integration is given as described in the following equation:

$$
m=\beta_{1}+\beta_{2} g+\beta_{3} p+\beta_{4}(g: p)+\varepsilon
$$

where $m$ : is the log-normalized metabolite abundance; $\beta_{1}$ : is the intercept; $\beta_{2} g$ : is the normalized and adjusted gene expression level; $\beta_{3} p$ : is the phenotype (FE group—high and low FE; or breed-Duroc and Landrace); $\beta_{4}(g: p)$ : is the interaction between gene expression and phenotype; $\varepsilon$ : is the residual effect associated with each observation $(\varepsilon=\mathrm{N}(0, \sigma))$.

A statistically significant two-tailed $p$-value of the gene-phenotype (g-p) interaction indicates the difference in the phenotype of FE groups (high and low) or breed (Duroc and Landrace) calculated by the slope relating gene-expression and metabolite abundance [23]. The two-tailed $p$-value indicates that the slope relating gene-expression and metabolite abundance is different from one phenotype compared to the other. Thus, it was used to identify gene-metabolite associations that are specific to a particular phenotype (breed-Duroc and Landrace, FE-low and high). We calculated the absolute difference in the Spearman correlation identified from IntLIM between the FE and breed groups to find the significant $\left(p<10^{-7}\right)$ gene-metabolite pairs. The absolute difference between the FE group was estimated as $\left(r_{\text {Low_cor }}-r_{\text {High_cor }}\right)$ where $\left(r_{\text {High_cor }}\right)$ is the correlation given for a gene-metabolite pair in high-FE group (Table 2) while $\left(r_{\text {Low_cor }}\right)$ is the correlation given for a gene-metabolite pair in the low-FE group (Table 2). The absolute difference in the correlation between the breeds was estimated as $\left(r_{\text {Landrace_cor }}-r_{\text {Duroc_cor }}\right)$, where, $\left(r_{\text {Duroc_cor }}\right)$ is the correlation for a given gene-metabolite pair in Duroc (Table 1) while $\left(r_{\text {Landrace_cor }}\right)$ is the correlation for a gene-metabolite pair in Landrace (Table 1).

\subsection{Pathway Over-Representation Analysis}

In the breed-specific and FE-specific group, the same metabolite can be related to more than one gene and vice-versa. So, we screened for the common metabolites and genes in the group and referred them as unique metabolites and unique genes respectively in this study. We analyzed the unique metabolites in each group (breed-specific and FE-specific) using Metaboanalyst 4.0 (www.metaboanalyst.ca) [58]. We used three parameters for the pathway analysis: the pathway library, algorithm for pathway over-representation analysis, and algorithm for topological analysis. For the current study, we selected the Homo sapiens (KEGG) pathway library to estimate the importance of the compound in a given metabolic pathway. For pathway over-representation and topology analysis, we used the hypergeometric test and relative-betweenness centrality algorithm, respectively, to measure the connections with the other nodes, including the number of shortest paths going through the node of interest.

Regarding the unique mapped (with chromosomal location information) genes, we carried out a co-functionality analysis using GeneMANIA [59] (www.genemania.org). GeneMANIA considers our query list of unique genes identified in each cluster (breed-specific and FE-specific) and allows us to predict the co-functional genes underlying similar functions. Thus, we analyzed the unique genes in each cluster (breed-specific-Duroc correlated cluster, and Duroc anti-correlated cluster, FE-specific-High-FE correlated cluster) to identify the co-functional genes in each cluster. Next, we used the unique genes, as well as the co-functional genes in each cluster of each group, to identify the GO terms using GOrilla (Gene ontology enrichment analysis and visualization tool) (http://cbl-gorilla.cs.technion.ac.il/) [60]. To this end, the Homo sapiens were used as the reference, and the entire set of identified and annotated genes in this study ( $n=15,187$ genes) was used as a background. Over-representation KEGG pathway analysis with the unique and co-functional genes in each cluster was performed using ClueGO version 2.5.4 [61] to cluster redundant terms with a kappa score of 0.4 and S. scrofa annotation as the background. The pathways were selected after filtering for group $p$-value corrected with Bonferroni step down $\leq 0.05$ and those with at-least two over-represented genes. 


\section{Conclusions}

This study applied a novel approach for metabolome-transcriptome data integration using the linear model unveiling potential gene-metabolite pairs affecting the biological processes related to FE in pigs. To the best of our knowledge, this is the first study to report the gene-metabolite interaction mechanisms that may determine nutrient partitioning and energy utilization and hence affect FE in pigs. The approach followed here provided many interesting genes and metabolites with significant $p$-values. While some of the metabolites and genes identified were known with their association for FE, others are novel and provide new avenues for further research. The unique metabolites were associated with valine-leucine-isoleucine biosynthesis/degradation and arginine-proline metabolism. The unique genes enriched for sphingolipid metabolism, valine-leucine-isoleucine degradation, alanine-aspartate-glutamate pathway (breed-specific), and cGMP-PKG signaling pathway (FE-specific). Further validation of genes, metabolites, and gene-metabolite interactions in a cohort with more animals with additional features such as alteration in dietary components, farm variations, and other environmental effects would help to establish a framework for future FE prediction using metabolomics biomarker profiles that could be practical to use in large populations other than genomic profiling. More data would also make it possible to model the complex relations in gene-metabolite profiles over time more accurately and will help to elucidate the regulatory mechanisms affecting the pathways underlying FE.

Supplementary Materials: The following are available online at http://www.mdpi.com/2218-1989/10/7/275/s1, Figure S1: The principal component analysis of metabolites and genes. A and B: PCA plot of genes and metabolites, respectively, in (1) Duroc and (2) Landrace; C and D: PCA plot of genes and metabolites, respectively, in high and low feed efficient groups, Table S1: Pathways enriched by unique gene-metabolite pairs. Spreadsheet tabs are divided into (a) metabolite enrichment analysis results by Metaboanalyst; (b) list of the co-functional genes by GeneMania; (c) gene ontology pathways enriched by Gorilla; (d) KEGG pathways enriched by ClueGO.

Author Contributions: H.N.K. conceived and designed this "FeedOMICS" project, obtained funding as the main applicant. V.A.O.C. and H.N.K designed blood sampling experiments, phenotype data collection, metabolite profiling, and RNA-Seq experiments. P.B. carried out biostatistical and bioinformatic data analysis and wrote the manuscript. All authors collaborated in the interpretation of results, discussion, and write up of the manuscript. All authors have read, reviewed, and approved the final manuscript. All authors have read and agreed to the published version of the manuscript.

Funding: This study was funded by the Independent Research Fund Denmark (DFF)-Technology and Production (FTP) grant (grant number: 4184-00268B).

Acknowledgments: This study was funded by the Independent Research Fund Denmark (DFF)—Technology and Production (FTP) grant (grant number: 4184-00268B). V.A.O.C. received partial Ph.D. stipends from the University of Copenhagen and Technical University of Denmark. Authors thank Wellison Jarles Da Silva Diniz for helping in the interpretation of results. Authors thank SEGES-Pig Research Centre (VSP) Denmark for access to blood samples and phenotype datasets used in this study.

Conflicts of Interest: The authors declare no conflict of interest. The funders had no role in the design of the study; in the collection, analyses, or interpretation of data; in the writing of the manuscript, or in the decision to publish the results.

Consent for Publication: The blood sampling and experiment were approved and carried out in accordance with the Ministry of Environment and Food of Denmark, Animal Experiments Inspectorate under the license number (tilladelsesnummer) 2016-15-0201-01123, and C-permit granted to the principal investigator/senior author (HK).

Availability of Data and Material: The metabolites dataset generated and analyzed during the current study is publicly available at the Metabolights database https://www.ebi.ac.uk/metabolights/MTBLS1384 with accession ID: MTBLS1384. (https://doi.org/10.1093/nar/gks1004; PubMed PMID: 2310955). The transcriptome dataset analyzed during the present study is also publicly available at the NCBI-GEO database with Accession ID GSE148889 and can be accessed at https://www.ncbi.nlm.nih.gov/geo/query/acc.cgi?acc=GSE148889.

\section{References}

1. Patience, J.F.; Rossoni-Serão, M.C.; Gutiérrez, N.A. A review of feed efficiency in swine: biology and application. J. Anim. Sci. Biotechnol. 2015, 6, 33. [CrossRef]

2. He, B.; Li, T.; Wang, W.; Gao, H.; Bai, Y.; Zhang, S.; Zang, J.; Li, D.; Wang, J. Metabolic characteristics and nutrient utilization in high-feed-efficiency pigs selected using different feed conversion ratio models. Sci. China Life Sci. 2019, 62, 959-970. [CrossRef] 
3. Carmelo, V.A.O.; Banerjee, P.; da Silva Diniz, W.J.; Kadarmideen, H.N. Metabolomic networks and pathways associated with feed efficiency and related-traits in Duroc and Landrace pigs. Sci. Rep. 2020, 10, 1-14. [CrossRef] [PubMed]

4. Godinho, R.M.; Bergsma, R.; Silva, F.F.; Sevillano, C.A.; Knol, E.F.; Lopes, M.S.; Lopes, P.S.; Bastiaansen, J.W.M.; Guimarães, S.E.F. Genetic correlations between feed efficiency traits, and growth performance and carcass traits in purebred and crossbred pigs. J. Anim. Sci. 2018, 96, 817-829. [CrossRef]

5. Ren, P.; Yang, X.J.; Cui, S.Q.; Kim, J.S.; Menon, D.; Baidoo, S.K. Effects of different feeding levels during three short periods of gestation on gilt and litter performance, nutrient digestibility, and energy homeostasis in gilts. J. Anim. Sci. 2017, 95, 1232-1242. [CrossRef] [PubMed]

6. Do, D.N.; Strathe, A.B.; Ostersen, T.; Pant, S.D.; Kadarmideen, H.N. Genome-wide association and pathway analysis of feed efficiency in pigs reveal candidate genes and pathways for residual feed intake. Front. Genet. 2014, 5, 307. [CrossRef] [PubMed]

7. Novais, F.J.; Dromms, R.A.; Alexandre, P.A.; Pires, P.R.L.; Styczynski, M.P.-W.; Ferraz, J.B.S.; Fukumasu, H.; Iglesias, A.H. Identification of a metabolomic signature associated with feed efficiency in beef cattle. BMC Genom. 2019, 20,1-10. [CrossRef] [PubMed]

8. Rohart, F.; Paris, A.; Laurent, B.; Canlet, C.; Molina, J.; Mercat, M.J.; Tribout, T.; Muller, N.; Iannuccelli, N.; Villa-Vialaneix, N.; et al. Phenotypic prediction based on metabolomic data for growing pigs from three main european breeds. J. Anim. Sci. 2012, 90, 4729-4740. [CrossRef]

9. D'Alessandro, A.; Marrocco, C.; Zolla, V.; D'Andrea, M.; Zolla, L. Meat quality of the longissimus lumborum muscle of Casertana and Large White pigs: Metabolomics and proteomics intertwined. J. Proteom. 2011, 75, 610-627. [CrossRef]

10. Bertram,H.C.; Oksbjerg, N.; Young, J.F. NMR-based metabonomics reveals relationship between pre-slaughter exercise stress, the plasma metabolite profile at time of slaughter, and water-holding capacity in pigs. Meat Sci. 2010, 84, 108-113. [CrossRef]

11. Jing, L.; Hou, Y.; Wu, H.; Miao, Y.; Li, X.; Cao, J.; Michael Brameld, J.; Parr, T.; Zhao, S. Transcriptome analysis of mRNA and miRNA in skeletal muscle indicates an important network for differential Residual Feed Intake in pigs. Sci. Rep. 2015, 5, 11953. [CrossRef] [PubMed]

12. Vincent, A.; Louveau, I.; Gondret, F.; Tréfeu, C.; Gilbert, H.; Lefaucheur, L. Divergent selection for residual feed intake affects the transcriptomic and proteomic profiles of pig skeletal muscle. J. Anim. Sci. 2015, 93, 2745-2758. [CrossRef]

13. Horodyska, J.; Hamill, R.M.; Reyer, H.; Trakooljul, N.; Lawlor, P.G.; Mccormack, U.M.; Wimmers, K. RNA-Seq of Liver From Pigs Divergent in Feed Efficiency Highlights Shifts in Macronutrient Metabolism, Hepatic Growth and Immune Response. Front. Genet. 2019, 10. [CrossRef]

14. Carmelo, V.A.O.; Kadarmideen, H.N. Genome Regulation and Gene Interaction Networks Inferred From Muscle Transcriptome Underlying Feed Efficiency in Pigs. Front. Genet. 2020, 11. [CrossRef]

15. Kadarmideen, H.N. Genomics to systems biology in animal and veterinary sciences: Progress, lessons and opportunities. Livest. Sci. 2014, 166, 232-248. [CrossRef]

16. Suravajhala, P.; Kogelman, L.J.A.; Kadarmideen, H.N. Multi-omic data integration and analysis using systems genomics approaches: methods and applications in animal production, health and welfare. Genet. Sel. Evol. 2016, 48, 38. [CrossRef]

17. Carrillo, J.A.; He, Y.; Li, Y.; Liu, J.; Erdman, R.A.; Sonstegard, T.S.; Song, J. Integrated metabolomic and transcriptome analyses reveal finishing forage affects metabolic pathways related to beef quality and animal welfare. Sci. Rep. 2016, 6, 25948. [CrossRef]

18. Cavill, R.; Jennen, D.; Kleinjans, J.; Briedé, J.J. Transcriptomic and metabolomic data integration. Brief. Bioinform. 2016, 17, 891-901. [CrossRef]

19. Wilkinson, J.M. Re-defining efficiency of feed use by livestock. Animal 2011, 5, 1014-1022. [CrossRef]

20. Do, D.N.; Strathe, A.B.; Jensen, J.; Mark, T.; Kadarmideen, H.N. Genetic parameters for different measures of feed efficiency and related traits in boars of three pig breeds. J. Anim. Sci. 2013, 91, 4069-4079. [CrossRef]

21. Morales, P.E.; Bucarey, J.L.; Espinosa, A. Muscle Lipid Metabolism: Role of Lipid Droplets and Perilipins. J. Diabetes Res. 2017, 2017, 1-10. [CrossRef] [PubMed]

22. Pedersen, B.K. Muscle as a Secretory Organ. In Comprehensive Physiology; John Wiley \& Sons, Inc.: Hoboken, NJ, USA, 2013. 
23. Siddiqui, J.K.; Baskin, E.; Liu, M.; Cantemir-Stone, C.Z.; Zhang, B.; Bonneville, R.; McElroy, J.P.; Coombes, K.R.; Mathé, E.A. IntLIM: integration using linear models of metabolomics and gene expression data. BMC Bioinform. 2018, 19, 81. [CrossRef] [PubMed]

24. Taylor, V.A.; Stone, H.K.; Schuh, M.P.; Zhao, X.; Setchell, K.D.; Erkan, E. Disarranged Sphingolipid Metabolism From Sphingosine-1-Phosphate Lyase Deficiency Leads to Congenital Nephrotic Syndrome. Kidney Int. Rep. 2019, 4, 1763-1769. [CrossRef] [PubMed]

25. Donati, C.; Cencetti, F.; Bruni, P. Sphingosine 1-phosphate axis: a new leader actor in skeletal muscle biology. Front. Physiol. 2013, 4. [CrossRef]

26. Lefaucheur, L.; Lebret, B.; Ecolan, P.; Louveau, I.; Damon, M.; Prunier, A.; Billon, Y.; Sellier, P.; Gilbert, H. Muscle characteristics and meat quality traits are affected by divergent selection on residual feed intake in pigs1. J. Anim. Sci. 2011, 89, 996-1010. [CrossRef]

27. Kajimoto, T.; Okada, T.; Yu, H.; Goparaju, S.K.; Jahangeer, S.; Nakamura, S. Involvement of Sphingosine-1-Phosphate in Glutamate Secretion in Hippocampal Neurons. Mol. Cell. Biol. 2007, 27, 3429-3440. [CrossRef]

28. Santos, L.S.; Miassi, G.M.; Tse, M.L.P.; Gomes, L.M.; Berto, P.N.; Denadai, J.C.; Caldara, F.R.; Dalto, D.B.; Berto, D.A. Growth performance and intestinal replacement time of $13 \mathrm{C}$ in newly weaned piglets supplemented with nucleotides or glutamic acid. Livest. Sci. 2019, 227, 160-165. [CrossRef]

29. Wang, M.; Zhang, X.; Kang, L.; Jiang, C.; Jiang, Y. Molecular characterization of porcine NECD, SNRPN and UBE3A genes and imprinting status in the skeletal muscle of neonate pigs. Mol. Biol. Rep. 2012, 39, 9415-9422. [CrossRef]

30. Wahl, M.C.; Will, C.L.; Lührmann, R. The Spliceosome: Design Principles of a Dynamic RNP Machine. Cell 2009, 136, 701-718. [CrossRef]

31. Bottje, W.G.; Lassiter, K.; Piekarski-Welsher, A.; Dridi, S.; Reverter, A.; Hudson, N.J.; Kong, B.-W. Proteogenomics Reveals Enriched Ribosome Assembly and Protein Translation in Pectoralis major of High Feed Efficiency Pedigree Broiler Males. Front. Physiol. 2017, 8. [CrossRef]

32. Zhang, S.; Zeng, X.; Ren, M.; Mao, X.; Qiao, S. Novel metabolic and physiological functions of branched chain amino acids: a review. J. Anim. Sci. Biotechnol. 2017, 8, 10. [CrossRef] [PubMed]

33. Harper, A.E.; Miller, R.H.; Block, K.P. Branched-chain amino acid metabolism. Annu. Rev. Nutr. 1984, 4, 409-454. [CrossRef] [PubMed]

34. Fu, L.; Xu, Y.; Hou, Y.; Qi, X.; Zhou, L.; Liu, H.; Luan, Y.; Jing, L.; Miao, Y.; Zhao, S.; et al. Proteomic analysis indicates that mitochondrial energy metabolism in skeletal muscle tissue is negatively correlated with feed efficiency in pigs. Sci. Rep. 2017, 7, 45291. [CrossRef]

35. Freund, H.R.; Hanani, M. The metabolic role of branched-chain amino acids. Nutrition 2002, 18, 287-288. [CrossRef]

36. Duarte, D.A.S.; Newbold, C.J.; Detmann, E.; Silva, F.F.; Freitas, P.H.F.; Veroneze, R.; Duarte, M.S. Genome-wide association studies pathway-based meta-analysis for residual feed intake in beef cattle. Anim. Genet. 2019, 50, 150-153. [CrossRef]

37. Hu, W.; Zhang, C.; Wu, R.; Sun, Y.; Levine, A.; Feng, Z. Glutaminase 2, a novel p53 target gene regulating energy metabolism and antioxidant function. Proc. Natl. Acad. Sci. USA 2010, 107, 7455-7460. [CrossRef] [PubMed]

38. Zampiga, M.; Flees, J.; Meluzzi, A.; Dridi, S.; Sirri, F. Application of omics technologies for a deeper insight into quali-quantitative production traits in broiler chickens: A review. J. Anim. Sci. Biotechnol. 2018, 9, 61. [CrossRef] [PubMed]

39. Grubbs, J.K.; Fritchen, A.N.; Huff-Lonergan, E.; Dekkers, J.C.M.; Gabler, N.K.; Lonergan, S.M. Divergent genetic selection for residual feed intake impacts mitochondria reactive oxygen species production in pigs1. J. Anim. Sci. 2013, 91, 2133-2140. [CrossRef] [PubMed]

40. Banerjee, P.; Carmelo, V.A.O.; Kadarmideen, H.N. Genome-Wide Epistatic Interaction Networks Affecting Feed Efficiency in Duroc and Landrace Pigs. Front. Genet. 2020, 11. [CrossRef]

41. Higgins, M.G.; Fitzsimons, C.; McClure, M.C.; McKenna, C.; Conroy, S.; Kenny, D.A.; McGee, M.; Waters, S.M.; Morris, D.W. GWAS and eQTL analysis identifies a SNP associated with both residual feed intake and GFRA2 expression in beef cattle. Sci. Rep. 2018, 8, 14301. [CrossRef]

42. Bijvelds, M.J.C.; Tresadern, G.; Hellemans, A.; Smans, K.; Nieuwenhuijze, N.D.A.; Meijsen, K.F.; Bongartz, J.-P.; Ver Donck, L.; de Jonge, H.R.; Schuurkes, J.A.J.; et al. Selective inhibition of intestinal guanosine $3^{\prime}, 5^{\prime}$-cyclic monophosphate signaling by small-molecule protein kinase inhibitors. J. Biol. Chem. 2018, 293, 8173-8181. [CrossRef] 
43. Valente, T.S.; Baldi, F.; Sant'Anna, A.C.; Albuquerque, L.G.; Paranhos da Costa, M.J.R. Genome-Wide Association Study between Single Nucleotide Polymorphisms and Flight Speed in Nellore Cattle. PLoS ONE 2016, 11, e0156956. [CrossRef] [PubMed]

44. Valentino, M.A.; Lin, J.E.; Snook, A.E.; Li, P.; Kim, G.W.; Marszalowicz, G.; Magee, M.S.; Hyslop, T.; Schulz, S.; Waldman, S.A. A uroguanylin-GUCY2C endocrine axis regulates feeding in mice. J. Clin. Investig. 2011, 121, 3578-3588. [CrossRef]

45. Rauw, W.M.; Portolés, O.; Corella, D.; Soler, J.; Reixach, J.; Tibau, J.; Prat, J.M.; Diaz, I.; Gómez-Raya, L. Behaviour influences cholesterol plasma levels in a pig model. Animal 2007, 1, 865-871. [CrossRef]

46. Zhang, L.; Rajbhandari, P.; Priest, C.; Sandhu, J.; Wu, X.; Temel, R.; Castrillo, A.; de Aguiar Vallim, T.Q.; Sallam, T.; Tontonoz, P. Inhibition of cholesterol biosynthesis through RNF145-dependent ubiquitination of SCAP. Elife 2017, 6. [CrossRef] [PubMed]

47. Holeček, M. Effect of starvation on branched-chain $\alpha$-keto acid dehydrogenase activity in rat heart and skeletal muscle. Physiol. Res. 2001, 50, 19-24. [PubMed]

48. Duan, Y.; Duan, Y.; Li, F.; Li, Y.; Guo, Q.; Ji, Y.; Tan, B.; Li, T.; Yin, Y. Effects of supplementation with branched-chain amino acids to low-protein diets on expression of genes related to lipid metabolism in skeletal muscle of growing pigs. Amino Acids 2016, 48, 2131-2144. [CrossRef] [PubMed]

49. Mukiibi, R.; Vinsky, M.; Keogh, K.; Fitzsimmons, C.; Stothard, P.; Waters, S.M.; Li, C. Liver transcriptome profiling of beef steers with divergent growth rate, feed intake, or metabolic body weight phenotypes1. J. Anim. Sci. 2019, 97, 4386-4404. [CrossRef] [PubMed]

50. Sung, Y.J.; Pérusse, L.; Sarzynski, M.A.; Fornage, M.; Sidney, S.; Sternfeld, B.; Rice, T.; Terry, J.G.; Jacobs, D.R.; Katzmarzyk, P.; et al. Genome-wide association studies suggest sex-specific loci associated with abdominal and visceral fat. Int. J. Obes. 2016, 40, 662-674. [CrossRef]

51. Valadkhan, S.; Gunawardane, L.S. Role of small nuclear RNAs in eukaryotic gene expression. Essays Biochem. 2013, 54, 79-90. [CrossRef]

52. Sun, J.S.; Manley, J.L. A novel U2-U6 snRNA structure is necessary for mammalian mRNA splicing. Genes Dev. 1995, 9, 843-854. [CrossRef] [PubMed]

53. Ravi, S.; Schilder, R.J.; Kimball, S.R. Role of Precursor mRNA Splicing in Nutrient-Induced Alterations in Gene Expression and Metabolism. J. Nutr. 2015, 145, 841-846. [CrossRef] [PubMed]

54. Mansouri, E.; Khorsandi, L.; Moaiedi, M.Z. Grape seed proanthocyanidin extract improved some of biochemical parameters and antioxidant disturbances of red blood cells in diabetic rats. Iran. J. Pharm. Res. 2015, 14, 329-334. [CrossRef] [PubMed]

55. Russell, J.R.; Sexten, W.J.; Kerley, M.S.; Hansen, S.L. Relationship between antioxidant capacity, oxidative stress, and feed efficiency in beef steers. J. Anim. Sci. 2016, 94, 2942-2953. [CrossRef] [PubMed]

56. Anders, S.; Huber, W. Differential expression analysis for sequence count data. Genome Biol. 2010, 11, R106. [CrossRef] [PubMed]

57. Ritchie, M.E.; Phipson, B.; Wu, D.; Hu, Y.; Law, C.W.; Shi, W.; Smyth, G.K. limma powers differential expression analyses for RNA-sequencing and microarray studies. Nucleic Acids Res. 2015, 43, e47. [CrossRef]

58. Chong, J.; Soufan, O.; Li, C.; Caraus, I.; Li, S.; Bourque, G.; Wishart, D.S.; Xia, J. MetaboAnalyst 4.0: Towards more transparent and integrative metabolomics analysis. Nucleic Acids Res. 2018, 46, W486-W494. [CrossRef]

59. Mostafavi, S.; Ray, D.; Warde-Farley, D.; Grouios, C.; Morris, Q. GeneMANIA: a real-time multiple association network integration algorithm for predicting gene function. Genome Biol. 2008, 9, S4. [CrossRef]

60. Eden, E.; Navon, R.; Steinfeld, I.; Lipson, D.; Yakhini, Z. GOrilla: a tool for discovery and visualization of enriched GO terms in ranked gene lists. BMC Bioinform. 2009, 10, 48. [CrossRef]

61. Bindea, G.; Mlecnik, B.; Hackl, H.; Charoentong, P.; Tosolini, M.; Kirilovsky, A.; Fridman, W.H.; Pagès, F.; Trajanoski, Z.; Galon, J. ClueGO: A Cytoscape plug-in to decipher functionally grouped gene ontology and pathway annotation networks. Bioinformatics 2009, 25, 1091-1093. [CrossRef] 



\title{
Article \\ Profiling of Metabolomic Changes in Plasma and Urine of Pigs Caused by Illegal Administration of Testosterone Esters
}

\author{
Kamil Stastny ${ }^{1, *}$, Kristina Putecova ${ }^{1}$, Lenka Leva ${ }^{1}$, Milan Franek ${ }^{1}$, Petr Dvorak ${ }^{2}$ and \\ Martin Faldyna ${ }^{1}$ \\ 1 Veterinary Research Institute, Hudcova 296/70, 62100 Brno, Czech Republic; putecova@vri.cz (K.P.); \\ leva@vri.cz (L.L.); franek@vri.cz (M.F.); faldyna@vri.cz (M.F.) \\ 2 Faculty of Veterinary Hygiene and Ecology, University of Veterinary and Pharmaceutical Sciences Brno, \\ Palackeho tr. 1946/1, 61242 Brno, Czech Republic; dvorakp@vfu.cz \\ * Correspondence: stastny@vri.cz
}

Received: 3 June 2020; Accepted: 6 July 2020; Published: 27 July 2020

\begin{abstract}
The use of anabolic steroid hormones as growth promoters in feed for farm animals has been banned in the European Union since 1988 on the basis of Council Directive 96/22/EC. However, there is still ongoing monitoring and reporting of positive findings of these banned substances in EU countries. The aim of this work was to investigate the efficacy and discriminatory ability of metabolic fingerprinting after the administration of $17 \beta$-testosterone esters to pigs. Plasma and urine samples were chromatographically separated on a Hypersil Gold C18 column. High resolution mass spectrometry metabolomic fingerprints were analysed on a hybrid mass spectrometer Q-Exactive. Three independent multivariate statistical methods, namely principal component analysis, clustre analysis, and orthogonal partial least squares discriminant analysis showed significant differences between the treated and control groups of pigs even 14 days after the administration of the hormonal drug. Plasma samples were also analysed by a conventional quantitative analysis using liquid chromatography with tandem mass spectrometry and a pharmacokinetic curve was constructed based on the results. In this case, no testosterone residue was detected 14 days after the administration. The results clearly showed that a metabolomics approach can be a useful and effective tool for the detection and monitoring of banned anabolic steroids used illegally in pig fattening.
\end{abstract}

Keywords: metabolomic; anabolic practices; testosterone; plasma; urine; pigs

\section{Introduction}

The use of hormones as growth promoters for fattening purposes in livestock has been banned in the European Union since 1988 by Council Directive 96/22/EC. However, the banned substances are still reported as positive in the European residue monitoring plans [1]. One of these banned substances is the anabolic and androgenic steroid testosterone, which naturally occurs in animal organisms. Testosterone is endogenously secreted by Leydig cells (testes) and is able to accelerate muscle growth (anabolic effect) and improve the development of male characteristics (androgenic effect). Testosterone is then secreted into the bloodstream where it primarily $(98 \%)$ binds to a specific protein beta-globulin termed sex hormone binding globulin (SHBG) and to a lesser extent to albumin. By this binding, testosterone is biologically protected from inactivation in the liver, and is subsequently transported to the target tissues via the bloodstream. A small amount of circulating testosterone is converted to estradiol, but the greater part of free testosterone is converted to 17-ketosteroids, particularly androsterone and its isomer etio-cholanolone (androsterone metabolites) [2]. In some target tissues, testosterone is reduced to $5 \alpha$-dihydrotestosterone (DHT) by the cytochrome $\mathrm{P}_{450}$ enzyme $5 \alpha$-reductase, an enzyme 
highly expressed in male sex organs, skin, and hair follicles. The inactivation and degradation of testosterone and its metabolites in cattle and pigs occurs mainly in the liver and, to a lesser extent, in the kidneys. These mechanisms of inactivation and degradation of testosterone occur with the participation of specific enzymes involved in the catalytic action of the partially transformed steroid molecule. Inactivation and degradation include the following: addition of two hydrogens (reduction) to a double bond or ketone group; removal of two hydrogens (oxidation) from a hydroxyl group; addition of a hydroxyl group (hydroxylation) to a carbon in the steroid molecule; and conjugation of testosterone by reaction of sulfuric acid or glucuronic acid with a hydroxyl group on the steroid molecule, forming testosterone sulphates and glucuronides, respectively. The sulfated or glucuronide conjugated form of testosterone is then excreted in the urine [3].

Testosterone (a natural steroid) is illegally administered to animals in the form of synthetic steroid esters, but these are rapidly hydrolysed to a natural steroid in vivo. For example, after oral administration of testosterone undecanoate, unchanged ester was found in athletes' plasma for only $6 \mathrm{~h} \mathrm{[4]}$. In analytical practice, it is difficult to distinguish between metabolites of natural endogenous testosterone, which is always present in body fluids (plasma, urine), and metabolites of identical exogenous testosterone derived from hydrolysed synthetically prepared esters [5]. In human doping control, this problem is usually solved by determining the urinary ratio of $17 \beta$-testosterone $/ 17 \alpha$-testosterone levels (T/EpT ratio) or by using gas chromatography with isotopic mass spectrometry (GC-IRMS) and application of the ${ }^{13} \mathrm{C} /{ }^{12} \mathrm{C}$ isotope ratio [6]. The World Anti-Doping Agency (WADA) has established a decision limit if a T/E ratio is equal to or greater than 4 , or an epitestosterone ( $17 \alpha$-testosterone) concentration is greater than $200 \mathrm{ng} \mathrm{mL}^{-1}$ which would require a testing procedure to confirm doping $[7,8]$. Although important in humans, these analytical parameters have failed in animals because of differences in their metabolism [9]. In food safety practices, relatively high or low levels of $17 \beta$-testosterone and $17 \alpha$-testosterone in urine are often ignored due to a lack of statistically valid reference data on naturally occurring endogenous background levels in animals. However, the EU Community Reference Laboratories (CRLs) for analytical methods recommended in the national monitoring control plans to limit concentrations for plasma $\left(\mathrm{CC}_{\alpha}\right.$ for confirmatory methods) to $0.5 \mu \mathrm{g} \mathrm{L}{ }^{-1}$ for heifers 18 months old, $10 \mu \mathrm{g} \mathrm{L}^{-1}$ for bullocks six months old, and $30 \mu \mathrm{g} \mathrm{L}{ }^{-1}$ for bulls 6-18 months old [10]. For other animals, no such recommendations exist for $17 \beta$-testosterone in plasma or urine.

Over time, a number of targeted analytical methods for the determination of testosterone in various biological samples (plasma, urine, muscles and hair) have been developed and described in the literature. In the 1990s, testosterone measurements were often performed by radioimmunoassay (RIA) [11] and immunoassays (ELISA) [12]. Immunological methods are fast, easy-to-perform, cheap, and have a short time to result for a large number of samples, so today they are preferably used in many laboratories primarily for screening. However, cross-reactivity and sensitivity in these assays is a common problem, so these methods are no longer good enough for the detection and quantitative determination of testosterone.

Gas chromatography (GC) and liquid chromatography (LC) are other alternatives for the targeted analysis of testosterone and its esters. GC methods coupled with mass spectrometry (MS) are usually applied for the determination of anabolic steroid levels ranging from micrograms to nanograms in biological samples [13]. The detection of testosterone esters at $1 \mathrm{ng} \mathrm{mL}^{-1}$ in human plasma by GC/MS has been reported [14]. However, GC-MS methods require a complicated, time-consuming, and expensive step of sample derivatization for steroid analysis. In general, these derivatives are unstable and are susceptible to thermal degradation during analysis, which, in particular, significantly affects the reproducibility of the method [15]. In contrast, LC-MS is a good solution for quantitative analysis of steroids because the included sample preparation step is easy, fast, economical and requires no further derivatization step. The high performance liquid chromatography with mass spectrometry (HPLC-MS) used for the analysis of steroid esters in plasma showed greater sensitivity than GC-MS [16]. HPLC is also a commonly used separation technique for the determination of testosterone and its esters in 
body fluids due to its sufficient sensitivity, good resolution, robustness and short analysis time [17]. Ultra-high performance liquid chromatography (UHPLC) coupled with tandem mass spectrometry (MS/MS) or today with high-resolution (HR) mass spectrometry is another powerful approach to significantly improve peak resolution, selectivity, sensitivity and speed of the analysis [18-20]. It should be noted that an interesting alternative to inconclusive urine analyses (endogenous testosterone vs. synthetic testosterone) at veterinary inspection may be the analysis of intact natural steroid esters in the hair by UHPLC-MS/MS [21,22] or DESI-MS (desorption ionizing mass spectrometry) [5].

Recently, new synthetic xenobiotic growth promoters have been designed and new ways of application employed, such as the administration of low dose cocktails. However, metabolomics approaches to non-targeted screening for the detection of anabolic practices with natural steroid hormones might change this situation in the future $[9,23]$. Indeed, several scientific studies have demonstrated the efficiency of mass spectrometry with high resolution based on urinary fingerprinting to discriminate anabolised animals from control ones. Rijk et al. [24] in their work showed the use of a novel untargeted metabolomics based strategy for the measurement of the anabolic steroid DHEA (dehydroepiandrosterone) and pregnenolone in bovine urine with liquid chromatography coupled with time-of-flight mass spectrometry (LCT Premier). In the same year, Kieken et al. [25] presented a metabolomics strategy involving the characterization of global metabolomic fingerprints in urine samples of non-treated and reGH (recombinant equine growth hormone)-treated horses by LC-HRMS (LTQ-Orbitrap) as a new screening tool for growth hormone abuse in horseracing.

Anizan et al. [26] presented in their study a metabolomics approach to 4-androstenedione (AED) detection after its administration to heifers. Using untargeted profiling by GC-MS, they identified $5 \alpha$-androst-2-en-17-one in urine as a new biomarker of anabolic AED abuse. From 2011 to the present, several studies have been conducted in cattle in relation to the administration of banned substances for fattening, which have confirmed the correctness of the research focus on non-targeted analyses based on metabolomic approaches [27-32]. However, all these studies were in all cases carried out only in cattle, although in many European countries, for example, pork was consumed significantly more than beef. The only metabolomic study published so far for another animal species was conducted in 2017 in pigs to which a banned beta-agonist substance, ractopamine, was administered [33].

The present study aimed to investigate the efficacy of metabolomic profiling of pig plasma and urine samples by high resolution mass spectrometry (HRMS) to discriminate between the testosterone ester group and the control group. The experiment was performed in two independent groups of pigs, where individual animals were assigned to groups based on randomization. Plasma and urine samples were continuously collected at specified time intervals, prepared and subsequently measured on a high-resolution hybrid tandem mass spectrometer (QExactive). The obtained metabolomic fingerprints were processed and statistically analyzed using principal component analysis (PCA) and orthogonal partial least squares discriminant analysis (OPLS-DA) multivariate methods. Furthermore, the results of the non-targeted metabolomic analysis obtained in this way were compared with the results of the targeted determination of $17 \beta$-testosterone in the same plasma and urine samples. All pigs in the experiment were weighed at weekly intervals and the anabolic effect of testosterone was studied based on the body weight gain.

\section{Results}

\subsection{Anabolic Effect of $17 \beta$-Testosterone (Esters)}

All animals from both groups were weighed at regular weekly intervals during the treatment experiment, and the body weight gain (BW) in kg is shown in Tables 1 and 2. The anabolic effect was expressed in a graphical form of the dependence of the body weight of experimental pigs on the time interval for both treatment and control groups. Two linear regression models were used to highlight the growth trends of both groups of pigs and the statistical assessment of the anabolic effect of $17 \beta$-testosterone (Figure 1). The average weekly body weight gains were calculated from the detected 
BW data for each group of pigs and are shown in Supplementary Materials Table S1 and Figure S1. All animals were clinically monitored during the experiment and were in good health until slaughter at the end of the experiment. The treatment experiment was performed without any problems.

Table 1. Body weight of the individual pigs treated with a hormone preparation containing a testosterone ester combination.

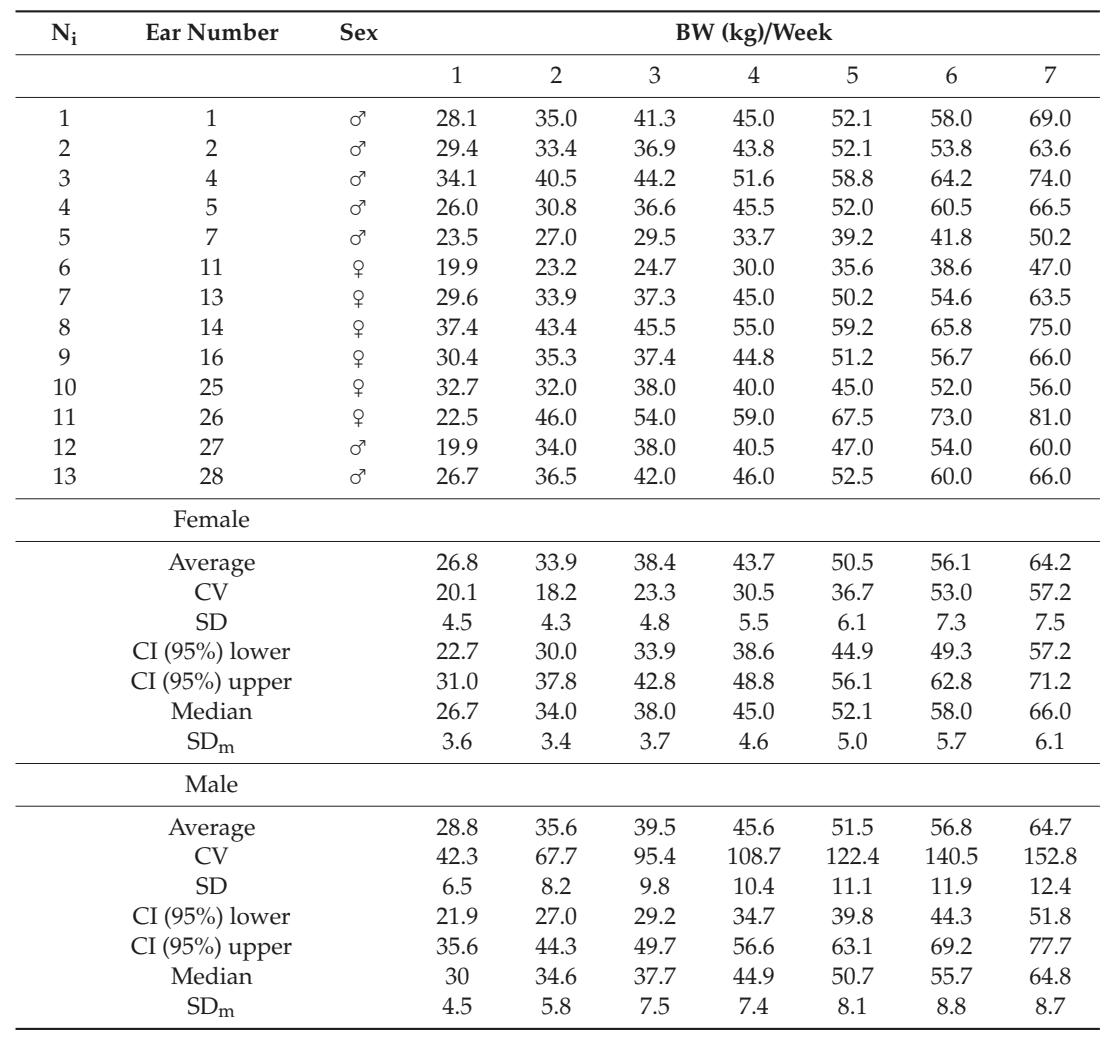

Table 2. Body weight of the individual pigs in the control group.

\begin{tabular}{|c|c|c|c|c|c|c|c|c|c|}
\hline \multirow[t]{2}{*}{$\mathrm{N}_{\mathrm{i}}$} & \multirow[t]{2}{*}{ Ear Number } & \multirow[t]{2}{*}{ Sex } & \multicolumn{7}{|c|}{ BW (kg)/Week } \\
\hline & & & 1 & 2 & 3 & 4 & 5 & 6 & 7 \\
\hline 1 & 8 & $0^{x}$ & 26.1 & 31.1 & 36.0 & 43.0 & 49.0 & 51.9 & 57.0 \\
\hline 2 & 9 & $\sigma^{\pi}$ & 26.3 & 30.2 & 34.6 & 38.6 & 44.2 & 48.1 & 54.5 \\
\hline 3 & 12 & q & 28.8 & 33.9 & 37.5 & 43.5 & 50.4 & 52.6 & 58.9 \\
\hline 4 & 15 & 우 & 29.2 & 33.8 & 36.1 & 43.5 & 50.0 & 54.2 & 60.5 \\
\hline 5 & 21 & 우 & 29.4 & 33.1 & 38.0 & 42.5 & 48.0 & 52.1 & 58.6 \\
\hline 6 & 22 & 우 & 30.4 & 36.0 & 43.0 & 45.5 & 51.0 & 55.0 & 60.0 \\
\hline 7 & 23 & $0^{7}$ & 30.9 & 34.5 & 40.5 & 44.0 & 48.0 & 52.5 & 60.5 \\
\hline 8 & 24 & $0^{7}$ & 24.9 & 35.0 & 39.5 & 42.0 & 44.5 & 51.5 & 58.1 \\
\hline & Average & & 28.3 & 33.5 & 38.2 & 42.8 & 48.1 & 52.2 & 58.5 \\
\hline & $\mathrm{CV}$ & & 4.8 & 3.8 & 7.5 & 4.0 & 6.6 & 4.2 & 4.1 \\
\hline & SD & & 2.2 & 1.9 & 2.7 & 2.0 & 2.6 & 2.1 & 2.0 \\
\hline & CI $(95 \%)$ lower & & 26.4 & 31.8 & 35.9 & 41.1 & 46.0 & 50.5 & 56.8 \\
\hline & CI (95\%) upper & & 30.1 & 35.1 & 40.4 & 44.5 & 50.3 & 54.0 & 60.2 \\
\hline & Median & & 29.0 & 33.9 & 37.8 & 43.3 & 48.5 & 52.3 & 58.8 \\
\hline & $\mathrm{SD}_{\mathrm{m}}$ & & 1.5 & 1.5 & 2.1 & 1.8 & 1.7 & 1.8 & 1.5 \\
\hline
\end{tabular}




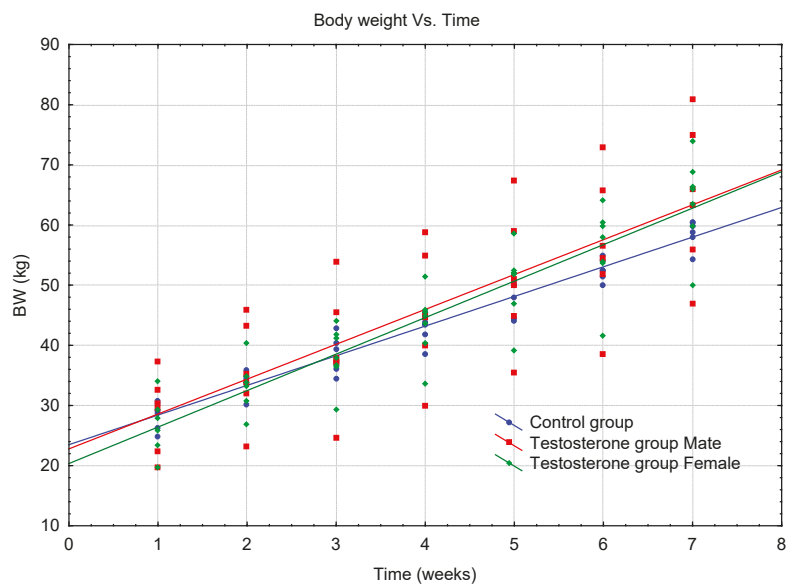

Figure 1. A combined diagram of two regression models for testosterone treated pigs (male and female) and the control group of pigs.

\subsection{Targeted Analysis of $17 \beta$-Testosterone in Plasma}

\subsubsection{Identification of Analytes}

Standards of $17 \beta$-testosterone, testosterone propionate, testosterone isocaproate, testosterone decanoate, and $17 \beta$-testosterone- $\mathrm{D}_{2}$ internal standard were always identified on the basis of the retention time (see Figure 2) obtained from the chromatogram and mass accuracy (MA) parameters calculated from mass spectra for precursor and product ions of each analyte by comparing the theoretical mass $m / z$ with the measured experimental mass $m / z$. The obtained results and the calculated MA values determined by the standards are presented in Supplementary Materials Table S3 and in the diagrams showing the detected experimental mass spectra of the analyte standards, always in comparison with the theoretical mass spectra (Supplementary Materials Figures S2 and S3).

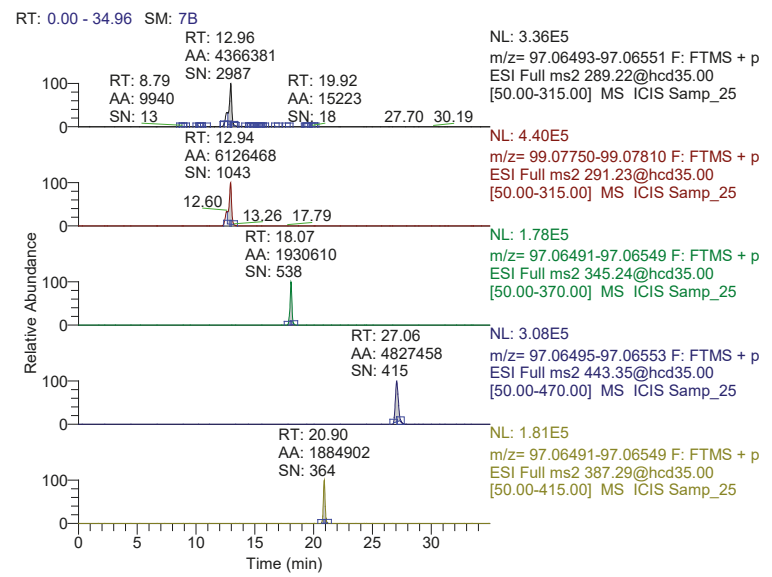

Figure 2. Chromatogram of the analysed plasma samples fortified with standards at $10 \mu \mathrm{g} \mathrm{L}-1$ (ppb), RT indicates retention time, AA indicates the peak area, $\mathrm{SN}$ indicates signal to noise ratio, black chromatogram represents $17 \beta$-testosterone, red chromatogram represents IS $17 \beta$-testosterone-D2, green chromatogram represents $17 \beta$-testosterone propionate, blue chromatogram represents $17 \beta$-testosterone decanoate and the yellow chromatogram represents $17 \beta$-testosterone isocaproate. 


\subsubsection{Study Validation}

The targeted quantitative method was in-house validated complies with model-dependent performance characteristic covering specificity, selectivity, precision, repeatability, within-laboratory reproducibility, the calibration curve, detection limit (LOD), limit of quantification (LOQ), decision limit $(C C \alpha)$, detection capability $(C C \beta)$, and ruggedness according to the recommendation defined in Commission Decision 2002/657 / EC [34] and the reference guidelines in VICH GL49 [35].

The linearity of the quantitative method was determined for testosterone and testosterone ester analytes that were fortified into real pig plasma samples with increasing concentrations. The model samples were prepared according to the procedure described in Section 4.5. A matrix calibration curve was constructed based on the measured peak area ratios (Std. area/IS area) and the corresponding concentration levels. The parameters of the linear regression models were calculated by the least squares method (with a weight coefficient $w=1 / 2$ ) based on ISO 11843: 2 [36]. Correlation coefficients $(\mathrm{r})$, linear regression model parameters $(\mathrm{y}=\mathrm{a}+\mathrm{bx})$ and critical curve limits (LOD, LOQ) were calculated and reported in Table 3 . The calibration curve for $17 \beta$-testosterone, which was used to back-estimate the results of real plasma samples obtained during the experiment, was shown graphically (Supplementary Materials Figure S4). The complete standard area and internal standard area data that was used to calculate the $17 \beta$-testosterone calibration curve are presented in Supplementary Materials Table S4.

To determine the precision and repeatability (within-laboratory reproducibility) of the targeted analysis method, the standard deviation (SD) and variation coefficient $(\mathrm{CV}, \%)$ were determined and calculated by repeated measurement of fortified plasma samples at two concentration levels. The calculated CV ( $n=12)$ was less than $3.09 \%$ for a concentration level of $5 \mathrm{ng} \mathrm{mL}^{-1} 17 \beta$-testosterone in plasma, demonstrating the good precision and repeatability required for confirmatory residual analyses by the Commission Decision 2002/657/EC. The results of the validation study for precision, repeatability, and other calculated statistics are shown in Supplementary Materials Table S5.

Table 3. Regression parameters of matrix calibration curves in the concentration range 0 to $80 \mathrm{ng} \mathrm{mL}^{-1}$.

\begin{tabular}{|c|c|c|c|c|c|c|}
\hline Analyte & Intercept $(a)$ & Slope $(b)$ & $\begin{array}{l}\text { SD of the } \\
\text { Slope }\end{array}$ & $\begin{array}{l}\text { Correlation } \\
\text { Coefficient } r\end{array}$ & $\begin{array}{c}\text { LOD } \\
\left(\mathrm{ng} \mathrm{mL}^{-1}\right)\end{array}$ & $\begin{array}{c}\mathrm{LOQ} \\
\left(\mathrm{ng} \mathrm{mL}^{-1}\right)\end{array}$ \\
\hline $17 \beta$-testosterone & 0.0518 & 0.0690 & 0.00096 & 0.9982 & 0.32 & 0.63 \\
\hline $17 \beta$-testosterone propionate & 0.0291 & 0.0482 & 0.00086 & 0.9991 & 0.19 & 0.52 \\
\hline $17 \beta$-testosterone decanoate & 0.0115 & 0.0713 & 0.00089 & 0.9979 & 0.21 & 0.54 \\
\hline $17 \beta$-testosterone isocaproate & 0.0248 & 0.0448 & 0.000073 & 0.9996 & 0.17 & 0.43 \\
\hline
\end{tabular}

Note: LOD and LOQ were estimated according to IUPAC (Direct Signal Method) methodology.

\subsubsection{Pharmacokinetic Profile of $17 \beta$-Testosterone}

The experiment included targeted analysis of the primary testosterone metabolite in porcine plasma after a single i.m. administration and subsequent determination of the pharmacokinetic curve. Plasma concentrations of free $17 \beta$-testosterone for individual pigs were determined based on an estimation from the matrix calibration curve (see Supplementary Materials Table S6). The resulting plasma concentrations of $17 \beta$-testosterone were used to construct a pharmacokinetic curve, and a graph of concentration versus time is shown in Figure 3. 


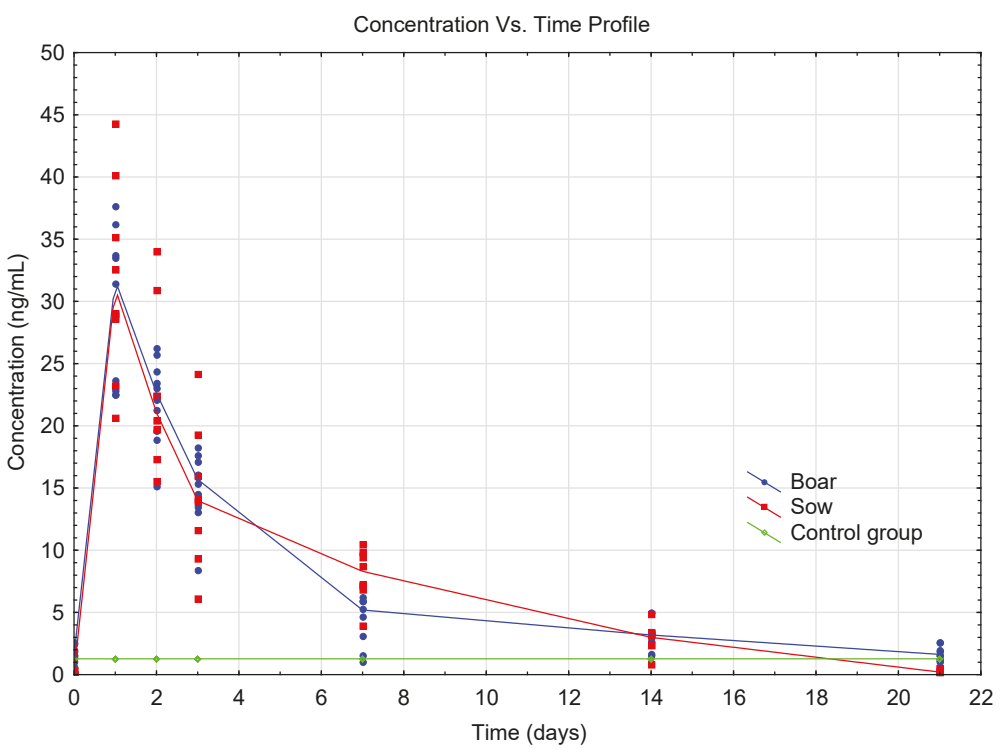

Figure 3. Plasma concentrations in pigs - time profile of testosterone after a single i.m. administration of $0.6 \mathrm{~mL}$ Sustanon $250 \mathrm{mg} / \mathrm{mL}$ inj.; the points on the curve represent the detected $17 \beta$-testosterone plasma concentrations in individual pigs.

\subsection{Metabolomic Study of Blood Plasma and Urine}

The obtained plasma and urine samples were processed in the laboratory as described in Section 4.2. The metabolomic profiles of the individual samples on day 14 after the administration of the hormonal preparation SUSTANON were measured as described in Section 4.4. Both groups of plasma and urine metabolomic profiles were processed for a comparison in XCMS software and, alternatively, using the SIEVE company software. Both variants of data processing identified the approximately corresponding number of ions $(\mathrm{m} / \mathrm{z})$ of peaks or metabolites: 2500 ions were found in plasma and 1400 ions were found in urine. In both cases, the original number of ions in these data sets was further reduced based on a $p$-volume $\leq 0.05$ for further statistical processing. The source data of sets $X(n \times m)$ after reduction each contained $n=21$ rows (animal objects) and $m=254$ columns of statistically significant identified peak areas or metabolites for plasma and $m=213$ columns for urine, respectively. Datasets were transformed using two different methods, i.e., column centering [37] and probabilistic quotient normalization $(\mathrm{PQN})$ [38], and a natural logarithm was applied for their scaling before the subsequent multivariate statistical analysis. The hotelling $\mathrm{T}^{2}$ test criterion did not identify any outlier in both data sets (Supplementary Materials Figures S5 and S6).

Multidimensional statistical methods such as principal component analysis (PCA), clustering analysis (CA) and orthogonal partial squares discriminant analysis (OPLS-DA) were applied for finding relationships between metabolomics datasets of plasma and urine. PCA score plots for plasma and urine samples (Figure 4) and a dendrogram from CA (Figure 5) visibly differentiated between the control group and the treated group of pigs after 17 beta-testosterone administration. The main graphical results from the OPLS-DA analysis of data matrix of $X$ mass spectra of plasma and urine samples versus data matrix $Y$ for binary variables ( $1=$ group of treated pigs, $2=$ control group) were generated by the proposed statistical model and are shown in Figure 6. Furthermore, the coefficients $R^{2}(X)=0.616, R^{2}(Y)=0.987$ for the fit and $Q^{2}(Y)=0.898$ for prediction of the model (according to cross validation) were calculated by OPLS-DA analysis for plasma and the coefficients $R^{2}(X)=0.469$, $R^{2}(Y)=0.997$ and $Q^{2}(Y)=0.879$ for urine data. The OPLS-DA permutation tests further confirmed that the proposed statistical models are correct and robust (Supplementary Materials Figures S7 and S8). 
A volcano plot and variable importance in the projection (VIP) plot and S-plot from OPLS-DA were employed to determine the most discriminating metabolites between the treatment group and the control group (Figure 7).

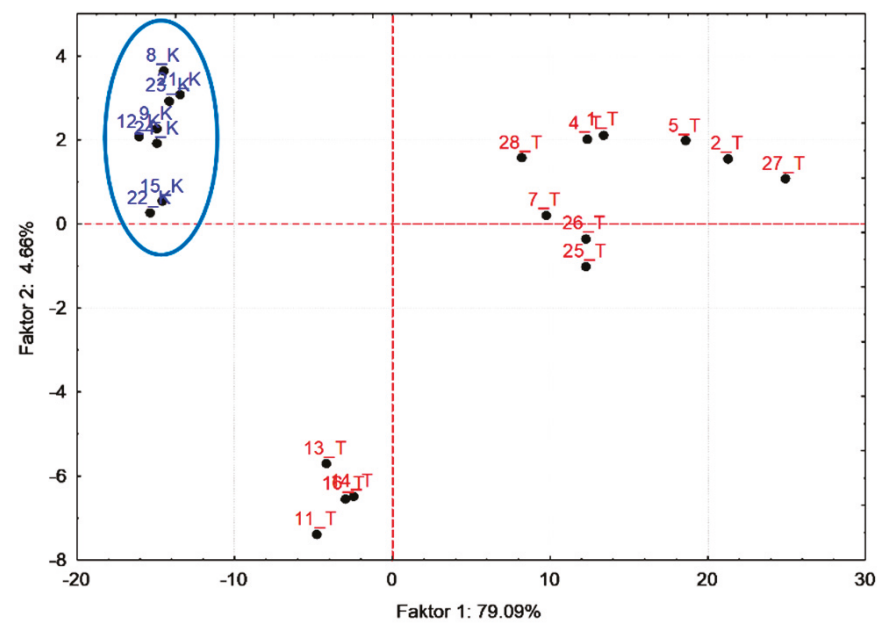

(A)

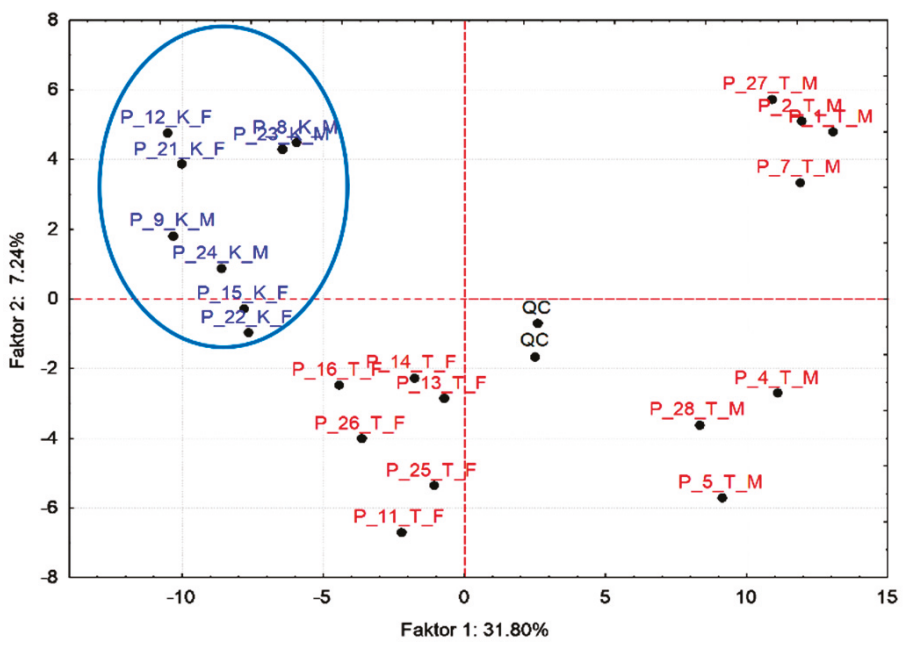

(B)

Figure 4. PCA Score plots for plasma (A) and urine (B) data matrix, blue ellipse, and blue point descriptions (K) represent statistically significantly different samples from the control group of pigs versus the treated $(\mathrm{T})$ group of pigs; added urine labelling: $\mathrm{M}-$ male and $\mathrm{F}-$ female (Centering, STATISTICA). 


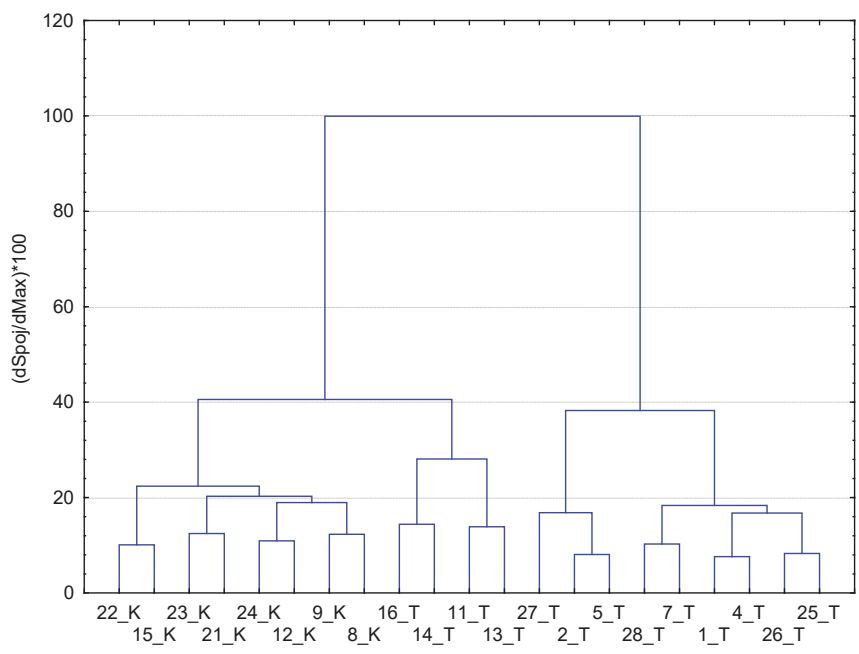

(A)

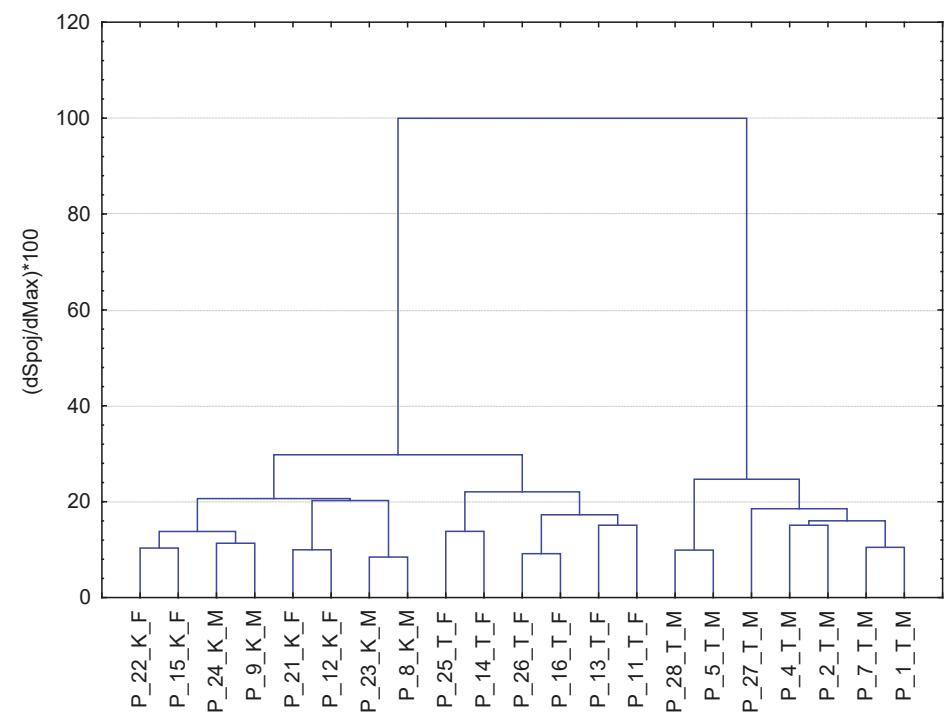

(B)

Figure 5. CA dendrogram of matrix data objects for plasma (A) and urine (B), labels: K-control group, T-treated group and $\mathrm{M}$-male, F-female (by Euclidean distance method, STATISTICA). 


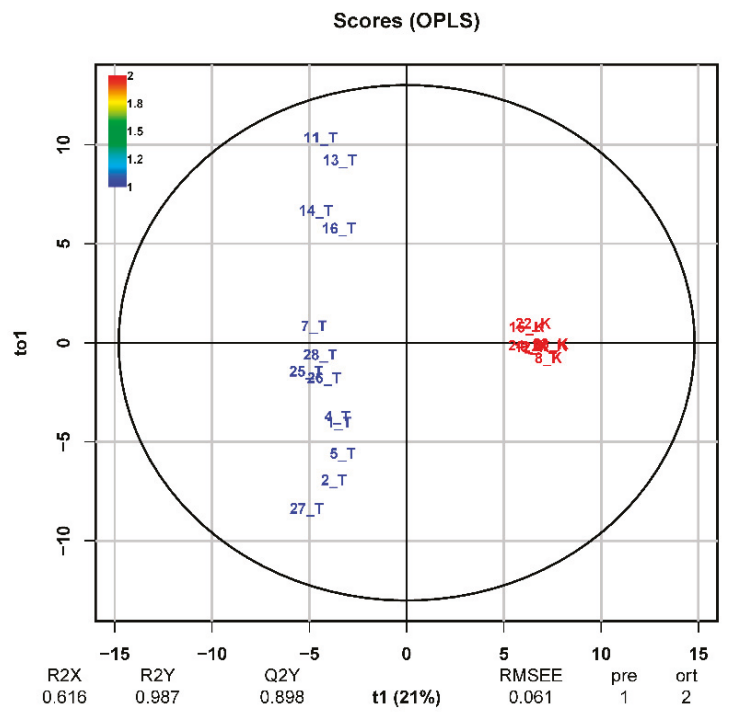

(A)

Scores (OPLS)

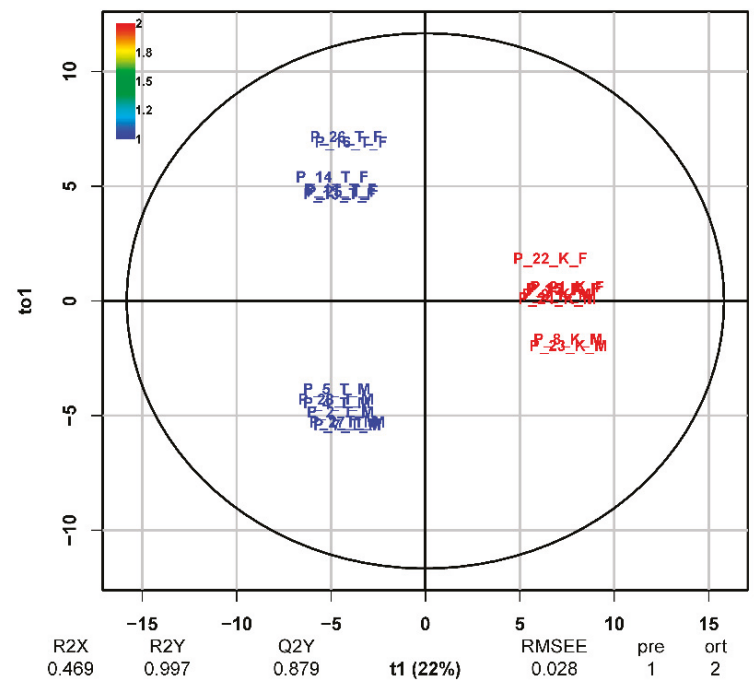

(B)

Figure 6. The OPLS-DA score plots for plasma (A) and urine (B) data matrix demonstrate robust discrimination between the control group of pigs marked with red colour and the group of treated pigs marked with blue colour (PQN scaling, R package). The control group indicated by red Pig number 8 , $9,12,15,21,22,23$, and 24 . The treated group indicated by blue Pig number 1, 2, 4, 5, 7, 11, 13, 14, 16, $25,26,27$, and 28 . 


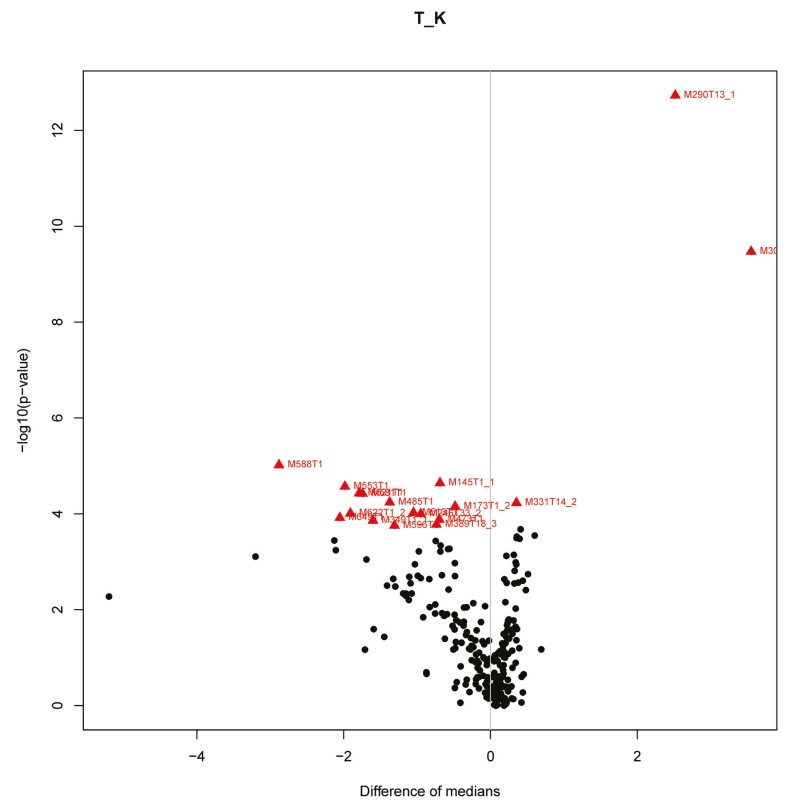

(A)

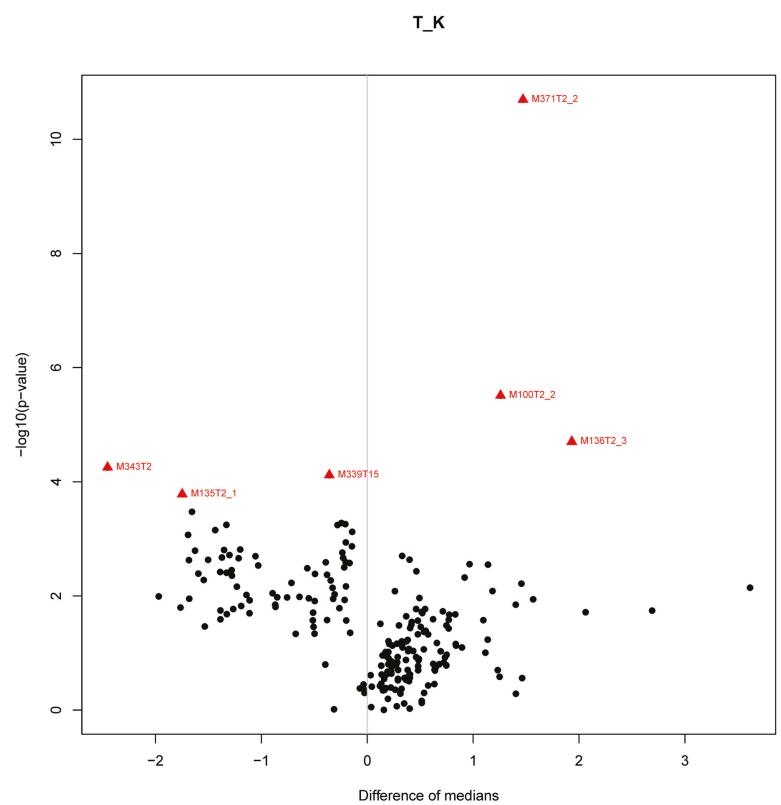

(B)

Figure 7. The OPLS-DA Vulcano plots for plasma (A) and urine (B) data matrix, only metabolites with the VIP scores above 2 were considered significant. A list of specific numbers of metabolites is given in Table 4 . 
Metabolites 2020, 10, 307

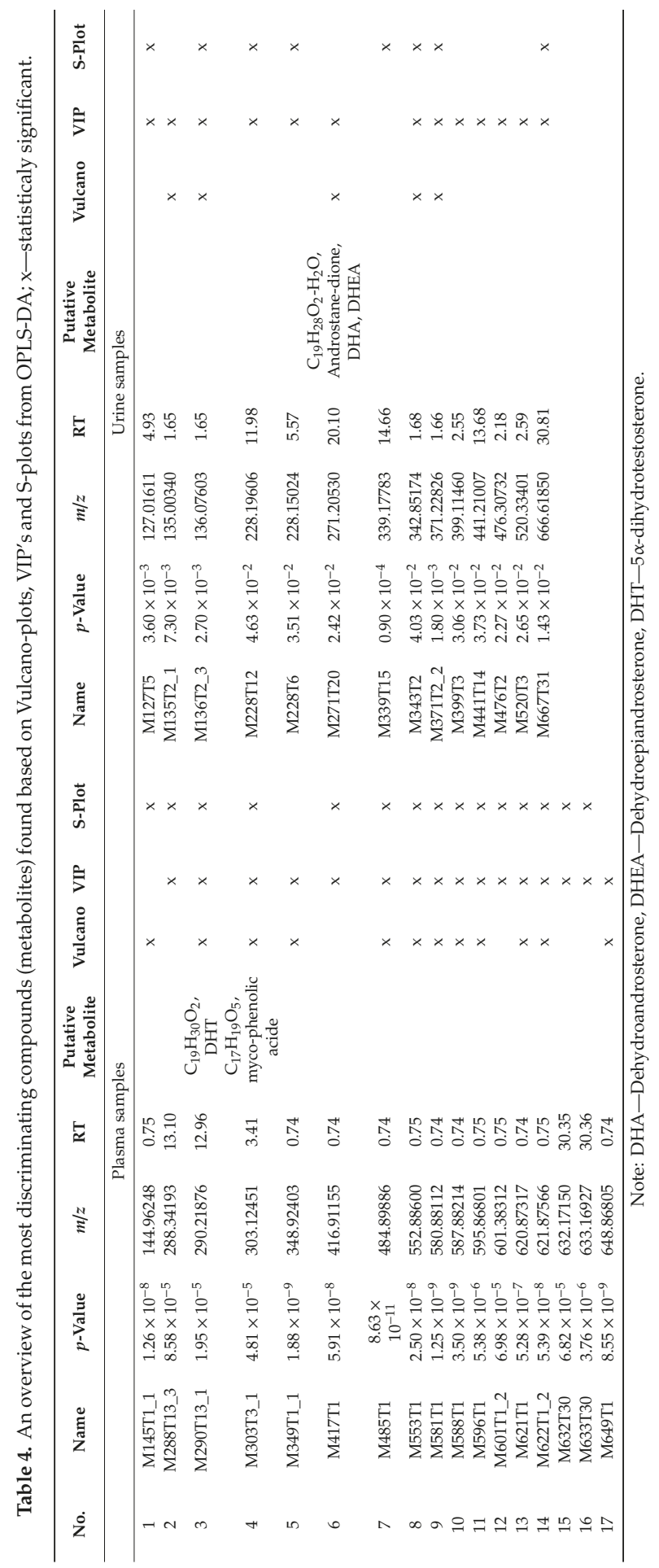




\subsection{QC Samples}

To control the quality of the metabolic profile (fingerprint) measurement, a constant amount of the internal 17 $\beta$-testosterone-D2 standard was added to each plasma and urine sample (Figure 8), and pooled QC samples were included in each measured acquisition. The RT (deviation up to $10 \%$ ), peak area (deviation up to $10 \%$ ) and $\mathrm{MA}(\Delta \mathrm{ppm}<3)$ were checked in each measured metabolomic profile.

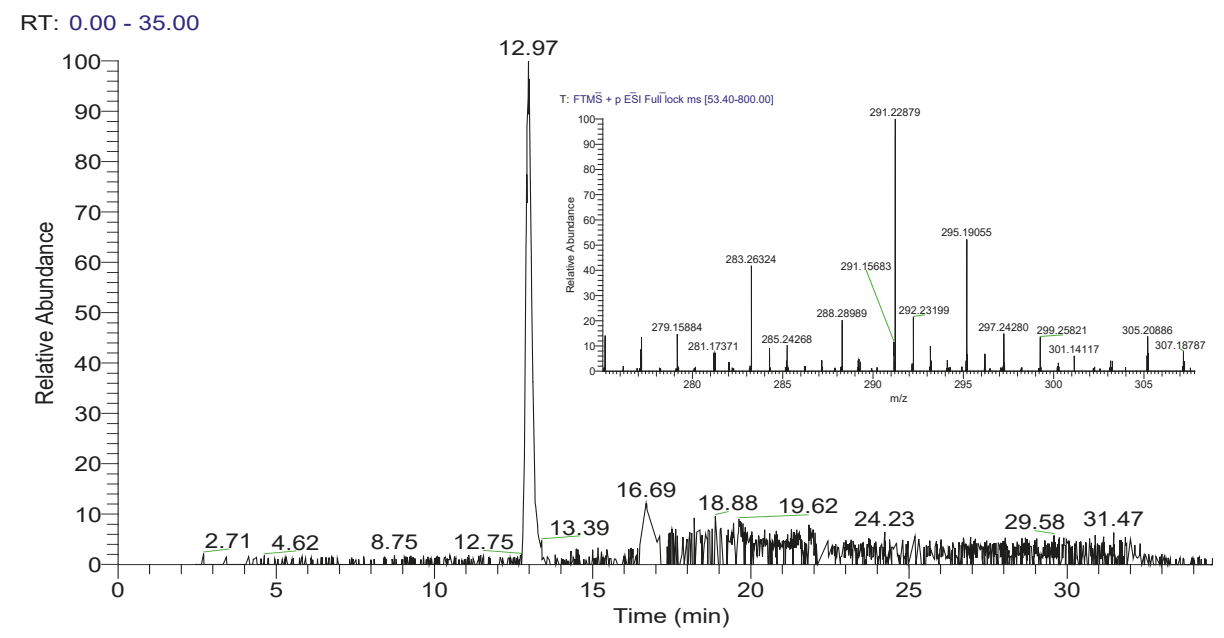

Figure 8. Pooled urine QC sample, control chromatogram for internal standard and corresponding mass spectrum (experimental $m / z=291.22879, \Delta p p m=0.1$ ).

\section{Discussion}

The experimental study was carried out in pigs with the main aim of demonstrating the suitability or unsuitability of metabolomic approaches and techniques for detecting the use of banned androgenic anabolic steroids in animal feed and food of animal origin in European countries. All experimental data obtained from the performed study were statistically evaluated using interactive computer-oriented approaches and specialized statistical software with the main emphasis on the correct interpretation of results and endeavour for obtaining a more comprehensive view of the analyzed and assessed topics of our study. The results were presented primarily in a graphical form, as opposed to the mathematical evaluation of the performed statistical analyses, because they generally have a higher predictive ability for the overall evaluation of the achieved effect.

\subsection{Anabolic Effect of $17 \beta$-Testosterone (Esters)}

From the obtained experimental data of BW measurements in a weekly time interval, estimates of standard univariate statistics (mean, variance, standard deviation, median, median standard deviation and $95 \%$ confidence interval) were calculated for each time period, separately for the treated pigs and the control group (Tables 1 and 2). The mean BW of pigs from the treated group was lower $($ mean $=27.7 \mathrm{~kg}, \mathrm{CI}=[24.5 ; 30.9]) 1$ week after $17 \beta$-testosterone administration than the mean BW of pigs from the control group (mean $=28.3 \mathrm{~kg}$, CI $[26.4 ; 30.1]$ ). At the end of the weighing at week 7 after the administration, this situation was reversed. The mean BW of pigs from the treated group $($ mean $=64.4 \mathrm{~kg}, \mathrm{CI}=[58.6 ; 70.2])$ was $9 \%$ higher than the mean BW of pigs from the control group (mean $=58.5 \mathrm{~kg}, \mathrm{CI}=[56.8 ; 60.2]$ ) and averages differed significantly ( $t$-test, $p<0.05$ ).

Two linear regression models of BW growth versus time were designed to test the anabolic effect, each model especially for the group of treated pigs and the data of the control group (Figure 1). Both models were tested by regression diagnostics. Testing of the regression triplet (data + model 
+ method) showed that the proposed linear models were significant and correct. Estimates of the regression parameters of both linear models are given in Supplementary Materials Table S2. Statistical testing of a comprehensive comparison of both models over the whole-time interval was performed. The Chow test [39] was used to test equality between sets of coefficients in two linear models, using the Fisher-Snedecor distribution with $m$ and $r$ degrees of freedom as statistics. The calculated statistic $\mathrm{F}_{\mathrm{Ch}}=13.583$ was greater than the critical value of $\mathrm{F}_{0.95(2.62)}=3.150$. It could be concluded that the hypothesis $\mathrm{H}_{0}$ was rejected at the significance level $\alpha=0.05$ and both models were not equal. Applying the conclusion of the Chow test, it can be concluded that the results of BW growth detected in pigs in relation to the time interval can be considered different for both groups of pigs, e.g., the effect of the anabolic effect of testosterone was demonstrated. Since the initial weights of the two groups of pigs did not differ significantly, it can be stated that in this case the slope of the two linear models compared is statistically significantly different. This partial conclusion of the study is also evident from the graphical comparison of the linear curves of both groups of pigs (see Figure 1). The anabolic effect, but in this case of nandrolone (19-nor $17 \beta$-testosterone), a synthetic analogue of testosterone, has recently been similarly demonstrated in treated barrows vs. control barrows [40].

\subsection{Targeted Determination of $17 \beta$-Testosterone in Plasma}

An analytical method for the targeted determination of $17 \beta$-testosterone in pig plasma based on LC-MS/(HR)MS was developed as part of this study to estimate the pharmacokinetic curve. The quantitative method of analysis was developed and validated as a confirmatory method as required by the European Directive for residues [34]. The correct identification of targeted analytes using the mass accuracy (MA) criterion [41,42] and quantification based on a matrix calibration curve with parameter estimates for precision and repeatability were part of the validation of the confirmation method; the results are given in Section 2.2. A detailed description of the methodology used for the identification and validation of targeted analytes has been previously described by Stastny et al. [43].

All calculated MA values (Supplementary Materials Table S2) ranged from 0.1 to $1.9(\Delta \mathrm{ppm})$ for the individual analytes determined, and these calculated values were lower than the allowed instrumental tolerance $\leq 3 \mathrm{ppm}$ for the QExactive mass spectrometer used. The tolerance of retention times (RT) was below $\pm 10 \%$ in all cases. Furthermore, a very good chromatographic separation of the analytical method from the chromatogram shown in Figure 2 is evident, where the signal-to-noise ratio (SN) value shown is significantly higher than the generally recommended value $(\mathrm{SN}>3)$, e.g., for $17 \beta$-testosterone, the ratio was $\mathrm{SN}=2987: 1$. Correlation coefficients $(\mathrm{r})$ estimated for the target analytes were $\geq 0.9991$ (17 $\beta$-testosterone), $\geq 0.9979$ (17 $\beta$-testosterone propionate) and 0.9996 (17 $\beta$-testosterone decanoate) in plasma (Table 4). The obtained regression models showed good linearity. The sensitivity of the method was determined by the critical values $\mathrm{LOD}=0.32 \mathrm{ng} \mathrm{mL}^{-1}$ and $\mathrm{LOQ}=0.63 \mathrm{ng} \mathrm{mL}{ }^{-1}$ for $17 \beta$-testosterone based on estimated from the matrix calibration curve model. The precision of the method as a simple repeatability, expressed as the value of the relative standard deviation (RSD), was $3.09 \%$ for $17 \beta$-testosterone to a concentration level of $5 \mathrm{ng} \mathrm{mL}^{-1}$. Based on the results obtained from the validation study, it was possible to conclude that the developed analytical method is suitable for the identification and quantification of free $17 \beta$-testosterone in pig plasma in the range of matrix calibration 0.5 to $80 \mathrm{ng} \mathrm{mL}^{-1}$.

The pharmacokinetic curve of free $17 \beta$-testosterone in pig plasma after the administration of $0.6 \mathrm{~mL} / \mathrm{pig}$ of the hormonal preparation SUSTANON 250 was constructed on the basis of the results of detected concentrations in selected time intervals (Figure 3). The application dosage of the hormonal drug used was calculated on the basis of the recommended dosage for this drug in human medicine. The pharmacokinetic curves for castrated boars and sows were identical. The maximum

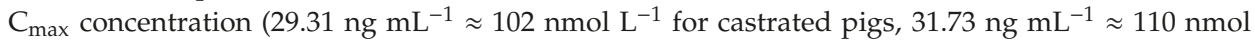
$\mathrm{L}^{-1}$ for sows) was reached $\mathrm{t}_{\max } 24 \mathrm{~h}$ after the administration. Plasma testosterone levels returned to the mean endogenous testosterone levels in castrated boars and sows in approximately 21 days. The PK results for pigs corresponded to the pharmacokinetic properties of i.m. administration of 
testosterone in men listed in the summary of product characteristics (SPC) of the hormonal drug used $\left(\mathrm{C}_{\max }=70 \mathrm{nmol} \mathrm{L}-1, \mathrm{t}_{\max }=24-48 \mathrm{~h}\right.$, elimination time approximately 21 days $)$. Although there are a number of published results on PK testosterone levels in human medicine, the authors of this study of testosterone pharmacokinetics could not compare their results with other authors because such results in animals (pigs) have not been yet published in veterinary medicine.

The determined concentrations of endogenous free $17 \beta$-testosterone in the plasma of castrated boars of the hybrid Lage White/Czech White breed (50/50) ranged from 0.55 to $2.58 \mathrm{ng} \mathrm{mL}-1$ (mean $=1.56 \mathrm{ng} \mathrm{mL}^{-1}$ ). These determined concentrations for endogenous testosterone corresponded to the values reported in the literature: White composite breed $4.0 \mathrm{ng} \mathrm{mL}^{-1}$ [44], Yorkshire 2.5-5.1 $\mathrm{ng} \mathrm{mL}^{-1}$, and Duroc $0.7-3.1 \mathrm{ng} \mathrm{mL}^{-1}$ [45]. The determined concentrations of endogenous free $17 \beta$-testosterone in sow plasma were below the limit of detection $(<\mathrm{LOD})$ of the analytical method.

The individual testosterone esters contained in SUSTANON 250 hydrolyzed very rapidly in the bloodstream of pigs and were no longer detecable $24 \mathrm{~h}$ after application, the concentration was $<$ LOD of the targeted quantitative method. Authors Rejtharová et al. [46] describes the methodology of targeted analysis of testosterone esters in model samples of bovine and porcine serum by LC-MS/MS. On the contrary, as the results of our study show, the targeted determination of testosterone esters in pig serum samples to directly demonstrate the illegal use of banned testosterone is not very suitable for a very short hydrolysis time after application in a real biological system.

\subsection{Metabolomic Profiling of Changes in Plasma and Urine}

The matrices of metabolomics data $X$ obtained from an experiment performed in pigs and measured on a high-resolution mass spectrometer contain data of the same type $m / z$, i.e., they are homogeneous matrices (low molecular weight compounds, metabolites). To search for latent structure and reveal interrelationships in characters (X-variables) and mainly in objects, cases (pigs) in metric scale, two generally used multivariate statistical methods for character reduction to latent variables were used: principal component analysis (PCA) and cluster analysis (CA). To investigate the dependences between the independent matrix $X$ of metabolomics data and the second dependent variable matrix $Y$ (single-column matrix with binary data, treated group $=1$ and control group $=2$ ), another powerful statistical method, partial least squares projection to latent structures PLS was used in the form of differential PLS-DA analysis and in the orthogonal variant O-PLS-DA. Unsupervised PCA and supervised OPLS-DA are today the most widely used multidimensional statistical methods in metabolomics for non-targeted monitoring of changes in biochemical pathways in various biological samples, for their ability to reduce data dimensions or reduce large numbers of variables without much loss of information contained in their first few principal components (most often 2-3).

The metabolomics study was performed in a total of 21 pigs (objects), which were allocated into two groups: a treated group $(n=13)$ and a control group $(n=8)$. The problem of the first metabolomics studies in the field of food safety and illegal use of prohibited substances in livestock fattening performed and published between 2009 and 2010 was mainly the small number of experimental animals [24-26]. The authors of these first metabolomic studies were aware of this problem and subsequent studies carried out and published since 2011 have already been performed in an adequate number of animals $(n>10)$, for example [28-31].

The main results of the PCA method were score plots for plasma and urine data matrix (Figure 4), which showed significant discrimination of all objects (pigs) into two large clusters for both cases of biological matrices. A compact cluster of control group pigs (indicated by a blue ellipse) against two clearly separated clusters (point descriptions marked in red) of pigs from the treated group was found. In the treated group of pigs after testosterone administration, there was another incomplete discrimination according to the characteristics of the sex. However, in the case of plasma, two sows (objects 25 and 25) remained assigned to a cluster of castrated boars, which means that they correlated more with this group. The above results lead to a significant partial conclusion that the built PCA models were able to clearly differentiate the group of tested pigs from the control group. 
A CA dendrogram of matrix data objects for plasma and urine (Figure 5) were constructed based on the average Euclidean distance and also showed a reliable distinction between the group of tested pigs and the control group, so CA analysis confirmed the previous partial conclusion from PCA. The following graphical outputs from the third supervised OPLS-DA method (Figure 6) scatter plots also confirmed the previous conclusions and were able to significantly differentiate the group of control pigs (marked in blue) from the group of tested pigs (descriptions marked in red). The $R^{2}(X), R^{2}(Y)$ and $\mathrm{Q}^{2}(\mathrm{Y})$ statistics for the OPLS-DA models were calculated. Multiple correlation $\mathrm{R}^{2}$ and cross-validated coefficient $Q^{2}$ for control vs. treated group $R^{2}(X)=0.616, R^{2}(Y)=0.987$ and $Q^{2}(Y)=0.898$ for plasma and $R^{2}(X)=0.469, R^{2}(Y)=0.997$ and $Q^{2}(Y)=0.879$ for urine, confirmed good class separation and a high predictive ability. Coefficients $\mathrm{Q}^{2}(\mathrm{Y})$ expressing the predictive abilities of the proposed model were calculated for $75 \%$ cross-validation.

The group of tested pigs, analogously as in the PCA and CA models, was further divided in the plane $t 1$ and to 1 into two clusters according to their sex. Therefore, the OPLS-DA model for urine was further designed and tested, where in matrix Y (two-column matrix with binary data; treated group $=1$, control group $=2$ and male group $=1$, female group $=2$ ) there was a differentiation according to gender (Figure 9). Statistics $\mathrm{R}^{2}(\mathrm{X})=0.417, \mathrm{R}^{2}(\mathrm{Y})=0.973$, and $\mathrm{Q}^{2}(\mathrm{Y})=0.85$ also showed good separation and high prediction. The observed differentiation of groups of pigs by sex in all three models used after testosterone administration brings a whole new dimension to the whole issue of using metabolomics profiling to prove illegal use of banned substances. This genetic factor will have to be taken into account and further investigated in additional metabolomics studies in pigs.

Scores (OPLS)

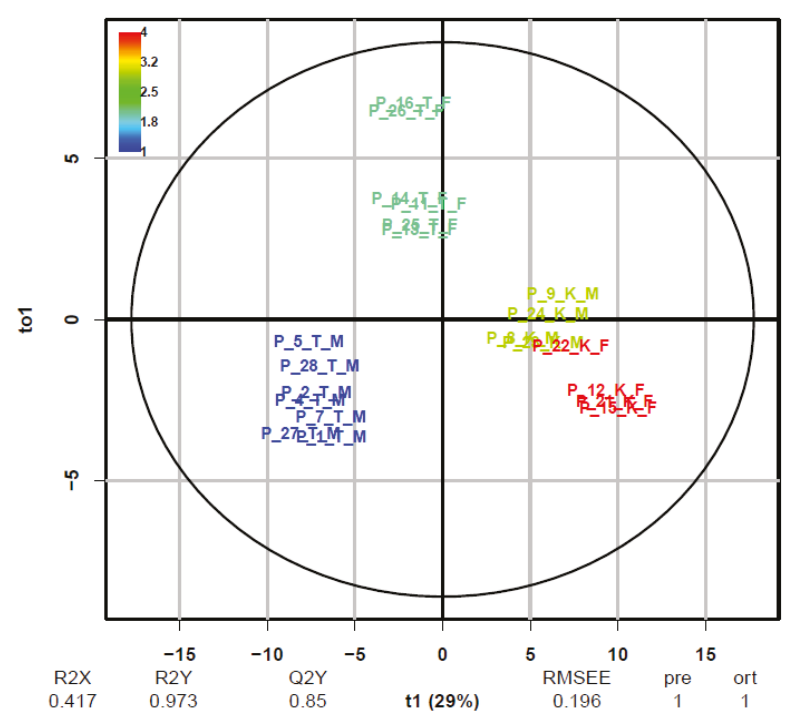

Figure 9. The OPLS-DA score plots for urine data matrix demonstrate discrimination between the control group of pigs and secondary discrimination by sex, objects (pigs) marked with: $\mathrm{K}$-control group, $\mathrm{T}$-treated group, $\mathrm{M}$-male and $\mathrm{F}$-female (PQN scaling, R package). A list of specific numbers of pigs is given in Tables 1 and 2.

All three used multivariable statistical methods, PCA, CA, and OPLS-DA, mathematically independent, were able to significantly differentiate the use of synthetic exogenous testosterone from naturally occurring (endogenous) testosterone in pigs of the same breed. This conclusion is in contrast to the findings published in the only study performed to date on ractopamine (a group of banned $\beta$-agonists) in 2017 [33]. Here, the authors of this study state that no significant difference between the 
samples from the control group and the ractopamine treated group was observed in the PCA analysis when all features were used. The use of metabolomics approaches and techniques also seems to be very promising from the point of view of the time of detection of banned anabolic steroids. Synthetic exogenous $17 \beta$-testosterone was demonstrably detected in plasma and urine in the treated group of pigs 14 days after administration. A similar conclusion was reached by the authors of a metabolomics study performed in cattle with $\beta$-agonists [31], when urine samples taken on days 27 and 48 after the administration could no longer be distinguished from the control group using PCA and OPLS-DA statistical models.

Supervised OPLS-DA models for urine and plasma, as one of the important practical results of this work, will be used for further testing of real samples to verify their predictive abilities. The models will be supplemented with other banned anabolic steroids and, especially, with increased numbers of test (training) data. Subsequently, the models will be verified by screening real plasma and urine samples taken as part of the monitoring of foreign substances in the Czech Republic. This is in line with the findings of other metabolomics studies [28,30-32], which also suggest increasing the number of samples used in statistical models and testing the proposed models on real samples obtained from national monitoring of contaminants in other EU countries (e.g., France, The Netherlands, Spain).

\subsection{Metabolomic Profiling for Identification of Biomarkers}

In the sequence of metabolomics multivariate statistical analysis, the last and often the most time-consuming step is to identify the most discriminating metabolites which are essential from the point of view of elucidating metabolomics pathways. A volcano plot with variable importance in projection plot (VIP) and S-plot from OPLS-DA were used to determine the most discriminating metabolites between the treatment group and control group (Figure 7 and Supplementary Materials Figure S9). The most discriminating compounds found were compared with the METLIN database, and their list and characteristics are shown in Table 4. Some very discriminating compounds have been identified with known human testostrone metabolites and confirmed against standards based on RT and MA criteria, such as M290T13_1 which corresponds to 5 $\alpha$-dihydrotestosterone. Nevertheless, further work needs to be done to identify the compounds and gain detailed explanation of their chemical structure.

\section{Materials and Methods}

\subsection{Animal Experiment and Urine/Plasma Sampling}

The animal experiments were performed at the Veterinary Research Institute in Brno, Czech Republic. Twenty clinically healthy 90-day-old male and female pigs (approximately $28 \mathrm{~kg}$ body weight) were randomly assigned to test (13 animals) and control ( 8 animals) groups. Animals from the test group were treated with an i.m. injection $(0.6 \mathrm{~mL} / \mathrm{pig})$ of the hormonal preparation (30 $\mathrm{mg} \mathrm{mL}^{-1} 17 \beta$-testosterone propionate, $60 \mathrm{mg} \mathrm{mL}^{-1} 17 \beta$-testosterone phenylpropionate, $60 \mathrm{mg} \mathrm{mL}^{-1}$ $17 \beta$-testosterone isocaproate, $100 \mathrm{mg} \mathrm{mL}^{-1} 17 \beta$-testosterone decanoate; Sustanon 250, N.V. Organon, CZ Reg.56/357/91-C). Experimental animals were grower pigs (hybrids of Large White $\times$ Landrace $($ sow $) \times$ Duroc (boar)) which were fed twice a day with a standard commercial diet according to the weight category. Pigs were housed in two separate pens (one pen/treatment and one pen/control) of $2.80 \times 2.00 \mathrm{~m}$.

The animals were injected on day 12 after the start of the experiment and were euthanized on day 90 of the experiment. Urine samples were collected from both groups 14, 28, 42 and 90 days after treatment and all samples were kept frozen until analysis at $-20^{\circ} \mathrm{C}$. Plasma samples were collected from day $1,2,3,4,5,7,14$, and 28 after treatment to day 90 . After blood clotting and $10 \mathrm{~min}$ of centrifugation at $6000 \times g$ of the samples, serum was removed and kept frozen until analysis at $-20^{\circ} \mathrm{C}$. All pigs were weighted every week within the experiment. All pigs were slaughtered at a body weight of $90-110 \mathrm{~kg}$ and the treated animal carcasses were destroyed. The study was performed in compliance 
with Act No. 246/1992 Coll. of the Czech National Council for the protection of animals against cruelty and with the agreement of the Branch Commission for Animal Welfare of the Ministry of Agriculture of the Czech Republic (permission no. MZe 17214).

\subsection{Reagents and Materials}

Reference analyte standards (17 $\beta$-testosterone, $17 \beta$-testosterone-D2 as isotopically labeled internal standards) were purchased from Sigma-Aldrich, Prague, Czech Republic. The standards were dissolved in methanol, diluted to a low concentration $\left(\mathrm{mg} \mathrm{mL}^{-1}\right)$ and used as working solutions. The organic solvents used were obtained from Merck (Darmstadt, Germany) and were in the SupraSolv ${ }^{\circledR}$ class. Used water prepared in an ultrapure water system of Golgman's water (Prague, Czech Republic). Centrifugal membrane filters Vivacon 500, cut off at $10 \mathrm{kDa}$, were obtained from Sartorius, Prague, Czech Republic.

\subsection{Sample Preparation}

\subsubsection{Plasma Samples for Targeted Analysis}

Each defrosted plasma sample $(500 \mu \mathrm{L})$ was transferred to a $15 \mathrm{~mL}$ centrifuge tube. After adding a methanol solution of $50 \mu \mathrm{L}$ of $17 \beta$-testosterone-D2 internal standard and $5 \mathrm{~mL}$ of ethyl acetate, the samples were shaken vigorously for $3 \mathrm{~min}$ on a vortex and then centrifuged at $4000 \times \mathrm{g}$ for $10 \mathrm{~min}$. The supernatant (ca. $4 \mathrm{~mL}$ ) was subsequently transferred to evaporator tubes. The samples were evaporated to dryness at $25{ }^{\circ} \mathrm{C}$ using a gentle stream of nitrogen $\left(99.99 \% \mathrm{~N}_{2}\right)$. The samples were reconstituted with $200 \mu \mathrm{L}$ in mobile phase solution (methanol: water, 70:30, v/v) and filtered through a $0.45 \mu \mathrm{m}$ (Hydrophilic PTFE) membrane centrifuge filter. The resulting samples were transferred to the insert in chromatography vials $(250 \mu \mathrm{L})$. Then, $10 \mu \mathrm{L}$ samples were injected directly the LC-MS/MS system.

\subsubsection{Samples for Metabolomics Profiling}

Plasma samples were defrosted at room temperature, homogenized and normalized by the creatinine concentration (Chemistry Analyzer BS-200, MINDRAY, Nanshen, China). Subsequently, $200 \mu \mathrm{L}$ of each sample was filtered through centrifugal devices (Vivacon 500, cut-off at $10 \mathrm{kDa}, 14,000 \times \mathrm{g}$, $\left.4{ }^{\circ} \mathrm{C}, 30 \mathrm{~min}\right)$ to remove high molecular weight proteins. Filtrates $(120 \mu \mathrm{L})$ were mixed with $30 \mu \mathrm{L}$ of internal standard (testosterone- $\mathrm{D}_{2}$ in methanol at the concentration of $1 \mathrm{ng} \mathrm{mL}^{-1}$ ). After thorough shaking, $5 \mu \mathrm{L}$ of each sample was injected into the chromatographic system.

Urine samples were defrosted at room temperature and normalized by specific density adjustment [47]. The specific density of the samples was measured using a refractometer (digital refractometer 30GS, Mettler-Toledo, Prague, Czech Republic) and, if necessary, adjusted to approximately $1.010-1.030 \mathrm{~kg} \mathrm{~m}^{-3}$ by dilution with deionized water. The urine samples $(500 \mu \mathrm{L})$ were then centrifuged through a centrifugal membrane filter (Vivacon 500, cut-off $10 \mathrm{kDa}$ ) at $14,000 \times \mathrm{g}$, $4{ }^{\circ} \mathrm{C}$ for $30 \mathrm{~min}$ to remove high molecular weight proteins. To the sample filtrate (ca. $450 \mu \mathrm{L}$ ), $50 \mu \mathrm{L}$ of $17 \beta$-testosterone-D2 internal standard was added. The urine samples were transferred to chromatographic vials and subsequently analysed.

QC samples for quality control of metabolomics profiling were prepared by the pool method so that they have the same or very similar (bio)-chemical varieties in the same range as individual samples of the study. The QC sample was prepared as a pool of all individual plasma or urine samples that were included in the study. Each QC sample was generated by mixing $20 \mu \mathrm{L}$ of the filtrate (cut-off) of each individual sample and was analysed at the same time as the study samples as part of the overall measurement sequence. 


\subsection{Targeted Quantitative Analytical Method}

\subsubsection{LC Condition}

Plasma samples were injected directly into a Thermo Fisher Scientific, (Waltham, MA, USA) LC system Accela 1200 equipped with an autosampler with a temperature controlled tray and column. Chromatographic separation was performed on Waters C18 XTerra MS analytical columns $(150 \times 2.1 \mathrm{~mm}$, size $3.5 \mu \mathrm{m})$ with a Waters C18 XTerra MS guard column $(10 \times 2.1 \mathrm{~mm}$, size $3.5 \mu \mathrm{m})$. The column and autosampler tray temperatures were set at $35^{\circ} \mathrm{C}$. The mobile phase consists of $0.1 \%$ formic acid in water: methanol $(95: 5, v / v)$ A and $0.1 \%$ formic acid in water: methanol $(5: 95, v / v) \mathrm{B}$, the flow rate was constant $300 \mu \mathrm{L} \mathrm{min}{ }^{-1}$. Gradient elution of 0-2 min with mobile phase $95 \% \mathrm{~A}$ and $5 \% \mathrm{~B}$ was started, 2.1-20 min linear gradient from 5\% to $90 \% \mathrm{~B}, 20.1-25 \mathrm{~min} 10 \% \mathrm{~A}$ and $90 \% \mathrm{~B}, 25.1-30 \mathrm{~min}$ linear gradient from $5 \%$ to $95 \% \mathrm{~A}$ and $30.1-35 \mathrm{~min} 95 \% \mathrm{~A}$ and $5 \% \mathrm{~B}$. The runtime of the method was $35 \mathrm{~min}$.

\subsubsection{MS/MS Parameters}

The tandem hybrid mass spectrometer Q Exactive (Thermo Fisher Scientific, (Massachusetts, USA) equipped with a heated electrospray ionisation probe measured in a positive mode (H-ESI+). For targeted quantification analysis, the mass spectrometer worked in the parallel reaction monitoring mode PRM (corresponding to the selected reaction monitoring mode SRM) with high resolution $\mathrm{RP}=17,500(\mathrm{FWHM})$ at $200 \mathrm{~m} / \mathrm{z}$. Before the start of each acquisition series, the mass spectrometer was externally calibrated to the mass accuracy with the positive ion calibration solution and the negative ion calibration solution (both Thermo Fisher Scientific). The "lock-mass" calibration was set to the molecular mass of $[\mathrm{M}+\mathrm{Na}]^{+}=64.01577 \mathrm{~g} \mathrm{~mol}^{-1}$ and $\left[\mathrm{M}_{2}+\mathrm{H}\right]^{+}=83.06037 \mathrm{~g} \mathrm{~mol}^{-1}$ for the acetonitrile ion, and was run continuously during the acquisition. Instrument and collision cell (HCD) parameters were optimised by direct syringe infusion of working solutions of $50 \mathrm{ng} \mathrm{mL} \mathrm{m}^{-1}$ of each targeted compound with a $5 \mu \mathrm{L} \mathrm{min}{ }^{-1}$ flow-rate. The mass spectrometer setting was as follows: sheath gas flow rate 30 (unit), aux gas flow rate 5 (unit), spray voltage $4.0 \mathrm{kV}$, capillary temperature $320^{\circ} \mathrm{C}$, heater temperature $220^{\circ} \mathrm{C}$, S-lens RF level 50, AGC target of $5 \times 10^{6}$, collision energy $35 \mathrm{eV}$, and a maximum injection time of $100 \mathrm{~ms}$.

The precursor ions and the four most intense product ions for each analyte were measured for quantification and identification (confirmation), respectively. The whole LC-(HR) MS system was controlled and the acquired data were stored and processed using Xcalibur 3.1 software, and then evaluated using Mass Frontier v. 7.0 for the identification.

\subsubsection{Method Validation}

The targeted quantitative method for the determination of $17 \beta$-testosterone and its esters in plasma has been validated to the extent required by European Directive 657/2002/EC [34] used for the determination of residues of foreign substances in biological matrices and according to the VICH GL49 [35] reference guide for validation method. To determine the validation characteristics of the matrix calibration curve, critical values detection limit (LOD), limit of quantification (LOQ), decision limit $\left(\mathrm{CC}_{\alpha}\right)$, detection capability $\left(\mathrm{CC}_{\beta}\right)$ and calibration range $0.5-80 \mathrm{ng} \mathrm{mL} \mathrm{m}^{-1}$ was used for $17 \beta$-testosterone and its esters. Samples of pig plasma (blank) were supplemented with the standards of $17 \beta$-testosterone and its esters at concentration corresponding to $0.5,1,5,10,20,40$, and $80 \mathrm{ng} \mathrm{mL}^{-1}$. The concentrations of the internal standards were constantly $10 \mathrm{ng} \mathrm{mL}^{-1}$. For each concentration level, two model samples were prepared and each sample was measured two times. Linear regression was carried out by plotting the peak area ratios of the analyte against the internal standard (dependent variable $\mathrm{Y}$ ) versus the analyte concentration (independent variable $\mathrm{X}$ ). To evaluate the precision of the method, repeatability and within-laboratory reproducibility, standard deviation (SD), and the coefficient of variation $(\mathrm{CV}, \%)$ were determined. Six model samples of pig plasma $(n=6)$ were 
prepared at concentrations of 5 and $80 \mathrm{ng} \mathrm{mL} \mathrm{m}^{-1}$ for all standards and measurements which were repeated two times for each sample on three different days $(3 \times 6, n=18)$.

\subsection{Metabolomic Profiling}

\subsubsection{LC Separation}

Plasma and urine samples prepared for non-targeted analysis (fingerprinting) were injected directly into the Accela 1200 LC system with a mass spectrometer. Chromatographic separation was performed on a C18 Hypersil GOLD $(50 \times 2.1 \mathrm{~mm}, 1.9 \mu \mathrm{m}$ size $)$ analytical column equipped with a C18 Hypersil GOLD $(10 \times 2.1 \mathrm{~mm}, 1.9 \mu \mathrm{m}$ size $)$ guard column, both from Thermo Fisher Scientific. The temperatures of the column and the autosampler tray were set at $35^{\circ} \mathrm{C}$ and $20^{\circ} \mathrm{C}$, respectively. The flow rate through the column was constantly set at $200 \mu \mathrm{L} \mathrm{min}{ }^{-1}$. The injection volume of the sample was $5 \mu \mathrm{L}$. The mobile phase consists of $0.1 \%$ formic acid in water: methanol $(95: 5, v / v) \mathrm{A}$ and $0.1 \%$ formic acid in water: methanol $(5: 95, v / v) \mathrm{B}$, the flow rate was constant $300 \mu \mathrm{Lmin}^{-1}$. Gradient elution of 0-2 min with mobile phase $95 \% \mathrm{~A}$ and $5 \% \mathrm{~B}$ was started, 2.1-20 min linear gradient from $5 \%$ to $90 \% \mathrm{~B}, 20.1-25 \mathrm{~min} 10 \% \mathrm{~A}$ and $90 \% \mathrm{~B}, 25.1-30 \mathrm{~min}$ linear gradient from $5 \%$ to $95 \% \mathrm{~A}$ and $30.1-35 \mathrm{~min}$ $95 \% \mathrm{~A}$ and $5 \% \mathrm{~B}$. The runtime of the method was $35 \mathrm{~min}$.

\subsubsection{Non-Targeted Mass Spectrometry}

For non-targeted metabolomic analysis, the tandem hybrid mass spectrometer $\mathrm{Q}$ Exactive worked in the positive full scan mode with resolving power (PR) =70,000 (FWHM) at $200 \mathrm{~m} / \mathrm{z}$ in the range 50 to $750 \mathrm{~m} / \mathrm{z}$ and in the centroid mode. Before the start of each acquisition series, the mass spectrometer was externally calibrated to the mass accuracy with a positive ion calibration solution and a negative ion calibration solution (both Thermo Fisher Scientific (Massachusetts, USA). The "lock-mass" calibration was set to the molecular mass of $[\mathrm{M}+\mathrm{Na}]^{+}=64.01577 \mathrm{~g} \mathrm{~mol}^{-1}$ and $\left[\mathrm{M}_{2}+\mathrm{H}\right]^{+}=83.06037 \mathrm{~g} \mathrm{~mol}^{-1}$ for the acetonitrile ion, and was run continuously during the acquisition. The mass spectrometer setting was as follows: sheath gas flow rate 30 (unit), aux gas flow rate 5 (unit), spray voltage $4.5 \mathrm{kV}$, capillary temperature $320^{\circ} \mathrm{C}$, heater temperature $180{ }^{\circ} \mathrm{C}$, S-lens RF level 50, AGC target of $6 \times 10^{6}$ and a maximum injection time of $200 \mathrm{~ms}$. The whole LC-(HR)MS system was controlled and the obtained data were stored and processed using Xcalibur 3.1 software.

\subsection{Data Processing}

The generated metabolomics profiling data sets were processed by the control software of the Xcalibur ${ }^{\circledR}$ mass spectrometer and saved in a specific data format $\left({ }^{*}\right.$.raw). The first step was to convert data from Excalibur-specific raw files to open format files ( ${ }^{*}$.mzXML) using MS Convertor software (ProteoWizard) [48]. Subsequently, metabolomics data were processed using the XCMS Online web version platform [49]. All results and images for processing were downloaded as zip files for offline analysis, including putative METLIN identities for each metabolite.

\subsection{Statistical Data Analysis}

Univariate and multivariate statistical analysis was performed in an interactive manner using statistical software Statistica (Version 13.3, TIBCO Software, Palo Alto, CA, USA) and R-statistic software in the Metabol package [50]. The data for processing was exported in the form of data matrix $X(n \times m)$ from an Excel file (output from data processing) to individual statistical programs. Before the application of multivariate statistical analysis, exploratory data analysis was conducted [51]. This included the assessment of primarily found outlier objects (or features thereof), assuming linear relationship and verifying the data provided (normality, non-correlation, homogeneity). Subsequently, the data matrices were standardized by two different methods. In the first case, the data were transformed by mean centering [37], and in the second case, they were transformed by probabilistic quotient normalization PQN $[38,52]$ and a natural logarithm was applied for their scaling before 
subsequent statistical analysis. Multidimensional statistical methods, such as principal component analysis (PCA), cluster analysis (CA), and orthogonal partial least squares discriminant analysis (OPLS-DA), were used to statistically evaluate data obtained from non-targeted metabolomics analyses.

\section{Conclusions}

The present study confirmed the ability of metabolomics approaches and techniques to significantly differentiate pigs administered an androgenic anabolic steroid which is on the list of banned substances (17 $\beta$-testosterone) in EU countries from control pigs. Using metabolomics profiling for plasma and urine samples, it was possible to differentiate the used synthetic exogenous testosterone from naturally occurring (endogenous) testosterone based on the results from three statistical models PCA, CA and OPLS-DA. The metabolomics workflow was designed with widely used multivariate statistical methods, analytical techniques, and equipment so that, based on the results of our study, it will be possible to develop validated methodologies for routine screening to prove the illegal use of prohibited substances in pig fattening. Furthermore, the anabolic effect of testosterone in pigs was demonstrated by comparing BW gains during the fattening period, and the targeted analysis of plasma testosterone levels provided data for the pharmacokinetic curves.

Supplementary Materials: The following are available online at http://www.mdpi.com/2218-1989/10/8/307/s1, Figure S1: Graph of average weekly weight gains in $\mathrm{kg}$, Figure S2: The measured experimental MS1 of the $17 \beta$-testosterone standards (up), comparison with the theoretical MS1 (down), Figure S3: The measured experimental MS1 of the 17 $\beta$-testosterone-D2 standards (up), comparison with the theoretical MS1 (down), Figure S4: Calibration curve of $17 \beta$-testosterone in plasma, Figure S5: Hotelling plot for identification of outliers objects in data source matrix $\mathrm{X}$ with calculated critical value T2 for plasma, ( $\mathrm{K}$ - control grup vs. T-treated grup), Figure S6: Hotelling plot for identification of outliers objects in data source matrix X with calculated critical value T2 for urine, Figure S7: The PLS-DA score plots for plasma (A) and urine (B) data matrix demonstrates robust discrimination between the control group of pigs marked with blue color and the group of teated pigs marked with red color (Centering, Statistica), Figure S8: The OPLS-DA permutation tests further confirmed that the proposed statistical models are correct and robust; A-plasma, B-urine, Figure S9: Variable importance in projection (VIP) and S-plots from OPLS-DA were used to determine the most discriminating metabolites between treatments and controls, A-plasma and B-urine, Table S1: The average weekly body weight gains, Table S2: The regression parameters of both linear models of BW growth versus time, Table S3: Identification and confirmation of $17 \beta$-testosterone and testosterone esters by mass accuracy (MA) for MS1, MS2 data, Table S4: Linearity of $17 \beta$-testosterone in plasma, Table S5: Precision, repeatability and within-laboratory reproducibility, Table S6: Pharmacokinetic of testosterone in plasma.

Author Contributions: Conceptualization, K.S., K.P., L.L., M.F. (Milan Franek), P.D. and M.F. (Martin Faldyna); methodology, K.S., K.P., L.L., M.F. (Milan Franek), P.D. and M.F. (Martin Faldyna); validation, K.S., K.P., L.L., M.F. (Milan Franek), P.D. and M.F. (Martin Faldyna); formal Analysis, K.S., K.P., L.L.; investigation, K.S., K.P., L.L., M.F. (Milan Franek), P.D. and M.F. (Martin Faldyna); resources, K.S., K.P., M.F. (Milan Franek), P.D. and M.F. (Martin Faldyna); writing - original draft preparation, K.S., K.P. and M.F. (Milan Franek); All authors have read and agreed to the published version of the manuscript.

Funding: This work was financially supported by the Ministry of Agriculture of the Czech (QK1910311 and RO1518) and by the Ministry of Education, Youth and Sports of the Czech Republic (project no. LO1218).

Conflicts of Interest: The authors declare no conflict of interest.

\section{References}

1. European Food Safety Authority. Report for 2018 on the results from the monitoring of veterinary medicinal product residues and other substances in live animals and animal products. EFSA Support. Publ. 2020, $17,1775 \mathrm{E}$.

2. Rosner, W. Plasma steroid-binding proteins. Endocrinol. Metab. Clin. N. Am. 1991, 20, 697-720. [CrossRef]

3. Westphal, U. Steroid-Protein Interactions Revisited. In Steroid-Protein Interactions II; Springer: Berlin/Heidelberg, Germany, 1986; pp. 1-7.

4. Peng, S.H.; Segura, J.; Farré, M.; González, J.C.; De La Torre, X. Plasma and urinary markers of oral testosterone undecanoate misuse. Steroids 2002, 67, 39-50. [CrossRef] 
5. Nielen, M.W.F.; Nijrolder, A.W.J.M.; Hooijerink, H.; Stolker, A.A.M. Feasibility of desorption electrospray ionization mass spectrometry for rapid screening of anabolic steroid esters in hair. Anal. Chim. Acta 2011, 700, 63-69. [CrossRef]

6. Van De Kerkhof, D.H.; De Boer, D.; Thijssen, J.H.H.; Maes, R.A.A. Evaluation of testosterone/epitestosterone ratio influential factors as determined in doping analysis. J. Anal. Toxicol. 2000, 24, 102-115. [CrossRef]

7. Wada Laboratory Expert Group. WADA Technical Document TD2014EAAS—Endogenous Anabolic Androgenic Steroids Measurement and Reporting; World Anti-Doping Agency: Montreal, QC, Canada, 2014; pp. 1-8.

8. Le Bizec, B.; Monteau, F.; Gaudin, I.; André, F. Evidence for the presence of endogenous 19-norandrosterone in human urine. J. Chromatogr. B Biomed. Sci. Appl. 1999, 723, 157-172. [CrossRef]

9. Pinel, G.; Weigel, S.; Antignac, J.P.; Mooney, M.H.; Elliott, C.; Nielen, M.W.F.; Le Bizec, B. Targeted and untargeted profiling of biological fluids to screen for anabolic practices in cattle. Trends Anal. Chem. 2010, 29, 1269-1280. [CrossRef]

10. Community Reference Laboratories. CRL Guidance Paper (7 December 2007)—CRL's View on State of the Art Analytical Methods for National Residue Control Plans; RIVM-National Institute for Public Health and the Environment: Bilthoven, The Netherlands, 2007; pp. 1-8.

11. Boschi, S.; De Iasio, R.; Mesini, P.; Bolelli, G.F.; Sciajno, R.; Pasquali, R.; Capelli, M. Measurement of steroid hormones in plasma by isocratic high performance liquid chromatography coupled to radioimmunoassay. Clin. Chim. Acta 1994, 231, 107-113. [CrossRef]

12. Di Benedetto, L.T.; Dimitrakopoulos, T.; Davy, R.M.; Iles, P.J. Testosterone determination using rapid heterogeneous competitive-binding for enzyme-linked immunosorbent assay in flow injection. Anal. Lett. 1996, 29, 2125-2139. [CrossRef]

13. Zhang, Z.; Duan, H.; Zhang, L.; Chen, X.; Liu, W.; Chen, G. Direct determination of anabolic steroids in pig urine by a new SPME-GC-MS method. Talanta 2009, 78, 1083-1089. [CrossRef]

14. De la Torre, X.; Segura, J.; Polettini, A.; Montagna, M. Detection of testosterone esters in human plasma. J. Mass Spectrom. 1995, 30, 1393-1404. [CrossRef]

15. He, C.; Li, S.; Liu, H.; Li, K.; Liu, F. Extraction of testosterone and epitestosterone in human urine using aqueous two-phase systems of ionic liquid and salt. J. Chromatogr. A 2005, 1082, 143-149. [CrossRef] [PubMed]

16. Shackleton, C.H.L.; Chuang, H.; Kim, J.; De La Torre, X.; Segura, J. Electrospray mass spectrometry of testosterone esters: Potential for use in doping control. Steroids 1997, 62, 523-529. [CrossRef]

17. Konieczna, L.; Plenis, A.; Oldzka, I.; Kowalski, P.; Bczek, T. Optimization of LC method for the determination of testosterone and epitestosterone in urine samples in view of biomedical studies and anti-doping research studies. Talanta 2011, 83, 804-814. [CrossRef]

18. Wang, G.; Hsieh, Y.; Cui, X.; Cheng, K.-C.; Korfmacher, W.A. Ultra-performance liquid chromatography/tandem mass spectrometric determination of testosterone and its metabolites in vitro samples. Rapid Commun. Mass Spectrom. 2006, 20, 2215-2221. [CrossRef]

19. You, Y.; Uboh, C.E.; Soma, L.R.; Guan, F.; Li, X.; Liu, Y.; Rudy, J.A.; Chen, J.; Tsang, D. Simultaneous separation and determination of 16 testosterone and nandrolone esters in equine plasma using ultra high performance liquid chromatography-tandem mass spectrometry for doping control. J. Chromatogr. A 2011, 1218, 3982-3993. [CrossRef]

20. Ponzetto, F.; Boccard, J.; Baume, N.; Kuuranne, T.; Rudaz, S.; Saugy, M.; Nicoli, R. High-resolution mass spectrometry as an alternative detection method to tandem mass spectrometry for the analysis of endogenous steroids in serum. J. Chromatogr. B Anal. Technol. Biomed. Life Sci. 2017, 1052, 34-42. [CrossRef]

21. Aqai, P.; Stolker, A.A.M.; Lasaroms, J.J.P. Effect of sample pre-treatment on the determination of steroid esters in hair of bovine calves. J. Chromatogr. A 2009, 1216, 8233-8239. [CrossRef]

22. Matraszek-Żuchowska, I.; Woźniak, B.; Sielska, K.; Posyniak, A. Determination of steroid esters in hair of slaughter animals by liquid chromatography with tandem mass spectrometry. J. Vet. Res. 2019, 63, 561-572. [CrossRef]

23. Dervilly-Pinel, G.; Courant, F.; Chéreau, S.; Royer, A.L.; Boyard-Kieken, F.; Antignac, J.P.; Monteau, F.; Le Bizec, B. Metabolomics in food analysis: Application to the control of forbidden substances. Drug Test. Anal. 2012, 4, 59-69. [CrossRef] 
24. Rijk, J.C.W.; Lommen, A.; Essers, M.L.; Groot, M.J.; Van Hende, J.M.; Doeswijk, T.G.; Nielen, M.W.F. Metabolomics approach to anabolic steroid urine profiling of bovines treated with prohormones. Anal. Chem. 2009, 81, 6879-6888. [CrossRef] [PubMed]

25. Kieken, F.; Pinel, G.; Antignac, J.P.; Monteau, F.; Paris, A.C.; Popot, M.A.; Bonnaire, Y.; Le Bizec, B. Development of a metabonomic approach based on LC-ESI-HRMS measurements for profiling of metabolic changes induced by recombinant equine growth hormone in horse urine. Anal. Bioanal. Chem. 2009, 394, 2119-2128. [CrossRef] [PubMed]

26. Anizan, S.; Bichon, E.; Duval, T.; Monteau, F.; Cesbron, N.; Antignac, J.-P.; Le Bizec, B. Gas chromatography coupled to mass spectrometry-based metabolomic to screen for anabolic practices in cattle: Identification of $5 \alpha$-androst-2-en-17-one as new biomarker of 4-androstenedione misuse. J. Mass Spectrom. 2012, 47, 131-140. [CrossRef] [PubMed]

27. Pinel, G.; Rambaud, L.; Monteau, F.; Elliot, C.; Le Bizec, B. Estranediols profiling in calves' urine after 17ß-nandrolone laureate ester administration. J. Steroid Biochem. Mol. Biol. 2010, 121, 626-632. [CrossRef]

28. Dervilly-Pinel, G.; Weigel, S.; Lommen, A.; Chereau, S.; Rambaud, L.; Essers, M.; Antignac, J.P.; Nielen, M.W.F.; Le Bizec, B. Assessment of two complementary liquid chromatography coupled to high resolution mass spectrometry metabolomics strategies for the screening of anabolic steroid treatment in calves. Anal. Chim. Acta 2011, 700, 144-154. [CrossRef]

29. Regal, P.; Anizan, S.; Antignac, J.P.; Le Bizec, B.; Cepeda, A.; Fente, C. Metabolomic approach based on liquid chromatography coupled to high resolution mass spectrometry to screen for the illegal use of estradiol and progesterone in cattle. Anal. Chim. Acta 2011, 700, 16-25. [CrossRef]

30. Blokland, M.H.; Van Tricht, E.F.; Van Rossum, H.J.; Sterk, S.S.; Nielen, M.W.F. Endogenous steroid profiling by gas chromatography-tandem mass spectrometry and multivariate statistics for the detection of natural hormone abuse in cattle. Food Addit. Contam. Part A Chem. Anal. Control Expo. Risk Assess. 2012, 29, 1030-1045. [CrossRef]

31. Dervilly-Pinel, G.; Chereau, S.; Cesbron, N.; Monteau, F.; Le Bizec, B. LC-HRMS based metabolomics screening model to detect various $\beta$-agonists treatments in bovines. Metabolomics 2015, 11, 403-411. [CrossRef]

32. Blokland, M.H.; van Tricht, E.F.; van Ginkel, L.A.; Sterk, S.S. Applicability of an innovative steroid-profiling method to determine synthetic growth promoter abuse in cattle. J. Steroid Biochem. Mol. Biol. 2017, 174, 265-275. [CrossRef]

33. Peng, T.; Royer, A.L.; Guitton, Y.; Le Bizec, B.; Dervilly-Pinel, G. Serum-based metabolomics characterization of pigs treated with ractopamine. Metabolomics 2017, 13, 77. [CrossRef]

34. European Commission. 2002/657/EC: Commission Decision of 12 August 2002 Implementing Council Directive 96/23EC Concerning the Performance of Analytical Methods and Interpretation of Results. Off. J. Eur. Communities 2002, 45, 8-36.

35. European Medicines Agency. VICH GL49 Studies to Evaluate the Metabolism and Residue Kinetics of Veterinary Drugsin Food-Producing Animals: Validation of Analytical Methods Used in Residue Depletion Studies; European Medicines Agency: Amsterdam, The Netherlands, 2015.

36. International Organization for Standardization. ISO 11843-1:1997. Capability of Detection-Part 1: Terms and Definitions; ISO: Geneva, Switzerland, 1997.

37. Worley, B.; Powers, R. Multivariate Analysis in Metabolomics. Curr. Metab. 2013, 1, 92-107.

38. Filzmoser, P.; Walczak, B. What can go wrong at the data normalization step for identification of biomarkers? J. Chromatogr. A 2014, 1362, 194-205. [CrossRef] [PubMed]

39. Chow, G.C. Tests of Equality Between Sets of Coefficients in Two Linear Regressions. Econometrica 1960, 28, 591-605. [CrossRef]

40. Groot, M.J.; Lasaroms, J.J.P.; van Bennekom, E.O.; Meijer, T.; Vinyeta, E.; van der Klis, J.D.; Nielen, M.W.F. Illegal treatment of barrows with nandrolone ester: Effect on growth, histology and residue levels in urine and hair. Food Addit. Contam. Part A Chem. Anal. Control Expo. Risk Assess. 2012, 29, 727-735. [CrossRef] [PubMed]

41. Holčapek, M.; Jirásko, R.; Lísa, M. Recent developments in liquid chromatography-mass spectrometry and related techniques. J. Chromatogr. A 2012, 1259, 3-15. [CrossRef]

42. Rochat, B.; Kottelat, E.; McMullen, J. The future key role of LC-high-resolution-MS analyses in clinical laboratories: A focus on quantification. Bioanalysis 2012, 4, 2939-2958. [CrossRef] 
43. Stastny, K.; Stepanova, H.; Hlavova, K.; Faldyna, M. Identification and determination of deoxynivalenol (DON) and deepoxy-deoxynivalenol (DOM-1) in pig colostrum and serum using liquid chromatography in combination with high resolution mass spectrometry (LC-MS/MS (HR)). J. Chromatogr. B Anal. Technol. Biomed. Life Sci. 2019, 1126-1127. [CrossRef]

44. McCoard, S.; Wise, T.; Ford, J. Endocrine and molecular influences on testicular development in Meishan and White Composite boars. J. Endocrinol. 2003, 178, 405-416. [CrossRef]

45. Park, C.S.; Yi, Y.J. Comparison of semen characteristics, sperm freezability and testosterone concentration between Duroc and Yorkshire boars during seasons. Anim. Reprod. Sci. 2002, 73, 53-61. [CrossRef]

46. Rejtharová, M.; Rejthar, L.; Čačková, K. Determination of testosterone esters and nortestosterone esters in animal blood serum by LC-MS/MS. Food Addit. Contam. Part A Chem. Anal. Control Expo. Risk Assess. 2018, 35, 233-240. [CrossRef]

47. Jacob, C.C.; Dervilly-Pinel, G.; Biancotto, G.; Le Bizec, B. Evaluation of specific gravity as normalization strategy for cattle urinary metabolome analysis. Metabolomics 2014, 10, 627-637. [CrossRef]

48. Adusumilli, R.; Mallick, P. Data conversion with proteoWizard msConvert. In Methods in Molecular Biology; Humana Press Inc.: Totowa, NJ, USA, 2017; Volume 1550, pp. 339-368.

49. Smith, C.A.; Want, E.J.; O’Maille, G.; Abagyan, R.; Siuzdak, G. XCMS: Processing mass spectrometry data for metabolite profiling using nonlinear peak alignment, matching, and identification. Anal. Chem. 2006, 78, 779-787. [CrossRef] [PubMed]

50. Gardlo, A.; Friedecký, D.; Najdekr, L.; Karlíková, R.; Adam, T. Metabol: The Statistical Analysis of Metabolomic Data; Laboratory for Inherited Metabolic Disorders, Faculty of Medicine and Dentistry, Palacky University in Olomouc, University Hospital Olomouc: Olomouc, Czech Republic, 2019.

51. Gelman, A. Exploratory data analysis for complex models. J. Comput. Graph. Stat. 2004, 13, 755-779. [CrossRef]

52. Cook, T.; Ma, Y.; Gamagedara, S. Evaluation of statistical techniques to normalize mass spectrometry-based urinary metabolomics data. J. Pharm. Biomed. Anal. 2020, 177, 112854. [CrossRef] [PubMed]

(C) 2020 by the authors. Licensee MDPI, Basel, Switzerland. This article is an open access article distributed under the terms and conditions of the Creative Commons Attribution (CC BY) license (http://creativecommons.org/licenses/by/4.0/). 


\title{
Gas Chromatography-Mass Spectrometry-Based Metabolomic Analysis of Wagyu and Holstein Beef
}

\author{
Tomoya Yamada *, Mituru Kamiya and Mikito Higuchi \\ Division of Livestock Feeding and Management, National Agriculture and Food Research Organization, \\ Nasushiobara, Tochigi 329-2793, Japan; m_kamiya@affrc.go.jp (M.K.); mikito@affrc.go.jp (M.H.) \\ * Correspondence: toyamada@affrc.go.jp; Tel.: +81-287-37-7811
}

Received: 6 February 2020; Accepted: 5 March 2020; Published: 6 March 2020

\begin{abstract}
Japanese Black cattle (Wagyu) beef is characterized by high intramuscular fat content and has a characteristic sweet taste. However, the chemical components for characterizing the sweet taste of Wagyu beef have been unclear. In this experiment, we conducted a metabolomic analysis of the longissimus muscle (sirloin) in Wagyu and Holstein cattle to determine the key components associated with beef taste using gas chromatography-mass spectrometry (GC-MS). Holstein sirloin beef was characterized by the abundance of components such as glutamine, ribose-5-phosphate, uric acid, inosine monophosphate, 5-oxoproline, and glycine. In contrast, Wagyu sirloin beef was characterized by the abundance of sugar components (maltose and xylitol). Dietary fat is known to increase the intensity of sweet taste. These results suggest that the sweet taste of Wagyu beef is due to the synergetic effects of higher sugar components and intramuscular fat.
\end{abstract}

Keywords: metabolome; beef; Wagyu; Holstein

\section{Introduction}

Food quality, especially the taste of food, is affected by numerous chemical components. Metabolomic analysis has been used to select biomarkers from numerous metabolites. Therefore, the relationship between metabolomic profiling and food quality has been investigated to identify quality-related components. Previous reports showed that the metabolomic profiles of foods such as fermented alcoholic beverages [1], soybeans [2], and tomatoes [3], strongly affect their taste.

Metabolomic analyses of meat have also been conducted to identify quality-related components. The relationships between metabolomic profiles and processing conditions of hams [4], the muscle type of pork [5], and the sensory perceptions of pork [6] have been reported. In addition, metabolome studies of beef have shown that metabolomic profiles were affected by geographical origin [7], breed [8,9], storage conditions [10], and aging periods [11].

Japanese Black cattle, also called Wagyu, are characterized by their great capacity for intramuscular adipose tissue accumulation [12,13]. The high intramuscular adipose tissue content of beef, called marbling, improves the texture, juiciness, and tenderness of Wagyu beef [13]. In sensory tests, Wagyu beef had significantly higher sensory characteristic scores than beef from other cattle breeds $[14,15]$. Interestingly, Wagyu beef has a characteristic sweet aroma and sweet taste that are not detected in other cattle breeds in sensory tests [15-17]. Previous reports indicated that the sweet aroma of Wagyu beef was affected by the lactone and decenal components $[18,19]$. However, the metabolomic biomarkers discerning the breed differences in beef, especially the characteristic sweet taste of Wagyu beef, have remained unclear. Holstein cattle are categorized as a dairy breed, and Holstein beef is characterized as lean meat $[20,21]$. Previous sensory test results have indicated breed differences between sensory characteristic scores of Wagyu and Holstein beef $[14,15]$. Therefore, to elucidate the breed differences in beef taste, a comparison of Wagyu and Holstein is thought to be the optimal model. In the present 
study, we conducted a metabolomic analysis of longissimus muscle (sirloin) samples from Wagyu and Holstein cattle to identify the metabolomic biomarkers characterizing the breed differences in beef taste.

\section{Results}

Gas chromatography-mass spectrometry (GC-MS) analysis detected 67 metabolites in the sirloin samples of Wagyu and Holstein. Full results are shown in Supplementary Table S1. The principal component analysis (PCA) score plots showed that the metabolomic profile was divided into Wagyu and Holstein groups (Figure 1). The heatmap of metabolites also showed a difference between Wagyu and Holstein groups (Figure 2). Metabolites contributing to cluster 7, which characterized the Holstein sample, were mainly composed of amino acids (proline and glycine), amino compounds (succinic acid, amino propanoic acid, creatinine, and pyruvic acid), and nucleic acid metabolites (inosine and ribose). In contrast, cluster 1 , which characterized the Wagyu sample, was mainly composed of sugar components (maltose and xylitol) and fatty acids (stearic acid, palmitic acid and nonanoic acid). Table 1 shows the differences in the relative quantity of the main metabolite compounds in Wagyu and Holstein samples. The amount of glutamine, ribose-5-phosphate, uric acid, inosine monophosphate, 5-oxoproline, and glycine in Holstein samples was significantly higher than in Wagyu samples. In contrast, the amount of maltose and xylitol in Wagyu samples was significantly higher than that in Holstein samples.

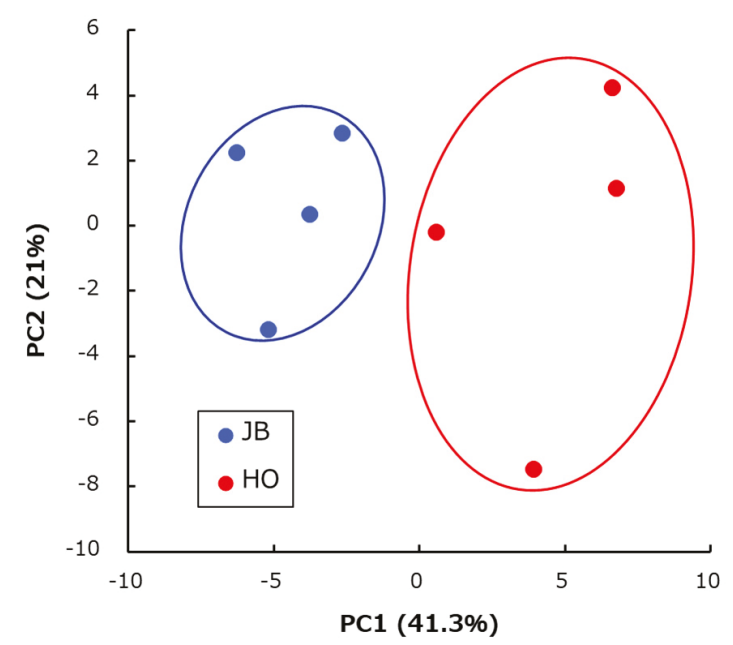

Figure 1. Principal component analysis (PCA) of metabolome data from Japanese Black Wagyu (JB) and Holstein $(\mathrm{HO})$ sirloin samples. $\bullet$ JB $(n=4), \bullet: \mathrm{HO}(n=4)$. 


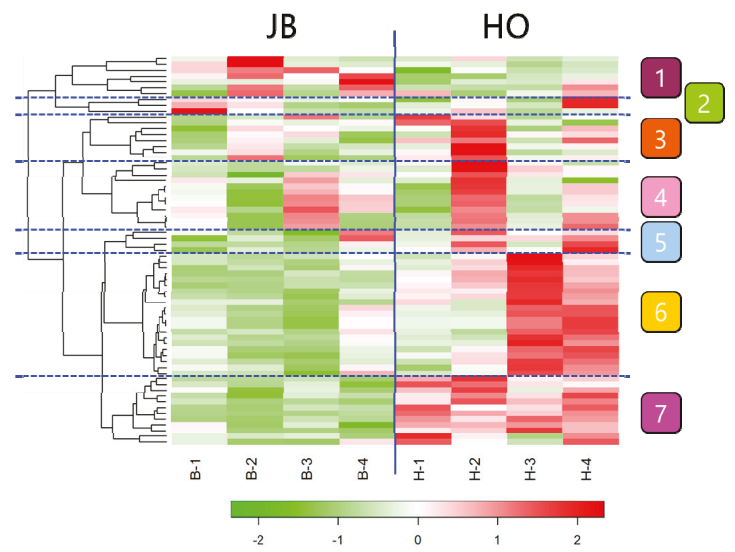

Figure 2. Heatmap of metabolites in Japanese Black Wagyu (JB) and Holstein (HO) sirloin samples. The upregulated metabolites are shown in red, and the downregulated metabolites are presented in green. Cluster 1, which characterized the Wagyu sample, was mainly composed of maltose, xylitol, stearic acid, palmitic acid, and nonanoic acid. Cluster 7, which characterized the Holstein sample, was mainly composed of proline, glycine, succinic acid, amino propanoic acid, creatinine, pyruvic acid, inosine, and ribose. B1-B4: Japanese Black Wagyu $(n=4)$; H1-H4: Holstein $(n=4)$.

Table 1. Main metabolite compounds in Japanese Black Wagyu and Holstein sirloin samples.

\begin{tabular}{|c|c|c|c|c|c|c|c|}
\hline \multirow{3}{*}{ Change } & \multirow{3}{*}{ Compound Name } & \multicolumn{4}{|c|}{ Relative Area } & \multirow{2}{*}{\multicolumn{2}{|c|}{$\begin{array}{c}\text { Comparative Analysis } \\
\text { JB/Ho }\end{array}$}} \\
\hline & & & & & & & \\
\hline & & Mean & S.D. & Mean & S.D. & Ratio & $p$-Value \\
\hline \multirow[t]{8}{*}{ Increase } & Maltose & $7.1 \times 10^{4}$ & $1.4 \times 10^{4}$ & $4.0 \times 10^{4}$ & $1.5 \times 10^{4}$ & 1.8 & $0.022 *$ \\
\hline & Xylitol & $2.7 \times 10^{4}$ & $4.4 \times 10^{3}$ & $1.8 \times 10^{4}$ & $2.3 \times 10^{3}$ & 1.5 & $0.022 *$ \\
\hline & Palmitic acid & $8.8 \times 10^{4}$ & $3.6 \times 10^{4}$ & $6.0 \times 10^{4}$ & $8.8 \times 10^{3}$ & 1.5 & 0.221 \\
\hline & Stearic acid & $6.7 \times 10^{4}$ & $4.0 \times 10^{4}$ & $5.2 \times 10^{4}$ & $1.3 \times 10^{4}$ & 1.3 & 0.513 \\
\hline & Ribose & $4.3 \times 10^{5}$ & $1.2 \times 10^{5}$ & $3.5 \times 10^{5}$ & $5.6 \times 10^{4}$ & 1.2 & 0.264 \\
\hline & Sedoheptulose7-phosphate & $7.9 \times 10^{4}$ & $3.2 \times 10^{4}$ & $6.5 \times 10^{4}$ & $2.3 \times 10^{4}$ & 1.2 & 0.513 \\
\hline & Mannose & $1.1 \times 10^{6}$ & $2.7 \times 10^{5}$ & $8.9 \times 10^{5}$ & $2.8 \times 10^{5}$ & 1.2 & 0.406 \\
\hline & Glycerol 3-phosphate & $2.4 \times 10^{4}$ & $7.9 \times 10^{3}$ & $2.1 \times 10^{4}$ & $3.3 \times 10^{3}$ & 1.1 & 0.623 \\
\hline \multirow[t]{8}{*}{ Decrease } & Glycine & $1.6 \times 10^{6}$ & $1.1 \times 10^{5}$ & $2.6 \times 10^{6}$ & $2.4 \times 10^{5}$ & 0.6 & $0.001 * *$ \\
\hline & Ornithine & $3.9 \times 10^{4}$ & $6.3 \times 10^{3}$ & $6.7 \times 10^{4}$ & $2.3 \times 10^{4}$ & 0.6 & 0.094 \\
\hline & 5-Oxoproline & $9.2 \times 10^{4}$ & $9.2 \times 10^{3}$ & $1.7 \times 10^{5}$ & $3.2 \times 10^{4}$ & 0.6 & 0.014 * \\
\hline & Inosine monophosphate & $1.8 \times 10^{5}$ & $7.9 \times 10^{4}$ & $3.3 \times 10^{5}$ & $1.4 \times 10^{4}$ & 0.5 & $0.030^{*}$ \\
\hline & Uric acid & $1.1 \times 10^{4}$ & $1.4 \times 10^{3}$ & $2.2 \times 10^{4}$ & $4.8 \times 10^{3}$ & 0.5 & $0.014 *$ \\
\hline & 2-Hydroxyglutaric acid & $5.8 \times 10^{3}$ & $2.0 \times 10^{3}$ & $1.3 \times 10^{4}$ & $5.3 \times 10^{3}$ & 0.5 & 0.075 \\
\hline & Ribose 5-phosphate & $3.2 \times 10^{4}$ & $5.4 \times 10^{3}$ & $6.9 \times 10^{4}$ & $1.2 \times 10^{4}$ & 0.5 & $0.004^{* *}$ \\
\hline & Glutamine & $3.5 \times 10^{4}$ & $3.8 \times 10^{3}$ & $9.8 \times 10^{4}$ & $3.1 \times 10^{4}$ & 0.4 & 0.026 * \\
\hline
\end{tabular}

Values are expressed as means and S.D. ratio: fold intensity of metabolite compounds (JB/Ho). Annotation and relative quantification of metabolites was measured by each peak using the gas chromatography-mass spectrometry (GC-MS) solution (Shimadzu) and GC/MS Metabolite Database Ver. 2 (Shimadzu). Japanese Black Wagyu (JB, $n=4)$, Holstein $(\mathrm{HO}, n=4) * p<0.05,{ }^{* *} p<0.01$.

\section{Discussion}

In the present study, we showed that the Holstein sirloin samples were characterized by amino acids, amino compounds and nucleic-acid metabolites. We also showed that the relative amount of glutamine, ribose-5-phosphate, uric acid, inosine monophosphate, 5-oxoproline, and glycine in the Holstein sirloin samples was significantly higher than in the Wagyu samples. The abundance of amino acids, amino compounds, and nucleic-acid metabolites clearly reflects the lean meat content of Holstein beef. Previous studies also showed the relationship between metabolomic profiling and beef quality [7-11]. These results indicate that metabolomic analysis is an optimum approach to identify quality-related chemical components of beef. The cooking of meat forms a characteristic taste via 
numerous chemical reactions. The Maillard reaction is one of the major chemical reactions of cooked meat that produces many flavoring components [22]. Amino acids and nucleic-acid metabolites are main meat-flavor precursors for the Maillard reaction [23]. Therefore, a higher amount of amino acid and nucleic-acid metabolites in Holstein beef indicates the abundance of a Maillard reaction substrate. These results suggest that breed differences in beef metabolomic profiles affect the taste of cooked meats. The elucidation of metabolomic profile differences between Wagyu and Holstein cooked meat is an important subject for further study.

We showed that the Wagyu sirloin samples were characterized by sugar components and fatty acids. The present study also showed that the relative amount of maltose and xylitol in Wagyu sirloin samples was significantly higher than that in Holstein samples. Ueda et al. reported that the relative amount of malic acid, maltose, trehalose, arabitol, isomaltose, n-acetylserine, and inositol in Wagyu beef was significantly higher than that in Holstein beef [9]. The aging periods of beef affect the meat quality and metabolomic profile [11]. The difference between the results of our study and those of Ueda et al. would be attributed to meat aging conditions. The meat aging period in the present study was at $4{ }^{\circ} \mathrm{C}$ for 7 days after slaughter. In contrast, the aging in Ueda et al. was at $4{ }^{\circ} \mathrm{C}$ for 20 days [9]. On the other hand, results showing a higher amount of maltose in Wagyu beef than in Holstein beef were common to both experiments. These results suggest that a higher maltose concentration is a primary feature of Wagyu beef, independent of the aging period. The causative substance of the sweet taste of Wagyu beef remains unclear. Amino acids have different taste properties depending upon their chemical structure [24]. Glycine is categorized as a "sweet" amino acid [24]. The present results showed that glycine was abundant in the Holstein sirloin samples. Therefore, the possibility that amino acids contribute to the sweet taste of Wagyu beef would be excluded. In contrast, the present study showed that maltose and xylitol, categorized as sugar components, are abundant in Wagyu beef. Wagyu is characterized by higher intramuscular fat content than Holstein [12,13]. Previous reports indicated that dietary fat increased the intensity of sweet taste $[25,26]$. These results suggest that the sweet taste of Wagyu beef is affected by the synergetic interaction between higher sugar components and intramuscular fat. Threshold sweetness concentrations of maltose and xylitol have been reported using sensory test methods $[27,28]$. Kearsley et al. reported that the threshold sweetness concentration of maltose was $1.07 \%(\mathrm{w} / \mathrm{v})$, and that of xylitol was $0.51 \%(\mathrm{w} / \mathrm{v})$ [27]. In molar volumes, Birch et al. showed that the threshold sweetness concentration of maltose was $21.0 \mathrm{mM} / \mathrm{l}$, and that of xylitol was also $21.0 \mathrm{mM} / 1$ [28]. However, the effect of dietary fat on the threshold sweetness concentration of sugar components has not been reported. The slaughter age of Wagyu (aged 29-30 months) and Holsteins (aged 21-22 months) in this study was defined in accordance with the commonly applied fattening periods of each breed in Japan. Ueda et al. also analyzed beef samples of Wagyu (aged 31-32 months) and Holsteins (aged 21 months) that were similar to the age of cattle used in this study [9]. Previous studies have shown that the slaughter age affects meat quality and sensory traits of beef [29,30]. To our knowledge, there are no previous studies examining the effects of slaughter age on metabolomic profiling of beef. Therefore, the differences between fattening periods of Wagyu and Holsteins in this study may affect the metabolomic profiling of beef. In addition, there is a possibility that other metabolites, which we could not detect in this study, might affect the sweetness of beef. Further studies are needed to clarify the effects of metabolites on the sweet taste of Wagyu beef.

\section{Materials and Methods}

\subsection{Animals}

Wagyu steers (aged 29-30 months, $n=4$ ) and Holstein steers (aged 21-22 months, $n=4$ ) were used in this study. They received a concentrate (78\% total digestible nutrients and $13 \%$ crude protein) and orchard grass hay (56\% total digestible nutrients and $8 \%$ crude protein) ad libitum from 10 months of age until they were slaughtered. Longissimus muscle (sirloin) samples were collected at slaughter. The sirloin samples $(1.5 \mathrm{~kg}$ ) were collected between the third and fourth lumbar vertebrae from the left 
side of the carcass. Samples were vacuum-packed and wet-aged at $4{ }^{\circ} \mathrm{C}$ for 7 days and then stored at $-80^{\circ} \mathrm{C}$ for later metabolome analysis. All animals received humane care as outlined in the Guide for the Care and Use of Experimental Animals (No.1631B004, National Agriculture and Food Research Organization).

\subsection{GC-MS Analysis}

Frozen sirloin samples were powdered with liquid nitrogen and weighed $(100 \mathrm{mg})$ in the frozen state. Frozen samples were plunged into 80\% methanol and homogenized using zirconia beads and an ultrasonic homogenizer for $5 \mathrm{~min}$. The samples were centrifuged at 15,000 rpm for $5 \mathrm{~min}$. The supernatant was filtered using a Mono-Spin C18 column (GL Science, Tokyo, Japan), and then the filtration $(50 \mu \mathrm{L})$ was dried by a nitrogen gas flow. Methoxyamine hydrochloride solubilized with pyridine $(20 \mathrm{mg} / \mathrm{mL}, 50 \mu \mathrm{L})$ was added to each sample, and oxime formation was achieved by reacting at $30^{\circ} \mathrm{C}$ for $90 \mathrm{~min}$. Trimethylsilyl-trifluoroacetamide (50 $\left.\mu \mathrm{L}\right)$ was then added to each sample, and trimethylsilylation was carried out by reacting at $37^{\circ} \mathrm{C}$ for $30 \mathrm{~min}$. Analyses were performed on a gas chromatography-mass spectrometer (GC-MS, QP2010Ultra, Shimadzu, Kyoto, Japan) using a DB-5 column (Agilent Technologies, Santa Clara, CA, USA) at the Kazusa DNA Research Institute. The carrier gas was helium in a flow of $1.1 \mathrm{~mL} / \mathrm{min}$. The injection temperature was $280^{\circ} \mathrm{C}$, and the injection volume was $0.5 \mu \mathrm{L}$. The temperature program was isothermal for 4 min at $100{ }^{\circ} \mathrm{C}$, then raised at a rate of $4{ }^{\circ} \mathrm{C} / \mathrm{min}$ to $320^{\circ} \mathrm{C}$ and held for $8 \mathrm{~min}$. The ion source temperature and scan speed were set to $200{ }^{\circ} \mathrm{C}$ and $2500 \mathrm{u} / \mathrm{sec}$, respectively. Sample peaks were recorded over the mass range of 45-600 m/z. The retention time correction of peaks was carried out based on the retention time of a standard alkane series mixture (C-7 to C-33). Annotation and relative quantification of metabolite was measured by each peak using the GC-MS solution (Shimadzu) and GC/MS Metabolite Database Ver. 2 (Shimadzu). The relative area was calculated using the peak area of each metabolite relative to the analyzed sample weight at Kazusa DNA Research Institute.

\subsection{Statistical Analysis}

Principal component analysis (PCA) was conducted using SampleStat software (Human Metabolome Technologies, Japan). Hierarchical cluster analysis (HCA) was performed and heatmap formation analyzed using PeakStat software (Human Metabolome Technologies). Differences between the relative quantity of metabolites in Wagyu and Holstein samples were evaluated using Welch's t-test. Results are presented as means, and S.D. values of $p<0.05$ were considered significant.

Supplementary Materials: The following are available online at http://www.mdpi.com/2218-1989/10/3/95/s1, Table S1: Metabolites of Wagyu and Holstein Beef.

Author Contributions: Conceptualization, T.Y. and M.H.; methodology, T.Y.; software, T.Y.; validation, T.Y., M.K., and M.H.; formal analysis, T.Y.; investigation, T.Y., M.K., and M.H.; resources, T.Y., M.K., and M.H.; data curation, T.Y., M.K., and M.H.; writing—original draft preparation, T.Y.; writing—review and editing, T.Y., M.K., and M.H.; visualization, T.Y.; supervision, T.Y.; project administration, T.Y.; funding acquisition, T.Y. All authors have read and agreed to the published version of the manuscript.

Funding: This work was supported in part by a Grant-in-Aid for Ito Memorial Foundation and JSPS KAKENHI (18K05958).

Acknowledgments: We would like to thank Kazuki Iguchi (Human Metabolome Technologies) for technical support.

Conflicts of Interest: The authors declare no conflict of interest.

\section{References}

1. Takahashi, K.; Kohno, H. Different polor metabolites and protein profiles between high- and low-quality Japanese Ginjo sake. PLoS ONE 2016, 11, e0150524. [CrossRef] [PubMed]

2. Sugimoto, M.; Goto, H.; Otomo, K.; Ito, M.; Onuma, H.; Suzuki, A.; Sugawara, M.; Abe, S.; Tomita, M.; Soga, T. Metabolomic profiles and sensory attributes of edamame under various storage duration and temperature conditions. J. Agric. Food Chem. 2010, 58, 8418-8425. [CrossRef] [PubMed] 
3. Malmendal, A.; Amoresano, C.; Trotta, R.; Lauri, I.; De Tito, S.; Novellino, E.; Randazzo, A. NMR spectrometers as "magic tongues": Prediction of sensory descriptors in canned Tomatos. J. Agric. Food Chem. 2011, 59, 10831-10838. [CrossRef] [PubMed]

4. Sugimoto, M.; Obiya, S.; Kaneko, M.; Enomoto, A.; Honma, M.; Wakayama, M.; Soga, T.; Tomita, M. Metabolic profiling as a possible reverse engineering tool for estimating processing conditions of dry-cured hams. J. Agric. Food Chem. 2017, 65, 402-410. [CrossRef] [PubMed]

5. Muroya, S.; Oe, M.; Nakajima, I.; Ojima, K.; Chikuni, K. CE-TOFMS-based metabolomic profiling revealed characteristic metabolic pathways in postmortem porcine fast and slow type muscles. Meat Sci. 2014, 98, 726-735. [CrossRef] [PubMed]

6. Straadt, I.K.; Aaslyng, M.D.; Bertram, H.C. An NMR-based metabolomics study of pork from different crossbreeds and relation to sensory perception. Meat Sci. 2014, 96, 719-728. [CrossRef] [PubMed]

7. Jung, Y.; Lee, J.; Kwon, J.; Lee, K.S.; Ryu, D.H.; Hwang, G.S. Discrimination of the geographical origin of beef by H NMR-based metabolomics. J. Agric. Food Chem. 2010, 58, 10458-10466. [CrossRef]

8. Ritota, M.; Casciani, L.; Failla, S.; Valentin, M. HRMAS-NMR spectroscopy and multivariate analysis meat characterization. Meat Sci. 2012, 92, 754-761. [CrossRef]

9. Ueda, S.; Iwamoto, E.; Kato, Y.; Shinohara, M.; Shirai, Y.; Yamanoue, M. Comparative metabolomics of Japanese Black cattle beef and other meats using gas chromatography-mass spectrometry. Biosci. Biotechnol. Biochem. 2019, 83, 137-147. [CrossRef]

10. Argyri, A.A.; Mallouchos, A.; Panagou, E.Z.; Nychas, G.J.E. The dynamics of the HS/SPME-GC-MS as a tool to assess the spoilage of minced beef stored under different packaging and temperature conditions. Int. J. Food Microbiol. 2015, 193, 51-58. [CrossRef]

11. Muroya, S.; Oe, M.; Ojima, K.; Watanabe, A. Metabolomic approach to key metabolites characterizing postmortem aged loin muscle of Japanese Black (Wagyu) cattle. Asian-Australas. J. Anim. Sci. 2019, 32, 1172-1185. [CrossRef] [PubMed]

12. Zembayashi, M.; Lunt, D.K. Distribution of intramuscular lipid throughout M. longissimus thoracis et lumborum in Japanese Black, Japanese Shorthorn, Holstein and Japanese Black crossbreds. Meat Sci. 1995, 211-216. [CrossRef]

13. Motoyama, M.; Sasaki, K.; Watanabe, A. Wagyu and the factors contributing to its beef quality: A Japanese industry overview. Meat Sci. 2016, 120, 10-18. [CrossRef] [PubMed]

14. Corbin, C.H.; O'Quinn, T.G.; Garmyn, A.J.; Legato, J.F.; Hunt, M.R.; Dinh, T.T.N.; Rathmann, R.J.; Brooks, J.C.; Miller, M.F. Sensory evaluation of tender beef strip loin steaks of varying marbling levels and quality treatments. Meat Sci. 2015, 100, 24-31. [CrossRef] [PubMed]

15. Sasaki, K.; Ooi, M.; Nagura, N.; Motoyama, M.; Narita, T.; Oe, M.; Nakajima, I.; Tatsuro, H.; Ojima, K.; Kobayashi, M.; et al. Classification and characterization of Japanese consumer's beef preferences by external preference mapping. J. Sci. Food. Agric. 2017, 97, 3453-3462. [CrossRef]

16. Watanabe, G.; Motoyama, M.; Orita, K.; Takita, K.; Aonuma, T.; Nakajima, I.; Tajima, A.; Abe, A.; Sasaki, K. Assessment of the dynamics of sensory perception of Wagyu beef strip loin prepared with different cooking methods and fattening periods using the temporal dominance of sensations. Food Sci. Nutr. 2019, 7, 3538-3548. [CrossRef]

17. Matsuishi, M.; Fujimori, M.; Okitani, A. Wagyu beef aroma in Wagyu (Japanese Black Cattle) beef preferred by the Japanese over imported beef. Anim. Sci. J. 2001, 72, 498-504. [CrossRef]

18. Matsuishi, M.; Kume, J.; Itou, Y.; Takahashi, M.; Arai, M.; Nagatomi, H.; Watanabe, K.; Hayase, F.; Okitani, A. Aroma components of Wagyu beef and imported beef. Nihon Chikusan Gakkaiho 2004, 75, 409-415. [CrossRef]

19. Inagaki, S.; Amano, Y.; Kumazawa, K. Identification and characterization of volatile components causing the characteristics flavor of Wagyu beef (Japanese Black cattle). J. Agric. Food. Chem. 2017, 65, 8691-8695. [CrossRef]

20. Matsuzaki, M.; Takizawa, S.; Ogawa, M. Plasma insulin metabolite concentrations and carcass characteristics of Japanese Black, Japanese Brown, and Holstein steers. J. Anim. Sci. 1997, 75, 3287-3293. [CrossRef]

21. Yamada, T.; Kawakami, S.; Nakanishi, N. Expression of adipogenic transcription factors in adipose tissue of fattening Wagyu and Holstein steers. Meat Sci. 2009, 81, 86-92. [CrossRef] [PubMed]

22. Kerth, C.R.; Miller, R.K. Beef flavor: A review from chemistry to consumer. J. Sci. Food Agric. 2015, 95, 2783-2798. [CrossRef] [PubMed] 
23. Khan, M.I.; Jo, C.; Tariq, M.R. Meat flavor precursors and factors influencing flavor precursors-A systematic review. Meat Sci. 2015, 110, 278-284. [CrossRef] [PubMed]

24. Kawai, M.; Sekine-Hayakawa, Y.; Okiyama, A.; Ninomiya, Y. Gustatory sensation of L- and D- amino acids in humans. Amino Acids. 2012, 43, 2349-2358. [CrossRef] [PubMed]

25. Tuoria, H.; Sommardahl, C.; Hyvönen, L.; Leporanta, K.; Merimma, P. Does fat affect the timing of flavor perception? a case study with yoghurt. Food Qual. Pref. 1995, 6, 55-58. [CrossRef]

26. Metcalf, K.L.; Vickers, Z.M. Taste intensities of oil-in-water emulsions with varying fat content. J. Sens. Stud. 2002, 17, 379-390. [CrossRef]

27. Kearsley, M.W.; Dziedzic, S.Z.; Birch, G.G.; Smith, P.D. The production and properties of glucose syrups III. Sweetness of glucose syrups and related carbohydrates. Starch 1980, 32, 244-247. [CrossRef]

28. Birch, G.G.; Munton, S.L. Evidence for constant number of available sweet receptor sites at threshold concentration of sugars. Experientia 1981, 37, 839-840. [CrossRef]

29. Bouton, P.E.; Ford, P.V.; Harris, P.V.; Shorthose, W.R.; Ratcliff, D.; Morgan, J.H.L. Influence of animal age on the tenderness of beef: Muscle differences. Meat Sci. 1978, 2, 301-311. [CrossRef]

30. Okumura, T.; Saito, K.; Sowa, T.; Sakuma, H.; Ohhashi, F.; Tameoka, N.; Hirayama, M.; Nakayama, S.; Sato, S.; Gogami, T.; et al. Changes in beef sensory traits as somatic-cell-cloned Japanese black steers increased in age from 20 to 30 months. Meat Sci. 2012, 90, 159-163. [CrossRef]

(C) 2020 by the authors. Licensee MDPI, Basel, Switzerland. This article is an open access article distributed under the terms and conditions of the Creative Commons Attribution (CC BY) license (http://creativecommons.org/licenses/by/4.0/). 

Article

\title{
Use of Large and Diverse Datasets for ${ }^{1} \mathrm{H}$ NMR Serum Metabolic Profiling of Early Lactation Dairy Cows
}

\author{
Timothy D. W. Luke ${ }^{1,2}$, Jennie E. Pryce ${ }^{1,2}$, Aaron C. Elkins ${ }^{1}$, William J. Wales ${ }^{3}$ \\ and Simone J. Rochfort ${ }^{1,2, *}$ \\ 1 Agriculture Victoria Research, AgriBio, Centre for AgriBioscience, Bundoora 3083, Australia; \\ tim.luke@agriculture.vic.gov.au (T.D.W.L.); jennie.pryce@agriculture.vic.gov.au (J.E.P.); \\ aaron.elkins@agriculture.vic.gov.au (A.C.E.) \\ 2 School of Applied Systems Biology, La Trobe University, Bundoora 3083, Australia \\ 3 Agriculture Victoria Research, Ellinbank Centre, Ellinbank 3821, Australia; bill.wales@agriculture.vic.gov.au \\ * Correspondence: simone.rochfort@agriculture.vic.gov.au
}

Received: 11 April 2020; Accepted: 27 April 2020; Published: 30 April 2020

\begin{abstract}
Most livestock metabolomic studies involve relatively small, homogenous populations of animals. However, livestock farming systems are non-homogenous, and large and more diverse datasets are required to ensure that biomarkers are robust. The aims of this study were therefore to (1) investigate the feasibility of using a large and diverse dataset for untargeted proton nuclear magnetic resonance $\left({ }^{1} \mathrm{H}\right.$ NMR) serum metabolomic profiling, and (2) investigate the impact of fixed effects (farm of origin, parity and stage of lactation) on the serum metabolome of early-lactation dairy cows. First, we used multiple linear regression to correct a large spectral dataset (707 cows from 13 farms) for fixed effects prior to multivariate statistical analysis with principal component analysis (PCA). Results showed that farm of origin accounted for up to $57 \%$ of overall spectral variation, and nearly $80 \%$ of variation for some individual metabolite concentrations. Parity and week of lactation had much smaller effects on both the spectra as a whole and individual metabolites $(<3 \%$ and $<20 \%$, respectively). In order to assess the effect of fixed effects on prediction accuracy and biomarker discovery, we used orthogonal partial least squares (OPLS) regression to quantify the relationship between NMR spectra and concentrations of the current gold standard serum biomarker of energy balance, $\beta$-hydroxybutyrate (BHBA). Models constructed using data from multiple farms provided reasonably robust predictions of serum BHBA concentration $(0.05 \leq$ RMSE $\leq 0.18)$. Fixed effects influenced the results biomarker discovery; however, these impacts could be controlled using the proposed method of linear regression spectral correction.
\end{abstract}

Keywords: NMR; metabotype; metabolomics; transition; ketosis; cattle; chemometrics; spectral correction

\section{Introduction}

Modern metabolomic techniques such as proton nuclear magnetic resonance $\left({ }^{1} \mathrm{H}\right.$ NMR) spectroscopy allow high-throughput, synchronous characterization of the small metabolites present in biological matrices [1]. In dairy cows, the metabolome gives a snapshot of the complex interactions between host genetics, the rumen microbiome, and the environment at a given time point. ${ }^{1} \mathrm{H}$ NMR-based metabolomics therefore offers exciting opportunities to better understand and characterize the complex physiological and biochemical challenges facing cows in the transition period (defined as the three weeks before and after calving [2,3]) which is the period of greatest disease risk [4]. This in turn can facilitate identification of new molecular phenotypes (metabotypes) for genetic selection for improved animal health. These "intermediate phenotypes," so-termed because they sit between the genome and external phenotype [5], can then be integrated with genomic data to improve 
genomic prediction accuracies of complex traits [6,7]. The aim of metabotype identification is therefore to identify biomarkers that represent inter-animal variation free of confounding environmental factors.

Another aim of dairy cattle metabolomic studies is to identify biomarkers which enable early identification of health disorders in the transition period such as ketosis [8,9], hypocalcemia [10] and displaced abomasa [11]. Of particular interest are studies that have identified biomarkers that are predictive of transition period disorders, such as that by Hailemariam et al. [12], who identified a panel of three metabolites that could predict the occurrence of peri-parturient disease up to four weeks before calving. If robust, such predictive biomarkers would enable producers and veterinarians to implement preventive nutritional, management and/or veterinary interventions before the onset of disease.Unlike metabotype biomarkers used for genetic selection, the aim of biomarkers used for management purposes is to predict the external phenotype, and these must therefore capture all sources of phenotypic variation (i.e., host genetics, rumen microbiome, and the environment).

To date, most serum ${ }^{1} \mathrm{H}$ NMR-based metabolomic studies of livestock have involved relatively small numbers of animals, often of a single breed, and often located on a single farm. In their review, Goldansaz et al. [13] identified limited sample size and diversity as limitations of many livestock metabolomics studies and highlighted the need for larger and more diverse datasets to ensure models and biomarkers are robust. However this needs to be balanced against the need for careful experimental design to account for potential confounding from systematic environmental effects such as diet/nutritional management, parity and stage of lactation, which are known to affect the metabolic status of cows [13]. However, in order to achieve large datasets, it may be necessary to obtain samples from multiple different farms, especially when the prevalence of the condition being investigated is low (e.g., displaced abomasa). Previous studies have reported differences in the milk metabolome of animals from different geographical regions [14], farms [15], and of different breeds [16]. However, given that there is not a strong relationship between blood and milk metabolomes $[17,18]$, these findings cannot be extrapolated to the blood serum/plasma metabolome. More information is therefore needed on the impact of systematic environmental effects on the serum metabolome of livestock.

Linear models are routinely used by quantitative geneticists to account for the influence of systematic environmental effects (also known as fixed effects) known to have significant effects on phenotypic variation [19], and thus disentangle genetic from non-genetic effects. Frequently used fixed effects include stage of lactation, parity, and herd-year-season. Similar approaches have recently been applied to metabolomic data, for example Wanichthanarak et al. [20], who used linear mixed-effects models and patient metadata to account for biological variation in metabolomics data, and Laine et al. [21], who used linear models to study the effect of pregnancy on mid-infrared spectral data derived from cows' milk.

The aim of this study was therefore to investigate the feasibility of using of large and diverse datasets in livestock metabolomics studies by examining the effects of fixed environmental and physiological effects on the ${ }^{1} \mathrm{H}$ NMR serum metabolome of clinically healthy dairy cows in early lactation. We propose a method that uses linear models to correct spectra for fixed effects and demonstrate its potential utility by quantifying the relationship between ${ }^{1} \mathrm{H}$ NMR spectra and the current gold-standard serum biomarker of energy balance, $\beta$-hydroxybutyrate (BHBA) $[22,23]$.

\section{Results}

\subsection{Dataset}

Serum samples were collected from 707 early lactation cows ( $<30 \mathrm{~d}$ in milk) from 13 farms located in southeastern Australia. Descriptive statistics of the animals included in the experiment, including herd of origin, stage of lactation (reported as days in milk, or the number of days post-calving), parity and serum BHBA concentrations and are summarized in Table 1. Of particular interest were the BHBA results obtained from Farm 1, which had a greater mean and standard deviation than observed in other farms. 
Table 1. Descriptive statistics of dataset used in this experiment, including farm details, number of cows (N), mean and standard deviation (shown in parentheses) of parity, days in milk (DIM), and serum $\beta$ hydroxybutyrate (BHBA) concentrations obtained from dairy cows in the first 30 days of lactation from 13 farms in south eastern Australia.

\begin{tabular}{cccccc}
\hline Farm & $\mathbf{N}$ & Location & Parity & DIM & BHBA \\
\hline 1 & 129 & Sth Gipp $^{1}$ & $2.9(1.1)$ & $19.4(7.2)$ & $1.25(0.69)$ \\
2 & 11 & Sth Gipp $^{2}$ & $2.6(1.2)$ & $20.4(8.1)$ & $0.34(0.12)$ \\
3 & 12 & W Gipp $^{2}$ & $2.6(1.4)$ & $22.8(5.7)$ & $0.33(0.10)$ \\
4 & 11 & W Gipp $^{2}$ & $3.1(1.2)$ & $17.9(10.2)$ & $0.54(0.15)$ \\
5 & 18 & MID $^{3}$ & $2.9(1.1)$ & $22.6(5.1)$ & $0.61(0.25)$ \\
6 & 248 & W Gipp $^{2}$ & $2.1(1.0)$ & $16.7(6.0)$ & $0.55(0.21)$ \\
7 & 9 & GV & $2.6(1.0)$ & $13.9(6.7)$ & $0.53(0.27)$ \\
8 & 24 & MID & $2.4(1.2)$ & $17.7(8.2)$ & $0.38(0.09)$ \\
9 & 33 & Sth Gipp & $2.5(1.2)$ & $18.3(7.2)$ & $0.55(0.33)$ \\
10 & 27 & Sth Gipp & $1.8(1.1)$ & $13.1(7.7)$ & $0.50(0.14)$ \\
11 & 50 & Tas & $2.6(1.3)$ & $18.6(7.3)$ & $0.42(0.17)$ \\
12 & 123 & MID & $2.8(1.2)$ & $15.8(8.6)$ & $0.38(0.15)$ \\
13 & 12 & Tas & $2.7(0.8)$ & $16.0(7.6)$ & $0.58(0.22)$ \\
ALL & 707 & - & $2.5(1.2)$ & $17.4(7.3)$ & $0.63(0.46)$ \\
\hline
\end{tabular}

${ }^{1}$ South Gippsland Region, ${ }^{2}$ West Gippsland Region, ${ }^{3}$ Macalister Irrigation District, ${ }^{4}$ Goulburn Valley Region,

${ }^{5}$ Tasmania.

\section{2. ${ }^{1}$ H NMR Spectroscopy of Serum Samples}

${ }^{1} \mathrm{H}$ NMR spectra were complex; however, more than 20 metabolites could be identified. Spectra were dominated by organic acids, amino acids, glucose and phospholipid intermediates (Figure S1 and Table S1).

\subsection{Preliminary Data Analysis Using Principal Component Analysis}

Preliminary data analysis and outlier identification was performed using principal component analysis (PCA). Plots of the first 2 principal components (PCs) identified several samples located outside the $95 \%$ confidence level. These spectra were manually inspected, and a single outlier with erroneous phasing was identified and removed from subsequent analyses.

PCA was repeated after outlier removal. The first 13 PCs explained greater than $90 \%$ of the variation in the spectra. Scores plots of the first three PCs, which explain $47.64 \%, 15.59 \%$, and $7.45 \%$ of variation, respectively, are shown in Figure 1a-c. There was obvious clustering of samples by herd of origin. Samples from Farm 1 showed greater variation than those from the other farms. The separation between farm clusters was most obvious along PC1 and PC2. Visual comparisons based on stage of lactation (defined as weeks in milk (WIM)) and parity were also performed, but no obvious clustering or separation was observed. Loadings plots of the first three PCs show that energy metabolites BHBA, lactate, acetate and glucose, have the largest influences on spectral differences (Figure $1 \mathrm{~d}-\mathrm{f}$ ), with smaller influences from the branched chain amino acids, lipoproteins, glycine, creatine, and betaine. 


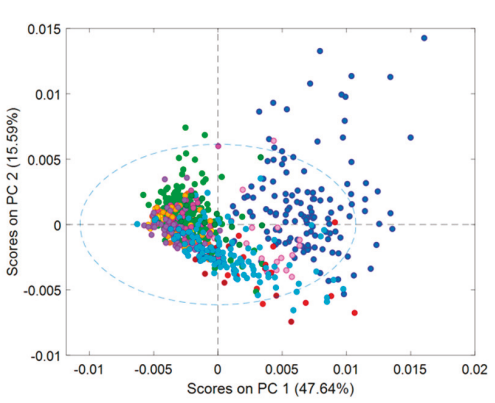

(a)

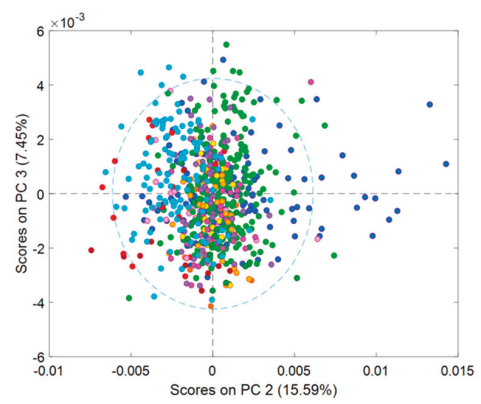

(c)

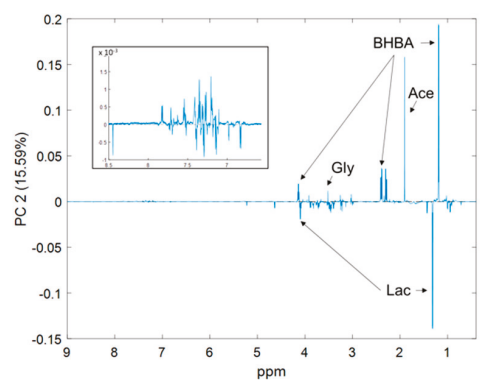

(e)
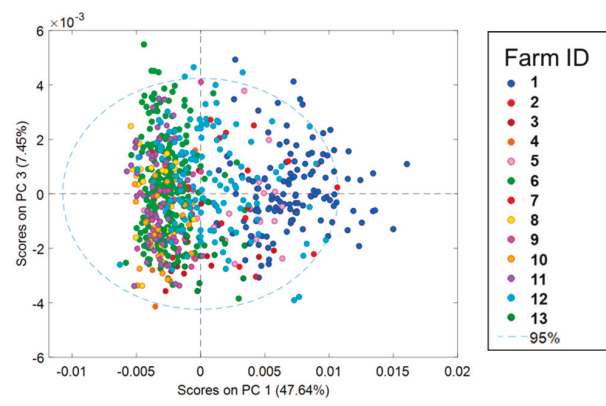

(b)

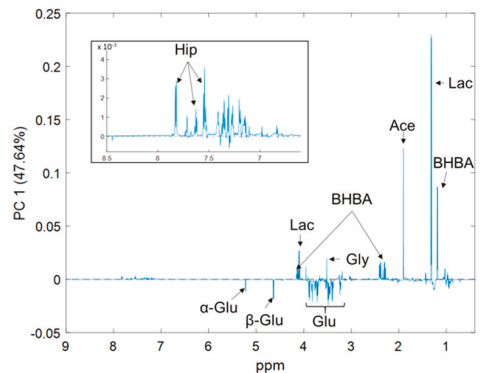

(d)

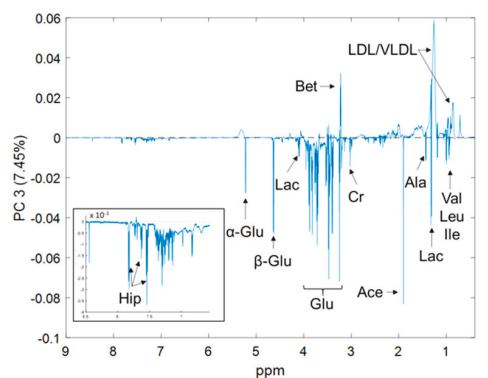

(f)

Figure 1. Results of principal component analysis (PCA) of 707 proton nuclear magnetic resonance $\left({ }^{1} \mathrm{H}\right.$ NMR) spectra of serum obtained from dairy cows in early lactation; (a) principal component (PC) 1 vs. PC 2 scores, (b) PC 1 vs. PC 3 scores, (c) PC 2 vs. PC 3 scores, (d) PC 1 loadings, (e) PC 2 loadings, and (f) PC 3 loadings plots. Scores plots are colored by farm of origin. The $\delta 6.5$ to 8.5 region of loadings plots have been magnified for clarity purposes. $\alpha$-Glu $=\alpha$ glucose, Ace $=$ acetate, Ala $=$ alanine, $\beta$-Glu $=\beta$ glucose, $\mathrm{Bet}=$ betaine, $\mathrm{BHBA}=\beta$ hydroxybutyrate, $\mathrm{Cr}=$ creatine, $\mathrm{Glu}=$ glucose, $\mathrm{Gly}=$ glycine, Hip = hippurate, Ile = isoleucine, Lac = lactate, Leu = leucine, Val = valine, VLDL/LDL = Very low density lipoprotein and low density lipoprotein.

\subsection{Principal Component Analysis of Spectra Corrected for Fixed Effects}

Principal component analysis (PCA) was repeated on spectra that had been corrected for (1) WIM, (2) Parity, (3) Herd, and (4) WIM, Parity and Herd simultaneously (hereafter referred to as all fixed effects) (Models 1 to 4). Results derived from spectra corrected separately for WIM and Parity are nearly identical to uncorrected spectra (Figures S2 and S3). By contrast, scores plots derived from PCA of spectra corrected for Herd (Figure S4), and spectra corrected for all fixed effects (Figure 2a-c), show no obvious clustering of samples by Herd, WIM or Parity. There is, however, still considerable 
separation of samples along all three PC axes, suggesting that significant inter-animal variation in the serum metabolome exists after accounting for fixed effects. Compared to the uncorrected data; (1) more PCs were required to explain $>90 \%$ of spectral variation (24 vs. 13), (2) the percentage of variation captured by PC1 was lower (25.70\% vs. $47.64 \%$ ), and (3) the percentage of variation captured by PC2 and PC3 was higher (16.92\% vs. $15.59 \%$ and $11.79 \%$ vs. $7.45 \%$, respectively). Loadings plots are shown in Figure 2d-f. Interestingly, separation of samples along PC1 (25.70\%) is due almost entirely to lactate. Loadings on PC2 and PC3 are similar to uncorrected data.

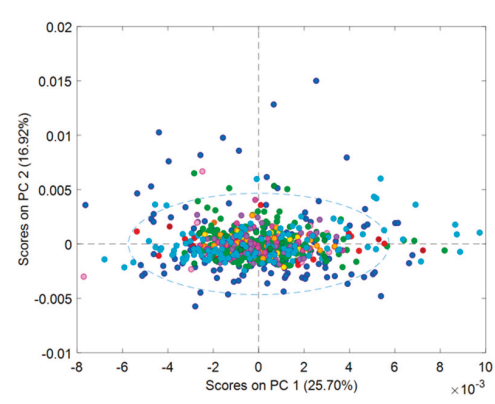

(a)

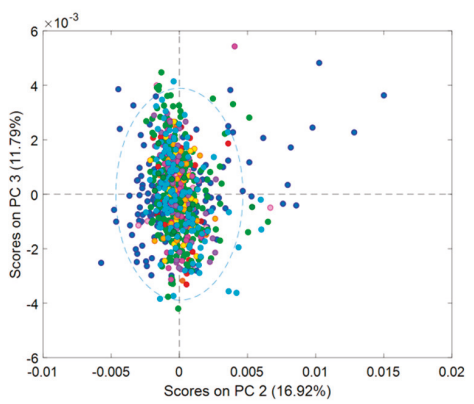

(c)

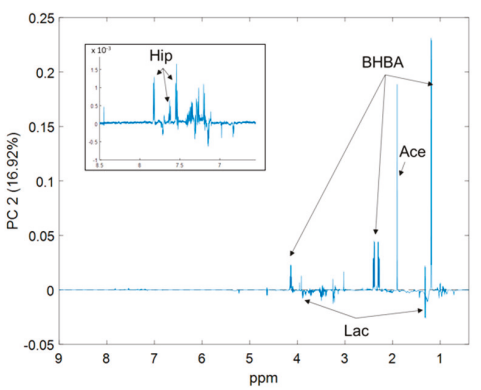

(e)

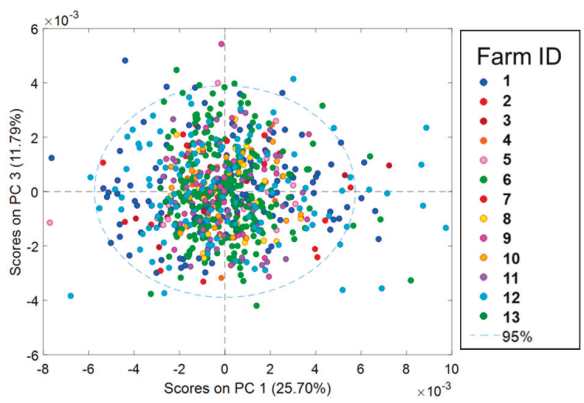

(b)

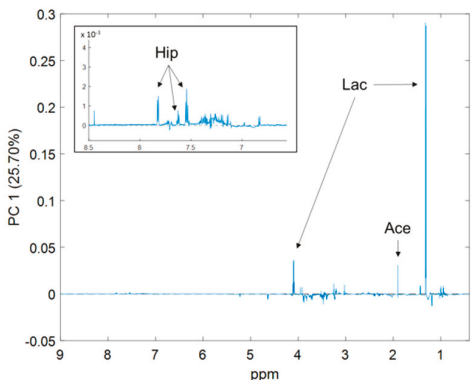

(d)

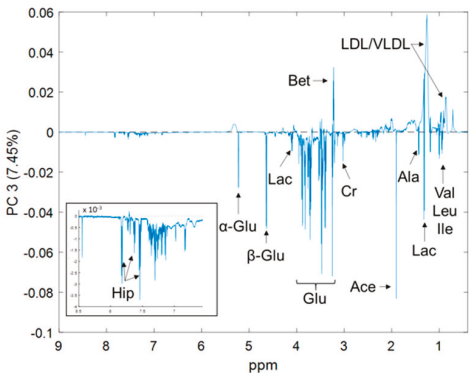

(f)

Figure 2. Results of PCA of $707{ }^{1} \mathrm{H}$ NMR spectra of serum, corrected for herd of origin, week of lactation, and parity obtained from dairy cows in early lactation; (a) PC 1 vs. PC 2 scores, (b) PC 1 vs. PC 3 scores, (c) PC 2 vs. PC 3 scores, (d) PC 1 loadings, (e) PC 2 loadings, and (f) PC 3 loadings plots. Scores plots are colored by farm of origin. The $\delta 6.5$ to 8.5 region of loadings plots have been magnified for clarity purposes. $\alpha$-Glu $=\alpha$ glucose, Ace $=$ acetate, Ala $=$ alanine, $\beta$-Glu $=\beta$ glucose, Bet $=$ betaine, $\mathrm{BHBA}=\beta$ hydroxybutyrate, $\mathrm{Cr}=$ creatine, $\mathrm{Glu}=$ glucose, $\mathrm{Gly}=$ glycine, $\mathrm{Ile}=$ isoleucine, $\mathrm{Lac}=$ lactate, Leu $=$ leucine, $\mathrm{Val}=$ valine, $\mathrm{VLDL} / \mathrm{LDL}=$ Very low density lipoprotein and low density lipoprotein. 


\subsection{Effect of Stage of Lactation, Parity, and Herd Effects on ${ }^{1} H$ NMR Spectra}

In order to quantify the effect of each fixed effect on NMR spectra, we calculated Pearson's correlations between scores for the first three PCs from the previously described PCAs (Figure 3). The largest differences (i.e., lowest correlations) were seen between uncorrected spectra, and spectra corrected for Herd $(\mathrm{r}=0.43)$. This suggests that there are significant differences between those 2 spectral datasets, and that Herd, therefore, has a significant effect on the serum NMR metabolome. This is consistent with the clustering of samples by farm in the original PCA (Figure 1a-c). By comparison, the correlations between PC scores derived from uncorrected spectra, and spectra corrected for WIM and Parity, were high (0.99 and 0.97 , respectively). This suggests that these spectra are nearly identical, and that these fixed effects have minimal influence on the serum metabolome. Correlations between PC2 scores were consistent with those observed between PC1 scores, and correlations between PC3 scores were all high $(\geq 0.89)$.

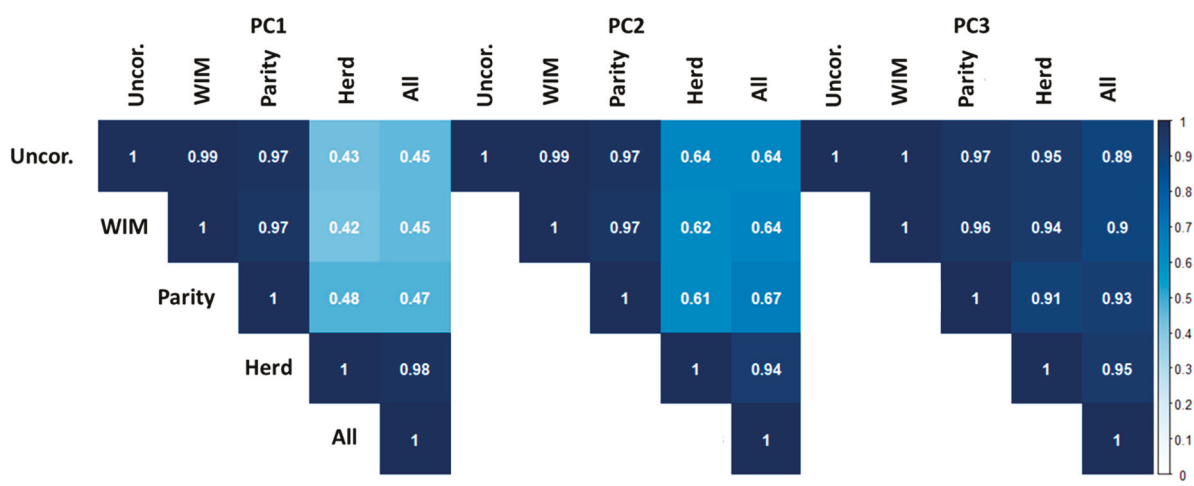

Figure 3. Pearson's correlations between scores derived from PCA of uncorrected ${ }^{1} \mathrm{H}$ NMR spectra of bovine serum, and the same spectra corrected using linear regression for week of lactation (WIM), parity, herd of origin, and WIM, parity, and herd simultaneously (All). Color map shows strength of Pearson's correlation.

To test the statistical significance of fixed effects on ${ }^{1} \mathrm{H}$ NMR spectra, we used conditional Wald F statistics derived from multiple linear regression models on the first three PC scores (Model 5). The higher the F statistic, the greater the effect of that variable on the PC score, and the lower the P value, the greater the statistical significance. Results derived from these models are summarized in Table 2. PC1 results were consistent with the results of Pearson's correlations, showing that Herd had the greatest effect. Interestingly, results for PC2 and PC3 differed slightly from Pearson's correlations. While Herd had a relatively large and significant $(\mathrm{P}<0.001)$ impact on both PC2 and PC3, the effect of Parity was nearly as great on PC2 scores and greater on PC3 scores.

Table 2. Results of multiple linear regression models of principal component (PC) scores derived from PCA of ${ }^{1} \mathrm{H}$ NMR spectra, against weeks in milk (WIM), parity, and herd of origin. Conditional Wald F statistics (F-con) and corresponding $P$ values describe the magnitude and statistical significance of each fixed effect, respectively.

\begin{tabular}{ccccccccc}
\hline & \multicolumn{2}{c}{ PC1 $(\mathbf{4 7 . 6 4} \%)$} & & \multicolumn{2}{c}{ PC2 $\mathbf{( 1 5 . 5 9 \% )}$} & & \multicolumn{2}{c}{ PC3 $\mathbf{( 7 . 4 5 \% )}$} \\
\cline { 2 - 3 } Fixed Effect & F-con & $\boldsymbol{P}$ Value & & F-con & $\boldsymbol{P}$ Value & & F-con & $\boldsymbol{P}$ Value \\
\hline WIM & 2.66 & 0.047 & & 5.42 & 0.001 & & 2.14 & 0.094 \\
Parity & 2.78 & 0.041 & & 20.39 & $<0.001$ & & 15.19 & $<0.001$ \\
Herd & 158.29 & $<0.001$ & & 26.78 & $<0.001$ & & 6.66 & $<0.001$ \\
\hline
\end{tabular}


$\mathrm{R}^{2}$ values obtained from models 1-4 were used to investigate which regions of the NMR spectra were most strongly influenced by the fixed effects. As the signal intensity at each chemical shift was treated as a separate response variable, the $\mathrm{R}^{2}$ values from Models 1,2 , and 3 describe the effect of WIM, parity, and herd on each of the 24,349 chemical shifts, respectively. These $\mathrm{R}^{2}$ values were color-coded, and overlaid on an average NMR spectrum. Plots showing the effects of WIM and Parity were unremarkable (all $\mathrm{R}^{2}<0.2$, Figure S5), however $\mathrm{R}^{2}$ values obtained from Model 2 showed that approximately $10-20 \%$ of the variation in glucose and acetate concentration could be explained by parity. The plot showing the effect of herd is shown in Figure 4. The strongest effect was seen in the downfield region of the spectrum, with close to $80 \%$ of variation in the concentration of some phenolic compounds being explained by Herd. Of these, hippurate could be clearly identified. Peaks at $\delta 7.31$ and 7.39 were tentatively assigned to 3 -phenyllactate, but the peak at $\delta 7.22$ could not be identified. Lactate, acetate, BHBA, betaine, pyruvate, glycine, and glucose concentrations were also strongly influenced by herd effect.

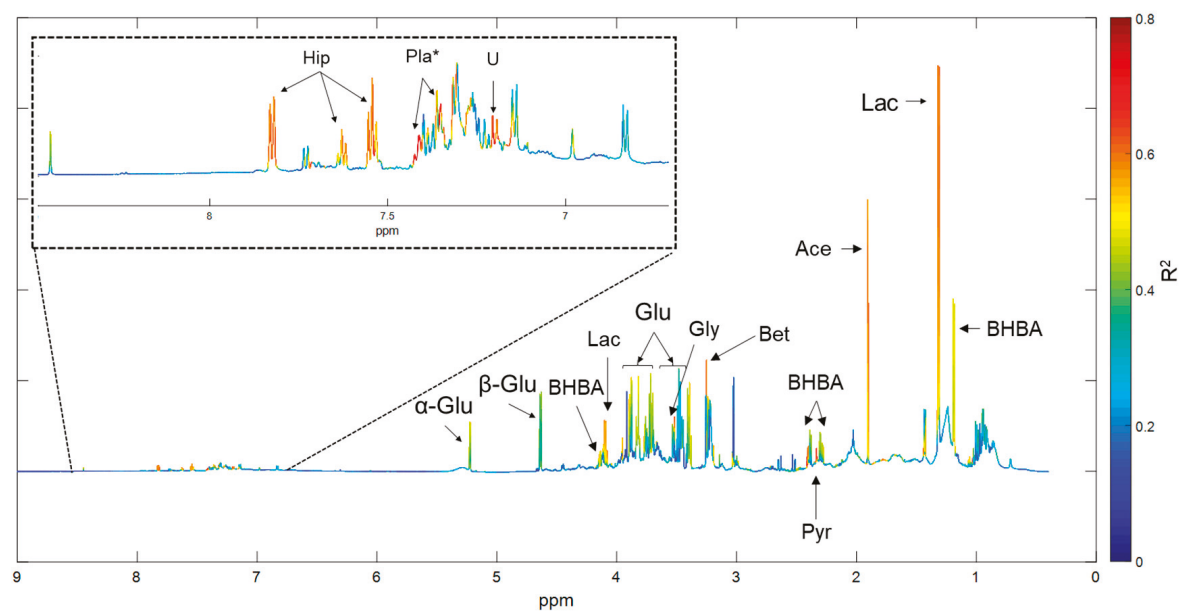

Figure 4. Average ${ }^{1} \mathrm{H}$ NMR spectrum of bovine serum. Color coding represents the percentage of variation in the signal at each chemical shift intensity that can be explained by herd of origin. The $\delta$ 6.5 to 8.5 region has been magnified for clarity purposes. Ace $=$ acetate, $\mathrm{Bet}=$ betaine, $\mathrm{BHBA}=\beta$ hydroxybutyrate, Gly = glycine, Hip = hippurate, Lac = lactate, Pla = 3-phenyllactate, Pyr = pyruvate, $\mathrm{U}=$ unidentified peak. * indicates tentative identification.

The results of ANOVA-simultaneous component analysis (ASCA) were consistent with results of linear regression spectral correction and are shown in Table S2. Herd had the greatest effect (43.99, $\mathrm{P}=0.02)$, followed by parity $(4.10, \mathrm{P}=0.02)$ and WIM $(1.37, \mathrm{P}=0.02)$. When ASCA was performed on corrected spectra, the effect of the fixed effect(s) was reduced to zero. For example, when ASCA was performed on spectra corrected for Herd, the effect of herd was zero $(\mathrm{P}=1.00)$, but the effects of WIM $(1.68, \mathrm{P}=0.02)$ and parity $(3.30, \mathrm{P}=0.02)$ were retained.

\subsection{Robustness of ${ }^{1} H$ NMR Predictions of Serum BHBA}

Our results show that ${ }^{1} \mathrm{H}$ NMR spectra can be used to predict serum BHBA concentration with good accuracy. This result is expected, as BHBA is directly quantifiable from NMR spectra. The overall robustness of our approach was assessed using a "leave-one-farm-out" external validation of OPLS models built using uncorrected data. This involved iteratively setting aside data from one farm, training models using data from the remaining 12 farms, then using the withheld data for external validation. $R^{2}$ results were variable $\left(0.30 \leq R^{2} \leq 0.99\right)$, however RMSE values remained relatively 
low $(\leq 0.18)$ (Table 3). Interestingly, RMSE values were considerably higher when Farm 1 data were withheld for validation.

Table 3. Results of leave-one-farm out external validation of orthogonal partial least squares (OPLS) regression models predicting serum BHBA concentration from uncorrected ${ }^{1} \mathrm{H}$ NMR spectra. Validation farm specifies the identity of the data used for validation, $N$ the number of animals used in calibration and validation datasets. The coefficient of determination $\left(\mathrm{R}^{2}\right)$ and root mean square error (RMSE) are reported for each calibration/validation subset.

\begin{tabular}{|c|c|c|c|c|c|c|c|c|c|c|}
\hline \multirow[b]{2}{*}{ Validation Farm } & \multirow[b]{2}{*}{$\mathbf{P}$} & \multirow[b]{2}{*}{ LV } & \multicolumn{3}{|c|}{ Calibration } & \multicolumn{2}{|c|}{ Cross Validation } & \multicolumn{3}{|c|}{ External Validation } \\
\hline & & & $\mathbf{N}$ & $\mathbf{R}^{2}$ & $\overline{R M S E}$ & $\mathbf{R}^{2}$ & RMSE & $\mathbf{N}$ & $\mathbf{R}^{2}$ & RMSE \\
\hline- & $<0.05$ & 3 & 707 & 0.95 & 0.10 & 0.95 & 0.10 & - & - & - \\
\hline 1 & $<0.05$ & 5 & 578 & 0.87 & 0.08 & 0.85 & 0.08 & 129 & 0.96 & 0.18 \\
\hline 2 & $<0.05$ & 3 & 696 & 0.95 & 0.10 & 0.95 & 0.10 & 11 & 0.59 & 0.10 \\
\hline 3 & $<0.05$ & 4 & 695 & 0.96 & 0.09 & 0.96 & 0.10 & 12 & 0.78 & 0.06 \\
\hline 4 & $<0.05$ & 3 & 696 & 0.95 & 0.10 & 0.95 & 0.10 & 11 & 0.93 & 0.09 \\
\hline 5 & $<0.05$ & 3 & 689 & 0.96 & 0.10 & 0.95 & 0.10 & 18 & 0.99 & 0.09 \\
\hline 6 & $<0.05$ & 3 & 459 & 0.96 & 0.11 & 0.96 & 0.11 & 248 & 0.87 & 0.10 \\
\hline 7 & $<0.05$ & 3 & 698 & 0.95 & 0.10 & 0.95 & 0.10 & 9 & 0.98 & 0.05 \\
\hline 8 & $<0.05$ & 3 & 683 & 0.95 & 0.10 & 0.95 & 0.10 & 24 & 0.30 & 0.07 \\
\hline 9 & $<0.05$ & 3 & 674 & 0.95 & 0.10 & 0.95 & 0.10 & 33 & 0.95 & 0.11 \\
\hline 10 & $<0.05$ & 3 & 680 & 0.95 & 0.10 & 0.95 & 0.10 & 27 & 0.85 & 0.09 \\
\hline 11 & $<0.05$ & 3 & 657 & 0.95 & 0.10 & 0.95 & 0.10 & 50 & 0.82 & 0.08 \\
\hline 12 & $<0.05$ & 3 & 584 & 0.97 & 0.09 & 0.96 & 0.09 & 123 & 0.52 & 0.12 \\
\hline 13 & $<0.05$ & 3 & 695 & 0.95 & 0.10 & 0.95 & 0.10 & 12 & 0.98 & 0.05 \\
\hline
\end{tabular}

\subsection{Influence of Fixed Effects on Interpretation of ${ }^{1} \mathrm{H}$ NMR Metabolomic Data}

The impact of fixed effects on the interpretation of ${ }^{1} \mathrm{H}$ NMR metabolomic data was determined by comparing the results of OPLS models built using (1) data from Farm 1 only (used as a control), (2) uncorrected data from all farms, and (3) data from all farms corrected for all fixed effects. Fixed effects appeared to have minimal effect on the predictive ability of models. We observed similar 10-fold cross validation prediction accuracies for all 3 datasets (Table 4). Interestingly, RMSE results were quite close to the results of the leave-one-farm out external validation $(0.05 \leq \mathrm{RMSE} \leq 0.18)$.

Table 4. Results obtained from 10-fold cross validation of OPLS regression models predicting serum BHBA concentration from ${ }^{1} \mathrm{H}$ NMR spectra using data from Farm 1 only, uncorrected data from all farms, and data from all farms corrected for the effect of herd. Number of cows (N), number of latent variables included in each mode (LV), coefficient of determination $\left(R^{2}\right)$ and root mean square error (RMSE) of calibration (C) and 10-fold cross validation (CV) are shown.

\begin{tabular}{cccccccc}
\hline Dataset & $\mathbf{N}$ & $\mathbf{L V s}$ & $\mathbf{P ~ V a l u e ~}^{\mathbf{1}}$ & $\mathbf{R}^{\mathbf{2}} \mathbf{\mathbf { R }}$ & $\mathbf{R S E}_{\mathbf{C}}$ & $\mathbf{R}^{\mathbf{2}} \mathbf{C V}$ & $\mathbf{R M S E}_{\mathbf{C V}}$ \\
\hline Farm 1 Uncorrected & 129 & 4 & $<0.001$ & 0.98 & 0.10 & 0.97 & 0.12 \\
All Data Uncorrected & 707 & 4 & $<0.001$ & 0.96 & 0.09 & 0.96 & 0.10 \\
All Data Corrected for Herd & 707 & 4 & $<0.001$ & 0.93 & 0.09 & 0.93 & 0.09 \\
\hline \multicolumn{7}{c}{${ }^{1}$ P-value derived from permutation testing (50 iterations) and pairwise Wilcoxon signed rank test. }
\end{tabular}

The influences of fixed effects on biomarker discovery were investigated by comparing loadings on LV1. Results obtained using only Farm 1 data were used as a reference and show a strong positive correlation between BHBA concentration and acetate, and strong negative correlations with lactate and glucose (Figure 5a,b). Loadings from the complete dataset corrected for all fixed effects were very similar (Figure 5e,f). Results from uncorrected data, however, were quite different (Figure 5c,d), with BHBA being positively correlated with lactate and glycine. Examination of scores plots shows obvious clustering and separation by herd (especially Farm 1) when uncorrected data are used (Figure S6a), but not when corrected data are used (Figure S6b). Results from the original PCA showed that samples from Farm 1 clustered at the positive end of PC1, and that lactate and glycine both had 
strong positive influences on PC1 loadings. Therefore, it is possible that OPLS results are confounded by a strong herd effect when uncorrected data are used.

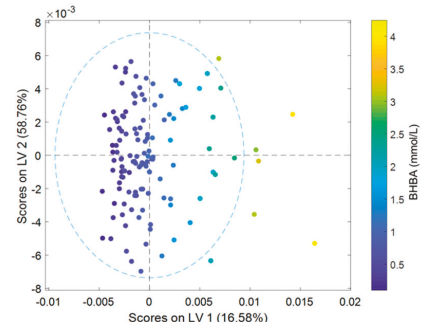

(a)

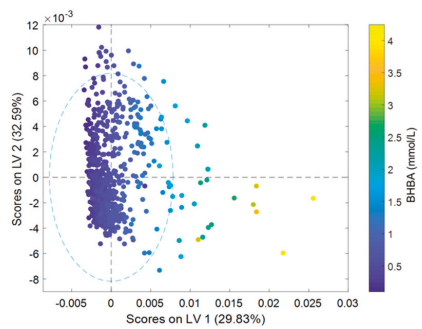

(c)

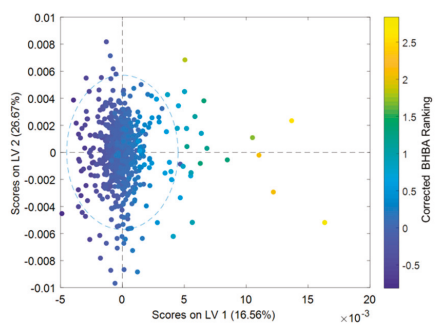

(e)

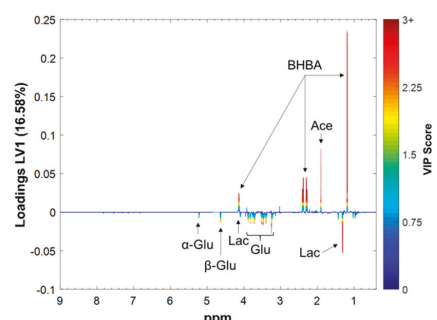

(b)

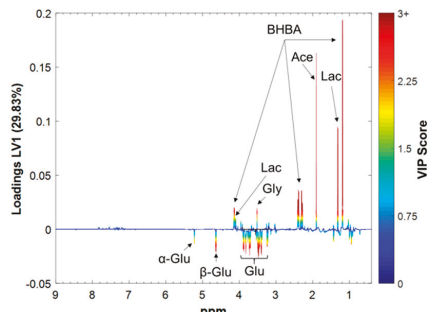

(d)

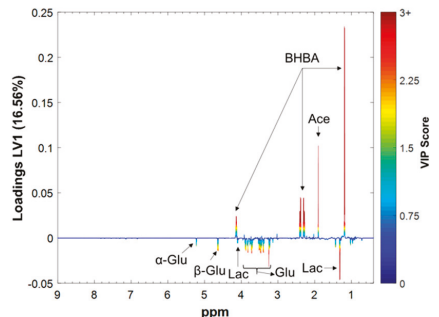

(f)

Figure 5. Results of OPLS regressions of serum BHBA concentration against ${ }^{1} \mathrm{H}$ NMR spectrum of bovine serum: (a) Farm 1 ( $\mathrm{N}=179)$ LV1 vs. LV2 scores and (b) LV1 loadings, (c) all farms ( $=707)$ uncorrected data LV1 vs. LV2 scores and (d) LV1 loadings, and (e) all farms data LV1 vs. LV2 scores and (d) LV1 loadings.

\section{Discussion}

To the best of the authors' knowledge, this is the first large-scale serum metabolomics study to investigate the impact of systematic environmental and physiological fixed effects on the ${ }^{1} \mathrm{H}$ NMR serum metabolome of clinically healthy dairy cattle. Our results indicate that herd-specific environmental factors have much greater effects on the serum metabolome of early lactation dairy cows than physiological factors such as WIM and parity. We demonstrate that, while confounding from herd effects can significantly influence the results of biomarker discovery, models built using data collected from multiple farms can give robust predictions of external phenotypes such as BHBA. In order to overcome the potential confounding of fixed effects on biomarker discovery, we propose a method to correct ${ }^{1} \mathrm{H}$ NMR spectra prior to multivariate analysis using multiple linear regression.

\subsection{Differences in ${ }^{1}$ H NMR Spectra Between Herds}

Our results clearly demonstrate that there are significant differences in the serum metabolomes of animals from different herds. The fact that energy metabolites BHBA, lactate, acetate and glucose 
dominated PCA loadings (Figure $2 \mathrm{~d}-\mathrm{f}$ ), and that herd effect accounted for a large percentage of the variation seen in lactate, acetate, pyruvate, glucose, and BHBA concentrations, (Figure 4) suggests that metabolic state, in particular energy balance, varied significantly between farms.

The importance of lactate was particularly interesting. Lactate was one of the most abundant metabolites identified in this experiment. This is very different to the findings of Sun et al. [8], who reported that lactate was one of the weakest signals in serum ${ }^{1} \mathrm{H}$ NMR spectra obtained from early-lactation cows fed a total mixed ration. One possible explanation for the very high concentrations of lactate seen in our dataset could be ruminal lactate production. During spring, dairy cows in pastoral farming systems of southeastern Australia are typically fed rations high in fermentable carbohydrate, and low in neutral detergent fiber. As a consequence, ruminal acidosis is common [24]. Serum concentrations of lactate, and in particular D-lactate from microbial fermentation, have been shown to increase following experimental induction of ruminal acidosis $[25,26]$. Without the use of a shift reagent and specialized experiments it is not possible to differentiate between the different lactate isomers by ${ }^{1} \mathrm{H}$ NMR [27]. We therefore plan to quantify the relative contributions of L- and D- lactate to better understand the cause of high lactate concentrations in our dataset.

The strong influence of Herd on the concentration of phenolic compounds could also be consistent with ruminal acidosis. Signal intensities in the downfield region of 2D spectra were weak, meaning clear identification of some of the phenolic peaks in our dataset was not possible. Our tentative identification of 3-phenyllactate is consistent with the findings of Yang et al. [26], who demonstrated that beef steers fed high starch (corn) diets had higher plasma concentrations of phenyllactate compared to those fed low starch diets. This study also identified L-phenyllalanine biosynthesis and metabolism as important metabolic pathways in high starch feeding. We plan to (1) enrich samples and repeat 2D analyses and (2) perform LCMS-based metabolomics on a subset of samples to identify these compounds.

Nearly $80 \%$ of the variation seen in hippurate concentration could be explained by herd effect (Figure 4). Hippurate is formed by the conjugation of glycine and benzoic acid, and has been associated with microbial degradation of dietary compounds [28]. Concentrations of hippurate increase with increased consumption of phenolic compounds [13], which are present in relatively high concentrations in pasture species. Milk hippurate concentration has been proposed as a biomarker of pasture/forage intake in goats [29], and it is possible that our results represent differences in feeding regimens between farms. Hippurate has also been proposed as a biomarker for gut microbiome diversity in humans [30], and our results may indicate differences in the gastrointestinal health of animals from different farms (i.e., ruminal acidosis). Detailed information of ration formulations is very difficult to define in grazing systems as pasture quality and intake vary considerably within and between herds. This information was therefore not available for the herds in our dataset and more data are required to further investigate this finding.

Results of the initial PCA showed that data from Farm 1 were significantly different to, and showed more variation than, data from the other farms. The reasons for these differences are hard to determine from our dataset, as Farm 1 differed in environment/management, breed and reference BHBA concentrations (and therefore it is assumed animal metabolic status). Given that we also observed clustering and separation of the 12 Holstein-Friesian herds in the initial PCA (Figure 2), it appears that herd-specific environmental factors have a larger effect on the serum metabolome than breed. However, Liao et al. [31] recently reported clear differences in the serum metabolomes (GC-MS) of three different breeds of beef steers, all the same age, fed the same ration, and managed under the same conditions. Further data are therefore required to investigate if there are differences between the serum metabolomes of different dairy breeds.

Pre-analytical sample handling and processing have been shown to have significant effects on human metabolomic data [32], and considerable efforts are made to streamline and standardize sample collection and processing protocols [33-35]. Standardizing protocols in livestock studies provides its own challenges, when relatively large number of samples are being taken at once, often in diverse, challenging and remote locations. While all attempts were made to ensure consistency, there were 
some unavoidable differences in the way samples from different herds were handled (for example time, between blood sample collection and centrifugation varied from approximately $2-4 \mathrm{~h}$ ). It is therefore possible that some of the variation between farms seen in our data could be due to pre-analytical sample handling. However, overall our results suggest that metabolomic differences between animals from different farms are due largely to differences in diet/nutritional management. We plan to collect more samples from animals receiving different diets to investigate this further.

\subsection{Effect of Lactation Stage and Parity on Serum Metabolome}

Our results suggest that stage of lactation appeared to have a minimal effect on the NMR spectra. This is consistent with the findings of Ilves et al. [17] who found that the mass spectrometry (MS) based plasma metabolome of dairy cows was more heavily influenced by animal individuality than by lactation stage. By contrast, several authors report that both the NMR and MS-derived milk metabolome changes across lactation $[17,36]$. This suggests that blood-based metabolomics may be more suitable for identification of individual animal-specific differences within a population, and therefore provide more robust metabotypes for genetic selection.

Parity appeared to have a small but significant $(\mathrm{P}<0.05)$ effect on the overall ${ }^{1} \mathrm{H}$ NMR serum metabolome. We could find no other reports in the literature describing the effect of parity on the entire serum metabolome. However, our results are consistent with other studies that showed parity has a significant effect on the concentration of several metabolites in serum including glucose, creatinine, urea and BHBA [37-39]. This suggests that parity should be taken into consideration when undertaking metabolomic studies in dairy cows.

\subsection{Accuracy of OPLS Models for Predicting Serum BHBA Concentration}

Despite the significant influence of fixed effects on the serum metabolome, results obtained from the leave-one-farm out external validation suggest that prediction models constructed with data from multiple farms are quite robust. $R^{2}$ values varied significantly depending on which farm was used for validation $\left(0.30 \leq R^{2} \leq 0.99\right)$; however, the $R^{2}$ is known to be affected by the range of the dataset, and RMSE is often considered to be a better predictor of model performance [40]. Promisingly, external validation RMSE results $\left(0.05 \leq R^{2} \leq 0.18\right)$ were close to those obtained from 10 -fold cross validation of models built using only Farm 1 data (RMSE $=0.12)$ and all data $($ RMSE $=0.10)$. The fact that prediction errors were highest when Farm 1 data were withheld for validation suggests that the increased variation observed in Farm 1 data represents valuable biological variation rather than confounding/noise.

Correcting data for fixed effects had very little impact on the predictive ability of OPLS models. Furthermore, when corrected spectra were used, y-values also had to be corrected, making interpretation of phenotypic values difficult. Interestingly, Wanichthanarak, et al. [20] found that "readjusting" mass spectroscopy metabolite signals using patient metadata and linear mixed models improved the sensitivity and specificity of classification of human tissue samples with and without colorectal cancer. Conversely, Posma et al. [41] found that adjusting NMR data for confounding factors lead to a loss of predictive power for cardiovascular risk in a large-scale human NMR metabolomic dataset. Whether using NMR spectra corrected using linear regression will improve the performance of classification models (as opposed to regression against a continuous variable as used in this study) requires further investigation. Overall, our results suggest that models constructed using uncorrected data collated from multiple farms may be appropriate for prediction of external phenotypes which are influenced by both genetic and environmental factors.

\subsection{Impact of Fixed Effects on the Interpretation of Metabolomic Data for Biomarker and Metabotype Discovery}

Loadings from OPLS models built using uncorrected spectra from Farm 1, and spectra from all farms corrected for fixed effects, were consistent with the literature. BHBA and glucose concentrations have been shown to be negatively correlated in the serum of cows in early lactation dairy cows [42]. L-lactate is an important gluconeogenic substrate in dairy cows $[43,44]$, so it follows that lactate 
concentration is also negatively correlated with BHBA concentration. Our results are also consistent with the findings of Sun et al. [8] who showed that cows with subclinical $(1.2<\mathrm{BHBA}<2.9 \mathrm{mmol} / \mathrm{L})$ and clinical ketosis (BHBA $>2.9 \mathrm{mmol} / \mathrm{L}$ ) had lower lactate and glucose concentrations and higher BHBA and acetate concentrations than the healthy controls.

The fact that loadings were different when uncorrected spectra from all farms were used demonstrates that herd-specific environmental effects can influence the results of biomarker discovery. How significant this is ultimately depends on the research question being asked. If the study aim is to identify biomarkers of external phenotypes (i.e., biomarkers that represent both genetic and environmental factors which are used for management purposes such as disease prediction), then the impact of environmental effects is important and must be captured. However, if the aim is to identify biomarkers indicative of inter-animal differences free of environmental confounding, or to understand biological processes, our results suggest that the influence of environmental effects could lead to erroneous results. This is consistent with the findings of Posma et al. [41] who showed that differences in fixed effects between subjects from the north and south of China explained some metabolite associations, which had previously been attributed to cardiovascular disease risk. This study also reported that adjusting metabolomic data for confounding using an algorithm called Covariate-Adjusted Projection to Latent Structures (CA-PLS) improved model interpretability and led to the identification of more robust biomarkers. Our results are also consistent with other studies that have explored the impacts of data pretreatments on the interpretation of metabolomics data. For example, van den Berg et al. [45] showed that pretreatment methods such as scaling, centering and transformations can greatly affect the outcome of metabolomic analyses (including the biological ranking of important metabolites) and have the potential to enhance biological interpretability. Similarly, Emwas et al. [46] concluded that the choice of spectral processing and post-processing depended on many factors including the aim of the experiment and the quality of data.

We believe that our approach has particular application in animal breeding, where the aim is to understand the biological processes that underpin economically important traits [47] and to identify metabotypes that represent inter-animal variation independent of confounding from systematic environment effects. Even with the advent of genomic selection, livestock genetic studies require relatively large numbers of animals to ensure there is adequate genetic variation in the study population $[48,49]$. The same is likely to be true for metabotype discovery studies. Such large datasets can be hard to compile, especially when the trait of interest is difficult and/or expensive to measure. As well as collecting data from multiple farms, another potential solution is data sharing through international collaboration. This is routinely done by geneticists; for example, de Haas et al. [50] used data from Holstein cattle in Europe, North America and Australasia to improve genomic prediction accuracies for feed intake. The ability to correct metabolomic data for factors such as experimental batch, diet, herd, year and season should allow similar collaborations in metabotype studies.

\section{Materials and Methods}

All procedures undertaken in this study were conducted in accordance with the Australian Code of Practice for the Care and Use of Animals for Scientific Purposes (National Health and Medical Research Council, 2013). Approval to proceed was granted by the Agricultural Research and Extension Animal Ethics Committee of the Department of Jobs, Precincts and Resources Animal Ethics Committee (DJPR, 475 Mickleham Road, Attwood, Victoria 3049, Australia), and the Tasmanian Department of Primary Industries, Parks, Water and Environment (DPIPWE Animal Biosecurity and Welfare Branch, 13 St Johns Avenue, New Town, Tasmania 7008, Australia). AEC project approval codes 2017-05 and 2018-07.

\subsection{Sample Collection}

A single $10 \mathrm{~mL}$ blood sample was taken from 708 clinical healthy cows, located on 13 farms in south-eastern Australia between September 2017 and July 2019. All cows had been calved 30 days 
or less at the time of sampling. Cows on all farms except Farm 1 were Australian Holstein-Friesians, while cows on Farm 1 were crossbred animals (including Holstein-Friesian, Jersey, and Australian Red breeds). All farms operated a feeding system reliant on grazed pasture plus other forages, and concentrates fed in the bail at milking time.

Blood samples were collected from the coccygeal vein into $10 \mathrm{~mL}$ serum clot activator vacutainer tubes (Becton Dickinson, Franklin Lakes, NJ, USA). Samples were allowed to clot at room temperature, before being centrifuged at $1000 \mathrm{~g}$ for $20 \mathrm{~min}$ at $20^{\circ} \mathrm{C}$. Sera were divided into two aliquots. The first aliquot was refrigerated at $4{ }^{\circ} \mathrm{C}$ then shipped on ice to a commercial laboratory for BHBA analysis. The second aliquot was stored at $-20^{\circ} \mathrm{C}$ until processing for NMR spectroscopy.

\subsection{Reference BHBA Measurements}

Serum BHBA concentrations were determined using a colorimetric enzymatic kinetic assay [51]. All assays were performed by Regional Laboratory Services (Benalla, Victoria, Australia) using a Kone 20 XT clinical chemistry analyzer (Thermo Fisher Scientific, Waltham, MA, USA). The uncertainty of measurement (at a $95 \%$ confidence level) was $\pm 0.060 \mathrm{mmol} / \mathrm{L}$ at $0.85 \mathrm{mmol} / \mathrm{L}$.

\subsection{Chemicals}

Methanol ( $>99.9 \%$ pure) and dipotassium hydrogen phosphate (anhydrous) were purchased from Fisher Chemical (Fair Lawn, NJ, USA). Sodium 2,2-dimethyl- 2-silapentane-5-sulfonate (DSS-d6, $98 \%)$ and deuterium oxide $\left(\mathrm{D}_{2} \mathrm{O}, 98 \%\right)$ were purchased from Cambridge Isotope Laboratories, Inc. (Tewksbury, MA, USA).

\subsection{Sample Preparation for NMR Spectroscopy}

Serum samples were thawed at room temperature for one hour and were prepared for NMR spectroscopy using a methanol protein precipitation method described by Nagana Gowda and Raftery [52]. Briefly, $300 \mu \mathrm{L}$ of serum was mixed with $600 \mu \mathrm{L}$ of methanol, vortexed (Ratek multi tube vortex mixer, MTV1), incubated at $-20^{\circ} \mathrm{C}$ for $20 \mathrm{~min}$, then centrifuged to pellet proteins $\left(11,360 \mathrm{~g}, 21^{\circ} \mathrm{C}\right.$, $30 \mathrm{~min})$. A $600 \mu \mathrm{L}$ aliquot of supernatant was then transferred to a clean $2 \mathrm{~mL}$ microcentrifuge tube and dried under vacuum at $21^{\circ} \mathrm{C}$ overnight using a SpeedVac Savant SPD 2010 Concentrator (Thermo Fisher Scientific, Waltham, MA, USA). Dried extracts were then reconstituted in a $\mathrm{D}_{2} \mathrm{O}$ phosphate buffer solution (100 $\left.\mathrm{mM} \mathrm{K}_{2} \mathrm{HPO}_{4}\right)$ containing $0.25 \mathrm{mM}$ DSS-d6 as an internal standard. A $550 \mu \mathrm{L}$ aliquot was transferred to $5 \mathrm{~mm} \mathrm{NMR}$ tube for analysis.

\section{5. ${ }^{1}$ H NMR Data Acquisition}

Routine 1D proton spectra were obtained on a Bruker Ascend $700 \mathrm{MHz}$ spectrometer equipped with cryoprobe and SampleJet automatic sample changer (Bruker Biospin, Rheinstetten, Germany). A Bruker noesypr1d pulse sequence was used over $-0.76 \mathrm{ppm}$ to $10.32 \mathrm{ppm}$ spectral range with 256 scans collected after eight dummy scans at $298 \mathrm{~K}$, with a total acquisition time of 2.11 seconds per increment and a relaxation delay (D1) of 2.00 seconds. The overall number of data points was 32,768. A line broadening of $0.3 \mathrm{~Hz}$ was applied to all spectra prior to Fourier transformation. Spectra were manually phased then baseline corrected in Topspin v.3.6.1 (Bruker Biospin, Rheinstetten, Germany). Samples were referenced to the internal standard (DSS-d6) at $\delta 0.00$.

\section{6. ${ }^{1}$ H NMR Spectral Processing \& Multivariate Statistical Analysis}

NMR spectra were imported into MatLab v.R2017b (Mathworks, Natick, WA, USA) using the ProMetab v.1.1 script [53]. Each raw spectrum consisted of 31,313 data points between -0.60 and $10.00 \mathrm{ppm}$.

Statistical analyses were performed in MatLab utilizing the PLS Toolbox v. 8.5.2 (Eigenvector Research Inc., Manson, WA, USA). The spectral region containing the residual water peak ( $\delta 4.68-5.00)$ 
was removed. Spectra were aligned using the correlation optimized warping algorithm [54] to account for chemical shift drift, then normalized to total signal area to account for inherent concentration differences between samples. After normalization, spectral regions containing methanol ( $\delta 3.32-3.36)$ and DSS-d6 ( $\delta$ 0.4--0.60) peaks, and the non-informative region beyond $9.00 \mathrm{ppm}$ were removed. Finally, spectra were baseline corrected using automatic weighted least squares, and scaled by mean centering. After editing, a total of 24,349 chemical shift datapoints were included in subsequent statistical analyses.

For multivariate analyses, unsupervised principal component analysis (PCA) was used. Peaks of interest were identified using the Chenomx NMR suite software v.8.4 (Chenomx Inc., Edmonton, AB, Canada), comparison to the literature, and 2D NMR analysis.

\subsection{Correction of ${ }^{1} H$ NMR Spectra for the Effects of Systematic Environemtal and Physiological Effects}

In order to investigate the effects on spectra of systematic environmental effects (also known as fixed effects) spectra were "corrected" using linear regression models. When correcting for a single categorical fixed effect, this is equivalent to scaling data using the "class centering" pre-processing step. Rather than mean centering, which involves subtracting the global mean from each variable, class centering subtracts the mean of each class. This allows investigation of intra-class variation by removing the effects of inter-class variation [55]. The advantage of using linear models rather than class centering is that the effect of multiple fixed effects or classes can be modelled simultaneously.

The approach we took was based on the principals of quantitative genetic models, where

Phenotypic observation $=$ environmental effects + genetic effects + residual effects

In this study, we only want to remove the effect of environmental factors (as it is the variation in NMR spectra under genetic influence that we are interested in), so the equation can be further simplified to

$$
\text { Phenotypic observation }=\text { genetic effects }+ \text { residual effects }
$$

The "corrected phenotype" (i.e., the phenotypic observation with the effects of the environmental effects removed) is defined as the residuals from the above model. For the purposes of this study each chemical shift was treated as a separate phenotype, with the signal intensity at each chemical shift being an individual phenotypic observation. The "corrected spectra" was a matrix of the residuals of each model.

A $707 \times 24,349$ matrix of signal intensities of pre-processed spectra was imported into the $\mathrm{R}$ statistical software package v 3.6.2 [56]. Each row in the matrix represented a single sample, and each column represented 1 of the 24,349 chemical shifts between $\delta 0.40$ and $\delta 8.99$ that made up an individual spectrum. The following 4 linear models were applied to each of the 24,349 columns in the matrix (i.e., the signal intensity at each chemical shift was treated as the response variable in a separate regression model):

$$
\begin{gathered}
\mathrm{y}_{\mathrm{il}}=\mu+\mathrm{WIM}_{\mathrm{i}}+\mathrm{e}_{\mathrm{il}}(\text { Model } 1) \\
\mathrm{y}_{\mathrm{jl}}=\mu+\mathrm{P}_{\mathrm{j}}+\mathrm{ej}_{\mathrm{jl}}(\text { Model } 2) \\
\mathrm{y}_{\mathrm{kl}}=\mu+\mathrm{H}_{\mathrm{k}}+\mathrm{e}_{\mathrm{kl}}(\text { Model } 3) \\
\mathrm{y}_{\mathrm{ijkl}}=\mu+\mathrm{WIM}_{\mathrm{i}}+\mathrm{P}_{\mathrm{j}}+\mathrm{H}_{\mathrm{k}}+\mathrm{e}_{\mathrm{ijkl}}(\text { Model } 4)
\end{gathered}
$$

where $y$ is the signal intensity at a given chemical shift, $\mu$ is the mean, WIM is weeks in milk (4 levels, defined as 1, 2, 3, or 4), $\mathrm{P}$ is parity (4 levels, defined as 1, 2, 3, or $\geq 4$ ), $\mathrm{H}$ is the effect of herd (13 levels, with a range of 9 to 248 cows per herd), and e is the random error term. This resulted in four separate $707 \times 24,349$ matrices containing spectra corrected for the effects of WIM, parity, herd, and all fixed effects, respectively. 
The $\mathrm{R}^{2}$ values from each regression model were stored in a separate vector. This resulted in four vectors each containing $24,349 R^{2}$ values; each value representing the percentage of variation in signal intensity explained by the fixed effect(s) at a given chemical shift.

\subsection{Quantifying the Effect of Stage of Lactation, Parity and Herd on ${ }^{1} H$ NMR Spectra}

A separate PCA was performed on each of the 4 corrected spectral datasets (as described in 4.7). Scores of the first three PCs were extracted for each model, and for the PCA model constructed using uncorrected data. We then calculated Pearson's correlations between scores derived from the 5 PCAs using the corrplot package [57] in R v 3.6.2 [56]. This resulted in three correlation matrices (one for each PC). The lower the Pearson's correlation coefficient, the greater the differences between PC scores, the greater the differences between the two spectral datasets and therefore the greater the significance of the fixed effect(s).

An alternative approach to investigating the influence of fixed effects is to use multiple linear regression on PC scores from uncorrected spectra. The advantage of this approach is that all fixed effects can be fitted simultaneously, and the statistical significance of each fixed effect can be calculated. The model used was

$$
\mathrm{y}_{\mathrm{ijk}}=\mu+\mathrm{WIM}_{\mathrm{i}}+\mathrm{P}_{\mathrm{j}}+\mathrm{H}_{\mathrm{k}}+\mathrm{e}_{\mathrm{ijkl}}(\text { Model 5) }
$$

where $\mathrm{y}$ is the PC score (on either PC1, PC2, or PC3) and $\mu, \mathrm{WIM}, \mathrm{P}, \mathrm{H}$, and e are the mean, fixed effect, and error terms described previously. The statistical significance of each fixed effect was determined using conditional Wald F statistics in ASReml v 4.2 (VSN International Ltd., Hemel Hempstead, UK). Conditional $\mathrm{F}$ statistics are used in multiple linear regression to infer the significance of a given fixed effect assuming that the effect of remaining predictor variables have been accounted for [58].

Finally, we validated our results using the analysis of variance (ANOVA) simultaneous component analysis (ASCA) method in the PLS Toolbox [55]. ASCA is a generalization of ANOVA used to quantify the variation induced by fixed experimental design factors on complex multivariate datasets [59]. ASCA was performed on all spectral datasets (corrected and uncorrected). Statistical significance was determined using permutation testing (50 iterations).

\subsection{The Relationships between ${ }^{1} H$ NMR Spectra and Existing Energy Balance Biomarker Concentrations}

In order to assess the utility of large and diverse datasets in livestock metabolomics studies, we used orthogonal partial least squares (OPLS) regression to compare ${ }^{1} \mathrm{H}$ NMR spectra to serum BHBA concentrations determined by colorimetric assay. The aims of this analysis were (1) to assess the robustness of OPLS models built using uncorrected data and (2) investigate the influence of systematic environmental effects on the interpretation of ${ }^{1} \mathrm{H}$ NMR spectra when used for untargeted metabolomic analyses.

\subsubsection{Robustness of OPLS Models to Predict External Phenotypes Using Uncorrected Data}

The robustness of OPLS models constructed using large and diverse datasets was assessed using a leave-one-farm-out external validation. This involved setting aside data from one farm, training OPLS models using data from the remaining 12 farms, then using the withheld data for external validation. This process was repeated until data from each farm was used as an external validation set once. Model performance was assessed using the $\mathrm{R}^{2}$ and RMSE of calibration, cross validation (venetian blind CV with 10 data splits, and one sample per split), and external validation. The statistical significance of OPLS models was determined using permutation testing (cross validated, Wilcoxon test). Only uncorrected data were used for this part of the analysis.

\subsubsection{Influence of Fixed Effects on Interpretation of ${ }^{1} \mathrm{H}$ NMR Metabolomic Data}

To assess the impact of fixed effects on the results of untargeted metabolomic analyses we compared the results of OPLS models constructed from (1) uncorrected data from Farm 1 only 
( $\mathrm{N}=129)$, (2) uncorrected data from all farms, and (3) data from all farms corrected for all fixed effects (Model 4). Farm 1 data was used to simulate a more "typical" metabolomics experiment in which confounding from environmental effects is controlled through experimental design.

When corrected spectra were used, reference BHBA concentrations were corrected for the same fixed effects (Model 4). The residuals of this model represent the "corrected BHBA" concentration, which is the expected BHBA concentration of an individual accounting for differences in WIM, Parity and Herd. This poses some challenges in terms of interpretation, as negative residual values (i.e., negative BHBA concentrations) are possible. However, for the purposes of genetic evaluations, the ranking of an animal, or the relative phenotypic value, is of more interest than an absolute value. The corrected value can therefore be considered a "corrected phenotypic ranking."

The impact of fixed effects on the ability of NMR spectra to predict external phenotypes (i.e., to classify animals or predict biomarker concentrations for management purposes) was assessed by comparing the predictive ability of OPLS models. The influences of fixed effects on biomarker discovery were investigated using scores and loadings on LV1 which show the magnitude and direction of relationships between BHBA concentration and ${ }^{1} \mathrm{H}$ NMR spectral features. Variable importance of projection (VIP) scores were used to identify the most statistically significant spectral features in each model. Variables with VIP scores greater than one were considered significant [60].

\section{Conclusions}

In this study we investigated the feasibility of using large and diverse datasets for untargeted ${ }^{1} \mathrm{H}$ NMR serum metabolomic profiling of clinically healthy dairy cows in early lactation. In particular, we investigated the effects of systematic environmental factors on the serum metabolome. We used linear regression to correct spectra for (1) herd of origin; (2) parity; (3) WIM; and (4) herd, parity, and WIM simultaneously. Corrected and uncorrected spectra were then analyzed using PCA. Comparison of PCA results showed that herd of origin had a much greater impact on the serum metabolome than either parity or WIM. In order to simulate the impact of these effects in untargeted metabolomics, we used OPLS regression to quantify the relationship between both corrected and uncorrected NMR spectra, and the current gold-standard biomarker of energy balance in dairy cows, BHBA. Our results showed that (1) models constructed using uncorrected data from multiple farms provided reasonably robust predictions of serum BHBA concentration, (2) environmental effects can alter the results of biomarker discovery, and (3) that correcting spectra for environmental effects using linear regression may be useful when the aim of analysis is to investigate phenotypic variation free of confounding from environmental effects (e.g., identification of metabotypes for genetic selection).

Supplementary Materials: The following are available online at http://www.mdpi.com/2218-1989/10/5/180/s1, Table S1. ${ }^{1} \mathrm{H}$ NMR chemical shifts $(\delta)$ and multiplicity of metabolites in bovine serum run in deuterated water (D2O); Table S2. Results of ANOVA-simultaneous component analysis (ASCA) of uncorrected ${ }^{1} \mathrm{H}$ NMR spectra of bovine serum.; Figure S1. Representative $700 \mathrm{MHz}{ }^{1} \mathrm{H}$ NMR spectrum ( $\delta 0.4$ to 9.0 ) of serum obtained from a Holstein-Friesian cow in early lactation.; Figure S2. Results of PCA of $707^{1} \mathrm{H}$ NMR spectra of serum obtained from dairy cows in early lactation, corrected for weeks in milk using linear regression; (a) PC 1 vs. PC 2 scores, (b) PC 1 vs. PC 3 scores, (c) PC 2 vs. PC 3 scores, (d) PC 1 loadings, (e) PC 2 loadings, and (f) PC 3 loadings plots.; Figure S3. Results of PCA of $707^{1} \mathrm{H}$ NMR spectra of serum obtained from dairy cows in early lactation, corrected for Parity using linear regression; (a) PC 1 vs. PC 2 scores, (b) PC 1 vs. PC 3 scores, (c) PC 2 vs. PC 3 scores, (d) PC 1 loadings, (e) PC 2 loadings, and (f) PC 3 loadings plots.; Figure S4. Results of PCA of $707{ }^{1} \mathrm{H}$ NMR spectra of serum obtained from dairy cows in early lactation, corrected for Herd using linear regression; (a) PC 1 vs. PC 2 scores, (b) PC 1 vs. PC 3 scores, (c) PC 2 vs. PC 3 scores, (d) PC 1 loadings, (e) PC 2 loadings, and (f) PC 3 loadings plots.; Figure S5. Average ${ }^{1} \mathrm{H}$ NMR spectrum of bovine serum. Color-coding represents the percentage of variation in the signal at each chemical shift intensity that can be explained by (a) WIM and (b) Parity: Figure S6: Results of OPLS regressions of serum BHBA concentration against ${ }^{1} \mathrm{H}$ NMR spectrum of bovine serum ( $\mathrm{n}=707)$ : (a) LV1 vs. LV2 scores for uncorrected data (b) CV predicted vs. measured BHBA (c) LV1 vs. LV2 scores for corrected data (d) CV predicted vs. measured corrected BHBA ranking. 
Author Contributions: Conceptualization, T.D.W.L., J.E.P., W.J.W., and S.J.R.; Formal analysis, T.D.W.L. and S.J.R.; Funding acquisition, J.E.P.; Investigation, T.D.W.L., A.C.E., and S.J.R.; Methodology, T.D.W.L., A.C.E., and S.J.R.; Project administration, J.E.P., W.J.W., and S.J.R.; Resources, S.J.R.; Supervision, J.E.P., W.J.W., and S.J.R.; Visualization, T.D.W.L.; Writing-original draft, T.D.W.L.; Writing一review and editing, J.E.P., A.C.E., W.J.W., and S.J.R. All authors have read and agreed to the published version of the manuscript.

Funding: DairyBio: jointly funded by Dairy Australia (Melbourne, Australia), the Gardiner Foundation (Melbourne, Australia), and Agriculture Victoria (Melbourne, Australia) funded this study and T.D.W.L.'s PhD project.

Acknowledgments: The authors thank Simone Vassiliadis for assistance with ${ }^{1} \mathrm{H}$ NMR protocol and metabolite identification, Di Mapleson, Brigid Ribaux and the staff at Ellinbank Dairy Research Centre (Ellinbank, Australia) for their technical expertise and assistance, Erika Oakes and the staff at Datagene (Bundoora, Australia) for their work coordinating this study, and the farmers who took part in this project.

Conflicts of Interest: The authors declare no conflict of interest.

\section{References}

1. Wishart, D.S. Metabolomics: Applications to food science and nutrition research. Trends Food Sci. Technol. 2008, 19, 482-493. [CrossRef]

2. Drackley, J.K. Biology of dairy cows during the transition period: The final frontier? J. Dairy Sci. 1999, 82, 2259-2273. [CrossRef]

3. Grummer, R.R. Impact of changes in organic nutrient metabolism on feeding the transition dairy cow. J. Anim. Sci. 1995, 73, 2820-2833. [CrossRef]

4. LeBlanc, S.J.; Lissemore, K.D.; Kelton, D.F.; Duffield, T.F.; Leslie, K.E. Major advances in disease prevention in dairy cattle. J. Dairy Sci. 2006, 89, 1267-1279. [CrossRef]

5. Houle, D.; Govindaraju, D.; Omholt, S. Phenomics: The next challenge. Nat. Rev. Gen. 2010, 11, 855-866. [CrossRef]

6. Daetwyler, H.D.; Xiang, R.; Yuan, Z.; Bolormaa, S.; Vander Jagt, C.J.; Hayes, B.J.; van der Werf, J.H.J.; Pryce, J.E.; Chamberlain, A.J.; Macleod, I.M.; et al. Integration of functional genomics and phenomics into genomic prediction raises its accuracy in sheep and dairy cattle. In Proceedings of the Association for the Advancement of Animal Breeding and Genetics, Armidale, NSW, Australia, 27 October-1 November 2019; pp. 11-14.

7. Xiang, R.; Berg, I.V.D.; Macleod, I.M.; Hayes, B.J.; Prowse-Wilkins, C.P.; Wang, M.; Bolormaa, S.; Liu, Z.; Rochfort, S.J.; Reich, C.M.; et al. Quantifying the contribution of sequence variants with regulatory and evolutionary significance to 34 bovine complex traits. Proc. Natl. Acad. Sci. USA 2019, 116, 19398-19408. [CrossRef]

8. Sun, L.W.; Zhang, H.Y.; Wu, L.; Shu, S.; Xia, C.; Xu, C.; Zheng, J.S. 1H-Nuclear magnetic resonance-based plasma metabolic profiling of dairy cows with clinical and subclinical ketosis. J. Dairy Sci. 2014, 97, 1552-1562. [CrossRef]

9. Zhang, G.; Dervishi, E.; Dunn, S.; Mandal, R.; Liu, P.; Han, B.; Wishart, D.; Ametaj, B. Metabotyping reveals distinct metabolic alterations in ketotic cows and identifies early predictive serum biomarkers for the risk of disease. Metabolomics 2017, 13,1-15. [CrossRef]

10. Sun, Y.; Xu, C.; Li, C.; Cheng, X.; Xu, C.; Wu, L.; Zhang, H. Characterization of the serum metabolic profile of dairy cows with milk fever using (1)H-NMR spectroscopy. Vet. Quart. 2014, 34, 1-18. [CrossRef]

11. Basoglu, A.; Baspinar, N.; Tenori, L.; Licari, C.; Gulersoy, E. Nuclear magnetic resonance (NMR)-based metabolome profile evaluation in dairy cows with and without displaced abomasum. Vet. Quart. 2020, 40, 1-15. [CrossRef]

12. Hailemariam, D.; Mandal, R.; Saleem, F.; Dunn, S.M.; Wishart, D.S.; Ametaj, B.N. Identification of predictive biomarkers of disease state in transition dairy cows. J. Dairy Sci. 2014, 97, 2680-2693. [CrossRef] [PubMed]

13. Goldansaz, S.A.; Guo, A.C.; Sajed, T.; Steele, M.A.; Plastow, G.S.; Wishart, D.S. Livestock metabolomics and the livestock metabolome: A systematic review. PLoS ONE 2017, 12, e0177675. [CrossRef] [PubMed]

14. Sacco, D.; Brescia, M.A.; Sgaramella, A.; Casiello, G.; Buccolieri, A.; Ogrinc, N.; Sacco, A. Discrimination between southern Italy and foreign milk samples using spectroscopic and analytical data. Food Chem. 2009, 114, 1559-1563. [CrossRef]

15. Tenori, L.; Santucci, C.; Meoni, G.; Morrocchi, V.; Matteucci, G.; Luchinat, C. NMR metabolomic fingerprinting distinguishes milk from different farms. Food Res. Int. 2018, 113, 131-139. [CrossRef] 
16. Sundekilde, U.K.; Frederiksen, P.D.; Clausen, M.R.; Larsen, L.B.; Bertram, H.C. Relationship between the metabolite profile and technological properties of bovine milk from two dairy breeds elucidated by NMR-based metabolomics. J. Agric. Food Chem. 2011, 59, 7360-7367. [CrossRef]

17. Ilves, A.; Harzia, H.; Ling, K.; Ots, M.; Soomets, U.; Kilk, K. Alterations in milk and blood metabolomes during the first months of lactation in dairy cows. J. Dairy Sci. 2012, 95, 5788-5797. [CrossRef]

18. Maher, A.D.; Hayes, B.; Cocks, B.; Marett, L.; Wales, W.J.; Rochfort, S.J. Latent biochemical relationships in the blood-milk metabolic axis of dairy cows revealed by statistical integration of $1 \mathrm{H}$ NMR spectroscopic data. J. Proteome Res. 2013, 12, 1428. [CrossRef]

19. Mrode, R.A. Linear Models for the Prediction of Animal Breeding Values, 3rd ed.; CABI: Wallingford, UK, 2014.

20. Wanichthanarak, K.; Jeamsripong, S.; Pornputtapong, N.; Khoomrung, S. Accounting for biological variation with linear mixed-effects modelling improves the quality of clinical metabolomics data. Comput. Struct. Biotechnol. J. 2019, 17, 611-618. [CrossRef]

21. Laine, A.; Bastin, C.; Grelet, C.; Hammami, H.; Colinet, F.G.; Dale, L.M.; Gillon, A.; Vandenplas, J.; Dehareng, F.; Gengler, N. Assessing the effect of pregnancy stage on milk composition of dairy cows using mid-infrared spectra. J. Dairy Sci. 2017, 100, 2863-2876. [CrossRef]

22. Oetzel, G.R. Monitoring and testing dairy herds for metabolic disease. Vet. Clin. North Am. Food Anim. Pr. 2004, 20, 651-674. [CrossRef]

23. Ospina, P.A.; Nydam, D.V.; Stokol, T.; Overton, T.R. Associations of elevated nonesterified fatty acids and $\beta$-hydroxybutyrate concentrations with early lactation reproductive performance and milk production in transition dairy cattle in the northeastern United States. J. Dairy Sci. 2010, 93, 1596-1603. [CrossRef] [PubMed]

24. Lean, I.; Westwood, C.T.; Playford, M.C. Livestock disease threats associated with intensification of pastoral dairy farming. N. Z. Vet. J. 2008, 56, 261-269. [CrossRef] [PubMed]

25. Schwaiger, T.; Beauchemin, K.A.; Penner, G.B. Duration of time that beef cattle are fed a high-grain diet affects the recovery from a bout of ruminal acidosis: Short-chain fatty acid and lactate absorption, saliva production, and blood metabolites. J. Anim. Sci. 2013, 91, 5743-5753. [CrossRef] [PubMed]

26. Yang, Y.; Dong, G.; Wang, Z.; Wang, J.; Zhang, Z.; Liu, J. Rumen and plasma metabolomics profiling by UHPLC-QTOF/MS revealed metabolic alterations associated with a high-corn diet in beef steers. PLoS ONE 2018, 13, e0208031. [CrossRef] [PubMed]

27. Zhang, L.; Martins, A.F.; Zhao, P.; Tieu, M.; Esteban-Gómez, D.; McCandless, G.T.; Platas-Iglesias, C.; Sherry, A.D. Enantiomeric recognition of d- and l-lactate by CEST with the aid of a paramagnetic shift reagent. J. Am. Chem. Soc. 2017, 139, 17431-17437. [CrossRef] [PubMed]

28. Lees, H.J.; Swann, J.R.; Wilson, I.D.; Nicholson, J.K.; Holmes, E. Hippurate: The natural history of a mammalian-microbial cometabolite. J. Proteome Res. 2013, 12, 1527-1546. [CrossRef] [PubMed]

29. Carpio, A.; Bonilla-Valverde, D.; Arce, C.; Rodríguez-Estévez, V.; Sánchez-Rodríguez, M.; Arce, L.; Valcárcel, M. Evaluation of hippuric acid content in goat milk as a marker of feeding regimen. J. Dairy Sci. 2013, 96, 5426-5434. [CrossRef]

30. Pallister, T.; Jackson, M.A.; Martin, T.C.; Zierer, J.; Jennings, A.; Mohney, R.P.; MacGregor, A.; Steves, C.J.; Cassidy, A.; Spector, T.D.; et al. Hippurate as a metabolomic marker of gut microbiome diversity: Modulation by diet and relationship to metabolic syndrome. Sci. Rep. 2017, 7, 13670. [CrossRef]

31. Liao, Y.; Hu, R.; Wang, Z.; Peng, Q.; Dong, X.; Zhang, X.; Zou, H.; Pu, Q.; Xue, B.; Wang, L. Metabolomics profiling of serum and urine in three beef cattle breeds revealed different levels of tolerance to heat stress. J. Agric. Food Chem. 2018, 66, 6926-6935. [CrossRef]

32. Yin, P.; Lehmann, R.; Xu, G. Effects of pre-analytical processes on blood samples used in metabolomics studies. Anal. Bioanal. Chem. 2015, 407, 4879-4892. [CrossRef]

33. Beckonert, O.; Keun, H.C.; Ebbels, T.M.; Bundy, J.; Holmes, E.; Lindon, J.C.; Nicholson, J.K. Metabolic profiling, metabolomic and metabonomic procedures for NMR spectroscopy of urine, plasma, serum and tissue extracts. Nat. Protoc. 2007, 2, 2692-2703. [CrossRef] [PubMed]

34. Emwas, A.-H.; Luchinat, C.; Turano, P.; Tenori, L.; Roy, R.; Salek, R.M.; Ryan, D.; Merzaban, J.S.; Kaddurah-Daouk, R.; Zeri, A.C.; et al. Standardizing the experimental conditions for using urine in NMR-based metabolomic studies with a particular focus on diagnostic studies: A review. Metabolomics 2015, 11, 872-894. [CrossRef] [PubMed] 
35. Jobard, E.; Trédan, O.; Postoly, D.; André, F.; Martin, A.-L.; Elena-Herrmann, B.; Boyault, S. A systematic evaluation of blood serum and plasma pre-analytics for metabolomics cohort studies. Int. J. Mol. Sci. 2016, 17, 2035. [CrossRef]

36. Klein, M.S.; Almstetter, M.F.; Schlamberger, G.; Nürnberger, N.; Dettmer, K.; Oefner, P.J.; Meyer, H.H.D.; Wiedemann, S.; Gronwald, W. Nuclear magnetic resonance and mass spectrometry-based milk metabolomics in dairy cows during early and late lactation. J. Dairy Sci. 2010, 93, 1539-1550. [CrossRef] [PubMed]

37. Cozzi, G.; Ravarotto, L.; Gottardo, F.; Stefani, A.L.; Contiero, B.; Moro, L.; Brscic, M.; Dalvit, P. Short communication: Reference values for blood parameters in holstein dairy cows: Effects of parity, stage of lactation, and season of production. J. Dairy Sci. 2011, 94, 3895-3901. [CrossRef] [PubMed]

38. Luke, T.D.W.; Rochfort, S.; Wales, W.J.; Bonfatti, V.; Marett, L.; Pryce, J.E. Metabolic profiling of early-lactation dairy cows using milk mid-infrared spectra. J. Dairy Sci. 2019, 102, 1747-1760. [CrossRef]

39. Morales Pineyrua, J.T.; Farina, S.R.; Mendoza, A. Effects of parity on productive, reproductive, metabolic and hormonal responses of Holstein cows. Anim. Reprod. Sci. 2018, 191, 9-21. [CrossRef]

40. Davies, A.; Fearn, T. Back to basics: Calibration statistics. Spectrosc. Eur. 2006, 18, 31-32.

41. Posma, J.M.; Garcia-Perez, I.; Ebbels, T.M.D.; Lindon, J.C.; Stamler, J.; Elliott, P.; Holmes, E.; Nicholson, J.K. Optimized phenotypic biomarker discovery and confounder elimination via covariate-adjusted projection to latent structures from metabolic spectroscopy data. J. Proteome Res. 2018, 17, 1586-1595. [CrossRef]

42. Lean, I.J.; Farver, T.B.; Troutt, H.F.; Bruss, M.L.; Galland, J.C.; Baldwin, R.L.; Holmberg, C.A.; Weaver, L.D. Time series cross-correlation analysis of postparturient relationships among serum metabolites and yield variables in Holstein cows. J. Dairy Sci. 1992, 75, 1891-1900. [CrossRef]

43. Aschenbach, J.R.; Kristensen, N.B.; Donkin, S.S.; Hammon, H.M.; Penner, G.B. Gluconeogenesis in dairy cows: The secret of making sweet milk from sour dough. IUBMB Life 2010, 62, 869-877. [CrossRef] [PubMed]

44. Drackley, J.K.; Overton, T.R.; Douglas, G.N. Adaptations of glucose and long-chain fatty acid metabolism in liver of dairy cows during the periparturient period. J. Dairy Sci. 2001, 84, E100-E112. [CrossRef]

45. Van den Berg, R.A.; Hoefsloot, H.C.J.; Westerhuis, J.A.; Smilde, A.K.; van der Werf, M.J. Centering, scaling, and transformations: Improving the biological information content of metabolomics data. BMC Genom. 2006, 7, 142. [CrossRef] [PubMed]

46. Emwas, A.-H.; Saccenti, E.; Gao, X.; McKay, R.; Santos, V.; Roy, R.; Wishart, D. Recommended strategies for spectral processing and post-processing of 1D $1 \mathrm{H}-\mathrm{NMR}$ data of biofluids with a particular focus on urine. Off. J. Metab. Soc. 2018, 14, 1-23. [CrossRef]

47. Fontanesi, L. Metabolomics and livestock genomics: Insights into a phenotyping frontier and its applications in animal breeding. Anim. Front. 2016, 6, 73-79. [CrossRef]

48. Boichard, D.; Brochard, M. New phenotypes for new breeding goals in dairy cattle. Anim. Int. J. Anim. Biosci. 2012, 6, 544-550. [CrossRef]

49. Egger-Danner, C.; Cole, J.B.; Pryce, J.E.; Gengler, N.; Heringstad, B.; Bradley, A.; Stock, K.F. Invited review: Overview of new traits and phenotyping strategies in dairy cattle with a focus on functional traits. Anim. Int. J. Anim. Biosci. 2015, 9, 191-207. [CrossRef]

50. De Haas, Y.; Pryce, J.E.; Calus, M.P.; Wall, E.; Berry, D.P.; Lovendahl, P.; Krattenmacher, N.; Miglior, F.; Weigel, K.; Spurlock, D.; et al. Genomic prediction of dry matter intake in dairy cattle from an international data set consisting of research herds in Europe, North America, and Australasia. J. Dairy Sci. 2015, 98, 6522-6534. [CrossRef]

51. McMurray, C.H.; Blanchflower, W.J.; Rice, D.A. Automated kinetic method for D-3-hydroxybutyrate in plasma or serum. Clin. Chem. 1984, 30, 421-425. [CrossRef]

52. Gowda, N.G.A.; Raftery, D. Quantitating metabolites in protein precipitated serum using NMR spectroscopy. Anal. Chem. 2014, 86, 5433-5440. [CrossRef]

53. Viant, M.R. Improved methods for the acquisition and interpretation of NMR metabolomic data. Biochem. Biophys. Res. Commun. 2003, 310, 943-948. [CrossRef] [PubMed]

54. Nielsen, N.-P.V.; Carstensen, J.M.; Smedsgaard, J. Aligning of single and multiple wavelength chromatographic profiles for chemometric data analysis using correlation optimised warping. J. Chromatogr. 1998, 805, 17-35. [CrossRef]

55. Eigenvector. PLS Toolbox, R2017b; Eigenvector Research Inc.: Manson, WA, USA, 2017.

56. Team, R.C. R: A Language and Environment for Statistical Computing; R Foundation for Statistical Computing: Vienna, Austria, 2019. 
57. Wei, T.; Simko, V. R Package "Corrplot": Visualization of a Correlation Matrix, (Version 0.84). Available online: https://github.com/taiyun/corrplot (accessed on 29 April 2020).

58. Gilmour, A.R.; Gogel, B.J.; Cullis, B.R.; Thompson, R. ASReml User Guide Release 4.1 Functional Specification, 4.1; HP1 1ES; VSN International Ltd.: Hemel Hempstead, UK, 2015; p. 67.

59. Smilde, A.; Jansen, J.; Hoefsloot, H.; Lamers, R.-J.; van Der Greef, J.; Timmerman, M. ANOVA-simultaneous component analysis (ASCA): A new tool for analyzing designed metabolomics data. Bioinformatics 2005, 21, 3043-3048. [CrossRef]

60. Chong, I.-G.; Jun, C.-H. Performance of some variable selection methods when multicollinearity is present. Chemom. Intellig. Lab. Syst. 2005, 78, 103-112. [CrossRef]

(C) 2020 by the authors. Licensee MDPI, Basel, Switzerland. This article is an open access article distributed under the terms and conditions of the Creative Commons Attribution (CC BY) license (http://creativecommons.org/licenses/by/4.0/). 
Article

\title{
A Tale of Two Biomarkers: Untargeted ${ }^{1} \mathrm{H}$ NMR Metabolomic Fingerprinting of BHBA and NEFA in Early Lactation Dairy Cows
}

\author{
Timothy D. W. Luke ${ }^{1,2}$, Jennie E. Pryce ${ }^{1,2}$, William J. Wales ${ }^{3,4}$ and Simone J. Rochfort ${ }^{1,2, *}$ \\ 1 Agriculture Victoria Research, AgriBio, Centre for AgriBioscience, Bundoora, VIC 3083, Australia; \\ tim.luke@agriculture.vic.gov.au (T.D.W.L.); jennie.pryce@agriculture.vic.gov.au (J.E.P.) \\ 2 School of Applied Systems Biology, La Trobe University, Bundoora, VIC 3083, Australia \\ 3 Agriculture Victoria Research, Ellinbank Centre, Ellinbank, VIC 3821, Australia; \\ bill.wales@agriculture.vic.gov.au \\ 4 Centre for Agricultural Innovation, School of Agriculture and Food, Faculty of Veterinary and Agricultural \\ Sciences, The University of Melbourne, Parkville, VIC 3010, Australia \\ * Correspondence: simone.rochfort@agriculture.vic.gov.au
}

Received: 21 May 2020; Accepted: 12 June 2020; Published: 15 June 2020

\begin{abstract}
Disorders of energy metabolism, which can result from a failure to adapt to the period of negative energy balance immediately after calving, have significant negative effects on the health, welfare and profitability of dairy cows. The most common biomarkers of energy balance in dairy cows are $\beta$-hydroxybutyrate (BHBA) and non-esterified fatty acids (NEFA). While elevated concentrations of these biomarkers are associated with similar negative health and production outcomes, the phenotypic and genetic correlations between them are weak. In this study, we used an untargeted ${ }^{1} \mathrm{H}$ NMR metabolomics approach to investigate the serum metabolomic fingerprints of BHBA and NEFA. Serum samples were collected from 298 cows in early lactation (calibration dataset $N=248$, validation $N=50$ ). Metabolomic fingerprinting was done by regressing ${ }^{1} \mathrm{H}$ NMR spectra against BHBA and NEFA concentrations (determined using colorimetric assays) using orthogonal partial least squares regression. Prediction accuracies were high for BHBA models, and moderately high for NEFA models ( $\mathrm{R}^{2}$ of external validation of 0.88 and 0.75 , respectively). We identified 16 metabolites that were significantly (variable importance of projection score $>1$ ) correlated with the concentration of one or both biomarkers. These metabolites were primarily intermediates of energy, phospholipid, and/or methyl donor metabolism. Of the significant metabolites identified; (1) two (acetate and creatine) were positively correlated with BHBA but negatively correlated with NEFA, (2) nine had similar associations with both BHBA and NEFA, (3) two were correlated with only BHBA concentration, and (4) three were only correlated with NEFA concentration. Overall, our results suggest that BHBA and NEFA are indicative of similar metabolic states in clinically healthy animals, but that several significant metabolic differences exist that help to explain the weak correlations between them. We also identified several metabolites that may be useful intermediate phenotypes in genomic selection for improved metabolic health.
\end{abstract}

Keywords: metabolic profile; ketosis; transition period; livestock; methyl donor; one-carbon metabolism; negative energy balance

\section{Introduction}

Most dairy cows experience a period of negative energy balance immediately after calving due to both a reduction in feed intake preceding calving [1], and an increase in energy requirements for milk production [2]. A successful transition from pregnancy to lactation requires a series of complex and 
coordinated changes in metabolism and nutrient partitioning, known as homeorhesis [3]. Failure of these homeorhetic controls can lead to the development of metabolic disorders such as ketosis and fatty liver [4]. These disorders can have significant negative effects on the health, welfare and profitability of early-lactation dairy cows due to their (1) relatively high incidence [5,6], (2) demonstrated association with other diseases $[4,7]$ and (3) their significant economic costs $[8,9]$.

Serum $\beta$-hydroxybutyrate (BHBA) and non-esterified fatty acids (NEFA) are biomarkers that are commonly used to evaluate the energy balance of dairy cows in the transition period $[6,10,11]$. One of the main physiological responses to reduced energy intake is the mobilization of stored energy from adipose tissue as NEFA. Serum NEFA concentration is a measure of the degree of lipolysis, and therefore an indicator of the magnitude of negative energy balance [12]. Once released, NEFA are transported via the bloodstream to the mammary gland for milk fat synthesis, or to the liver where they undergo either (1) complete oxidation via the TCA cycle, (2) partial oxidation to ketone bodies (BHBA, acetone and acetoacetate), or (3) re-esterification to form triglycerides which can either be stored or exported as very low density lipoprotein (VLDL). BHBA is the most stable of the three ketone bodies [13], and is commonly used as a biomarker of energy balance [14].

Mild elevations in serum BHBA and/or NEFA concentration during the transition period are considered normal [15], but marked elevations are indicative of excessive negative energy balance and/or perturbed metabolism [16]. Elevated concentrations of both BHBA and NEFA can be observed in clinically healthy animals (i.e., showing no visible signs of illness), and are associated with (1) reduced reproductive performance [11,17], (2) an increased incidence of clinical diseases such as displaced abomasa and metritis $[15,17,18]$, (3) decreased milk production $[6,11,19]$ and $(4)$ an increased risk of culling $[6,15,20]$. However, despite these similarities, both the phenotypic $[21,22]$ and genetic [23] correlations between these two biomarkers are low. This is not necessarily important if biomarkers are being used for management purposes (such as the identification of sick animals or the assessment of nutritional status) but may be significant if the biomarkers are used as phenotypes for genetic selection for improved animal health and resilience. There is therefore a need to better understand the metabolic states represented by BHBA and NEFA.

Untargeted metabolomics combines high throughput molecular analytical techniques such as proton nuclear magnetic resonance $\left({ }^{1} \mathrm{H}\right.$ NMR) spectroscopy with multivariate statistical modelling, to characterize the metabolic response of a biological system to pathophysiological stimuli [24]. Examples in dairy cattle include studies of ketosis [25,26], fatty liver [27], hypocalcaemia [28] and displaced abomasa [29]. The collective metabolic features of a given state or condition can be described as its "metabolomic fingerprint". As well improving our understanding of the biological processes, metabolomic studies can uncover intermediate molecular phenotypes (metabotypes) associated with complex animal health traits such as metabolic resilience. These metabotypes can then be integrated with genomic data to (1) elucidate the genetic architecture of these traits, and (2) improve genomic prediction accuracies $[30,31]$.

The aim of this study was therefore to use an untargeted ${ }^{1} \mathrm{H}$ NMR metabolomic approach to investigate the metabolomic fingerprints of serum BHBA and NEFA concentrations in clinical healthy dairy cows in early lactation, and in so doing (1) identify common and differential metabolic pathways, and (2) identify novel metabotypes for application to genetic selection for improved metabolic health.

\section{Results}

\subsection{Analysis of Experimental Metadata}

Descriptive statistics of the datasets used in this experiment are shown in Table 1. BHBA concentrations were significantly higher in Dataset 1 than in Dataset $2(p<0.001)$. The differences in all other parameters were not statistically significant $(p>0.05)$. The correlation between BHBA and NEFA concentrations was 0.45 in Dataset 1 and 0.40 in Dataset 2. 
Table 1. Descriptive statistics of the datasets used in this experiment, including number of animals (N), stage of lactation defined as days in milk (DIM), age in years, and $\beta$-hydroxybutyrate (BHBA) and non-esterified fatty acid (NEFA) concentrations $(\mathrm{mmol} / \mathrm{L})$ in the serum obtained from clinically healthy dairy cows.

\begin{tabular}{|c|c|c|c|c|c|c|c|}
\hline \multirow[b]{2}{*}{ Variable } & \multicolumn{3}{|c|}{ Dataset $1(N=248)$} & \multicolumn{3}{|c|}{ Dataset $2(N=50)$} & \multirow[b]{2}{*}{$p^{1}$} \\
\hline & Min & Max & $\begin{array}{c}\text { Mean } \\
\text { (SD) }\end{array}$ & Min & Max & $\begin{array}{c}\text { Mean } \\
\text { (SD) }\end{array}$ & \\
\hline DIM (days) & 4 & 30 & $16.7(6.0)$ & 4 & 30 & $18.6(7.3)$ & 0.09 \\
\hline Age (years) & 2 & 12 & $3.7(2.0)$ & 2 & 9 & $3.9(1.8)$ & 0.22 \\
\hline BHBA (mmol/L) & 0.22 & 1.86 & $0.55(0.21)$ & 0.23 & 0.94 & $0.42(0.17)$ & $<0.001$ \\
\hline NEFA (mmol/L) & 0.11 & 2.18 & $0.75(0.32)$ & 0.14 & 1.91 & $0.67(0.36)$ & 0.07 \\
\hline
\end{tabular}

${ }^{1}$ Statistical significance of the differences between Datasets 1 and 2 were determined using paired $t$-test for DIM, and a paired Wilcoxon signed-rank test for age, BHBA and NEFA.

\section{2. ${ }^{1} H$ NMR Spectra}

Twenty-four metabolites could be clearly identified from the ${ }^{1} \mathrm{H}$ NMR spectra. Two metabolites, cholate and 3-phenyllactate, were tentatively identified. Figure 1 shows representative spectra from animals in Dataset 1 with (a) elevated BHBA concentration, (b) elevated NEFA concentration and (c) normal BHBA and NEFA concentrations. Upfield regions of spectra were dominated by branched-chain amino acids (leucine, isoleucine and valine), organics acids (BHBA, lactate, acetate) and the methyl and methylene groups of low density (LDL) and very low density lipoproteins (VLDL) at $\delta 0.86 \mathrm{ppm}$ and $\delta 1.25 \mathrm{ppm}$, respectively [32]. We also observed a prominent peak at $\delta 2.03 \mathrm{ppm}$ which was consistent with the $\mathrm{N}$-acetyl groups of glycoproteins [33]. The singlet at $\delta 3.14 \mathrm{ppm}$ was identified as dimethyl sulfone $\left(\mathrm{DMSO}_{2}\right)[34,35]$. The middle of the spectrum was complex and dominated by glucose. Signal overlap and weak 2D signal strength meant that hippurate was the only compound that could be clearly identified in the downfield region. Relative chemical shifts and the multiplicity of identified peaks are available in the supplementary material (Table S1).

Unsupervised analysis of the data using PCA showed no obvious clustering of samples by dataset. Results of ANOVA-simultaneous component analysis showed that fixed effects (cow age, herd of origin and days in milk (DIM)) explained only $13.94 \%$ of the spectral variation (Table S2). Only the effect of age was statistically significant $(p<0.05)$. This suggests that most spectral variation is due to differences between individual animals. 


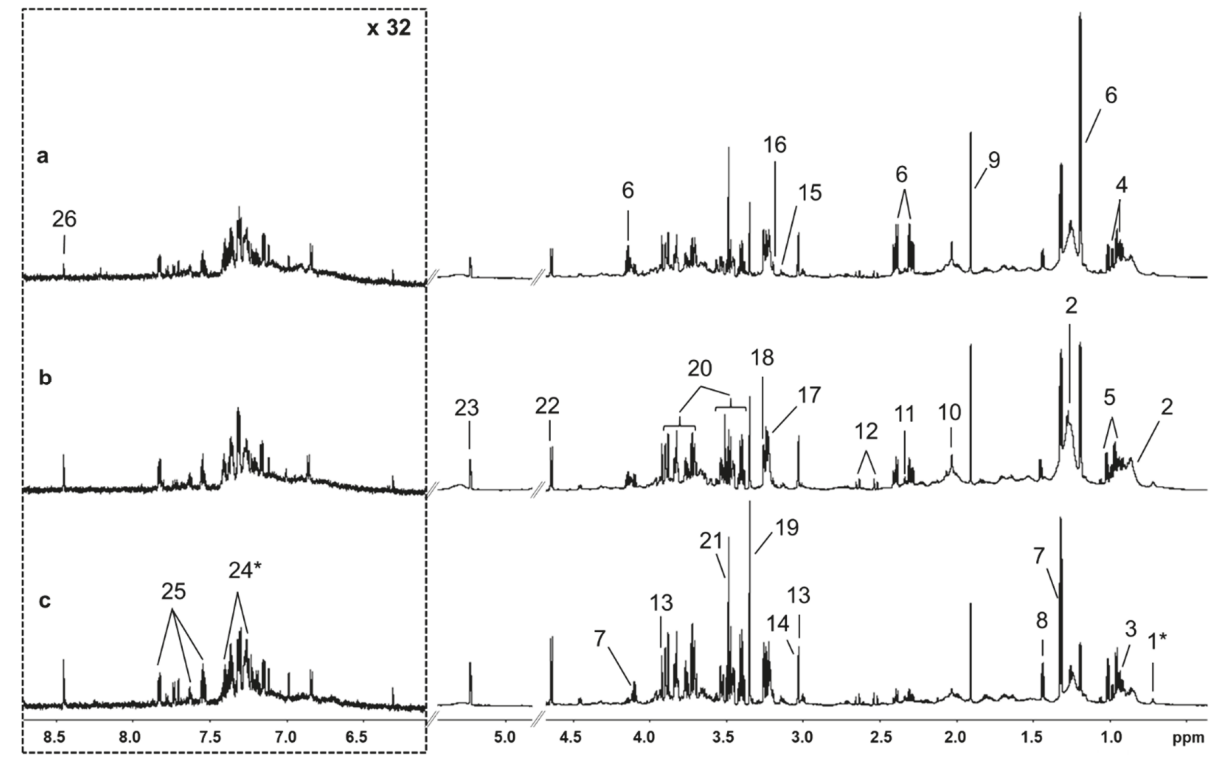

Figure 1. Representative $700 \mathrm{MHz}{ }^{1} \mathrm{H}$ nuclear magnetic resonance spectra of serum samples from early lactation dairy cows with (a) elevated $\beta$-hydroxybutyrate (BHBA), (b) elevated non-esterified fatty acid (NEFA), and (c) normal BHBA and NEFA concentrations. Downfield regions were vertically expanded 32 times for clarity. Legend: 1, cholate; 2, very low density lipoprotein/low density lipoprotein; 3 , leucine; 4 , isoleucine; 5 , valine; 6 , $\beta$-hydroxybutyrate; 7 , lactate; 8 , alanine; 9 , acetate; 10 , N-acetyl glycoprotein; 11 , pyruvate; 12 , citrate; 13 , creatine; 14 , creatine phosphate; 15 , dimethyl sulfone $\left(\mathrm{DMSO}_{2}\right) ; 16$, choline; 17 , phosphocholine; 18 , betaine; 19 , methanol; 20 , glucose; 21 , glycine; $22, \beta-G l u$; 23, $\alpha$-Glu; 24, 3-phenyllactate; 25, hippurate; 26; formate. * = tentative identification.

\subsection{Accuracy and Robustness of Prediction Models}

The robustness of the orthogonal partial least squares (OPLS) regression models built using data from Dataset 1 was assessed using (1) 10-fold cross-validation (Figure 2a,c) and (2) external validation with data from Dataset 2 (Figure $2 b$,d). Prediction accuracies derived from external validation were high for BHBA $\left(R^{2}=0.88\right)$, and moderately high for NEFA $\left(R^{2}=0.75\right)$. BHBA models were remarkably robust, with external validation $\mathrm{R}^{2}$ and RMSE results almost identical to cross-validation results. Models predicting serum NEFA concentration were less accurate than those predicting BHBA (NRMSE 0.32 and 0.50 , respectively), but external validation results indicated that these models were still quite robust. $p$-values derived from permutation testing were $<0.001$ for all models, indicating that models were not over-fitted. 


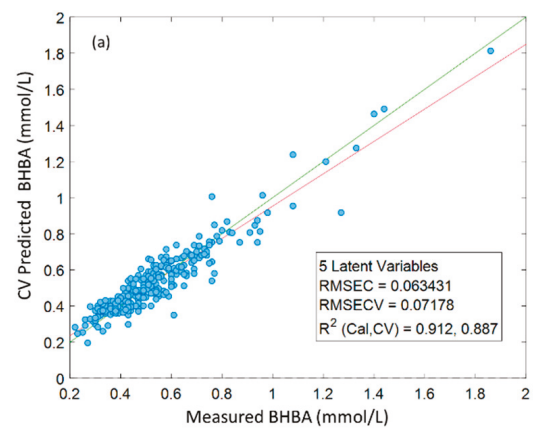

(a)

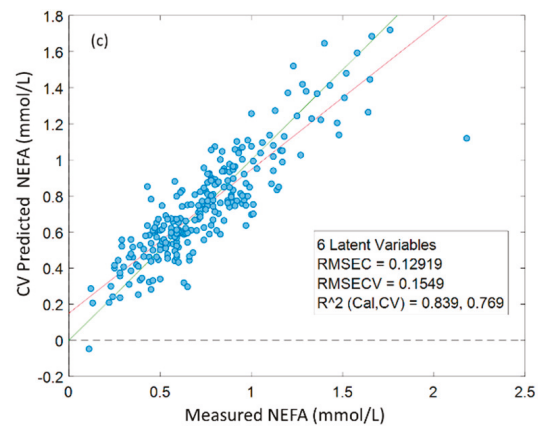

(c)

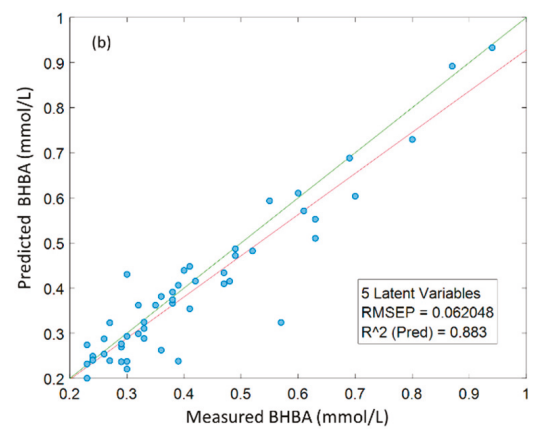

(b)

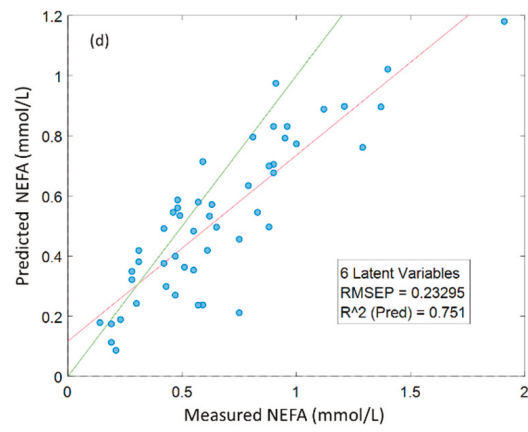

(d)

Figure 2. Accuracy of orthogonal partial least squares (OPLS) regression models predicting serum $\beta$-hydroxybutyrate (BHBA) and non-esterified fatty acid (NEFA) concentrations from ${ }^{1} \mathrm{H}$ NMR spectra, built using data from Dataset $1(N=248)$; (a) 10-fold cross-validation (CV)-predicted BHBA vs. measured BHBA; (b) external validation ( $N=50)$-predicted BHBA vs. actual BHBA; (c) CV-predicted NEFA vs. measured NEFA; (d) external validation-predicted NEFA vs measured NEFA.

\subsection{Metabolomic Fingerprints of BHBA and NEFA}

The metabolomic fingerprints associated with BHBA and NEFA were investigated using OPLS regression. Larger scores on the first latent variable (LV1) correspond to higher concentrations of both BHBA and NEFA (Figure 3a,b). LV1 loadings plots were used to identify which spectral features contributed most to the variation in the reference biomarker concentrations [36] (Figure 3c,d). Spectral features with positive loadings correspond to metabolites that are positively correlated with reference biomarker concentrations, and vice-versa. Peaks with a variable importance of projection (VIP) score greater than one were considered statistically significant [37] (Figure S2). 


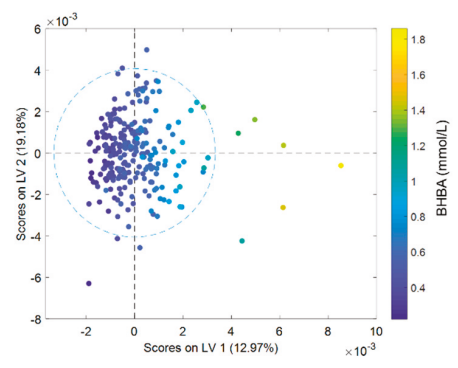

(a)

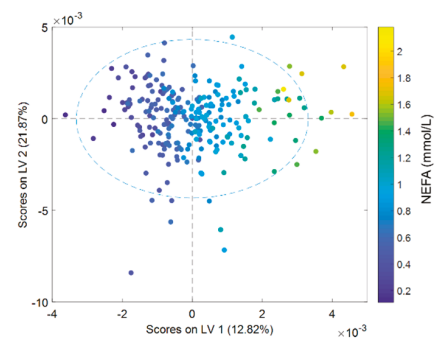

(b)

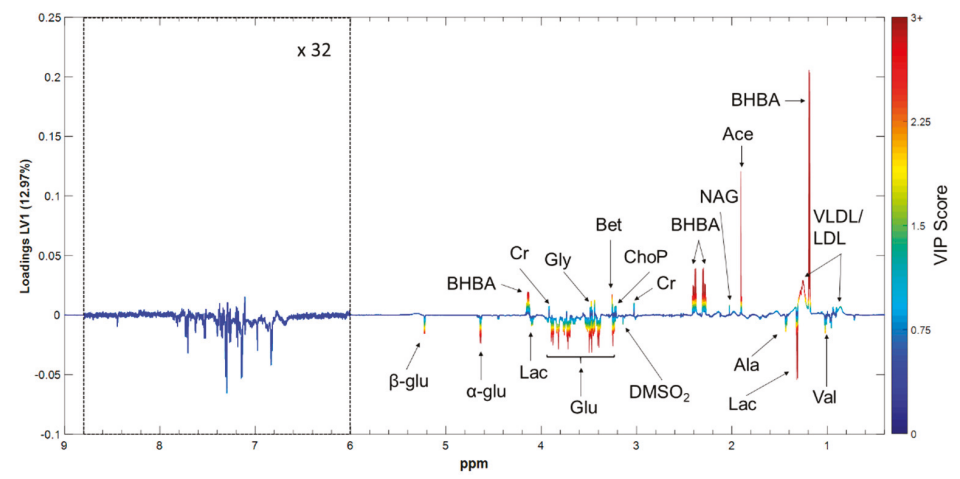

(c)

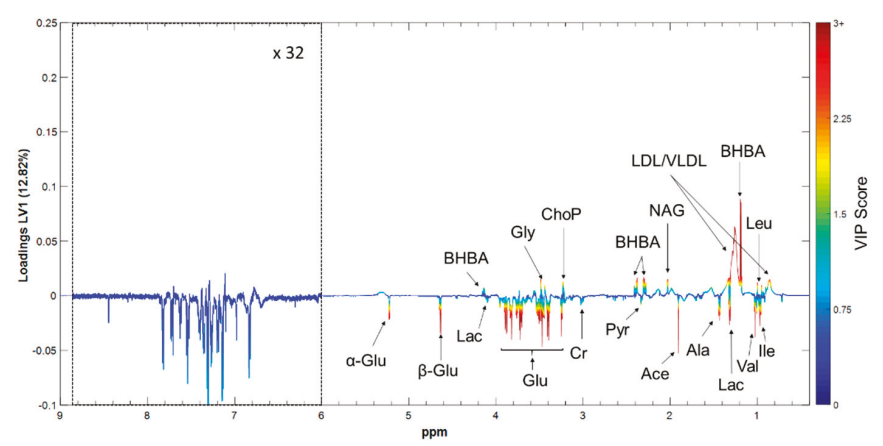

(d)

Figure 3. Results of the orthogonal partial least squares (OPLS) regression models predicting serum BHBA and NEFA concentrations from ${ }^{1} \mathrm{H}$ NMR spectra; (a) First latent variable (LV1) vs. second latent variable (LV2) scores for the BHBA prediction model; (b) LV1 vs. LV2 scores for the NEFA prediction model; (c) LV1 loadings for the BHBA prediction model; (d) LV1 loadings for the NEFA prediction model. Scores plots color-coded by reference biomarker concentration, loadings plots by VIP score. $\alpha$-Glu $=\alpha$ glucose, $\beta$-Glu $=\beta$ glucose, Ace $=$ acetate, Ala $=$ alanine, Bet $=$ betaine, $\mathrm{BHBA}=\beta$ hydroxybutyrate, $\mathrm{Cr}=$ creatine, $\mathrm{DMSO}_{2}=$ dimethyl sulfone, $\mathrm{Glu}=$ glucose, $\mathrm{Gly}=$ glycine, Ile $=$ isoleucine, $\mathrm{Lac}=$ lactate, $\mathrm{Leu}=$ leucine $\mathrm{NAG}=\mathrm{N}$-acetyl glycoprotein, $\mathrm{ChoP}=$ phosphocholine, Pyr $=$ pyruvate, $\mathrm{Val}=$ valine, $\mathrm{LDL}=$ low density lipoprotein; $\mathrm{VLDL}=$ very low density lipoprotein. 


\subsubsection{Commonalities in the Metabolomic Fingerprints of BHBA and NEFA}

The results of this study show that several metabolites showed similar co-variances with both BHBA and NEFA concentrations. The largest effect we observed was from peaks assigned to glucose, which were negatively correlated with both biomarkers. Other metabolites with common co-variances included lactate, valine and alanine (negatively correlated), and glycine and phosphocholine (positively correlated). Spectral regions attributed to lipoproteins (LDL and VLDL) and glycoproteins were positively correlated with both BHBA and NEFA concentrations.

\subsubsection{Differences between the Metabolomic Fingerprints of BHBA and NEFA}

Figure 4 highlights the differences we observed between the metabolomic fingerprints of BHBA and NEFA. Acetate and creatine were positively correlated with BHBA, and negatively correlated with NEFA. A small number of metabolites showed significant co-variance with only one of the biomarkers. BHBA concentration was positively correlated with betaine, and negatively correlated with dimethyl sulfone $\left(\mathrm{DMSO}_{2}\right)$, while NEFA concentration was positively correlated with isoleucine and negatively correlated with leucine.

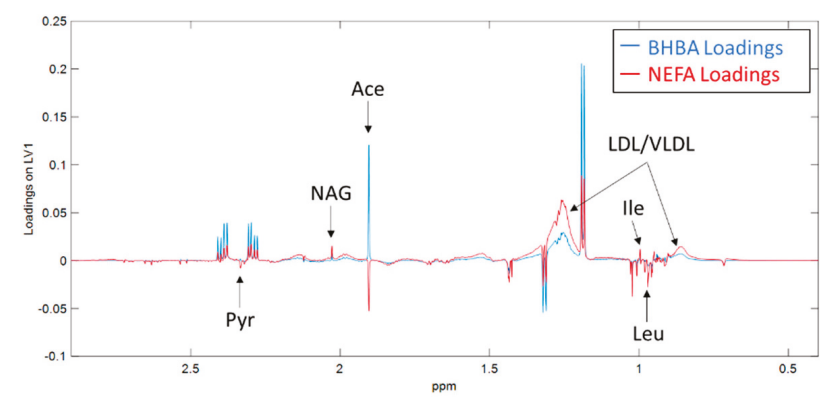

(a)

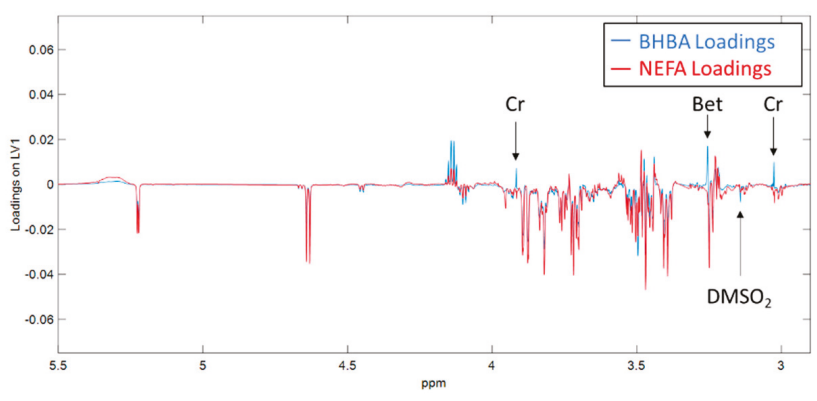

(b)

Figure 4. Loadings on the first latent variable (LV1) derived from orthogonal partial least squares (OPLS) regression of ${ }^{1} \mathrm{H}$ NMR spectra against serum BHBA (blue) and NEFA (red) concentrations in early lactation dairy cows. Spectral regions between (a) $\delta 0.2 \mathrm{ppm}$ to $2.9 \mathrm{ppm}$ and (b) $\delta 2.9 \mathrm{ppm}$ to $5.5 \mathrm{ppm}$ are shown. Figure $(\mathbf{b})$ has been for clarity purposes. Ace $=$ acetate, Bet $=$ betaine, $\mathrm{ChoP}=$ Phosphocholine, $\mathrm{Cr}=$ creatine, $\mathrm{DMSO}_{2}=$ dimethyl sulfone, Ile = isoleucine, Leu = leucine, LDL/VLDL = low/very low-density lipoprotein, NAG = N-acetyl glycoprotein, $\mathrm{Pyr}=$ pyruvate . 


\section{Discussion}

\subsection{Similarities between BHBA and NEFA}

Not surprisingly, many of the metabolites identified as having common co-variance with both BHBA and NEFA concentrations are involved in hepatic energy metabolism. These relationships are summarized in Figure 5. Most obvious was the negative relationship between both biomarkers and glucose. Hypoglycaemia has been widely reported in early lactation dairy cows due to the massive demand for glucose for lactogenesis [3,38]. More recently, NMR metabolomics studies have identified serum glucose concentration as being (1) directly correlated to energy balance $(r=0.84)$ [39], and (2) lower in cows with clinical and subclinical ketosis [25] and fatty liver [27] when compared to healthy controls. Our results offer further evidence of the pivotal role glucose plays in the early lactation metabolic health in dairy cows.

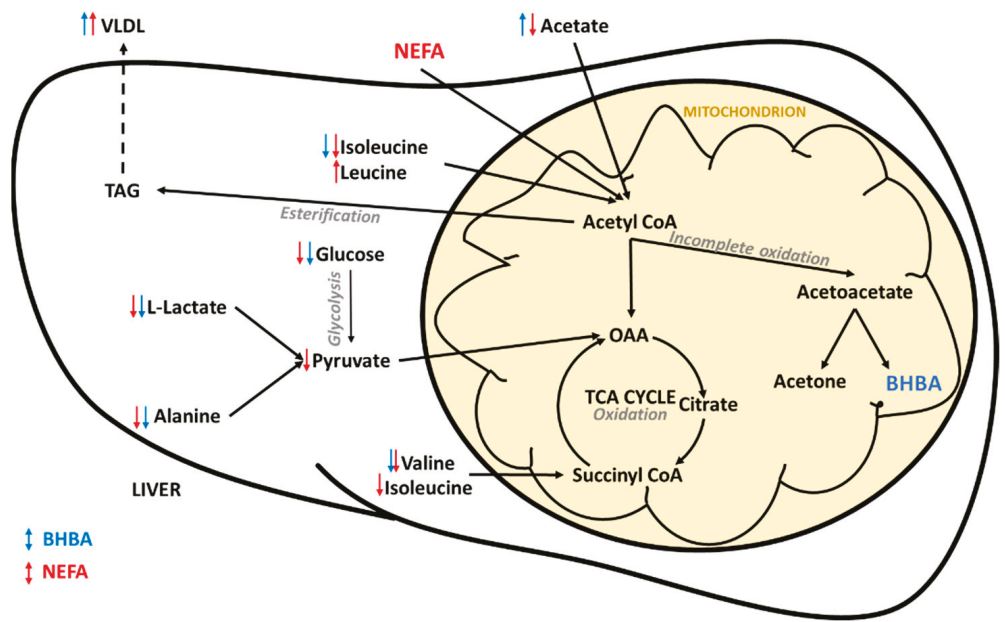

Figure 5. Summary of hepatic energy metabolism in early lactation dairy cows. Arrows indicate the direction of the relationship between the metabolites and the reference BHBA (blue) and non-esterified fatty acid (NEFA) (red) concentrations. BHBA = $\beta$-hydroxybutyrate; OAA = oxaloacetate; $\mathrm{TAG}=$ triglyceride, $\mathrm{TCA}=$ tricarboxylic acid, $\mathrm{VLDL}=$ very low density lipoprotein.

Lactate and alanine, important gluconeogenic substrates in ruminants [40,41], were also negatively associated with both BHBA and NEFA, as was valine (another gluconeogenic amino acid). Interestingly, $\mathrm{Xu}$ et al. [39] found no correlation between calculated energy balance in early lactation dairy cows and the concentrations of any of the branched-chain amino acids or lactate. Conversely, when compared to healthy controls, cows with fatty liver and displaced abomasa have been shown to have lower serum alanine concentrations [27,29], and cows with ketosis have lower lactate and alanine concentrations $[25,42]$. This suggests that alterations in glucogenic precursors, in particular lactate and alanine, are indicative of a perturbed metabolism, not simply negative energy balance. We previously showed that lactate concentration in pasture-fed dairy cows is heavily influenced by herd-specific management factors [43], and as such may not be heavily influenced by genetic factors. Alanine has been shown to be the most important glucogenic amino acid, and the most important gluconeogenic precursor after lactate and propionate, in dairy cows [41]. Therefore, genetic selection for cows with higher serum concentrations of alanine in early lactation may help to increase endogenous glucose supply.

Spectral features attributed to VLDL and LDL were positively correlated with the concentrations of both BHBA and NEFA. These results need to be interpreted with caution as the methanol extraction 
used in this study removed much of the protein from the samples and may have introduced experimental artefacts. Interestingly, ${ }^{1} \mathrm{H}$ NMR spectroscopy has recently been shown capable of providing high-throughput and accurate quantification of lipoprotein subclasses in human serum and plasma samples [32,44]. It is important to note that these protocols used different pulse sequences and involved the dilution of plasma/serum in a deuterated water/phosphate buffer solution without any metabolite extraction, such as the one used in our study. The findings of these studies cannot, therefore, be applied directly to our results. However, lipoprotein metabolism is central to early lactation health in dairy cows, and impaired VLDL production in the liver can result in hepatic triglyceride (TAG) accumulation (Figure 4) and the development of fatty liver [45]. Dyslipoproteinaemia is also an important feature of metabolic syndrome in humans, and the quantification of lipoprotein subclasses is considered critical to the better understanding of this disease [44]. We believe that the investigation of serum lipoproteins using ${ }^{1} \mathrm{H}$ NMR spectroscopy holds great promise in the research of early lactation metabolic health in dairy cows, and we plan to validate the aforementioned protocols on bovine serum and plasma samples.

The region of the spectrum associated with glycoproteins was also significantly positively correlated with both NEFA and BHBA concentrations. Glycoproteins are acute phase proteins which can be used as indicators of inflammation in cattle [46]. In dairy cattle, increased serum NEFA concentrations in early lactation are associated with uncontrolled inflammation, and this inflammatory dysfunction is hypothesized to be a central link between metabolic and infectious disorders [14,47]. ${ }^{1} \mathrm{H}$ NMR spectroscopy is showing promise for the quantification of glycoprotein A (GlcA) in human research into metabolic diseases such obesity, diabetes mellitus and the metabolic syndrome [33]. Given that these syndromes have much in common with early lactation metabolic disease in dairy cows (e.g., insulin resistance), we believe that further research into GlcA as a biomarker for early lactation health is warranted. Overall, our results offer further evidence that inflammation plays an important role in early lactation metabolic health of dairy cows.

Glycine was positively correlated with the concentrations of both BHBA and NEFA. Metabolomics studies comparing healthy and ketotic dairy cows have reported (1) no change in glycine concentrations [25], (2) increased glycine concentrations in cows with sub-clinical ketosis [26], (3) increased glycine concentrations in cows with clinical ketosis [48] and (4) decreased glycine concentrations in cows with clinical ketosis [26] and fatty liver [49]. Glycine concentration has also been shown to increase in response to lipolysis [50]. These differing results suggest that changes in glycine concentration may be dependent on the severity of the metabolic disorder (i.e., increased in mild cases, and decreased in more severe cases). Most interesting are the findings of a recent metabolomics study that showed that glycine concentrations in plasma and milk were strongly negatively correlated with energy balance in early lactation dairy cows ( $r=-0.80$ and $r=-0.79$, respectively) [39]. The authors of this study hypothesized that this relationship was due to an increase in one-carbon or methyl donor metabolism, specifically an increase in the conversion of choline to glycine. Given that all cows in our study were clinically healthy, our results are consistent with glycine being an indicator of negative energy balance, lipolysis, and/or sub-clinical ketosis. Further work is required to better understand the role of glycine metabolism in clinical metabolic disease.

The positive correlations between phosphocholine and both BHBA and NEFA concentrations, and between betaine and BHBA concentration, are consistent with an increase in methyl donor metabolism in cows experiencing negative energy balance. Methyl donor metabolism and nutrition are receiving a great deal of attention in dairy science due to links with early-lactation cow health (including fatty liver), milk production and immune function [51]. Betaine, phosphocholine and glycine are intermediates in several important one-carbon metabolic pathways including the folate and methionine cycles, and the cytidine diphosphate (CDP)-choline pathway [51] (Figure 6a). The positive correlation between NEFA and phosphocholine may be due to increased breakdown of phosphatidylcholine (Figure 6a). This is consistent with the findings of Imhasly et al. [52] who showed that serum concentrations of lyso-phosphatidylcholines and phosphatidylcholines increase in response to negative energy balance 
in post-partum dairy cows. The positive association observed between betaine and BHBA could be due to increased oxidation of choline. A detailed description of these pathways is beyond the scope of this study, however our results suggest that methyl donor metabolism has an important influence on both BHBA and NEFA concentrations in early-lactation dairy cows.

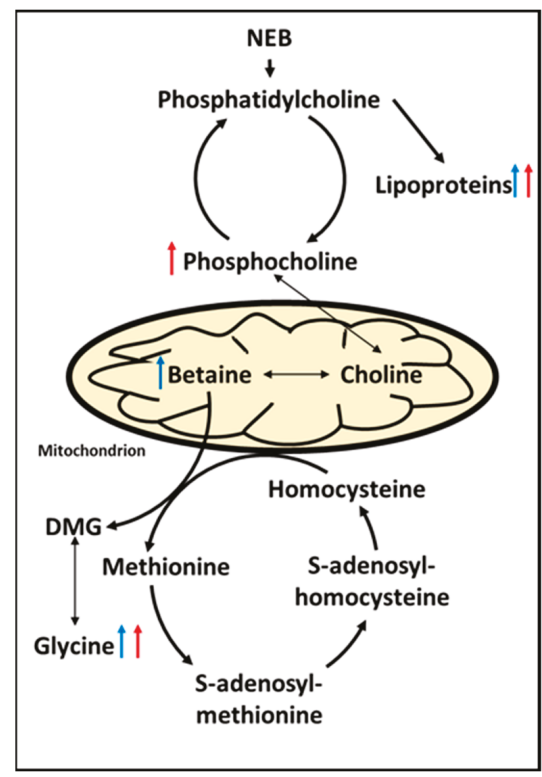

(a)

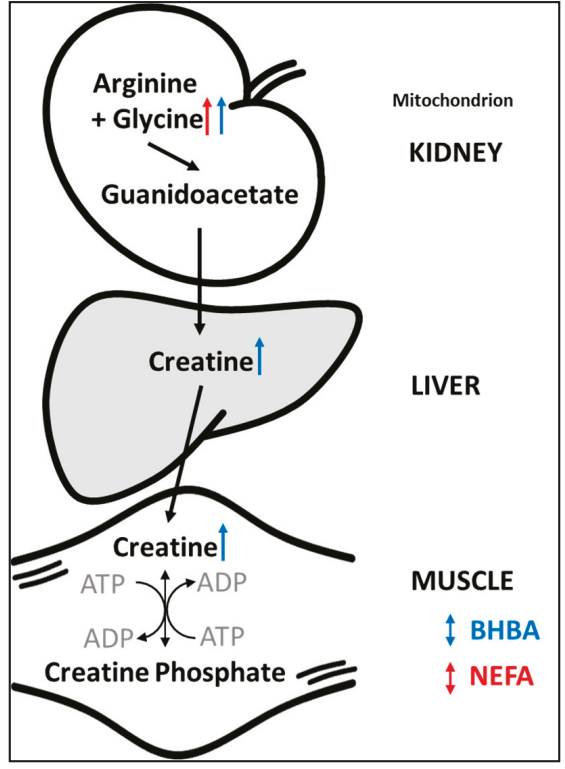

(b)

Figure 6. Summary of (a) phospholipid and one-carbon/methyl donor metabolism [53,54], and (b) creatine metabolism in early lactation dairy cows. Arrows indicate the direction of the relationship between the metabolites identified using untargeted ${ }^{1} \mathrm{H}$ NMR metabolomics, and reference BHBA (blue) and non-esterified fatty acids (NEFA) (red) concentrations. ADP = adenosine diphosphate; $\mathrm{ATP}=$ adenosine triphosphate; $\mathrm{DMG}=$ dimethylglycine; NEB = negative energy balance.

\subsection{Differences between $B H B A$ and NEFA}

Despite many similarities, we observed some significant differences between the metabolomic fingerprints of BHBA and NEFA. Most obvious was the difference in the direction of correlation between acetate and the two biomarkers. Acetate is a volatile fatty acid produced by microbial fermentation of feedstuffs in the rumen, and is an important energy source [55] (via oxidation or the partial oxidation of acetyl-CoA in the liver) and substrate for de novo milk fat synthesis [56] in cows. The negative relationship we observed between acetate and NEFA is consistent with the findings of Bielak et al. [57], who reported a negative correlation $(r=0.44)$ between plasma NEFA and acetate concentrations in early lactation dairy cows, possibly due to the down-regulation of the active transport of acetate across the rumen wall. The positive association between acetate and BHBA is consistent with previously discussed metabolomic studies of ketosis and fatty liver $[25,27]$. These results suggest that differences in acetate metabolism may help to explain the weak correlation between serum BHBA and NEFA concentrations in early lactation dairy cows.

The positive correlation between creatine and BHBA concentration is consistent with previous reports that creatine is a potentially useful biomarker of ketosis and severe energy deficiency in dairy cows $[25,26,39]$. Creatine is an important intermediate in energy metabolism, and this result may represent increased breakdown of creatine phosphate in skeletal muscle and the release of high-energy 
phosphate for the conversion of adenosine diphosphate (ADP) to adenosine triphosphate (ATP) (Figure 6b). Interestingly, creatine concentration was negatively correlated with NEFA concentration (albeit weakly and non-significantly $(\mathrm{VIP}<1)$ ). That mobilization of energy from skeletal muscle is a feature of the BHBA metabolomic fingerprint, but not that of NEFA, suggests that elevated BHBA concentrations are indicative of a more severe energy deficiency than are elevated NEFA concentrations. However, the ability to rapidly mobilize energy from skeletal muscle may be advantageous to early-lactation dairy cows, and we believe the role of creatine metabolism in transition cow health warrants further investigation. We therefore plan to undertake genome-wide association studies to better understand the genetic relationships between hepatic and skeletal muscle energy metabolism.

The significant negative correlation between $\mathrm{DMSO}_{2}$ and $\mathrm{BHBA}$ concentration was an interesting finding of this study. $\mathrm{DMSO}_{2}$ concentration in the milk and rumen fluid of dairy cows has been shown to vary according to feeding system; higher in pasture-fed cows managed outdoors than in cows fed a total mixed ration indoors [58]. Maher et al. [59] showed that the concentrations of $\mathrm{DMSO}_{2}$ in milk and plasma are highly correlated $(\mathrm{r}=0.69)$, so serum $\mathrm{DMSO}_{2}$ may also be an indicator of pasture intake. Given that all animals in this experiment were fed pasture, the negative association we observed between $\mathrm{DMSO}_{2}$ and $\mathrm{BHBA}$ concentration may indicate that hyperketonemic cows are consuming less feed.

\section{Materials and Methods}

All procedures undertaken in this study were conducted in accordance with the Australian Code of Practice for the Care and Use of Animals for Scientific Purposes (National Health and Medical Research Council, 2013). Approval to proceed was granted by the Agricultural Research and Extension Animal Ethics Committee of the Department of Jobs, Precincts and Regions Animal Ethics Committee (DJPR, 475 Mickleham Road, Attwood, Victoria 3049, Australia), and the Tasmanian Department of Primary Industries, Parks, Water and Environment (DPIPWE Animal Biosecurity and Welfare Branch, 13 St Johns Avenue, New Town, Tasmania 7008, Australia). AEC project approval code 2017-05.

\subsection{Animals and Datasets}

A total of 298 Holstein-Friesian cows were used in this experiment. The calibration dataset (Dataset 1) was collected between August and September 2017 from 248 animals located at the Ellinbank Dairy Research Centre, Ellinbank, Victoria, Australia. An independent validation dataset (Dataset 2) was collected in September 2018, from 50 cows located on a commercial dairy farm in Smithton, Tasmania, Australia. All cows were clinically healthy, and had been calved for between 4 and 30 days at the time of sampling. Feeding systems on Australian dairy farms are diverse but can be classified into 5 main feeding systems [60]. Both farms operated under feeding system 2; grazed pasture plus moderate to high level concentrate feeding ( $>1.0$ tonne of concentrate fed in the milking parlour per cow per year).

\subsection{Blood Sample Collection and Reference Biomarker Measurements}

A single serum sample was taken from each cow immediately after morning milking (approximately 07:00) according to the protocol described in Luke et al. [43]. Cows were fed their concentrate ration as soon as they entered the milking parlour, meaning that samples were collected approximately $10 \mathrm{~min}$ after grain feeding.

An aliquot of each serum sample was shipped on ice to Regional Laboratory Services (Benalla, Victoria, Australia) for BHBA and NEFA analyses. Colorimetric assays were performed using a Kone 20 XT clinical chemistry analyser (Thermo Fisher Scientific, Waltham, MA, USA); an enzymatic kinetic assay for BHBA (McMurray et al., 1984) and enzymatic end point assay for NEFA (Randox Laboratories, Crumlin, UK). The uncertainty of measurement (at a $95 \%$ confidence level) was $\pm 0.060 \mathrm{mmol} / \mathrm{L}$ at $0.85 \mathrm{mmol} / \mathrm{L}$ for BHBA, and $\pm 0.031 \mathrm{mM}$ at $1.45 \mathrm{mM}$ for NEFA. A second aliquot was stored at $-20^{\circ} \mathrm{C}$ until processing for NMR spectroscopy. 


\subsection{Sample Preparation for NMR Spectroscopy}

Details of the sample preparation and metabolite extraction protocols used in this study can be found in Luke et al. [43]. Briefly, $300 \mu \mathrm{L}$ of serum was (1) mixed with $600 \mu \mathrm{L}$ of methanol, (2) vortexed, (3) incubated at $-20{ }^{\circ} \mathrm{C}$ for $20 \mathrm{~min}$, and (4) centrifuged at $11,360 \times g$ at $21{ }^{\circ} \mathrm{C}$ for $30 \mathrm{~min}$ to pellet proteins. A $600 \mu \mathrm{L}$ aliquot of the supernatant was then transferred to a clean $2 \mathrm{~mL}$ microcentrifuge tube, dried under vacuum at $21^{\circ} \mathrm{C}$ overnight using a SpeedVac Savant SPD 2010 Concentrator (Thermo Fisher Scientific, Waltham, MA, USA) then reconstituted in a $\mathrm{D}_{2} \mathrm{O}$ phosphate buffer solution (100 mM $\mathrm{K}_{2} \mathrm{HPO}_{4}$ ) containing $0.25 \mathrm{mM}$ DSS-d6 as an internal standard. A $550 \mu \mathrm{L}$ aliquot of reconstituted extract was transferred to a $5 \mathrm{~mm}$ NMR tube for analysis.

\section{4. ${ }^{1}$ H NMR Data Acquisition and Pre-Processing}

One-dimensional proton spectra were acquired using a Bruker Ascend $700 \mathrm{MHz}$ spectrometer equipped with cryoprobe and SampleJet automatic sample changer (Bruker Biospin, Rheinstetten, Germany). A Bruker noesypr1d pulse sequence was used over a $-0.76-10.32 \mathrm{ppm}$ spectral range with the following acquisition parameters; (1) a temperature of $298 \mathrm{~K}$, (2) 256 scans after eight dummy scans (3) acquisition time per increment of $2.11 \mathrm{~s}$, and (4) relaxation delay (D1) of $2.00 \mathrm{~s}$. This resulted in 32,768 data points. A line broadening of $0.3 \mathrm{~Hz}$ was applied to all spectra prior to Fourier transformation. Spectra were manually phased, baseline corrected and referenced to the internal standard (DSS-d6) at $\delta 0.00$ ppm using the Topspin v.3.6.1 software (Bruker Biospin, Rheinstetten, Germany).

Data pre-processing was performed in MatLab v.R20017b (Mathworks, Natick, MA, USA). Spectra were imported as a matrix of signal intensities using the ProMetab v.1.1 script [61]. Spectral pre-processing involved (1) deletion of the residual water peak region $(\delta 4.68-5.00 \mathrm{ppm})$, (2) spectral alignment using the correlation optimized warping algorithm [62], (3) normalization to total signal area (area $=1),(4)$ deletion of methanol $(\delta 3.32-3.36 \mathrm{ppm})$ and DSS-d6 $(\delta 0.4-0.60 \mathrm{ppm})$ peak regions, and the non-informative region beyond $9.00 \mathrm{ppm},(5)$ baseline correction using automatic weighted least squares and (6) mean centering.

\subsection{Statistical Analysis}

Statistical analysis of experimental metadata was performed in R v3.6.2 [63]. Differences between the 2 datasets were analysed using a paired $t$-test or a Wilcoxon signed-rank test depending on the normality of the data.

Multivariate statistical analyses were performed using the PLS Toolbox v. 8.5.2 (Eigenvector Research Inc., Manson, WA, USA). Preliminary data analysis and outlier detection was performed using an unsupervised PCA. Examination of PC1 vs. PC2 scores plot showed 14 samples from Dataset 1 outside the 95\% confidence level ellipse (Figure S1). These samples were individually examined, and a single spectrum with poor water suppression and baseline correction was removed from subsequent analyses. The influences of fixed effects (DIM, age and herd) on spectra were investigated using ANOVA simultaneous component analysis with 1000 permutations [64]. Untargeted metabolomic fingerprinting was done by regressing reference NEFA and BHBA concentrations against ${ }^{1} \mathrm{H}$ NMR spectra using supervised OPLS regression. Variable importance of projection (VIP) scores for the first latent variable were used to identify the most statistically significant peaks in each model. Peaks of interest were identified using the Chenomx NMR suite software v.8.4 (Chenomx Inc., Edmonton, AB, Canada), comparison to the literature, 2D NMR analysis (COSY, gHMBC and gHSQC), and statistical total correlation spectroscopy [65].

OPLS models were constructed using data from Dataset 1 . The robustness of models was assessed using (1) cross-validation using a venetian blind technique (10 sample splits with 1 sample per blind) and (2) external validation using data from Dataset 2. The prediction accuracy of OPLS models was assessed using the coefficient of determination $\left(\mathrm{R}^{2}\right)$ and root mean square error (RMSE). Normalized 
RMSE (NRMSE) values, calculated as external validation RMSE divided by the interquartile interval of the observed data, were used to compare RMSE estimates for NEFA and BHBA predictions. Permutation testing (50 iterations and statistical significance determined using a Wilcoxon signed-rank test) was performed to ensure that models were not over-fitted.

\section{Conclusions}

In this study we used an untargeted ${ }^{1} \mathrm{H}$ NMR metabolomics approach to investigate the serum metabolic fingerprints of the two most common biomarkers of energy balance in dairy cows, BHBA and NEFA. Our results suggest that while BHBA and NEFA are indicative of similar metabolic states in early-lactation dairy cows, there are significant differences between the two biomarkers. Metabolites with common co-variances were intermediates of energy, phospholipid, and methyl donor metabolism. The most significant differences in the metabolomic fingerprints were related to acetate and creatine metabolism. We also identified several intermediate metabotypes which, when combined with genomic data, will enable further the investigation of the genetic architecture of metabolic health in early lactation dairy cows.

Supplementary Materials: The following are available online at http://www.mdpi.com/2218-1989/10/6/247/s1, Table S1: ${ }^{1} \mathrm{H}$ NMR chemical shifts $(\delta)$ and multiplicity of metabolites in bovine serum run in deuterated water $\left(\mathrm{D}_{2} \mathrm{O}\right)$. Clearly observed resonances are indicated in bold text. s, singlet; d, doublet; dd, doublet of a doublet; m, multiplet; $t$, triplet. The right two columns show the direction of the relationship with serum $\beta$-hydroxybutyrate (BHBA) and non-esterified fatty acid (NEFA) concentrations determined by colorimetric assays, Table S2: Results of ANOVA-simultaneous component analysis (ASCA) of ${ }^{1} \mathrm{H}$ NMR spectra of bovine serum $(N=298)$. Effect describes the relative influence of each variable (herd, age and days in milk (DIM)) on spectra. $p$-value is derived from permutation testing (1000 iterations), Figure S1: Results of PCA of ${ }^{1} \mathrm{H}$ NMR spectra of serum obtained from 298 dairy cows in early lactation from the Ellinbank research farm (Dataset $1, N=248$ ) and a commercial dairy farm in Tasmania (Dataset 2, $N=50$ ), Figure S2: VIP scores from OPLS regressions of ${ }^{1} \mathrm{H}$ NMR spectra of serum obtained from 298 dairy cows in early lactation against (a) BHBA concentration and (b) NEFA concentration.

Author Contributions: Conceptualization, T.D.W.L., J.E.P., W.J.W., and S.J.R.; formal analysis, T.D.W.L. and S.J.R.; funding acquisition, J.E.P.; investigation, T.D.W.L. and S.J.R.; methodology, T.D.W.L. and S.J.R.; project administration, J.E.P., W.J.W. and S.J.R.; resources, S.J.R.; supervision, J.E.P., W.J.W., and S.J.R.; visualization, T.D.W.L.; writing—original draft, T.D.W.L.; writing-review and editing, T.D.W.L, J.E.P., W.J.W., and S.J.R. All authors have read and agreed to the published version of the manuscript.

Funding: DairyBio: jointly funded by Dairy Australia (Melbourne, Australia), the Gardiner Foundation (Melbourne, Australia), and Agriculture Victoria (Melbourne, Australia) funded this study and T.D.W.L.'s PhD project.

Acknowledgments: The authors thank Aaron Elkins and Simone Vassiliadis for assistance with ${ }^{1} \mathrm{H}$ NMR protocol and metabolite identification, Di Mapleson, Brigid Ribaux and the staff at Ellinbank Dairy Research Centre (Ellinbank, Australia) for their technical expertise and assistance, Erika Oakes and the staff at Datagene (Bundoora, Australia) for their work coordinating this study, and the farmers who took part in this project.

Conflicts of Interest: The authors declare no conflict of interest.

\section{References}

1. Grant, R.J.; Albright, J.L. Feeding behavior and management factors during the transition period in dairy cattle. J. Anim. Sci. 1995, 73, 2791-2803. [CrossRef] [PubMed]

2. Bell, A.W. Regulation of organic nutrient metabolism during transition from late pregnancy to early lactation. J. Anim. Sci. 1995, 73, 2804-2819. [CrossRef] [PubMed]

3. Bauman, D.E.; Bruce Currie, W. Partitioning of Nutrients During Pregnancy and Lactation: A Review of Mechanisms Involving Homeostasis and Homeorhesis. J. Dairy Sci. 1980, 63, 1514-1529. [CrossRef]

4. Herdt, T.H. Ruminant Adaptation to Negative Energy Balance: Influences on the Etiology of Ketosis and Fatty Liver. Vet. Clin. N. Am. Food Anim. Pract. 2000, 16, 215-230. [CrossRef]

5. Compton, C.; McDougall, S.; Young, L.; Bryan, M. Prevalence of subclinical ketosis in mainly pasture-grazed dairy cows in New Zealand in early lactation. N. Z. Vet. J. 2013, 62, 30-37. [CrossRef]

6. McArt, J.A.A.; Nydam, D.V.; Oetzel, G.R. Epidemiology of subclinical ketosis in early lactation dairy cattle. J. Dairy Sci. 2012, 95, 5056-5066. [CrossRef] 
7. Cameron, R.E.B.; Dyk, P.B.; Herdt, T.H.; Kaneene, J.B.; Miller, R.; Bucholtz, H.F.; Liesman, J.S.; Vandehaar, M.J.; Emery, R.S. Dry Cow Diet, Management, and Energy Balance as Risk Factors for Displaced Abomasum in High Producing Dairy Herds. J. Dairy Sci. 1998, 81, 132-139. [CrossRef]

8. Drackley, J.K. Biology of Dairy Cows During the Transition Period: The Final Frontier? J. Dairy Sci. 1999, 82, 2259-2273. [CrossRef]

9. McArt, J.A.A.; Nydam, D.V.; Overton, M.W. Hyperketonemia in early lactation dairy cattle: A deterministic estimate of component and total cost per case. J. Dairy Sci. 2015, 98, 2043-2054. [CrossRef]

10. Chapinal, N.; Leblanc, S.J.; Carson, M.E.; Leslie, K.E.; Godden, S.; Capel, M.; Santos, J.E.; Overton, M.W.; Duffield, T.F. Herd-level association of serum metabolites in the transition period with disease, milk production, and early lactation reproductive performance. J. Dairy Sci. 2012, 95, 5676-5682. [CrossRef]

11. Ospina, P.; Nydam, D.; Stokol, T.; Overton, T. Associations of elevated nonesterified fatty acids and $\beta$-hydroxybutyrate concentrations with early lactation reproductive performance and milk production in transition dairy cattle in the northeastern United States. J. Dairy Sci. 2010, 93, 1596-1601. [CrossRef] [PubMed]

12. Adewuyi, A.A.; Gruys, E.; Van Eerdenburg, F.J.C.M. Non esterified fatty acids (NEFA) in dairy cattle. A review. Vet. Q. 2005, 27, 117-126. [CrossRef] [PubMed]

13. Laffel, L. Ketone bodies: A review of physiology, pathophysiology and application of monitoring to diabetes. Diabetes Metab. Res. Rev. 1999, 15, 412-426. [CrossRef]

14. Sordillo, L.M.; Raphael, W. Significance of Metabolic Stress, Lipid Mobilization, and Inflammation on Transition Cow Disorders. Vet. Clin. N. Am. Food Anim. Pract. 2013, 29, 267-278. [CrossRef] [PubMed]

15. Ospina, P.A.; Nydam, D.V.; Stokol, T.; Overton, T.R. Evaluation of nonesterified fatty acids and $\beta$-hydroxybutyrate in transition dairy cattle in the northeastern United States: Critical thresholds for prediction of clinical diseases. J. Dairy Sci. 2010, 93, 546-554. [CrossRef]

16. McArt, J.A.A.; Nydam, D.V.; Oetzel, G.R.; Overton, T.R.; Ospina, P.A. Elevated non-esterified fatty acids and $\beta$-hydroxybutyrate and their association with transition dairy cow performance. Vet. J. 2013, 198, 560-570. [CrossRef] [PubMed]

17. Compton, C.; Young, L.; McDougall, S. Subclinical ketosis in post-partum dairy cows fed a predominantly pasture-based diet: Defining cut-points for diagnosis using concentrations of beta-hydroxybutyrate in blood and determining prevalence. N. Z. Vet. J. 2014, 63, 241-248. [CrossRef]

18. LeBlanc, S.J.; Leslie, K.E.; Duffield, T.F. Metabolic predictors of displaced abomasum in dairy cattle. J. Dairy Sci. 2005, 88, 159-170. [CrossRef]

19. Duffield, T.F.; Lissemore, K.D.; McBride, B.W.; Leslie, K.E. Impact of hyperketonemia in early lactation dairy cows on health and production. J. Dairy Sci. 2009, 92, 571-580. [CrossRef]

20. Seifi, H.A.; Leblanc, S.J.; Leslie, K.E.; Duffield, T.F. Metabolic predictors of post-partum disease and culling risk in dairy cattle. Vet. J. 2011, 188, 216-220. [CrossRef]

21. Luke, T.D.W.; Rochfort, S.; Wales, W.J.; Bonfatti, V.; Marett, L.; Pryce, J.E. Metabolic profiling of early-lactation dairy cows using milk mid-infrared spectra. J. Dairy Sci. 2019, 102, 1747-1760. [CrossRef] [PubMed]

22. McCarthy, M.M.; Mann, S.; Nydam, D.V.; Overton, T.R.; McArt, J.A.A. Short communication: Concentrations of nonesterified fatty acids and $\beta$-hydroxybutyrate in dairy cows are not well correlated during the transition period. J. Dairy Sci. 2015, 98, 6284-6290. [CrossRef]

23. Luke, T.D.W.; Nguyen, T.T.T.; Rochfort, S.; Wales, W.J.; Richardson, C.M.; Abdelsayed, M.; Pryce, J.E. Genomic prediction of serum biomarkers of health in early lactation. J. Dairy Sci. 2019, 102, 11142-11152. [CrossRef]

24. Nicholson, J.K.; Lindon, J.C.; Holmes, E. 'Metabonomics': Understanding the metabolic responses of living systems to pathophysiological stimuli via multivariate statistical analysis of biological NMR spectroscopic data. Xenobiotica 1999, 29, 1181-1189. [CrossRef] [PubMed]

25. Sun, L.W.; Zhang, H.Y.; Wu, L.; Shu, S.; Xia, C.; Xu, C.; Zheng, J.S. 1H-Nuclear magnetic resonance-based plasma metabolic profiling of dairy cows with clinical and subclinical ketosis. J. Dairy Sci. 2014, 97, 1552-1562. [CrossRef] [PubMed]

26. Wang, Y.; Gao, Y.; Xia, C.; Zhang, H.; Qian, W.; Cao, Y. Pathway analysis of plasma different metabolites for dairy cow ketosis. Ital. J. Anim. Sci. 2016, 15, 545-551. [CrossRef]

27. Xu, C.; Sun, L.-W.; Xia, C.; Zhang, H.-Y.; Zheng, J.-S.; Wang, J.-S. (1)H-Nuclear Magnetic Resonance-Based Plasma Metabolic Profiling of Dairy Cows with Fatty Liver. Asian-Australas. J. Anim. Sci. 2016, 29, 219-229. [CrossRef] 
28. Sun, Y.; Xu, C.; Li, C.; Xia, C.; Xu, C.; Wu, L.; Zhang, H. Characterization of the serum metabolic profile of dairy cows with milk fever using 1H-NMR spectroscopy. Vet. Q. 2014, 34, 159-163. [CrossRef]

29. Basoglu, A.; Baspinar, N.; Tenori, L.; Licari, C.; Gulersoy, E. Nuclear magnetic resonance (NMR)-based metabolome profile evaluation in dairy cows with and without displaced abomasum. Vet. Q. 2020, 40, 1-15. [CrossRef]

30. Daetwyler, H.D.; Xiang, R.; Yuan, Z.; Bolormaa, S.; Vander Jagt, C.J.; Hayes, B.J.; van der Werf, J.H.J.; Pryce, J.E.; Chamberlain, A.J.; Macleod, I.M.; et al. Integration of functional genomics and phenomics into genomic prediction raises its accuracy in sheep and dairy cattle. In Proceedings of the Association for the Advancement of Animal Breeding and Genetics, Armidale, NSW, Australia, 27 October-1 November 2019; pp. 11-14.

31. Xiang, R.; Berg, I.v.D.; Macleod, I.M.; Hayes, B.J.; Prowse-Wilkins, C.P.; Wang, M.; Bolormaa, S.; Liu, Z.; Rochfort, S.J.; Reich, C.M.; et al. Quantifying the contribution of sequence variants with regulatory and evolutionary significance to 34 bovine complex traits. Proc. Natl. Acad. Sci. USA 2019, 116, 19398-19408. [CrossRef]

32. Aru, V.; Lam, C.; Khakimov, B.; Hoefsloot, H.C.J.; Zwanenburg, G.; Lind, M.V.; Schäfer, H.; van Duynhoven, J.; Jacobs, D.M.; Smilde, A.K.; et al. Quantification of lipoprotein profiles by nuclear magnetic resonance spectroscopy and multivariate data analysis. Trends Anal. Chem 2017, 94, 210-219. [CrossRef]

33. Fuertes-Martin, R.; Correig, X.; Vallve, J.C.; Amigo, N. Human Serum/Plasma Glycoprotein Analysis by (1)H-NMR, an Emerging Method of Inflammatory Assessment. J. Clin. Med. 2020, 9, 354. [CrossRef] [PubMed]

34. Engelke, U.F.; Tangerman, A.; Willemsen, M.A.; Moskau, D.; Loss, S.; Mudd, S.H.; Wevers, R.A. Dimethyl sulfone in human cerebrospinal fluid and blood plasma confirmed by one-dimensional (1) $\mathrm{H}$ and two-dimensional (1)H-(13)C NMR. NMR Biomed. 2005, 18, 331-336. [CrossRef]

35. Maher, A.D.; Coles, C.; White, J.; Bateman, J.F.; Fuller, E.S.; Burkhardt, D.; Little, C.B.; Cake, M.; Read, R.; McDonagh, M.B.; et al. 1H NMR spectroscopy of serum reveals unique metabolic fingerprints associated with subtypes of surgically induced osteoarthritis in sheep. J. Proteome Res. 2012, 11, 4261-4268. [CrossRef] [PubMed]

36. Worley, B.; Powers, R. Multivariate Analysis in Metabolomics. Curr. Metabolomics 2013, 1, 92-107. [CrossRef]

37. Chong, I.-G.; Jun, C.-H. Performance of some variable selection methods when multicollinearity is present. Chemom. Intell. Lab. Syst. 2005, 78, 103-112. [CrossRef]

38. Annison, E.F.; Bickerstaffe, R.; Linzell, J.L. Glucose and fatty acid metabolism in cows producing milk of low fat content. J. Agric. Sci 1974, 82, 87-95. [CrossRef]

39. Xu, W.; Vervoort, J.; Saccenti, E.; Kemp, B.; van Hoeij, R.J.; van Knegsel, A.T.M. Relationship between energy balance and metabolic profiles in plasma and milk of dairy cows in early lactation. J. Dairy Sci. 2020, 103, 4795-4805. [CrossRef]

40. Aschenbach, J.R.; Kristensen, N.B.; Donkin, S.S.; Hammon, H.M.; Penner, G.B. Gluconeogenesis in dairy cows: The secret of making sweet milk from sour dough. IUBMB Life 2010, 62, 869-877. [CrossRef]

41. Drackley, J.K.; Overton, T.R.; Douglas, G.N. Adaptations of Glucose and Long-Chain Fatty Acid Metabolism in Liver of Dairy Cows during the Periparturient Period. J. Dairy Sci. 2001, 84, E100-E112. [CrossRef]

42. Zhang, H.; Wu, L.; Xu, C.; Xia, C.; Sun, L.; Shu, S. Plasma metabolomic profiling of dairy cows affected with ketosis using gas chromatography/mass spectrometry. BMC Vet. Res. 2013, 9, 186. [CrossRef] [PubMed]

43. Luke, T.D.W.; Pryce, J.E.; Elkins, A.C.; Wales, W.J.; Rochfort, S.J. Use of Large and Diverse Datasets for ${ }^{1}$ H NMR Serum Metabolic Profiling of Early Lactation Dairy Cows. Metabolites 2020, 10, 180. [CrossRef] [PubMed]

44. Jimenez, B.; Holmes, E.; Heude, C.; Tolson, R.F.; Harvey, N.; Lodge, S.L.; Chetwynd, A.J.; Cannet, C.; Fang, F.; Pearce, J.T.M.; et al. Quantitative Lipoprotein Subclass and Low Molecular Weight Metabolite Analysis in Human Serum and Plasma by (1)H NMR Spectroscopy in a Multilaboratory Trial. Anal. Chem. 2018, 90, 11962-11971. [CrossRef]

45. Grummer, R.R. Etiology of Lipid-Related Metabolic Disorders in Periparturient Dairy Cows. J. Dairy Sci. 1993, 76, 3882-3896. [CrossRef]

46. Horadagoda, N.U.; Knox, K.M.G.; Gibbs, H.A.; Reid, S.W.J.; Horadagoda, A.; Edwards, S.E.R.; Eckersall, P.D. Acute phase proteins in cattle: Discrimination between acute and chronic inflammation. Vet. Rec. 1999, 144, 437-441. [CrossRef] 
47. Sordillo, L.M. Nutritional strategies to optimize dairy cattle immunity. J. Dairy Sci. 2016, 99, 4967-4982. [CrossRef]

48. Li, Y.; Xu, C.; Xia, C.; Zhang, H.; Sun, L.; Gao, Y. Plasma metabolic profiling of dairy cows affected with clinical ketosis using LC/MS technology. Vet. Q. 2014, 34, 152-158. [CrossRef]

49. Imhasly, S.; Naegeli, H.; Baumann, S.; Von Bergen, M.; Luch, A.; Jungnickel, H.; Potratz, S.; Gerspach, C. Metabolomic biomarkers correlating with hepatic lipidosis in dairy cows. BMC Vet. Res. 2014, 10, 122. [CrossRef]

50. Humer, E.; Khol-Parisini, A.; Metzler-Zebeli, B.U.; Gruber, L.; Zebeli, Q. Alterations of the Lipid Metabolome in Dairy Cows Experiencing Excessive Lipolysis Early Postpartum. PLoS ONE 2016, 11, e0158633. [CrossRef]

51. McFadden, J.W.; Girard, C.L.; Tao, S.; Zhou, Z.; Bernard, J.K.; Duplessis, M.; White, H.M. Symposium review: One-carbon metabolism and methyl donor nutrition in the dairy cow. J. Dairy Sci. 2020. [CrossRef]

52. Imhasly, S.; Bieli, C.; Naegeli, H.; Nyström, L.; Ruetten, M.; Gerspach, C. Blood plasma lipidome profile of dairy cows during the transition period. BMC Vet. Res. 2015, 11, 252. [CrossRef] [PubMed]

53. Artegoitia, V.M.; Middleton, J.L.; Harte, F.M.; Campagna, S.R.; de Veth, M.J. Choline and Choline Metabolite Patterns and Associations in Blood and Milk during Lactation in Dairy Cows. PLoS ONE 2014, 9, e103412. [CrossRef] [PubMed]

54. Zhao, G.; He, F.; Wu, C.; Li, P.; Li, N.; Deng, J.; Zhu, G.; Ren, W.; Peng, Y. Betaine in Inflammation: Mechanistic Aspects and Applications. Front. Immunol. 2018, 9, 1070. [CrossRef] [PubMed]

55. Bergman, E. Energy Contributions of Volatile Fatty Acids from the Gastrointestinal Tract in Various Species. Physiol. Rev. 1990, 70, 567. [CrossRef] [PubMed]

56. Urrutia, N.L.; Harvatine, K.J. Acetate Dose-Dependently Stimulates Milk Fat Synthesis in Lactating Dairy Cows. J. Nutr 2017, 147, 763-769. [CrossRef]

57. Bielak, A.; Derno, M.; Tuchscherer, A.; Hammon, H.M.; Susenbeth, A.; Kuhla, B. Body fat mobilization in early lactation influences methane production of dairy cows. Sci. Rep. 2016, 6, 28135. [CrossRef]

58. O'Callaghan, T.F.; Vázquez-Fresno, R.; Serra-Cayuela, A.; Dong, E.; Mandal, R.; Hennessy, D.; McAuliffe, S.; Dillon, P.; Wishart, D.S.; Stanton, C.; et al. Pasture Feeding Changes the Bovine Rumen and Milk Metabolome. Metabolites 2018, 8, 27. [CrossRef]

59. Maher, A.D.; Hayes, B.; Cocks, B.; Marett, L.; Wales, W.J.; Rochfort, S.J. Latent biochemical relationships in the blood-milk metabolic axis of dairy cows revealed by statistical integration of $1 \mathrm{H}$ NMR spectroscopic data. J. Proteome Res. 2013, 12, 1428-1435. [CrossRef]

60. Little, S. Feeding Systems Used by Australian Dairy Farmers; Dairy Australia: Melbourne, Australia, 2010.

61. Viant, M.R. Improved methods for the acquisition and interpretation of NMR metabolomic data. Biochem. Biophys. Res. Commun. 2003, 310, 943-948. [CrossRef]

62. Nielsen, N.-P.V.; Carstensen, J.M.; Smedsgaard, J. Aligning of single and multiple wavelength chromatographic profiles for chemometric data analysis using correlation optimised warping. J. Chromatogr. 1998, 805, 17-35. [CrossRef]

63. R Core Team. R: A Language and Environment for Statistical Computing; R Foundation for Statistical Computing: Vienna, Austria, 2019.

64. Smilde, A.; Jansen, J.; Hoefsloot, H.; Lamers, R.-J.; van Der Greef, J.; Timmerman, M. ANOVA-simultaneous component analysis (ASCA): A new tool for analyzing designed metabolomics data. Bioinformatics 2005, 21, 3043-3048. [CrossRef] [PubMed]

65. Cloarec, O.; Dumas, M.-E.; Craig, A.; Barton, R.H.; Trygg, J.; Hudson, J.; Blancher, C.; Gauguier, D.; Lindon, J.C.; Holmes, E.; et al. Statistical total correlation spectroscopy: An exploratory approach for latent biomarker identification from metabolic ${ }^{1} \mathrm{H}$ NMR data sets. Anal. Chem. 2005, 77, 1282-1289. [CrossRef] [PubMed]

(C) 2020 by the authors. Licensee MDPI, Basel, Switzerland. This article is an open access article distributed under the terms and conditions of the Creative Commons Attribution (CC BY) license (http://creativecommons.org/licenses/by/4.0/). 


\title{
Metabolic Profiling Provides Unique Insights to Accumulation and Biosynthesis of Key Secondary Metabolites in Annual Pasture Legumes of Mediterranean Origin
}

\author{
Sajid Latif ${ }^{1,2, *}$, Paul A. Weston ${ }^{1,3}$, Russell A. Barrow ${ }^{1,4}$, Saliya Gurusinghe ${ }^{1}$, John W. Piltz ${ }^{5}$ and \\ Leslie A. Weston ${ }^{1,3}$ \\ 1 Graham Center for Agricultural Innovation, Locked Bag 588, Wagga Wagga, NSW 2678, Australia; \\ pweston@csu.edu.au (P.A.W.); rubarrow@csu.edu.au (R.A.B.); sgurusinghe@csu.edu.au (S.G.); \\ leweston@csu.edu.au (L.A.W.) \\ 2 School of Animal and Veterinary Sciences, Charles Sturt University, Wagga Wagga, NSW 2678, Australia \\ 3 School of Agriculture and Wine Sciences, Charles Sturt University, Wagga Wagga, NSW 2678, Australia \\ 4 Plus 3 Australia Pty Ltd., P.O. Box 4345, Hawker, ACT 2614, Australia \\ 5 New South Wales Department of Primary Industries, Wagga Wagga, NSW 2678, Australia; \\ john.piltz@dpi.nsw.gov.au \\ * Correspondence: slatif@csu.edu.au
}

Received: 27 May 2020; Accepted: 19 June 2020; Published: 28 June 2020

\begin{abstract}
Annual legumes from the Mediterranean region are receiving attention in Australia as alternatives to traditional pasture species. The current study employed novel metabolic profiling approaches to quantify key secondary metabolites including phytoestrogens to better understand their biosynthetic regulation in a range of field-grown annual pasture legumes. In addition, total polyphenol and proanthocyanidins were quantified using Folin-Ciocalteu and vanillin assays, respectively. Metabolic profiling coupled with biochemical assay results demonstrated marked differences in the abundance of coumestans, flavonoids, polyphenols, and proanthocyanidins in annual pasture legume species. Genetically related pasture legumes segregated similarly from a chemotaxonomic perspective. A strong and positive association was observed between the concentration of phytoestrogens and upregulation of the flavonoid biosynthetic pathway in annual pasture legumes. Our findings suggest that evolutionary differences in metabolic dynamics and biosynthetic regulation of secondary metabolites have logically occurred over time in various species of annual pasture legumes resulting in enhanced plant defense.
\end{abstract}

Keywords: pasture legumes; phytoestrogens; flavonoids; coumestans; polyphenols; proanthocyanidins; metabolic profiling; biosynthesis

\section{Introduction}

Broadacre farming frequently occurs with livestock production throughout southeastern Australia, with the pasture phase of crop rotation sustaining both lamb and cattle enterprises [1]. Lamb and beef production account for the majority of livestock-related income in southeastern Australia (AU $\$ 22$ billion in 2017) and global demand is projected to dramatically increase over the next decade (Australian Bureau of Statistics, 2018). Legumes are integral to livestock pasture production systems through provision of high quality forage for grazing livestock. Establishment of pasture species that are non-toxic, persistent, and high in nutritional quality is therefore critical for continued improvement of livestock productivity. 
Traditionally, subterranean clover (Trifolium subterraneum L.) and lucerne (Medicago sativa L.) are the most widely utilized pasture species in prime lamb, wool, and cattle producing regions across southeastern Australia. Subterranean clover is compatible across various soil types and is tolerant of $\mathrm{pH}$ extremes [2,3]. Lucerne is a deep-rooted perennial species frequently established across diverse rainfall regions and is suitable for neutral or mildly alkaline soils. Recently the establishment of both pasture species has proven challenging to sustain livestock production due to a range of biotic and abiotic factors. For example, lucerne, which contains high protein content, has been shown to undergo rapid fermentation in the rumen resulting in increased incidence of bloat and potential loss of nitrogen due to excretion [4]. In addition, ingestion of lucerne and subterranean clover is associated with metabolic disorders including acute inflammation of both the small and large intestine (red gut), sodium deficiency and pregnancy-related toxemia [5-7].

Increased risk of metabolic disorders, the significant cost of establishment, and low persistence of traditional legume pastures in low-rainfall regions $[8,9]$ has led to the introduction of novel annual pasture legume species originating from Mediterranean regions of Europe and northern Africa to Australia [3]. These include Biserrula pelecinus L. (biserrula), Ornithopus sativus Brot. (French serradella), Ornithopus compressus L. (yellow serradella), Trifolium glanduliferum Boiss. (gland clover), Trifolium spumosum L. (bladder clover), and Trifolium vesiculosum Savi. (arrowleaf clover). These accessions are characterized by their adaptation to deep, acid and sandy soils, drought tolerance, weed suppressive potential, and prolonged availability as feed for livestock $[10,11]$. While the nutritive value of traditional pasture legumes has been studied in southeastern and Western Australia, a detailed investigation of the phenolic chemistry of annual legumes has not been performed with respect to livestock production [2].

Legumes have evolved chemical defense mechanisms mediated by secondary metabolites, including phytoestrogens, to deter herbivores and plant pests [12,13]. In terms of phenolic chemistry and key secondary metabolites in pasture legumes, flavonoids represent a distinct class of secondary products with both positive and negative impacts on plant-microbial and plant-livestock interactions $[14,15]$. In general, they are classified by their chemical structure into subgroups including anthocyanidins and anthoxanthins (flavanones, flavans, and flavanonols) [16].

Significant levels of phytoestrogens are produced in pasture legumes including lucerne as well as various clover species and when present at significant levels can seriously reduce reproductive efficiency and livestock fertility $[15,17,18]$. Coumestans and isoflavone phytoestrogens are stable, non-steroidal secondary metabolites that mimic mammalian estrogen, an endogenous female sex hormone $[15,19]$. The affinity of these phytoestrogens in binding estrogen receptor- $\beta$ can result in reproductive abnormalities during embryo development, and infertility in both sexes of grazing livestock [20]. Isoflavone phytoestrogens are typically stored either as glycosides or aglycones in pasture legumes [15,21].

Coumestans are non-flavonoid phytoestrogens, and include coumestrol and 4'-methoxycoumestrol, first isolated from white clover (Trifolium repens L.) and lucerne (Medicago sativa L.) in 1957 [22]. These polycylic aromatic metabolites are closely related biosynthetically to flavonoids (Figure 1). Elevated concentrations of phytoestrogens, including isoflavones, coumestrol, and related metabolites, from ingestion of fodder or feedstock have been implicated in estrogenic clinical signs in livestock as edematous vaginal and cervical tissue, hypertrophy of mammary glands, and milky secretions from elongated teats. Phytoestrogens in forage may also cause adverse effects in ovarian function resulting in loss of fecundity or early embryonic death [23]. Livestock grazing various Trifolium species exhibit varying tolerance to coumestans, which typically range in concentration from 25 to $200 \mathrm{mg} \mathrm{kg}^{-1}$ dry matter (DM) [15]. The presence of coumestrol at concentrations greater than $\approx 40 \mathrm{mg} \mathrm{kg}^{-1} \mathrm{DM}$ in plant tissues is associated with reproductive inefficiency in sheep and cattle through disruption of several endocrine mechanisms $[24,25]$. 
CLASS<smiles>[R9]Oc1ccc2c(c1)OC1c3ccc(O)cc3OC(=O)C21</smiles>

isoflavone<smiles>[R20]c1ccc(-c2coc3cc(O)cc([R])c3c2=O)cc1</smiles>

diadzein $\quad \mathrm{R}_{1}=\mathrm{H} \quad \mathrm{R}_{2}=\mathrm{H}$

formononetin $\quad \mathrm{R}_{1}=\mathrm{H} \quad \mathrm{R}_{2}=\mathrm{CH}_{3}$

genistein $\mathrm{R}_{1}=\mathrm{OH} \quad \mathrm{R}_{2}=\mathrm{H}$

Figure 1. Phytoestrogens found in abundance in lucerne (Medicago sativa) and subterranean clover (Trifolium subterraneum).

Isoflavonoids are 3-phenylchromen-4-ones (3-phenyl-1,4-benzopyrone) and are important in regulating numerous interactions in higher plants. The isoflavone biosynthetic pathway is one of the most well elaborated pathways in plant secondary metabolism due to the importance of isoflavones as chemoattractants for rhizobia and their involvement in plant defense. Isoflavones are mainly derived from the phenylpropanoid pathway but can be generated through multiple pathways in many plant species [26]. Legumes possess a unique enzyme, isoflavone synthase (IFS), which is a cytochrome P450 monooxygenase that catalyzes the 2, 3 migration of the B-ring of naringenin or liquiritigenin, resulting in the production of various biologically active isoflavonoids [27]. The genes encoding enzymes in this pathway are tissue specific and are regulated both spatially and temporally in legumes [28]. Such catalytic enzymes are induced by various stress factors influencing plant condition, including climate, temperature, soil moisture availability, nutrient deficiency, and herbivory [29].

Polyphenolic compounds, including condensed tannins (proanthocyanidins), are another key group of metabolites possessing a range of biological and nutritional properties in grazing livestock. These compounds vary with regard to chemical structure, plant source, and target animal species [30]. For example, monomeric phenolic acids in forages are associated with enhanced milk production and acid-base imbalance in the rumen following microbial degradation [31]. Traditionally, the proanthocyanidins, which are oligomeric polyphenols, have been considered as anti-nutritional factors leading to reduced palatability of forages, but recent research has shown they provide several potential advantages to grazing livestock, including reduced risk of bloat at moderate intake of 3-4\% of dry matter (DM) [32] and increased weight gain, while reducing greenhouse gas emissions [33-35] and parasite burden $[36,37]$. However, feed composition, age and physiological status of the animal are some of the key factors need to be taken into account while studying the effects of plant secondary metabolites on livestock [38,39].

There is a marked lack of information on the phytochemical profiles of aerial tissues of annual pasture legumes, particularly those recently introduced to the southern hemisphere from the Mediterranean. Recent advances in quadrupole time-of-flight mass spectrometry (MS-QToF) instrumentation have resulted in increasing usage of time-of-flight mass spectrometry (MS-ToF) instruments as quantitation tools as well as prediction of molecular formulae because of their high resolving power and wide dynamic range [40,41]. To broaden our understanding of the secondary chemistry of novel pasture legumes in Australia, we took a metabolomics approach using LC-MS-QToF to investigate (a) the distribution of secondary metabolites that may impact livestock performance, including flavonoids and phytoestrogens (coumestrol, $4^{\prime}$-methoxycoumestrol, formononetin, genistein and daidzein), in above-ground tissues of selected traditional and newly introduced annual pasture legumes grown under field conditions in southern Australia; (b) the total polyphenol and proanthocyanidin content to better delimit their prevalence in these pasture 
species, and (c) the biosynthetic pathways associated with the production of flavonoids and certain phytoestrogens in these plants.

\section{Results}

\subsection{Quantification of Phytoestrogens}

Concentration of coumestans in foliar tissues of all species ranged between 0.13 and $48.4 \mathrm{mg} \mathrm{kg}^{-1}$ and varied significantly across pasture species (Table 1). Both coumestrol and $4^{\prime}$-methoxycoumestrol accumulated at higher concentrations in leaf and stem compared to inflorescence tissues. Bladder clover possessed significantly higher concentrations of both coumestrol and $4^{\prime}$-methoxycoumestrol in leaf tissue compared to other pasture legumes ( 48.4 and $24.8 \mathrm{mg} \mathrm{kg}^{-1}$, respectively) while in stem tissue, bladder clover had the highest concentration of coumestrol $\left(39.6 \mathrm{mg} \mathrm{kg}^{-1}\right)$. Lucerne contained the highest concentration of $4^{\prime}$-methoxycoumestrol $\left(27.7 \mathrm{mg} \mathrm{kg}^{-1}\right)$ in any tissue of all species, and lucerne inflorescence tissue contained the highest concentrations of coumestrol and $4^{\prime}$-methoxycoumestrol ( 0.5 and $0.2 \mathrm{mg} \mathrm{kg}^{-1}$, respectively) of all annual pasture species examined.

Table 1. The concentration of coumestrol and $4^{\prime}$-methoxycoumestrol $\left(\mathrm{mg} \mathrm{kg}^{-1}\right)$ at physiological maturity in selected annual pasture legume species.

\begin{tabular}{|c|c|c|c|c|}
\hline Tissue Type & Pasture Species & Coumestrol & $4^{\prime}$-Methoxycoumestrol & Total Coumestans \\
\hline \multirow{10}{*}{ Leaf } & Arrowleaf clover & $14.7 \pm 2.2$ & $5.9 \pm 1.5$ & $20.6 \pm 5.1$ \\
\hline & Biserrula cv.* Casbah & $13.1 \pm 2.7$ & $5.2 \pm 1.7$ & $18.2 \pm 6.5$ \\
\hline & Biserrula cv. Mauro & $14.6 \pm 2.4$ & $7.4 \pm 5.5$ & $22.0 \pm 4.2$ \\
\hline & Bladder clover & $48.4 \pm 10.6$ & $24.8 \pm 17.7$ & $73.2 \pm 13.6$ \\
\hline & French serradella & ND & ND & ND \\
\hline & Gland clover & $27.1 \pm 11.1$ & $21.1 \pm 5.6$ & $48.2 \pm 3.5$ \\
\hline & Lucerne & $31.8 \pm 14.4$ & $20.2 \pm 7.5$ & $52.0 \pm 6.7$ \\
\hline & Subterranean clover & $25.1 \pm 8.6$ & $12.5 \pm 4.5$ & $37.6 \pm 7.3$ \\
\hline & Yellow serradella & ND & ND & ND \\
\hline & $\operatorname{LSD}(p<0.05)$ & 9.3 & 5.4 & 13.1 \\
\hline \multirow{10}{*}{ Stem } & Arrowleaf clover & $24.2 \pm 5.3$ & $6.1 \pm 4.6$ & $30.3 \pm 10.5$ \\
\hline & Biserrula cv. Casbah & $\mathrm{ND}$ & ND & ND \\
\hline & Biserrula cv. Mauro & ND & ND & ND \\
\hline & Bladder clover & $39.6 \pm 7.7$ & $18.7 \pm 3.6$ & $58.3 \pm 12.1$ \\
\hline & French serradella & ND & ND & ND \\
\hline & Gland clover & $26.2 \pm 14.4$ & $3.6 \pm 1.9$ & $29.8 \pm 13.0$ \\
\hline & Lucerne & $36.6 \pm 16.5$ & $27.7 \pm 12.8$ & $64.3 \pm 5.1$ \\
\hline & Subterranean clover & $25.4 \pm 7.5$ & $7.1 \pm 1.6$ & $32.5 \pm 10.6$ \\
\hline & Yellow serradella & ND & ND & ND \\
\hline & $\operatorname{LSD}(p<0.05)$ & 9.4 & 14.5 & 17.5 \\
\hline \multirow{10}{*}{ Inflorescence } & Arrowleaf clover & ND & $0.1 \pm 0.1$ & $<0.1$ \\
\hline & Biserrula cv. Casbah & ND & ND & ND \\
\hline & Biserrula cv. Mauro & ND & $0.2 \pm 0.1$ & $<0.1$ \\
\hline & Bladder clover & $0.2 \pm 0.2$ & $<0.1$ & $<0.1$ \\
\hline & French serradella & ND & ND & $\mathrm{ND}$ \\
\hline & Gland clover & 0.1 & ND & $<0.1$ \\
\hline & Lucerne & $0.5 \pm 0.1$ & $0.2 \pm 0.1$ & $0.7 \pm 0.1$ \\
\hline & Subterranean clover & $0.3 \pm 0.1$ & ND & $0.3 \pm 0.1$ \\
\hline & Yellow serradella & ND & ND & ND \\
\hline & $\operatorname{LSD}(p<0.05)$ & 0.2 & 0.1 & 0.2 \\
\hline
\end{tabular}

ND denotes compounds not detected. Least significant difference (LSD) differentiated between concentration means.

Values represent the arithmetic mean of five replicates \pm standard deviation. ${ }^{*} \mathrm{cv}$. is acronym for cultivar.

Three isoflavones-daidzein, formononetin and genistein-are commonly found in pasture legumes and were also profiled in this study (Table 2). Isoflavone content in leaf (113.3-443.9 $\left.\mathrm{mg} \mathrm{kg}^{-1}\right)$, stem (37.5-968.1 mg kg-1$)$, and inflorescence (29.8-614.1 $\mathrm{mg} \mathrm{kg}^{-1}$ ) varied significantly by both species and tissue (Table 2). Gland clover had the highest concentration of total isoflavones in leaf tissue $\left(443.9 \mathrm{mg} \mathrm{kg}^{-1}\right)$ while bladder clover had the highest concentration in stem and inflorescence tissue 
(968.1 and $604.1 \mathrm{mg} \mathrm{kg}^{-1}$, respectively). In terms of individual phytoestrogenic isoflavones in aerial tissues, formononetin concentration was greatest overall, followed by genistein at physiological maturity (Table 2). The production of phytoestrogenic isoflavones was greatest in clover species; specifically, daidzein concentration was highest in leaf tissues of gland clover at $120.2 \mathrm{mg} \mathrm{kg}^{-1}$. Production of these compounds in stems was greatest in bladder clover and subterranean clover (112.0 and $107.8 \mathrm{mg} \mathrm{kg}^{-1}$, respectively) when compared to other species. Subterranean clover contained the highest levels of daidzein $\left(128.9 \mathrm{mg} \mathrm{kg}^{-1}\right)$ in inflorescence tissue when compared to other annual pasture species.

Table 2. The concentration of daidzein, formononetin and genistein $\left(\mathrm{mg} \mathrm{kg}^{-1}\right)$ in annual pasture legume species at physiological maturity, post-flowering.

\begin{tabular}{|c|c|c|c|c|c|}
\hline Tissue Type & Pasture Species & Daidzein & Formononetin & Genistein & Total \\
\hline \multirow{10}{*}{ Leaf } & Arrowleaf clover & $55.1 \pm 26.4$ & $222.9 \pm 84.4$ & $28.3 \pm 6.8$ & $306.3 \pm 94.3$ \\
\hline & Biserrula cv. Casbah & $11.5 \pm 3.5$ & $73.6 \pm 51.3$ & $116.8 \pm 2.8$ & $192.5 \pm 18.7$ \\
\hline & Biserrula cv. Mauro & $6.1 \pm 0.7$ & $58.9 \pm 17.3$ & $124.7 \pm 12.5$ & $189.7 \pm 24.0$ \\
\hline & Bladder clover & $95.0 \pm 2.1$ & $152.3 \pm 18.9$ & $184.1 \pm 26.3$ & $431.4 \pm 32.8$ \\
\hline & French serradella & $47.7 \pm 12.5$ & $35.5 \pm 48.6$ & $130.1 \pm 7.6$ & $113.3 \pm 8.1$ \\
\hline & Gland clover & $120.3 \pm 16.1$ & $226.1 \pm 20.5$ & $97.5 \pm 10.8$ & $443.9 \pm 61.4$ \\
\hline & Lucerne & $48.2 \pm 13.3$ & $329.4 \pm 192.7$ & $61.5 \pm 10.1$ & $439.1 \pm 141.9$ \\
\hline & Subterranean clover & $109.1 \pm 14.8$ & $157.6 \pm 47.3$ & $72.1 \pm 244.3$ & $318.8 \pm 40.5$ \\
\hline & Yellow serradella & $89.0 \pm 13$ & $91.6 \pm 16.6$ & $118.4 \pm 5.4$ & $219.1 \pm 43.0$ \\
\hline & LSD $(p<0.05)$ & 24.9 & 37.5 & 34.8 & - \\
\hline \multirow{10}{*}{ Stem } & Arrowleaf clover & $9.8 \pm 3.2$ & $147.7 \pm 58.2$ & $27.6 \pm 5.2$ & $185.1 \pm 67.1$ \\
\hline & Biserrula cv. Casbah & $7.5 \pm 0.9$ & $23.9 \pm 12.2$ & $6.1 \pm 1.9$ & $37.5 \pm 8.9$ \\
\hline & Biserrula cv. Mauro & $5.1 \pm 1.4$ & $60.4 \pm 18.4$ & $9.9 \pm 3.7$ & $75.4 \pm 27.4$ \\
\hline & Bladder clover & $112.0 \pm 33.5$ & $829.8 \pm 144.9$ & $126.1 \pm 5.4$ & $968.1 \pm 394.7$ \\
\hline & French serradella & $75.2 \pm 23.3$ & $96.7 \pm 34.4$ & $104.1 \pm 18.9$ & $276.0 \pm 13.4$ \\
\hline & Gland clover & $3.4 \pm 0.5$ & $239.5 \pm 18.4$ & $88.1 \pm 37.4$ & $331.0 \pm 107.0$ \\
\hline & Lucerne & $7.5 \pm 2.3$ & $120.6 \pm 35.3$ & $15.9 \pm 5.1$ & $144.0 \pm 56.4$ \\
\hline & Subterranean clover & $107.8 \pm 18.9$ & $36.2 \pm 6.4$ & $18.2 \pm 8.3$ & $162.2 \pm 42.4$ \\
\hline & Yellow serradella & $42.5 \pm 7.2$ & $77.7 \pm 14.2$ & $20.3 \pm 6.9$ & $140.5 \pm 25.9$ \\
\hline & $\mathrm{LSD}(p<0.05)$ & 20.8 & 47.9 & 19.8 & - \\
\hline \multirow{10}{*}{ Inflorescence } & Arrowleaf clover & $1.3 \pm 0.3$ & $98.2 \pm 24.5$ & $18.4 \pm 4.6$ & $117.9 \pm 45.3$ \\
\hline & Biserrula cv. Casbah & $2.2 \pm 0.5$ & $39.6 \pm 9.9$ & $10.2 \pm 2.5$ & $52.0 \pm 17.6$ \\
\hline & Biserrula cv. Mauro & $1.7 \pm 0.4$ & $18.5 \pm 4.6$ & $9.6 \pm 4.1$ & $29.8 \pm 7.5$ \\
\hline & Bladder clover & $19.3 \pm 4.8$ & $435.8 \pm 108.9$ & $158.9 \pm 37.2$ & $614.1 \pm 190.6$ \\
\hline & French serradella & $10.5 \pm 2.6$ & $98.8 \pm 24.7$ & $19.9 \pm 4.9$ & $129.2 \pm 43.4$ \\
\hline & Gland clover & $68.3 \pm 17.1$ & $184.8 \pm 46.2$ & $68.3 \pm 17.1$ & $321.0 \pm 60.1$ \\
\hline & Lucerne & $25.5 \pm 6.4$ & $182.6 \pm 45.6$ & $25.7 \pm 6.4$ & $233.8 \pm 81.1$ \\
\hline & Subterranean clover & $128.9 \pm 32.2$ & $187.6 \pm 46.9$ & $148.7 \pm 39.8$ & $465.2 \pm 26.3$ \\
\hline & Yellow serradella & $14.6 \pm 3.6$ & $17.6 \pm 4.4$ & $25.8 \pm 6.6$ & $58.00 \pm 5.2$ \\
\hline & $\mathrm{LSD}(p<0.05)$ & 16.8 & 39.2 & 24.5 & - \\
\hline
\end{tabular}

Least significant difference (LSD) was used to differentiate between concentration means. Values represent the arithmetic mean of five replicates \pm standard deviations.

Formononetin concentration in leaf tissue was significantly higher in the perennial legume lucerne (329.4 $\mathrm{mg} \mathrm{kg}^{-1}$ ) than the other species, while for stem tissue, formononetin concentration was greatest in bladder clover $\left(829.8 \mathrm{mg} \mathrm{kg}^{-1}\right)$. Interestingly, genistein levels were greatest in all three tissues types in bladder clover compared to other species.

\subsection{Effect of Biserrula Cultivar and Growth Stage on Phytoestrogen Levels}

Given the strong potential of biserrula to produce large quantities of biomass and suppress weeds successfully over time, further evaluation was performed to examine temporal effects on the accumulation of phytoestrogens in aerial tissues in both of the commercially available cultivars of biserrula in Australia. Analysis of tissues of Casbah and Mauro analyzed at five different growth stages showed that total concentrations of the phytoestrogens differed significantly between cultivars (Figure 2a,b). Phytoestrogen concentrations reached their maxima at either $50 \%$ bloom or full bloom 
while the lowest concentrations were observed at crop senescence. Significant differences in the concentration of coumestans between the two cultivars were limited to coumestrol at pre-bloom and $50 \%$ bloom stages; cv. Mauro was observed to produce greater levels of coumestans than cv. Casbah. The phytoestrogenic isoflavones formononetin and daidzein were more abundant in tissues of Casbah than Mauro, while the opposite was true for genistein.

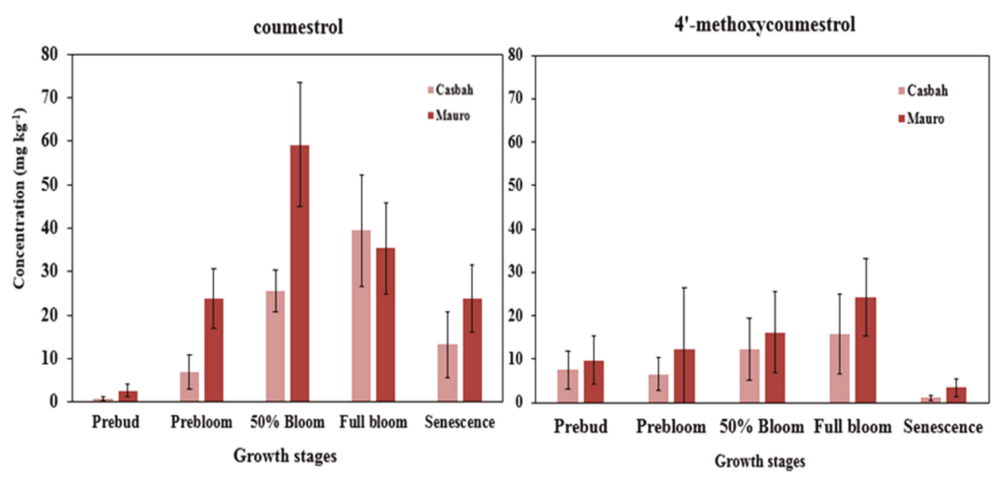

(a)
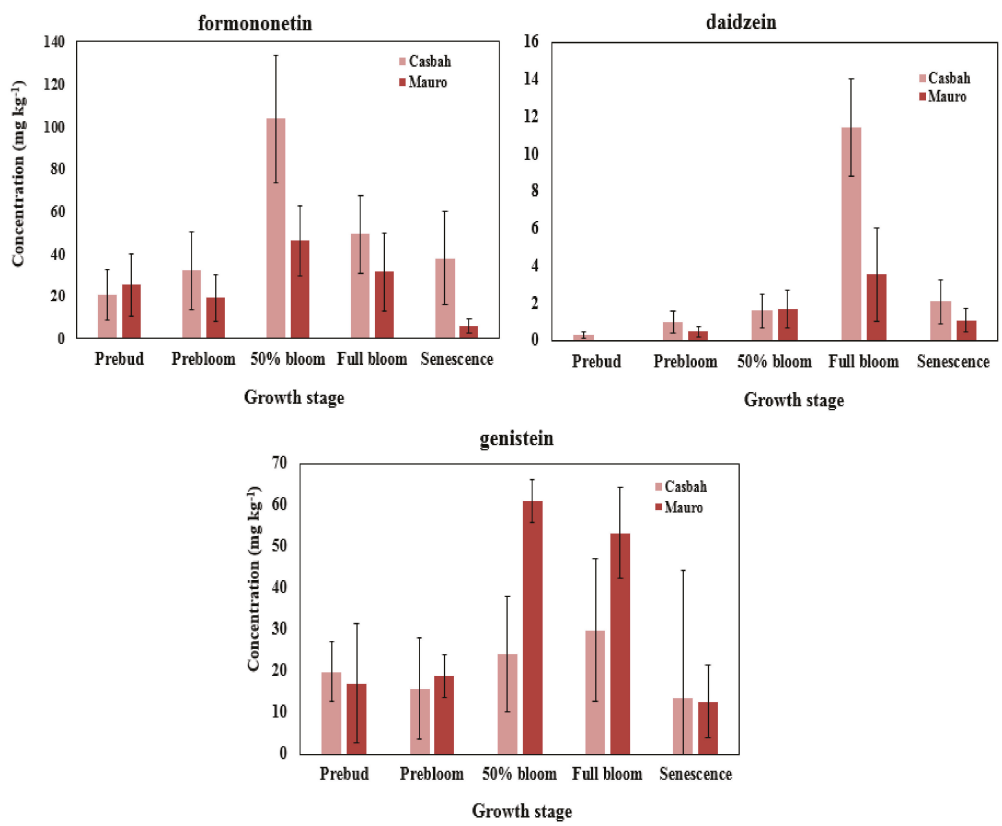

(b)

Figure 2. (a) The concentration of coumestrol and $4^{\prime}$-methoxycoumestrol at five growth stages i.e., pre bud, pre bloom, 50\% bloom, full bloom, and senescence of field-grown biserrula cv. Casbah and cv. Mauro averaged over two years. Error bars indicate standard deviation; (b) the concentration of selected isoflavones at five growth stages i.e., pre bud, pre bloom, 50\% bloom, full bloom, and senescence in biserrula cv. Casbah and cv. Mauro samples averaged over two years. Error bars indicate standard deviation. 


\subsection{Quantification of Total Polyphenols and Proanthocyanidins}

Extractable TPC (total polyphenol content) ranged from 4.40 to 13.84 GAE and TPAC (total proanthocyanidins) ranged from 1.73 to $6.49 \mathrm{mg} 100^{-1} \mathrm{~g} \mathrm{CE}$ (Table 3). Gland clover contained significantly higher extractable TPC levels $\left(13.84 \mathrm{mg} 100^{-1} \mathrm{~g}\right)$ compared to all other pasture species. Extractable and bound TPAC was significantly higher in biserrula cv. Casbah compared to other species, while bound TPAC was only detected in the two biserrula cultivars (Casbah and Mauro) and the perennial legume, lucerne.

Table 3. Concentration of extractable and bound total polyphenol and total proanthocyanidins in foliar tissues of pasture legumes.

\begin{tabular}{ccccc}
\hline \multirow{2}{*}{ Pasture Species } & \multicolumn{2}{c}{ TPC } & \multicolumn{2}{c}{ TPAC } \\
\cline { 2 - 5 } & Extractable & Bound & Extractable & Bound \\
\hline Arrowleaf clover & $10.50^{\mathrm{b}}$ & $0.24^{\mathrm{de}}$ & $3.71^{\mathrm{c}}$ & $\mathrm{ND}$ \\
Biserrula cv. Casbah & $7.69^{\mathrm{cde}}$ & $0.43^{\mathrm{cd}}$ & $6.49^{\mathrm{a}}$ & $8.87^{\mathrm{a}}$ \\
Biserrula cv. Mauro & $6.24^{\mathrm{def}}$ & $0.11^{\mathrm{e}}$ & $4.29^{\mathrm{b}}$ & $4.06^{\mathrm{b}}$ \\
Bladder clover & $6.67^{\mathrm{def}}$ & $0.45^{\mathrm{bc}}$ & $1.73^{\mathrm{c}}$ & $\mathrm{ND}$ \\
French serradella & $8.29^{\mathrm{bcd}}$ & $0.35^{\mathrm{cd}}$ & $1.94^{\mathrm{c}}$ & $\mathrm{ND}$ \\
Gland clover & $13.84^{\mathrm{a}}$ & $0.57^{\mathrm{bc}}$ & $2.12^{\mathrm{c}}$ & $\mathrm{ND}$ \\
Lucerne & $4.40^{\mathrm{f}}$ & $0.11^{\mathrm{de}}$ & $2.05^{\mathrm{c}}$ & $0.33^{\mathrm{c}}$ \\
Subterranean clover & $9.39^{\mathrm{bc}}$ & $0.62^{\mathrm{bc}}$ & $4.52^{\mathrm{ab}}$ & $\mathrm{ND}$ \\
Yellow serradella & $5.17^{\mathrm{ef}}$ & $0.72^{\mathrm{b}}$ & $1.94^{\mathrm{c}}$ & $\mathrm{ND}$ \\
LSD & 2.62 & 0.28 & 2.16 & 1.95 \\
\hline
\end{tabular}

Values represent arithmetic mean of five replicates. Values followed by the same superscript in each column are not significantly different $(p<0.05)$ as determined by ANOVA.

\subsection{Abundance of Flavonoids and Their Glycosides}

The clustering of molecular features profiled through non-targeted metabolic profiling revealed that molecular entities varied between species but were similar in species of the same genera (Supplementary Materials Figure S1). Over 5000 molecular features in total were successfully profiled in legume leaf tissues, with 1727 in stem and 1503 in inflorescence tissues (Figure S2). Interestingly, flavonoids and their glycosides accounted for the majority of constituents among all annotated major classes of secondary metabolites, based on verification with analytical standards as well as METLIN database comparisons. The relative abundance of various flavonoids and their glycosides in selected pasture legumes is presented in Figure 3. Interestingly, the total number of molecular features characterized in this study was highest in biserrula followed by French and yellow serradella, while gland clover exhibited the fewest total number of molecular features (Supplementary Materials Figure S1). However, gland clover, a recent introduction to Australia obtained from native pastures in the Mediterranean, exhibited a considerably higher abundance of secondary metabolites including flavonoids and their glycosides profiled in this study compared to other species (Figure 3). Both Casbah and Mauro cultivars of biserrula presented chemically similar profiles and exhibited relatively low abundance of flavonoids and their glycosides, in contrast to subterranean and gland clover. 


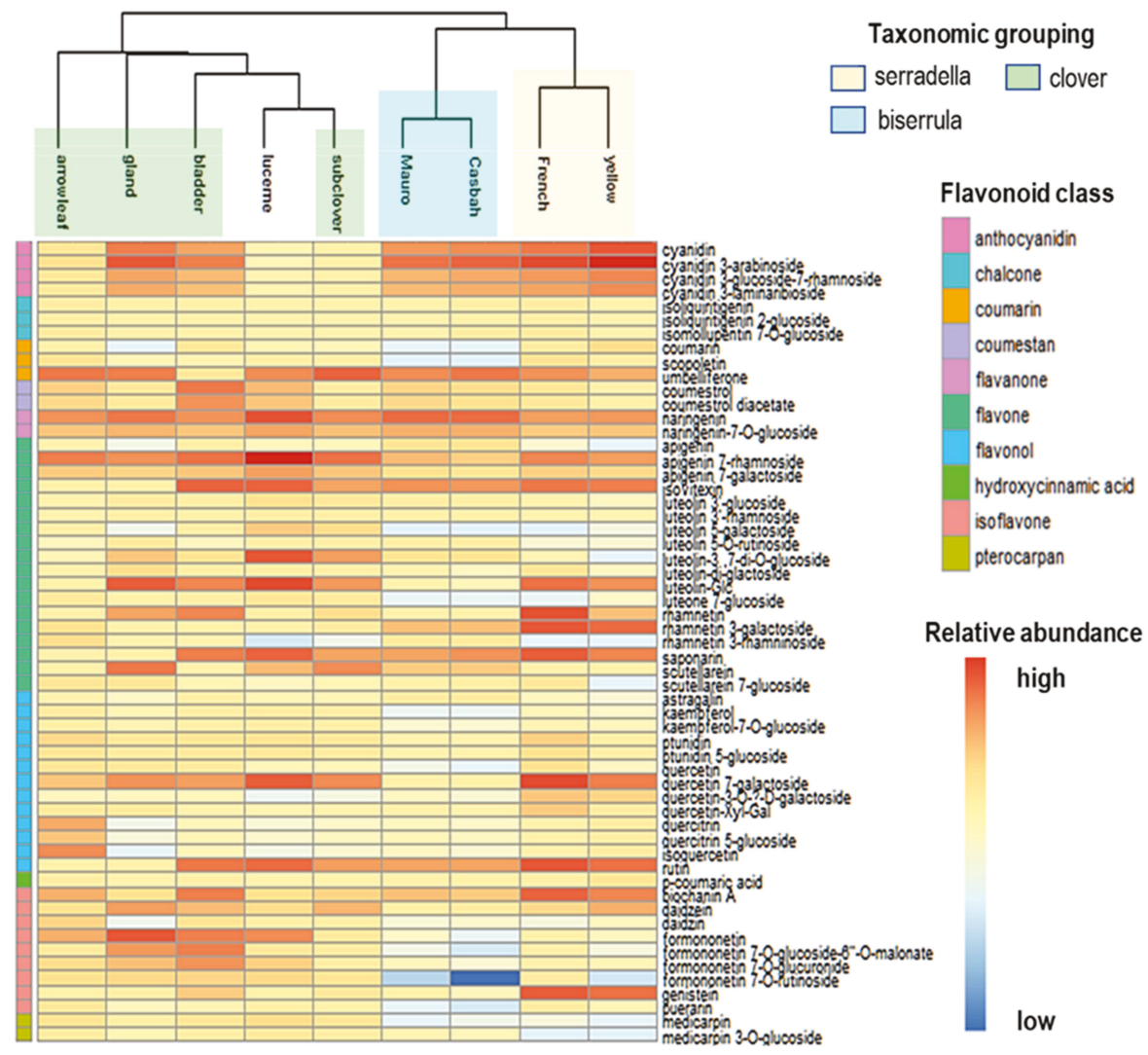

Figure 3. Hierarchal clustering of relative abundance of flavonoids, their glycosides, and coumestrol in leaf tissue in pasture legumes collected in 2016. Hierarchical clustering algorithm and Euclidean distance metric were used on normalized abundance using Mass Profiler Professional MPP (ver. 14.5 Agilent Santa Clara, CA, USA).

\section{Discussion}

This study represents the first published report on quantification and distribution of secondary products, including flavonoids, phenolics, proanthocyanidins, and phytoestrogens, in aerial tissues of several annual pasture species (Tables 1-5, Figure 3). The metabolic profiling and metabolomics analyses employed in this study provided a comprehensive overview of the specific metabolites pertaining to the biosynthesis and regulation of flavonoids and related metabolites, particularly those with adverse impacts on grazing livestock. The accurate identification of secondary metabolites was facilitated by the use of quantitative liquid chromatography quadrupole time-of-flight mass spectrometry (LC-MS-QToF), allowing their detection and quantification at low concentrations $\left(\mathrm{ng} \mathrm{kg}^{-1}\right)$, with compounds identified by comparison with authentic standards or annotated using the METLIN library of secondary metabolites. 
Table 4. Scientific, common, and cultivar names of annual pasture legumes evaluated in this study.

\begin{tabular}{ccc}
\hline Scientific Name & Common Name & Cultivar \\
\hline Trifolium vesiculosum Savi. & Arrowleaf clover & Cefalu \\
Biserrula pelecinus L. & Biserrula & Casbah, Mauro \\
Trifolium spumosum L. & Bladder clover & Bartolo \\
Ornithopus sativus Brot. & French serradella & Margarita \\
Trifolium glanduliferum Boiss. & Gland clover & Prima \\
Trifolium subterraneum L. & Subterranean clover & Seaton Park \\
Ornithopus compressus L. & Yellow serradella & Santorini \\
Medicago sativa L. & Lucerne & Aurora \\
\hline
\end{tabular}

Along with various isoflavonoids, coumestans are produced in large quantities by members of the Fabaceae, commonly known as legumes, and most of these metabolites contribute to plant defense. Plant-produced coumestans are known to be associated with various biological activities, many of which can be attributed to their function as phytoestrogens and polyphenols [42]. 4- methoxycoumestrol has quantified using coumestan as surrogate standard [43,44]. Our findings suggest that the greatest concentration of coumestans occurs in leaf and stem tissues, in contrast to floral tissues which exhibited only trace quantities. This is in agreement with previous findings in traditional pasture legumes [45]. In addition, our results suggest that consumption of pure stands of novel annual pasture legumes such as biserrula, French serradella, yellow serradella, and arrowleaf clover at physiological maturity would likely pose no threat to herd fertility given the lower levels of coumestans than threshold suggested in the literature. In direct contrast, gland and bladder clover exhibited concentrations of coumestans at levels above the suggested tolerance limit of $\approx 40 \mathrm{mg} \mathrm{kg}^{-1}$ in leaf tissues at approximately 48 and $70 \mathrm{mg} \mathrm{kg}^{-1}$, respectively.

Table 5. Phytoestrogens from pasture legume species identified in this study by LC-MS-QToF in positive ionization mode.

\begin{tabular}{cccc}
\hline Name & Molecular Formula & $\mathbf{M}+\mathbf{H}$ & Basis for Identification $^{\text {a }}$ \\
\hline Isoflavones & & & \\
Daidzein & $\mathrm{C}_{15} \mathrm{H}_{10} \mathrm{O}_{4}$ & 255.0652 & STD \\
Formononetin & $\mathrm{C}_{16} \mathrm{H}_{12} \mathrm{O}_{4}$ & 269.0808 & STD \\
Genistein & $\mathrm{C}_{15} \mathrm{H}_{10} \mathrm{O}_{5}$ & 271.0601 & STD \\
Coumestans & & & \\
Coumestrol & $\mathrm{C}_{15} \mathrm{H}_{8} \mathrm{O}_{5}$ & 269.0444 & STD \\
$4^{\prime}$-methoxycoumestrol & $\mathrm{C}_{16} \mathrm{H}_{10} \mathrm{O}_{5}$ & 283.0579 & AM \\
\hline
\end{tabular}

\footnotetext{
a Basis for identification codes: AM [43,44] — match to accurate mass/molecular formula and MS/MS spectra;
} STD - match to accurate mass and retention time of analytical standards.

The most prevalent traditional pasture legumes in Australia, lucerne and subterranean clover, have been reported to consistently accumulate higher concentrations of coumestans at flowering in both leaf and stem tissues, but recurrent selection by plant breeders has recently resulted in reduced levels in many commercial cultivars. Our results indicated that both lucerne and subterranean clover cultivars selected in this study produced substantial concentrations of both metabolites, leading to a total concentration of 52 and $38 \mathrm{mg} \mathrm{kg}^{-1}$, respectively; levels which may exceed livestock tolerance limits $\left(\approx 40 \mathrm{mg} \mathrm{kg}^{-1} \mathrm{DM}\right)$. Considerably higher concentrations of coumestans in leaf and stem tissues of gland and bladder clover suggest that these cultivars have experienced relatively limited genetic improvement through breeding at this stage (Angelo Loi, personal communication), and produce significant levels of secondary products that may adversely impact livestock health. This observation further supports the hypothesis that coumestans play a role in plant defense against herbivory when established in natural settings [46]. Our findings also imply that these cultivars should be avoided as sole sources of forage for grazing livestock. 
Naturally occurring isoflavones found predominately in the Fabaceae have been well described, largely based on their pharmacological activities, including estrogenic effects, in humans and animals. The isoflavonoids profiled in the present study demonstrated clear intra-species variation as well as variation associated with tissue type. Other researchers have also noted some chemical variance within the genus Trifolium [47]. The ability of plants to regulate production of secondary metabolites in response to biotic and abiotic stressors, including climate change, has been well documented [48-51] and recent studies have shown that these abilities extend to production of flavonoids $[12,13]$.

Qualitative and quantitative variation in flavonoids and associated phytoestrogens profiled in our study is an indicator of both species and tissue specific adaptations to the environment, resulting in modulation in the expression of associated genes [52]. Although recent pasture studies have suggested that isoflavones are highly abundant in Medicago and Trifolium spp. and are frequently highest before full-bloom [53], considerable variation in total concentration has been reported in mature Trifolium pratense pastures, with those surveyed ranging between 9000 and $27,000 \mathrm{mg} \mathrm{kg}^{-1}$ DM $[48,54,55]$. Variations in isoflavone concentration may have been due to environmentally induced responses, time of sampling, extraction efficiency and recovery, and previous analytical workflows employed to profile individual isoflavones [21]. We specifically attempted to address these issues noted in previous published works by conducting experimentation in uniform and replicated field sites, creating composite replicated samples for each pasture plot, and performing sampling at similar stages of physiological maturity in both 2016 and 2017 growing seasons. In addition, we utilized an automated high-pressure extraction device to rapidly and uniformly extract all samples, thereby performing uniform extraction across treatments and replicates, and reducing the possibility of plant to plant variation or inefficiencies associated with manual extraction protocols.

Several phytoestrogenic flavonoids, such as formononetin, the most abundant isoflavone detected followed by and genistein, were prevalent at physiological maturity (50\% flowering) in all pasture legumes. These observations are in agreement with previous reports pertaining to various Trifolium species [48,56-58]. Recent findings also suggested a greater abundance of phytoestrogens and isoflavonoids in leaf tissues when compared to stems and inflorescence [53]. Biosynthesis and subsequent distribution of isoflavones among leaf, stem, and floral tissues was impacted by cultivar, physiological growth stage, and climatic conditions under which plants were maintained [59]. In Trifolium pratense (perennial red clover), isoflavone concentrations were also found to be impacted by growth stage, with inflorescence tissue containing equivalent concentrations of isoflavones to those in leaf tissue in initial bloom stages, with subsequent declines in floral tissue as the crop entered full bloom stage [48]. The disproportionately high levels of formononetin in bladder clover observed in our study suggests that modulation of the phenylpropanoid pathway may, in fact, be species specific and is important in determination of terminal isoflavone accumulation in the pathway.

Only arrowleaf clover, gland clover, bladder clover, and subterranean clover exhibited total isoflavone concentrations above the livestock-safe threshold level of $\approx 280 \mathrm{mg} \mathrm{kg}^{-1}$ DM (Table 4) and therefore, all four of these Trifolium spp., could theoretically adversely impact the reproductive performance of livestock. Hashem et al., 2016 similarly reported that cattle fed solely on Trifolium alexandrinum (berseem clover) containing isoflavones at high concentrations of $\approx 280 \mathrm{~kg}^{-1} \mathrm{DM}$ exhibited hormonal disruption and reduced fertility, comparable to similar cattle grazing other species [18]. Interestingly, lambs grazing Trifolium pratense with high levels of isoflavones exhibited weight gain compared to cultivars low in isoflavones, but macromolecular interactions impacting plant nutrition and subsequent weight gain were not fully explored in that study [60]. Future studies investigating the production of phytoestrogens, including coumestans and isoflavones at various growth stages will be important to optimize seasonal grazing. 
Isoflavones are synthesized as part of the phenylpropanoid pathway (Figure 4), which has multiple branches common to both legumes and non-legumes. The phenylpropanoid pathway leads to the generation of lignins, anthocyanins, phytoalexins and flavonoids, including isoflavones, as a means of plant protection against stress or predation [29]. Encoding enzymes in this pathway are both developmentally and tissue-specifically regulated, and environmental stressors such as exposure to UV light, drought, prolonged cold, pathogen attack, and nutrient deficiency may also influence end products. Isoflavone synthase (IFS) is a key enzyme involved in the production of an array of isoflavones from naringenin, a common phenylpropanoid pathway intermediate [27]. Legumes can produce both daidzein from the intermediate compound liquiritigenin, and genistein from the intermediate naringenin chalcone in alternative branches of the phenylpropanoid pathway (Figure 4). The presence of multiple copies of enzymes such as chalcone synthase and IFS in species within the Fabaceae allows for the differential regulation of isoflavone biosynthesis in response to both developmental and environmental stimuli [61,62].

The flavonoid precursors and various isoflavones profiled in this study varied qualitatively and quantitatively among the annual pasture species investigated, suggesting that these species differ with respect to their metabolic dynamics and ability to regulate flavonoid biosynthesis. Daidzein and formononetin are produced from one branch of the phenylpropanoid pathway while genistein is produced from another branch. Both branches originate from $p$-coumaroyl-CoA, but the bias of the pathway towards one branch or the other is typically determined by the equilibrium between the enzymes chalcone synthase (CS) and chalcone reductase (CR) [27]. Greater production of formononetin in the pasture legume extracts profiled in this study, in contrast to the other isoflavones (daidzein and genistein) (Table 2), suggests that flavonoid biosynthesis in some pasture legumes is biased towards the branch terminating in production of formononetin as opposed to the alternate pathway ending in genistein. These findings are also consistent with previous observations [27,63]. Of note, close metabolite clustering and a higher concentration of genistein in both cultivars of biserrula and serradella species (as opposed to the more typical daidzein and formononetin) suggested potential overexpression of the gene encoding CS for the conversion of $p$-coumaroyl-CoA to naringenin chalcone in biserrula and serradella (Figure 4). This observation is consistent with recent results of a study investigating isoflavone profiles in food-related species in the Fabaceae family where higher genistein concentrations discriminated the genera Biserrula and Ornithopus from other members of the family [52]. 
CS-chalcone synthase
CR-chlacone reductase

Cl-chalcone isomerase

IFS-isoflavone synthase<smiles>O=C([O-])C=Cc1ccccc1</smiles>

trans-cinnamic acid<smiles>O=C(C=Cc1ccc(O)cc1)[Se]O</smiles>

p-coumaroyl-CoA

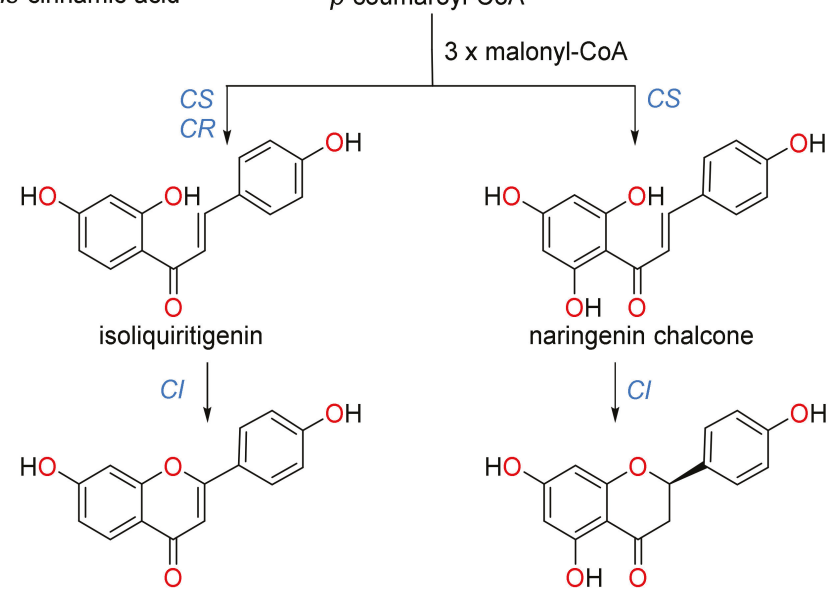

(S)-naringenin<smiles>O=C1c2c(O)cc(O)cc2OC(O)C1c1ccc(O)cc1</smiles>

2,4',5,7-tetrahydroxyisoflavanone

2,4',7-trihydroxyisoflavanone

DH-idehydratase

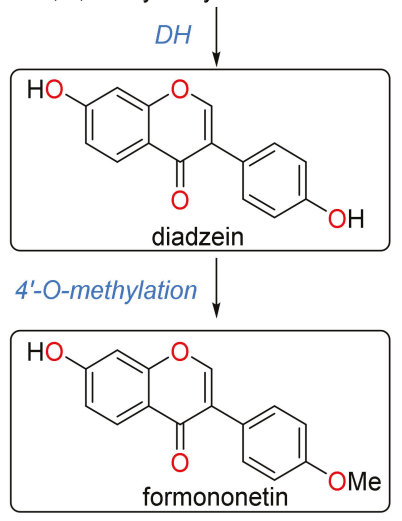

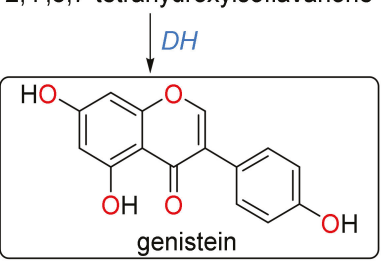

Figure 4. Biosynthetic pathway leading to the formation of various isoflavones including phytoestrogenic isoflavones (boxed) $[29,64]$.

Isoflavones tend to accumulate at highest concentrations at physiological maturity of the plant in Trifolium spp. as they commence and complete flowering [65]. To assess the impact of growth stage on the abundance of key phytoestrogens, we further profiled phytoestrogens in two common biserrula cultivars, first commercialized in Australia in 2001, at five different growth stages. Interestingly, we observed no significant differences in coumestan concentrations between the two cultivars, 
despite the fact that the cultivars were initially isolated from geographically separate locations in the Mediterranean. This suggests that the phenylproponoid pathway was highly conserved in this species, particularly in early growth stages, and was not affected by genotypic differences. However, the production of phytoestrogenic isoflavones varied significantly at $50 \%$ bloom and full bloom stages (Figure 2a,b). A previous study also described a significant difference in the accumulation of isoflavonoids (formononetin and biochanin A) in different cultivars of red clover, peaking at $50 \%$ bloom and full bloom stage [48]. Our results demonstrated that concentrations of all phytoestrogens assessed were lowest at senescence, and are in agreement with earlier observations in red clover [65]. As noted previously, the genes encoding enzymes for the biosynthesis of isoflavones are developmentally regulated and are frequently influenced by environmental stressors and various biotic factors $[29,49,62]$, some of which are likely experienced in southeastern Australia during a typical growing season. Breeding programs in annual pasture legumes can therefore exploit the bias of the phenylpropanoid pathway by targeted manipulation of genes involved in the biosynthesis of genistein and daidzein through ectopic expression of specific transcription factors over various growth stages [66].

Pasture legume samples collected from multi-year field trials were also subjected to quantification of extractable and bound total polyphenol content (TPC) and total proanthocyanidin content (TPAC) (Table 3). The important role of TPAC in pasture legumes in reducing herbivory by impacting palatability attributes has been elucidated previously [46]. This is the first study to report on both TPC and TPAC in annual self-regenerating pasture legumes at physiological maturity and our findings support previous reports suggesting a similar range for other related pasture legumes $[33,67,68]$.

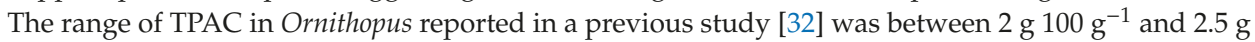
$100 \mathrm{~g}^{-1}$, which is also in agreement with the current study. However, proanthocyanidins in Onobrychis viciifolia (sainfoin) were reported to be up to 10 times higher than measured in this study and this discrepancy could be associated with species, methodology, or growth stage of sampling [69]. Of note is that previous studies using similar assays did not detect measurable TPAC in lucerne [70,71]. This could be due to differences in cultivar genetics and growth conditions; however, we also observed that lucerne exhibited the lowest extractable TPC and TPAC levels of all legumes surveyed. A high concentration of extractable TPAC was noted in biserrula cv. Casbah; both cultivars also exhibited high levels of bound TPAC in their cell walls. High TPAC levels suggest the potential for biserrula to limit pathogen and herbivore attack or reduced palatability to grazing livestock [72], but may potentially offer a cost-effective opportunity for this species to be integrated into multi-species mixtures to reduce parasite burden, an outcome suggested by recent replicated trials with biserrula on grazing sheep. At this time, specific bioactive proanthocyanidins in biserrula remain unidentified.

This study employed metabolic profiling approaches for identification and quantification of a number of more common secondary plant metabolites, particularly flavonoids. Our results clearly demonstrate that (a) this collection of annual pasture legumes produces a diverse array of flavonoids and other phytochemicals associated with plant defense, and in some cases less desirable phytoestrogens or proanthocyanidins and (b) the regulation of the biosynthesis of flavonoids and related metabolites such as coumestans through the phenylpropanoid pathway occurs at multiple branch sites and as reported could be impacted by elicitation in response to various biotic factors including predation and herbivory. Plants have thus developed various evolutionary adaptations for making regulatory decisions to produce an array of secondary metabolites which can provide them with multiple ecological benefits. In some cases, a single compound or related family of compounds can exhibit multiple biological functions in plants $[64,73]$. Despite significant differences in biological functions of various plant metabolites which are frequently concentration dependent, related compounds often share common biosynthetic pathways while others, including flavonoids, can originate from diverse biosynthetic pathways and precursors [51,74]. Evolutionarily, this chemical diversity or flexibility provides higher plants with a cost-effective strategy for further resource allocation or reallocation. As an example, our results suggest that both lucerne and gland clover have the ability to upregulate the synthesis of 
formononetin from daidzein while bladder clover evolved to upregulate biosynthesis of genistein from $2^{\prime}, 4^{\prime}, 5,7$ tetrahydroxy-isoflavone (Figure 4).

Our study results also support the hypothesis that high concentrations of key phytoestrogens, TPC and TPAC in annual pasture legumes are associated with flavonoid abundance. Interestingly, the relative abundance of total molecular features profiled through non-targeted metabolic profiling was highest in biserrula leaf tissues followed by serradella, while gland clover leaf tissue exhibited the fewest molecular features. In contrast, gland clover exhibited a higher concentration of flavonoids and their glycosides, suggesting that trade-offs in metabolite production and regulation occur in pasture legume species; i.e., if flavonoid synthesis is upregulated, then the expression of TPAC, TPC, and other polyphenols may be downregulated.

\section{Materials and Methods}

\subsection{Chemicals}

Analytical grade acetone, acetic acid, anhydrous sodium acetate, ethanol, hexane, hydrochloric acid, potassium chloride, sodium carbonate, and sulfuric acid were obtained from Chem Supply (Port Adelaide, South Australia, Australia). High pressure liquid chromatography (HPLC) grade methanol, acetonitrile, formic acid, and ammonium acetate as well as analytical standards (Folin-Ciocalteu reagent, vanillin, gallic acid, cyanidin-3-O-glucoside, (+)-catechin, coumestrol, formononetin, genistein, and naringenin) were purchased from Sigma Aldrich (Australia).

\subsection{Plant Material}

Monocultures of selected pasture legumes listed in Table 4 were established in 2016 and 2017 (Experiment 1 ) at the Charles Sturt University research farm in Wagga Wagga, NSW, Australia ( $35.04^{\circ} \mathrm{S}$, $147.36^{\circ} \mathrm{E}$ ) on a red sodosol soil [75]. Each planting was arranged as a randomized complete block design with five replications. Individual pasture legumes were established in plots by direct-seeding with a drill adapted for small-seeded legumes in late May to early June of each year, with plots measuring approximately $4 \times 20 \mathrm{~m}$. Following on from experimentation to assess legume establishment and performance [75], above-ground plant tissues (leaf, stem, and flower) were collected from established plots in the third week of October in 2016 and 2017 after $>50 \%$ flowering was achieved in each species. This corresponded to the growth stage in which peak concentrations of phytoestrogen have been reported in vegetative tissue [76]. An additional trial was also established at Charles Sturt University research farm in 2016 and 2017 which focused on chemical composition in two newly released biserrula cultivars (cv. Casbah and Mauro) using four replicates per cultivar (Experiment 2). In this case, composite fresh plant samples were collected from each plot (two sub-samples per cultivar) at pre-bud, pre-bloom, 50\% bloom, full bloom, and senescence between mid-July to mid-November in 2016 and 2017, approximately every three weeks. All plant material was placed on ice at collection and subsequently stored at $-20^{\circ} \mathrm{C}$ until extraction.

\subsection{LC-QToF-MS Analysis}

Metabolic profiling of plant tissues was performed using an Agilent 1290 Infinity LC system equipped with a quaternary pump, diode array detector (DAD), degasser, temperature-controlled column $\left(25^{\circ} \mathrm{C}\right)$, and cooled auto-sampler compartment $\left(4^{\circ} \mathrm{C}\right)$ which was coupled to an Agilent 6530 quadrupole time-of-flight (QToF) mass spectrometer (MS) with an Agilent Dual Jet Stream ionization source (Agilent Technologies, Melbourne, Australia). Full-scan mass spectra were acquired over an $\mathrm{m} / \mathrm{z}$ range of 100-1700 Da at a rate of two spectra/second in both positive and negative ion modes. Chromatographic separation was achieved using a reverse-phase $\mathrm{C}_{18}$ Poroshell column $(2.1 \times 100 \mathrm{~mm}$, $2.7 \mu \mathrm{m}$ particle size) (Agilent Technologies, Santa Clara, CA, USA) equipped with a $\mathrm{C}_{18}$ guard column $\left(2.1 \times 12.5 \mathrm{~mm}, 5 \mu \mathrm{m}\right.$ particle size) (Agilent Technologies, CA, USA) using a flow rate of $0.3 \mathrm{~mL} \mathrm{~min}^{-1}$. The column was equilibrated for $40 \mathrm{~min}$ prior to analysis. Separation was obtained with a gradient of 
solvent A, [water (Milli-Q, TKA-GenPure, Germany) + 0.1\% formic acid (LC-MS grade, LiChropur ${ }^{\circledR}$, 98-100\%, Sigma-Aldrich, MO, USA)] and solvent B [95\% HPLC-grade acetonitrile (RCI Labscan, Bangkok, Thailand) $+0.1 \%$ formic acid]. The solvent gradient was as follows: $5 \%$ B for $0.5 \mathrm{~min}$ increasing to $100 \%$ B over the next $16.5 \mathrm{~min}$, then holding at $100 \% \mathrm{~B}$ for $23 \mathrm{~min}$ and returning to $5 \%$ B from $23.1 \mathrm{~min}$ to $29 \mathrm{~min}$. The DAD monitored absorbance across a range of wavelengths from 210 to $635 \mathrm{~nm}$. Injection volume was $10 \mu \mathrm{L}$ for each sample. Nitrogen was used as the drying gas at $250{ }^{\circ} \mathrm{C}$ and a flow rate of $9 \mathrm{~L} \mathrm{~min}^{-1}$. Five biological replicates for each treatment were analyzed. Phytoestrogens (isoflavones and coumestans) identified in annual pasture legumes in the current study are summarized in Table 5.

\subsection{Extraction of Polyphenols}

Foliar and inflorescence samples $(1 \mathrm{~g})$ from each pasture legume were freeze-dried and ground manually to a fine powder $(\approx 1-2 \mathrm{~mm}$ size) using a mortar and pestle. Samples were extracted as described previously [77] with minor modifications. Briefly, ground foliar tissue was defatted with $n$-hexane, and residues were extracted three times with $20 \mathrm{~mL}$ of solvent (acetone:water:acetic acid 70:29.5:0.5 v/v/v). The three aliquots were combined, and solvent was removed using a rotary evaporator. The residue was lyophilized and reconstituted in $50 \%$ aqueous methanol to a final concentration of $1 \mathrm{~g} \mathrm{~mL}^{-1}$ for storage at $-20^{\circ} \mathrm{C}$ until required.

\subsection{Quantification of Total Polyphenol Content}

Total free phenolic content was determined as described previously [78] with minor alterations. Briefly, $125 \mu \mathrm{L}$ of the extract was mixed with $125 \mu \mathrm{L}$ of Folin-Ciocalteu reagent and $500 \mu \mathrm{L}$ of deionized water and incubated in the dark for $6 \mathrm{~min}$. The solution was neutralized by adding $1.5 \mathrm{~mL} 7 \%$ aqueous sodium carbonate solution and further incubated in the dark for $90 \mathrm{~min}$, after which absorbance was measured at $725 \mathrm{~nm}$ using a UV/Vis spectrophotometer (FLUOstar Omega, BMG Labtech, Offenburg,

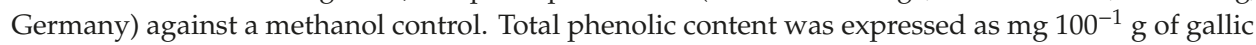
acid equivalent (GAE). The experiment was repeated twice with three technical replicates.

\subsection{Quantification of Total Proanthocyanidin Content (TPAC)}

TPAC was quantified using a vanillin assay as described previously [79,80]. Briefly, $0.2 \mathrm{~mL}$ of extract was added to $0.5 \mathrm{~mL}$ of $1 \%(w / v)$ vanillin in methanol and $0.5 \mathrm{~mL}$ of $25 \%$ sulfuric acid in methanol. The extract was thoroughly blended using a vortex mixer and placed in a water bath at $37^{\circ} \mathrm{C}$ for $15 \mathrm{~min}$. Absorbance was measured at room temperature at a wavelength of $500 \mathrm{~nm}$.

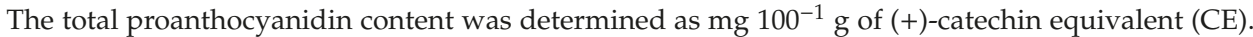
The experiment was performed with three technical replicates and the experiment was repeated twice.

\subsection{Statistical Analysis}

A matrix of molecular features characterized by mass to charge ratio $(\mathrm{m} / \mathrm{z})$ and retention time (RT) was generated using MassHunter Workstation Qualitative software version B07.00, MassHunter Profinder (version B.08.00), Mass Profiler Professional (MPP version 14.5) and Personal Compound Database Library (PCDL) (Agilent Technologies, CA, USA). Molecular features were extracted and binned/aligned using parameters as follows: peak height $\geq 10,000$ counts, compound ion count threshold of two or more ions, compound alignment tolerances $0.00 \%+0.15 \mathrm{~min}$ for RT and $20.00 \mathrm{mg} \mathrm{kg}^{-1} \pm$ $2.00 \mathrm{mDa}$ for mass using Profinder. Molecular features which were present only in three samples out of five were included in the analysis. Compounds were tentatively identified by matching molecular entities with PCDL entries having similar accurate mass, RT, and mass spectra (generated from analytical standards) where possible and the METLIN metabolomics database (version B 07.00, Agilent Technologies, CA, USA) otherwise. All descriptive statistical analyses were performed using Statistix (STATISTIX software, version 4.1; Analytical Software, Tallahassee, FL, USA) and standard deviations were calculated and reported where possible. 


\section{Conclusions}

In conclusion, this study has extended our understanding of secondary metabolism associated with the biosynthesis of phytoestrogens and proanthocyanidins in annual and perennial legumes. Metabolic profiling performed with a variety of hardseeded annual pasture legumes demonstrated that phytoestrogens quantified in this study were at concentrations insufficient to pose negative impact on livestock production with the exception of gland clover, bladder clover, and lucerne. Results of the study also suggest that annual hardseeded pasture legumes of Mediterranean origin offer viable and sustainable alternative pasture options for mixed farming systems of southeastern New South Wales. In the future, the use of an integrated experimental approach including multi-omics platforms could also potentially provide deeper insights into pathway dynamics and regulation of associated genes important in the production of secondary metabolites in pasture legumes. Our findings along with those of Butkut et al., 2018 suggest strong potential to improve legume-based forage quality through recurrent selection or engineering for species- or cultivar-specific phytochemicals [53]. In addition, optimization of livestock management to reduce health and reproductive issues by selective grazing through timing of animal movement, manipulation of plant growth stage at harvest, and appropriate selection of pasture species mixtures is also warranted.

Supplementary Materials: The following are available online at http://www.mdpi.com/2218-1989/10/7/267/s1, Figure S1: Hierarchal clustering of molecular features in leaf tissue of annual pasture legumes acquired using UHPLC-QToF-MS in positive and negative mode. Hierarchical clustering algorithm and Euclidean distance metric were used on normalized abundance using MPP (ver. 14.5 Agilent Santa Clara, CA, USA), Figure S2: Hierarchal clustering of relative abundance of flavonoids, their glycosides, and coumestrol in inflorescence tissue in pasture legumes collected in 2016. Hierarchical clustering algorithm and Euclidean distance metric were used on normalized abundance using $\mathrm{R}$ package.

Author Contributions: Conceptualization, S.L., P.A.W. and L.A.W.; Methodology, S.L., J.W.P., P.A.W. and L.A.W.; Formal analysis, S.L., P.A.W. and L.A.W.; Writing, S.L., S.G., R.A.B., P.A.W. and L.A.W.; Original draft preparation, S.L.; Review and editing, S.G., R.A.B., P.A.W., J.W.P. and L.A.W. Funding acquisition, J.W.P. and L.A.W. All authors have read and agreed to the published version of the manuscript.

Funding: This research was funded by Meat and Livestock Australia (MLA) (Grant: B.WEE.0146 awarded to L.A. Weston and J. Piltz).

Acknowledgments: The authors acknowledge funding support from Meat and Livestock Australia (MLA) (Grant: B.WEE.0146 awarded to L.A. Weston and J. Piltz) for field trials and the Australian Center for International Agricultural Research (ACIAR) for sponsoring the PhD fellowship awarded to S. Latif for studies associated with this project. The authors also wish to acknowledge Ulrike Mathesius of The Australian National University (Acton, ACT, Australia) for providing analytical standards of flavonoids used in this study, and Willian Brown, Graeme Heath, and Simon Flinn for their support in data collection.

Conflicts of Interest: Authors declare no conflict of interest, there is no connection between Plus 3 Australia Pty Ltd. and the subject of this manuscript.

\section{References}

1. Whitbread, A.M.; Hall, C.A.; Pengelly, B.C. A novel approach to planting grasslegume pastures in the mixed farming zone of southern inland Queensland, Australia. Crop Pasture Sci. 2009, 60, 1147-1155. [CrossRef]

2. Hackney, B.; Dear, B.; Li, G.; Rodham, C.; Tidd, J. Current and future use of pasture legumes in central and southern NSW-Results of a farmer and advisor survey. In Proceedings of the 14th Australian Society of Agronomy Conference, Adelaide, Australia, 21-25 September 2008.

3. Loi, A.; Howieson, J.G.; Nutt, B.J.; Carr, S.J. A second generation of annual pasture legumes and their potential for inclusion in Mediterranean-type farming systems. Aust. J. Exp. Agric. 2005, 45, 289-299. [CrossRef]

4. Burggraaf, V.; Waghorn, G.; Woodward, S.; Thom, E. Effects of condensed tannins in white clover flowers on their digestion in vitro. Anim. Feed Sci. Technol. 2008, 142, 44-58. [CrossRef]

5. Hall, D.; Wolfe, E.; Cullis, B. Performance of breeding ewes on lucerne-subterranean clover pastures. Aust. J. Exp. Agric. 1985, 25, 758-765. [CrossRef]

6. Humphries, A. Future applications of lucerne for efficient livestock production in southern Australia. Crop Pasture Sci. 2013, 63, 909-917. [CrossRef] 
7. Mulholland, J. Animal production from lucerne-based pastures. In Realising the Potential of Pastures, the Proceedings of the 16th Riverina Outlook Conference, Wagga Wagga, Australia, 15 July, 1987; Riverina-Murray Institute of Higher Education: Wagga Wagga, Australia, 1987; pp. 57-66.

8. Loi, A.; Howieson, J.H.; Carr, S.J. Register of Australian herbage plant cultivars. Biserrula pelecinus L. (biserrula) cv. Casbah. Aust. J. Exp. Agric. 2001, 41, 841-842. [CrossRef]

9. Hackney, B.; Nutt, B.; Loi, A.; Yates, R.; Quinn, J.; Piltz, J.; Jenkins, J.; Weston, L.; O’Hare, M.; Butcher, A. "On-demand" hardseeded pasture legumes-a paradigm shift in crop-pasture rotations for southern Australian mixed farming systems. In Building Productive, Diverse and Sustainable Landscapes, Proceedings of the 17th Australian Agronomy Conference, Hobart, Australia, 20-24 September 2015; Acuña, T., Moeller, C., Parsons, D., Harrison, M., Eds.; Australian Agronomy society: Hobart, Australia, 2015.

10. Dear, B.; Ewing, M.A. The search for new pasture plants to achieve more sustainable production systems in southern Australia. Aust. J. Exp. Agric. 2008, 48, 387-396. [CrossRef]

11. Latif, S.; Gurusinghe, S.; Weston, P.A.; Brown, W.B.; Quinn, J.C.; Piltz, J.W.; Weston, L.A. Performance and weed-suppressive potential of selected pasture legumes against annual weeds in south-eastern Australia. Crop Pasture Sci. 2019, 70, 147-158. [CrossRef]

12. Mathesius, U. Flavonoid Functions in Plants and Their Interactions with Other Organisms. Plants 2018, 7, 30. [CrossRef]

13. Weston, L.A.; Mathesius, U. Flavonoids: Their structure, biosynthesis and role in the rhizosphere, including allelopathy. J. Chem. Ecol. 2013, 39, 283-297. [CrossRef]

14. Hassan, S.; Mathesius, U. The role of flavonoids in root-rhizosphere signalling: Opportunities and challenges for improving plant-microbe interactions. J. Exp. Bot. 2012, 63, 3429-3444. [CrossRef]

15. Reed, K.F.M. Fertility of herbivores consuming phytoestrogen-containing Medicago and Trifolium species. Agriculture 2016, 6, 35. [CrossRef]

16. Tiwari, B.K.; Brunton, N.P.; Brennan, C. Handbook of Plant Food Phytochemicals: Sources, Stability and Extraction; John Wiley \& Sons: Hoboken, NJ, USA, 2013.

17. Wocławek-Potocka, I.; Mannelli, C.; Boruszewska, D.; Kowalczyk-Zieba, I.; Waśniewski, T.; Skarżyński, D.J. Diverse effects of phytoestrogens on the reproductive performance: Cow as a model. Int. J. Endocrinol. 2013, 2013, 650984. [CrossRef] [PubMed]

18. Hashem, N.; El-Azrak, K.; Sallam, S. Hormonal concentrations and reproductive performance of Holstein heifers fed Trifolium alexandrinum as a phytoestrogenic roughage. Anim. Reprod. Sci. 2016, 170, 121-127. [CrossRef] [PubMed]

19. Hloucalová, P.; Skládanka, J.; Horký, P.; Klejdus, B.; Pelikán, J.; Knotová, D. Determination of Phytoestrogen Content in Fresh-Cut Legume Forage. Animals 2016, 6, 43. [CrossRef]

20. Burton, J.; Wells, M. The effect of phytoestrogens on the female genital tract. J. Clin. Pathol. 2002, 55, 401-407. [CrossRef] [PubMed]

21. Cvejić, J.; Bursać, M.; Atanacković, M. Phytoestrogens: “Estrogene-Like" Phytochemicals. In Studies in Natural Products Chemistry; Elsevier: Amsterdam, The Netherlands, 2012; Volume 38, pp. 1-35.

22. Bickoff, E.; Booth, A.; Lyman, R.; Livingston, A.; Thompson, C.; Deeds, F. Coumestrol, a new estrogen isolated from forage crops. Science 1957, 126, 969-970. [CrossRef] [PubMed]

23. Mostrom, M.; Evans, T.J. Phytoestrogens; Elsevier: Amsterdam, The Netherlands, 2011; pp. 707-722.

24. Woclawek-Potocka, I.; Okuda, K.; Acosta, T.; Korzekwa, A.; Pilawski, W.; Skarzynski, D. Phytoestrogen metabolites are much more active than phytoestrogens themselves in increasing prostaglandin F $2 \alpha$ synthesis via prostaglanin F2 $\alpha$ synthase-like 2 stimulation in bovine endometrium. Prostaglandins Other Lipid Mediat. 2005, 78, 202-217. [CrossRef]

25. Lookhart, G. Analysis of coumestrol, a plant estrogen, in animal feeds by high-performance liquid chromatography. J. Agric. Food Chem. 1980, 28, 666-667. [CrossRef]

26. Dixon, R.A.; Achnine, L.; Kota, P.; Liu, C.J.; Reddy, M.S.; Wang, L. The phenylpropanoid pathway and plant defence-A genomics perspective. Mol. Plant Pathol. 2002, 3, 371-390. [CrossRef]

27. Yu, O.; Jung, W.; Shi, J.; Croes, R.A.; Fader, G.M.; McGonigle, B.; Odell, J.T. Production of the Isoflavones Genistein and Daidzein in Non-Legume Dicot and Monocot Tissues. Plant Physiol. 2000, 124, 781-794. [CrossRef] [PubMed] 
28. Gupta, O.P.; Dahuja, A.; Sachdev, A.; Jain, P.K.; Kumari, S.; Praveen, S. Cytosine Methylation of Isoflavone Synthase Gene in the Genic Region Positively Regulates Its Expression and Isoflavone Biosynthesis in Soybean Seeds. DNA Cell Biol. 2019, 38, 510-520. [CrossRef] [PubMed]

29. Dixon, R.A.; Harrison, M.J.; Paiva, N.L. The isoflavonoid phytoalexin pathway from enzymes to genes to transcription factors. Physiol. Plant. 1995, 93, 385-392. [CrossRef]

30. Piluzza, G.; Sulas, L.; Bullitta, S. Tannins in forage plants and their role in animal husbandry and environmental sustainability: A review. Grass Forage Sci. 2014, 69, 32-48. [CrossRef]

31. Salami, S.A.; Luciano, G.; O'Grady, M.N.; Biondi, L.; Newbold, C.J.; Kerry, J.P.; Priolo, A. Sustainability of feeding plant by-products: A review of the implications for ruminant meat production. Anim. Feed Sci. Technol. 2019, 251, 37-55. [CrossRef]

32. Aerts, R.J.; Barry, T.N.; McNabb, W.C. Polyphenols and agriculture: Beneficial effects of proanthocyanidins in forages. Agric. Ecosyst. Environ. 1999, 75, 1-12. [CrossRef]

33. Douglas, G.; Stienezen, M.; Waghorn, G.; Foote, A.; Purchas, R. Effect of condensed tannins in birdsfoot trefoil (Lotus corniculatus) and sulla (Hedysarum coronarium) on body weight, carcass fat depth, and wool growth of lambs in New Zealand. N. Z. J. Agric. Res. 1999, 42, 55-64. [CrossRef]

34. Ramírez-Restrepo, C.; Barry, T. Alternative temperate forages containing secondary compounds for improving sustainable productivity in grazing ruminants. Anim. Feed Sci. Technol. 2005, 120, 179-201. [CrossRef]

35. Kingston-Smith, A.H.; Edwards, J.E.; Huws, S.A.; Kim, E.J.; Abberton, M. Plant-based strategies towards minimising 'livestock's long shadow'. Proc. Nutr. Soc. 2010, 69, 613-620. [CrossRef]

36. Tzamaloukas, O.; Athanasiadou, S.; Kyriazakis, I.; Huntley, J.; Jackson, F. The effect of chicory (Cichorium intybus) and sulla (Hedysarum coronarium) on larval development and mucosal cell responses of growing lambs challenged with Teladorsagia circumcincta. Parasitology 2006, 132, 419-426. [CrossRef]

37. Hoste, H.; Torres-Acosta, J.; Quijada, J.; Chan-Perez, I.; Dakheel, M.; Kommuru, D.; Mueller-Harvey, I.; Terrill, T. Interactions between nutrition and infections with Haemonchus contortus and related gastrointestinal nematodes in small ruminants. In Advances in Parasitology; Elsevier: Amsterdam, The Netherlands, 2016; Volume 93, pp. 239-351.

38. Min, B.R.; Barry, T.N.; Attwood, G.T.; McNabb, W.C. The effect of condensed tannins on the nutrition and health of ruminants fed fresh temperate forages: A review. Anim. Feed Sci. Technol. 2003, 106, 3-19. [CrossRef]

39. Mueller-Harvey, I.; Bee, G.; Dohme-Meier, F.; Hoste, H.; Karonen, M.; Kölliker, R.; Lüscher, A.; Niderkorn, V.; Pellikaan, W.F.; Salminen, J.-P.; et al. Benefits of condensed tannins in forage legumes fed to ruminants: Importance of structure, concentration, and diet composition. Crop. Sci. 2019, 59, 861. [CrossRef]

40. Weston, L.A.; Skoneczny, D.; Weston, P.A.; Weidenhamer, J.D. Metabolic profiling: An overview-New approaches for the detection and functional analysis of biologically active secondary plant products. J. Allelochem. Interact. 2015, 1, 15-27.

41. Williamson, L.N.; Bartlett, M.G. Quantitative liquid chromatography/time-of-flight mass spectrometry. Biomed. Chromatogr. 2007, 21, 567-576. [CrossRef]

42. Nehybova, T.; Smarda, J.; Benes, P. Plant coumestans: Recent advances and future perspectives in cancer therapy. Anti Cancer Agents Med. Chem. 2014, 14, 1351-1362. [CrossRef] [PubMed]

43. Christ, B.; Hauenstein, M.; Hörtensteiner, S. A liquid chromatography-mass spectrometry platform for the analysis of phyllobilins, the major degradation products of chlorophyll in Arabidopsis thaliana. Plant. J. 2016, 88, 505-518. [CrossRef]

44. Jiang, H.; Liao, X.; Wood, C.M.; Xiao, C.-W.; Feng, Y.-L. A robust analytical method for measurement of phytoestrogens and related metabolites in serum with liquid chromatography tandem mass spectrometry. J. Chromatogr. B 2016, 1012-1013, 106-112. [CrossRef]

45. Smith, J.; Jagusch, K.; Brunswick, L.; Kelly, R. Coumestans in lucerne and ovulation in ewes. N. Z. J. Agric. Res. 1979, 22, 411-416. [CrossRef]

46. Molyneux, R.J.; Ralphs, M.H. Plant toxins and palatability to herbivores. Rangel. Ecol. Manag. J. Range Manag. Arch. 1992, 45, 13-18. [CrossRef]

47. Oleszek, W.; Stochmal, A.; Janda, B. Concentration of isoflavones and other phenolics in the aerial parts of Trifolium species. J. Agric. Food Chem. 2007, 55, 8095-8100. [CrossRef]

48. Sivesind, E.; Seguin, P. Effects of the environment, cultivar, maturity, and preservation method on red clover isoflavone concentration. J. Agric. Food Chem. 2005, 53, 6397-6402. [CrossRef] [PubMed] 
49. Mahmood, K.; Khan, M.B.; Song, Y.Y.; Ijaz, M.; Luo, S.M.; Zeng, R.S. UV-irradiation enhances rice allelopathic potential in rhizosphere soil. Plant Growth Regul. 2013, 71, 21-29. [CrossRef]

50. Iannucci, A.; Fragasso, M.; Platani, C.; Papa, R. Plant growth and phenolic compounds in the rhizosphere soil of wild oat (Avena fatua L.). Front. Plant Sci. 2013, 4. [CrossRef] [PubMed]

51. Einhellig, F.A. Allelopathy-A natural protection, allelochemicals. In Handbook of Natural Pesticides: Methods; CRC Press: Boca Raton, FL, USA, 2018; pp. 161-200.

52. Visnevschi-Necrasov, T.; Barreira, J.C.; Cunha, S.C.; Pereira, G.; Nunes, E.; Oliveira, M.B.P. Phylogenetic insights on the isoflavone profile variations in Fabaceae spp.: Assessment through PCA and LDA. Food Res. Int. 2015, 76, 51-57. [CrossRef]

53. Butkutè, B.; Padarauskas, A.; Cesevičienè, J.; Taujenis, L.; Norkevičienè, E. Phytochemical composition of temperate perennial legumes. Crop. Pasture Sci. 2018, 69, 1020-1030. [CrossRef]

54. Tsao, R.; Papadopoulos, Y.; Yang, R.; Young, J.C.; McRae, K. Isoflavone profiles of red clovers and their distribution in different parts harvested at different growing stages. J. Agric. Food Chem. 2006, 54, 5797-5805. [CrossRef]

55. Wu, Q.; Wang, M.; Simon, J.E. Determination of isoflavones in red clover and related species by high-performance liquid chromatography combined with ultraviolet and mass spectrometric detection. J. Chromatogr. 2003, 1016, 195-209. [CrossRef]

56. Butkutè, B.; Lemežienè, N.; Dabkevičienè, G.; Jakštas, V.; Vilčinskas, E.; Janulis, V. Source of variation of isoflavone concentrations in perennial clover species. Pharmacogn. Mag. 2014, 10, S181. [CrossRef]

57. Zgórka, G. Ultrasound-assisted solid-phase extraction coupled with photodiode-array and fluorescence detection for chemotaxonomy of isoflavone phytoestrogens in Trifolium L. (Clover) species. J. Sep. Sci. 2009, 32, 965-972.

58. Ramos, G.P.; Dias, P.M.; Morais, C.B.; Fröehlich, P.E.; Dall'Agnol, M.; Zuanazzi, J.A. LC determination of four isoflavone aglycones in red clover (Trifolium pratense L.). Chromatographia 2008, 67, 125-129. [CrossRef]

59. Saviranta, N.M.; Anttonen, M.J.; von Wright, A.; Karjalainen, R.O. Red clover (Trifolium pratense L.) isoflavones: Determination of concentrations by plant stage, flower colour, plant part and cultivar. J. Sci. Food Agric. 2008, 88, 125-132. [CrossRef]

60. Bennetau-Pelissero, C. Risks and benefits of phytoestrogens: Where are we now? Curr. Opin. Clin. Nutr. Metab. Care 2016, 19, 477-483. [CrossRef] [PubMed]

61. Dubery, I.A.; Mienie, C. Tissue-specific expression of the chalcone synthase multigene family in Phaseolus vulgaris: Development of a RT-PCR method for the expression profiling of the chs isogenes. J. Plant Physiol. 2001, 158, 115-120. [CrossRef]

62. Martin, C. Structure, function, and regulation of the chalcone synthase. In International Review of Cytology; Elsevier: Amsterdam, The Netherlands, 1993; Volume 147, pp. 233-284.

63. Jung, W.; Yu, O.; Lau, S.-M.C.; O'Keefe, D.P.; Odell, J.; Fader, G.; McGonigle, B. Identification and expression of isoflavone synthase, the key enzyme for biosynthesis of isoflavones in legumes. Nat. Biotechnol. 2000, 18, 208. [CrossRef] [PubMed]

64. Dixon, R.A.; Liu, C.; Jun, J.H. Metabolic engineering of anthocyanins and condensed tannins in plants. Curr. Opin. Biotechnol. 2013, 24, 329-335. [CrossRef]

65. McMurray, C.H.; Laidlaw, A.S.; McElroy, M. The effect of plant development and environment on formononetin concentration in red clover (Trifolium pratense L.). J. Sci. Food Agric. 1986, 37, 333-340. [CrossRef]

66. Kim, H.-K.; Jang, Y.-H.; Baek, I.-S.; Lee, J.-H.; Park, M.J.; Chung, Y.-S.; Chung, J.-I.; Kim, J.-K. Polymorphism and expression of isoflavone synthase genes from soybean cultivars. Mol. Cells 2005, 19, 67-73.

67. Jonker, A.; Peiqiang, Y. The Role of Proanthocyanidins Complex in Structure and Nutrition Interaction in Alfalfa Forage. Int. J. Mol. Sci. 2016, 17, 793. [CrossRef]

68. Wang, Y.; McAllister, T.A.; Acharya, S. Condensed tannins in sainfoin: Composition, concentration, and effects on nutritive and feeding value of sainfoin forage. Crop. Sci. 2015, 55, 13-22. [CrossRef]

69. Azuhnwi, B.N.; Boller, B.; Dohme-Meier, F.; Hess, H.D.; Kreuzer, M.; Stringano, E.; Mueller-Harvey, I. Exploring variation in proanthocyanidin composition and content of sainfoin (Onobrychis viciifolia). J. Sci. Food Agric. 2013, 93, 2102-2109. [CrossRef] 
70. Terrill, T.H.; Rowan, A.M.; Douglas, G.B.; Barry, T.N. Determination of extractable and bound condensed tannin concentrations in forage plants, protein concentrate meals and cereal grains. J. Sci. Food Agric. 1992, 58, 321-329. [CrossRef]

71. Jackson, F.S.; McNabb, W.C.; Barry, T.N.; Foo, Y.L.; Peters, J.S. The Condensed Tannin Content of a Range of Subtropical and Temperate Forages and the Reactivity of Condensed Tannin with Ribulose- 1,5-bis-phosphate Carboxylase (Rubisco) Protein. J. Sci. Food Agric. 1996, 72, 483-492. [CrossRef]

72. Johnson, M.T.; Smith, S.D.; Rausher, M.D. Plant sex and the evolution of plant defenses against herbivores. Proc. Natl. Acad. Sci. USA 2009, 106, 18079-18084. [CrossRef] [PubMed]

73. Sumner, L.; Paiva, N.; Dixon, R.; Geno, P. HPLC-Continuous-flow liquid secondary ion mass spectrometry of flavonoid glycosides in leguminous plant extracts. J. Mass Spectrom. 1996, 31, 472-485. [CrossRef]

74. Neilson, E.H.; Goodger, J.Q.D.; Woodrow, I.E.; Møller, B.L. Plant chemical defense: At what cost? Trends Plant Sci. 2013, 18, 250-258. [CrossRef]

75. Latif, S.; Gurusinghe, S.; Weston, P.A.; Quinn, J.C.; Piltz, J.W.; Weston, L.A. Metabolomic approaches for the identification of flavonoids associated with weed suppression in selected Hardseeded annual pasture legumes. Plant Soil 2019, 447, 199-218. [CrossRef]

76. Dedio, W.; Clark, K. Biochanin A and formononetin content in red clover varieties at several maturity stages. Can. J. Plant Sci. 1968, 48, 175-181. [CrossRef]

77. Zhou, Z.; Chen, X.; Zhang, M.; Blanchard, C. Phenolics, flavonoids, proanthocyanidin and antioxidant activity of brown rice with different pericarp colors following storage. J. Stored Prod. Res. 2014, 59, 120-125. [CrossRef]

78. Qiu, Y.; Liu, Q.; Beta, T. Antioxidant properties of commercial wild rice and analysis of soluble and insoluble phenolic acids. Food Chem. 2010, 121, 140-147. [CrossRef]

79. Min, B.; McClung, A.M.; Chen, M.H. Phytochemicals and antioxidant capacities in rice brans of different color. J. Food Sci. 2011, 76, C117-C126. [CrossRef]

80. Sun, B.; Ricardo-da-Silva, J.M.; Spranger, I. Critical factors of vanillin assay for catechins and proanthocyanidins. J. Agric. Food Chem. 1998, 46, 4267-4274. [CrossRef]

(C) 2020 by the authors. Licensee MDPI, Basel, Switzerland. This article is an open access article distributed under the terms and conditions of the Creative Commons Attribution (CC BY) license (http://creativecommons.org/licenses/by/4.0/). 


\title{
Effects of Cyclic High Ambient Temperature and Dietary Supplementation of Orotic Acid, a Pyrimidine Precursor, on Plasma and Muscle Metabolites in Broiler Chickens
}

\author{
Saki Shimamoto ${ }^{1,2}$, Kiriko Nakamura ${ }^{1}$, Shozo Tomonaga ${ }^{3}$, Satoru Furukawa ${ }^{4}$, Akira Ohtsuka ${ }^{1}$ \\ and Daichi Ijiri ${ }^{1, *}$ \\ 1 Department of Agricultural Sciences and Natural Resources, Kagoshima University, Korimoto, \\ Kagoshima 890-0065, Japan; shimamoto@agr.niigata-u.ac.jp (S.S.); k5023091@kadai.jp (K.N.); \\ ohtsuka@chem.agri.kagoshima-u.ac.jp (A.O.) \\ 2 Graduate School of Science and Technology, Niigata University, Nishi-ku, Niigata 950-2181, Japan \\ 3 Division of Applied Biosciences, Graduate School of Agriculture, Kyoto University, Sakyo-ku, \\ Kyoto 606-8502, Japan; tomonaga.shozo.4n@kyoto-u.ac.jp \\ 4 Furukawa Research Office Co. Ltd., Setagaya-ku, Tokyo 157-0066, Japan; furukawa@furukawa-res.co.jp \\ * Correspondence: ijiri@chem.agri.kagoshima-u.ac.jp; Tel.: +81-99-285-8654
}

Received: 7 April 2020; Accepted: 10 May 2020; Published: 12 May 2020

\begin{abstract}
The aim of this study was to evaluate the effects of high ambient temperature (HT) and orotic acid supplementation on the plasma and muscle metabolomic profiles in broiler chickens. Thirty-two 14-day-old broiler chickens were divided into four treatment groups that were fed diets with or without $0.7 \%$ orotic acid under thermoneutral $\left(25 \pm 1{ }^{\circ} \mathrm{C}\right)$ or cyclic HT $\left(35 \pm 1{ }^{\circ} \mathrm{C}\right.$ for $8 \mathrm{~h} /$ day) conditions for 2 weeks. The chickens exposed to HT had higher plasma malondialdehyde concentrations, suggesting an increase in lipid peroxidation, which is alleviated by orotic acid supplementation. The HT environment also affected the serine, glutamine, and tyrosine plasma concentrations, while orotic acid supplementation affected the aspartic acid, glutamic acid, and tyrosine plasma concentrations. Untargeted gas chromatography-triple quadrupole mass spectrometry (GC-MS/MS)-based metabolomics analysis identified that the HT affected the plasma levels of metabolites involved in purine metabolism, ammonia recycling, pyrimidine metabolism, homocysteine degradation, glutamate metabolism, urea cycle, $\beta$-alanine metabolism, glycine and serine metabolism, and aspartate metabolism, while orotic acid supplementation affected metabolites involved in pyrimidine metabolism, $\beta$-alanine metabolism, the malate-aspartate shuttle, and aspartate metabolism. Our results suggest that cyclic HT affects various metabolic processes in broiler chickens, and that orotic acid supplementation ameliorates HT-induced increases in lipid peroxidation.
\end{abstract}

Keywords: chickens; heat stress; lipid peroxidation; metabolomics; orotic acid

\section{Introduction}

Ambient temperatures above the thermoneutral zone cause environmental heat stress. Chickens are more vulnerable to heat stress than other domestic animals, because they lack sweat glands and have higher body temperatures [1,2]. Under such high ambient temperature (HT) conditions, the generation of reactive oxygen species increases in various body tissues as the heat load increases [3]. This results in a range of physiological changes that can severely depress growth performance and meat yield $[4,5]$ and reduce meat quality, accompanied by increasing lipid peroxidation levels [6,7] and drip loss [8,9], decreasing the share force value $[10,11]$ and changing meat color [12-14]. 
Gas chromatography-mass spectrometry (GC-MS)-based, untargeted metabolomics analysis has been used to comprehensively characterize the effects of HT on the physiology of chickens [15], and has found that the plasma levels of 38 metabolites changed significantly when chickens were exposed to chronic heat $\left(38^{\circ} \mathrm{C}\right)$ for 4 days. These altered metabolites indicated that such heat exposure affected 35 metabolic processes, including the sulfur amino acid metabolic pathway, the kynurenine pathway in tryptophan metabolism, and nucleic acid metabolism [15]. In agreement with this observation, dietary supplementation with either a sulfur amino acid (methionine) or tryptophan, which is mainly metabolized to kynurenine, has been reported to alleviate the negative effects of HT [16,17]. However, the involvement of nucleic acids and their metabolites in the metabolic changes that occur in chickens kept in HT environments remains unclear.

Orotic acid, which is found in high concentrations in cow's milk, is a key intermediate in the pyrimidine biosynthesis pathway [18]. Pyrimidine nucleotides are important constituents of RNA and of the phospholipids present in cell membranes. Orotic acid can enter the de novo synthesis pathway for pyrimidines beyond the rate-limiting step, and thereby improve throughput. In chickens, the plasma level of orotic acid was found to significantly decrease, by approximately $60 \%$, in response to short-term chronic heat exposure [15], suggesting that the plasma orotic acid concentration may be important under HT conditions.

In this study, we evaluated the effects of prolonged heat exposure and feeding orotic acid at $0.7 \%$ of the diet on the growth performance, plasma and muscle lipid peroxidation levels, and plasma and muscle metabolite concentrations of broiler chickens. To mimic realistic HT conditions, a cyclic HT environment $\left(35 \pm 1{ }^{\circ} \mathrm{C}\right.$ for $8 \mathrm{~h}$ /day) was used. In addition, a food-grade orotic acid (Lactoserum; Matsumoto Trading Co., Ltd., Tokyo, Japan), which contains more than $98 \%$ of orotic acid monohydrate, was used for dietary supplementation.

\section{Results}

The final body weight, body weight gain, and feed conversion ratio were not affected by either the HT or orotic acid supplementation, whereas the feed intake was significantly depressed in the chickens kept under the HT conditions (Table 1). In addition, the body temperatures of the chickens kept in the HT environment were significantly increased compared with those of the chickens kept under thermoneutral conditions. However, orotic acid supplementation alleviated the increase in body temperature under the HT conditions.

Although the weights of the leg muscles were not affected by the rearing temperature, the weights of the breast muscles, breast tender muscles, livers, and hearts were lower in the chickens kept under the HT conditions (Table 2). The yield of the breast muscles (the ratio of breast muscles weight to body weight) was the highest in the chickens supplemented with orotic acid and kept in the thermoneutral environment compared to the other three groups of chickens (Table S1). However, the yield of neither the breast tender muscles nor the leg muscles were different among these four groups. Furthermore, the weight of the abdominal fat tissue was increased by the HT environment.

The malondialdehyde (MDA) concentration, which serves as an index for the lipid peroxidation level, was significantly affected by either temperature or orotic acid supplementation. The plasma MDA concentration of chickens fed a control diet and kept under the HT condition was significantly increased compared with the chickens kept in the thermoneutral environment (Figure 1). However, under the HT condition, the dietary supplementation of orotic acid decreased the plasma MDA concentration compared to the control diet. On the other hand, the HT condition did not significantly increase the muscle MDA concentration of chickens fed a control diet. Furthermore, dietary supplementation of orotic acid did not alleviate that of chickens under the HT condition. 
A

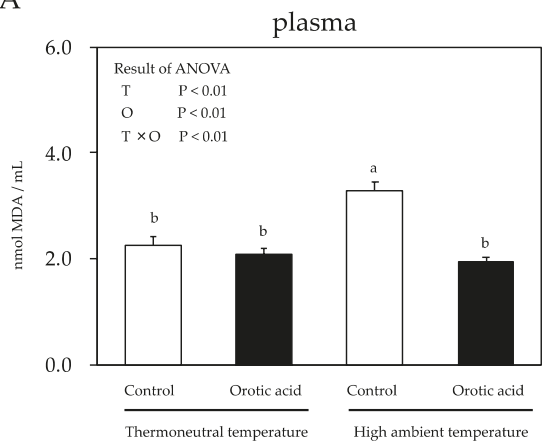

B

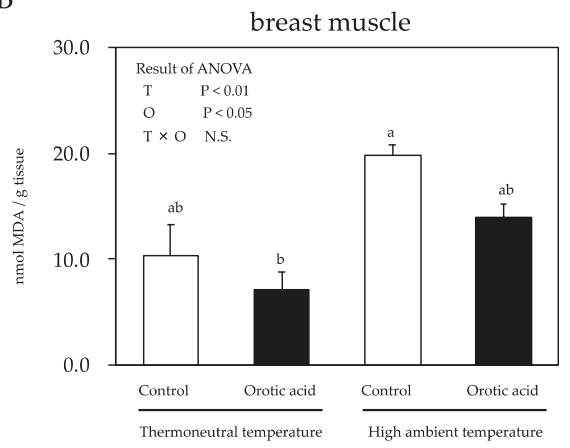

Figure 1. Effects of a cyclic high ambient temperature and feeding orotic acid on the plasma (A) and muscle (B) malondialdehyde (MDA) concentrations of broiler chickens. Results are expressed as mean \pm standard error of the mean (SEM) $(n=8)$. Means with the same superscript letter within columns are not significantly different at $p<0.05$. T: the effect of high ambient temperature; $\mathrm{O}$ : the effect of feeding orotic acid; $\mathrm{T} \times \mathrm{O}$ : the statistical interaction between high ambient temperature and feeding orotic acid.

Table 3 shows the plasma-free amino acid concentrations of the chickens kept under thermoneutral or HT conditions. There were significant effects of ambient temperature on the plasma serine, glutamine, and tyrosine concentrations, while orotic acid supplementation significantly affected the plasma aspartic acid, glutamic acid, and tyrosine concentrations. The two-way ANOVA revealed no significant interaction between the HT and orotic acid supplementation on these 18 plasma-free amino acids.

Untargeted gas chromatography-triple quadrupole mass spectrometry (GC-MS/MS)-based metabolomics analysis identified a total of 172 metabolites in the plasma of the chickens (Table 4 and Table S2). Of these metabolites, 23 were significantly affected by HT, of which 11 were significantly increased and 12 were decreased (Table 4). On the other hand, 12 were affected by orotic acid supplementation, of which 11 were significantly increased, and 1 was decreased. However, the two-way ANOVA revealed no significant interaction between the temperature and dietary treatments for the plasma metabolites.

The enrichment analysis indicated that nine and four metabolic pathways were affected by the HT environment and the orotic acid supplementation, respectively (Table 5).

The free amino acid and carnosine concentrations in the broiler chickens' breast muscles are shown in Table 6. The HT treatment significantly affected the serine, glutamine, arginine, methionine, and phenylalanine concentrations, while the orotic acid supplementation significantly affected the histidine, methionine, and carnosine concentrations. The two-way ANOVA revealed no significant interaction between the temperature and dietary treatments for the concentrations of the 18 free amino acids and carnosine in the muscle. 


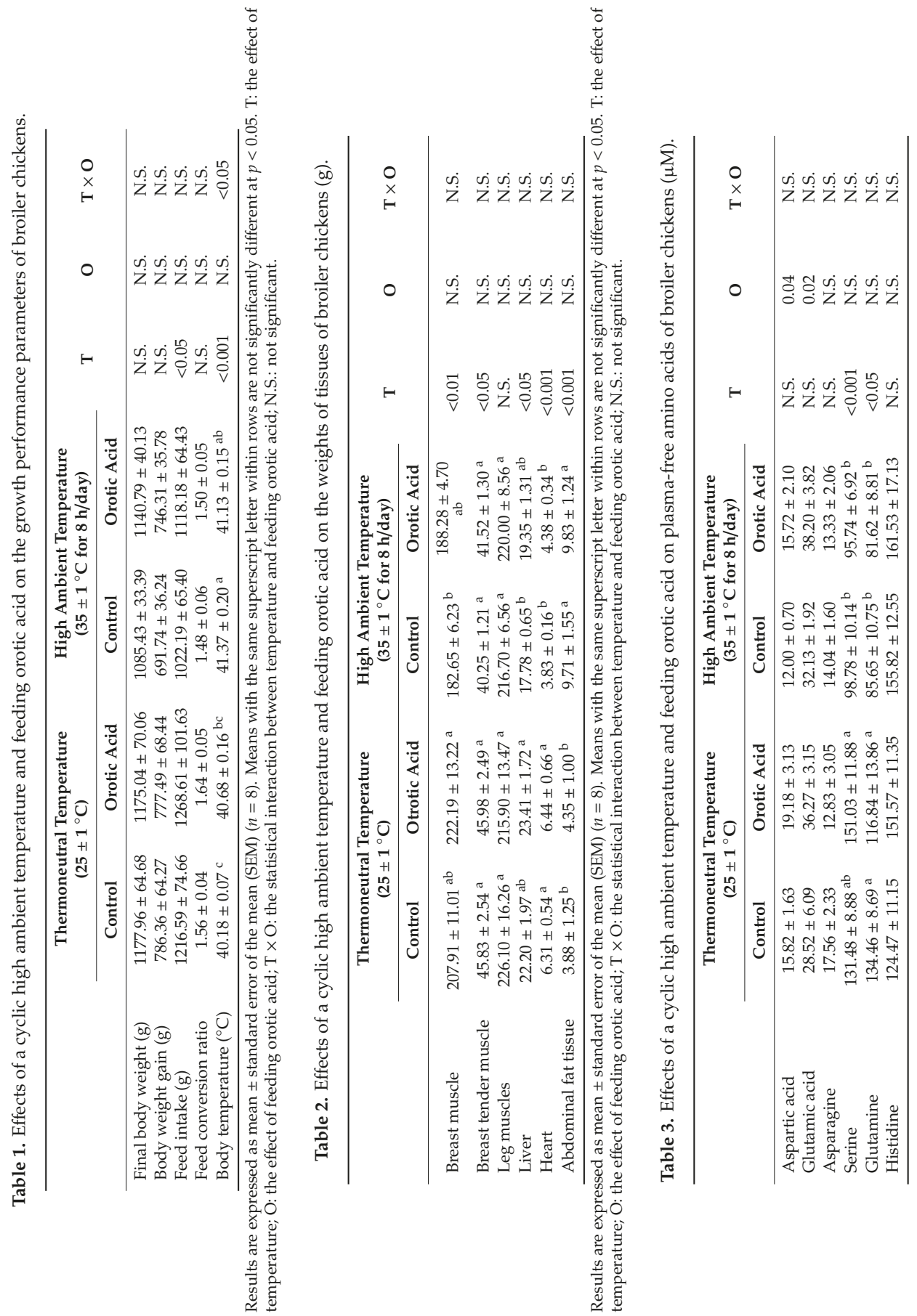




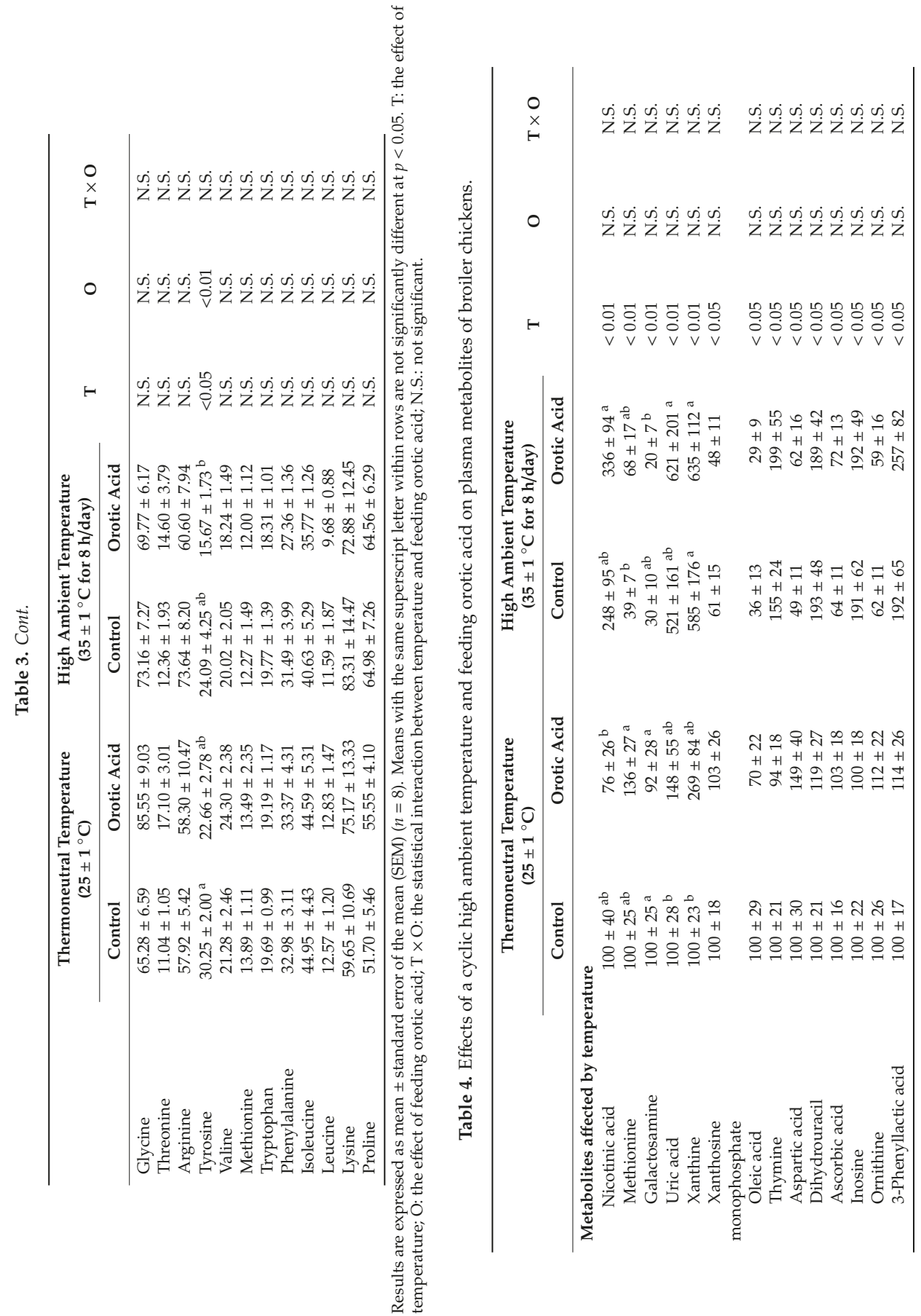




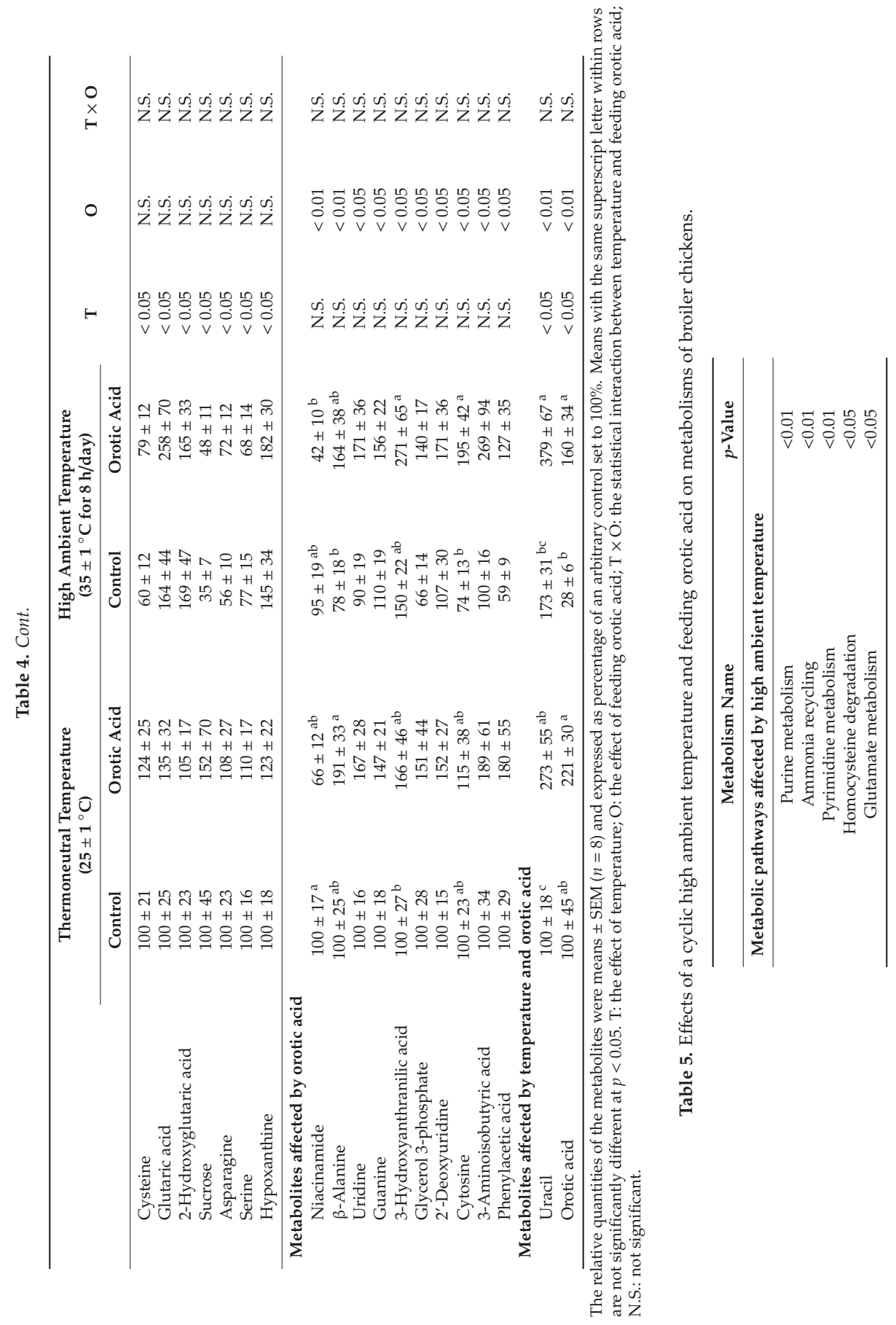




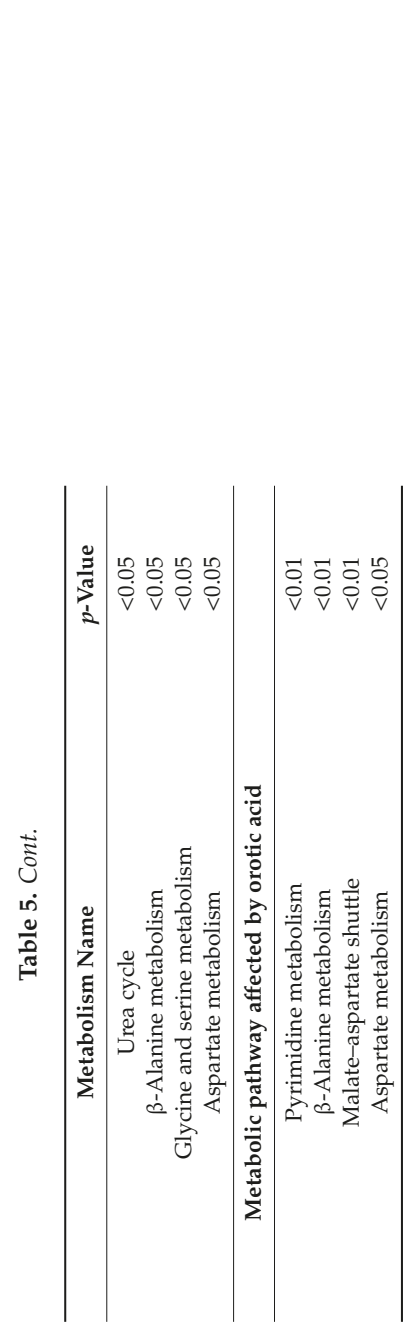

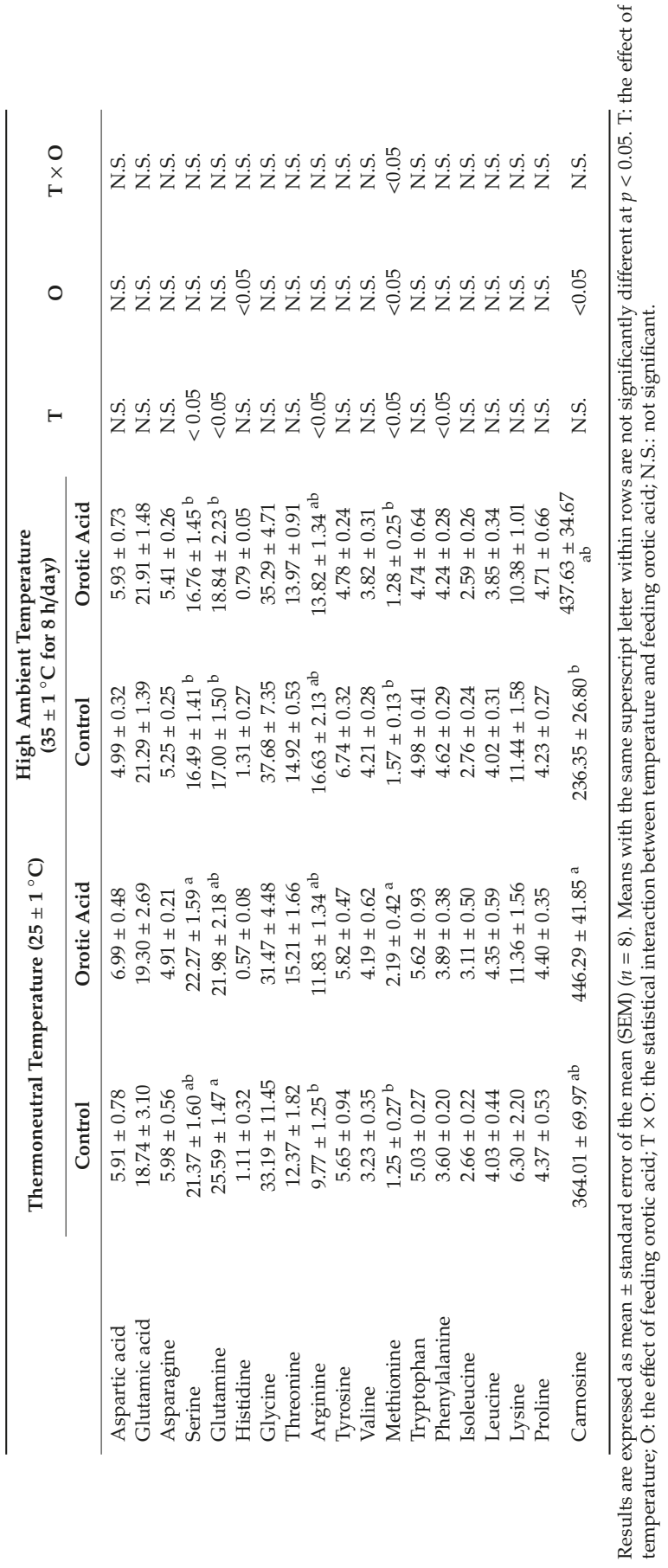




\section{Discussion}

Reactive oxygen species (ROS), such as singlet oxygen, hydrogen peroxide, and hydroxyl radicals, are highly reactive molecules produced by mitochondria [19]. Under high ambient temperature conditions, ROS generation increases in various body tissues of chickens as the heat load elevates [3], and consequently oxidizes and impairs lipids, proteins, and DNA [20]. Because chicken muscle has a high polyunsaturated fatty acid content, making it more sensitive to oxidative deterioration [21], the oxidation of such lipids negatively influences their industrial values (i.e., growth performance, meat yield, and meat quality) [4-14].

Under HT conditions, chickens have been known to show lower growth rate and feed efficiency, accompanied by decreasing meat yield $[4,5]$. In this study, although the HT environment did not affect the final body weight, body weight gain, or feed conversion ratio of the broiler chickens, it significantly depressed their feed intake and increased their average body temperature. These results concur with those of a previous study, which examined the effects of a similar HT environment [13]. In addition, the HT environment affected the weights of the breast muscles, breast tender muscles, livers, hearts, and abdominal fat tissue. In particular, the yield of breast muscle was significantly decreased by the HT environment. Furthermore, the plasma lipid peroxidation level was higher in the chickens kept under the HT conditions than those kept in the thermoneutral environment. Therefore, these results suggest that the HT environment used in this study could realistically induce the negative effects commonly observed in broiler chickens kept in heat stress-inducing environments.

In this study, most of the plasma amino acid concentrations were unaffected by HT, with the exception of the serine, tyrosine, and glutamine concentrations. This was particularly notable in the chickens fed the basal diet, in which the HT environment significantly decreased the plasma glutamine concentration. This concurred with the results of a previous study, which reported that chickens kept under HT conditions had lower plasma glutamine concentrations, in addition to poorer performance and carcass characteristics [22]. Although glutamine is a non-essential amino acid, it has been reported to promote enterocyte proliferation and survival, and regulate intestinal barrier function under a variety of stress conditions [23]. Furthermore, intestinal morphology and permeability were found to be disrupted under HT conditions, with this being accompanied by an increase in the plasma endotoxin concentration in broiler chickens [24]. These results suggest that glutamine is utilized to maintain intestinal integrity in broiler chickens under heat-stress conditions.

In addition, glutamine is known to be a precursor for the synthesis of purine and pyrimidine nucleotides, which are essential for DNA synthesis and the proliferation of cells [25]. In rats, GC-MS and liquid chromatography-mass spectrometry-based metabolomics analysis of the plasma indicated that short-term chronic heat exposure $\left(37^{\circ} \mathrm{C}\right.$ for $\left.48 \mathrm{~h}\right)$ altered pyrimidine and purine degradation [26]. To investigate the effects of an HT environment on metabolite profiles in the plasma of chickens, we performed untargeted GC-MS/MS-based metabolomics analysis. This analysis identified 172 metabolites in the plasma, of which 23 were significantly affected by the HT environment. The HT environment particularly affected the plasma levels of purine- (inosine, hypoxanthine, xanthine, xanthosine monophosphate, and uric acid) and pyrimidine-related (uracil, dihydrouracil, and thymine) metabolites. The changes in these metabolites are illustrated in Figure 2, with a general increase in concentration in response to the HT environment being apparent. 


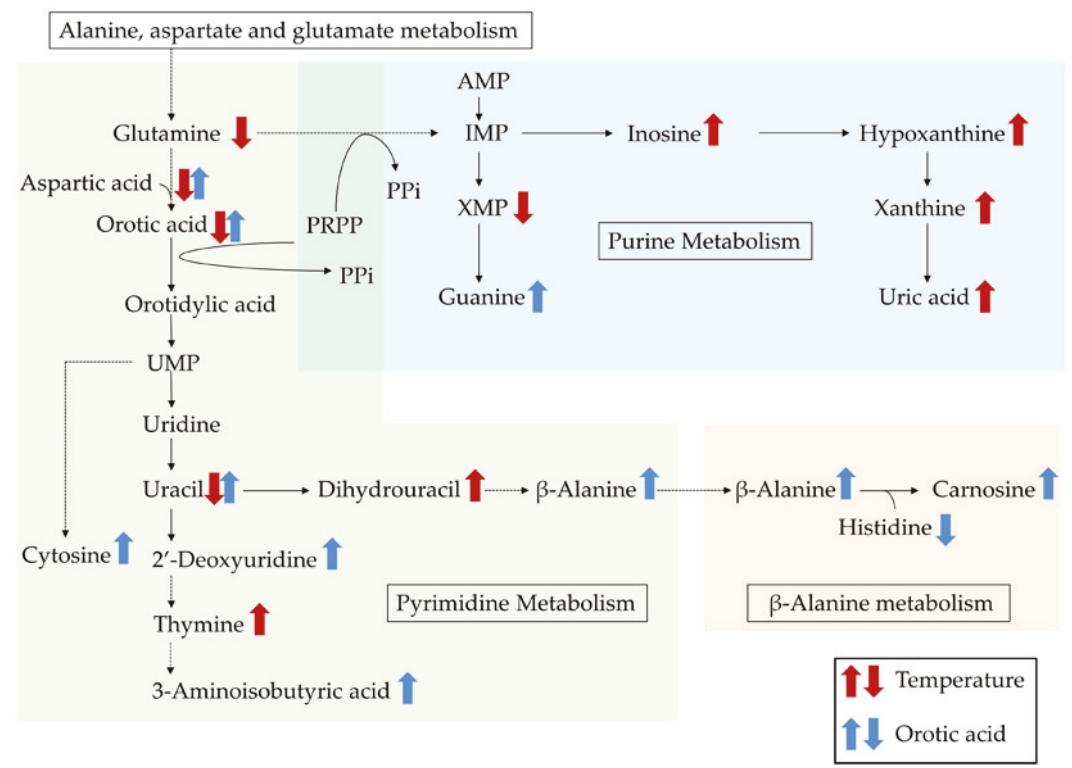

Figure 2. Integrated overview of the metabolic changes induced by either a cyclic high ambient temperature or by feeding orotic acid. Red arrows indicate high ambient temperature-induced changes in the metabolites, and blue arrows indicate orotic acid-induced changes. The metabolic scheme was based on information gathered from the KEGG PATHWAY Database (http://www.genome.jp/kegg/ pathway.html).

One possible explanation for the changes in the metabolite concentrations observed may be the higher oxidative stress levels in the broiler chickens kept under the HT conditions. Uric acid is known to act as an antioxidant in the plasma [27], and is thought to stabilize ascorbic acid [28]. However, the concentration of ascorbic acid was lower in the plasma of the chickens kept in the HT environment than in the chickens kept under thermoneutral conditions. Another possibility is that these changes were linked the higher energy expenditure of the broiler chickens kept at HT. When broiler chickens are exposed to environmental temperatures above $31^{\circ} \mathrm{C}$, they increase heat production [29], suggesting an increase in adenosine triphosphate (ATP) production. Adenylate kinase, which acts as a regulator for phosphate nucleotide levels inside cells, converts two adenosine diphosphate (ADP) molecules to one ATP molecule and one adenosine monophosphate (AMP) molecule [30]. Since the concentration of AMP is maintained at a much lower level than that of either ADP or ATP [31], the generated AMP may be catabolized to form hypoxanthine, xanthine, or uric acid.

The enrichment analysis identified that the HT environment induced changes in purine metabolism, ammonia recycling, pyrimidine metabolism, homocysteine degradation, glutamate metabolism, the urea cycle, $\beta$-alanine metabolism, glycine and serine metabolism, and aspartate metabolism. Seven of these pathways (excluding homocysteine degradation and aspartate metabolism) concur with the findings of a previous study on the effects of short-term chronic heat exposure on the plasma metabolomic profiles of chicks [15]. Furthermore, the plasma homocysteine concentration is considered an oxidative stress marker [32]. In Japanese quail, HT increased both the homocysteine concentration and the lipid peroxidation level in the plasma [33,34], and it has been suggested that chickens kept under HT conditions have higher plasma homocysteine concentrations. Furthermore, the untargeted GC-MS/MS-based metabolomics analysis indicated that the HT environment decreased the plasma level of aspartic acid, which is a precursor for the synthesis of pyrimidines. These results suggest that although purine and pyrimidine synthesis pathways in chickens were enhanced in response to the HT 
conditions, the levels of related metabolites (e.g., glutamine, aspartic acid, and ascorbic acid) may have been disrupted.

It is noteworthy that the HT environment changed not only pyrimidine metabolism, but also aspartate metabolism and $\beta$-alanine metabolism, because both pyrimidine metabolism and aspartate metabolism are closely related to $\beta$-alanine metabolism. $\beta$-alanine is one of constituents of carnosine ( $\beta$-alanyl-L-histidine) and anserine ( $\beta$-alanyl-1-methyl-L-histidine), which are known to be present at high concentrations in the breast muscle of chickens and to exert antioxidant activity [35]. In this study, we found that the chickens fed the basal diet and kept at a HT had the lowest carnosine content in the breast muscle of the three treatment groups. This low carnosine content may explain the higher lipid peroxidation level in the breast muscle samples from this group.

An HT-induced decrease in the muscle carnosine content was also observed by Yang et al. [36], while Tomonaga et al. [15] reported that the constituents of carnosine ( $\beta$-alanine and histidine) in chicks decreased in response to short-term chronic heat exposure. In this study, although the plasma dihydrouracil concentration was the highest, the plasma level of $\beta$-alanine was the lowest in the chickens fed the basal diet and kept under the HT conditions. Therefore, these results suggest that the conversion of dihydrouracil to $\beta$-alanine may be disrupted in chickens kept under HT conditions.

This study also evaluated the effects of feeding orotic acid to chickens, and found that it did not affect growth performance, as indicated by the final body weight, body weight gain, feed conversion ratio, feed intake, and tissue weights. However, orotic acid did ameliorate the HT-induced increases in lipid peroxidation level in the plasma of the chickens. This amelioration may have partially been due to the antioxidative activity of orotic acid [37], with the plasma level of orotic acid decreasing under the HT conditions and increasing in response to the orotic acid supplementation. The GC-MS/MS-based metabolomics analysis of the plasma indicated that 12 metabolites were affected by orotic acid supplementation, some of which have been reported to show antioxidative activity (e.g., uridine and 3-hydroxyanthranilic acid) [38,39] and appeared to be increased by orotic acid supplementation. Furthermore, the high-performance liquid chromatography analysis revealed that orotic acid significantly affected the plasma concentrations of aspartic acid, glutamic acid, and tyrosine. Of these three amino acids, aspartic acid and glutamic acid appeared to be increased by the orotic acid supplementation. Aspartic acid is converted into glutamic acid via the citric acid cycle [40], and glutamic acid is a component amino acid of glutathione, a well-known natural antioxidant [41,42]. These results suggest that alterations in the concentrations of these metabolites and amino acids may contribute to the amelioration of the HT environment-induced increase in the plasma lipid peroxidation level. Furthermore, orotic acid supplementation significantly affected the carnosine content of the breast muscle of the chickens. As previously mentioned, carnosine plays a role as an antioxidant [35], and the reduction of the lipid peroxidation level in the breast muscle tissue may therefore be linked to the orotic acid-induced increase in the carnosine content.

The GC-MS/MS-based metabolomics analysis indicated that orotic acid affected the plasma levels of pyrimidine-related metabolites (orotic acid, uracil, uridine, $2^{\prime}$-deoxyuridine, and $\beta$-alanine). This effect was likely linked to the entry of orotic acid into the de novo synthesis pathway for pyrimidines beyond the rate-limiting step. Furthermore, the de novo synthesis pathways for both pyrimidines and purines require 5-phosphoribosyl-1-pyrophosphate (PRPP), and the synthesis of PRPP by PRPP synthetase is the rate-limiting step for both pathways. In rats, it has been reported that orotic acid stimulates hepatic purine biosynthesis [43]. These results suggest that orotic acid may up-regulate the de novo synthesis of purines by providing additional PRPP. However, there was no significant interaction between the temperature and dietary treatments for the plasma metabolites. Further studies are required to understand the effects of orotic acid supplementation on the regulation of the de novo synthesis pathways for pyrimidines and purines in chickens.

The enrichment analysis identified changes in not only pyrimidine metabolism, but also in $\beta$-alanine metabolism, the malate-aspartate shuttle, and aspartate metabolism, in response to supplementing orotic acid. Of these, $\beta$-alanine metabolism and aspartate metabolism may be 
particularly important for the alleviation of HT-induced negative effects, as they were also found to be altered by HT conditions. Orotic acid supplementation was found to significantly increase the plasma level of $\beta$-alanine and the carnosine content of the breast muscles of the chickens. In chicks, orally administered $\beta$-alanine increased the carnosine content of the brain and muscles in a dose-dependent manner [44]. Therefore, these results suggest that orotic acid increases the muscle carnosine content by altering $\beta$-alanine metabolism, and thereby maintains the antioxidative capacity of broiler chickens under HT conditions. However, the mechanisms by which orotic acid supplementation increased the plasma level of $\beta$-alanine, even under HT conditions, remain unclear. Further research evaluating the effects of $\mathrm{HT}$ and orotic acid supplementation on the gene expression and activities of enzymes related to the conversion of dihydrouracil to $\beta$-alanine (i.e., dihydropyrimidinase and $\beta$-ureidopropionase) in chickens is necessary if we are to improve our insight into the reason for the HT-induced decrease in the muscle carnosine content and the alleviating effects of orotic acid supplementation.

As mentioned above, HT conditions severely increase lipid peroxidation levels [6,7], and consequently reduce meat quality (i.e., increase in drip loss, decrease in share force value, and change in meat color) [8-14]. The breast muscles of the chickens kept under cyclic HT conditions showed the highest lipid peroxidation levels, in conjunction with the lowest carnosine content, suggesting that their meat was of lower quality than that of the control chickens. In contrast, orotic acid increased the muscle carnosine content, possibly via pyrimidine metabolism and $\beta$-alanine metabolism, and consequently alleviated the HT environment-induced increase in the muscle lipid peroxidation levels. Cong et al. [45] reported that dietary supplementation of carnosine improved meat quality, antioxidant capacity, and lipid peroxidation status in broiler chickens. In addition, carnosine exerts antioxidant activity, even in cocked chicken meat [35]. These results suggest that orotic acid supplementation may have a positive effect on either carnosine content in meat or the meat quality of chickens.

\section{Materials and Methods}

\subsection{Animals and Experimental Design}

All experimental protocols and procedures were reviewed and approved by the Animal Care and Use Committee of Kagoshima University (approval number A18010). One hundred 1-day-old male broiler chicks (Chunky strain ROSS 308) were obtained from a commercial hatchery (Kumiai Hina Center, Kagoshima, Japan). Chicks were housed in an electrically-heated battery brooder and provided with water and a commercial diet (23\% crude protein, $12.8 \mathrm{MJ} / \mathrm{kg}$; Nichiwa Sangyou Company, Hyogo, Japan) until they were 14 days old. On day 14,32 chicks were randomly selected from the group of 100 . These chicks were housed individually in wire-bottomed aluminum cages $(50 \times 40 \times 60 \mathrm{~cm})$ and fed the basal diet (Table 7) for 3 days until the start of the main experimental period. The chicks were then randomly allocated to one of four groups, with the main experimental factors being diet and ambient temperature, in a $2 \times 2$ factorial design. The dietary treatments consisted of the basal diet or the basal diet supplemented with $0.7 \%$ of Lactoserum (Matsumoto Trading Co., Ltd., Tokyo, Japan), a food grade orotic acid that contains more than $98 \%$ of orotic acid as monohydrate form (i.e., more than $87.9 \%$ of orotic acid), and the temperature treatments consisted of either a thermoneutral environment at $25 \pm 1{ }^{\circ} \mathrm{C}$ or an HT environment at $35 \pm 1{ }^{\circ} \mathrm{C}$. The experiment was conducted in a temperature-controlled room with $24 \mathrm{~h}$ of light and $50-70 \%$ relative humidity. The chicks assigned to the HT treatment groups were kept at $35 \pm 1{ }^{\circ} \mathrm{C}$ for $8 \mathrm{~h}$ every day to mimic a realistic summer environment. At 32 days old, all the chickens were weighed, anesthetized by carbon dioxide, and killed by cervical dislocation. The chickens were then dissected, and the weights of the breast muscles (pectoralis major muscle), breast tender muscles (pectoralis minor muscle), leg muscles (thigh and drumstick), livers, hearts, and abdominal fat tissue depots were recorded. Blood samples were collected in heparinized test tubes, centrifuged at $5900 \times \mathrm{g}$ for $10 \mathrm{~min}$ at $4{ }^{\circ} \mathrm{C}$ to separate the plasma, and stored at $-30^{\circ} \mathrm{C}$ until analysis. 
Table 7. Composition and analysis of the basal diet.

\begin{tabular}{|c|c|}
\hline & Ingredients $(\mathrm{g} / 100 \mathrm{~g})$ \\
\hline Corn meal & 57.90 \\
\hline Soybean meal & 34.00 \\
\hline Corn oil & 4.30 \\
\hline $\mathrm{CaCO}_{3}$ & 0.66 \\
\hline $\mathrm{CaHPO}_{4}$ & 2.00 \\
\hline $\mathrm{NaCl}$ & 0.50 \\
\hline DL-Methionine & 0.14 \\
\hline Mineral and vitamin premix ${ }^{1}$ & 0.50 \\
\hline \multicolumn{2}{|l|}{ Calculated analysis } \\
\hline Crude protein $(\%)$ & 20.00 \\
\hline Metabolizable energy (Mcal/kg) & 3.10 \\
\hline
\end{tabular}

${ }^{1}$ Content per kg of the vitamin and mineral premix: vitamin A $=90 \mathrm{mg}$, vitamin D3 = $1 \mathrm{mg}$, DL-alpha-tocopherol acetate $=2000 \mathrm{mg}$, vitamin K3 $=229 \mathrm{mg}$, thiamin nitrate $=444 \mathrm{mg}$, riboflavin $=720 \mathrm{mg}$, calcium d-pantothenate $=2174 \mathrm{mg}$, nicotinamide $=7000 \mathrm{mg}$, pyridoxine hydrochloride $=700 \mathrm{mg}$, biotin $=30 \mathrm{mg}$, folic acid $=110 \mathrm{mg}$, cyanocobalamine $=2 \mathrm{mg}$, calcium iodinate $=108 \mathrm{mg}, \mathrm{MgO}=198,991 \mathrm{mg}, \mathrm{MnSO}_{4}=32,985 \mathrm{mg}, \mathrm{ZnSO}_{4}=19,753 \mathrm{mg}$, $\mathrm{FeSO}_{4}=43,523 \mathrm{mg}, \mathrm{CuSO}_{4}=4019 \mathrm{mg}$, and choline chloride $=299,608 \mathrm{mg}$.

\subsection{Determination of MDA Concentration}

To evaluate the oxidative stress levels, the MDA concentrations in the breast muscles and plasma were determined colorimetrically, using the thiobarbituric acid reactive substances assay, as described by Yagi [46] and Ohkawa et al. [47].

\subsection{Determination of Free Amino Acid Concentrations}

The analysis of the free amino acid and carnosine concentrations in the plasma and breast muscle tissue samples from the chickens was performed using a pre-column technique with liquid chromatography, according to previously reported methods [48]. A liquid chromatography system with automated pre-column derivatization functionality was used in the analysis (Nexera X2; Shimadzu Corporation, Kyoto, Japan). A total of 21 compounds were measured in the analysis, including the following basic amino acids and associated molecules: alanine, anserine, arginine, asparagine, aspartic acid, carnosine, glutamic acid, glutamine, glycine, histidine, isoleucine, leucine, lysine, methionine, phenylalanine, proline, serine, threonine, tryptophan, tyrosine, and valine. The concentrations of the amino acids in the plasma are expressed in $\mu \mathrm{mol} / \mathrm{L}$, and those in the breast muscle are expressed in $\mathrm{mg} / 100 \mathrm{~g}$. In this study, alanine and anserine were not separated, and carnosine was not detected in the plasma.

\subsection{Sample Preparation for GC-MS/MS Analysis}

Fifty $\mu \mathrm{L}$ aliquots of plasma were suspended in $250 \mu \mathrm{L}$ of methanol/chloroform/water (5:2:2), with $5 \mu \mathrm{L}$ of $1 \mathrm{mg} / \mathrm{mL}$ 2-isopropylmalic acid as the internal standard. The samples were then mixed in a shaker at $1200 \mathrm{rpm}$ at $37^{\circ} \mathrm{C}$ for $30 \mathrm{~min}$, and then centrifuged at $16,000 \times g$ at $4{ }^{\circ} \mathrm{C}$ for $5 \mathrm{~min}$. Next, $225 \mu \mathrm{L}$ of the supernatant was mixed with $200 \mu \mathrm{L}$ of distilled water and vortex-mixed, followed by centrifugation at $16,000 \times g$ at $4{ }^{\circ} \mathrm{C}$ for $5 \mathrm{~min}$. Subsequently, $250 \mu \mathrm{L}$ of the supernatant was dried under a vacuum using a centrifugal evaporator (RD-400; Yamato Scientific, Tokyo, Japan), after cooling at $-80^{\circ} \mathrm{C}$ for $10 \mathrm{~min}$. Methoxyamine hydrochloride in pyridine $(20 \mathrm{mg} / \mathrm{mL}, 40 \mu \mathrm{L})$ was then added to the tubes, and they were vortex-mixed, then shaken at $1200 \times \mathrm{g}$ at $30^{\circ} \mathrm{C}$ for $90 \mathrm{~min}$ in the dark to allow oximation. $\mathrm{N}$-methyl-N-trimethylsilyltrifluoroacetamine $(20 \mu \mathrm{L})$ was then added to each tube, and the contents were vortex-mixed. To prepare trimethylsilyl derivatives, the tubes were shaken at $1200 \times g$ at $37^{\circ} \mathrm{C}$ for $45 \mathrm{~min}$ in the dark. 


\subsection{GC-MS/MS Analysis and Data Processing}

GC-MS/MS analysis was performed as previously described [49], using a GCMS-TQ8050 (Shimadzu Corporation, Kyoto, Japan). A $30 \mathrm{~m} \times 0.25 \mathrm{~mm}$ (internal diameter) BPX-5 column (SGE, Melbourne, Australia) with a $0.25 \mu \mathrm{m}$ film thickness was used, according to the method described in the Smart Metabolites Database (Shimadzu, Kyoto, Japan).

Data processing was performed using the Smart Metabolites Database (Shimadzu, Kyoto, Japan), MS-DIAL version 3.08 [50], and the MRMPROBS program version 2.42 [51]. Peaks were recorded for the $45-600 \mathrm{~m} / \mathrm{z}$ mass range, and were automatically detected via MS-DIAL using the peak detection option of a minimum peak height of 2000. A data quality check was conducted using the thresholds of $-10<\mathrm{RI}<10$, dot production $>0.8$, and presence $>0.6$, and the remaining data was then manually checked. Ultimately, 172 metabolites were identified in the plasma samples. The relative quantities of the metabolites were calculated using the peak areas of each metabolite relative to that of the internal standard (2-isopropylmalic acid), and expressed as a percentage of an arbitrary control set to $100 \%$.

\subsection{Statistical Analysis}

The data were analyzed using two-way ANOVA, with individual comparisons being made using Tukey's multiple comparison test. All analyses were performed using R [52]. Statistical significance was set at $p<0.05$, and the data are expressed as the means \pm standard error of the mean (SEM).

Quantitative enrichment analysis using the pathway-associated metabolite sets included in MetaboAnalyst 4.0 [53], which is an established tool for metabolite set enrichment analysis, was performed using the plasma metabolite compounds that were significantly affected by either the HT treatment or orotic acid supplementation. Differences were considered significant at $p<0.05$.

\section{Conclusions}

A cyclic HT environment altered nucleic acid metabolism and increased lipid peroxidation levels in the plasma and breast muscle of broiler chickens. Feeding the pyrimidine precursor orotic acid to the chickens altered the plasma concentrations of metabolites mainly related to pyrimidine metabolism and $\beta$-alanine metabolism, and ameliorated the increases in lipid peroxidation levels caused by rearing the broiler chickens under HT conditions.

Supplementary Materials: The following is available online at http://www.mdpi.com/2218-1989/10/5/189/s1, Table S1. Effects of a cyclic high ambient temperature and feeding orotic acid on the muscle yields of broiler chickens. Table S2. Metabolites identified in the plasma of broiler chickens reared under either thermoneutral or high ambient temperature (HT) conditions, and fed either a control or orotic acid-supplemented diet.

Author Contributions: Conceptualization, S.S., K.N., S.F., A.O., and D.I.; methodology, D.I. and S.T.; software, S.T. and D.I.; validation, S.S. and S.T.; formal analysis, S.S. and K.N; investigation, S.S. and K.N.; resources, S.F; original draft preparation, S.S. and K.N; manuscript review and editing, S.S., S.T., S.F., A.O., and D.I.; supervision, A.O.; project administration, D.I. All authors have read and agreed to the published version of the manuscript.

Funding: This study was funded by the Furukawa Research Office Co., Ltd., and a Grant-in-Aid for Scientific Research (C) (No. 19K06357) from The Japan Society for the Promotion of Science (JSPS) to D. Ijiri., Kagoshima University, and received no specific grants from any funding agency in the public, commercial, or not-for-profit sectors.

Conflicts of Interest: The authors declare no conflict of interest. 


\section{References}

1. Ensminger, M.E.; Oldfield, J.E.; Heineman, W.W. Feeds and Nutrition, 2nd ed.; Ensminger Pub Co: Prentice Hall: Upper Saddle River, NJ, USA, 1990; pp. 8-110.

2. Sahin, K.; Sahin, N.; Kucuk, O.; Hayirili, A.; Prasad, A.S. Role of dietary zinc in heat stressed poultry: A review. Poult. Sci. 2009, 88, 2176-2183. [CrossRef] [PubMed]

3. Khan, R.U.; Naz, S.; Nikousefat, Z.; Selvaggi, M.; Laudadio, V.; Tufarelli, V. Effect of ascorbic acid in heat-stressed poultry. Worlds Poult. Sci. J. 2012, 68, 477-489. [CrossRef]

4. Yalçin, S.; Settar, P.; Ozkan, S.; Cahaner, A. Comparative evaluation of three commercial broiler stocks in hot versus temperate climates. Poult. Sci. 1997, 76, 921-929. [CrossRef] [PubMed]

5. Yunis, R.; Cahaner, A. The effects of naked neck (Na) and frizzle (F) genes on growth and meat yields of broilers and their interactions with ambient temperatures and potential growth rate. Poult. Sci. 1999, 78, 1347-1352. [CrossRef]

6. Whitehead, C.C.; Keller, T. An update on ascorbic acid in poultry. Worlds Poult. Sci. J. 2003, 59, 161-184. [CrossRef]

7. Zeferino, C.P.; Komiyama, C.M.; Pelícia, V.C.; Fascina, V.B.; Aoyagi, M.M.; Coutinho, L.L.; Sartori, J.R.; Moura, A.S.A.M.T. Carcass and meat quality traits of chickens fed diets concurrently supplemented with vitamins $C$ and $E$ under constant heat stress. Animal 2016, 10, 163-171. [CrossRef]

8. Zhu, X.; Ruusunen, M.; Gusella, M.; Zhou, G.; Puolanne, E. High post-mortem temperature combined with rapid glycolysis induces phosphorylase denaturation and produces pale and exudative characteristics in broiler pectoralis major muscles. Meat Sci. 2011, 89, 181-188. [CrossRef]

9. Wang, R.H.; Liang, R.R.; Lin, H.; Zhu, L.X.; Zhang, Y.M.; Mao, Y.W.; Dong, P.C.; Niu, L.B.; Zhang, M.H.; Luo, X.X. Effect of acute heat stress and slaughter processing on poultry meat quality and postmortem carbohydrate metabolism. Poult. Sci. 2017, 96, 738-746. [CrossRef]

10. Sosnicki, A.A.; Greaser, M.L.; Pieterzak, M.; Pospiech, E.; Sante, V. PSE-like syndrome in breast muscle of domestic turkeys: A review. J. Muscle Foods 1998, 9, 13-23. [CrossRef]

11. Hashizawa, Y.; Kubota, M.; Kadowaki, N.; Fujimura, S. Effect of dietary vitamin E on broiler meat qualities, color, water-holding capacity and shear force value, under heat stress conditions. Anim. Sci. J. 2013, 84, 732-736. [CrossRef]

12. Petracci, M.; Betti, M.; Cavani, C. Color variation and characterization of broiler breast meat during processing in Italy. Poult. Sci. 2004, 83, 2085-2092. [CrossRef]

13. Inoue, H.; Simamoto, S.; Takahashi,H.; Kawashima, Y.; Sato, W.; Ijiri, D.; Ohtsuka, A. Effects of astaxanthin-rich dried cell powder from paracoccus carotinifaciens on carotenoid composition and lipid peroxidation in skeletal muscle of broiler chickens under thermo-neutral or realistic high temperature conditions. Anim. Sci. J. 2019, 90, 229-236. [CrossRef] [PubMed]

14. Al-Sagan, A.A.; Khalil, S.; Hussein, E.O.S.; Attia, Y.A. Effects of fennel seed powder supplementation on growth performance, carcass characteristics, meat quality, and economic efficiency of broilers under thermoneutral and chronic heat stress conditions. Animals (Basel) 2020, 10, 206. [CrossRef]

15. Tomonaga, S.; Okuyama, H.; Tachibana, T.; Makino, R. Effects of high ambient temperature on plasma metabolomic profiles in chicks. Anim. Sci. J. 2018, 89, 448-455. [CrossRef] [PubMed]

16. Tabiri, H.Y.; Sato, K.; Takahashi, K.; Toyomizu, M.; Akiba, Y. Effects of heat stress and dietary tryptophan on performance and plasma amino acid concentrations of broiler chickens. Asian Austral. J. Anim. 2002, 15, 247-253. [CrossRef]

17. Del Vesco, A.P.; Gasparino, E.; Grieser, D.O.; Zancanela, V.; Voltolini, D.M.; Khatlab, A.S.; Guimarães, S.E.; Soares, M.A.; Oliveira Neto, A.R. Effects of methionine supplementation on the expression of protein deposition-related genes in acute heat stress-exposed broilers. PLoS ONE 2015, 25, e0115821. [CrossRef] [PubMed]

18. Löffler, M.; Carrey, E.A.; Zameitat, E. Orotic acid, more than just an intermediate of pyrimidine de novo synthesis. J Genet. Genom. 2015, 42, 207-219. [CrossRef]

19. Freeman, B.A.; Crapo, J.D. Biology of disease: Free radicals and tissue injury. Lab. Investig. 1982, 47, 412-426.

20. Liu, D.; Wen, J.; Liu, J.; Li, L. The roles of free radicals in amyotrophic lateral sclerosis: Reactive oxygen species and elevated oxidation of protein, DNA, and membrane phospholipids. FASEB J. 1999, 13, 2318-2328. [CrossRef] 
21. Grashorn, M.A. Functionality of poultry meat. J. Appl. Poult. Res. 2007, 16, 99-106. [CrossRef]

22. Dai, S.F.; Gao, F.; Zhang, W.H.; Song, S.X.; Xu, X.L.; Zhou, G.H. Effects of dietary glutamine and gamma-aminobutyric acid on performance, carcass characteristics and serum parameters in broilers under circular heat stress. Anim. Feed Sci. Technol. 2011, 168, 51-60. [CrossRef]

23. Wang, B.; Wu, G.; Zhou, Z.; Dai, Z.; Sun, Y.; Ji, Y.; Li, W.; Wang, W.; Liu, C.; Han, F.; et al. Glutamine and intestinal barrier function. Amino Acids 2015, 47, 2143-2154. [CrossRef] [PubMed]

24. Nanto-Hara, F.; Kikusato, M.; Ohwada, S.; Toyomizu, M. Heat stress directly affects intestinal integrity in broiler chickens. J. Poult. Sci. 2019. [CrossRef]

25. Wu, G. Intestinal mucosal amino acid catabolism. Nutr. J. 1998, 128, 1249-1252. [CrossRef] [PubMed]

26. Ippolito, D.L.; Lewis, J.A.; Yu, C.; Leon, L.R.; Stallings, J.D. Alteration in circulating metabolites during and after heat stress in the conscious rat: Potential biomarkers of exposure and organ-specific injury. BMC Physiol. 2014, 14, 14. [CrossRef]

27. Ames, B.N.; Cathcart, R.; Schwiers, E.; Hochstein, P. Uric acid provides an antioxidant defense in humans against oxidant- and radical-caused aging and cancer: A hypothesis. Proc. Natl. Acad. Sci. USA 1981, 78, 6858-6862. [CrossRef]

28. Sevanian, A.; Davies, K.J.; Hochstein, P. Serum urate as an antioxidant for ascorbic acid. Am. J. Clin. Nutr. 1991, 54, 1129S-1134S. [CrossRef]

29. Yunianto, V.D.; Hayashi, K.; Kaneda, S.; Ohtsuka, A.; Tomita, Y. Effect of environmental temperature on muscle protein turnover and heat production in tube-fed broiler chickens. Br. J. Nutr. 1997, 77, 897-909. [CrossRef]

30. Dzeja, P.P.; Zeleznikar, R.J.; Goldberg, N.D. Adenylate kinase: Kinetic behavior in intact cells indicates it is integral to multiple cellular processes. Mol. Cell. Biochem. 1998, 184, 169-182. [CrossRef]

31. Beis, I.; Newsholme, E.A. The contents of adenine nucleotides, phosphagens and some glycolytic intermediates in resting muscles from vertebrates and invertebrates. Biochem. J. 1975, 152, 23-32. [CrossRef]

32. Miller, J.W.; Nadeau, M.R.; Smith, J.; Smith, D.; Selhub, J. Folate-deficiency-induced homocysteinaemia in rats: Disruption of S-adenosylmethionine's co-ordinate regulation of homocysteine metabolism. Biochem. J. 1994, 298, 415-419. [CrossRef] [PubMed]

33. Sahin, K.; Onderci, M.; Sahin, N.; Gursu, M.F.; Kucuk, O. Dietary vitamin C and folic acid Supplementation ameliorates the detrimental effects of heat stress in Japanese quail. J. Nutr. 2003, 133, 1882-1886. [CrossRef] [PubMed]

34. Sahin, K.; Onderci, M.; Sahin, N.; Gursu, M.F.; Khachik, F.; Kucuk, O. Effects of lycopene supplementation on antioxidant status, oxidative stress, performance and carcass characteristics in heat-stressed Japanese quail. J. Therm. Biol. 2006, 31, 307-312. [CrossRef]

35. O'Neill, L.M.; Galvina, K.; Morrisseya, P.A.; Buckleyb, D.J. Inhibition of lipid oxidation in chicken by carnosine and dietary $\alpha$-tocopherol supplementation and its determination by derivative spectrophotometry. Meat Sci. 1998, 50, 479-488. [CrossRef]

36. Yang, P.; Hao, Y.; Feng, J.; Lin, H.; Feng, Y.; Wu, X.; Yang, X.; Gu, X. The expression of carnosine and its effect on the antioxidant capacity of longissimus dorsi muscle in finishing pigs exposed to constant heat stress. Asian-Australas. J. Anim. Sci. 2014, 27, 1763-1772. [CrossRef]

37. Prachayasittikul, S.; Wongsawatkul, O.; Worachartcheewan, A.; Ruchirawat, S.; Prachayasittikul, V. Vasorelaxation and superoxide scavenging activities of orotic acid. Int. J. Pharmacol. 2010, 6, 413-418.

38. Chadha, R.; Mahal, H.S.; Mukherjee, T.; Kapoor, S. Evidence for a possible role of 3-hydroxyanthranilic acid as an antioxidant. J. Phys. Org. Chem. 2008, 22, 349-354. [CrossRef]

39. Lu, X.; Li, N.; Qiao, X.; Qiu, Z.; Liu, P. Composition analysis and antioxidant properties of black garlic extract. J. Food Drug Anal. 2017, 25, 340-349. [CrossRef]

40. Simon, G.; Drori, J.B.; Cohen, M.M. Mechanism of conversion of aspartate into glutamate in cerebral-cortex slices. Biochem. J. 1967, 102, 153-162. [CrossRef]

41. Hopkins, G.F.; Dixon, M. On glutathione. II. A thermostable oxidation-reduction system. J. Biol. Chem. 1992, $54,527-563$.

42. Hunter, G.; Eagles, B.A. Glutathione: A critical study. J. Biol. Chem. 1927, 72, 147-166.

43. Windmueller, H.G.; Spaeth, A.E. Stimulation of hepatic purine biosynthesis by orotic acid. J. Biol. Chem. 1965, 240, 4398-4405. [PubMed] 
44. Tomonaga, T.; Matsumoto, M.; Furuse, M. $\beta$-Alanine enhances brain and muscle carnosine levels in broiler chicks. J. Poult. Sci. 2012, 4, 308-312. [CrossRef]

45. Cong, J.; Zhang, L.; Li, J.; Wang, S.; Gao, F.; Zhou, G. Effects of dietary supplementation with carnosine on meat quality and antioxidant capacity in broiler chickens. Br. Poult. Sci. 2017, 58, 69-75. [CrossRef]

46. Yagi, K. A simple fluorometric assay for lipoperoxide in blood plasma. Biochem. Med. 1976, 15, $212-216$. [CrossRef]

47. Ohkawa, H.; Ohishi, N.; Yagi, K. Assay for lipid peroxides in animal tissues by thiobarbituric acid reaction. Anal. Biochem. 1979, 95, 351-358. [CrossRef]

48. Azuma, K.; Hirao, Y.; Hayakawa, Y.; Murahata, Y.; Osaki, T.; Tsuka, T.; Imagawa, T.; Okamoto, Y.; Ito, N. Application of pre-column labeling liquid chromatography for canine plasma-free amino acid analysis. Metabolites 2016, 6, 3. [CrossRef]

49. Goto, T.; Mori, H.; Shiota, S.; Tomonaga, S. Metabolomics approach reveals the effects of breed and feed on the composition of chicken eggs. Metabolites 2019, 9, 224. [CrossRef]

50. Lai, Z.; Tsugawa, H.; Wohlgemuth, G.; Mehta, S.; Mueller, M.; Zheng, Y.; Ogiwara, A.; Meissen, J.; Showalter, M.; Takeuchi, K.; et al. Identifying metabolites by integrating metabolome databases with mass spectrometry cheminformatics. Nat. Methods 2018, 15, 53-56. [CrossRef]

51. Tsugawa, H.; Kanazawa, M.; Ogiwara, A.; Arita, M. MRMPROBS suite for metabolomics using large-scale MRM assays. Bioinformatics 2014, 30, 2379-2380. [CrossRef]

52. R Core Team. R: A Language and Environment for Statistical Computing; R Foundation for Statistical Computing: Vienna, Austria, 2019; Available online: https://www.R-project.org/ (accessed on 17 December 2019).

53. Chong, J.; Soufan, O.; Li, C.; Caraus, I.; Li, S.; Bourque, G.; Wishart, D.S.; Xia, J. MetaboAnalyst 4.0: Towards more transparent and integrative metabolomics analysis. Nucleic Acids Res. 2018, 46, 486-494. [CrossRef] [PubMed]

(C) 2020 by the authors. Licensee MDPI, Basel, Switzerland. This article is an open access article distributed under the terms and conditions of the Creative Commons Attribution (CC BY) license (http://creativecommons.org/licenses/by/4.0/). 


\title{
Metabolomics Approach Reveals the Effects of Breed and Feed on the Composition of Chicken Eggs
}

\author{
Tatsuhiko Goto ${ }^{1,2, *}$, Hiroki Mori ${ }^{2}$, Shunsuke Shiota ${ }^{3}$ and Shozo Tomonaga ${ }^{3}$ \\ 1 Research Center for Global Agromedicine, Obihiro University of Agriculture and Veterinary Medicine, \\ Obihiro, Hokkaido 080-8555, Japan \\ 2 Department of Life and Food Sciences, Obihiro University of Agriculture and Veterinary Medicine, Obihiro, \\ Hokkaido 080-8555, Japan; s27224@st.obihiro.ac.jp \\ 3 Graduate School of Agriculture, Kyoto University, Kyoto 606-8502, Japan; \\ shiota.shunsuke.68v@st.kyoto-u.ac.jp (S.S.); shozo@kais.kyoto-u.ac.jp (S.T.) \\ * Correspondence: tats.goto@obihiro.ac.jp; Tel.: +81-155-49-5426
}

Received: 24 September 2019; Accepted: 10 October 2019; Published: 13 October 2019

\begin{abstract}
Chicken eggs provide essential nutrients to consumers around the world. Although both genetic and environmental factors influence the quality of eggs, it is unclear how these factors affect the egg traits including egg metabolites. In this study, we investigated breed and feed effects on 10 egg traits, using two breeds (Rhode Island Red and Australorp) and two feed conditions (mixed feed and fermented feed). We also used gas chromatography-mass spectrometry (GC-MS/MS) to analyze 138 yolk and 132 albumen metabolites. Significant breed effects were found on yolk weight, eggshell weight, eggshell colors, and one albumen metabolite (ribitol). Three yolk metabolites (erythritol, threitol, and urea) and 12 albumen metabolites (erythritol, threitol, ribitol, linoleic acid, isoleucine, dihydrouracil, 4-hydroxyphenyllactic acid, alanine, glycine, N-butyrylglycine, pyruvic acid, and valine) were significantly altered by feed, and a significant interaction between breed and feed was discovered in one albumen metabolite (N-butyrylglycine). Yolk and albumin had higher levels of sugar alcohols when hens were fed a fermented diet, which indicates that sugar alcohol content can be transferred from diet into eggs. Linoleic acid was also enriched in albumen under fermented feed conditions. This study shows that yolk and albumen metabolites will be affected by breed and feed, which is the first step towards manipulating genetic and environmental factors to create "designer eggs."
\end{abstract}

Keywords: albumen; breed; chicken; feed; metabolome; yolk

\section{Introduction}

Approximately 80 million tons of chicken eggs are produced every year, and they are a crucial source of animal protein in many developing countries [1,2]. Globally, 821 million people are malnourished [3], so international efforts to increase egg production are of high priority. In more developed countries, consumers are increasingly concerned with the quality of eggs and the bioavailability of favorable functional ingredients $[4,5]$. Increasing the quantity and quality of eggs around the world is key not only to alleviating hunger, but also to providing important dietary nutrients and keeping up with a rapidly changing international livestock industry. Currently, huge scientific effort is devoted to modifying agricultural produce traits to create "designer foods" [6]; eggs are no exception, and considerable effort is currently underway to modify egg traits and produce "designer eggs" to meet consumer demand $[7,8]$.

Both genetic and environmental factors influence the quantity and quality of eggs [9-11]. Heritability estimates of egg traits such as overall weight, albumen weight, and yolk weight, are around $0.30-0.70$ [12-14], which indicates that genetic factors are crucial to the regulation of egg 
traits. These general egg traits, which are mass and quality of egg components (yolk, albumen, and eggshell), are important for quality of egg itself as the product of egg market. Environmental factors including age, nutrition, stress, disease, medication, and production system also have important roles in modifying egg traits $[9,10]$. Thus, studies seeking to enhance egg traits should be cognizant of both genetic and environmental factors.

Egg enrichment with omega-3 polyunsaturated fatty acids (n-3 PUFA), which provide various human health benefits, is of current interest [15]. Evidence suggests that fatty acids quantity can be altered through changes to hens' diet [16]. Other research suggests that responses to dietary enrichment and conversion into egg metabolites may be breed-specific [17]. Both breed and feed appear to regulate the abundance of metabolites in egg yolk and albumen. Recently, we also have reported that egg yolk amino acid was modified by both breed and feed [18]. This is very likely to be the tip of the iceberg regarding the effects of genetic and environmental factors on egg composition.

Metabolomics, the study of the set of metabolites present in various tissues, is used to identify novel metabolites or changes in metabolite ratios in tissues [19]. Metabolomic analyses are used in a variety of fields, including biomedicine [20,21], plant science [19], food science [22,23], and ecological/environmental science [24,25]. Typical metabolite analyses use gas chromatography- mass spectrometry (GC-MS), liquid chromatography-mass spectrometry (LC-MS), capillary electrophoresismass spectrometry (CE-MS), and nuclear magnetic resonance (NMR). Metabolome analyses have previously been used to identify changes caused by stress and/or feed in mice using CE-MS [26] and GC-MS [27], so we expect a metabolomics approach to provide useful insight into the role of breed and feed in determining egg composition.

As studies into the metabolomes of livestock animals accumulate, a central database-the Livestock Metabolome Database- - has been developed to compile this information. Research into cattle metabolic constitutes a large proportion of this work (76 of 149 articles), as does research into animal health, nutrition, and production (97 of 149 papers; [28]). For instance, effects of long-distance transportation in serum metabolites have been studied in cattle [29] and heat stress-induced metabolomic changes have been investigated in chicks [30]. Relatively few reports target non-major livestock breeds, and there is a need to populate the Livestock Metabolome Database with basic metabolome data for all livestock breeds.

In this study, we analyzed the yolk and albumen metabolome of eggs and general egg traits from two different breeds of hens under two different feed conditions. We used the GC-MS/MS technique to measure metabolome content, and tested how egg metabolites are influenced by specific genetic and environmental factors, i.e., breed and feed, respectively.

\section{Results}

\subsection{Egg Traits}

To test the effects of breed and feed on egg traits, 10 traits were investigated (Table 1). Two-way mixed design analysis of variance (ANOVA) revealed a significant effect of breed on yolk weight $\left(\mathrm{F}_{1,12}=8.098, P=0.0147\right)$, eggshell weight $\left(\mathrm{F}_{1,12}=7.287, P=0.0193\right)$, lightness of eggshell color $\left(\mathrm{F}_{1,12}=6.022, P=0.0304\right)$, redness of eggshell color $\left(\mathrm{F}_{1,12}=10.818, P=0.0065\right)$, and yellowness of eggshell color $\left(\mathrm{F}_{1,12}=14.394, P=0.0026\right)$. Rhode Island Red (RIR) eggs showed higher yolk weight and lower eggshell weight than Australorp (AUS) eggs. Eggshell color in RIR eggs had less lightness, and more redness and yellowness than that of AUS. There were no significant effects of feed or interaction terms between breed and feed on any of the egg traits. 


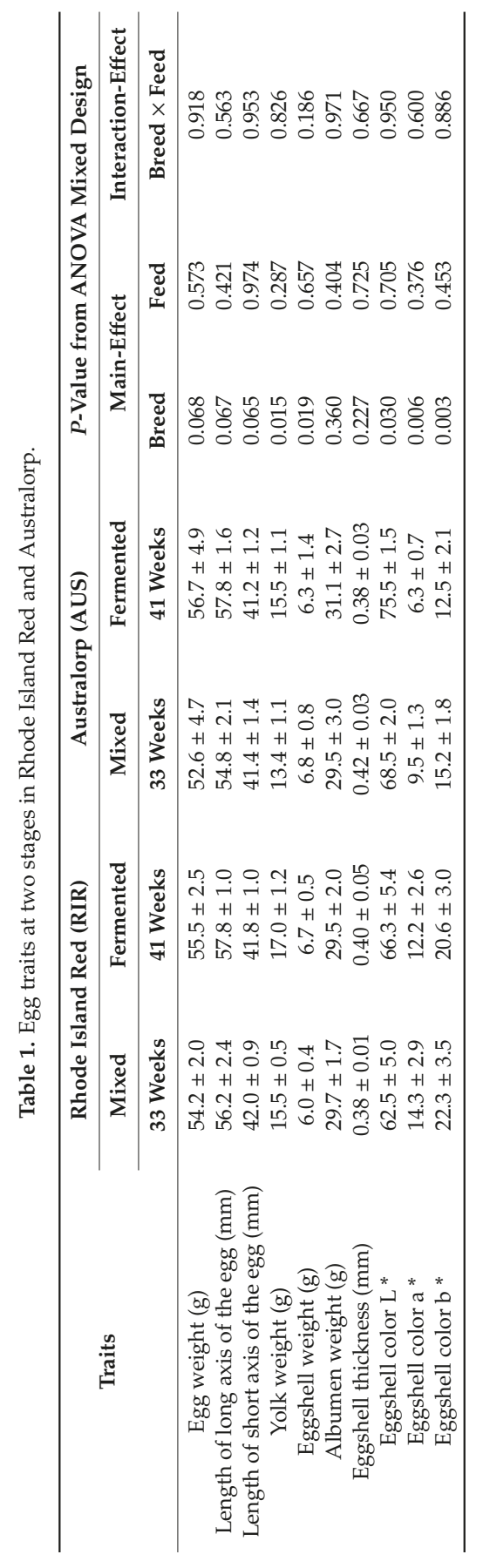




\subsection{Egg Metabolite Traits}

Egg metabolite traits from yolk (138 metabolites) and albumen (132 metabolites) were semi-quantified using GC-MS/MS. Full results were shown for yolk and albumen in Supplementary Tables S1 and S2, respectively. Controlling for multiple comparisons in each sample, some metabolites were found to be metabolome-wide significantly altered by breed and feed $(Q<0.1)$.

Albumen ribitol was significantly affected by breed (Table 2), with RIR eggs containing significantly higher ribitol levels than AUS eggs. Three metabolites in yolk and 12 metabolites in albumen had significant effects of feed (Table 3). Erythritol and threitol were significantly altered by feed in both the yolk and the albumen. Urea was altered in the yolk samples only, whereas isoleucine, dihydrouracil, linoleic acid, 4-hydroxyphenyllactic acid, alanine, glycine, N-butyrylglycine, pyruvic acid, ribitol, and valine were altered in albumen samples only. For threitol, erythritol, dihydrouracil, linoleic acid, pyruvic acid, and ribitol, the fermented feed group in both chicken breeds showed significantly higher metabolite content than mixed feed. On the other hand, the fermented feed group had significantly lower contents of urea, isoleucine, 4-hydroxyphenyllactic acid, alanine, glycine, N-butyrylglycine, and valine than the mixed feed group. There was a significant interaction between breed and feed on $\mathrm{N}$-butyrylglycine in the albumen; N-butyrylglycine content in RIR chickens was higher with mixed feed than with fermented feed, but this effect was reversed in the AUS samples (Table 4). 

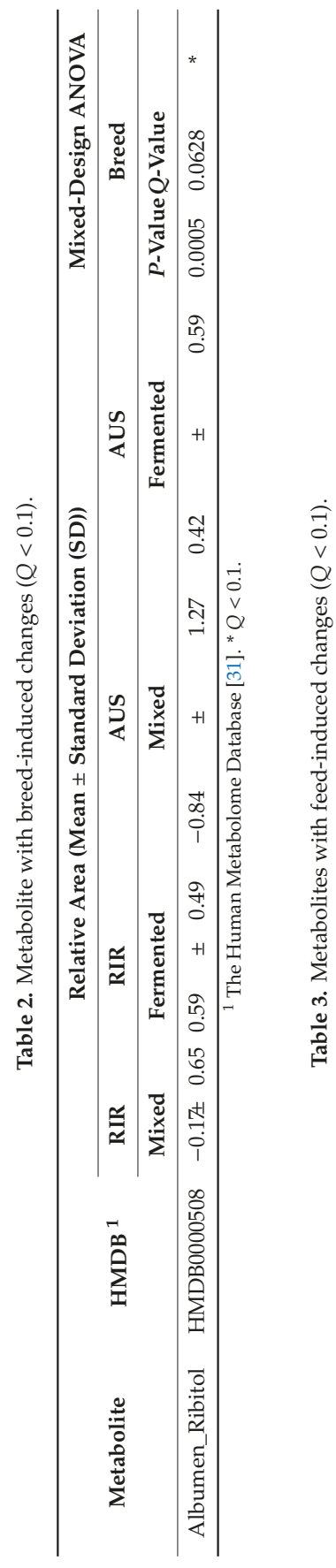

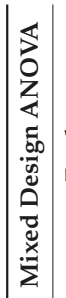

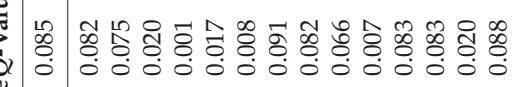

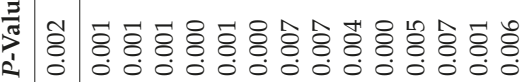

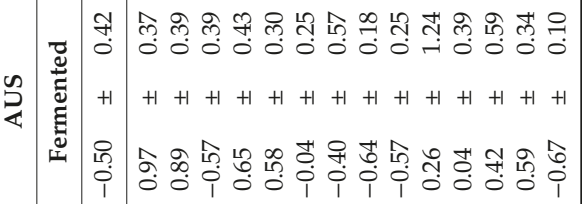
苞 की 0 远 $+1+1+1+1+1+1+1+1+1+1+1+1+1$

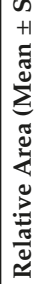
$\dot{2}$

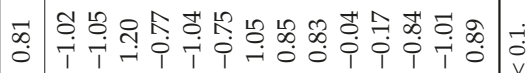
$\cong$ 芯

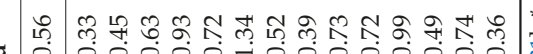
$+1+1+1+1+1+1+1+1+1+1+1+1+1$

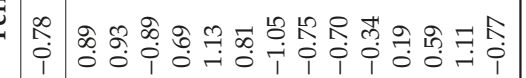
สุ่ กิ

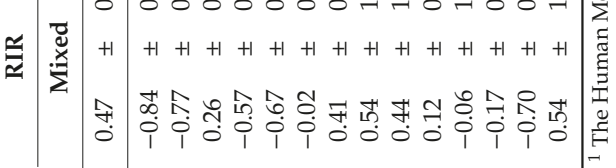

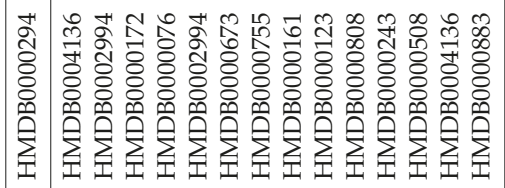

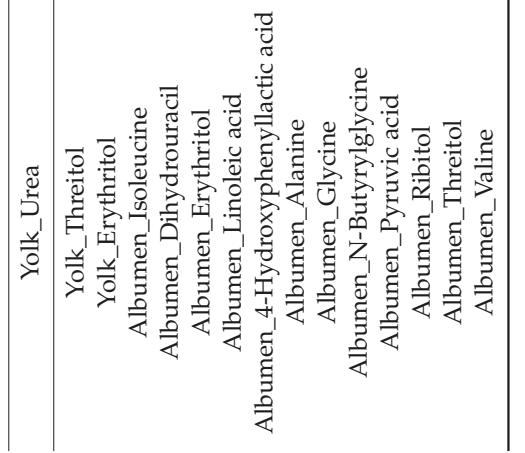




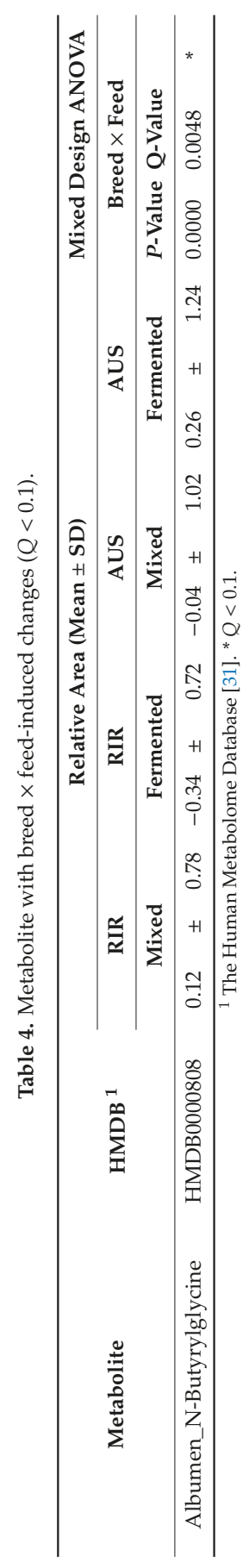




\section{Discussion}

This study was designed to test the effects of breed and feed on 10 egg traits, 138 yolk metabolite traits, and 132 albumen metabolite traits, in RIR/AUS hens fed with either mixed feed or fermented feed. There were significant breed effects on yolk weight, eggshell weight, eggshell colors, and one albumen metabolite. Three yolk metabolites and 12 albumen metabolites were significantly altered by feed, and a significant interaction between breed and feed affected levels of one albumen metabolite. Using the metabolome technique, this study has demonstrated that certain egg properties, including metabolites in the yolk and albumen, can be changed by both genetic and environmental factors.

Since general egg traits, which are mass and quality of egg components (yolk, albumen, and eggshell), are crucial to maintain egg quality, we investigated 10 egg traits in this study. Egg traits including weight, length, width, albumen weight, and eggshell thickness showed no significant differences between RIR and AUS hens in this study, although AUS eggshell weight was higher than RIR. Average body weight at 35 weeks of age in RIR hens was more than twice that of AUS hens (3.69 and $1.58 \mathrm{~kg}$ in RIR and AUS, respectively). Yolk weight in RIR was significantly greater than that in AUS, corresponding to differences in body weight. We previously reported that Oh-Shamo $(2.91 \mathrm{~kg})$, and classical type of White Leghorn hens $(1.54 \mathrm{~kg})$ with average body weight at 36 weeks of age produced $53.8 \pm 4.2 \mathrm{~g}$ and $47.4 \pm 2.3 \mathrm{~g}$ of egg weight at 300 days of age, respectively [32,33]. Egg weight in AUS hens was $56.7 \pm 4.9 \mathrm{~g}$ at around 300 days (41 weeks). Thus, AUS hens have a potential to produce eggs that are relatively larger than expected from their body weight [18]. On the other hand, significant breed effects were found on eggshell colors (lightness, redness, and yellowness), with RIR hens laying darker brown eggs than AUS hens. White to brown variation in eggshell color is a heritable quantitative trait [9-11], and eggshell colors of Oh-Shamo, White Leghorn, Hy-line Brown, and Onagadori chickens were reported [33-35]. Comparing this study to previous work, eggs appear to decrease in color from brown to white in the order of Hy-Line Brown $>$ RIR $>$ Oh-Shamo $>$ AUS $>$ Onagadori $>$ White Leghorn [33-35]. Further efforts must be needed to reveal the genetic basis of eggshell coloration in chickens using population genomics and genome-wide scan.

The present metabolome analyses revealed significant changes in the amount of sugar alcohols (polyols) present in eggs. Erythritol and threitol in the yolk and albumen appear to be affected by dietary changes, specifically moving to a fermented diet. Sugar alcohols are naturally found in small quantities in fruits, vegetables, mushrooms, and fermented foods such as wine, beer, sake, and soy sauce [36], and can be produced by several yeasts and fungi [37]. The fermented feed used in this study was made with wheat, pumpkin, yam, soybean, potato, rice bran, fish meal, beet lees, scallop shell, and other materials, and lactic acid bacteria were used to ferment the mixture [18]; the amount of sugar alcohols in fermented feed would be higher than in mixed feed. Eggs produced under the fermented feed treatment had high sugar alcohol content, which is likely due to the transfer of these nutrients from feed to eggs. The same trend was also apparent for ribitol, which was found at a higher concentration in eggs produced on the fermented diet. There was also a significant breed effect, and ribitol levels in RIR eggs were higher than those of AUS eggs. These results indicate that different genetic backgrounds can affect the transfer of nutrients into eggs.

After 7 weeks on the fermented feed treatment, erythritol contents in both the yolk and albumen were significantly higher than on mixed feed. Erythritol is a four-carbon sugar alcohol (polyol) that has a sweetness $60 \%$ to $80 \%$ that of sucrose, and is used as a low-calorie sweetener [38]. This may indicate that yolk and albumen taste are affected by feed type. In humans, erythritol is fast absorbed through the small intestine, and $>90 \%$ is excreted intact in urine. The remaining $10 \%$ is fermented in the large intestine by colonic microorganisms [36]. Polyols act as probiotics like a fiber, and can help normalize intestine function [36]. There are massive evidences that the intake of erythritol would not cause adverse effects in humans [38]. Artificial sweeteners and/or polyols may be helpful in diabetes and weight control, and eggs enriched with erythritol may be useful as a functional food.

Linoleic acid levels in egg albumen were significantly increased by the fermented feed diet. Linoleic acid is a long-chain polyunsaturated fatty acid (PUFA), and an essential nutrient in wide 
range of animals including humans. PUFA is vital for body functions and plays an important role in the formation and functioning of cell membranes, cell physiology, signaling, immunity, and reproduction [39]. Many animals lack the ability to synthesize linoleic acid de novo, so dietary intake of linoleic acid is necessary [39]. Enrichment of eggs with PUFAs, including linoleic acid, is relatively common and increases the nutritional functionality of enriched eggs [15]. Linoleic acid is found at relatively high levels in seed oils [40]. Fermented feed may derive its high linoleic acid content from its constituent plant matter, which includes pumpkin seeds, or from its relatively high proportion of crude fat. However, although isoleucine, alanine, glycine, and valine levels in the fermented feed were also higher than those of mixed feed, albumin isoleucine, alanine, glycine, and valine contents of eggs under the fermented feed diet were significantly lower than those of the mixed feed diet. More works are required to understand the changes to these amino acids levels in albumen.

There was a significant interaction between breed and feed on albumin N-butyrylglycine levels. $\mathrm{N}$-butyrylglycine is an acylglycine [41], which is formed by the conjugation of acyl-CoA esters with glycine [42]. Acylglycines are used as diagnostic markers of inborn errors of metabolism [41], and previous studies have shown that acylglycine levels are sensitive in the urine of spontaneously hypertensive rats on a high-fat diet [42]. In this study, we found that acylglycine content was higher under the mixed feed treatment than fermented feed in RIR hens, but the opposite was true for AUS hens. Since N-butyrylglycine is involved in fatty acid metabolism [42], this may indicate that the combination of breed and feed can affect fatty acid metabolism. Actually, linoleic acid in albumen was significantly altered in this study. Such interaction effects of breed and feed have been documented in the enriched fatty acid levels of yolks in other studies [17]. In the modern chicken industry, hybrid chickens rather than pure breeds are often used for egg and meat production. This study indicates that the combination of breed and feed should be considered to modulate metabolite levels in yolk and albumen.

In conclusion, we found significant breed and feed effects on yolk weight, eggshell weight, eggshell colors, and several metabolites in the yolk and albumen. Feed alone had a notable impact on sugar alcohol and fatty acid levels, which were enriched in both yolk and albumen under the fermented feed treatment. We illustrated that both genetic and environmental factors are critical to determining egg composition and should be considered in the efforts to meet consumer needs and develop nutritionally functional designer eggs.

\section{Materials and Methods}

\subsection{Study Animals}

Rhode Island Red (RIR; $n=5$ ) and Australorp (AUS; $n=5$ ) hens were procured from the Animal Research Center of the Hokkaido Research Organization, Japan. They were introduced to the experimental farm of the Obihiro University of Agriculture and Veterinary Medicine, Japan, at 22 weeks of age. After introduction, all hens were reared in individual cages under a photoperiod cycle of $16 \mathrm{~h}$ light and $8 \mathrm{~h}$ dark, with free access to diet and water. Body weight at 35 weeks of age was $3.69 \pm 0.57$ and $1.58 \pm 0.09 \mathrm{~kg}$ (mean \pm standard deviation) for RIR and AUS, respectively $\left(\mathrm{F}_{1,8}=67.324, P=3.6 \times 10^{-5}\right)$. Animal management was carried out following the Guide for the Use of Experimental Animals in Universities (The Ministry of Education, Science, Sports, and Culture, Tokyo, Japan 1987) and the Standards Related to the Care and Management of Experimental Animals (Prime Ministers' Office, Tokyo, Japan, 1980). This study (authorization number 18-15) was approved from the Experimental Animal Committee of the Obihiro University of Agriculture and Veterinary Medicine.

\subsection{Experimental Conditions and Sampling}

To test the effects of breed and feed on egg metabolome, RIR and AUS hens were reared under two different feed conditions. Mixed feed (Rankeeper; Marubeni Nisshin Feed Co., Ltd., Tokyo, Japan) was provided for 11 weeks, from introduction until 33 weeks of age. Fermented feed (Kusanagi Farm 
Limited Company, Obihiro, Japan) was provided for 9 weeks, from 34 weeks of age until the end of the experiment. The fermented feed was made from many feed materials such as potato peel, cottons and seeds of pumpkin, sake lees, and wheat, especially using a silage preparation additive, WS360 (Protocol Japan Ltd., Obihiro, Japan), which contains lactic acid bacteria and cellulolytic enzyme [18]. The ingredients of both mixed and fermented feeds were analyzed at the Institute of Chemurgy, in the Tokachi Federation of Agricultural Cooperatives, Japan (Supplementary Table S3). Eggs from each breed (RIR and AUS) were collected at two stages: at the end of the mixed feed period ( 33 weeks of age) and near the end of the fermented feed period (41 weeks of age, see Figure 1). Five eggs were collected from each breed at each stage, totaling 20 eggs over the course of the study.

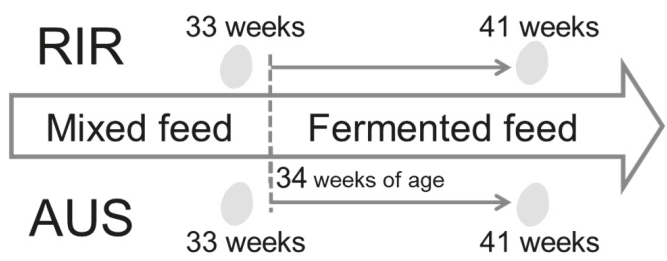

Figure 1. Experimental design. Eggs from Rhode Island Red (RIR) and Australorp (AUS) hens, which had been fed mixed feed, were collected at 33 weeks of age (mixed feed). Feed was switched at 34 weeks of age to fermented feed. Eggs were again collected at 41 weeks of age (fermented feed). Five eggs were collected at each stage from each breed; egg traits and yolk and albumen metabolite traits were measured in the 20 eggs. These data were analyzed using two-way mixed design analysis of variance (ANOVA) with breed group as the between-subjects factor and feed group as the within-subject factor.

\subsection{Measuring Egg Properties}

Ten egg traits were measured, including egg weight, length of the long axis, length of the short axis, eggshell weight, yolk weight, albumen weight, eggshell thickness, and eggshell lightness $\left(L^{*}\right)$, redness $\left(\mathrm{a}^{*}\right)$, and yellowness $\left(\mathrm{b}^{*}\right)$. Size was measured using a digital caliper (P01 110-120; ASONE, Japan). Eggshell colors were measured using a chromameter (CR-10 Plus Color Reader; Konica Minolta Japan, Inc., Tokyo, Japan), and eggshell thickness was measured with a Peacock dial pipe gauge P-1 (Ozaki MFG Co., Ltd., Tokyo, Japan). After weighing, yolk and albumen were kept separately at $-30{ }^{\circ} \mathrm{C}$ pending further analysis.

\subsection{Metabolomic Analysis of Egg Yolk and Albumen}

Prior to metabolomic analysis, yolk and albumen samples were dried using a freeze dryer (FD-550P, Eyela; Tokyo Rikakikai Co. Ltd., Tokyo, Japan). Samples were crushed into a powder form, and $10 \mathrm{mg}$ of each sample was used for metabolomic analysis. Powder samples were mixed with $250 \mu \mathrm{L}$ of pretreatment liquid (methanol/ $\mathrm{H}_{2} \mathrm{O} /$ chloroform 5:2:2) and $5 \mu \mathrm{L}$ of internal standard solution (2-isopropylmalic acid, $1 \mathrm{mg} / \mathrm{mL}$ ). After vortex mixing, the sample tubes were shaken at $1200 \mathrm{rpm}$ at $37^{\circ} \mathrm{C}$ for $30 \mathrm{~min}$ under dark light conditions. After centrifugation at $16,000 \times g, 4{ }^{\circ} \mathrm{C}$ for $5 \mathrm{~min}$, the supernatants $(160 \mu \mathrm{L})$ were collected and placed in new tubes with $200 \mu \mathrm{L}$ of $\mathrm{H}_{2} \mathrm{O}$ and mixed using a vortex. The tubes were again centrifuged at $16,000 \times g, 4{ }^{\circ} \mathrm{C}$ for $5 \mathrm{~min}$. The supernatants $(200 \mu \mathrm{L})$ were collected into new tubes and centrifuged at room temperature in a vacuum (Centrifugal Evaporator RD400; Yamato Scientific Co. Ltd., Tokyo, Japan) for $20 \mathrm{~min}$. Then, the tubes were placed in $-80{ }^{\circ} \mathrm{C}$ until freezing. The tubes were centrifuged at room temperature in a vacuum for $7-8 \mathrm{~h}$. Methoxyamine hydrochloride in pyridine $(20 \mathrm{mg} / \mathrm{mL}, 40 \mu \mathrm{L})$ was added to each tube and mixed using the vortex. The tubes were shaken for $90 \mathrm{~min}$ at $1200 \mathrm{rpm}, 30^{\circ} \mathrm{C}$, under dark conditions, for oximation. $\mathrm{N}$-methyl-N-trimethylsilyltrifluoroacetamine (MSTFA; $20 \mu \mathrm{L}$ ) was added to each tube and vortex-mixed. The tubes were shaken at $1200 \mathrm{rpm}$ at $37^{\circ} \mathrm{C}$ for $45 \mathrm{~min}$ in the dark to prepare trimethylsilyl (TMS) derivatives. GC-MS/MS analyses were carried out using a GCMS-TQ8050 (Shimadzu, Kyoto, Japan) 
with BPX-5 column (Length $30 \mathrm{~m}$; 0.25 mm I.D.; df = $0.25 \mu \mathrm{m}$ ) (SGE, Melbourne, Australia) according to the method in Smart Metabolites Database (Shimadzu, Kyoto, Japan).

Data processing was performed with Smart Metabolites Database (Shimadzu, Kyoto, Japan), MS-DIAL ver 3.08 [43] and MRMPROBS program ver. 2.42 [44]. Peaks of 40 samples (20 eggs $\times$ 2 samples) were recorded over the mass range 45-600 m/z. Peaks were automatically detected via MS-DIAL with peak detection options that minimum peak height is 2000. A data quality check was conducted using thresholds, which are $-10<\mathrm{RI}<10$, dot prod $>0.8$, and presence $>0.6$ and then manually checked. Finally, 138 and 132 metabolites for yolk and albumen were identified, respectively. Relative quantity of metabolites was calculated using the peak area of each metabolite relative to an internal standard (2-isopropylmalic acid).

\subsection{Statistical Methods}

To identify the effects of breed and feed on egg properties, data were analyzed using a two-way mixed-design analysis of variance (ANOVA) with breed group (RIR and AUS) as the between-subjects factor and feed group (mixed feed and fermented feed) as the within-subject (repeated) factor [18,45-47]. Main- and interaction-effects of breed and feed on egg properties were tested $(P<0.05)$. Data are presented as the mean and standard deviation. Statistical analyses were conducted using R [48]. In metabolites in particular, individual values were standardized with their mean and standard deviation.

To control the $P$ value for multiple comparisons in metabolites, the false discovery rate was determined using the approach of Benjamini and Hochberg [49]. The $P$ values were adjusted using the Benjamini-Hochberg correction, and post-adjustment are referred to as $Q$ values. The metabolome-wide significance threshold was set at $Q<0.1$, following previous studies [26,27,50,51].

Supplementary Materials: The following are available online at http://www.mdpi.com/2218-1989/9/10/224/s1: Table S1: All yolk metabolites, Table S2: All albumen metabolites, Table S3: Ingredient analysis of mixed feed and fermented feed.

Author Contributions: Conceptualization, T.G. and S.T.; methodology, T.G. and S.T.; software, S.S. and S.T.; validation, T.G., H.M., S.S. and S.T.; formal analysis, T.G. and H.M.; investigation, T.G., H.M., S.S. and S.T.; resources, T.G. and S.T.; data curation, T.G.; writing — original draft preparation, T.G.; writing-review and editing, T.G. and S.T.; visualization, T.G.; supervision, T.G.; project administration, T.G.; funding acquisition, T.G.

Funding: This work was supported in part by Grants-in-Aid from The Akiyama Life Science Foundation and Obihiro City Office to T.G.

Acknowledgments: We thank Masaaki Hanada and Masafumi Tetsuka for the use of laboratory equipments, and all members of the Animal Breeding Research Group at the Obihiro University of Agriculture and Veterinary Medicine for their continuous support.

Conflicts of Interest: The authors declare no conflict of interest.

\section{References}

1. FAOSTAT. 2019. Available online: http://www.fao.org/faostat/en/\#home (accessed on 20 September 2019).

2. Goto, T.; Fernandes, A.F.A.; Tsudzuki, M.; Rosa, G.J.M. Causal phenotypic networks for egg traits in an $\mathrm{F}_{2}$ chicken population. Mol. Genet. Genom. 2019, 1-8. [CrossRef] [PubMed]

3. Hunger Map. 2018. Available online: https://www.wfp.org/content/2018-hunger-map (accessed on 20 September 2019).

4. Kralik, G.; Kralik, Z. Poultry products enriched with nutricines have beneficial effects on human health. Med. Glas. 2017, 14, 1-7. [CrossRef]

5. Laudodio, V.; Lorusso, V.; Lastella, N.M.B.; Dhama, K.; Karthik, K.; Tiwari, R.; Alam, G.M.; Tufarelli, V. Enhancement of nutraceutical value of table eggs through poultry feeding strategies. Int. J. Pharm. 2015, 11, 201-212. [CrossRef]

6. Rajasekaran, A.; Kalaivani, M. Designer foods and their benefits: A review. J. Food Sci. Technol. 2013, 50, 1-16. [CrossRef]

7. Zaheer, K. An updated review on chicken eggs: Production, consumption, management aspects and nutritional benefits to human health. Food Nutr. Sci. 2015, 6, 1208-1220. [CrossRef] 
8. Alagawany, M.; Farag, M.R.; Dhama, K.; Patra, A. Nutritional significance and health benefits of designer eggs. Worlds Poult. Sci. J. 2018, 74, 317-330. [CrossRef]

9. Roberts, J. Factors affecting egg internal quality and egg shell quality in laying hens. J. Poult. Sci. 2004, 41, 161-177. [CrossRef]

10. Wilson, P.B. Recent advances in avian egg science: A review. Poult. Sci. 2017, 96, 3747-3754. [CrossRef] [PubMed]

11. Goto, T.; Tsudzuki, M. Genetic mapping of quantitative trait loci for egg production and egg quality traits in chickens: A review. J. Poult. Sci. 2017, 54, 0160121. [CrossRef]

12. Wolc, A.; White, I.M.S.; Hill, W.G.; Olori, V.E. Inheritance of hatchability in broiler chickens and its relationship to egg quality traits. Poult. Sci. 2010, 89, 2334-2340. [CrossRef]

13. Wolc, A.; Arango, J.; Settar, P.; O'Sullivan, N.P.; Olori, V.E.; White, I.M.S.; Hill, W.G.; Dekkers, J.C.M. Genetic parameters of egg defects and egg quality in layer chickens. Poult. Sci. 2012, 91, 1292-1298. [CrossRef] [PubMed]

14. Zhang, L.C.; Ning, Z.H.; Xu, G.Y.; Hou, Z.C.; Yang, N. Heritabilities and genetic and phenotypic correlations of egg quality traits in brown-egg dwarf layers. Poult. Sci. 2005, 84, 1209-1213. [CrossRef] [PubMed]

15. Fraeye, I.; Bruneel, C.; Lemahieu, C.; Buyse, J.; Muylaert, K.; Foubert, I. Dietary enrichment of eggs with omega-3 fatty acids: A review. Food Res. Int. 2012, 48, 961-969. [CrossRef]

16. Surai, P.F.; Sparks, N.H.C. Designer eggs: From improvement of egg composition to functional food. Trends. Food Sci. Technol. 2001, 12, 7-16. [CrossRef]

17. Yin, J.D.; Shang, X.G.; Li, D.F.; Wang, F.L.; Guan, Y.F.; Wang, Z.Y. Effects of dietary conjugated linoleic acid on the fatty acid profile and cholesterol content of egg yolks from different breeds of layers. Poult. Sci. 2008, 87, 284-290. [CrossRef] [PubMed]

18. Mori, H.; Takaya, M.; Nishimura, K.; Goto, T. Breed and feed affect amino acid contents of egg yolk and eggshell color in chickens. Poult. Sci. 2019. [CrossRef]

19. Weckwerth, W. Metabolomics in systems biology. Annu. Rev. Plant Biol. 2003, 54, 669-689. [CrossRef] [PubMed]

20. Griffiths, W.J.; Koal, T.; Wang, Y.; Kohl, M.; Enot, D.P.; Deigner, H.P. Targeted metabolomics for biomarker discovery. Angew. Chem. Int. Ed. Engl. 2010, 26, 5426-5445. [CrossRef]

21. Li, B.; He, X.; Jia, W.; Li, H. Novel applications of metabolomics in personalized medicine: A mini-review. Molecules 2017, 22, 1173. [CrossRef]

22. Wishart, D.S. Metabolomics: Applications to food science and nutrition research. Trends Food Sci. Technol. 2008, 19, 482-493. [CrossRef]

23. Cubero-Leon, E.; Peñalver, R.; Maquet, A. Review on metabolomics for food authentication. Food Res. Int. 2014, 60, 95-107. [CrossRef]

24. Bundy, J.G.; Davey, M.P.; Viant, M.R. Environmental metabolomics: A critical review and future perspectives. Metabolomics 2009, 5, 3. [CrossRef]

25. Macel, M.; Van Dam, N.M.; Keurentjes, J.J. Metabolomics: The chemistry between ecology and genetics. Mol. Ecol. Resour. 2010, 10, 583-593. [CrossRef] [PubMed]

26. Goto, T.; Kubota, Y.; Toyoda, A. Plasma and liver metabolic profiles in mice subjected to subchronic and mild social defeat stress. J. Proteome Res. 2015, 14, 1025-1032. [CrossRef] [PubMed]

27. Goto, T.; Tomonaga, S.; Toyoda, A. Effects of diet quality and psychosocial stress on the metabolic profiles of mice. J. Proteome Res. 2017, 16, 1857-1867. [CrossRef] [PubMed]

28. Goldansaz, S.A.; Guo, A.C.; Sajed, T.; Steele, M.A.; Plastow, G.S.; Wishart, D.S. Livestock metabolomics and the livestock metabolome: A systematic review. PLoS ONE 2017, 12, e0177675. [CrossRef] [PubMed]

29. Takemoto, S.; Tomonaga, S.; Funaba, M.; Matsui, T. Effect of long-distance transportation on serum metabolic profiles of steer calves. Anim. Sci. J. 2017, 88, 1970-1978. [CrossRef]

30. Tomonaga, S.; Okuyama, H.; Tachibana, T.; Makino, R. Effects of high ambient temperature on plasma metabolomic profiles in chicks. Anim. Sci. J. 2018, 89, 448-455. [CrossRef]

31. Wishart, D.S.; Feunang, Y.D.; Marcu, A.; Guo, A.C.; Liang, K.; Vázquez-Fresno, R.; Sajed, T.; Johnson, D.; Li, C.; Karu, N.; et al. HMDB 4.0: The human metabolome database for 2018. Nucleic Acid Res. 2017, 46, D608-D617. [CrossRef]

32. Goto, T.; Ishikawa, A.; Nishibori, M.; Tsudzuki, M. A longitudinal quantitative trait locus mapping of chicken growth traits. Mol. Genet. Genom. 2019, 294, 243-252. [CrossRef] 
33. Goto, T.; Ishikawa, A.; Yoshida, M.; Goto, N.; Umino, T.; Nishibori, M.; Tsudzuki, M. Quantitative trait loci mapping for external egg traits in $\mathrm{F}_{2}$ chickens. J. Poult. Sci. 2014, 51, 118-129. [CrossRef]

34. Goto, T.; Shiraishi, J.I.; Bungo, T.; Tsudzuki, M. Characteristics of egg-related traits in the Onagadori (Japanese Extremely Long Tail) breed of chickens. J. Poult. Sci. 2015, 52, 81-87. [CrossRef]

35. Sirri, F.; Zampiga, M.; Berardinelli, A.; Meluzzi, A. Variability and interaction of some egg physical and eggshell quality attributes during the entire laying hen cycle. Poult. Sci. 2018, 97, 1818-1823. [CrossRef] [PubMed]

36. Grembecka, M. Sugar alcohols—-their role in the modern world of sweeteners: A review. Eur. Food Res. Technol. 2015, 241, 1-14. [CrossRef]

37. Wisselink, H.W.; Weusthuis, R.A.; Eggink, G.; Hugenholtz, J.; Grobben, G. Mannitol production by lactic acid bacteria: A review. Int. Dairy J. 2002, 12, 151-161. [CrossRef]

38. Bernt, W.O.; Borzelleca, J.F.; Flamm, G.; Munro, I.C. Erythritol: A review of biological and toxicological studies. Regul. Toxicol. Pharm. 1996, 24, S191-S197. [CrossRef] [PubMed]

39. Malcicka, M.; Visser, B.; Ellers, J. An evolutionary perspective on linoleic acid synthesis in animals. Evol. Biol. 2018, 45, 15-26. [CrossRef] [PubMed]

40. Abedi, E.; Sahari, M.A. Long-chain polyunsaturated fatty acid sources and evaluation of their nutritional and functional properties. Food Sci. Nutr. 2014, 2, 443-463. [CrossRef] [PubMed]

41. Stanislaus, A.; Guo, K.; Li, L. Development of an isotope labeling ultra-high performance liquid chromatography mass spectrometric method for quantification of acylglycines in human urine. Anal. Chim. Acta 2012, 750, 161-172. [CrossRef]

42. Čermáková, M.; Pelantová, H.; Neprašová, B.; Šedivá, B.; Maletínská, L.; Kuneš, J.; Tomášová, P.; Železná, B.; Kuzma, M. Metabolomic study of obesity and its treatment with palmitoylated prolactin-releasing peptide analog in spontaneously hypertensive and normotensive rats. J. Proteome Res. 2019, 18, 1735-1750. [CrossRef]

43. Lai, Z.; Tsugawa, H.; Wohlgemuth, G.; Mehta, S.; Mueller, M.; Zheng, Y.; Ogiwara, A.; Meissen, J.; Showalter, M.; Takeuchi, K.; et al. Identifying metabolites by integrating metabolome databases with mass spectrometry cheminformatics. Nat. Methods 2018, 15, 53-56. [CrossRef] [PubMed]

44. Tsugawa, H.; Kanazawa, M.; Ogiwara, A.; Arita, M. MRMPROBS suite for metabolomics using large-scale MRM assays. Bioinformatics 2014, 30, 2379-2380. [CrossRef] [PubMed]

45. Olejnik, S.; Algina, J. Generalized eta and omega squared statistics: Measures of effect size for some common research designs. Psychol. Methods 2003, 4, 434-447. [CrossRef] [PubMed]

46. Franz, V.H.; Loftus, G.R. Standard errors and confidence intervals in within-subjects designs: Generalizing Loftus and Masson (1994) and avoiding the biases of alternative accounts. Psychon. Bull. Rev. 2012, 19, 395-404. [CrossRef] [PubMed]

47. Nikiforuk, A.; Potasiewicz, A.; Kos, T.; Popik, P. The combination of memantine and galantamine improves cognition in rats: The synergistic role of the $\alpha 7$ nicotinic acetylcholine and NMDA receptors. Behav. Brain Res. 2016, 313, 214-218. [CrossRef] [PubMed]

48. R Core Team. R: A language and Environment for Statistical Computing; R Foundation for Statistical Computing: Vienna, Austria, 2019; Available online: https://www.R-project.org/ (accessed on 20 September 2019).

49. Benjamini, Y.; Hochberg, Y. Controlling the false discovery rate: A practical and powerful approach to multiple testing. J. R. Stat. Soc. Ser. B 1995, 57, 298-300. [CrossRef]

50. McClay, J.L.; Adkins, D.E.; Vunck, S.A.; Batman, A.M.; Vann, R.E.; Clark, S.L.; Beardsley, P.M.; van den Oord, E.J. Large-scale neurochemical metabolomics analysis identifies multiple compounds associated with methamphetamine exposure. Metabolomics 2013, 9, 392-402. [CrossRef]

51. Pedersen, H.K.; Gudmundsdottir, V.; Nielsen, H.B.; Hyotylainen, T.; Nielsen, T.; Jensen, B.A.; Forslund, K.; Hildebrand, F.; Prifti, E.; Falony, G.; et al. Human gut microbes impact host serum metabolome and insulin sensitivity. Nature 2016, 535, 376-381. [CrossRef] [PubMed] 
Article

\title{
Exercise Induced Changes in Salivary and Serum Metabolome in Trained Standardbred, Assessed by ${ }^{1} \mathrm{H}-\mathrm{NMR}$
}

\author{
Marilena Bazzano ${ }^{1}$, Luca Laghi ${ }^{2, *}$, Chenglin Zhu ${ }^{2}$, Enrica Lotito ${ }^{1}$, Stefano Sgariglia ${ }^{3}$, \\ Beniamino Tesei ${ }^{1}$ and Fulvio Laus ${ }^{1}$ \\ 1 School of Biosciences and Veterinary Medicine, University of Camerino, Via Circonvallazione, 93/95, \\ 62024 Matelica, Italy; marilena.bazzano@unicam.it (M.B.); lotitoenrica@gmail.com (E.L.); \\ beniamino.tesei@unicam.it (B.T.); fulvio.laus@unicam.it (F.L.) \\ 2 Department of Agricultural and Food Sciences, University of Bologna, 47521 Cesena, Italy; \\ chenglin.zhu2@unibo.it \\ 3 Practitioner, 63900 Fermo, Italy; stefano.sgariglia@libero.it \\ * Correspondence: 1.laghi@unibo.it
}

Received: 16 June 2020; Accepted: 20 July 2020; Published: 21 July 2020

\begin{abstract}
In the present study, data related to the metabolomics of saliva and serum in trained standardbred horses are provided for the first time. Metabolomic analysis allows to analyze all the metabolites within selected biofluids, providing a better understanding of biochemistry modifications related to exercise. On the basis of the current advances observed in metabolomic research on human athletes, we aimed to investigate the metabolites' profile of serum and saliva samples collected from healthy standardbred horses and the relationship with physical exercise. Twelve trained standardbred horses were sampled for blood and saliva before $\left(\mathrm{T}_{0}\right)$ and immediately after $\left(\mathrm{T}_{1}\right)$ standardized exercise. Metabolomic analysis of both samples was performed by ${ }^{1} \mathrm{H}-\mathrm{NMR}$ spectroscopy. Forty-six metabolites in serum and 62 metabolites in saliva were detected, including alcohols, amino acids, organic acids, carbohydrates and purine derivatives. Twenty-six and 14 metabolites resulted to be significantly changed between $T_{0}$ and $T_{1}$ in serum and saliva, respectively. The findings of 2-hydroxyisobutyrate and 3-hydroxybutyrate in serum and GABA in equine saliva, as well as their modifications following exercise, provide new insights about the physiology of exercise in athletic horses. Glycerol might represent a novel biomarker for fitness evaluation in sport horses.
\end{abstract}

Keywords: horse; metabolomic; metabolism; exercise; saliva

\section{Introduction}

Metabolic changes related to energy production and cell growth have been observed in response to exercise in humans [1-4]. Although these studies have contributed to the understanding of the metabolic response after physical exercise, the limited number of metabolites taken into account does not represent the broader metabolic response caused by exercise [2,4,5]. Human serum accounts for about 4000 metabolites [6] that interact in a large and complex network [7]. Therefore, utilizing a more efficient and comprehensive method to analyze all the metabolites within selected biofluids could provide a better understanding of the metabolic response in the context of biochemistry modifications related to exercise.

In this context, the employment of metabolomic analysis can be essential, as demonstrated in a study by Berton and colleagues (2017) [8] that focused on the possibility of using serum as a valuable biofluid to investigate metabolomic modifications induced by exercise in sports players [8].

However, serum is only one of the biofluids suitable for scientific research in the field of sport medicine. A special interest was recently pointed towards the use of saliva as a diagnostic fluid both 
in human and veterinary medicine $[9,10]$. It is well known that saliva composition can be affected by systemic disorders and may reflect general metabolic changes [11]. When compared with other biological samples, saliva has the advantage of being easily collected by non-invasive and non-stressful procedures, which is extremely important when sampling animals [9]. The composition of saliva is 99\% water but it also contains several compounds such as hormones, glucose, lactate, fatty acids, triglycerides, cholesterol, urea, uric acid and phosphorus [9]. Metabolites, enzymes, proteins and minerals have been found in saliva samples collected from humans, pigs, sheep and horses [9,12-17]. Some of these metabolites have been found to change as a result of pathological conditions like lameness, stress, abdominal pain, inflammation and kidney diseases, and could be eligible as biomarkers in humans and animals $[9,12,13,16,18,19]$. A proteomic approach was also applied to saliva samples from humans, horses, cattle, dogs, sheep, rabbits and rats with the aim to establish specific proteome signatures of mammals' saliva [20]. Recent studies focused on the possibility of using saliva as a valuable biofluid to investigate enzymatic [17] and metabolomic [21] modifications induced by exercise in soccer players. Exercise testing and monitoring of training sessions have an important value in the assessment of poor performance, fitness and performance potential in athletic horses [22-24]. However, despite a continuous search for novel methods to evaluate the equine athlete, little is known about its metabolomic profile $[25,26]$, and no information is available on the metabolome of standardbred horses

On the basis of the current advances observed in metabolomic research, we aimed to investigate the metabolomic profile of serum and saliva samples collected from healthy standardbred horses and the relationship with physical exercise. Closely monitoring fitness, workload and injuries in sport horses is a major matter to better understand the effects of training methods, so to reduce injuries [24]. That is why the evaluation of indices of fitness (starting from blood parameter, velocity and/or heart rate) is important to adapt training programs with the double aim to improve performance and preserve horses' welfare [24]. Metabolomics applied to athletes could provide new insight in the adaption of horses to exercise, evaluating in detail which metabolites are altered, trying to give an explanation and apply any practical corrective measures. Among the high-throughput platforms employed for metabolomics, we decided to employ proton nuclear magnetic resonance $\left({ }^{1} \mathrm{H}-\mathrm{NMR}\right)$ spectroscopy, that ensures data traceability, reproducibility and interoperability, because the only variables modulating an NMR spectrum are magnetic field, solvent and pulse sequence [27].

\section{Results}

All the horses included in the study were accustomed to the training program and showed no clinical sign of disease during the experimental period. The saliva collection method herein used was non-invasive and well tolerated by all horses.

${ }^{1} \mathrm{H}-\mathrm{NMR}$ spectroscopy allowed to quantify 46 metabolites in serum and 62 metabolites in saliva, including, among others, alcohols, amino acids, organic acids, carbohydrates and purine derivatives (Figures 1 and 2), with a spectrum of substances broadly in line with those found in the human metabolome $[8,28]$.

Despite the detection of 30 shared metabolites, serum and salivary metabolomes resulted to be different, with 16 metabolites found only in serum, and 32 metabolites found only in saliva.

The concentrations of the metabolites that resulted to be statistically different between the samples collected before $\left(\mathrm{T}_{0}\right)$ and after $\left(\mathrm{T}_{1}\right)$ exercise in serum are reported in Table 1. $p$-values for all non-significant metabolites are reported in Supplementary Materials Table S1. 

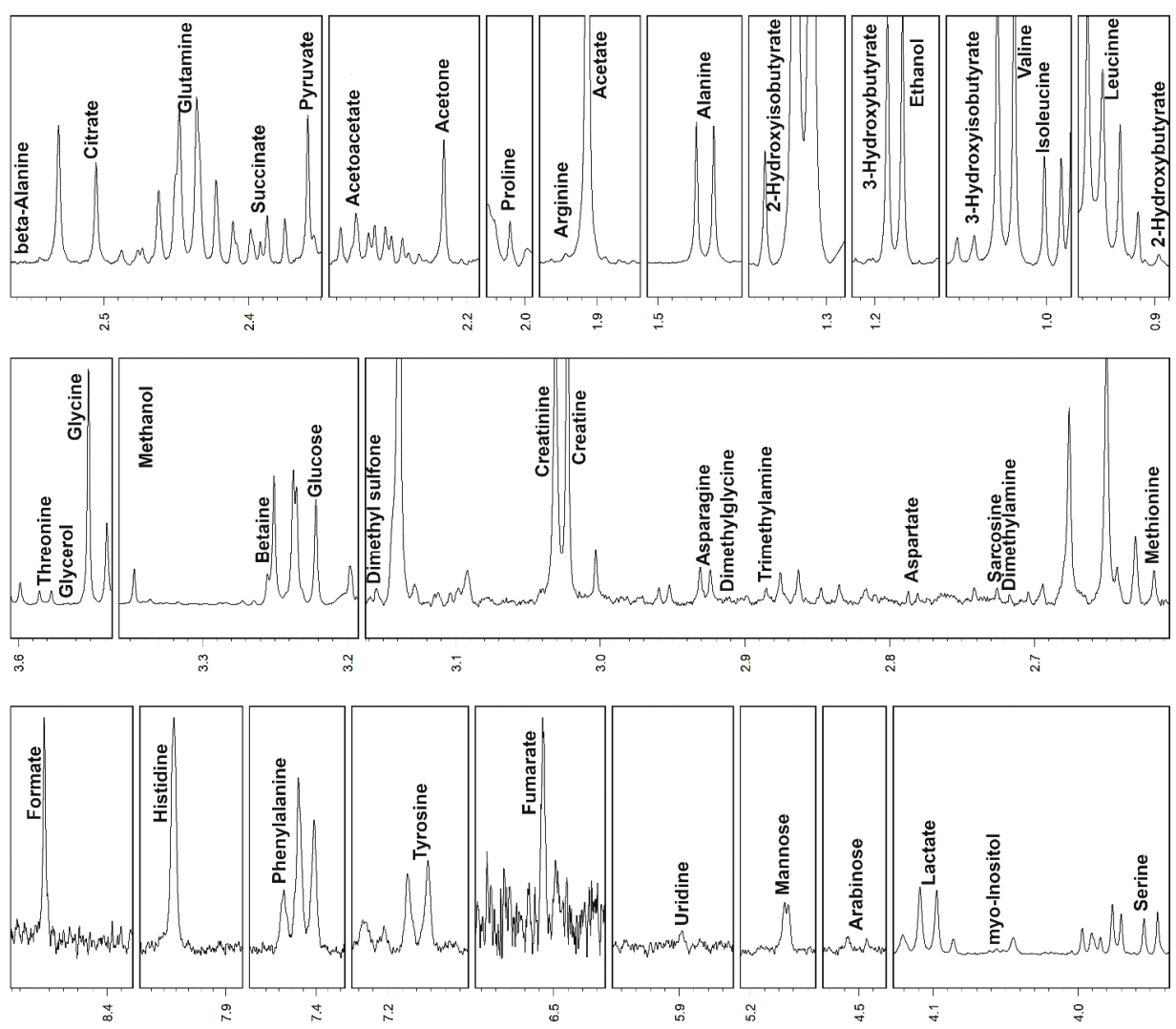

Chemical shift (ppm)

Figure 1. Portions of ${ }^{1} \mathrm{H}-\mathrm{NMR}$ spectra from typical serum samples. Assignments appear on the signals used for molecules quantification. The vertical scale of each portion is conveniently set to ease the signals observation.

To observe the overall variations intrinsic to the samples in the space constituted by this restricted group of metabolites, we calculated on their concentrations a robust Principal Component Analysis (rPCA) model (Figure 3). Three principal components (PCs) were accepted, the first of which accounted for $57.5 \%$ of the samples' variance represented by the model. Such a PC nicely accounted for the differences among the samples connected to exercise and showed that lactate, pyruvate, succinate, glycerol, fumarate and alanine mostly characterized the samples collected after exercise, while myo-inositol, histidine, proline, asparagine, glutamine and mannose mostly characterized the samples collected before exercise. 

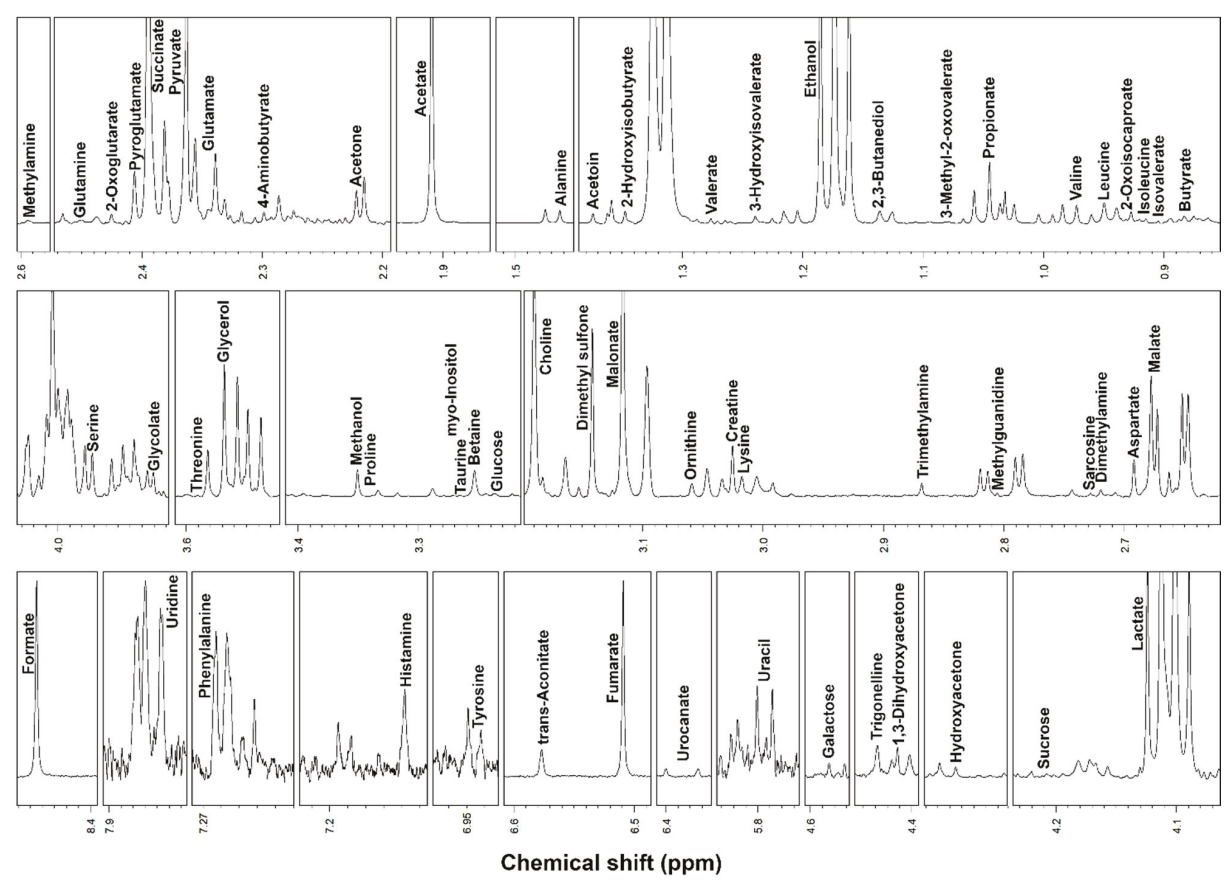

Figure 2. Portions of ${ }^{1} \mathrm{H}-\mathrm{NMR}$ spectra from typical saliva samples. Assignments appear on the signals used for molecules quantification. The vertical scale of each portion is conveniently set to ease the signals observation.
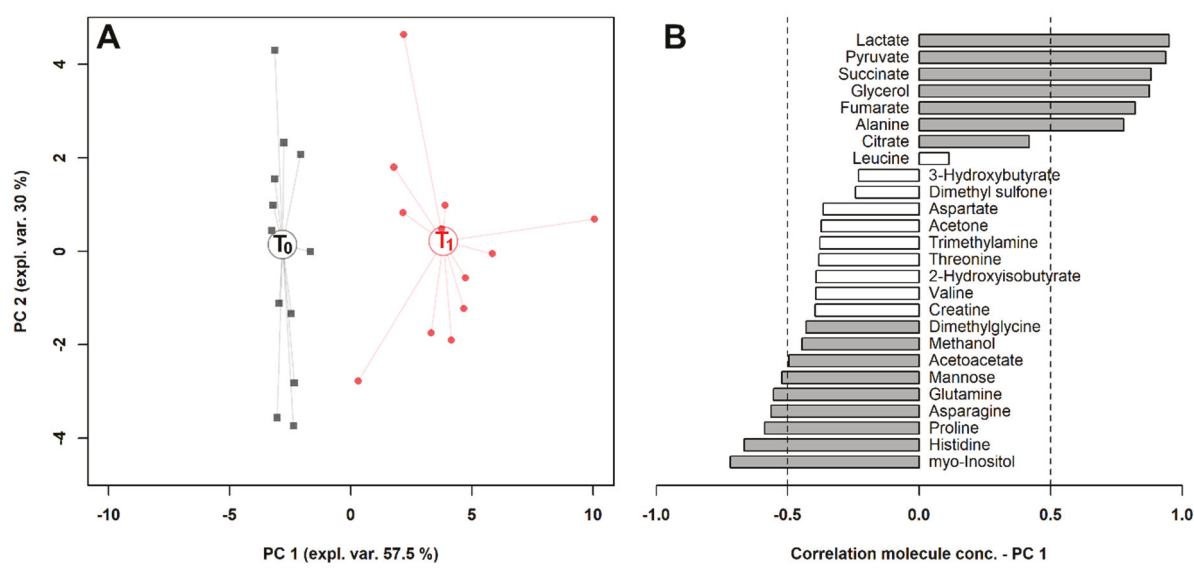

Figure 3. rPCA model built on the space constituted by the concentration of the molecules significantly different in serum, listed in Table 1. (A) In the score plot, samples collected at $T_{0}$ and $T_{1}$ are represented with black squares and red circles, respectively. The wide, empty circles represent the median of the groups. (B) The loading plot reports the correlation between the concentration of each substance and its importance over principal component (PC) 1. Significant correlations $(p<0.05)$ are highlighted with gray bars. 
Table 1. Serum metabolites with significantly different concentration $(\mu \mathrm{mol} / \mathrm{L} ;$ mean $\pm \mathrm{SD})$ before $\left(\mathrm{T}_{0}\right)$ and after $\left(\mathrm{T}_{1}\right)$ exercise.

\begin{tabular}{|c|c|c|c|c|}
\hline Molecule & $\mathrm{T}_{0}$ & $\mathrm{~T}_{1}$ & $p$ & Trend * \\
\hline 3-Hydroxybutyrate & $2.09 \pm 1.57$ & $1.52 \pm 1.33$ & $4.58 \times 10^{-2}$ & $\downarrow$ \\
\hline 2-Hydroxyisobutyrate & $1.64 \pm 0.96$ & $0.95 \pm 1.19$ & $6.19 \times 10^{-5}$ & $\downarrow$ \\
\hline Acetoacetate & $3.73 \pm 0.73$ & $3.21 \pm 0.58$ & $3.20 \times 10^{-4}$ & $\downarrow$ \\
\hline Acetone & $3.31 \pm 1.42$ & $2.56 \pm 0.85$ & $1.45 \times 10^{-2}$ & $\downarrow$ \\
\hline Alanine & $66.34 \pm 10.37$ & $104.87 \pm 11.85$ & $4.87 \times 10^{-8}$ & $\uparrow$ \\
\hline Asparagine & $10.36 \pm 1.93$ & $7.44 \pm 1.82$ & $7.92 \times 10^{-5}$ & $\downarrow$ \\
\hline Aspartate & $2.17 \pm 1.17$ & $1.53 \pm 0.61$ & $2.23 \times 10^{-2}$ & $\downarrow$ \\
\hline Citrate & $11.24 \pm 5.88$ & $16.40 \pm 7.51$ & $1.79 \times 10^{-2}$ & $\uparrow$ \\
\hline Creatine & $22.76 \pm 7.67$ & $17.94 \pm 5.91$ & $2.95 \times 10^{-4}$ & $\downarrow$ \\
\hline Dimethyl sulfone & $14.74 \pm 8.56$ & $11.09 \pm 5.96$ & $4.32 \times 10^{-3}$ & $\downarrow$ \\
\hline Dimethylglycine & $0.36 \pm 0.13$ & $0.28 \pm 0.09$ & $3.91 \times 10^{-3}$ & $\downarrow$ \\
\hline Fumarate & $0.84 \pm 0.21$ & $1.50 \pm 0.73$ & $8.82 \times 10^{-3}$ & $\uparrow$ \\
\hline Glutamine & $71.51 \pm 11.48$ & $58.14 \pm 6.78$ & $6.18 \times 10^{-5}$ & $\downarrow$ \\
\hline Glycerol & $4.03 \pm 3.01$ & $45.51 \pm 22.46$ & $1.84 \times 10^{-5}$ & $\uparrow$ \\
\hline Histidine & $29.91 \pm 4.34$ & $23.46 \pm 3.35$ & $1.52 \times 10^{-3}$ & $\downarrow$ \\
\hline Lactate & $141.26 \pm 50.20$ & $\begin{array}{c}1143.45 \pm \\
582.63\end{array}$ & $1.03 \times 10^{-4}$ & $\uparrow$ \\
\hline Leucine & $35.68 \pm 6.37$ & $39.22 \pm 6.87$ & $9.62 \times 10^{-3}$ & $\uparrow$ \\
\hline Mannose & $11.20 \pm 2.71$ & $7.85 \pm 2.09$ & $1.33 \times 10^{-5}$ & $\downarrow$ \\
\hline Methanol & $16.98 \pm 10.21$ & $10.26 \pm 8.25$ & $1.49 \times 10^{-3}$ & $\downarrow$ \\
\hline myo-Inositol & $10.35 \pm 1.68$ & $7.37 \pm 2.06$ & $8.38 \times 10^{-4}$ & $\downarrow$ \\
\hline Proline & $10.57 \pm 2.45$ & $8.02 \pm 1.54$ & $4.19 \times 10^{-4}$ & $\downarrow$ \\
\hline Pyruvate & $8.72 \pm 2.37$ & $26.61 \pm 7.59$ & $4.38 \times 10^{-6}$ & $\uparrow$ \\
\hline Succinate & $1.12 \pm 0.21$ & $4.24 \pm 1.79$ & $6.11 \times 10^{-5}$ & $\uparrow$ \\
\hline Threonine & $37.30 \pm 5.45$ & $33.26 \pm 6.98$ & $4.93 \times 10^{-3}$ & $\downarrow$ \\
\hline Trimethylamine & $0.16 \pm 0.05$ & $0.14 \pm 0.05$ & $2.73 \times 10^{-3}$ & $\downarrow$ \\
\hline Valine & $54.09 \pm 6.79$ & $50.40 \pm 9.04$ & $1.85 \times 10^{-2}$ & $\downarrow$ \\
\hline
\end{tabular}

* Increasing $(\uparrow)$ or decreasing $(\downarrow)$ trends from $\mathrm{T}_{0}$ to $\mathrm{T}_{1}$.

About the salivary metabolome, the concentrations of five molecules significantly increased after exercise, while nine significantly decreased between $\mathrm{T}_{0}$ and $\mathrm{T}_{1}$ (Table 2). $p$-values for all non-significant metabolites are reported in Supplementary Materials Table S2.

Table 2. Salivary metabolites with different concentrations ( $\mu$ mol/L; mean $\pm \mathrm{SD}$ ) before $\left(\mathrm{T}_{0}\right)$ and after $\left(\mathrm{T}_{1}\right)$ exercise.

\begin{tabular}{ccccc}
\hline Molecule & $\mathrm{T}_{\mathbf{0}}$ & $\mathrm{T}_{\mathbf{1}}$ & $p$ & Trend * \\
\hline 4-Aminobutyrate & $30.09 \pm 15.39$ & $10.87 \pm 16.55$ & $4.35 \times 10^{-3}$ & $\downarrow$ \\
Betaine & $143.58 \pm 86.06$ & $67.19 \pm 52.51$ & $1.23 \times 10^{-2}$ & $\downarrow$ \\
Creatine & $36.68 \pm 19.11$ & $62.11 \pm 26.59$ & $1.28 \times 10^{-2}$ & $\uparrow$ \\
Fumarate & $94.70 \pm 58.46$ & $23.20 \pm 29.32$ & $8.35 \times 10^{-4}$ & $\downarrow$ \\
Galactose & $21.68 \pm 10.18$ & $12.55 \pm 12.66$ & $1.98 \times 10^{-2}$ & $\downarrow$ \\
Malate & $550.72 \pm 395.87$ & $138.69 \pm 166.50$ & $2.11 \times 10^{-3}$ & $\downarrow$ \\
Malonate & $193.93 \pm 107.17$ & $46.84 \pm 35.06$ & $5.10 \times 10^{-4}$ & $\downarrow$ \\
Methanol & $131.26 \pm 73.70$ & $73.05 \pm 50.01$ & $5.07 \times 10^{-3}$ & $\downarrow$ \\
Ornithine & $22.36 \pm 12.53$ & $46.91 \pm 21.66$ & $3.24 \times 10^{-3}$ & $\uparrow$ \\
Phenylalanine & $28.22 \pm 11.81$ & $53.82 \pm 23.36$ & $6.10 \times 10^{-3}$ & $\uparrow$ \\
Pyruvate & $70.77 \pm 32.43$ & $49.34 \pm 24.69$ & $7.41 \times 10^{-2}$ & $\downarrow$ \\
Sarcosine & $2.65 \pm 1.64$ & $4.48 \pm 2.94$ & $2.48 \times 10^{-2}$ & $\uparrow$ \\
Succinate & $210.91 \pm 78.35$ & $120.47 \pm 80.66$ & $3.59 \times 10^{-2}$ & $\downarrow$ \\
Tyrosine & $33.32 \pm 18.13$ & $66.07 \pm 46.48$ & $3.29 \times 10^{-2}$ & $\uparrow$ \\
\hline
\end{tabular}

* Increasing $(\uparrow)$ or decreasing $(\downarrow)$ trends from $\mathrm{T}_{0}$ to $\mathrm{T}_{1}$. 
To observe the overall trends associated with the samples, we calculated on their concentrations the rPCA model outlined in Figure 4. Three principal components (PCs) were accepted, the first of which accounted for $84.2 \%$ of the samples' variance represented by the model. Such PCs summarized the differences among the samples connected to exercise. The salivary metabolome of horses after exercise was mainly characterized by creatine, ornithine, phenylalanine and tyrosine, while horses before exercise were mainly characterized by fumarate, malate, malonate, 4-aminobutyrate, betaine and galactose. In Figure 5, the metabolic pathways overrepresented by the molecules significantly affected by exercise in serum and saliva are reported.
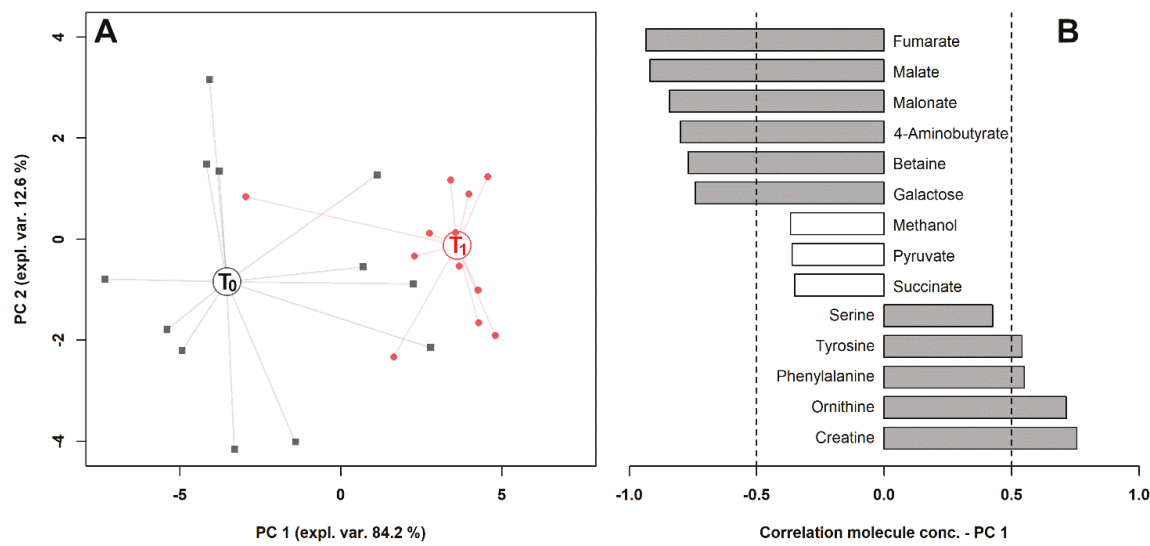

Figure 4. rPCA model built on the space constituted by the concentration of the molecules significantly different in saliva, listed in Table 2. (A) In the score plot, samples collected at $T_{0}$ and $T_{1}$ are represented with black squares and red circles, respectively. The wide, empty circles represent the median of the groups. (B) The loading plot reports the correlation between the concentration of each substance and its importance over PC 1. Significant correlations $(p<0.05)$ are highlighted with gray bars.

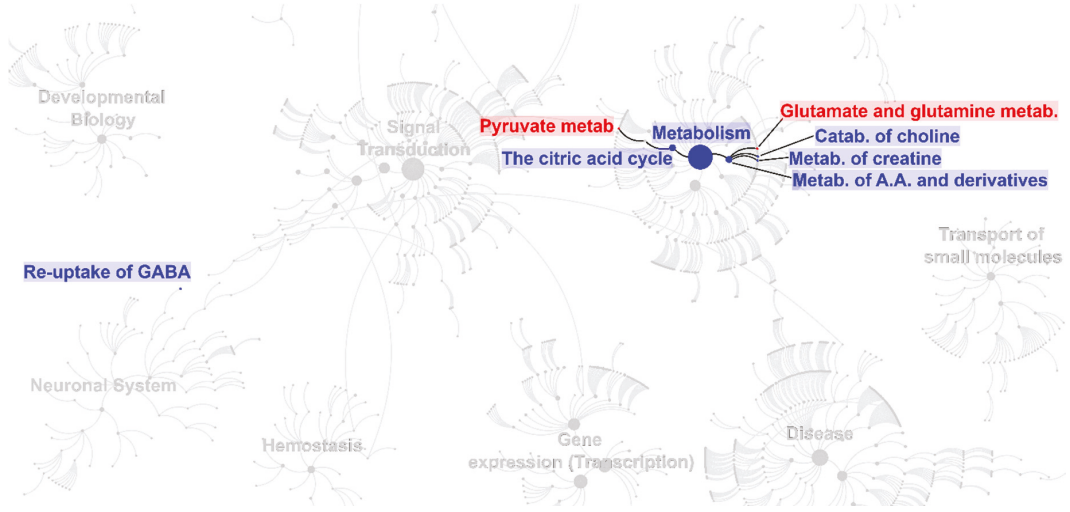

Figure 5. Biomolecular pathways overrepresentation analysis performed on the molecules listed in Table 1 for serum (red) and Table 2 for saliva (blue). The figure replicates a convenient portion of the biomolecular pathways overview according to Reactome, modified to show the main pathways and sub-pathways overrepresented as a consequence of exercise. 


\section{Discussion}

The results showed in the present paper highlight for the first time the changes occurring in the metabolomic profile of trained standardbred horses following exercise in both serum and saliva specimens.

Biochemical constituents of the tricarboxylic acid cycle (TCA or Krebs cycle) including succinate and fumarate were significantly increased in serum after exercise. This is in agreement with results obtained by other authors in human and equine athletes $[25,29,30]$ and could be explained by the need to maintain and/or increase the Krebs cycle flow during exercise [8]. On the contrary, succinate and fumarate, together with malate, another constituent of the TCA cycle, have been found to decrease in saliva after exercise. Among salivary metabolites, malonate was found to have a very strong inhibitory effect on the TCA cycle in complex metabolic systems. Malonate is not normally present in cells [31] and the reason for its presence in saliva, its decrease after exercise and its inhibitory effect on the TCA cycle in horses during exercise need further investigations. Notwithstanding the paucity of data on salivary metabolomics during exercise, we can speculate that the decreased salivary level of succinate, fumarate and malate might reflect the imbalance of the aerobic pathways in favor of the anaerobic production of energy during exercise.

The increase in pyruvate and lactate serum concentrations was expected, since it reflects the anaerobic pathway activation during exercise [8,25]. The high energy demand required during exercise can overload the mitochondria's ability to oxidize pyruvate that is converted to lactate, supplying energy by the Cori cycle [8]. In our study, serum lactate increased up to 11.6-fold following exercise, and this result was beyond the physiological range usually reported for human athletes of a 9.3-9.6-fold increase $[8,32]$. Probably, the greater muscle mass of horses with respect to humans can account for this difference. Although not significant, pyruvate and lactate slightly decreased in saliva after exercise (Supplemental Materials Table S1), demonstrating once again that salivary metabolic changes do not follow the same metabolic changes observed in serum, at least regarding the energy supply.

Among the metabolites detected in plasma, there are also two carbohydrates: mannose and arabinose.

The concentration of mannose resulted more concentrated in post-exercise equine plasma. This monosaccharide is found in small amounts in the diet, and after conversion to fructose-6-phosphate, it can be used in both glycolysis and gluconeogenesis [33]. Unlike mannose, the concentration of arabinose in serum appeared as decreased by exercise. Arabinose is known to have an inhibitory effect on intestinal sucrase [34], but its role in horse during exercise in not clear.

Galactose was the only carbohydrate with a changed concentration in saliva, as it decreased at $\mathrm{T}_{1}$. In a study on soccer players, Pitti et al. [21] found an increased level of galactose in blood after exercise. Galactose is an important source of glucose and its role in liver glycogen restoration after exercise has been well recognized in cyclists $[33,35]$. The reason for galactose reduction in the saliva of horses could reflect a different energetic role of this metabolite compared with humans.

Among amino acids, alanine and histidine increased significantly after exercise. Serum alanine increased 1.6-fold soon after exercise, in agreement with the results obtained in humans by Berton et al. [8] but in contrast with Nieman et al. [29]. Alanine in muscle derives from pyruvate, in turn originated from glucose breakdown [8]. This metabolic mechanism is in line with the rise in serum pyruvate found in our study. Furthermore, alanine has a detoxing function by transporting to the liver the large amount of ammonia typically produced during short and high-intensity exercise derived from branched chain amino acids $[8,36]$. Similarly, the increase in gluconeogenic amino acid histidine could be linked to the higher energetic demand during acute exercise.

The decreased levels of glutamine, asparagine and proline observed in horses after training were similar to the findings in human athletes $[29,30]$ thus supporting the hypothesis of an enhanced muscle amino acid oxidation during exercise to sustain the energetic demand.

In equine saliva, amino acids like tyrosine and phenylalanine rose almost 2-fold after exercise. Tyrosine results from hydroxylation of phenylalanine, an essential amino acid [37], and it is metabolized 
to acetoacetate and fumarate or used as a precursor of catecholamines that play an important role in athletic performance during exercise [38]. We can speculate that the rise in salivary tyrosine after exercise could reflect an increased production to encounter the need for catecholamines during exercise, but the lack of similar changes in serum needs to be clarified.

Sarcosine (N-methylglycine) is a non-proteinogenic amino acid derivative and occurs in the body as a product of the metabolism of glycine and creatine [39]. In addition to its multiple functions in the body and its use as a potential marker in various diseases, it has been an ingredient in toothpaste for decades as it prevents tooth decay and causes foaming [39]. The reason for the slight rising of sarcosine and creatine in equine saliva after exercise is not clear, but its physiological function could be protective for teeth health.

Ornithine levels increased up to 2.1-fold in saliva but not in serum, differently from what has been observed in human athletes where it is down regulated 60-70 min after exercise as a consequence of the accelerated urea cycle due to the higher production of ammonia $[8,30]$. However, the differences in the sampling times of our study compared with others might explain this discrepancy. Betaine, a small molecule acting as a methyl donor in minor pathways [37], decreased in saliva after exercise, but this finding needs further investigations.

The marked elevation in serum glycerol in our horses following exercise resulted from an extensive lipolysis and is consistent with the study by Lewis et al. [30], who found higher serum glycerol up-regulation after exercise in fitter athletes. Considering that all the horses included in our study were well trained, we could consider glycerol a marker of adaptation to training also in equine species.

Salivary methanol was reduced 1.8 times by exercise. The same result was recently found in the saliva of soccer players, and the change was ascribed to the higher evaporation of volatile compounds in the mouth during exercise [21]. In a recent study, methanol was considered a marker of inflammation in horses affected by equine asthma when detected in exhaled breath condensate (EBC) [40].

Uracil nucleotide, together with cytosine, are the major pyrimidine components of RNA. Uracil can be utilized in glycogen synthesis [41] and its rise in saliva could be related to the higher energetic demand during exercise.

2-hydroxyisobutyrate was up-regulated in the serum of standardbred horses following exercise. This metabolite is a normal constituent of human serum and saliva [42] that has been proposed as a biomarker for glycogen storage disease type $1 \mathrm{a}$ in juveniles and acute coronary syndrome $[43,44]$. It was also found decreased in plasma of Alzheimer's disease patients vs. controls [45], however no information is available about its role during sport activity.

3-hydroxybutyrate decreased in serum after exercise. An elevation of this ketone was documented in marathon runners due to ketone production [30]. It was also found to be increased in runners after a three-day intensified exercise as a result of fatty acid oxidation [29]. On the other hand, Berton et al. [8] did not find any change in 3-hydroxybutyrate after a leg press resistance exercise. Our result might suggest that this metabolic pathway is of negligible importance in trained standardbred horses performing acute exercise of short duration.

4-aminobutyrate ( $\gamma$-Aminobutiryc acid or GABA) is the most inhibitory neurotransmitter in the central nervous system [46] and its presence has been already demonstrated in human saliva [47] as well as in salivary glands of men and rats $[48,49]$, being associated with its biosynthetic and metabolic enzymes. However, this is the first report showing the presence of GABA in equine saliva and the down-regulatory effect of exercise in this species. GABA and its receptors can be found in other non-neuronal organs but its role in peripheral tissue remains to be established, although it is known to be involved in cellular proliferation [48]. Authors suggested a role of GABA(A)-R in the suppression of salivary secretion in rat salivary glands [50]. Similarly, the down-regulation of salivary GABA during exercise in horses could be associated with this regulatory function of saliva secretion, possibly linked to thermoregulation. 
Significantly lower levels of myo-inositol have been found both in the serum and saliva of horses after exercise. Myo-inositol promotes the maturation of pulmonary surfactants and supports respiratory function [51], modulates cytoskeleton dynamics, thus allowing alveolar cells to counteract collapsing forces and promoting mechanical stabilization of cell shape [52], and it recruits water and organic compounds in the alveolar space, decreasing surface tension through the formation of a biofilm layer at the interface [53]. Myo-inositol is also the most effective allosteric effector identified to date, being able to increase the tissue delivering of oxygen bound to hemoglobin [54]. Since its administration can improve sport performance in laboratory mice, its analogues have been suspected to be abused in the horse racing industry [55]. Up to now, no study about its effect in equine species has been performed. Recently, reduced concentrations of myo-inositol have been found in the bronchoalveolar lavage fluid of horses affected by equine asthma in comparison with healthy horses [56]. The exact role of this metabolite in physical exercise is not known, however, the decrease observed in both serum and saliva could indicate an impairment in the normal respiratory function during exercise.

\section{Materials and Methods}

\subsection{Animals}

Twelve clinically healthy standardbred horses (7 males and 5 females), mean age 6.7 years old, mean body condition score 3 out of 5 [57], were included in the study with informed owner consent.

All horses were stabled in individual boxes and were fed the same polyphyte hay at a ratio of $2 \mathrm{~kg} / 100 \mathrm{~kg}$ BW, and commercial horse feed in the amount of $0.8 \mathrm{~kg} / 100 \mathrm{~kg}$ of BW (protein $11 \%$; fat $4 \%$; fiber $8.56 \%$; Ca/P 2:1) twice a day (at 10 am and at 5 pm). Clean potable drinking water was offered ad libitum.

The sampling herein described was part of a normal procedure for monitoring the training status of the animals. All procedures were approved by the Animal Care Committee of Camerino University (Registration number E81AC.10/Ac) and were in accordance with the standard recommended by the EU Directive 2010/63/EU for experimental animals.

\subsection{Experimental Procedure}

All horses were trained 6 days per week and the training program consisted of 7 runs of $1000 \mathrm{~m}$ each at different speed. Briefly, the first warm-up run clockwise at light trot (mean speed $5 \mathrm{~m} / \mathrm{s}$ ) was followed by 3 counter clockwise runs (mean speed $10 \mathrm{~m} / \mathrm{s}$ ), then 1 recovery run at walking and 2 runs at full speed trot (mean speed $12 \mathrm{~m} / \mathrm{min}$ ), and finally 2 runs at light trot (mean speed $5 \mathrm{~m} / \mathrm{s}$ ). All animals were sampled the day before the rest-day.

Saliva and blood samples were collected before $\left(T_{0}\right)$ and immediately after full speed exercise $\left(\mathrm{T}_{1}\right)$. Saliva samples were collected by using cotton swabs and Salivette ${ }^{\circledR}$ tubes (Sarstedt AG \& Co., Nümbrecht, Germany). The cotton swab was grasped with a surgical clamp, inserted at the angle of the lips into the mouth of the horse and placed gently on the tongue surface for $5 \mathrm{~min}$ and then inserted into the Salivette ${ }^{\circledR}$ tube.

Venous blood samples were collected at $\mathrm{T}_{0}$ and $\mathrm{T}_{1}$ by jugular venepuncture into $4 \mathrm{~mL}$ vacutainer sterile tubes containing EDTA (Vacuette ${ }^{\circledR}$ Greiner Bio-One, Cassina de Pecchi, Italy) and $9 \mathrm{~mL}$ vacutainer sterile tubes containing clot activators (Vacuette ${ }^{\circledR}$ Greiner Bio-One, Cassina de Pecchi, Italy). A complete blood count $(\mathrm{CBC})$ and biochemical profile was performed on blood and serum samples to exclude systemic disorders.

Whole blood was directly tested in the field for blood lactate concentration by using a portable device (Accutrend Plus ${ }^{\circledR}$, Roche, Mannheim, Germany) at $T_{0}$ and $T_{1}$ as a part of the fitness evaluation routine.

All the sampling procedures were performed before feeding, at the same time of the day (06:00-8:00 a.m.) and, immediately after collection, all the samples were stored at $5{ }^{\circ} \mathrm{C}$ and delivered to the lab within $2 \mathrm{~h}$. 


\subsection{Laboratory Analysis.}

Saliva samples were obtained by centrifugation of Salivette ${ }^{\circledR}$ tubes (10 min at $\left.1000 \mathrm{~g}\right)$ (Universal 32 , Hettich Zentrifugen, Tuttlingen, Germany) and $2 \mathrm{~mL}$ aliquots of supernatant were stored at $-20^{\circ} \mathrm{C}$ until metabolomic analysis.

Blood samples with clot activators were centrifuged for $10 \mathrm{~min}$ at $1000 \mathrm{~g}$ (Universal 32, Hettich Zentrifugen, Tuttlingen, Germany) and the obtained sera were stored at $-20{ }^{\circ} \mathrm{C}$ until analysis.

For ${ }^{1} \mathrm{H}-\mathrm{NMR}$ analysis, we created a stock solution composed of 3-(trimethylsilyl)-propionic-2,2,3,3-d4 acid sodium salt (TSP) $10 \mathrm{mmol} / \mathrm{L}$ and $\mathrm{NaN}_{3} 2 \mathrm{mmol} / \mathrm{L}$ in $\mathrm{D}_{2} \mathrm{O}$. The former served as the NMR spectra chemical-shift reference, while the latter avoided bacteria proliferation. The solution was set to $\mathrm{pH}$ at $7.00 \pm 0.02$ by phosphate buffer $(1 \mathrm{M})$. Both serum and saliva samples were prepared for ${ }^{1} \mathrm{H}-\mathrm{NMR}$ by thawing and centrifuging $1 \mathrm{~mL}$ of each sample at $4{ }^{\circ} \mathrm{C}$ for $15 \mathrm{~min}$ at $18,630 \mathrm{~g}$. The supernatant $(700 \mu \mathrm{L})$ was added to $100 \mu \mathrm{L}$ of the NMR analysis solution and centrifuged again.

The spectra were recorded with an AVANCE III spectrometer (Bruker, Milan, Italy), controlled by the Topspin software (Ver. 3.5), at a frequency of $600.13 \mathrm{MHz}$ and a temperature of $298 \mathrm{~K}$. The residual signal from the water was suppressed by pre-saturation, while broad signals from large molecules were reduced by a CPMG-filter, set as outlined by Zhu et al. [58]. Each spectrum was acquired by summing up 256 transients registering $32 \mathrm{~K}$ data points over a $7184 \mathrm{~Hz}$ spectral window, with an acquisition time of $2.28 \mathrm{~s}$ and relaxation delay of $5 \mathrm{~s}$.

In Topspin, a manual correction phase was applied to each spectrum, together with a line-broadening of $0.3 \mathrm{~Hz}$. The subsequent steps were performed in R computational language by means of scripts developed in-house. The spectra were aligned toward the right peak of the alanine doublet, set to $1.473 \mathrm{ppm}$. Baseline was then corrected, after having removed the residual water signal, by isolating irregularities of the baseline by peak detection, according to the "rolling ball" principle [59].

For signals' assignment, chemical shift and multiplicity were compared with the Chenomx software library (Chenomx Inc., Edmonton, AB, Canada, ver 8.3). The added TSP was employed as internal standard in the first sample analyzed. Differences in water content among samples were then taken into consideration by probabilistic quotient normalization [27]. Rectangular integration was employed to quantify each molecule, by focusing on one signal per molecule free from superimpositions.

\subsection{Statistical Analysis}

Differences between the two groups were looked for by means of a paired $t$-test applied to the concentrations of each molecule, transformed by the Box-Cox algorithm for normal distribution [60].

We highlighted any trend characterizing the samples with robust principal component analysis (rPCA) models [61], based on the molecules accepted by the univariate analysis. To this purpose, we employed the PcaHubert algorithm implemented in the rrcov package. The main features of each rPCA model are summarized by a score plot and by a Pearson correlation plot. The former is the projection of the samples in the PC space and highlights the underlying structure of the data. The latter relates the concentration of each variable to the components of the model.

Metabolic pathway analysis was conducted by relying on the Reactome pathway knowledgebase [62], with overrepresentation analysis (ORA) based on a hypergeometric test [63].

\section{Conclusions}

In the present study, data related to the metabolomics of saliva and serum in trained standardbred horses have been provided for the first time. Several molecules are common to metabolic pathways already described in human athletes (e.g., energetic metabolism). However, the findings of 2-hydroxyisobutyrate and 3- hydroxybutyrate in serum and GABA in equine saliva, as well as their modifications following exercise, provide new insights about the physiology of exercise in athletic horses. Furthermore, the use of metabolomic analysis allowed to identify metabolites like glycerol that 
might represent novel biomarkers for fitness evaluation in sport horses. However, further investigations are needed to validate the metabolites herein found as indices of fitness levels in equine species.

Supplementary Materials: The following are available online at http:/www.mdpi.com/2218-1989/10/7/298/s1, Table S1: Serum metabolites with no significant difference in concentrations before and after exercise $(\mu \mathrm{mol} / \mathrm{L}$; mean \pm SD), Table S2: Salivary metabolites with no significant difference in concentrations before and after exercise $(\mu \mathrm{mol} / \mathrm{L} ;$ mean $\pm \mathrm{SD})$.

Author Contributions: Conceptualization, F.L; L.L and M.B; methodology, F.L., C.Z. and L.L.; formal analysis, L.L. and C.Z.; investigation, E.L. and S.S.; resources, S.S and B.T.; writing-original draft preparation, F.L., L.L. and M.B.; writing-review and editing, F.L., L.L., M.B. and B.T.; supervision, F.L. All authors have read and agreed to the published version of the manuscript.

Funding: This research received no external funding.

Acknowledgments: Chenglin Zhu gratefully acknowledges financial support from Chinese Scholarship Council (grant number 201606910076).

Conflicts of Interest: The authors declare no conflict of interest.

\section{References}

1. Dunn, W.B.; Broadhurst, D.I.; Atherton, H.J.; Goodacre, R.; Griffin, J.L. Systems level studies of mammalian metabolomes: The roles of mass spectrometry and nuclear magnetic resonance spectroscopy. Chem. Soc. Rev. 2011, 40, 387-426. [CrossRef] [PubMed]

2. Gorostiaga, E.M.; Navarro-Amezqueta, I.; Calbet, J.A.; Hellsten, Y.; Cusso, R.; Guerrero, M.; Granados, C.; González-Izal, M.; Ibañez, J.; Izquierdo, M. Energy metabolism during repeated sets of leg press exercise leading to failure or not. PLOS ONE 2012, 7, e40621. [CrossRef]

3. Schoenfeld, B.J. Potential mechanisms for a role of metabolic stress in hypertrophic adaptations to resistance training. Sports Med. 2013, 43, 179-194. [CrossRef] [PubMed]

4. Tesch, P.A.; Colliander, E.B.; Kaiser, P. Muscle metabolism during intense, heavy-resistance exercise. Eur. J. Appl. Physiol. Occup. Physiol. 1986, 55, 362-366. [CrossRef] [PubMed]

5. Mendez-Villanueva, A.; Edge, J.; Suriano, R.; Hamer, P.; Bishop, D. The recovery of repeated-sprint exercise is associated with PCr resynthesis, while muscle pH and EMG amplitude remain depressed. PLoS ONE 2012, 7, e51977. [CrossRef] [PubMed]

6. Psychogios, N.; Hau, D.D.; Peng, J.; Guo, A.C.; Mandal, R.; Bouatra, S.; Young, N. The human serum metabolome. PLoS ONE 2011, 6, 16957. [CrossRef]

7. Ryan, D.; Robards, K. Metabolomics: The greatest omics of them all? Anal. Chem. 2006, 78, 7954-7958. [CrossRef]

8. Berton, R.; Conceição, M.S.; Libardi, C.A.; Canevarolo, R.R.; Gáspari, A.F.; Chacon-Mikahil, M.P.; Zeri, A.C.; Cavaglieri, C.R. Metabolic time-course response after resistance exercise: A metabolomics approach. J. Sports Sci. 2017, 35, 1211-1218. [CrossRef]

9. Contreras-Aguilar, M.D.; Escribano, D.; Martínez-Subiela, S.; Martín-Cuervo, M.; Lamy, E.; Tecles, F.; Cerón, J.J. Changes in saliva analytes in equine acute abdominal disease: A sialochemistry approach. BMC Vet. Res. 2019, 15, 187. [CrossRef]

10. Ghizoni, J.S.; Nichele, R.; de Oliveira, M.T.; Pamato, S.; Pereira, J.R. The utilization of saliva as an early diagnostic tool for oral cancer: microRNA as a biomarker. Clin. Transl. Oncol. 2020, 22, 804-812. [CrossRef]

11. Greabu, M.; Battino, M.; Mohora, M.; Totan, A.; Didilescu, A.; Spinu, T. Saliva-A diagnostic window to the body, both in health and in disease. J. Med. Life 2009, 2, 124-132. [PubMed]

12. Tecles, F.; Escribano, D.; Martínez-Miró, S.; Hernández, F.; Contreras, M.D.; Cerón, J.J. Cholinesterase in porcine saliva: Analytical characterization and behavior after experimental stress. Res. Vet. Sci. 2016, 106, 23-28. [CrossRef] [PubMed]

13. Tecles, F.; Contreras-Aguilar, M.D.; Martínez-Miró, S.; Tvarijonaviciute, A.; Martínez-Subiela, S.; Escribano, D.; Cerón, J.J. Total esterase measurement in saliva of pigs: Validation of an automated assay, characterization and changes in stress and disease conditions. Res. Vet. Sci. 2017, 114, 170-176. [CrossRef] [PubMed]

14. Tecles, F.; Rubio, C.P.; Contreras-Aguilar, M.D.; Lopez-Arjona, M.; Martinez-Miro, S.; Martinez-Subiela, S.; Cerón, J.J. Adenosine deaminase activity in pig saliva: Analytical validation of two spectrophotometric assays. J. Vet. Diagn. Investig. 2018, 30, 175-179. [CrossRef] [PubMed] 
15. Contreras-Aguilar, M.D.; Escribano, D.; Quiles, A.; López-Arjona, M.; Cerón, J.J.; Martínez-Subiela, S.; Hevia, M.L.; Tecles, F. Evaluation of new biomarkers of stress in saliva of sheep. Animal 2019, 13, 1278-1286. [CrossRef]

16. Contreras-Aguilar, M.D.; Escribano, D.; Martín-Cuervo, M.; Tecles, F.; Cerón, J.J. Salivary alpha-amylase activity and cortisol in horses with acute abdominal disease: A pilot study. BMC Vet. Res. 2018, 14, 156. [CrossRef]

17. Barranco, T.; Tvarijonaviciute, A.; Tecles, F.; Carrillo, J.M.; Sanchez-Resalt, C.; Jimenez-Reyes, P.; Rubio, M.; García-Balletbó, M.; Cerón, J.; Ramon Cugat, R. Changes in creatine kinase, lactate dehydrogenase and aspartate aminotransferase in saliva samples after an intense exercise: A pilot study. J. Sports Med. Phys. 2018, 58, 910-916.

18. Jacobsen, S.; Top Adler, D.M.; Bundgaard, L.; Sørensen, M.A.; Andersen, P.H.; Bendixen, E. The use of liquid chromatography tandem mass spectrometry to detect proteins in saliva from horses with and without systemic inflammation. Vet. J. 2014, 202, 483-488.

19. Bilancio, G.; Cavallo, P.; Lombardi, C.; Guarino, E.; Cozza, V.; Giordano, F.; Palladino, G.; Cirillo, M. Salivary levels of phosphorus and urea as indices of their plasma levels in nephropathic patients. J. Clin. Lab. Anal. 2018, 32, 1-6. [CrossRef]

20. de Sousa-Pereira, P.; Cova, M.; Abrantes, J.; Ferreira, R.; Trindade, F.; Barros, A.; Gomes, P.; Colaço, B.; Amado, F.; Esteves, P.J.; et al. Cross-species comparison of mammalian saliva using an LC-MALDI based proteomic approach. Proteomics 2015, 15, 1598-1607. [CrossRef]

21. Pitti, E.; Petrella, G.; Di Marino, S.; Summa, V.; Perrone, M.; D'Ottavio, S.; Bernardini, A.; Cicero, D.O. Salivary Metabolome and Soccer Match: Challenges for Understanding Exercise induced Changes. Metabolites 2019, 9, 141. [CrossRef] [PubMed]

22. Navas de Solis, C. Cardiovascular Response to Exercise and Training, Exercise Testing in Horses. Vet. Clin. N. Am. Equine Pract. 2019, 35, 159-173. [CrossRef] [PubMed]

23. Bazzano, M.; Giudice, E.; Rizzo, M.; Congiu, F.; Zumbo, A.; Arfuso, F.; Di Pietro, S.; Bruschetta, D.; Piccione, G. Application of a combined global positioning and heart rate monitoring system in jumper horses during an official competition-A preliminary study. Acta Vet. Hung. 2016, 64, 189-200. [PubMed]

24. Munsters, C.C.; van Iwaarden, A.; van Weeren, R.; Sloet van Oldruitenborgh-Oosterbaan, M.M. Exercise testing in Warmblood sport horses under field conditions. Vet. J. 2014, 202, 11-19. [PubMed]

25. Luck, M.M.; Le Moyec, L.; Barrey, E.; Triba, M.N.; Bouchemal, N.; Savarin, P.; Robert, C. Energetics of endurance exercise in young horses determined by nuclear magnetic resonance metabolomics. Front. Physiol. 2015, 6, 198.

26. Mach, N.; Ramayo-Caldas, Y.; Clark, A.; Moroldo, M.; Robert, C.; Barrey, E.; Lòpez, J.M.; Le Moyec, L. Understanding the response to endurance exercise using a systems biology approach: Combining blood metabolomics, transcriptomics and miRNomics in horses. BMC Genom. 2017, 18, 187. [CrossRef]

27. Laghi, L.; Picone, G.; Capozzi, F. Nuclear magnetic resonance for foodomics beyond food anaysis. Trend Anal. Chem. 2014, 59, 93-102.

28. Wishart, D.S.; Jewison, T.; Guo, A.C.; Wilson, M.; Knox, C.; Liu, Y.; Scalbert, A. HMDB 3.0—The human metabolome database in 2013. Nucleic Acids Res. 2013, 41, D801-D807. [CrossRef]

29. Nieman, D.C.; Shanely, R.A.; Gillitt, N.D.; Pappan, K.L.; Lila, M.A. Serum metabolic signatures induced by a three-day intensified exercise period persist after $14 \mathrm{~h}$ of recovery in runners. J. Proteome Res. 2013, 12, 4577-4584.

30. Lewis, G.D.; Farrell, L.; Wood, M.J.; Martinovic, M.; Arany, Z.; Rowe, G.C.; Souza, A.; Cheng, S.; McCabe, E.L.; Yang, E.; et al. Metabolic signatures of exercise in human plasma. Sci. Transl. Med. 2010, 2, 33-37.

31. Nelson, D.L.; Cox, M.M. Lehninger Principles of Biochemistry, 7th ed.; International ed.; W.H. Freeman \& Company: New York, NY, USA, USA; 2016; p. 612.

32. Gorostiaga, E.M.; Navarro-Amezqueta, I.; Calbet, J.A.; Sanchez-Medina, L.; Cusso, R.; Guerrero, M.; Izquierdo, M. Blood ammonia and lactate as markers of muscle metabolites during leg press exercise. J. Strength Cond. Res. 2014, 28, 2775-2785. [CrossRef] [PubMed]

33. Harris, R.A. Carbohydrate Metabolism: Major Metabolic Pathways and their Control. In Textbook of Biochemistry with Clinical Correlation, 4th ed.; Devlin, T.M., Ed.; Wiley-Liss: New York, NY, USA, 1997; pp. 268-317. 
34. Krog-Mikkelsen, I.; Hels, O.; Tetens, I.; Holst, J.J.; Andersen, J.R.; Bukhave, K. The effects of L-arabinose on intestinal sucrase activity: Dose-response studies in vitro and in humans. Am. J. Clin. Nutr. 2011, 94, 472-478. [CrossRef]

35. Décombaz, J.; Jentjens, R.; Ith, M.; Scheurer, E.; Buehler, T.; Jeukendrup, A.; Boesch, C. Fructose and galactose enhance postexercise human liver glycogen synthesis. Med. Sci. Sports Exerc. 2011, 43, 1964-1971. [PubMed]

36. Felig, P.W. Amino acid metabolism in exercising man. J. Clin. Investig. 1971, 50, 2703-2714. [CrossRef]

37. Coomes, M.W. Amino Acid Metabolism. In Textbook of Biochemistry with Clinical Correlation, 4th ed.; Devlin, T.M., Ed.; Wiley-Liss: New York, NY, USA, 1997; pp. 446-483.

38. Sutton, E.E.; Coill, M.R.; Deuster, P.A. Ingestion of tyrosine: Effects on endurance, muscle strength, and anaerobic performance. Int. J. Sport Nutr. Exerc. Metab. 2005, 15, 173-185. [CrossRef] [PubMed]

39. Pundir, C.S.; Deswal, R.; Kumar, P. Quantitative analysis of sarcosine with special emphasis on biosensors: A review. Biomarkers 2019, 24, 415-422.

40. Bazzano, M.; Laghi, L.; Zhu, C.; Magi, G.E.; Serri, E.; Spaterna, A.; Tesei, B.; Laus, F. Metabolomics of tracheal wash samples and exhaled breath condensates in healthy horses and horses affected by equine asthma. J. Breath Res. 2018, 12, 046015. [CrossRef]

41. Olson, M.S. Bioenergetics and Oxidative Metabolism. In Textbook of Biochemistry with Clinical Correlation, 4th ed.; Devlin, T.M., Ed.; Wiley-Liss: New York, NY, USA, 1997; pp. 218-261.

42. Dame, Z.T.; Aziat, F.; Mandal, R.; Krishnamurthy, R.; Bouatra, S.; Borzouie, S.; Guo, A.C.; Tanvir Sajed, T.; Deng, L.; Lin, H.; et al. The human saliva metabolome. Metabolomics 2015, 11, 1864-1883. [CrossRef]

43. Duarte, I.F.; Goodfellow, B.J.; Barros, A.; Jones, J.G.; Barosa, C.; Diogo, L.; Garcia, P.; Gil, A.M. Metabolic characterisation of plasma in juveniles with glycogen storage disease type 1a (GSD1a) by high-resolution (1)H NMR spectroscopy. NMR Biomed. 2007, 20, 401-412. [CrossRef]

44. Laborde, C.M.; Mourino-Alvarez, L.; Posada-Ayala, M.; Alvarez-Llamas, G.; Serranillos-Reus, M.G.; Moreu, J.; Vivanco, F.; Padial, L.R.; Barderas, M.G. Plasma metabolomics reveals a potential panel of biomarkers for early diagnosis in acute coronary syndrome. Metabolomics 2014, 10, 414-424. [CrossRef]

45. Trushina, E.; Dutta, T.; Persson, X.M.; Mielke, M.M.; Petersen, R.C. Identification of altered metabolic pathways in plasma and CSF in mild cognitive impairment and Alzheimer's disease using metabolomics. PLoS ONE 2013, 8, e63644. [CrossRef] [PubMed]

46. Koolman, J.; Roehm, K.H. Color. Atlas of Biochemistry, 2nd ed.; Thieme: Stuttgart, Germany, 2005; p. 352.

47. Marukawa, H.; Shimomura, T.; Takahashi, K. Salivary substance P, 5-hydroxytryptamine, and gamma-aminobutyric acid levels in migraine and tension-type headache. Headache 1996, 36, 100-104. [CrossRef] [PubMed]

48. Jezewska, E.; Scinska, A.; Kukwa, W.; Sobolewska, A.; Turzynska, D.; Samochowiec, J.; Bienkowski, P. Gamma-aminobutyric acid concentrations in benign parotid tumours and unstimulated parotid saliva. J. Laryngol. Otol. 2011, 125, 492-496. [CrossRef] [PubMed]

49. Sawaki, K.; Ouchi, K.; Sato, T.; Kawaguchi, M. Existence of gamma-aminobutyric acid and its biosynthetic and metabolic enzymes in rat salivary glands. Jpn. J. Pharmacol. 1995, 67, 359-363. [CrossRef] [PubMed]

50. Okubo, M.; Kawaguchi, M. Rat submandibular gland perfusion method for clarifying inhibitory regulation of GABAA receptor. J. Pharmacol. Sci. 2013, 122, 42-50. [CrossRef] [PubMed]

51. Bizzarri, M.; Fuso, A.; Dinicola, S.; Cucina, A.; Bevilacqua, A. Pharmacodynamics and pharmacokinetics of inositol(s) in health and disease. Expert Opin. Drug Metab. Toxicol. 2016, 12, 1181-1196. [CrossRef]

52. Gilmore, A.P.; Burridge, K. Regulation of vinculin binding to talin and actin by phosphatidylinositol-4-5-bisphosphate. Nature 1996, 381, 531-535. [CrossRef]

53. Hallman, M.; Spragg, R.; Harrell, J.H.; Moser, K.M.; Gluck, L. Evidence of lung surfactant abnormality in respiratory failure. Study of bronchoalveolar lavage phospholipids, surface activity, phospholipase activity, and plasma myoinositol. J. Clin. Investig. 1982, 70, 673-683. [CrossRef]

54. Benesch, R.; Benesch, R.E. The effect of organic phosphates from the human erythrocyte on the allosteric properties of hemoglobin. Biochem. Biophys. Res. Commun. 1967, 26, 162-167. [CrossRef]

55. Lam, G.; Zhao, S.; Sandhu, J.; Yi, R.; Loganathan, D.; Morrissey, B. Detection of myo-inositol tris pyrophosphate (ITPP) in equine following an administration of ITPP. Drug Test. Anal. 2014, 6, 268-276.

56. Bazzano, M.; Laghi, L.; Zhu, C.; Magi, G.E.; Tesei, B.; Laus, F. Respiratory metabolites in bronchoalveolar lavage fluid (BALF) and exhaled breath condensate $(\mathrm{EBC})$ can differentiate horses affected by severe equine asthma from healthy horses. BMC Vet. Res. 2020. under review. [CrossRef] [PubMed] 
57. Carroll, C.L.; Huntington, P.J. Body condition scoring and weight estimation of horses. Equine Vet. J. 1988, 20, 41-45. [CrossRef] [PubMed]

58. Zhu, C.; Faillace, V.; Laus, F.; Bazzano, M.; Laghi, L. Characterization of trotter horses urine metabolome by means of proton nuclear magnetic resonance spectroscopy. Metabolomics 2018, 14, 106. [CrossRef] [PubMed]

59. Kneen, M.A.; Annegarn, H.J. Algorithm for fitting XRF, SEM and PIXE X-ray spectra backgrounds. Nucl. Instrum. Methods Phys. Res. Sect. B Beam Interact. Mater. At. 1996, 109-110, 209-213. [CrossRef]

60. Box, G.E.P.; Cox, D.R. An Analysis of Transformations. J. R. Stat. Soc. Ser. B 2018, 26, 211-243. [CrossRef]

61. Hubert, M.; Rousseeuw, P.J.; Vanden Branden, K. ROBPCA: A new approach to robust principal component analysis. Technometrics 2005, 47, 64-79. [CrossRef]

62. Croft, D.; Mundo, A.F.; Haw, R.; Milacic, M.; Weiser, J.; Wu, G.; Jassal, B. The Reactome pathway knowledgebase. Nucleic Acids Res. 2014, 42, D472-D477. [CrossRef]

63. Johnson, N.L.; Kotz, S.; Kemp, A.W. Univariate Discrete Distributions, 2nd ed.; Wiley: New York, NY, USA, 2005.

(C) 2020 by the authors. Licensee MDPI, Basel, Switzerland. This article is an open access article distributed under the terms and conditions of the Creative Commons Attribution (CC BY) license (http://creativecommons.org/licenses/by/4.0/). 
Article

\title{
First Insights into the Urinary Metabolome of Captive Giraffes by Proton Nuclear Magnetic Resonance Spectroscopy
}

\author{
Chenglin Zhu ${ }^{1,+}$, Sabrina Fasoli ${ }^{2, \dagger}$, Gloria Isani ${ }^{2}$ and Luca Laghi ${ }^{1, *}$ \\ 1 Department of Agro-Food Science and Technology, University of Bologna, 47521 Cesena, Italy; \\ chenglin.zhu2@unibo.it \\ 2 Department of Veterinary Medical Sciences, University of Bologna, Ozzano Emilia, 40064 Bologna, Italy; \\ sabrina.fasoli2@unibo.it (S.F.); gloria.isani@unibo.it (G.I.) \\ * Correspondence: 1.laghi@unibo.it; Tel.: +39-0547-338105 \\ + These authors contributed equally to this work.
}

Received: 13 March 2020; Accepted: 15 April 2020; Published: 17 April 2020

\begin{abstract}
The urine from 35 giraffes was studied by untargeted ${ }^{1} \mathrm{H}-\mathrm{NMR}$, with the purpose of obtaining, for the first time, a fingerprint of its metabolome. The metabolome, as downstream of the transcriptome and proteome, has been considered as the most representative approach to monitor the relationships between animal physiological features and environment. Thirty-nine molecules were unambiguously quantified, able to give information about diet, proteins digestion, energy generation, and gut-microbial co-metabolism. The samples collected allowed study of the effects of age and sex on the giraffe urinary metabolome. In addition, preliminary information about how sampling procedure and pregnancy could affect a giraffe's urinary metabolome was obtained. Such work could trigger the setting up of methods to non-invasively study the health status of giraffes, which is utterly needed, considering that anesthetic-related complications make their immobilization a very risky practice.
\end{abstract}

Keywords: captive giraffes; urine; metabolomics; ${ }^{1} \mathrm{H}-\mathrm{NMR}$

\section{Introduction}

According to the International Union for Conservation of Nature (IUCN), giraffe (Giraffa camelopardalis) is declared a vulnerable species [1]. Moreover, different measures have been taken to monitor and protect giraffe population. For example, the International Union for Conservation of Nature (IUCN) Species Survival Commission (SSC) Giraffe and Okapi Specialist Group (GOSG) was established with the aim of studying and guaranteeing the conservation needs of this species (https://www.giraffidsg.org/). In addition, from November 26, 2019, giraffes are included in Appendix II of the CITES (Convention on International Trade in Endangered Species of Wild Fauna and Flora) to improve its protection, subjecting it to strict regulation (https://www.cites.org/).

Zoos represent a significant part of the protection strategy for giraffes, with projects explicitly aimed at protecting endangered species and pursuing high standards of animal welfare [2]. In these structures, however, giraffes may be subjected to sources of stress that reverberate negatively on individual and social behaviors [3]. Causes of stress could be represented by the presence of visitors and attendants [4]. Among the efforts that have been made to reduce the stressors, some are devoted to developing protocols to evaluate their general health status that do not involve immobilization, but are based on indirect methods [3]. In fact, giraffes are particularly prone to anesthetic-related complications and death, due to their unique cardiovascular system, making immobilization a risky practice $[5,6]$. 
The possibility of obtaining information from urine collected from the ground seems particularly attractive from this point of view, but the literature on this type of sampling is absent for giraffes and it has been only reported in okapi [7]. Indeed giraffes have been studied more for their iconic height and the mechanisms existing at the cardiovascular level to counterbalance the consequent state of primary hypertension [8-10].

Among the completely unexplored characteristics of giraffe urine is its metabolome, the ensemble of low weight molecules produced by the cellular metabolism. Studies carried out by liquid chromatography-mass spectrometry (LC/MS) or by proton nuclear magnetic resonance spectroscopy $\left({ }^{1} \mathrm{H}-\mathrm{NMR}\right)$ on humans and other animals suggest that the giraffe's urinary metabolome may be particularly informative about the general health of the animal. In horse urine, molecules revealing the action of the intestinal microbiota were found in micromolar concentrations [11,12]. Molecular patterns of the urinary metabolome linked to inflammatory processes have been identified in humans [13]. Urinary profile responses to the calorie content of the diet were identified in rat [14]. The effects of heat stress were studied in cattle by metabolomic profiling of urine [15]. Indeed, the use of urine as a source of biological data in giraffes could be a suitable alternative, due to its non-invasive approach that could avoid the immobilization of animals.

Among the analytical platforms capable of fulfilling the requirements, proton nuclear magnetic resonance spectroscopy $\left({ }^{1} \mathrm{H}-\mathrm{NMR}\right)$ has been widely used for the investigation of urine metabolomes, taking advantage of its high reproducibility and minimal sample preparation.

In the present study, we wanted to verify the feasibility of ${ }^{1} \mathrm{H}-\mathrm{NMR}$ based metabolomic studies focusing on the urine of giraffes. For this purpose, we characterized the molecular profile of healthy giraffes held in captivity to obtain preliminary quantitative values that could be applied for the diagnosis of diseases affecting this animal. Moreover, the samples collected gave the opportunity to have a first insight about the influence of important physiological factors, such as the sex and age of the subjects, on the urinary metabolomic profile.

\section{Results}

\subsection{Urinary Metabolites Identification by Untargeted ${ }^{1} \mathrm{H}-\mathrm{NMR}$}

A representative spectrum of the metabolites identified in the giraffe's urine is reported in Figure 1. In this study, we identified 39 molecules (Table S1). These molecules mainly pertain to the classes of amino acids and derivatives and organic acids and derivatives. Hippurate $(30.63 \%)$, creatinine $(25.17 \%)$, and phenylacetylglycine $(12.64 \%)$ were the most represented metabolites.

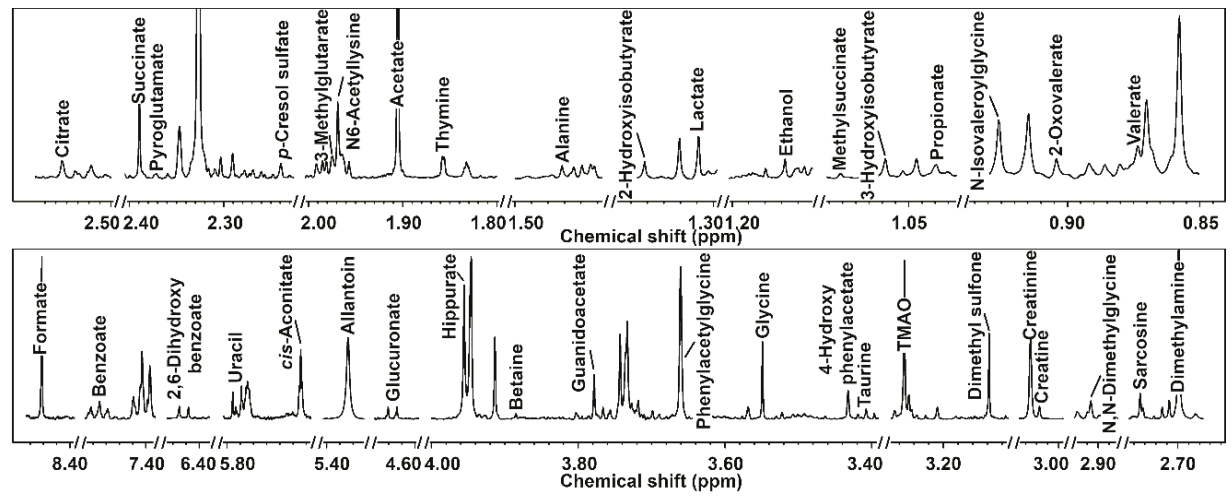

Figure 1. Portions of ${ }^{1} \mathrm{H}-\mathrm{NMR}$ spectra, representative of all the spectra obtained in this study. Each molecule's name appears over the NMR peak used for its quantification. To ease the visual inspection of each portion, a different spectrum with a convenient signal-to-noise ratio has been selected. 


\subsection{Effects of Sampling Procedure and Location}

To check the potential influence of the different sampling methods, we wanted to collect pairs of samples during the same voiding, one directly and one from the ground. Unfortunately, we only succeeded in this task for one individual (Ronny). Among 39 quantified compounds, four molecules showed a variation of concentration higher than 50\%, namely $p$-cresol sulfate, citrate, glycine, and benzoate. ${ }^{1} \mathrm{H}-\mathrm{NMR}$ signals for these compounds are reported in Figure 2. In detail, benzoate and glycine were more concentrated in the urine collected from the ground, while citrate and $p$-cresol sulfate showed the opposite trend. Overall, the 39 molecules showed a median difference between the two samples of $4.8 \%$. As these observations were based only on one pair of samples from a single individual, we decided not to exclude these molecules from the subsequent analyses.

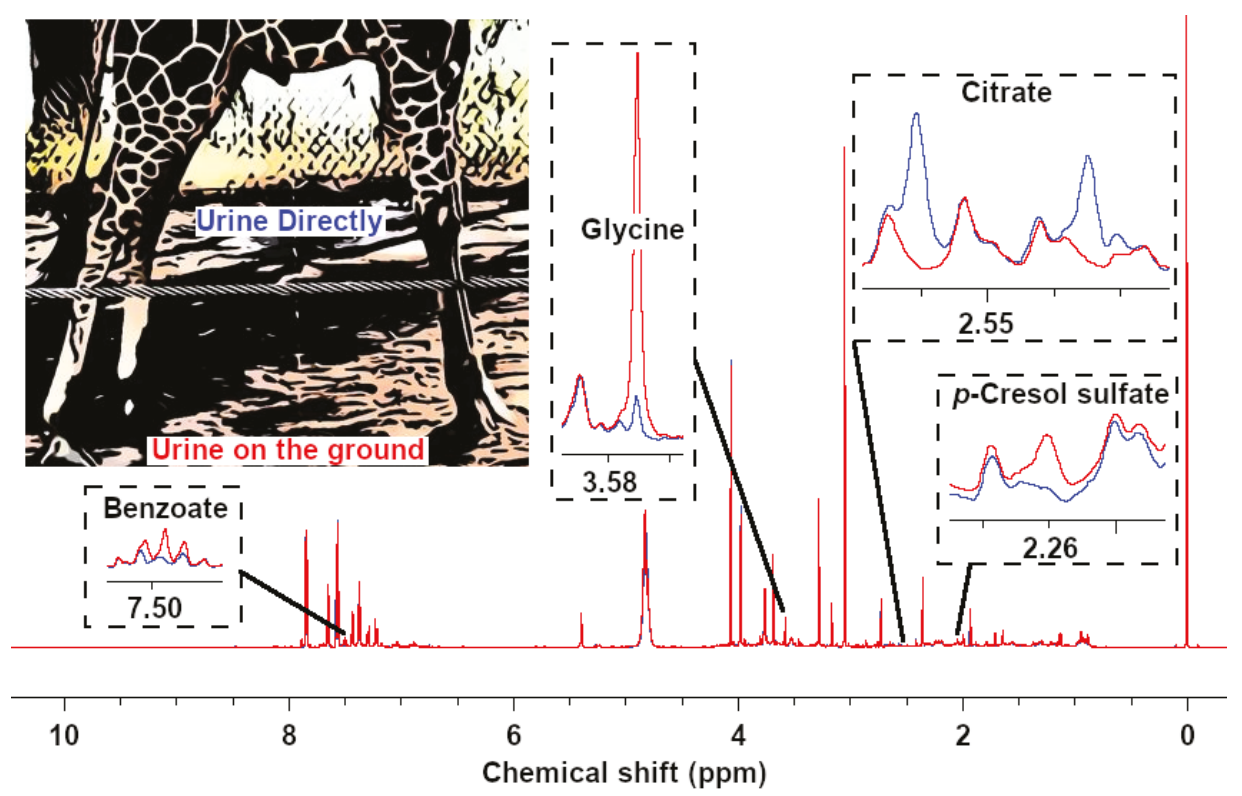

Figure 2. Representative sections of two spectra obtained from analyzing urine from the same giraffe (Ronny), collected directly (blue line) and from the ground (red line) during one urination, respectively.

To obtain hints about the potential effects of location on the metabolome of giraffe urine, we selected the samples from the locations BG (Parco Faunistico Le Cornelle) and FA (Zoosafari Fasanolandia), where most of the samples had been collected, and we set up a three-way ANOVA analysis aiming at excluding any effect related to gender or age. None of the molecules quantified appeared as significantly different in relation to zoo, so this variable was not considered in the subsequent analyses.

\subsection{Sex Affects the Giraffe Urine Molecular Profile}

To obtain preliminary data on the effect of sex on the urinary metabolome, we focused on samples collected from adult, non-pregnant individuals. Six molecules were found to be significantly $(p<0.05)$ affected by sex, as shown in Table 1 . 
Table 1. Metabolite concentrations ( $\mathrm{mmol} / \mathrm{L}$, median $(\mathrm{IQR}))$ in the adult group were significantly $(p<0.05)$ affected by sex, as assessed by $t$-test.

\begin{tabular}{ccccc}
\hline & Females (6) & Males (7) & Trend & $p$ Value \\
\hline Acetate & $2.04\left(5.23 \times 10^{-1}\right)$ & $1.33\left(9.04 \times 10^{-1}\right)$ & $\downarrow$ & 0.034 \\
Hippurate & $13.50(10.70)$ & $19.30(19.50)$ & $\uparrow$ & 0.047 \\
Lactate & $2.77 \times 10^{-1}\left(8.90 \times 10^{-2}\right)$ & $1.28 \times 10^{-1}\left(7.35 \times 10^{-2}\right)$ & $\downarrow$ & 0.003 \\
Phenylacetylglycine & $7.82(2.41)$ & $15.20(5.53)$ & $\uparrow$ & 0.014 \\
Succinate & $2.48 \times 10^{-1}\left(3.00 \times 10^{-2}\right)$ & $1.66 \times 10^{-1}\left(8.80 \times 10^{-2}\right)$ & $\downarrow$ & 0.006 \\
Thymine & $1.77 \times 10^{-1}\left(4.94 \times 10^{-2}\right)$ & $2.86 \times 10^{-1}\left(1.79 \times 10^{-1}\right)$ & $\uparrow$ & 0.043 \\
\hline
\end{tabular}

To have an overall view of the data, a robust principal component analysis (rPCA) model was calculated on their concentration, as shown in Figure 3.
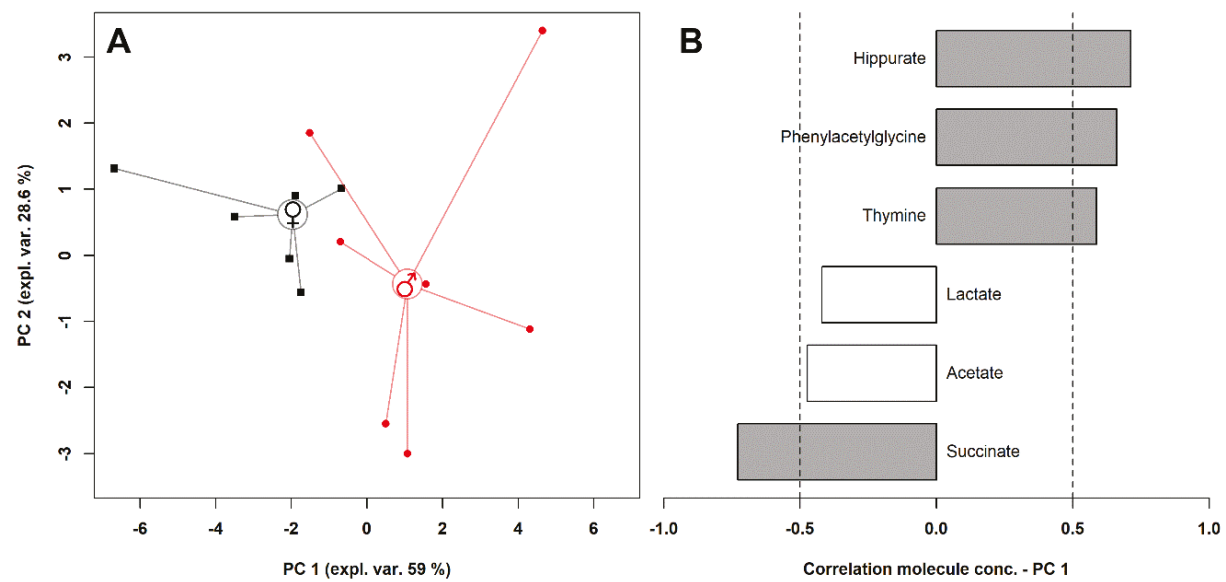

Figure 3. rPCA model calculated on the concentration of the significantly different molecules between male and female giraffes. The scoreplot (A) represents with squares and circles females and males, respectively. The median of each sample group is represented by wide circles. The loading plot (B) reports the correlation between the importance of each substance over principal component 1 and its concentration. Gray bars highlight significant correlations $(p<0.05)$.

Three principal components (PCs) were accepted by the algorithm to depict the overall data features. PC 1, accounting for 59\% of the variance thus represented, indeed significantly summarized the peculiarities connected to sex $(p<0.05)$, with female and male individuals appearing respectively at low and high PC scores. Among these molecules, hippurate, phenylacetylglycine, and thymine were more abundant in the urine of male individuals, while lactate, acetate, and succinate were more concentrated in the females' urine.

\subsection{Effect of Age on the Urinary Metabolome}

Age was found to significantly affect $(p<0.05)$ the concentration of three urinary metabolites, namely formate, alanine, and valerate, (Figure 4). To understand if their evolution was part of a trend spanning over the entire life of the giraffe, these molecules were used as a base for an rPCA model (Figure 5). 

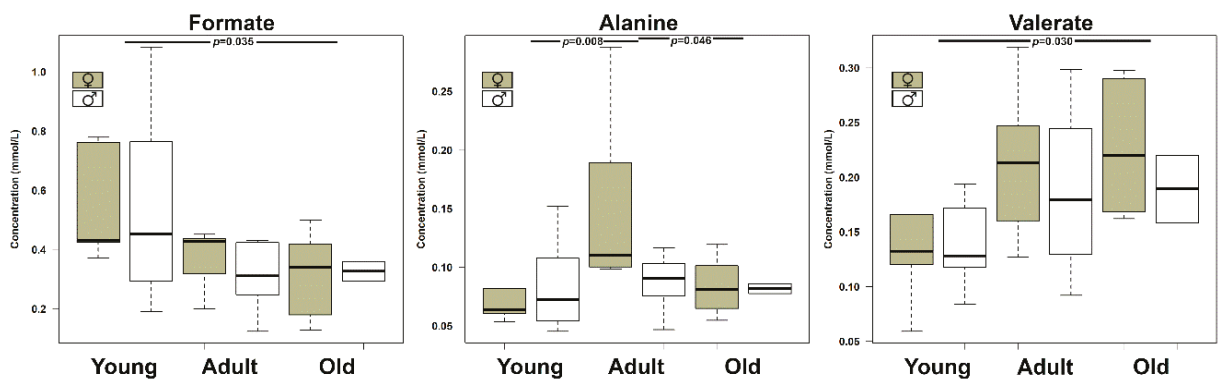

Figure 4. Boxplots showing the concentration of molecules significantly $(p<0.05)$ affected by age, as assessed by two-way ANOVA followed by Tukey post-hoc test.

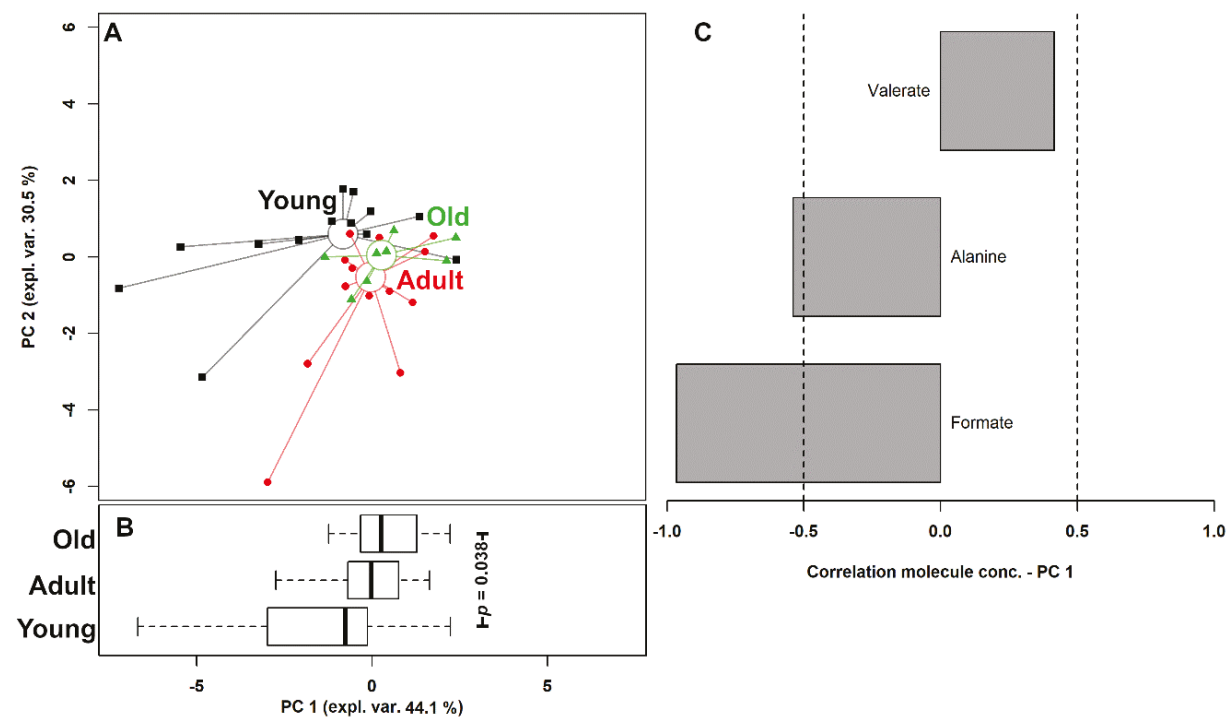

Figure 5. rPCA model of the concentration of the molecules showing a significant difference among the giraffes grouped by age. The scoreplot (A) shows the samples from the three groups with squares (Young), circles (Adult), and triangles (Old). The median of each sample group is represented by wide circles. The boxplot (B) summarizes the positions of the samples along PC1 and compares them by two-way ANOVA, followed by Tukey post-hoc test. The loading plot (C) reports the correlation between the importance of each substance over PC 1 and its concentration. Gray bars highlight significant correlations $(p<0.05)$.

Three PCs were accepted by the algorithm to depict the overall data features. PC 1, accounting for $44.1 \%$ of the variance thus represented, summarized effectively the peculiarities connected to age $(p<0.05)$, with Young, Adult, and Old individuals appearing respectively at low, intermediate, and high PC scores. Among these molecules, formate and alanine were more abundant in young individuals, while valerate showed an opposite trend.

\subsection{Pregnancy Related Urinary Metabolome}

Urine samples were obtained from two female giraffes during and after pregnancy (Table S2). Despite the limited number of samples, it was possible to observe a variation of five metabolites during the pregnancy. These molecules showed consistent trends in the samples from both giraffes. 
All these molecules showed a relevant increase in concentration during the pregnancy, except for phenylacetylglycine, as shown in Table 2.

Table 2. Urinary metabolites (mmol/L) affected by pregnancy consistently across the two giraffes observed.

\begin{tabular}{ccccc}
\hline & \multicolumn{2}{c}{ Giulietta } & \multicolumn{2}{c}{ Nicole } \\
& Not Pregnant & Pregnant $^{\mathbf{1}}$ & Not Pregnant $^{\text {Pregnant }}$ \\
\hline Phenylacetylglycine & 10.20 & $3.52 \downarrow$ & 10.40 & $5.02 \downarrow$ \\
Benzoate & 2.14 & $3.88 \uparrow$ & 2.46 & $12.22 \uparrow$ \\
Glycine & 1.06 & $3.04 \uparrow$ & 1.79 & $11.65 \uparrow$ \\
Taurine & $1.75 \times 10^{-1}$ & $2.93 \times 10^{-1} \uparrow$ & $7.98 \times 10^{-2}$ & $1.33 \times 10^{-1} \uparrow$ \\
$p$-Cresol sulfate & $1.46 \times 10^{-2}$ & $2.15 \times 10^{-2} \uparrow$ & $6.37 \times 10^{-2}$ & $3.50 \times 10^{-1} \uparrow$ \\
\hline
\end{tabular}

${ }^{1}$ For readability, only molecules changing by more than $40 \%$ for both giraffes are shown.

\section{Discussion}

The present paper describes one of the first studies ever devoted to the urinary metabolome of nonfarmed animals, and the very first focusing on the giraffe metabolome. Due to such paucity of studies on the topic, a key point that needs to be addressed before giraffe urine can be used for metabolomics studies is the possibility of relying on samples collected from the ground. Several aspects, in fact, make the collection of urine directly from the individual during urination highly impractical. To obtain a first insight on this point, we managed to collect the same urine sample either at the start of a spontaneous voiding or from the ground with a syringe at the end the voiding. The corresponding ${ }^{1} \mathrm{H}-\mathrm{NMR}$ spectra were highly superimposable, except for four molecules, namely benzoate, citrate, $p$-cresol sulfate, and glycine. The fact that the non-volatile glycine showed the greatest differences gave hints that the discrepancies could be mainly connected to dynamic variations in composition during urination, in agreement with Sink and Weinstein [16]. Modifications induced by the collection method could therefore be considered a confounding factor of lower entities than inhomogeneity in the composition of urine during voiding.

The 39 molecules identified give information about protein digestion, diet, gut-microbial co-metabolism, and energy production. Their quantitative observation therefore offers a handy perspective of the health status of giraffes, through a quintessentially non-invasive sampling method.

Comparisons with the urinary metabolome of other animals are also possible, giving indirect information about the differences in metabolism. An example of this possibility is offered by allantoin. This molecule is the fourth most concentrated in giraffe urine (Table S1), identically to yak (Bos grunniens) [17] and horse [18]. Differently from these strictly herbivorous animals, this molecule is the most concentrated in the urine of the giant panda [19], even if the giant panda consumes an amount of vegetables in relation to body weight (as much as 30\%) much higher than ruminants or horses, which should lead to the lowest concentration of urinary allantoin [20]. This apparent contradiction leads to speculate that the main mechanism determining the concentration of allantoin in the urine of the above-mentioned animals is likely to be its renal reabsorption, which is very effective in strictly herbivorous animals [20].

\subsection{Sex Affects the Giraffe Urine Molecular Profile}

In the current study acetate, succinate, and lactate concentrations appeared to be significantly higher in female giraffe urine, while hippurate, phenylacetylglycine, and thymine were more concentrated in male urine. For acetate, two of the authors of the present paper identified a similar situation in horse urine [18]. For the other molecules, indirect connections with published findings can be devised. There is an abundance of references, focusing on humans, showing that exercise leads to higher concentrations of acetate, succinate, and lactate in urine, and lower concentrations of thymine and hippurate [21-23]. Ginnett et al. showed that female giraffes spend more time walking, foraging, 
feeding, and traveling than males [24]. The two observations seem to suggest that the sex-related differences observed in the urine of males and females may be partly due to the different daily activities. Contrary to the previously reported molecules, phenylacetylglycine is mainly a co-metabolite of gut microorganisms, derived from valine, leucine, phenylalanine, lysine, or ornithine [25]. Its different concentration in relation to sex may therefore reflect peculiarities in gut microbiota profiles or different foraging behaviors, similarly to what was recently observed in the giant panda [19]. Ginnett et al., in fact, demonstrated that males prefer larger bites than females, with potential consequences on the food, and in turn urine, metabolome profile [24]. It is tantalizing to speculate that the length of the neck, which is higher in males [5], may play a role too. In fact, Schüßler and Greven [26] found an allometric direct relationship between rumen-to-mouth distance and the duration of rumination intercycles, influencing in turn the digestive action of ruminal microorganisms.

\subsection{Effect of Age}

By removing the gender effect by two-way ANOVA, it was possible to focus on the effect of age. In parallel with previous studies in rats and humans $[27,28]$, formate and alanine were negatively related to age. The trend observed for formate is very likely related to the gut microbiome. In fact, in the gut microbiota of the juvenile giraffes there is a prevalence of Bacteroides and Acinetobacter genera, responsible for the degradation of starch and cellulose to formate [29], while in the gut of adult giraffes other genera tend to prevail, such as Treponema [30].

The concentration of amino acids in urine has been consistently linked to the turnover of muscle amino acids [18,31], with urinary concentration of alanine specifically related to exercise [32]. Therefore, the difference in the concentration of alanine could be ascribed to a variation of daily activity intensity along age.

\subsection{Effect of Pregnancy}

Early identification of pregnant giraffes with maximum accuracy is an important issue for optimizing their management. Although some diagnostic methods (e.g., ultrasonography) have been described in domestic animals [33], their application to wild or captive animals is hindered by practical reasons. Metabolomics approaches seem in principle promising for setting up diagnostic methods that might be more convenient in specific contexts, due to the possibility to quantify a high number of molecules at the same time. However, previous studies performed in domestic animals were focused on serum [34,35], a sub-optimal sample from the point of view of non-invasivity. Therefore, despite the restricted number of samples analyzed in the present study, the obtained data can provide a preliminary urinary fingerprint of pregnancy in giraffes.

Taurine is an important amino acid during pregnancy and lactation, because it satisfies the needs of both the fetus and suckling infant. In our research, taurine excretion through urine increased during early pregnancy, consistent with human studies [36]. Taurine is rarely found in plants [37], so that herbivores cannot obtain a sufficient amount taurine from the diet. Remarkably, in ruminants the urinary taurine concentration is strongly diet-dependent, as can be inferred from the works of Bristow et al. on cows fed with maize silage compared to free grazing cows [38]. Diet is therefore likely to trigger biosynthetic pathways, such as the one leading to taurine from methionine [39]. Moreover, a specific pathway, converting homocysteine to taurine and glycine through cysteine, is known to become effective in early pregnancy [39]. This latter mechanism is a likely reason for the increasing trend of taurine excretion we found in the present work.

A further contribution to urine metabolome profile modifications may be due to changes in the gut microbiota. In fact, among the molecules showing the greatest changes we found $p$-cresol sulfate and phenylacetylglycine, mainly described as gut microorganism co-metabolites [11,25], absorbed at the intestinal level and then expelled through urine. Interestingly, the change in the concentration of both has been related, in humans, with alterations in the microbiota profile linked to inflammatory states [13,40], in which pregnancy is known to play a role [41]. Despite the very limited number of cases 
here, these observations support the compelling possibility to use the urine metabolome to gain specific information about giraffe inflammatory status during pregnancy, as modulated by the gut microbiota.

\section{Materials and Methods}

\subsection{Compliance with Ethical Requirements}

All the procedures related to animals respected the Directive 2010/63/EU of the European Parliament and of the Council of September 22, 2010 on the protection of animals used for scientific purposes (Article 1, Paragraph 1, Letter b) and the Italian legislation (D. Lgs. n. 26/2014, Article 2, Paragraph 1, Letter b).

\subsection{Sample Collection}

A total of 35 captive giraffes (Giraffa camelopardalis) were involved in the current study. Based on physical examinations, giraffes did not show symptoms of diseases both before and during the urine sampling period. The giraffes were housed in five Italian zoos: Zoosafari Fasanolandia (FA) $(\mathrm{N}=11)$, Safari Ravenna (RA) ( $=4)$, Giardino Zoologico di Pistoia (PT) $(\mathrm{N}=1)$, Parco Natura Viva (VR) $(\mathrm{N}=4)$, and Parco Faunistico Le Cornelle $(\mathrm{BG})(\mathrm{N}=15)$.

The details for each giraffe are reported in Table 3. Their age ranged from a minimum of 6 months to a maximum of 20 years. The giraffes were categorized in 3 age classes: Young (from 6 months to 6 years old, $N=14$ ), Adult (from 6 to 15 years old, $N=16$ ), and Old (older than 15, $N=9$ ), according to the following information. In female giraffes the first birth is at about 6.4 years old, even if sexual maturity is reached at $3-4$ years $[42,43]$. Giraffe males are considered as adults when older than 6 years old, according to Lee et al. [44].

Table 3. Animal information.

\begin{tabular}{|c|c|c|c|c|}
\hline Sample ID & Name & Sex & Age (years) & Zoo \\
\hline N.01 & Ronny & Male & 14 & FA \\
\hline N.02 & Nicole & Female & 14 & FA \\
\hline N.03 & Giulietta & Female & 17 & FA \\
\hline N.04 & Marcello & Male & 9 & FA \\
\hline N.05 & Italia & Female & 8 & FA \\
\hline N.06 & Carlos & Male & 2 & RA \\
\hline N.07 & Daniele & Male & 11 & RA \\
\hline N.08 & Cleopatra & Female & 20 & PT \\
\hline N.09 & Alto & Male & 2 & FA \\
\hline N.10 & Congo & Male & 0.3 & FA \\
\hline N.11 & Roberto & Male & 0.6 & RA \\
\hline N.12 & Martina & Female & 0.6 & RA \\
\hline N.13 & Linda & Female & 16 & BG \\
\hline N.14 & Sandy & Female & 16 & $\mathrm{BG}$ \\
\hline N.15 & Raffa & Female & 7 & BG \\
\hline N.16 & Telete & Female & 2 & BG \\
\hline N.17 & Rusman & Male & 16 & $\mathrm{BG}$ \\
\hline N.18 & Akuna & Female & 10 & $\mathrm{BG}$ \\
\hline N.19 & Ciokwe & Male & 5 & BG \\
\hline N.20 & Miro & Male & 9 & BG \\
\hline N.21 & Lucia & Female & 16 & $\mathrm{BG}$ \\
\hline N.22 & Nuvola & Female & 7 & BG \\
\hline N.23 & Sahel & Female & 2 & BG \\
\hline N.24 & Russel & Male & 16 & BG \\
\hline N.25 & Ramiro & Male & 3 & BG \\
\hline N.26 & Madiba & Male & 6 & BG \\
\hline N.27 & Nasanta & Female & 2 & BG \\
\hline
\end{tabular}


Table 3. Cont.

\begin{tabular}{lclcl}
\hline N.28 & Macchia & Male & 5 & VR \\
N.29 & Secondo & Male & 11 & VR \\
N.30 & Akasha & Male & 7 & VR \\
N.31 & Quarto & Male & 9 & VR \\
N.32 & Luna & Female & 15 & FA \\
N.33 & Kenya & Female & 20 & FA \\
N.34 & Alessia & Female & 4 & FA \\
N.35 & Mina & Female & 14 & FA \\
\hline
\end{tabular}

The samples were collected between 10:00 a.m. and 2:00 p.m., in connection to the daily activities of the keepers. Urine samples were collected with a syringe from the ground. To limit the soil contaminants, only the upper part of the urine was collected, immediately after the spontaneous voiding, before it was absorbed by the soil. A sample from one male was also collected directly into a sterile beaker, preventing the sample from touching the ground. Four urine samples were collected from two females during and after pregnancy. After collection, the urine samples were centrifuged at $1500 \times \mathrm{g}$ for $10 \mathrm{~min}$, to further remove potential ground contaminants, and the supernatants were frozen at $-80^{\circ} \mathrm{C}$.

\subsection{Metabolomic Analysis}

We prepared urine samples for NMR by thawing and centrifuging them for $15 \mathrm{~min}$ at $18,630 \times \mathrm{g}$ at $4{ }^{\circ} \mathrm{C}$. We added the supernatant $(350 \mu \mathrm{L})$ to bi-distilled water $(350 \mu \mathrm{L})$ and to a $\mathrm{D}_{2} \mathrm{O}$ solution $(200 \mu \mathrm{L})$ of TSP (3-(trimethylsilyl)-propionic-2,2,3,3-d4 acid) $10 \mathrm{mM}$ and of $\mathrm{NaN}_{3} 2 \mathrm{mM}$. A $1 \mathrm{M}$ phosphate buffer had been used to set the $\mathrm{D}_{2} \mathrm{O}$ solution to $\mathrm{pH}$ of $7.00 \pm 0.02$. After a further centrifugation, we recorded ${ }^{1} \mathrm{H}-\mathrm{NMR}$ spectra at $298 \mathrm{~K}$ with an AVANCE III spectrometer (Bruker, Milan, Italy), at a frequency of 600.13 MHz, equipped with Topspin software (Ver. 3.5).

According to Zhu et al. [17], we suppressed the signals from broad resonances using a CPMG-(Carr-Purcell-Meiboom-Gill) filter composed of 400 echoes with a of $400 \mathrm{~s}$ and a $180^{\circ}$ pulse of $24 \mathrm{~s}$, for a total filter of $330 \mathrm{~ms}$. We also applied pre-saturation, to reduce the signal from water. We employed Topspin software to apply a line broadening of $0.3 \mathrm{~Hz}$ and to adjust the phase of each spectrum. We set the recycle delay to $5 \mathrm{~s}$, by considering the relaxation time of the protons under investigation. We employed R computational language [45] for any further processing of spectra, quantification of molecules, and data mining, with custom scripts.

We aligned the spectra by using the TSP signal as a reference $(-0.017 \mathrm{ppm})$. We adjusted the baseline of each spectrum by distinguishing irregularities of the baseline from genuine signals, according to the "rolling ball" idea [46], implemented in the R package "baseline" [47]. We performed the assignment of the signals by comparing chemical shift and multiplicity with the libraries (Ver. 10) of Chenomx software (Chenomx Inc., Canada, v. 8.3).

According to Dieterle et al. [48], water intake behavior can change the dilution of urine as much as five times, obscuring any trend in metabolite concentrations. We removed this confounding factor by calculating, for each sample, the ratio between the area of TSP peak and the intensity of the spectrum. This allowed us to estimate the dilution of each sample and to select the one with the mostly representative dilution. We used this sample as a reference by quantifying the molecules from the added TSP. We then normalized the other samples towards the reference by probabilistic quotient normalization (PQN) [48].

\subsection{Statistical Analysis}

We conducted the statistical analysis in R computational language [45] and we refined the artwork by GIMP (version 2.10, www.gimp.org). Prior to univariate analysis, we transformed the data to normality by BoxCox transformation [49]. To investigate the effects of sex on urinary metabolites, 
we considered only adult, non-pregnant giraffes. This allowed us to reduce potential interferences due to different age classes. We then highlighted any difference by t-test. To investigate age related effects, by removing sex effect, we applied a two-way ANOVA test followed by Tukey-HSD, by taking advantage of the "aov" function of the R package "stats" [50]. For the above statistical tests, we accepted a cut-off $p$-value of 0.05 .

In agreement with Bazzano et al. [51], we highlighted any trend characterizing the samples with robust principal component analysis (rPCA) models [52], using the molecules accepted by univariate analysis as a base. We took advantage of the PcaHubert algorithm implemented in the "rrcov" package. The algorithm grants robustness with a two-steps approach. In the first step outlying samples are detected according to their distance from the others along and orthogonally to the PCA plane. A second step determines the optimal number of principal components (PCs). The main features of each rPCA model are summarized by a scoreplot and by a Pearson correlation plot. The former is the projection of the samples in the PC space and highlights the underlying structure of the data. The latter relates the concentration of each variable to the components of the model.

\section{Conclusions}

This work represents a primer in giving quantitative information about the urinary metabolome of captive giraffes, as detected by untargeted ${ }^{1} \mathrm{H}-\mathrm{NMR}$. Foraging behaviors and daily activity could be considered as one of the main reasons for the differences we highlighted that are linked to sex and age. A preliminary observation conducted on two female giraffes suggests that ${ }^{1} \mathrm{H}-\mathrm{NMR}$ based metabolomics could be conveniently applied to monitor modifications occurring during pregnancy, some of which are potentially related to inflammatory status triggered by modification of the microbiota profile.

Supplementary Materials: The following are available online at http://www.mdpi.com/2218-1989/10/4/157/s1, Table S1: Concentration (mmol/L, median (IQR)) of the molecules quantified by ${ }^{1} \mathrm{H}-\mathrm{NMR}$ in all the samples studied in the present investigation, sorted by abundance. Table S2. Concentration $(\mathrm{mmol} / \mathrm{L})$ of the molecules quantified by ${ }^{1} \mathrm{H}-\mathrm{NMR}$ in the samples collected during and after pregnancy.

Author Contributions: C.Z., L.L., S.F., and G.I. conceived and designed the research. C.Z. and L.L. performed metabolomics analysis. S.F. collected the samples. C.Z., L.L., S.F., and G.I. wrote the manuscript. All authors have read and agreed to the published version of the manuscript.

Funding: This research received no external funding.

Acknowledgments: Chenglin Zhu gratefully acknowledges financial support from Chinese Scholarship Council (grant $n^{\circ}$ 201606910076). Part of the samples used in this study was collected with the financial Grant won by Sabrina Fasoli and donated by the Zebra Foundation for Veterinary Zoological Education in 2019. The authors would like to thank Bandoli Francesca, Cordon Rossana, Cotignoli Chiara, Laguardia Daniele, Laricchiuta Pietro, Sandri Camillo, Schneider Rainer, and Spiezio Caterina for their support. The authors also would like to thank all staff of the zoos involved in this study for their precious help during the sampling and Vito Barnaba, Pietro Ciaccia, Giacomo Melani, and Fulvio Pendezza for their fundamental assistance.

Conflicts of Interest: The authors declare no conflict of interest.

\section{References}

1. Muller, Z.; Bercovitch, F.; Brand, R.; Brown, D.; Brown, M.; Bolger, D.; Carter, K.; Deacon, F.; Doherty, J.B.; Fennessy, J.; et al. Giraffa Camelopardalis. The IUCN Red List of Threatened Species 2016: e.T9194A51140239. Available online: https://www.iucnredlist.org/species/9194/109326950 (accessed on 17 April 2020).

2. Paul-Murphy, J.; Molter, C. Overview of Animal Welfare in Zoos. In Fowler's Zoo and Wild Animal Medicine Current Therapy; Miller, R.E., Lamberski, N., Calle, P.P., Eds.; Elsevier: St. Louis, MO, USA, 2019; Volume 9 , pp. 64-72. ISBN 978-0-323-55228-8.

3. Hosey, G.; Melfi, V.; Pankhurst, S. Animal Welfare. In Zoo Animals: Behaviour, Management, and Welfare, 2nd ed.; Oxford University Press: Oxford, UK, 2013; pp. 212-250. ISBN 0199693528.

4. Normando, S.; Pollastri, I.; Florio, D.; Ferrante, L.; Macchi, E.; Isaja, V.; de Mori, B. Assessing animal welfare in animal-visitor interactions in zoos and other facilities. A pilot study involving giraffes. Animals 2018, 8, 153. [CrossRef] [PubMed] 
5. Dagg, A.I. Physiology. In Giraffe: Biology, Behaviour and Conservation; Dagg, A.I., Ed.; Cambridge University Press: New York, NY, USA, 2014; pp. 117-134. ISBN 9781139542302.

6. Gage, L.J. Giraffe Husbandry and Welfare. In Fowler's Zoo and Wild Animal Medicine Current Therapy; Miller, R.E., Lamberski, N., Calle, P.P., Eds.; Elsevier: St. Louis, MO, USA, 2019; Volume 9, pp. 619-622. ISBN 978-0-323-55228-8.

7. Glatston, A.R.; Smit, S. Analysis of the urine of the okapi (Okapia johnstoni). Acta Zool. Pathol. Antverp. 1980, 75, 49-58.

8. Zhang, Q. Hypertension and Counter-Hypertension Mechanisms in Giraffes. Cardiovasc. Hematol. Disord. Targets 2012, 6, 63-67. [CrossRef] [PubMed]

9. Hargens, A.R.; Millard, R.W.; Pettersson, K.; Johansen, K. Gravitational haemodynamics and oedema prevention in the giraffe. Nature 1987, 329, 59-60. [CrossRef]

10. Agaba, M.; Ishengoma, E.; Miller, W.C.; McGrath, B.C.; Hudson, C.N.; Bedoya Reina, O.C.; Ratan, A.; Burhans, R.; Chikhi, R.; Medvedev, P.; et al. Giraffe genome sequence reveals clues to its unique morphology and physiology. Nat. Commun. 2016, 7, 1-8. [CrossRef]

11. Patel, K.P.; Luo, F.J.G.; Plummer, N.S.; Hostetter, T.H.; Meyer, T.W. The production of p-Cresol sulfate and indoxyl sulfate in vegetarians versus omnivores. Clin. J. Am. Soc. Nephrol. 2012, 7, 982-988. [CrossRef]

12. Laghi, L.; Zhu, C.; Campagna, G.; Rossi, G.; Bazzano, M.; Laus, F. Probiotic supplementation in trained trotter horses: Effect on blood clinical pathology data and urine metabolomic assessed in field. J. Appl. Physiol. 2018, 125, 654-660. [CrossRef]

13. Barbara, G.; Scaioli, E.; Barbaro, M.R.; Biagi, E.; Laghi, L.; Cremon, C.; Marasco, G.; Colecchia, A.; Picone, G.; Salfi, N.; et al. Gut microbiota, metabolome and immune signatures in patients with uncomplicated diverticular disease. Gut 2017, 66, 1252-1261. [CrossRef]

14. Kok, D.E.G.; Rusli, F.; van der Lugt, B.; Lute, C.; Laghi, L.; Salvioli, S.; Picone, G.; Franceschi, C.; Smidt, H.; Vervoort, J.; et al. Lifelong calorie restriction affects indicators of colonic health in aging C57Bl/6J mice. J. Nutr. Biochem. 2018, 56, 152-164. [CrossRef]

15. Liao, Y.; Hu, R.; Wang, Z.; Peng, Q.; Dong, X.; Zhang, X.; Zou, H.; Pu, Q.; Xue, B.; Wang, L. Metabolomics Profiling of Serum and Urine in Three Beef Cattle Breeds Revealed Different Levels of Tolerance to Heat Stress. J. Agric. Food Chem. 2018, 66, 6926-6935. [CrossRef] [PubMed]

16. Sink, C.A.; Weinstein, N.M. Specimen procurement. In Practical Veterinary Urinalysis; John Wiley \& Sons: Chichester, West Sussex, UK, 2012; pp. 9-18.

17. Zhu, C.; Li, C.; Wang, Y.; Laghi, L. Characterization of yak common biofluids metabolome by means of proton nuclear magnetic resonance spectroscopy. Metabolites 2019, 9, 41. [CrossRef] [PubMed]

18. Zhu, C.; Faillace, V.; Laus, F.; Bazzano, M.; Laghi, L. Characterization of trotter horses urine metabolome by means of proton nuclear magnetic resonance spectroscopy. Metabolomics 2018, 14, 106. [CrossRef] [PubMed]

19. Zhu, C.; Laghi, L.; Zhang, Z.; He, Y.; Wu, D.; Zhang, H.; Huang, Y.; Li, C.; Zou, L. First Steps toward the Giant Panda Metabolome Database: Untargeted Metabolomics of Feces, Urine, Serum, and Saliva by $1 \mathrm{H}$ NMR. J. Proteome Res. 2020, 19, 1052-1059. [CrossRef]

20. Chen, X.; Kyle, D.; Orskov, E.; Hovell, F. Renal clearance of plasma allantoin in sheep. Exp. Physiol. 1991, 76, 59-65. [CrossRef]

21. Sheedy, J.R.; Gooley, P.R.; Nahid, A.; Tull, D.L.; McConville, M.J.; Kukuljan, S.; Nowson, C.A.; Daly, R.M.; Ebeling, P.R. 1H-NMR analysis of the human urinary metabolome in response to an 18-month multi-component exercise program and calcium-vitamin-D3 supplementation in older men. Appl. Physiol. Nutr. Metab. 2014, 39, 1294-1304. [CrossRef]

22. Mukherjee, K.; Edgett, B.A.; Burrows, H.W.; Castro, C.; Griffin, J.L.; Schwertani, A.G.; Gurd, B.J.; Funk, C.D. Whole blood transcriptomics and urinary metabolomics to define adaptive biochemical pathways of highintensity exercise in 50-60 year old masters athletes. PLOS ONE 2014, 9, e92031. [CrossRef]

23. Enea, C.; Seguin, F.; Petitpas-Mulliez, J.; Boildieu, N.; Boisseau, N.; Delpech, N.; Diaz, V.; Eugène, M.; Dugué, B. 1H NMR-based metabolomics approach for exploring urinary metabolome modifications after acute and chronic physical exercise. Anal. Bioanal. Chem. 2010, 396, 1167-1176. [CrossRef]

24. Ginnett, T.F.; Demment, M.W. Sex differences in giraffe foraging behavior at two spatial scales. Oecologia 1997, 110, 291-300. [CrossRef] 
25. Mayneris-Perxachs, J.; Bolick, D.T.; Leng, J.; Medlock, G.L.; Kolling, G.L.; Papin, J.A.; Swann, J.R.; Guerrant, R.L. Protein-and zinc-deficient diets modulate the murine microbiome and metabolic phenotype. Am. J. Clin. Nutr. 2016, 104, 1253-1262. [CrossRef]

26. Schüßler, D.; Greven, H. Quantitative aspects of the ruminating process in giraffes (Giraffa camelopardalis) fed with different diets. Zoo Biol. 2017, 36, 407-412. [CrossRef] [PubMed]

27. Schnackenberg, L.K.; Sun, J.; Espandiari, P.; Holland, R.D.; Hanig, J.; Beger, R.D. Metabonomics evaluations of age-related changes in the urinary compositions of male Sprague Dawley rats and effects of data normalization methods on statistical and quantitative analysis. In Proceedings of the BMC Bioinformatics, Hong Kong, China, 27-30 August 2007.

28. Slupsky, C.M.; Rankin, K.N.; Wagner, J.; Fu, H.; Chang, D.; Weljie, A.M.; Saude, E.J.; Lix, B.; Adamko, D.J.; Shah, S.; et al. Investigations of the effects of gender, diurnal variation, and age in human urinary metabolomic profiles. Anal. Chem. 2007, 79, 6995-7004. [CrossRef] [PubMed]

29. Theodorou, M.K.; France, J. Rumen microorganisms and their interactions. In Quantitative Aspects of Ruminant Digestion and Metabolism; Dijkstra, J., Forbes, J., France, J., Eds.; CAB International: Wallingford, Oxfordshire, UK, 2009; Volume 2, pp. 207-228.

30. Schmidt, J.M.; Henken, S.; Dowd, S.E.; McLaughlin, R.W. Analysis of the Microbial Diversity in the Fecal Material of Giraffes. Curr. Microbiol. 2018, 75, 323-327. [CrossRef] [PubMed]

31. Soupart, P. Urinary excretion of free amino acids in normal adult men and women. Clin. Chim. Acta 1959, 4, 265-271. [CrossRef]

32. Pechlivanis, A.; Kostidis, S.; Saraslanidis, P.; Petridou, A.; Tsalis, G.; Mougios, V.; Gika, H.G.; Mikros, E.; Theodoridis, G.A. 1H NMR-based metabonomic investigation of the effect of two different exercise sessions on the metabolic fingerprint of human urine. J. Proteome Res. 2010, 9, 6405-6416. [CrossRef] [PubMed]

33. Karen, A.; Szabados, K.; Reiczigel, J.; Beckers, J.F.; Szenci, O. Accuracy of transrectal ultrasonography for determination of pregnancy in sheep: Effect of fasting and handling of the animals. Theriogenology 2004, 61, 1291-1298. [CrossRef]

34. Sun, L.; Guo, Y.; Fan, Y.; Nie, H.; Wang, R.; Wang, F. Metabolic profiling of stages of healthy pregnancy in Hu sheep using nuclear magnetic resonance (NMR). Theriogenology 2017, 92, 121-128. [CrossRef]

35. Kenéz, Á.; Dänicke, S.; Rolle-Kampczyk, U.; von Bergen, M.; Huber, K. A metabolomics approach to characterize phenotypes of metabolic transition from late pregnancy to early lactation in dairy cows. Metabolomics 2016, 12, 165. [CrossRef]

36. Diaz, S.O.; Barros, A.S.; Goodfellow, B.J.; Duarte, I.F.; Carreira, I.M.; Galhano, E.; Pita, C.; Almeida, M.D.C.; Gil, A.M. Following healthy pregnancy by nuclear magnetic resonance (NMR) metabolic profiling of human urine. J. Proteome Res. 2013, 12, 969-979. [CrossRef]

37. Bouckenooghe, T.; Remacle, C.; Reusens, B. Is taurine a functional nutrient? Curr. Opin. Clin. Nutr. Metab. Care 2006, 9, 728-733. [CrossRef]

38. Bristow, A.W.; Whitehead, D.C.; Cockburn, J.E. Nitrogenous constituents in the urine of cattle, sheep and goats. J. Sci. Food Agric. 1992, 59, 387-394. [CrossRef]

39. Dasarathy, J.; Gruca, L.L.; Bennett, C.; Parimi, P.S.; Duenas, C.; Marczewski, S.; Fierro, J.L.; Kalhan, S.C. Methionine metabolism in human pregnancy. Am. J. Clin. Nutr. 2010, 91, 357-365. [CrossRef] [PubMed]

40. Sarosiek, I.; Schicho, R.; Blandon, P.; Bashashati, M. Urinary metabolites as noninvasive biomarkers of gastrointestinal diseases: A clinical review. World J. Gastrointest. Oncol. 2016, 8, 459. [CrossRef] [PubMed]

41. Edwards, S.M.; Cunningham, S.A.; Dunlop, A.L.; Corwin, E.J. The Maternal Gut Microbiome during Pregnancy. MCN Am. J. Matern. Nurs. 2017, 42, 310-316. [CrossRef] [PubMed]

42. Bercovitch, F.B.; Berry, P.S.M. Reproductive life history of Thornicroft's giraffe in Zambia. Afr. J. Ecol. 2010, 48, 535-538. [CrossRef]

43. Bercovitch, F.B.; Berry, P.S.M. Giraffe birth locations in the South Luangwa National Park, Zambia: Site fidelity or microhabitat selection? Afr. J. Ecol. 2015, 53, 206-213. [CrossRef]

44. Lee, D.E.; Bond, M.L.; Bolger, D.T. Season of birth affects juvenile survival of giraffe. Popul. Ecol. 2017, 59, 45-54. [CrossRef]

45. R Development Core Team. R: A Language and Environment for Statistical Computing; Vienna, Austria, 2011; Volume 1. Available online: http://www.r-project.org (accessed on 24 March 2020).

46. Kneen, M.A.; Annegarn, H.J. Algorithm for fitting XRF, SEM and PIXE X-ray spectra backgrounds. Nucl. Instrum. Methods Phys. Res. Sect. B Beam Interact. Mater. Atoms 1996, 109-110, 209-213. [CrossRef] 
47. Liland, K.H.; Almøy, T.; Mevik, B.H. Optimal choice of baseline correction for multivariate calibration of spectra. Appl. Spectrosc. 2010, 64, 1007-1016. [CrossRef]

48. Dieterle, F.; Ross, A.; Schlotterbeck, G.; Senn, H. Probabilistic quotient normalization as robust method to account for dilution of complex biological mixtures. Application in $1 \mathrm{H}$ NMR metabonomics. Anal. Chem. 2006, 78, 4281-4290. [CrossRef]

49. Box, G.E.P.; Cox, D.R. An Analysis of Transformations. J. R. Stat. Soc. Ser. B 2018, 26, 211-243. [CrossRef]

50. Chambers, J.M.; Freeny, A.; Heiberger, R.M. Analysis of variance; designed experiments. In Statistical Models in S; Wadsworth and Brooks/Cole Advanced Books and Software; Springer: Pacific Grove, CA, USA, 1992; pp. 145-193.

51. Bazzano, M.; Laghi, L.; Zhu, C.; Magi, G.E.; Serri, E.; Spaterna, A.; Tesei, B.; Laus, F. Metabolomics of tracheal wash samples and exhaled breath condensates in healthy horses and horses affected by equine asthma. J. Breath Res. 2018, 12, 46015. [CrossRef] [PubMed]

52. Hubert, M.; Rousseeuw, P.J.; Vanden Branden, K. ROBPCA: A new approach to robust principal component analysis. Technometrics 2005, 47, 64-79. [CrossRef]

(C) 2020 by the authors. Licensee MDPI, Basel, Switzerland. This article is an open access article distributed under the terms and conditions of the Creative Commons Attribution (CC BY) license (http://creativecommons.org/licenses/by/4.0/). 



\title{
Characterization of Lipid Profiles after Dietary Intake of Polyunsaturated Fatty Acids Using Integrated Untargeted and Targeted Lipidomics
}

\author{
Satoko Naoe ${ }^{1}$, Hiroshi Tsugawa ${ }^{1,2, *}$, Mikiko Takahashi ${ }^{2}$, Kazutaka Ikeda ${ }^{1,3}$ and \\ Makoto Arita 1,3,4,* \\ 1 Laboratory for Metabolomics, RIKEN Center for Integrative Medical Sciences, Yokohama 230-0045, Japan; \\ satoko.naoe@mochida.co.jp (S.N.); kazutaka.ikeda@riken.jp (K.I.) \\ 2 Metabolome informatics research team, RIKEN Center for Sustainable Resource Science, Yokohama 230-0045, \\ Japan; mikiko.takahashi@riken.jp \\ 3 Cellular and Molecular Epigenetics Laboratory, Graduate School of Medical Life Science, Yokohama City \\ University, Tsurumi, Yokohama 230-0045, Japan \\ 4 Division of Physiological Chemistry and Metabolism, Graduate School of Pharmaceutical Sciences, Keio \\ University, Minato-ku, Tokyo 105-8512, Japan \\ * Correspondence: hiroshi.tsugawa@riken.jp (H.T.); makoto.arita@riken.jp (M.A.); Tel.: +81-45-503-9618 (H.T.); \\ +81-45-503-7055 (M.A.)
}

Received: 28 August 2019; Accepted: 17 October 2019; Published: 21 October 2019

\begin{abstract}
Illuminating the comprehensive lipid profiles after dietary supplementation of polyunsaturated fatty acids (PUFAs) is crucial to revealing the tissue distribution of PUFAs in living organisms, as well as to providing novel insights into lipid metabolism. Here, we performed lipidomic analyses on mouse plasma and nine tissues, including the liver, kidney, brain, white adipose, heart, lung, small intestine, skeletal muscle, and spleen, with the dietary intake conditions of arachidonic acid (ARA), eicosapentaenoic acid (EPA), and docosahexaenoic acid (DHA) as the ethyl ester form. We incorporated targeted and untargeted approaches for profiling oxylipins and complex lipids such as glycerol (phospho) lipids, sphingolipids, and sterols, respectively, which led to the characterization of 1026 lipid molecules from the mouse tissues. The lipidomic analysis indicated that the intake of PUFAs strongly impacted the lipid profiles of metabolic organs such as the liver and kidney, while causing less impact on the brain. Moreover, we revealed a unique lipid modulation in most tissues, where phospholipids containing linoleic acid were significantly decreased in mice on the ARA-supplemented diet, and bis(monoacylglycero)phosphate (BMP) selectively incorporated DHA over ARA and EPA. We comprehensively studied the lipid profiles after dietary intake of PUFAs, which gives insight into lipid metabolism and nutrition research on PUFA supplementation.
\end{abstract}

Keywords: arachidonic acid; omega-3 fatty acids; lipidomics; mass spectrometry; dietary fat; fatty acid metabolism

\section{Introduction}

Polyunsaturated fatty acids (PUFAs) are essential nutrients that have a range of biological effects such as on brain function, cardiovascular disease, obesity, cancer, and bone health in humans [1,2], and also affect the reproduction quality of livestock animals [3]. Among PUFAs, arachidonic acid (ARA), eicosapentaenoic acid (EPA), and docosahexaenoic acid (DHA) are known as long chain PUFAs, and their physicochemical properties and metabolisms provide various functions in mammalian cells. ARA and EPA/DHA are also known as $\omega 6$ and $\omega 3$ polyunsaturated fatty acids, respectively, and their biological functions and the importance of $\omega 3 / \omega 6$ fatty acid balance in maintaining homeostasis have attracted increasing attentions [1,2]. Mammals cannot synthesize ARA, EPA, and DHA from their 
precursors in sufficient quantities, i.e., linoleic acid (LA) and alpha-linoleic acid (ALA), and they must rely on dietary intake. Therefore, the dietary intake of ARA, EPA, and DHA is essential to maintain human health, and it is well known that livestock animals and marine foods are the primary nutrient sources for ARA and EPA/DHA, respectively.

The lipid profiles in tissues are affected by different balances of dietary PUFAs [4-9]. In fact, the intake of $\omega 3$ PUFAs increases tissue levels of $\omega 3$ PUFAs and their oxidized forms, namely oxylipins, while the amount of $\omega 6$ PUFAs and their oxidized forms is decreased [4,5]. Moreover, a previous study revealed that dietary intake of $\omega 3$ PUFA inhibits ARA biosynthesis from LA by the suppression of fatty acid desaturase 2 (FADS2) expression [6], and competes with ARA for membrane phospholipid remodeling. In addition, several $\omega 3$ PUFA intervention studies have shown that the supplementation of $\omega 3$ PUFA increases $\omega 3$-PUFA-derived oxylipins while decreasing ARA-derived oxylipins in human peripheral blood [7]. Although oxylipin profiles under different dietary conditions have been reported $[4,5,8,9]$, there have been few studies that comprehensively investigated the lipid profile after the dietary intake of ARA, EPA, or DHA side-by-side in various tissues, which would be necessary to grasp the effects of different PUFA-containing diets.

After dietary intake, PUFAs are actively incorporated into cells as acyl chains of membrane phospholipids and other lipid classes, such as triacylglycerol and cholesteryl esters. Intake of ARA, EPA, and DHA affects several physiological functions, such as membrane scaffold formation to harmonize biomolecule interactions [10], energy storage and related functions to maintain metabolism [11], and signal transduction via bioactive lipid mediators produced by cyclooxygenases (COX), lipoxygenases (LOX), and cytochrome P450 (CYP) [12]. Since these functions are accomplished by a molecular diversity of lipid species, the comprehensive profiling of lipids in cells and tissues is important to understanding the mechanisms underlying the effect of different PUFA supplementations.

Liquid chromatography coupled with high resolution tandem mass spectrometry (LC-HR-MS/MS) and triple quadruple mass spectrometry (LC-QqQ/MS) are popular techniques for untargeted and targeted lipidomic analysis, respectively $[13,14]$. LC-QqQ/MS-based targeted lipidomics has high sensitivity, and has often been used for the profiling of oxylipins, where the concentrations in plasma range from $10 \mathrm{pM}$ to $100 \mathrm{nM}$ [14]. In contrast, LC-HR-MS/MS, which has high resolution and scanning speed, has often been used for the profiling of glycerol (phospho) lipids, sphingolipids, and sterols, where concentrations in plasma range from $10 \mathrm{nM}$ to $100 \mu \mathrm{M}$ [14]. The current informatics technique for untargeted lipidomics enabled us to characterize more than 90 lipid classes by untangling the MS/MS spectrum [15]. Mass-spectrometry-based lipidomics, therefore, has the potential to comprehensively examine tissue lipid profiles in detail, and this lipidomic profiling could give us new insights into dietary PUFA distribution and metabolism in the body.

In this study, we investigated the lipid profiles in plasma and tissues including the liver, kidney, white adipose, skeletal muscle, heart, small intestine, lung, brain, and spleen after dietary intake of the ethyl ester form of ARA (ARA-E), EPA (EPA-E) or DHA (DHA-E) in mice. In total, 1026 molecular species of lipid were identified, of which 915 and 111 were annotated in untargeted and targeted analyses, respectively.

\section{Materials and Methods}

\subsection{Standard Chemicals}

ARA-d8, 15-HETE-d8, $\mathrm{LTB}_{4}$-d4 and PGE 2 -d4 were obtained from Cayman Chemical (Ann Arbor, MI, USA). Highly purified EPA ethyl ester (EPA-E) (> 98\%), DHA ethyl ester (DHA-E) (> 97\%), and ethyl arachidonate (ARA-E) (> 99\%) were obtained from Nippon Suisan Kaisha, Ltd. (Tokyo, Japan), Harima foods, Inc. (Osaka, Japan) and NuChek Prep, Inc. (Elysian, MN, USA), respectively. All solvents of LC/MS grade were obtained from Wako (Tokyo, Japan). 


\subsection{Animals}

Male C57BL/6J mice (Japan SLC, Inc., Shizuoka, Japan) were purchased at 10 weeks of age, and housed under controlled temperature and lighting (12 h light/dark cycle) with free access to water and a controlled diet (fish-meal-free F1, Funabashi Farm, Chiba, Japan). The fatty acid composition and other nutritional factors for fish-meal-free F1 are described in Supplementary Table S1. In this study, the fish-meal-free F1 was used as an ARA-, EPA-, and DHA-free diet, and each PUFA was supplemented into the feed in its ethyl ester form. After 1 week of acclimation, mice were assigned to four groups $(n=5)$ and fed control diet (control group), control diet supplemented with $1 \%$ EPA-E $(w / w)$ (EPA-fed group), 1\% DHA-E (w/w) (DHA-fed group), or 1\% ARA-E (w/w) (ARA-fed group) for 2 weeks. Animals were dissected under isoflurane anesthesia, after which organs, such as the liver, kidney, white adipose, skeletal muscle, heart, small intestine, lung, brain, and spleen, were collected and plasma was isolated. For the lipidome analyses, four biological replicates $(n=4)$ were used for liver tissue, and five biological replicates $(n=5)$ were used for the other tissues and plasma. Two of the white adipose samples from the EPA-fed group and one of the skeletal muscle samples from the ARA-fed group in were excluded from the targeted lipidomics results owing to LC-MS analysis failure. All animal experiments were carried out in accordance with the guidelines for the use and care of laboratory animals of Mochida Pharmaceutical, and were approved by the Institutional Animal Care and Use Committee (identification code: PMS14-041).

\subsection{Determination of Plasma Triglyceride, Cholesterol, and Fatty Acid Composition}

Standard enzymatic methods were used to determine plasma total cholesterol (TC) and triglyceride (TAG) with commercially available kits purchased from Wako Pure Chemical Industries Ltd. Total fatty acids in plasma were analyzed by gas chromatography (Japan SLC, Inc., Shizuoka, Japan). C16:0, C16:1, C18:0, C18:1, C18:2, C20:3, C20:4, C20:5, C22:5, and C22:6 levels were determined using the standard substances: palmitic acid, palmitoleic acid, stearic acid, oleic acid, linoleic acid, dihomo- $\gamma$-linoleic acid, arachidonic acid, eicosapentaenoic acid, docosapentaenoic acid, and docosahexaenoic acid, respectively.

\subsection{Untargeted LC-MS/MS-Based Lipidomics}

Total lipids from organs were extracted as previously described [16] using internal standards: 1 $\mu$ M TAG (8:0/8:0/8:0)- ${ }^{13}$ C3 (Larodan, Inc., Monroe, MI, USA), $0.5 \mu$ M phosphatidyl glycerol (17:0/14:1) (Avanti Polar Lipids, Inc., Alabaster, AL, USA), $0.5 \mu \mathrm{M}$ lyso phosphatidylcholine (17:1) (Avanti Polar Lipids, Inc.), $5 \mu \mathrm{M}$ acylcarnitine (18:0)-d3 (Larodan, Inc., Monroe, MI, USA), $25 \mu \mathrm{M}$ palmitic acid-d3 (Olbracht Serdary Research Laboratories, Toronto, ON, Canada), $25 \mu \mathrm{M}$ stearic acid-d3 (Olbracht Serdary Research Laboratories, Toronto, ON, Canada), $5 \mu \mathrm{M}$ cholic acid-d4 (Cambridge Isotope Laboratories, Inc., Tewksbury, MA, USA), and 0.5 $\mu \mathrm{M}$ Cer/Sph mixture I (Avanti Polar Lipids, Inc., Alabaster, AL, USA) (Supplementary Table S2). Untargeted analysis was performed using an ACQUITY UPLC system (Waters, Milford, MA, USA) coupled with a quadruple time-of-flight/MS (TripleTOF 5600+, SCIEX, Framingham, MA, USA). LC separation was performed using a reverse-phase column (Acquity UPLC BEH peptide C18; $2.1 \times 50 \mathrm{~mm}, 1.7 \mu \mathrm{m}$ particle size; Waters, Milford, MA, USA) with a gradient elution of mobile phase $\mathrm{A}$ (methanol: acetonitrile: water $=1: 1: 3, v / v / v$ for volume ratio containing $5 \mathrm{mM}$ ammonium acetate and $10 \mathrm{nM}$ EDTA) and mobile phase $\mathrm{B}$ (100\% isopropanol containing $5 \mathrm{mM}$ ammonium acetate and $10 \mathrm{nM}$ EDTA), and the composition was produced by mixing those solvents. LC gradient consisted of holding solvent (A/B: 100/0) for $1 \mathrm{~min}$, then linearly converting to solvent (A/B: 60/40) for $4 \mathrm{~min}$, linearly converting to solvent (A/B:36/64) for $2.5 \mathrm{~min}$ and holding for $4.5 \mathrm{~min}$, then linearly converting to solvent (A/B: 17.5/82.5) for $0.5 \mathrm{~min}$, linearly converting to solvent (A/B: 15/85) for $8.5 \mathrm{~min}$, and linearly converting to solvent (A/B: 5/95) for $1 \mathrm{~min}$ followed by returning to solvent (A/B: 100/0) and holding for $5 \mathrm{~min}$ for re-equilibration. The flow rate and column temperature were set to $0.3 \mathrm{~mL} / \mathrm{min}$ and $45^{\circ} \mathrm{C}$, respectively. Data dependent MS/MS acquisition mode was applied as previously described [16]. Briefly, the temperature and ion spray voltage floating were set to $300{ }^{\circ} \mathrm{C}$ 
and $-5.5 \mathrm{kV}$, respectively. The accumulation times for MS1 and MS/MS were set to $100 \mathrm{~ms}$ and $50 \mathrm{~ms}$, respectively, for scanning a mass range from $\mathrm{m} / \mathrm{z} 75$ to $\mathrm{m} / \mathrm{z} 1250$. The collision energy (CE) was set to $35 \mathrm{eV}$ with a $\mathrm{CE}$ spread of $15 \mathrm{eV}$ in high-resolution mode, and other settings of DDA mode were as follows: 10 most intense ions, 100 cps intensity threshold, and $100 \mathrm{sec}$ exclusion time.

\subsection{Targeted LC-MS/MS-Based Lipidomics}

The LC-MS/MS analysis was performed as described previously [12]. Samples were extracted by solid-phase extraction using Sep-Pak Vac 3cc C18 cartridges (Waters) with deuterium-labeled internal standards at a final concentration of $10 \mathrm{pg} / \mu \mathrm{L}$ ARA-d8, $10 \mathrm{pg} / \mu \mathrm{L}$ 15-HETE-d8, $10 \mathrm{pg} / \mu \mathrm{L}$ $\mathrm{LTB}_{4}-\mathrm{d} 4,10 \mathrm{pg} / \mu \mathrm{L} \mathrm{PGE}_{2}-\mathrm{d} 4$ per samples. The targeted analysis was performed using a UPLC system (Waters UPLC, Waters, Milford, MA, USA) with a triple quadruple linear ion trap mass spectrometer (QTRAP 5500; SCIEX, Framingham, MA, USA), equipped with Acquity UPLC BEH C18 column (1.0 $\times 150 \mathrm{~mm}, 1.7 \mu \mathrm{m}$ particle size; Waters, Milford, MA, USA). Samples were eluted with a mobile phase composed of water/acetate $(100: 0.1, v / v)$ and acetonitrile/methanol $(4: 1, v / v)(73: 27)$ for $5 \mathrm{~min}$, and ramped to 30:70 over $15 \mathrm{~min}$, to 20:80 over $25 \mathrm{~min}$ and held for $8 \mathrm{~min}$, ramped to 0:100 over $35 \mathrm{~min}$, and held for $10 \mathrm{~min}$ with flow rates of $70 \mu \mathrm{L} / \mathrm{min}(0-30 \mathrm{~min}), 80 \mu \mathrm{L} / \mathrm{min}$ (30-33 $\mathrm{min})$, and $100 \mu \mathrm{L} / \mathrm{min}$ (33-45 min). The MS/MS analyses were performed in negative ion mode, and oxylipins were identified and quantified by multiple reaction monitoring (Supplementary Table S3). Compounds were quantified by using stable internal standards. The calibration curves of all compounds were acquired in triplicate (Supplementary Figure S1). The extraction and matrix recovery were calculated with internal standards (IS) and the compound quantification was conducted with the calibration curves. The lipid compounds of (group 1) free fatty acids, (group 2) monohydrides and epoxides, (group 3) diols, leukotrienes, thromboxanes, lipoxines, and resolvins, and (group 4) prostaglandins and others were corrected with ARA-d8, 15-HETE-d8, $\mathrm{LTB}_{4}-\mathrm{d} 4$, and $\mathrm{PGE}_{2}-\mathrm{d} 4$, respectively. The compound concentrations were calculated as:

$$
\text { Compound concentration }=\frac{(\text { target compound peak area }) /(\text { slope of calibration curve })}{(\text { IS peak area }) /(\text { IS slope of calibration curve })}
$$

\subsection{Data Analysis}

The targeted analysis data was analyzed using MultiQuant software (SCIEX, Framingham, MA, USA). The chromatogram peaks were manually curated. The untargeted analysis data was analyzed using MS-DIAL software $[17,18]$ version 2.90 (http://prime.psc.riken.jp/) with the following parameters: retention time start: 0 min; retention time end: 100 min; mass range start: 0 Da; mass range end: $5000 \mathrm{Da}$; accurate mass tolerance (MS1) tolerance: 0.01 Da; MS2 tolerance: $0.025 \mathrm{Da}$; maximum charge number: two; smoothing method: linear weighted moving average; smoothing level: 3; minimum peak width: five scans; minimum peak height: 1000; mass slice width: $0.1 \mathrm{Da}$; sigma window value: 0.5 ; MS2Dec amplitude cut off: 0; exclude after precursor: true; keep isotope until: $0.5 \mathrm{Da}$; keep original precursor isotopes: false; exclude after precursor: true; retention time tolerance for identification: $10 \mathrm{~min}$; MS1 for identification: $0.01 \mathrm{Da}$; accurate mass tolerance (MS2) for identification: $0.05 \mathrm{Da}$; identification score cut off: $80 \%$; using retention time for scoring: true; relative abundance cut off: 0 ; top candidate report: true; retention time tolerance for alignment: $0.1 \mathrm{~min}$; MS1 tolerance for alignment: 0.015 Da; peak count filter: 0; adduct ion setting: $[\mathrm{M}+\mathrm{H}]^{+},\left[\mathrm{M}+\mathrm{NH}_{4}\right]^{+}$, $[\mathrm{M}+\mathrm{Na}]^{+},\left[\mathrm{M}+\mathrm{C}_{2} \mathrm{H}_{3} \mathrm{~N}+\mathrm{H}\right]^{+},\left[\mathrm{M}+\mathrm{H}-\mathrm{H}_{2} \mathrm{O}\right]^{+},\left[\mathrm{M}+\mathrm{C}_{3} \mathrm{H}_{8} \mathrm{O}+\mathrm{H}\right]^{+},\left[\mathrm{M}+\mathrm{C}_{2} \mathrm{H}_{3} \mathrm{~N}+\mathrm{Na}\right]^{+},\left[\mathrm{M}-\mathrm{C}_{6} \mathrm{H}_{10} \mathrm{O}{ }_{5}+\mathrm{H}\right]^{+}$, $[2 \mathrm{M}+\mathrm{H}]^{+},\left[2 \mathrm{M}+\mathrm{NH}_{4}\right]^{+},[2 \mathrm{M}+\mathrm{Na}]^{+}$in positive ion mode and $[\mathrm{M}-\mathrm{H}]^{-},\left[\mathrm{M}-\mathrm{H}_{2} \mathrm{O}-\mathrm{H}\right]^{-},\left[\mathrm{M}+\mathrm{CH}_{3} \mathrm{COO}\right]^{-}$, $\left[\mathrm{M}-\mathrm{C}_{6} \mathrm{H}_{10} \mathrm{O}_{5}-\mathrm{H}\right]^{-},[2 \mathrm{M}-\mathrm{H}]^{-}$, and $\left[2 \mathrm{M}+\mathrm{CH}_{3} \mathrm{COO}\right]^{-}$in negative ion mode. All lipid classes supported in the MS-DIAL version were used for the targeted lipids. The annotation was performed using the in silico spectral library of lipids, and the false positive annotations were manually curated by mass spectrometry experts. The principal component analysis and circus plots were executed in the R language with the packages of "prcomp" and "OmicCircos". Our untargeted lipidomic 
data were discussed in terms of relative quantification, defined by lipidomic standard initiative (https://lipidomics-standards-initiative.org/), among dietary conditions in each tissue. All of the mass spectrometry data are available at RIKEN DROP Met (http://prime.psc.riken.jp/menta.cgi/prime/drop_ index, $\mathrm{ID}=\mathrm{DM} 0030$ ).

\section{Results}

\subsection{Lipid Profiles after Dietary Intake of ARA, EPA, or DHA}

We first examined the profiles of total cholesterol, triacylglycerol (TAG), and total fatty acids in mouse plasma. Dietary intake of 1\% (w/w) ARA-EE, EPA-EE, or DHA-EE for 2 weeks significantly decreased plasma cholesterol and decreased triacylglycerol levels as compared to the levels of the control group (Figure 1a,b). There were no body weight loss, suggesting that PUFA supplementation did not affect the amount of dietary consumption (Figure 1c). Total fatty acid levels, including C16:0 (palmitic acid), C18:1, and C18:2, were decreased after dietary intake of PUFAs, while C20:4 (ARA), C20:5 (EPA), and C22:6 (DHA) were increased in the respective dietary conditions (Figure 1d). Here, abbreviations of C18:1 and C18:2 were used because of the unclear chromatogram separation from several isomers including vaccenic acid (18:1 n-7), oleic acid (18:1 n-6), linoleic acid (n-6), and conjugated linoleic acids. In this study, we fed PUFAs in synthetic ethyl ester form (PUFA-EE) because this method of producing ethyl esters can achieve a high degree of fatty acid purity. However, the bioavailability of the ethyl ester forms has been reported to be lower than the TAG form, which is the major form of PUFAs in natural fish oil $[19,20]$. Dietary PUFA-EE and TAG are both hydrolyzed to free fatty acid by pancreatic lipase and passively transported into enterocytes. In the enterocytes, free fatty acids are re-esterified to TAG and then transferred into lymph and blood circulation. Among those processes, the digestion of the ester bond and re-esterification may cause the difference of bioavailability [21]. However, the plasma PUFA levels were increased as expected after dietary intake of PUFA-EEs (Figure 1d), supporting the validity of this study design.
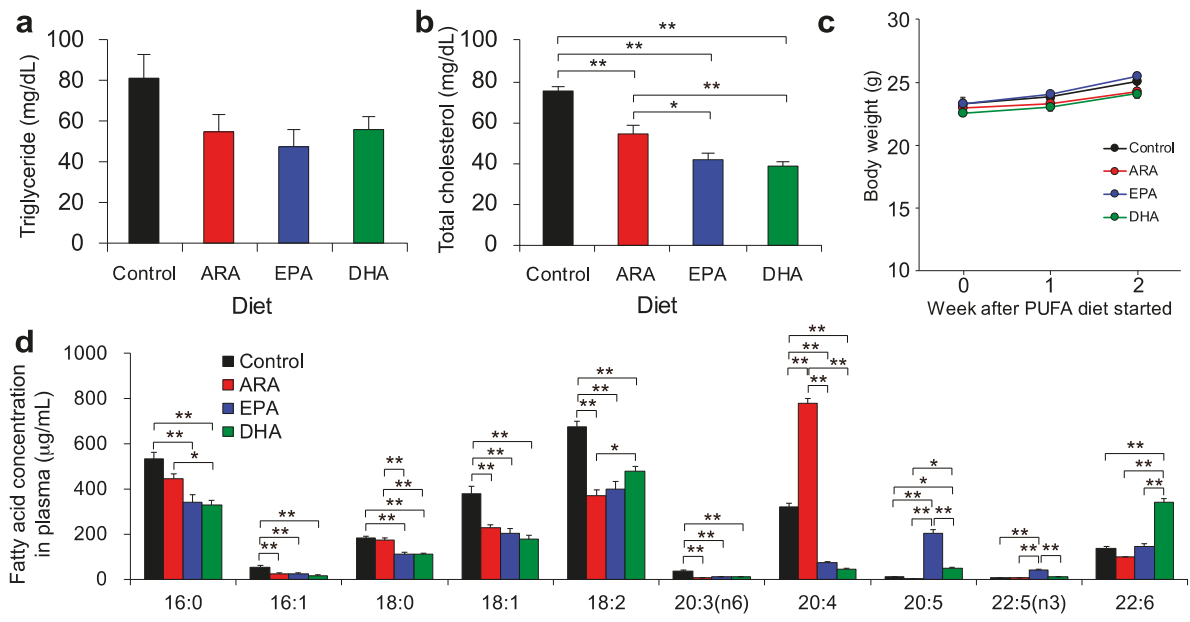

Figure 1. Effects on mouse plasma and body tissues following dietary supplementation with polyunsaturated fatty acids (PUFAs). (a) The total triglyceride and (b) total cholesterol in plasma were analyzed after two weeks of each PUFA diet. (c) The body weight in each PUFA diet condition was traced for two weeks, where the intake was started at 11 weeks of age. (d) The concentrations of total fatty acids in plasma were analyzed. The abbreviations ARA, EPA, and DHA, refer to arachidonic acid, eicosapentaenoic acid, and docosahexaenoic acid, respectively. The statistical significance was evaluated by Tukey's testing $\left({ }^{*} P<0.05\right.$ and $\left.{ }^{* *} P<0.01\right)$ where one-way ANOVA (analysis of variance) showed the statistical significance among groups $(P<0.05)$. 
Next, we performed untargeted and targeted lipidomic analysis on the plasma and the nine tissues. A total of 915 molecules from 33 lipid classes were characterized in the untargeted analysis, while 111 oxylipins derived from LA, ALA, ARA, EPA, and DHA were identified in the targeted analysis (Table 1) The score plots of the principal component analysis (PCA) from untargeted lipidomics were clearly clustered by dietary-fed group in the metabolic organs, such as the liver and kidney, and the factor loadings showed that the profiles of glycerolipids and glycerophospholipids contributed to the principal components (Figure 2a and Supplementary Figure S2). On the other hand, the lipidome in the brain and muscle tissues, including the heart and skeletal muscle, was not significantly changed between different dietary conditions. Interestingly, the score plots of DHA dietary intake were classified independently from other conditions in the skeletal muscle, adipose, and heart. According to the factor loadings, the profile of DHA-containing lipids substantially contributed to these score plot dimensions. In fact, the muscle tissues were enriched in DHA present in membrane phospholipids [22], and our results suggested that the efficiency of DHA incorporation into membrane phospholipids was higher in muscle tissues compared to other tissues, and that the PC, PE, and PI lipid classes highly incorporated DHA as an acyl chain component in both the skeletal muscle and heart. In contrast, the membrane phospholipids incorporating DHA and the score plots of dietary DHA intake were not significantly changed in the brain.

a
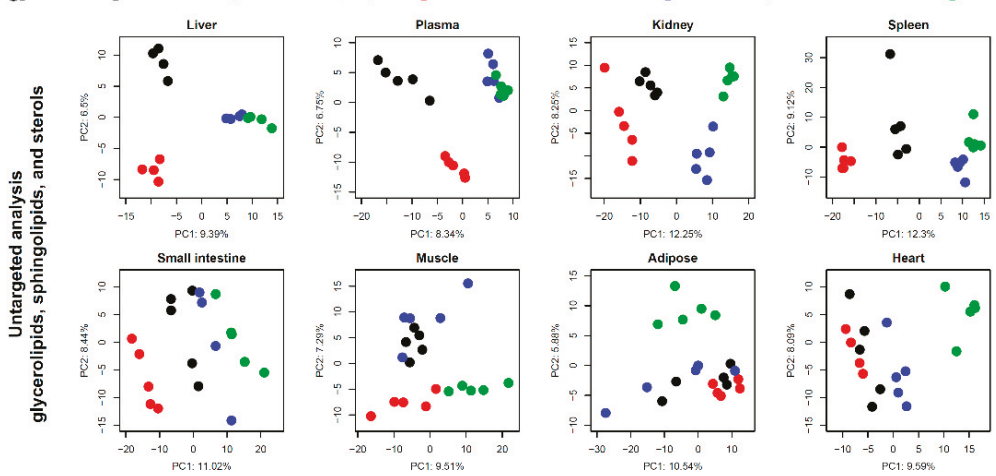

$1 \%$ Docosahexaenoic acid $+F 1$
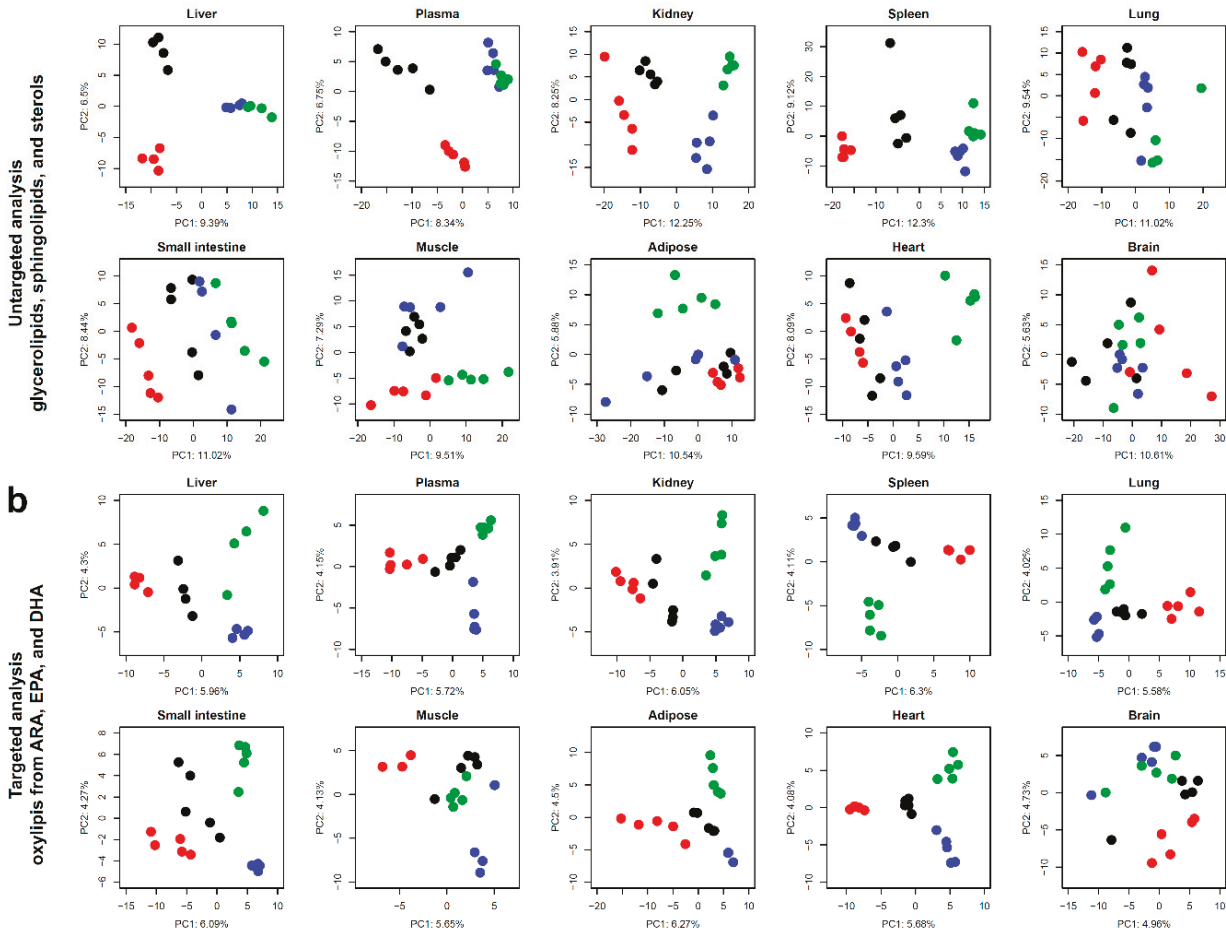

Plasm
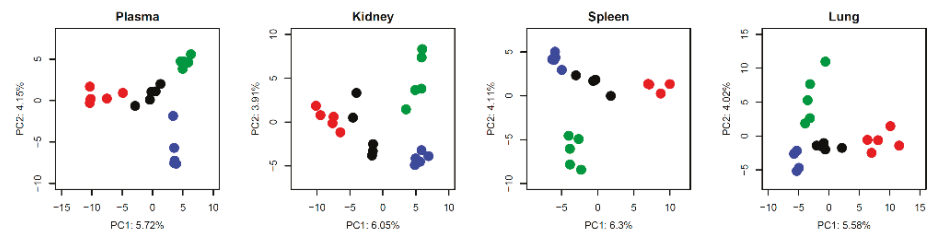

Muscle
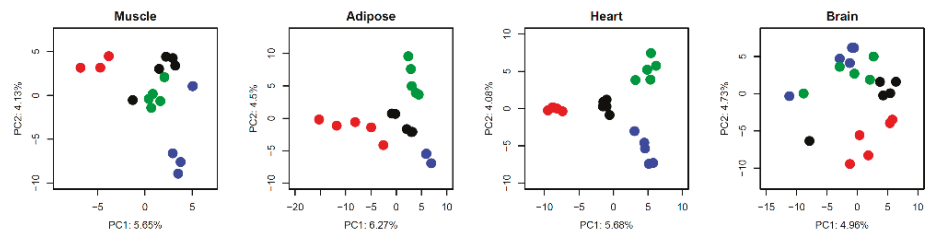

Figure 2. Principal component analyses (PCA) in untargeted and targeted lipidomics data. (a) The first and second principal components of PCA were described for plasma and all tissue metabolites obtained in the untargeted analysis. (b) The first and second components were also shown by the targeted lipidomics data. F1: fish-meal-free diet not containing ARA, EPA, or DHA. 
Table 1. Summary of characterized lipids in untargeted and targeted lipidomics.

\begin{tabular}{|c|c|c|c|c|c|c|c|c|c|c|c|}
\hline Method & Class & Liver & Plasma & Kidney & Spleen & Lung & Small Intestine & Muscle & Adipose & Heart & Brain \\
\hline Untargeted & FA & 12 & 16 & 18 & 17 & 15 & 22 & 15 & 14 & 13 & 14 \\
\hline Untargeted & ACar & 2 & 2 & 9 & 7 & 10 & 7 & 14 & 0 & 12 & 6 \\
\hline Untargeted & MAG & 1 & 2 & 2 & 2 & 3 & 4 & 2 & 1 & 0 & 3 \\
\hline Untargeted & DAG & 14 & 8 & 14 & 15 & 18 & 29 & 13 & 28 & 6 & 5 \\
\hline Untargeted & TAG & 70 & 80 & 73 & 88 & 116 & 96 & 80 & 156 & 116 & 6 \\
\hline Untargeted & $\mathrm{CE}$ & 7 & 10 & 4 & 6 & 2 & 5 & 3 & 0 & 3 & 1 \\
\hline Untargeted & LPC & 7 & 13 & 8 & 7 & 8 & 7 & 7 & 1 & 7 & 6 \\
\hline Untargeted & LPE & 4 & 6 & 10 & 9 & 8 & 8 & 6 & 0 & 4 & 7 \\
\hline Untargeted & LPG & 0 & 0 & 0 & 1 & 1 & 0 & 0 & 0 & 0 & 0 \\
\hline Untargeted & LPS & 0 & 0 & 1 & 0 & 0 & 1 & 0 & 0 & 0 & 0 \\
\hline Untargeted & LPI & 2 & 0 & 3 & 1 & 0 & 2 & 0 & 0 & 1 & 4 \\
\hline Untargeted & PC & 33 & 30 & 25 & 43 & 45 & 38 & 47 & 6 & 33 & 22 \\
\hline Untargeted & EtherPC & 0 & 1 & 18 & 17 & 9 & 3 & 4 & 0 & 7 & 2 \\
\hline Untargeted & $\mathrm{PE}$ & 27 & 9 & 34 & 29 & 32 & 28 & 31 & 4 & 28 & 25 \\
\hline Untargeted & EtherPE & 8 & 3 & 24 & 40 & 35 & 26 & 16 & 2 & 21 & 35 \\
\hline Untargeted & PG & 5 & 1 & 13 & 23 & 22 & 9 & 7 & 1 & 8 & 11 \\
\hline Untargeted & BMP & 1 & 0 & 3 & 6 & 7 & 7 & 0 & 0 & 1 & 0 \\
\hline Untargeted & PS & 2 & 0 & 6 & 20 & 11 & 2 & 1 & 0 & 1 & 10 \\
\hline Untargeted & PI & 15 & 14 & 18 & 25 & 18 & 16 & 15 & 1 & 12 & 10 \\
\hline Untargeted & OxPC & 2 & 0 & 9 & 0 & 0 & 5 & 5 & 0 & 8 & 0 \\
\hline Untargeted & OxPE & 13 & 0 & 29 & 11 & 2 & 16 & 10 & 0 & 18 & 2 \\
\hline Untargeted & OxPG & 0 & 0 & 0 & 0 & 0 & 0 & 0 & 0 & 0 & 0 \\
\hline Untargeted & OxPI & 2 & 0 & 5 & 0 & 0 & 2 & 1 & 0 & 0 & 0 \\
\hline Untargeted & OxPS & 0 & 0 & 7 & 2 & 2 & 1 & 0 & 0 & 1 & 0 \\
\hline Untargeted & Cer-NS & 10 & 6 & 19 & 16 & 24 & 12 & 12 & 6 & 13 & 12 \\
\hline Untargeted & Cer-NDS & 0 & 0 & 1 & 2 & 1 & 4 & 1 & 0 & 0 & 1 \\
\hline Untargeted & Cer-NP & 0 & 0 & 4 & 0 & 4 & 8 & 0 & 0 & 0 & 1 \\
\hline Untargeted & Cer-AS & 0 & 0 & 2 & 0 & 6 & 0 & 2 & 0 & 0 & 5 \\
\hline Untargeted & Cer-AP & 0 & 0 & 0 & 0 & 1 & 5 & 0 & 0 & 0 & 0 \\
\hline Untargeted & HexCer-NS & 4 & 4 & 8 & 11 & 12 & 5 & 4 & 2 & 2 & 13 \\
\hline Untargeted & HexCer-AP & 0 & 0 & 4 & 0 & 0 & 15 & 0 & 0 & 0 & 0 \\
\hline Untargeted & SHexCer & 0 & 0 & 5 & 0 & 2 & 0 & 2 & 0 & 0 & 14 \\
\hline Untargeted & $\mathrm{SM}$ & 6 & 6 & 13 & 13 & 18 & 11 & 6 & 2 & 9 & 5 \\
\hline Targeted & FFA & 17 & 17 & 17 & 16 & 17 & 17 & 17 & 17 & 17 & 16 \\
\hline Targeted & LA-O & 7 & 7 & 5 & 6 & 6 & 7 & 7 & 7 & 7 & 7 \\
\hline Targeted & ALA-O & 3 & 3 & 3 & 3 & 3 & 3 & 3 & 3 & 2 & 2 \\
\hline Targeted & GLA-O & 1 & 0 & 0 & 0 & 0 & 1 & 1 & 0 & 0 & 0 \\
\hline Targeted & DGLA-O & 1 & 2 & 1 & 2 & 2 & 4 & 4 & 3 & 1 & 1 \\
\hline Targeted & MA-O & 1 & 2 & 1 & 2 & 0 & 2 & 2 & 1 & 1 & 1 \\
\hline Targeted & ARA-O & 26 & 23 & 28 & 27 & 30 & 29 & 27 & 30 & 28 & 27 \\
\hline Targeted & EPA-O & 16 & 13 & 13 & 15 & 14 & 19 & 13 & 19 & 14 & 13 \\
\hline Targeted & DHA-O & 15 & 17 & 15 & 16 & 16 & 17 & 18 & 16 & 15 & 15 \\
\hline
\end{tabular}

The nomenclature of lipid classes in untargeted analysis is described in RIKEN PRIMe website (http://prime.psc. riken.jp/). FFA: free fatty acid; LA-O, ALA-O, GLA-O, DGLA-O, MA-O, ARA-O, EPA-O, and DHA-O: oxylipins derived from linoleic acid, alpha-linolenic acid, gamma-linolenic acid, dihomo-gamma-linolenic acid, mead acid, arachidonic acid, eicosapentaenoic acid, and docosahexaenoic acid, respectively.

\subsection{Oxylipin Profiles in Targeted Lipidomics}

According to the PCA score plots of the targeted lipidomic analysis, the oxylipins and their related molecules of plasma and tissues, except for the brain, were significantly affected by the dietary intake of PUFAs (Figure 2b and Supplementary Figure S2). Further detail of the oxylipin profiles is given in Figure 3. In most organs, except for the small intestine, lung, and spleen, the concentrations of 11,12-epoxyeicosatrienoic acid (EET) and 14,15-EET were higher than those of other oxylipins in ARA-derived metabolites, including epoxides, hydroxides, prostaglandins (PGs), and others. In contrast, COX-derived PGs such as $\mathrm{PGE}_{2}, \mathrm{PGD}_{2}$, and 6-keto- $\mathrm{PGF}_{1 \alpha}$ were enriched in the small intestine, lung, and spleen, and these tissues contained higher amounts of hydroxyeicosatetraenoic acids (HETEs). 


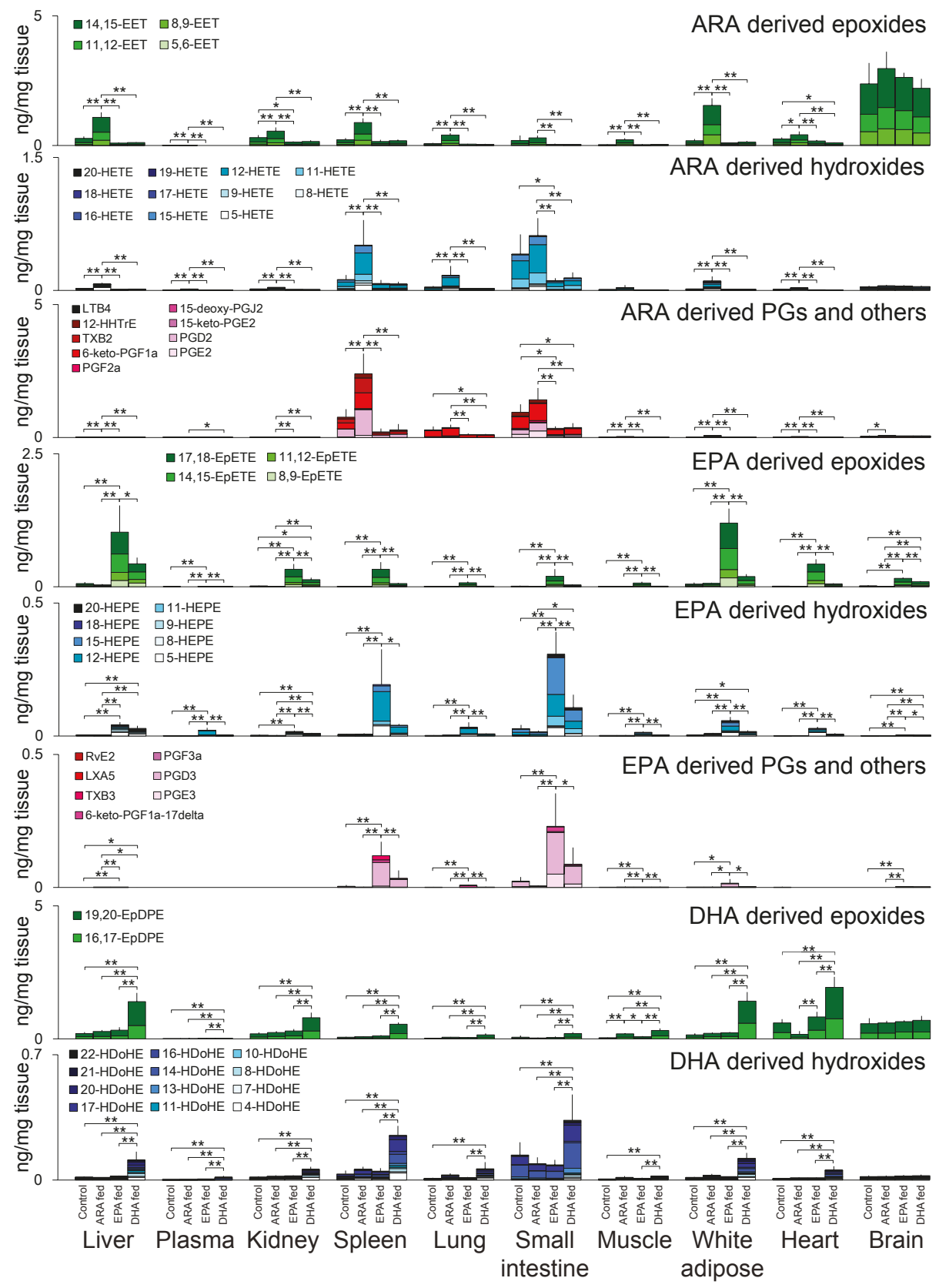

Figure 3. Oxylipin profiles in mouse tissues under each dietary condition. The total amounts of monoepoxides, hydroxides, and cyclized oxidized fatty acids including prostaglandins derived from ARA, EPA, and DHA are described. The results are given for each tissue in the four groups, i.e., the control, ARA-fed, EPE-fed, and DHA-fed groups. The ratio of oxidized fatty acids in each metabolite group is described by the stacked bar chart. The error bar indicates the standard deviation from the total amount values, and the statistical significance was evaluated by Tukey's testing $\left({ }^{*} P<0.05\right.$ and $* *$ $P<0.01)$ where one-way ANOVA showed the statistical significance among groups $(P<0.05)$. For plasma, the $\mathrm{y}$-axis unit becomes $\mathrm{ng} / \mu \mathrm{L}$ plasma instead of $\mathrm{ng} / \mathrm{mg}$ tissue. 
The levels of EPA and EPA-derived oxylipins were in fact lower than those of ARA and DHA in plasma and all tissues (Figure 1d and Supplementary Figure S3), while their amounts were substantially increased by dietary EPA intake as indicated by EPA-derived metabolites (Figure 3). Among the EPA-derived oxylipins, epoxyeicosatetraenoic acids (EpETEs) were the primary oxylipins present in the liver, kidney, white adipose tissue, skeletal muscle, heart, lung, and brain, while PGs and hydroxyeicosapentaenoic acids (HEPEs) were enriched in the small intestine and spleen. A similar pattern was observed in dietary DHA intake; epoxydocosapentaenoic acid (EpDPE) and hydroxydocosahexaenoic acid (HDoHE) were characteristically present in tissues.

The ARA-, EPA-, and DHA-derived epoxides, which are known as CYP metabolites, were enriched in metabolic tissues such as the liver, kidney, and white adipose tissue. ARA-derived epoxides activate various signaling pathways, and elicit functional responses such as vasorelaxation and anti-inflammation [23]. EPA-derived epoxides have anti-allergic and anti-inflammatory properties: 17,18-EpETE inhibits mast cell degranulation [24,25] and 12-hydroxy-17,18-EpETE suppresses neutrophil infiltration and eosinophilic inflammation $[26,27]$. These epoxides are converted into diols by soluble epoxide hydrolase, and the levels of diols and the diols/epoxide ratio were high in the small intestine (Supplementary Figure S4).

The $\omega 3$ oxylipins of EPA were increased following the dietary intake of DHA, while the amounts of ARA-derived oxylipins were decreased by $\omega 3$ PUFA dietary intake. Similar observations have also been reported in several other studies $[5,7,28]$. Interestingly, the increase in brain EPA levels following DHA intake was relatively high, and this result may represent the retro-conversion from DHA to EPA [29]. In contrast, ARA metabolism was competitively suppressed by a rich intake of EPA and DHA.

\subsection{Lipidomic Signatures in Untargeted Analyses}

We used circus plots to display the result of untargeted lipidomics (Figure 4) for the liver and brain, the metabolites of which were the most and least affected by the dietary intakes of PUFAs, respectively (Figure 2a). The heatmap layer and correlation linkages in the circus plot clearly showed that the PUFAs in liver were incorporated into glycerolipids, glycerophospholipids, and cholesterol esters, while the profile of sphingolipids was not affected by any dietary supplement. Moreover, the heatmap layer and the detail of fatty acid compositions indicating the existence of 18:2, 20:4/22:4, 20:5/22:5, and 22:6 as the acyl chain composition in a certain lipid class showed that glycerol (phospho) lipids containing 22:4 and 22:5 were increased in most tissues after the dietary intake of ARA and EPA, respectively. This result indicated that ARA and EPA were elongated to docosatetraenoic acid (DTA, 22:4) and docosapentaenoic acid (DPA, 22:5) by elongases such as elongation of very long chain fatty acids protein (ELOVL) 2 and 5 [30]. In contrast, an increase in lipids containing the elongated product of DHA, i.e., 24:6, was not observed following DHA dietary supplementation in the tissues examined.

A few differences in lipid profiles were observed in the mouse brain: free fatty acids (FA) of ARA, EPA, and DHA were affected by dietary intake (Figure 4). Moreover, several phospholipids, including phosphatidylcholine (PC), phosphatidylethanolamine (PE), phosphatidylserine (PS), and ether linked PE (EtherPE), partially reflected the changes in dietary conditions according to the heatmap layer. Interestingly, the amounts of most lipid classes, including sphingolipids, were increased in the ARA-supplemented mice. Moreover, untargeted lipidomics identified the mono-galactocyl/glycosyl-diacylglycerol (MGDG) lipids, the main component of plant membrane lipids, in the mouse brain, where the MGDG lipids were composed of saturated or monounsaturated fatty acids. A few studies have also shown the existence of MGDG in the brain, and its biosynthesis and physiological role have been examined in several in vitro studies, although their mechanisms are still unclear [31-33]. These results suggest that the annotation of untargeted lipidomics should be performed first in an unbiased manner. 

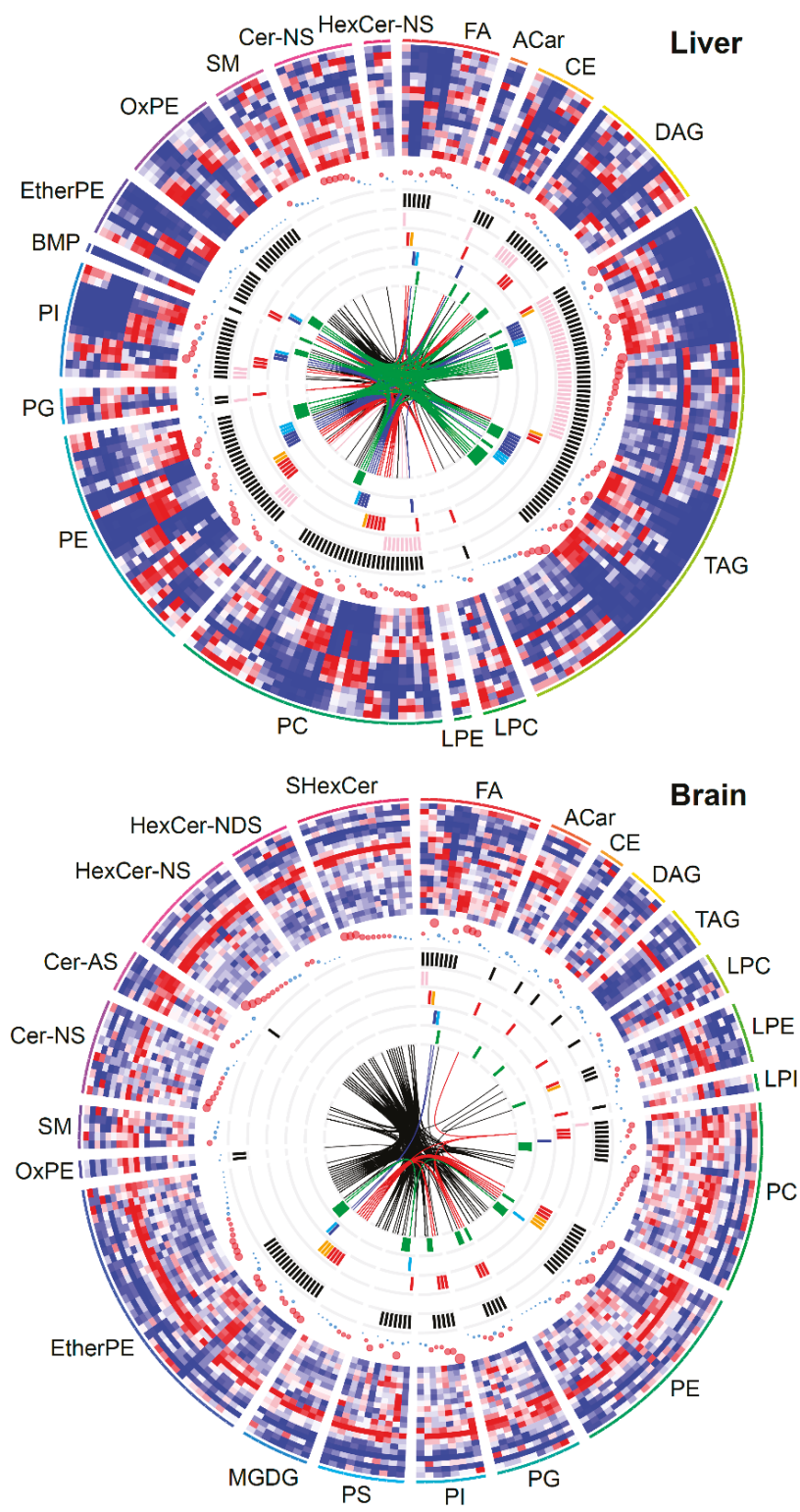

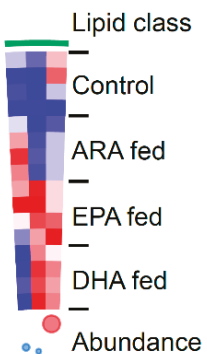

III PUFA contained

18:2 contained

|| 20:4/22:4 contained

| 20:5/22:5 contained

22:6 contained

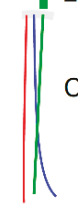

Figure 4. Lipid profiles in the liver and brain from the untargeted lipidomics data. In the circus plot, the lipid profiles are described separately by lipid class. The profile has been scaled in each metabolite from -1 to 1 in the heatmap. The ion abundance of each lipid is described by the circle size, where the average value in all conditions was utilized for the ion abundance size. Any lipid with more than two double bonds in an acyl chain was defined as a PUFA-containing metabolite. Lipids containing 18:2, 20:4, 22:4, 20:5, 22:5, and 22:6 are marked by pink, red, orange, blue, sky blue, and green colors, respectively. Metabolites were linked if the ion abundance correlation of two metabolites in biological samples was greater than 0.9 . 
Untargeted lipidomics also revealed unique lipid features in the other tissues. The diacylglycerol (DAG) and TAG lipids were the most abundant species in the white adipose tissue, as has previously been described [34], and the DAG and TAG profiles were significantly affected in most tissues. Interestingly, however, we found that the profiles of DAG and TAG were not affected in the spleen, although the profiles of free fatty acids, glycerophospholipids, and cholesteryl esters did reflect the intakes of ARA, EPA, and DHA (Supplementary Figure S5). This result suggested that the metabolic pathway has a limited ability to incorporate dietary PUFAs into TAG in the spleen. Indeed, diglyceride acyltransferase (DGAT) activity has been reported to be low in the spleen [35]. Our results also showed that the ether-linked PC and PE (EtherPC and EtherPE) incorporating $O$-alkenyl or $O$-alkyl chains were enriched in the kidney, spleen, small intestine, muscle, heart, and brain (Table 1), while the acyl chain profiles of EtherPC and EtherPE reflected the dietary PUFA in all tissues and plasma. In contrast, the low variety of $O$-alkenyl or $O$-alkyl chains containing $\mathrm{PC}$ and $\mathrm{PE}$ in the liver can be explained by the low expression of the alkyldihydroxyacetonephosphate synthase (AGPS) [36].

In addition, we found that the levels of phospholipids containing LA (LA-PLs) were substantially decreased after the dietary intake of ARA (Figure 5a), and profound changes were observed in PC and PE. This may have resulted from competition for the PL remodeling enzyme lysophospholipid acyltransferase 3 (LPCAT3), which has a high affinity for both ARA and LA [37]. Since free LA was decreased by dietary intake of ARA, in addition to LA-PLs (Supplementary Figure S5), the decrease of LA under ARA supplementation could reflect a potential mechanism whereby total $\omega 6$ fatty acid amount, i.e., LA + ARA, is maintained in mammalian cells: in fact, a previous study provided a complementary report in which an ARA decrease is observed in plasma following a higher intake of LA [38]. We also found that the BMP containing DHA (DHA-BMP) was substantially increased under DHA supplementation, while an increase in BMP containing ARA and EPA was not observed in ARA or EPA supplementation (Figure 5b): the annotation was performed by curating both positive and negative ion MS/MS spectra (Figure 5c). While DHA is known to be the predominant fatty acyl chain of BMP in the brain [39], our study suggested that there is a selective mechanism in which DHA is preferably incorporated into BMP in several tissues, including the small intestine, lung, heart, kidney, spleen, and liver. Although the BMP biosynthesis mechanism is still unclear, one study reported that lysocardiolipin acyltransferase 8 (AGPAT8), preferring unsaturated fatty acids as the acyl donor, catalyzed the acylation of BMP [40,41], and the gene expression level of AGPAT8 was high in the small intestine, heart, liver, and kidney in the BioGPS dataset (http://ds.biogps.org/?dataset=GSE10246\&gene=225010). Importantly, these results indicate the importance of untargeted lipidomics, where unexpected and novel insights can be discovered in a data-driven manner. 

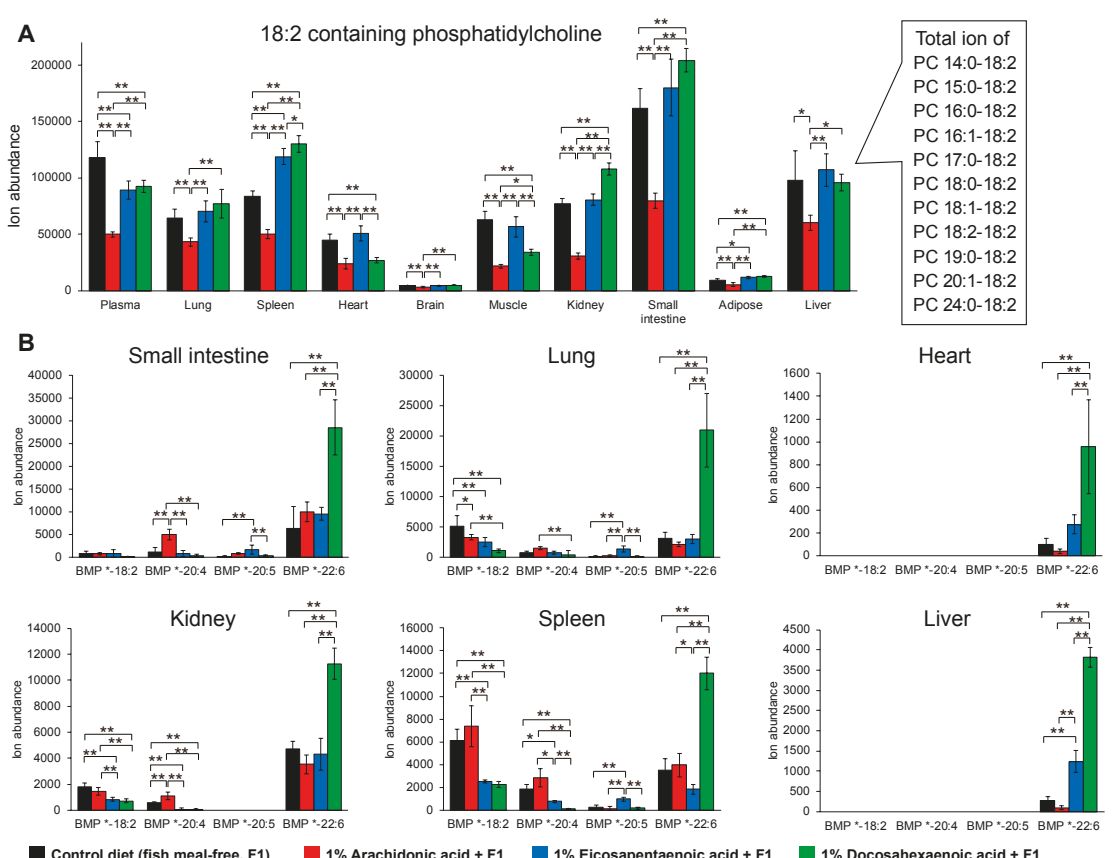

Control diet (fish meal-free, F1) $1 \%$ Arachidonic acid + F1
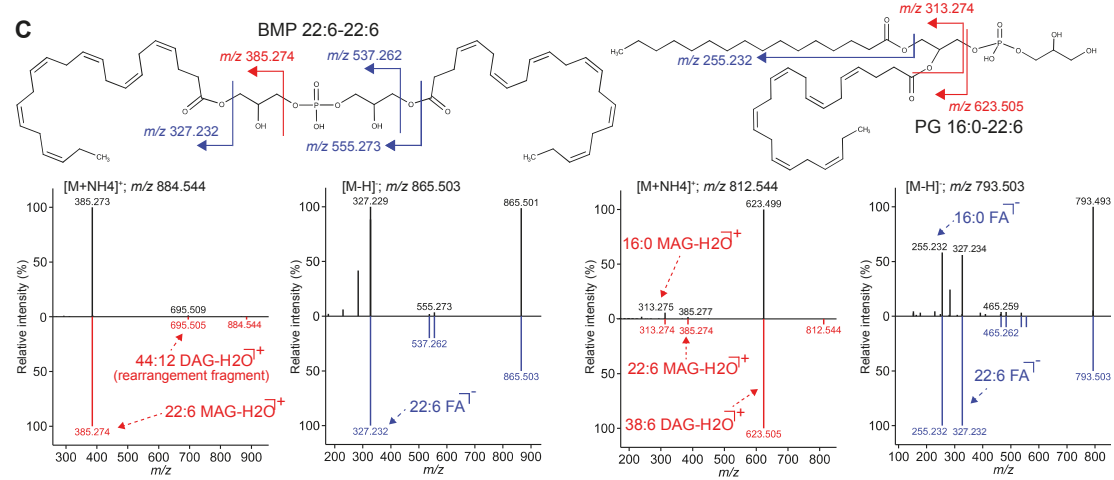

Figure 5. Unique lipid modulations in ARA or DHA dietary intakes. (a) The profile of phosphatidylcholines containing linoleic acid (18:2) as the longest PUFA chain and the ion abundances of each molecule. Note that a hyphen has been used for the description of acyl chains, e.g., 18:0-18:2, because the $s n 1 / s n 2$ acyl chain structural isomers were not distinguished in this study. (b) The profile of bis(monoacylglycero)phosphate (BMP): the asterisk $\left(^{*}\right)$ means any of the fatty acids and the ion abundances of lipids containing 18:2, 20:4, 20:5, or 22:6 were summed for quantification. Black, red, blue, and green represent the dietary conditions: control, ARA, EPA, and DHA supplementation, respectively. The statistical significance was evaluated by Tukey's testing $\left({ }^{*} P<0.05\right.$ and $\left.{ }^{* *} P<0.01\right)$ where one-way ANOVA showed the statistical significance among groups $(P<0.05)$. (c) The experimental positive and negative ion mode MS/MS spectra (black color) of BMP 22:6-22:6 and PG 16:0-22:6 with the in silico reference spectrum (red color for ESI(+)-MS/MS and blue color for ESI(-)-MS/MS). The cleaved places generating the major fragment ions are described by red color for ESI(+)-MS/MS and blue color for ESI(-)-MS/MS. The product ion of $m / z 695.5$ from BMP 22:6-22:6 in positive ion mode was interpreted as the result of structural rearrangement in the fragmentation process. 


\section{Discussion}

In this study, we applied lipidomics techniques to monitor the comprehensive lipid profiles of mouse plasma and tissues, and their changes following the dietary intake of different PUFAs: ARA, EPA, and DHA. Importantly, we examined the effect of dietary supplementation of pure ARA, EPA, or DHA instead of dietary oils such as fish, linseed, and sunflower oils, and investigated the plasma and nine organs to better understand the common and/or differential lipid modulations in tissues. Mouse tissues, except for the brain, effectively incorporated the dietary PUFAs into glycerolipids and glycerophospholipids, and tissue levels of free fatty acids and oxylipins were well correlated with dietary PUFA intakes. It should be noted that our lipidomics data provided the lipidome result from "bulk" cells summing the heterogeneous nature in each tissue, and the results mainly reflect the lipid profiles of the major cell type or the major part of tissue. As for the brain, Arnold et al. reported similar results [42] when they examined cerebral cortex upon dietary EPA and DHA intake; the lipid changes in cerebral cortex were compared with other tissues including liver, kidney, heart, lung, and pancreas. Since cerebral cortex is the largest part of the brain, our whole brain data would have been significantly by the cerebral cortex. This was also the case for other tissues such as liver, white adipose, and skeletal muscle. Therefore, the lipidomes of different cell populations in each tissue should be investigated to further understand the mechanism of PUFA dietary intake in more detail.

ARA, EPA, and DHA supplementation induced various changes in lipid profiles. Dietary PUFA intake substantially increased the levels of free PUFAs and oxylipins, as well as the incorporation of PUFAs in phospholipids and triglycerides. In contrast, this side-by-side study revealed the unique features of ARA, EPA, and DHA metabolism. The basal amount of EPA was much lower than that of ARA and DHA in the plasma and tissues. This may have been because EPA easily undergoes degradation and/or conversion in tissues $[43,44]$. Dietary supplementation of EPA for 2 weeks elevated tissue EPA levels, and the profile of EPA-derived oxylipins was similar to that of ARA-derived oxylipins. However, the profile of DHA-derived oxylipins was a little bit different from those of ARA and EPA. These results may suggest that EPA and DHA have different metabolic compartments, which may be associated with their difference in biological effects.

In addition, dietary DHA was selectively incorporated into a unique phospholipid BMP. BMP is known as an acyl chain positional isomer of phosphatidylglycerol (PG), in which one acyl moiety is incorporated into the glycerol polar head, and the structures can be distinguished by positive ion mode MS/MS spectra, while the MS/MS spectra are the same in negative ion mode (Figure 5c). The biosynthetic and degradation pathways of BMP are still unclear; however, several enzymes, such as AGPAT8 and $\alpha / \beta$ hydrolase domain-containing 6 (ABHD6), have been reported as candidate enzymes involved in these pathways $[40,41,45,46]$. The subcellular localization of BMP is in the multivesicular membranes of late endosomes, where it enhances sphingolipid activator protein activity, resulting in (glyco) sphingolipid degradation [47-49]. In addition, BMP lipids contribute to membrane deformation, fusion, and transportation, as well as the incorporation of proteins and lipids [50], and one study reported that DHA-rich BMP is prone to peroxidation to prevent cholesterol oxidation [51]. However, the biological significance of DHA-BMP in endosome function and regulation is not well understood.

PCA analysis (Figure 2) and circus plots (Figure 4 and Supplementary Figure S5) displayed the metabolic fingerprints of different tissues. In the immune organs, the spleen, small intestine, and lung, high levels of PGs, TXs and fatty acid hydroxides were detected. In contrast, PUFA epoxides were the major metabolites in plasma and other tissues. CYPs are responsible for fatty acid epoxidation, and ARA-, EPA-, and DHA-derived epoxides have various actions, such as vasodilation, anti-inflammation, anticoagulation, and anti-allergy activity [23-27].

The comprehensive lipidomics approach revealed many findings about dietary PUFA metabolism. Our untargeted/targeted lipidomics data incorporating 1026 unique lipid molecules (Supplementary Tables S2 and S3) are powerful when combined with other omics layers such as proteomics and transcriptomics. Recently, the lipidomics standards initiative (LSI) proposed three types of quantification in mass-spectrometry-based lipidomics: level 1, matching internal standards (IS) together 
with consideration of species-specific analytical response (essentially stating that stable-isotope-labeled lipids are preferred); level 2, matching IS where the lipid class between analyte and internal standard is identical; and level 3, non-matching IS where analytes are normalized with other lipid class molecules [52]. Although our untargeted lipidomics data have been displayed as the relative quantification in each tissue, our lipidome table can be normalized as level 3 and partially level 2 quantifications by using the internal standards. A consortium with the advances in analytical chemistry and informatics research would contribute to facilitating open data sciences through the reanalysis of deposited data [15].

This experimental design to investigate the PUFA metabolisms is expandable for further nutrient researches, and we believe that this study is a fundamental step towards clarification of the effects of dietary PUFA intakes in mice. Our lipidomics study offers a comprehensive picture of dietary PUFA metabolism in different tissues, and could provide an opportunity for data-driven hypotheses and biological insights into the molecular mechanisms of how different PUFA balances affect human and livestock health and disease.

Supplementary Materials: The following are available online at http://www.mdpi.com/2218-1989/9/10/241/s1, Figure S1. Calibration curves of standard compounds including internal standards. All of calibration curves of 111 metabolites and four internal standards was described. Three technical replicates were obtained for each concentration. The R-square and coefficient value used for metabolite quantification were also described. The compound ID delimited by double underbars " _" is matched with MRM transition ID described in Supplementary Table S2. The $95 \%$ confidence interval was also described by gray color. Figure S2. Factor loadings of PCA described in Figure 2. The top 20 factor loadings from the maximum and minimum values for first- and second principal components were described for each PCA result. Figure S3. Concentration of free fatty acids in each tissue after dietary intakes. Black, red, blue, and green colors represent the dietary conditions; control, ARA, EPA, and DHA supplementation respectively. The statistical significance was evaluated by Tukey's testing $\left({ }^{*} P<0.05\right.$ and $\left.{ }^{* *} P<0.01\right)$ where one-way ANOVA showed the statistical significance among groups $(P<0.05)$. Figure $S 4$. Detail of several oxylipin profiles under different dietary conditions. (a) The concentrations of 14,15-EET in control and ARA intake condition were described. (b) The concentrations of 17,18-EpETE in the control and following EPA intake were described. (c) The concentrations of 19,20-EpDPE in the control and following DHA intake were described. (d) The concentrations of 14,15-DHT in the control and following ARA intake were described. (e) The concentrations of 17,18-diHETE in the control and following EPA intake were described. (f) The concentrations of 19,20-diHDoPE in the control and following DHA intake were described. (g) The ratio of 14,15-DHT and 14,15-EET in ARA intake condition was described. (h) The ratio of 17,18-diHETE and 17,18-EpETE in EPA intake condition was described. (i) The ratio of 19,20-diHDoPE and 19,20-EpDPE in DHA intake condition was described. The significance was evaluated as $* P<0.05$ and $* * P<0.01$. Figure S5. Circus plots for plasma, adipose, kidney, small intestine, spleen, lung, muscle, and heart. Table S1. Details of fish-meal free F1 diet. Table S2. Details of untargeted lipidomics results. The false discovery rate (FDA) was corrected by Benjamini-Hochberg (BH) method, and the Q-value was also described. Table S3. Details of targeted lipidomics results. The false discovery rate (FDA) was corrected by Benjamini-Hochberg $(\mathrm{BH})$ method, and the Q-value was also described.

Author Contributions: S.N. and M.A. designed the research. S.N. performed the experiments, and K.I. supported them. S.N. performed most of the data analysis for targeted lipidomics, and H.T. and M.T. performed the analysis for untargeted and targeted lipidomics. S.N., H.T., and M.A. primarily wrote the manuscript, and all authors approved the final manuscript.

Funding: This work was partially supported by JSPS KAKENHI $(15 \mathrm{H} 05897,15 \mathrm{H} 05898,18 \mathrm{H} 02432,18 \mathrm{~K} 19155)$ and JST National Bioscience Database Center (NBDC).

Acknowledgments: We thank to Yuuya Senoo, Aya Hori, and Mie Honda (RIKEN) for LC-MS analyses, and Yasutaka Jinno and Hiroyuki Kawano (Mochida Pharmaceutical Co.) for study supports.

Conflicts of Interest: Part of this study was funded by Mochida Pharmaceutical Co., Ltd., which S.N. belongs to Mochida Pharmaceutical Co. was in charge of animal experiments.

\section{References}

1. Saini, R.K.; Keum, Y.S. Omega-3 and omega-6 polyunsaturated fatty acids: Dietary sources, metabolism, and significance-A review. Life Sci. 2018, 203, 255-267. [CrossRef] [PubMed]

2. Zárate, R.; el Jaber-Vazdekis, N.; Tejera, N.; Pérez, J.A.; Rodríguez, C. Significance of long chain polyunsaturated fatty acids in human health. Clin. Transl. Med. 2017, 6, 25. [CrossRef] [PubMed]

3. Wathes, D.C.; Abayasekara, D.R.; Aitken, R.J. Polyunsaturated fatty acids in male and female reproduction. Biol. Reprod. 2007, 77, 190-201. [CrossRef] [PubMed] 
4. Ostermann, A.I.; Schebb, N.H. Effects of omega-3 fatty acid supplementation on the pattern of oxylipins: A short review about the modulation of hydroxy-, dihydroxy-, and epoxy-fatty acids. Food Funct. 2017, 8 , 2355-2367. [CrossRef] [PubMed]

5. Ostermann, A.I.; Waindok, P.; Schmidt, M.J.; Chiu, C.Y.; Smyl, C.; Rohwer, N.; Weylandt, K.H.; Schebb, N.H. Modulation of the endogenous omega-3 fatty acid and oxylipin profile in vivo-A comparison of the fat-1 transgenic mouse with C57BL/6 wildtype mice on an omega-3 fatty acid enriched diet. PLOS ONE 2017, 12, e0184470. [CrossRef] [PubMed]

6. Wijendran, V.; Downs, I.; Srigley, C.T.; Kothapalli, K.S.; Park, W.J.; Blank, B.S.; Zimmer, J.P.; Butt, C.M.; Salem, N.; Brenna, T. Dietary arachidonic acid and docosahexaenoic acid regulate liver fatty acid desaturase (FADS) alternative transcript expression in suckling piglets. Prostaglandins Leukot. Essent. Fat. Acids 2013, 89, 345-350. [CrossRef] [PubMed]

7. Arterburn, L.M.; Hall, E.B.; Oken, H. Distribution, interconversion, and dose response of n-3 fatty acids in humans. Am. J. Clin. Nutr. 2006, 83, 1467S-1476S. [CrossRef]

8. Balvers, M.G.J.; Verhoeckx, K.C.M.; Bijlsma, S.; Rubingh, C.M.; Meijerink, J.; Wortelboer, H.M.; Witkamp, R.F. Fish oil and inflammatory status alter the n- 3 to $n-6$ balance of the endocannabinoid and oxylipin metabolomes in mouse plasma and tissues. Metabolomics 2012, 8, 1130-1147. [CrossRef]

9. Wood, J.T.; Williams, J.S.; Pandarinathan, L.; Janero, D.R.; Lammi-Keefe, C.J.; Makriyannis, A. Dietary docosahexaenoic acid supplementation alters select physiological endocannabinoid-system metabolites in brain and plasma. J. Lipid Res. 2010, 51, 1416-1423. [CrossRef]

10. Harayama, T.; Riezman, H. Understanding the diversity of membrane lipid composition. Nat. Rev. Mol. Cell Biol. 2018, 19, 281-296. [CrossRef]

11. Olzmann, J.A.; Carvalho, P. Dynamics and functions of lipid droplets. Nat. Rev. Mol. Cell Biol. 2019, 20, 137-155. [CrossRef] [PubMed]

12. Arita, M. Mediator lipidomics in acute inflammation and resolution. J. Biochem. 2012, 152, 313-319. [CrossRef] [PubMed]

13. Tsugawa, H.; Ikeda, K.; Arita, M. The importance of bioinformatics for connecting data-driven lipidomics and biological insights. Biochim. Biophys. Acta Mol. Cell Biol. Lipids 2017, 1862, 762-765. [CrossRef] [PubMed]

14. Burla, B.; Arita, M.; Arita, M.; Bendt, A.K.; Cazenave-Gassiot, A.; Dennis, E.A.; Ekroos, K.; Han, X.; Ikeda, K.; Liebisch, G.; et al. MS-based lipidomics of human blood plasma: A community-initiated position paper to develop accepted guidelines. J. Lipid Res. 2018, 59, 2001-2017. [CrossRef] [PubMed]

15. Tsugawa, H.; Satoh, A.; Uchino, H.; Cajka, T.; Arita, M.; Arita, M. Mass Spectrometry Data Repository Enhances Novel Metabolite Discoveries with Advances in Computational Metabolomics. Metabolites 2019, 9 , 119. [CrossRef] [PubMed]

16. Tsugawa, H.; Ikeda, K.; Tanaka, W.; Senoo, Y.; Arita, M.; Arita, M. Comprehensive identification of sphingolipid species by in silico retention time and tandem mass spectral library. J. Cheminformatics 2017, 9, 1-12. [CrossRef]

17. Tsugawa, H.; Cajka, T.; Kind, T.; Ma, Y.; Higgins, B.; Ikeda, K.; Kanazawa, M.; VanderGheynst, J.; Fiehn, O.; Arita, M. MS-DIAL: Data-independent MS/MS deconvolution for comprehensive metabolome analysis. Nat. Methods 2015, 12, 523-526. [CrossRef]

18. Tsugawa, H.; Nakabayashi, R.; Mori, T.; Yamada, Y.; Takahashi, M.; Rai, A.; Sugiyama, R.; Yamamoto, H.; Nakaya, T.; Yamazaki, M.; et al. A cheminformatics approach to characterize metabolomes in stable-isotope-labeled organisms. Nat. Methods 2019, 16, 295-298. [CrossRef]

19. Hansen, J.B.; Olsen, J.O.; Wilsgard, L.; Lyngmo, V.; Svensson, B. Comparative effects of prolonged intake of highly purified fish oils as ethyl ester or triglyceride on lipids, haemostasis and platelet function in normolipaemic men. Eur. J. Clin. Nutr. 1993, 47, 497-507.

20. Neubronner, J.; Schuchardt, J.P.; Kressel, G.; Merkel, M.; von Schacky, C.; Hahn, A. Enhanced increase of omega-3 index in response to long-term n-3 fatty acid supplementation from triacylglycerides versus ethyl esters. Eur. J. Clin. Nutr. 2011, 65, 247-254. [CrossRef]

21. Schuchardt, J.P.; Hahn, A. Bioavailability of long-chain omega-3 fatty acids. Prostaglandins Leukot. Essent. Fat. Acids 2013, 89, 1-8. [CrossRef] [PubMed]

22. Hishikawa, D.; Valentine, W.J.; Iizuka-Hishikawa, Y.; Shindou, H.; Shimizu, T. Metabolism and functions of docosahexaenoic acid-containing membrane glycerophospholipids. FEBS Lett. 2017, 591, 2730-2744. [CrossRef] [PubMed] 
23. Spector, A.A.; Norris, A.W. Action of epoxyeicosatrienoic acids on cellular function. Am. J. Physiol. Physiol. 2007, 292, C996-C1012. [CrossRef] [PubMed]

24. Nagatake, T.; Shiogama, Y.; Inoue, A.; Kikuta, J.; Honda, T.; Tiwari, P.; Kishi, T.; Yanagisawa, A.; Isobe, Y.; Matsumoto, N.; et al. The 17,18-epoxyeicosatetraenoic acid-G protein-coupled receptor 40 axis ameliorates contact hypersensitivity by inhibiting neutrophil mobility in mice and cynomolgus macaques. J. Allergy Clin. Immunol. 2018, 142, 470-484. [CrossRef]

25. Kunisawa, J.; Arita, M.; Hayasaka, T.; Harada, T.; Iwamoto, R.; Nagasawa, R.; Shikata, S.; Nagatake, T.; Suzuki, H.; Hashimoto, E.; et al. Dietary $\omega 3$ fatty acid exerts anti-allergic effect through the conversion to 17,18-epoxyeicosatetraenoic acid in the gut. Sci. Rep. 2015, 5, 9750. [CrossRef]

26. Mochimaru, T.; Fukunaga, K.; Miyata, J.; Matsusaka, M.; Kabata, H.; Ueda, S.; Suzuki, Y.; Goto, T.; Urabe, D.; Inoue, M.; et al. 12-OH-17,18-Epoxyeicosatetraenoic acid alleviates eosinophilic airway inflammation in murine lungs. Allergy 2018, 73, 369-378. [CrossRef]

27. Kubota, T.; Arita, M.; Isobe, Y.; Iwamoto, R.; Goto, T.; Yoshioka, T.; Urabe, D.; Inoue, M.; Arai, H. Eicosapentaenoic acid is converted via $\omega-3$ epoxygenation to the anti-inflammatory metabolite 12-hydroxy-17,18-epoxyeicosatetraenoic acid. FASEB J. 2014, 28, 586-593. [CrossRef]

28. Dasilva, G.; Pazos, M.; García-Egido, E.; Pérez-Jiménez, J.; Torres, J.L.; Giralt, M.; Nogués, M.R.; Medina, I. Lipidomics to analyze the influence of diets with different EPA:DHA ratios in the progression of Metabolic Syndrome using SHROB rats as a model. Food Chem. 2016, 205, 196-203. [CrossRef]

29. Grønn, M.; Christensen, E.; Hagve, T.A.; Christophersen, B.O. Peroxisomal retroconversion of docosahexaenoic acid (22:6(n-3)) to eicosapentaenoic acid (20:5(n-3)) studied in isolated rat liver cells. Biochim. Biophys. Acta (BBA) Lipids Lipid Metab. 1991, 1081, 85-91. [CrossRef]

30. Zhang, J.Y.; Kothapalli, K.S.D.; Brenna, J.T. Desaturase and elongase limiting endogenous long chain polyunsaturated fatty acid biosynthesis. Curr. Opin. Clin. Nutr. Metab. Care 2016, 19, 103-110. [CrossRef]

31. Schmidt-Schultz, T.; Althaus, H.H. Monogalactosyl Diglyceride, a Marker for Myelination, Activates Oligodendroglial Protein Kinase C. J. Neurochem. 1994, 62, 1578-1585. [CrossRef] [PubMed]

32. Murray, R.K.; Narasimhan, R.; Levine, M.; Pinteric, L.; Shirley, M.; Lingwood, C.; Schacter, H. Galactoglycerolipids of Mammalian Testis, Spermatozoa, and Nervous Tissue; ACS Symposium Series; American Chemical Society: Washington, DC, USA, 1980; pp. 105-125.

33. Påhlsson, P.; Spitalnik, S.L.; Spitalnik, P.F.; Fantini, J.; Rakotonirainy, O.; Ghardashkhani, S.; Lindberg, J.; Konradsson, P.; Larson, G. Characterization of galactosyl glycerolipids in the HT29 human colon carcinoma cell line. Arch. Biochem. Biophys. 2001, 396, 187-198. [CrossRef] [PubMed]

34. Jain, M.; Ngoy, S.; Sheth, S.A.; Swanson, R.A.; Rhee, E.P.; Liao, R.; Clish, C.B.; Mootha, V.K.; Nilsson, R. A systematic survey of lipids across mouse tissues. Am. J. Physiol. Metab. 2014, 306, E854-E868. [CrossRef] [PubMed]

35. Yen, C.L.E.; Stone, S.J.; Koliwad, S.; Harris, C.; Farese, R.V. Thematic Review Series: Glycerolipids. DGAT enzymes and triacylglycerol biosynthesis. J. Lipid Res. 2008, 49, 2283-2301. [CrossRef] [PubMed]

36. Wu, C.; Jin, X.; Tsueng, G.; Afrasiabi, C.; Su, A.I. BioGPS: Building your own mash-up of gene annotations and expression profiles. Nucleic Acids Res. 2016, 44, D313-D316. [CrossRef] [PubMed]

37. Hashidate-Yoshida, T.; Harayama, T.; Hishikawa, D.; Morimoto, R.; Hamano, F.; Tokuoka, S.M.; Eto, M.; Tamura-Nakano, M.; Yanobu-Takanashi, R.; Mukumoto, Y.; et al. Fatty acyl-chain remodeling by LPCAT3 enriches arachidonate in phospholipid membranes and regulates triglyceride transport. Elife 2015, 4, e06328. [CrossRef]

38. Adam, O.; Wolfram, T.G. Impact of linoleic acid intake on arachidonic acid formation and eicosanoid biosynthesis in humans. Prostaglandins Leukot. Essent. Fat. Acids 2008, 75, 177-181. [CrossRef]

39. Akgoc, Z.; Sena-Esteves, M.; Martin, D.R.; Han, X.; d'Azzo, A.; Seyfried, T.N. Bis(monoacylglycero)phosphate: A secondary storage lipid in the gangliosidoses. J. Lipid Res. 2015, 56, 1006-1013. [CrossRef]

40. Cao, J.; Shen, W.; Chang, Z.; Shi, Y. ALCAT1 is a polyglycerophospholipid acyltransferase potently regulated by adenine nucleotide and thyroid status. Am. J. Physiol. Metab. 2008, 296, E647-E653. [CrossRef]

41. Yamashita, A.; Hayashi, Y.; Nemoto-Sasaki, Y.; Ito, M.; Oka, S.; Tanikawa, T.; Waku, K.; Sugiura, T. Acyltransferases and transacylases that determine the fatty acid composition of glycerolipids and the metabolism of bioactive lipid mediators in mammalian cells and model organisms. Prog. Lipid Res. 2014, 53, 18-81. [CrossRef] 
42. Arnold, C.; Markovic, M.; Blossey, K.; Wallukat, G.; Fischer, R.; Dechend, R.; Konkel, A.; von Schacky, C.; Luft, F.C.; Muller, D.N.; et al. Arachidonic Acid-metabolizing Cytochrome P450 Enzymes Are Targets of $\omega-3$ Fatty Acids. J. Biol. Chem. 2010, 285, 32720-32733. [CrossRef] [PubMed]

43. Igarashi, M.; Chang, L.; Ma, K.; Rapoport, S.I. Kinetics of eicosapentaenoic acid in brain, heart and liver of conscious rats fed a high n-3 PUFA containing diet. Prostaglandins Leukot. Essent. Fat. Acids 2013, 89, 403-412. [CrossRef] [PubMed]

44. Chen, C.T.; Bazinet, R.P. $\beta$-oxidation and rapid metabolism, but not uptake regulate brain eicosapentaenoic acid levels. Prostaglandins Leukot. Essent. Fat. Acids 2015, 92, 33-40. [CrossRef] [PubMed]

45. Scherer, M.; Schmitz, G. Metabolism, function and mass spectrometric analysis of bis(monoacylglycero)phosphate and cardiolipin. Chem. Phys. Lipids 2011, 164, 556-562. [CrossRef] [PubMed]

46. Pribasnig, M.A.; Mrak, I.; Grabner, G.F.; Taschler, U.; Knittelfelder, O.; Scherz, B.; Eichmann, T.O.; Heier, C.; Grumet, L.; Kowaliuk, J.; et al. $\alpha / \beta$ hydrolase domain-containing 6 (ABHD6) degrades the late Endosomal/Lysosomal lipid Bis(Monoacylglycero)phosphate. J. Biol. Chem. 2015, 290, 29869-29881. [CrossRef]

47. Matsuo, H.; Chevallier, J.; Mayran, N.; Le Blanc, I.; Ferguson, C.; Fauré, J.; Blanc, N.S.; Matile, S.; Dubochet, J.; Sadoul, R.; et al. Role of LBPA and Alix in Multivesicular Liposome Formation and Endosome Organization. Science 2004, 303, 531-534. [CrossRef]

48. Kobayashi, T.; Stang, E.; Fang, K.S.; De Moerloose, P.; Parton, R.G.; Gruenberg, J. A lipid associated with the antiphospholipid syndrome regulates endosome structure and function. Nature 1998, 392, 193-197. [CrossRef]

49. Kolter, T.; Sandhoff, K. Principles of lysosomal membrane digestion: Stimulation of sphingolipid degradation by sphingolipid activator proteins and anionic lysosomal lipids. Annu. Rev. Cell Dev. Biol. 2005, 21, 81-103. [CrossRef]

50. Bissig, C.; Gruenberg, J. Lipid sorting and multivesicular endosome biogenesis. Cold Spring Harb. Perspect. Biol. 2013, 5, a016816. [CrossRef]

51. Bouvier, J.; Zemski Berry, K.A.; Hullin-Matsuda, F.; Makino, A.; Michaud, S.; Geloën, A.; Murphy, R.C.; Kobayashi, T.; Lagarde, M.; Delton-Vandenbroucke, I. Selective decrease of bis(monoacylglycero)phosphate content in macrophages by high supplementation with docosahexaenoic acid. J. Lipid Res. 2009, 50, $243-255$. [CrossRef]

52. Liebisch, G.; Ahrends, R.; Arita, M.; Arita, M.; Bowden, J.A.; Ejsing, C.S.; Griffiths, W.J.; Holcapek, M.; Kofeler, H.; Mitchell, T.W.; et al. Lipidomics needs more standardization. Nat. Metab. 2019, 1, 745-747.

(C) 2019 by the authors. Licensee MDPI, Basel, Switzerland. This article is an open access article distributed under the terms and conditions of the Creative Commons Attribution (CC BY) license (http://creativecommons.org/licenses/by/4.0/). 



\title{
MEATabolomics: Muscle and Meat Metabolomics in Domestic Animals
}

\author{
Susumu Muroya ${ }^{1, *}$, Shuji Ueda ${ }^{2}$, Tomohiko Komatsu ${ }^{3}$, Takuya Miyakawa ${ }^{4}$ and Per Ertbjerg ${ }^{5}$ \\ 1 NARO Institute of Livestock and Grassland Science, Tsukuba, Ibaraki 305-0901, Japan \\ 2 Graduate School of Agricultural Science, Kobe University, Hyogo 657-8501, Japan; \\ uedas@people.kobe-u.ac.jp \\ 3 Livestock Research Institute of Yamagata Integrated Research Center, Shinjo, Yamagata 996-0041, Japan; \\ komatsutom@pref.yamagata.jp \\ 4 Graduate School of Agricultural and Life Sciences, University of Tokyo, Bunkyo-ku, Tokyo 113-8657, Japan; \\ atmiya@mail.ecc.u-tokyo.ac.jp \\ 5 Department of Food and Nutrition, University of Helsinki, 00014 Helsinki, Finland; per.ertbjerg@helsinki.fi \\ * Correspondence: muros@affrc.go.jp; Tel.: +81-29-838-8686
}

Received: 26 March 2020; Accepted: 7 May 2020; Published: 11 May 2020

\begin{abstract}
In the past decades, metabolomics has been used to comprehensively understand a variety of food materials for improvement and assessment of food quality. Farm animal skeletal muscles and meat are one of the major targets of metabolomics for the characterization of meat and the exploration of biomarkers in the production system. For identification of potential biomarkers to control meat quality, studies of animal muscles and meat with metabolomics (MEATabolomics) has been conducted in combination with analyses of meat quality traits, focusing on specific factors associated with animal genetic background and sensory scores, or conditions in feeding system and treatments of meat in the processes such as postmortem storage, processing, and hygiene control. Currently, most of MEATabolomics approaches combine separation techniques (gas or liquid chromatography, and capillary electrophoresis)-mass spectrometry (MS) or nuclear magnetic resonance (NMR) approaches with the downstream multivariate analyses, depending on the polarity and/or hydrophobicity of the targeted metabolites. Studies employing these approaches provide useful information to monitor meat quality traits efficiently and to understand the genetic background and production system of animals behind the meat quality. MEATabolomics is expected to improve the knowledge and methodologies in animal breeding and feeding, meat storage and processing, and prediction of meat quality.
\end{abstract}

Keywords: authentication; biomarker; breed; feeding; meat quality traits; metabolite; postmortem aging; processing; skeletal muscle

\section{Introduction}

In the last two decades, the metabolomic approach has been employed widely in the research fields of animal and plant nutrition, physiology, breeds, environment, post-harvest storage and processing [1,2] with the advantages of its high-throughput capacity. The target molecules of food metabolomics are animal and plant metabolites, including low molecular-weight hydrophilic and hydrophobic compounds. These compounds include key metabolites such as flavor-associated compounds, nutrients, and functionality-associated compounds in food, some of which have a molecular weight of $>1000$. Such compounds characterize nutritional and sensory properties, and therefore the metabolome of a food provides important phenotypic information. In this context, metabolomics is a powerful tool to obtain a deeper understanding of the biologically and agriculturally 
meaningful information in the global metabolome profiles and the changes caused by factors in food production processes [3].

Currently, two major types of platforms have been applied for metabolomic studies so far: mass spectrometry (MS)-based [4] and non-MS-based techniques such as nuclear magnetic resonance (NMR) [2]. Moreover, various types of separation techniques are incorporated in most of the MS-based approaches, depending on the lipophilicity and polarity of the metabolites of interest. Combined with advanced statistical analyses, multivariate analyses, and bioinformatic databases, metabolomics provides clues not only to discover biomarkers for monitoring and assessment of food quality but also to uncover molecular pathways in which enzymatic reaction generates key metabolites [5]. Correlation analysis between sensory evaluation scores and metabolomic profiles potentially leads to key compounds that are associated with eating quality, such as flavor and texture [6-8], which may enable us to predict the palatability of the foods by using the biomarker metabolites in pre- and post-harvest materials. Metabolomic profile data have also been utilized to explore the responsible genes for specific metabolite-featured phenotypes in genome-wide association studies (GWAS) $[9,10]$.

In recent years, studies in muscle and meat science have utilized metabolomics as in other fields [11-13]. Skeletal muscle characteristics are designed by a functionally cooperative set of genes specific to the spatiotemporal requirement in each muscle. The gene expression is further modulated at levels of transcription, post-transcription, translation, and protein modification during development, growth, and maturation stages of the muscle. Accordingly, muscle metabolites determine the physiological muscle characteristics and meat quality traits as the major phenotypic components. The history of the muscle in the developmental and physiological specialization, feeding processes of animals, circumstances in slaughtering, postmortem aging, and processing processes all have influences on transcriptomic and/or proteomic profiles, and finally on the muscle metabolome profile. This makes the understanding of mechanisms behind meat quality traits by the use of molecular markers more difficult. Nevertheless, the metabolome profiles have been successfully distinguished with metabolites of biomarker candidates in comparisons such as between physiologically different types of skeletal muscles [14-16], between at-slaughter and postmortem muscles [14,15,17-19], between meats produced by different animal feeding conditions [20-26], and between different types of meat processing [7,27-30]. Thus, to better understand factors determining muscle characteristics and meat quality, the recent metabolomics approach has great advantages, which potentially supports to capture biomarkers of the phenotype. Since raw and cooked meat are rich in flavor-associated volatile compounds and precursors, MEATabolomics studies in combination with sensory evaluation have been conducted to explore biomarker candidates associated with eating quality of meat.

Especially in studies of postmortem muscle and meat quality, MEATabolomics is expected to provide a clue for drawing maps of the metabolic network in postmortem muscle aging and flavor development during cooking, which cannot be replaced by the other biological or chemical approaches. Muscles, especially beef and pork, are more or less aged after death for the tenderization and flavor component accumulation. During the aging, the muscles experience drastic and irreversible physico-chemical and metabolic changes [31]. These metabolic changes have a large impact on subsequent beef and pork quality, under influences of physiological background before slaughter. Despite the impact on meat quality, the metabolic changes have not easily predicted, because coordinated metabolism in live muscle is no longer maintained due to lack of energy supply and arrest of de novo gene expression after animal death. Postmortem metabolic maps drawn by MEATabolomics could lead to a finding of the metabolic factors responsible for meat quality traits and thereby contribute to the exploration of biomarkers in quality monitoring, processing, and authentication of meat including chicken. Thus, postmortem muscle metabolism and the factors associated with meat quality have been the major and unique challenges to be addressed for MEATabolomics.

This review provides an overview of past applications, recent findings, and topics achieved by MS- and NMR-based MEATabolomics approaches associated with animal muscle physiology and meat quality traits. We especially focus on studies of meat quality traits and the factors influencing meat 
quality, such as postmortem aging, as well as animal breed and feeding, processing, spoilage, and authentication. Using those keywords, a total of 78 studies were collected by search in databases such as PubMed, ScienceDirect, and Springer Link, from which 56 studies were selected and featured.

\section{MEATabolomic Methodologies and Approaches}

The MS-based metabolomics need a separation step suitable for target metabolites of interest (Table 1). Of the current separation techniques, capillary electrophoresis (CE)-MS has high performance in the acquisition of polar and charged metabolite information with high resolution and sensitivity, but not in non-ionic molecules [32,33]. CE has an advantage over gas chromatography (GC) and high-performance liquid chromatography (HPLC; LC) for the resolution of ionic compounds, including their isomers, owing to separation in CE by their charge-to-mass ratio. GC-MS has been widely used for decades due to its established high-separation efficiency, selective and sensitive mass detection, and a broad range of target molecules, mainly fatty acids and sugars, although it needs derivatization of sample molecules, especially for non-volatile compounds. Derivatization artifacts may be generated by decomposition of thermolabile molecules in GC, during the analysis after enhancement of volatility of molecules. On the other hand, LC-MS targets compounds with a relatively narrow range but has the flexibility to change the type of targets with replaceable separation columns. As the common stationary columns in LC, C18 reversed-phase is the most frequently used for separation of hydrophobic molecules, while the polar phase, such as silica and amide, is used for hydrophilic molecules. Running time of LC separation can be much reduced to less than $10 \mathrm{~min}$ in the case of ultra HPLC (UHPLC, UPLC).

Table 1. Features of the most commonly used separation techniques in mass spectrometry (MS)-based metabolomics.

\begin{tabular}{|c|c|c|c|}
\hline & $\mathrm{CE}$ & GC & LC \\
\hline Favorable target metabolites & Polar, charged & Mainly volatile & Non-polar, neutral \\
\hline Sample derivatization & Unnecessary & $\begin{array}{l}\text { Required for non-volatile } \\
\text { compounds }\end{array}$ & Unnecessary \\
\hline Number of theoretical plate & $10^{5} \sim 10^{6}$ & $10^{4} \sim 10^{5}$ & $10^{4}$ \\
\hline Separation of structural isomer & High & High & Low \\
\hline Running time & $>20 \mathrm{~min}$ & $20-40 \mathrm{~min}$ & $15-40 \mathrm{~min}$ \\
\hline Downstream ionization & ESI & EI, CI & ESI, APCI \\
\hline
\end{tabular}

The separated molecules are required to be ionized generally by electrospray ionization (ESI) in $\mathrm{CE}$, electron ionization (EI) or chemical ionization (CI) in GC, and ESI or atmospheric pressure chemical ionization (APCI; API) in LC. MS detection techniques have been developed to various types, and, currently, the most frequently used technique for matching to the upstream CE, GC, and LC separation steps is time-of-flight (TOF) to achieve higher sensitivity, accuracy, rate of measurement, and mass and dynamic range for the acquisition of more metabolite information [4]. Fourier transform (FT) type MS also has a high-throughput performance with high resolution and a broad range of target molecules and is thereby used for metabolomics. Of the FTMS, FT-ion cyclotron resonance (FT-ICR) MS utilizes a magnetic field to detect resonance of cyclotron motion of metabolite ion, while the orbitrap type is based on a system using an electric field. In some cases, tandem MS (MS/MS) is employed to acquire more structural information for the characterization of compounds. These MS-based approaches are utilized not only for targeted metabolomics but also for untargeted metabolomics [26,34,35]. Recently, rapid evaporative ionization mass spectrometry (REIMS) has been introduced in MEATabolomics [26,36]. This method allows direct MS analysis of a biological sample with no preparative steps, under normal atmospheric conditions, due to being based on the ambient ionization $[37,38]$. A trend of MEATabolomic approach can be seen in the times of employment for each technique in the 56 studies collected in this review: CE-MS, GC-MS, LC-MS, NMR, and REIMS approaches have been employed in 5, 17, 17, 22, and 1 studies, respectively, with some cases combining multiple approaches (Table 2). 


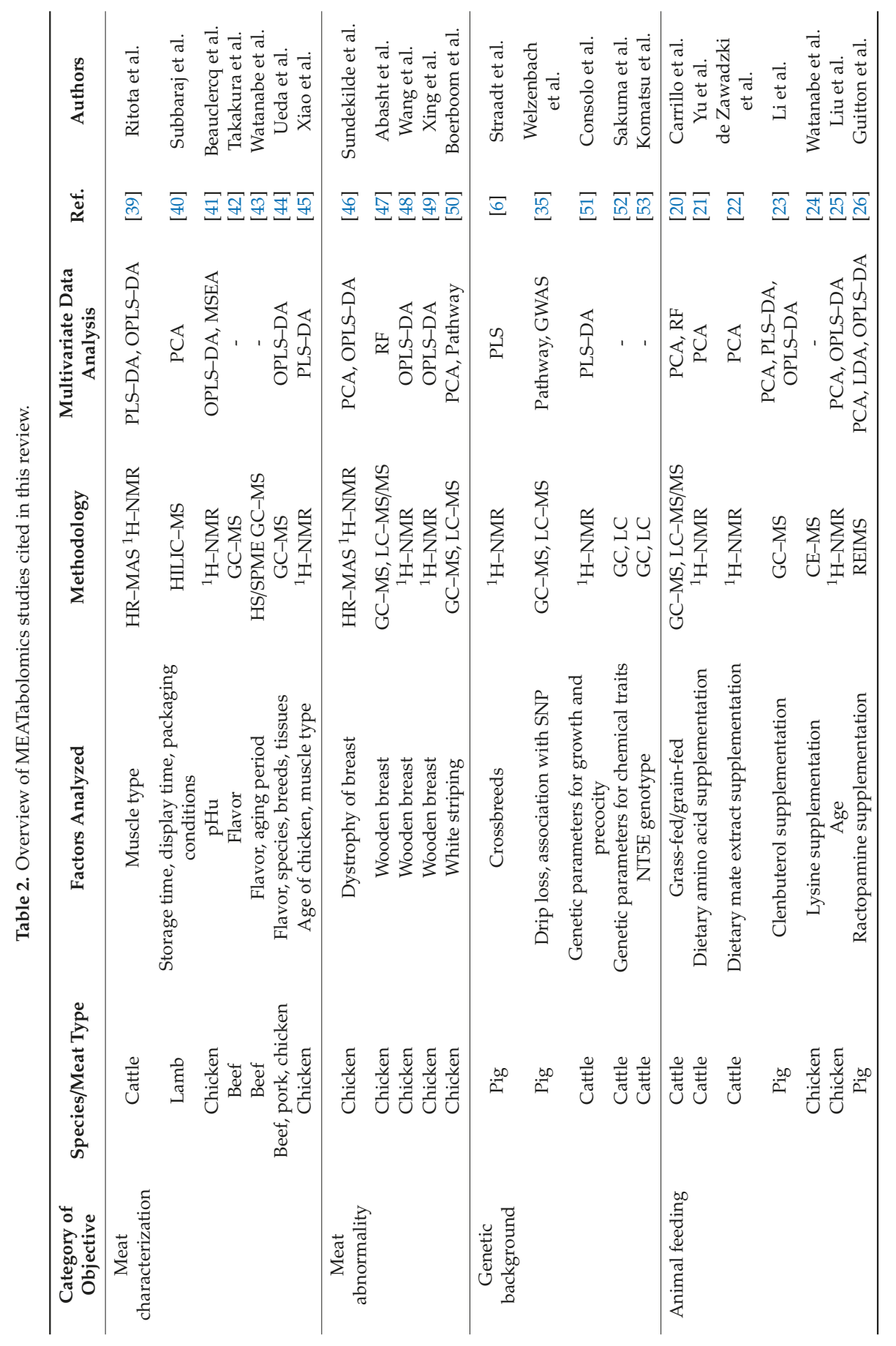




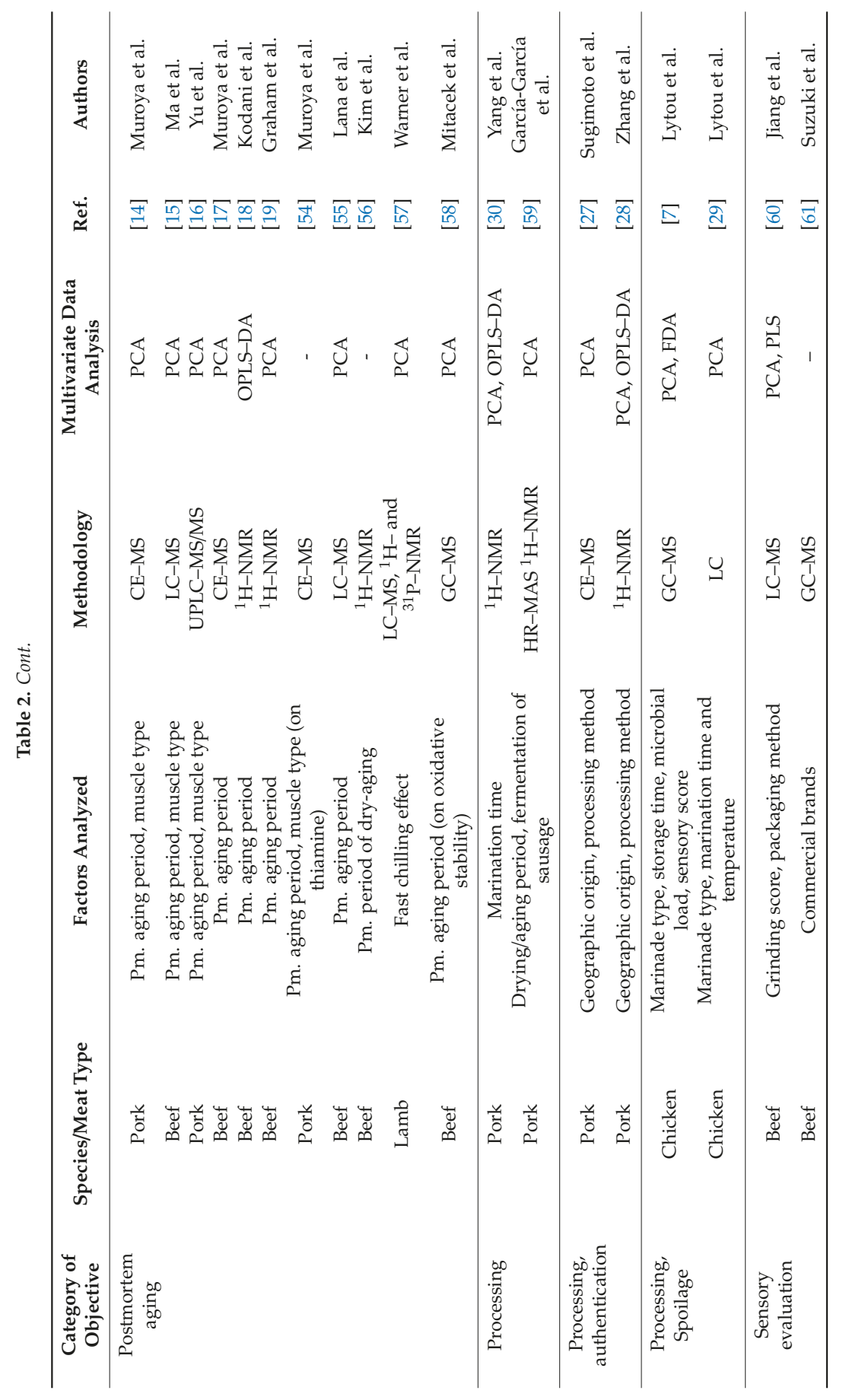




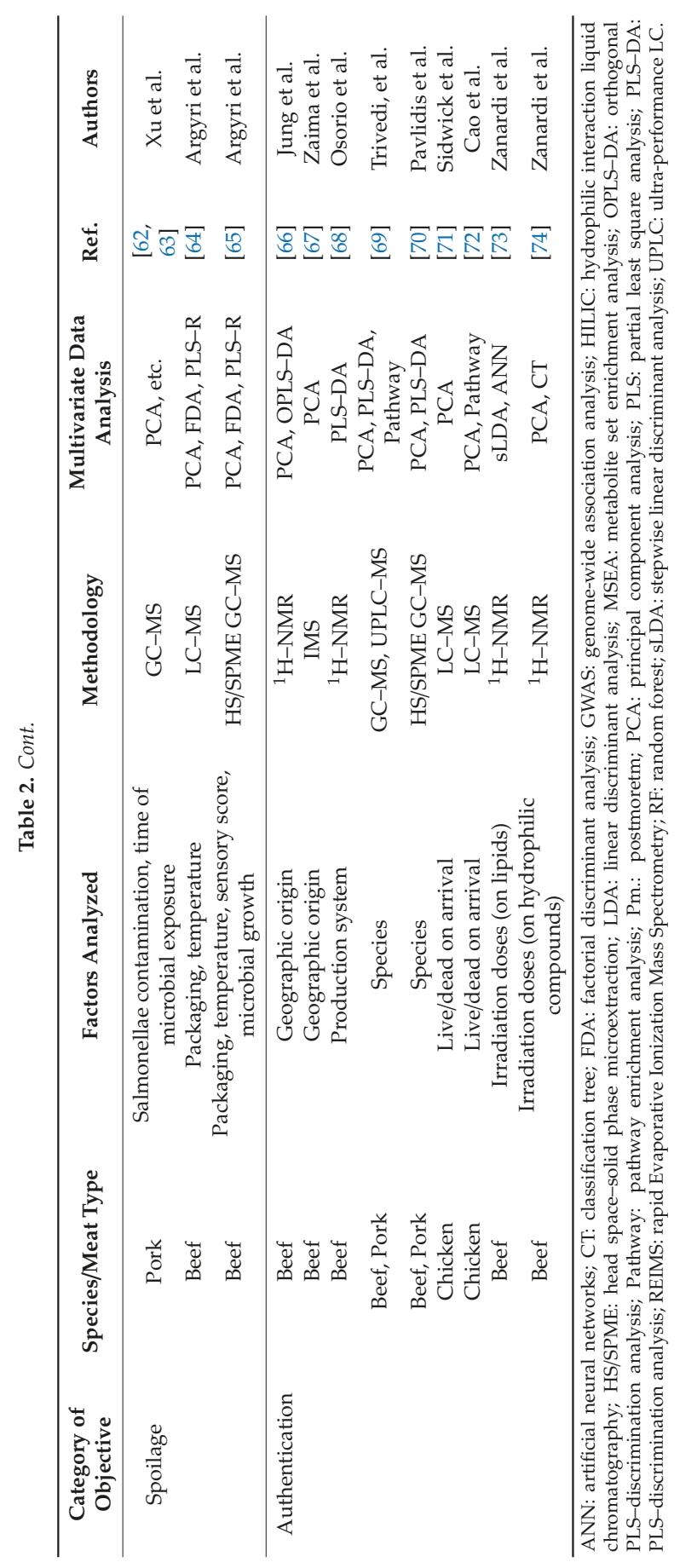


After MS measurement, initial putative metabolite can be identified on the basis of the accurate mass-to-charge ratio $(\mathrm{m} / \mathrm{z})$ of the mass spectral ion in MS-based metabolomics. This is assisted by the use of metabolite databases such as METLIN (https://metlin.scripps.edu/), the Human Metabolome Database (HMDB; http://www.hmdb.ca/), and MassBank (http://www.massbank.jp/).

NMR is also highlighted as a method for practical use, such as authentication purposes, in an analytical routine. This technique can provide rapid and reproducible measurements in complex mixtures without a time-consuming pretreatment. Although NMR has relatively low ability to profile metabolites compared to the MS-based techniques due to its low resolution and sensitivity, it has the ability to collect distinct information that the other metabolomics cannot access in a non-destructive and non-biased way [2]. Especially, approaches with ${ }^{1} \mathrm{H}$-high-resolution magic angle spinning (HR-MAS) have also been applied to characterize meat quality $[39,46,59]$. Unlike the other types of NMR, this NMR enables us to investigate intact tissue specimens $(10-50 \mathrm{mg})$ and allows the spectra to be obtained with a high resolution compatible to that obtained in liquid samples in less than $30 \mathrm{~min}$.

Once the data matrix is produced from the collected raw data, subsequent statistical analyses and data mining are often performed to identify samples or variables (metabolites) that characterize the variations between datasets and may represent biologically meaningful determinants. In most cases, the statistical sample classification is conducted to overview pattern recognition of sample categories, by multivariate analyses such as principal component analysis (PCA), clustering analysis, partial least square analysis (also called projection to latent structures, PLS), PLS-discriminant analysis (PLS-DA), and random forests (RF) $[5,75]$.

PCA is an unsupervised statistical method that reduces dimensions of high dimensional data to visualize sample distribution and grouping on the principal component (PC) plot based on the pattern of the metabolite dataset and thereby is employed by most of the metabolomic studies as the first step of data analysis [75]. Clustering analysis, especially hierarchical clustering analysis (HCA), is also widely used to generate a snapshot profiling of dataset. In HCA, the algorithm divides the measured datasets into subgroups so that datasets with similar metabolomic profiles are placed in each group. PCA and HCA are frequently used for visualization of classification in omics studies, including metabolomics.

PLS has been developed as a supervised extension of PCA [75]. This regression-based method is especially useful when fewer samples are available than measured metabolites. PLS-DA is used to elucidate the metabolites that carry the information of classification, which screens highly contributing metabolites to the classification. PLS and PLS-DA have often been employed to sharpen the separation between the groups, especially when the groups are not sufficiently separated in PCA. Orthogonal PLS (OPLS)-DA is an extension of PLS-DA to cover the defect of PLS-DA, owing to its robustness against noise. RF is a machine learning method used to discriminate two groups. Different from conventional methods such as PCA and PLS-DA, RF allows data structure understood without dimensional reduction with its low bias and low variance. As with other data mining analyses, the application of support vector machine (SVM) and neural networks are attempted for some of MEATabolomic studies [34]. These analytical methods have been applied for sample characterization and determination of biomarker candidates.

Classical statistical analyses such as Student's t-test and analysis of variance (ANOVA) are also used to compare metabolite levels between sample groups, with care about the detection of false-positive metabolites in multiple comparisons of the datasets. To reduce false-positive detection caused by the familywise error rate (FWER) in multiple comparisons, procedures of false discovery rate (FDR), Holm, Bonferroni, or Benjamini-Hochberg are applied to adjust the levels of significance detection in metabolomic studies [5]. Obviously, there are advantages and disadvantages to every method and database. Further information and details in these statistical analyses are described for reference in methodological reviews $[5,75]$.

Moreover, recent progress of bioinformatics analytic tools and databases largely contributes to compound annotation, metabolic pathway finding, and biological data interpretation of the 
extracted compounds (see the websites of The Metabolomics Society, Inc. for more detail information; http://metabolomicssociety.org/). HMDB (http://www.hmdb.ca/) and PubChem (https://pubchem. ncbi.nlm.nih.gov/) are among the most widely used databases, and they play important roles in the annotation of compound signals.

The Kyoto Encyclopedia of Genes and Genomes (KEGG; https://www.genome.jp/kegg/) is frequently used to connect the metabolome profile with genomic, transcriptomic, enzymatic, and pathway information, which supports prediction and pathway mapping of potential molecular factors responsible for the phenotypic events. Software for analyses of metabolic pathways such as MetaboAnalyst (https://www.metaboanalyst.ca/) has also often been used for biological interpretation in recent years. MetaboAnalyst provides a variety of statistical analysis tools, including visualizing programs, multivariate analyses, and metabolite pathway analyses. These metabolomic approaches, especially CE-MS, GC-MS, LC-MS, and NMR, in combination with subsequent data analyses have recently been increasingly used in animal-based food research fields [10-12,76].

\section{Metabolomes Associated with Meat Quality Traits}

Metabolomics is used for the exploration of key compounds contributing to the physico-chemical properties and sensory evaluation scores, and thereby it contributes to accounting for meat palatability and quality traits such as color and water-holding capacity (WHC). As described below, the contents of skeletal muscle metabolites, including amino acids and sugars, are affected by animal genetic background, feeding, muscle type, postmortem aging, and meat processing. These metabolomic changes are linked with the physico-chemical properties of muscles and meat, as shown in variations in meat color and WHC between different types of muscles or between differently treated muscles. Accordingly, the metabolites are beneficial indicators to predict the physico-chemical meat quality traits, which indicate a result more or less from the influences of animal genetic background, feeding, and postmortem processes (Table 2).

\subsection{Meat Color, WHC, and pH Decline}

The color of meat is one of the commercially important meat quality traits since the appearance of meat has a great influence on the consumer's desire to purchase [77]. The redness and discoloration are determined by the chemical status of myoglobin, which is affected by multiple factors that are inherent to both live animal and postmortem conditions. With hydrophilic interaction liquid chromatography-mass spectrometry (HILIC-MS)-based metabolomics, influences of postmortem aging time, packaging atmosphere, and display time on lamb meat color was investigated to identify the metabolites affected by those postmortem conditions [40]. Many compounds were found to change with time of aging and display or packaging atmosphere, which indicated the contribution of the compounds, including amino acids, sugars, nucleotides, and organic acids, to the sample discrimination. Furthermore, the abundance of reducing or antioxidant compounds such as L-glutathione and taurine contributed to color stability. Intriguingly, boron complexes of sugar and malate, presumably color stability-related compounds present in plants but not in animals, were detected, although the role of the compounds remains unknown [40]. WHC is also one of the most important traits of meat quality. Various genetic and environmental factors can induce myofibrillar protein denaturation and thereby lower the WHC of meat. Meat with lower WHC generally has higher drip loss, which has a negative impact on juiciness and palatability of the meat. To assess the responsible genes for WHC in pork, metabolomics was applied to characterize pork with high drip loss via metabolic pathway enrichment analysis in an integrative omics approach, as described below (see Section 4.1) [35].

Due to the large impact on meat color, WHC, and other final meat quality traits, postmortem $\mathrm{pH}$ decline is also an important factor to be controlled. In a study investigating two chicken lines differing in ultimate $\mathrm{pH}(\mathrm{pHu})$, the pectoralis major muscles of the two lines were discriminated against by high-resolution NMR metabolomics [41]. Subsequent metabolite set enrichment analysis (MSEA) of the data showed that carbohydrate metabolism in the low $\mathrm{pHu}$ line and metabolism of amino acid and 
protein in the high $\mathrm{pHu}$ line were over-representative pathways. The difference in the metabolome profile between high and low $\mathrm{pHu}$ chicken might be due to the ability of the muscles of glycogen storage and carbohydrate use.

\subsection{Flavor and Palatability}

Skeletal muscle metabolites include amino acids and sugars that are precursors of volatile compounds associated with meat aroma. The contents of these compounds are altered, depending on animal feeding, the genetic background, and the postmortem aging process. This could cause variations in meat flavor between meats from different animal production or postmortem conditions. Therefore, muscle and meat metabolites are useful indices to predict or evaluate meat flavor and overall palatability in a comparison between meats of different animal breeds, feeding conditions, and/or postmortem processes. After postmortem aging, amino acids and sugars in muscles make a large contribution to the quality of cooked meat, both as intact forms and as products by the Maillard reaction that occurs between amino compounds and reducing sugars during heat treatment. One of the final volatile products, 2,5-dimethyl-4-hydroxy-3(2H)-furanone (DMHF; furaneol), has been identified as a key aroma compound in beef extract by aroma extract dilution analysis (AEDA) [42]. Even though the aroma of furaneol depends on its concentration, it has great influences on sensory characteristics as a flavor component in meat as well as other foods [78]. In fact, furaneol was detected on Japanese Shorthorn beef during cooking at $180^{\circ} \mathrm{C}$ by analysis with headspace solid-phase micro-extraction (HS/SPME) GC-MS, as well as other flavor-associated volatile compounds [43]. Moreover, inosine- $5^{\prime}$-monophosphate (5'-IMP) greatly contributes to the enhanced flavor of the meat due to its primary role as an umami compound, in a different manner from aroma compounds [79,80]. Thus, the MEATabolomics approach provides tools to access these palatability-associated compounds with the high-throughput analytic systems.

In a beef study combined with flavor-associated evaluation scores, the longissimus thoracis (LT) muscles of Japanese Black (JB) and Holstein cattle were discriminated by the metabolome profiles especially with decanoic acid and glutamine [44], indicating that these compounds were associated with the difference in flavor between the two breeds. Metabolites in beef affecting the sensory attributes have also been explored using commercial ground beef differing in grinding and packaging methods [60]. The study resulted in the determination of 33 metabolites differentiating the grinding and 22 metabolites associated with beef flavor and off-flavor. Results from a pork study of differences between breeds indicated that high carnosine content in meat was associated with a low flavor/taste score in pork [6]. Sensory score-associated variation of sugar content and composition of fatty acids, amino acids, and nucleotides were also observed in a comprehensive analysis of the metabolites in seven brands of JB beef [61]. The results of these studies suggest the influences of animal genetic and feeding factors on sensory evaluation scores via the meat metabolites. It is quite likely that altered contents of amino acids and sugars in the animal and meat-producing processes cause changes in the content of volatile flavor-associated compounds, including furaneol in the cooked meat. Even in the presence of complicated biological and manufactural factors, these studies revealed that metabolomic information is expected to provide indices to predict sensory phenotypes of meat.

\subsection{Chicken Meat Quality Traits}

Chicken meat quality traits are one of the most intensively focused targets in recent MEATabolomics, due to the globally growing market. A Chinese local breed, Wuding chicken meat, was analyzed by the ${ }^{1} \mathrm{H}$ NMR-based approach [45], in which the age of chicken affected the levels of metabolites such as lactate, creatine, IMP, glucose, carnosine, anserine, and taurine. Moreover, the abnormal meat quality of chicken is currently an important issue in the poultry industry. Increasing incidents of muscle abnormalities, including white striping (WS), wooden breast (WB), and spaghetti meat (SM) phenotypes, have been linked to a drastic improvement of breast muscle mass [81]. Metabolomics approaches have been applied to especially elucidate the mechanisms underlying the hardness of 
the WB phenotype. As WB-related biomarker candidates, Abasht et al. [47] identified compounds associated with protein levels, muscle protein degradation, and altered glucose metabolism, using GC-MS and LC-MS/MS followed by RF procedure analysis. Another study with ${ }^{1} \mathrm{H}-\mathrm{NMR}$ approach revealed that WB-affected broiler chicken breasts had a higher content of leucine, valine, alanine, glutamine, lysine, lactate, succinate, taurine, glucose, and IMP, but lower histidine, $\beta$-alanine, acetate, creatine, creatinine, and anserine compared to normal fillets [48]. In another attempt to explore WB myopathy biomarkers, muscle exudate was used as a sample. Linked with discrimination of the samples between WB and non-WB phenotypes, amino acids, nucleotides, and organic acids were identified as the WB-associated metabolites by an NMR approach [49], suggesting the usefulness of those metabolites as the WB markers. This result was partly consistent with the previous study using breast samples with/without WB [48]. WS, on the other hand, has been associated with altered metabolism related to carbohydrates, long-chain fatty acids, and carnitine in a study with GC-MS and LC-MS approaches, suggesting the involvement of altered $\beta$-oxidation in the WS phenotype [50]. A HR-MAS NMR approach revealed that muscle dystrophy of pectoralis in chicken is associated with low content of anserine and carnosine [46]. Thus, the results of these MEATabolomics studies have suggested that the chicken breast muscle abnormalities could be caused by the altered metabolism of carbohydrates, protein and amino acids, and $\beta$-oxidation.

\section{Factors Affecting Muscle and Meat Metabolomes}

As mentioned above, meat is originally skeletal muscles of livestock; consequently, meat quality is greatly affected by factors in the livestock production system, such as animal genetic background, the feeding and stress that animals experienced, but also the physiological characteristics of live muscle, postmortem aging, processing, and spoilage of meat. All the influences of these factors can be assessed by the muscle or meat metabolome profile that is the phenotype resulting from animal experiences through the expression of gene transcription, translation, and the protein modifications as well.

\subsection{Animal Species, Breeds, and Genetic Backgrounds}

A variety of characteristics in meat depends on species or breeds of animals to a great extent. The genetic background of animals thus has an influence on the phenotype expression resulting in an inherent metabolome profile. For the purpose of investigating the effect of genetic background, metabolomics has so far been applied to capture muscle and meat phenotypic differences between species (beef, pork, chicken) [44] or breeds in beef cattle [44] and pigs [6]. The meat of those species was discriminated by PCA of GC-MS metabolome profiles with specific compounds being characteristic to each species [44], although different postmortem muscle metabolism might affect the profile. In comparison between Holstein and the highly marbled JB cattle that differ in oily flavor, sweet flavor, wagyu beef aroma, and the overall evaluation in a sensory test, their LT muscles were discriminated by OPLS-DA [44]. A higher level of decanoic acid in JB than in Holstein cattle, and differences between skeletal muscle, inter-, and intramuscular fat tissues of JB cattle were also observed.

Regarding cattle, in the comparison between different strains of Nellore cattle genetically selected by precocity, a genetic effect on longissimus lumborum (LL) muscle metabolome was observed by ${ }^{1} \mathrm{H}-\mathrm{NMR}$ [51]. The purpose of that study was to investigate changes in metabolites associated with muscle physiology and quality of the LL meat, under the established improving effect of high growth or precocity background on daily gain, carcass characteristics, and other industrial meaningful traits. In the subsequent PLS-DA using the detected compounds (metabolites related to glycolysis and the citric acid cycle, amino acids, organic acids, nucleotides, and sugars), the muscles were discriminated between high and low precocity groups. Pathway analysis in the study highlighted the association of the muscle protein metabolism with the intensity of the selection. Thus, these MEATabolomic studies have revealed their potential to uncover species- or breed-specific differences in metabolites, through which differences in meat quality between breeds can be assessed at the molecular level. 
A study comparing ${ }^{1} \mathrm{H}-\mathrm{NMR}$ metabolome and sensory evaluation scores between five crossbreds of pigs observed an inter-crossbreed effect on metabolites, such as amino acids, lactate, IMP, glycerol, and choline-containing compounds. Some of those compounds were associated with meat quality, including sensory scores [6]. The results also suggested the association of live muscle metabolism, membrane properties, muscle fiber glycolytic potential, lipolysis, and proteolysis with the metabolomic difference.

Furthermore, in a study on pork investigating the relationship between metabolites and drip loss, metabolomics was applied in an integrative way to understand the association of a high drip loss phenotype with the genetic background [35]. In this single nucleotide polymorphism (SNP)-based genome-wide association study (GWAS), a region of candidate genes was identified on chromosome 18 as one associated with drip loss, and the metabolite glycine. Compared to conventional genetic studies using quantitative trait loci (QTL) and candidate genes for drip loss in pigs, GWAS is expected to improve the efficiency of candidate gene detection and accuracy of genomic prediction by avoiding detection of false-positive associations. In beef, GWAS was conducted on JB cattle to explore genes responsible for the palatability of beef, which revealed that SNPs in the ecto-5'-nucleotidase (NT5E) gene affected the content of IMP in the postmortem aged beef, due to the modulating effects of NT5E enzymatic activity. This is a result of which GC- and LC-based metabolomics contributed to GWAS on meat [52,53]. In these comprehensive omics approaches such as GWAS, metabolomics is useful to capture phenotypic metabolites for the candidate genes, due to its wide range of phenotypic molecular measurements.

\subsection{Animal Feeding}

Feeding conditions, one of the environmental factors for animals, have great influences on animal physiology, including skeletal muscle growth and maturation, and the final meat yield and quality. As the feeding factors, the nutritional condition and stress originating from the farming system are considered as the most important ones [82,83], due to their influences on downstream meat production. The influence of feeding on animals are expected to be observed as metabolome changes via gene expression and physiological alteration in tissues. For these reasons, metabolomics approaches have attempted to capture metabolite signatures of feeding systems aiming at the improvement of meat production.

In beef cattle, to address an increasing demand by consumers for beef produced in a sustainable farming system, the effect of differences in feeding on the muscle metabolome was assessed by metabolomic approaches with GC-MS and LC-MS/MS [20]. Between grass- and grain-fed Angus steers, significant variations between the cattle groups was observed in a variety of lipids, including polyunsaturated fatty acid (PUFA) in the latissimus dorsi muscle, showing higher $\omega 3$ and lower $\omega 6$ fatty acids in grass-fed cattle than in the grain-fed. The PCA classified these samples by the diet, and RF analysis based on the metabolites resulted in a predictive accuracy of $100 \%$ between the dietary conditions, in which lipids and amino acids were potential biomarkers discriminating the two groups of cattle. Intriguingly, the cortisol level was lower in grass-fed cattle, suggesting they might experience less stress than the grain-fed [20]. These results indicated that metabolite signatures could be utilized as indices not only in feeding-originated beef quality but also in animal welfare and authentication. In other studies, the effects of dietary amino acids and mate extract administration on beef were also investigated by NMR approaches followed by PCA [21,22]. Biopsy semitendinosus muscle samples of dairy calves fed protein-based milk replacer at 7 weeks of age were classified by amino acid supplementation [21]. Additionally, in a study of feedlot Nellore cattle, the dietary addition of mate extract, one of the antioxidant additives administered to broiler chickens, resulted in increased content of IMP, creatine, carnosine, and conjugated linoleic acid in the LT muscle, and some of these were in a dose-dependent manner [22]. Coupled with redox status analysis, the results further indicated that the beef of mate extract had increased oxidative stability, tenderness, and consumer acceptability. Thus, not only the global feeding conditions but also nutrients and ingredients in the animal feed cause 
changes in muscle and meat metabolomes, which can be assessed by MEATabolomics. In such studies, metabolites can be screened and be identified as potential biomarkers of the feeding.

Regarding pork, a GC-MS approach was applied to the screening of pigs fed with Clenbuterol, a $\beta$-adrenergic agonist [23]. The biceps femoris muscles of pigs treated with Clenbuterol were discriminated from the counterparts in the subsequent PLS-DA by compounds associated with fatty acids and amino acids. Recently, a similar study was conducted using a REIMS approach [26], in which carcasses of pigs fed with ractopamine, another $\beta$-agonist, were discriminated by the lipid profiles of REIMS with high accuracy of classification in three different types of muscle. Due to the high-throughput and accurate performance, this methodology can be widely applied to fields of practical meat research.

Such nutrimetabolomic approaches have also been attempted in chicken, of which growth performance, breast yield, and meat quality are improved with dietary lysine supplementation [12]. Aiming at an increase in flavor compounds in chicken, the effect of dietary lysine supplementation at a 1.5-fold level of a standard requirement on the breast muscle was investigated by a CE-MS approach. Supplementation resulted in increased levels of lysine degradation products, such as saccharopine, $\alpha$-amino adipic acid, and glutamate, the latter being one of the most important taste-improving amino acids in meat [24]. On the other hand, in a study investigating the effect of duck aging on the meat metabolome, duck meat samples of different ages were classified in PCA and PLS-DA by NMR metabolite data, showing increased levels of lactate and anserine and decreased levels of fumarate, betaine, taurine, and inosine with increasing age [25].

\subsection{Muscle Type}

The type of skeletal muscle of pig and cattle is associated with meat quality (WHC, meat color, and tenderness) $[84,85]$ and sensory traits $[86,87]$. This is due to the differences in physiological characteristics $[88,89]$ and compositions of proteins $[90,91]$ and metabolites $[14,54,92,93]$ between different types of muscles that have distinct compositions of fast and slow type muscle fibers. Due to the different physiological properties of the muscle fibers, muscles with different fast/slow fiber type composition undergo different postmortem aging processes, as shown in protein $[94,95]$ and metabolite $[14,16]$ degradation, which could be the cause of the intermuscular difference in meat quality. Contents of flavor-associated metabolites such as amino acids, sugar, and nucleotides differed between porcine LL and vastus intermedius (VI) muscles in a CE-MS metabolomics study [14], and between bovine muscles in an LC-MS [15] or a UPLC-MS/MS study [16]. These results indicate the association of fast and slow type fiber composition with contents of flavor-associated metabolites in aged meat $[14,15]$. It is noticeable that the rate and extent of the postmortem metabolite changes including glycolysis, metabolism of amino acids and nucleotides are different between the muscle types, as shown in different accumulation of lactate in glycolysis, hypoxanthine in purine metabolism, and aliphatic amino acids and methionine in amino acid metabolism [14]. During postmortem aging of pork, thiamine is accumulated, while thiamine triphosphate level declines, which is another difference between muscle types [54]. In addition, the metabolomic difference between longissimus and semitendinosus muscles of four cattle breeds was investigated by an HR-MAS NMR approach, which resulted in a good classification of the two muscles in Chianina and Buffalo, but not in Holstein Friesian and Maremmana cattle [39]. Such intermuscular differences may be associated with differences in meat quality and sensory traits between those muscles.

\subsection{Postmortem Aging and Storage}

Aging and storage of postmortem muscle have great influences on meat quality, as well as animal genetic background and feeding conditions. Even when an animal has muscles of high meat quality potential, the final meat quality could be lowered by inappropriate management, resulting in meat deterioration such as spoilage and discoloration in parallel with abnormal metabolome changes. Inversely, the development of metabolites in meat during postmortem aging under the appropriate condition has a beneficial influence on the final meat quality. 
MEATabolomics has contributed to postmortem meat aging studies to account for influences of postmortem aging on meat quality in terms of metabolites. In pork, postmortem changes in the LL and VI muscles were investigated by a CE-MS approach [14]. The result of this study indicated that a variety of postmortem muscle metabolisms were activated, especially within $24 \mathrm{~h}$ after slaughter. The PCA classified the samples primarily into LL and VI muscles, and secondarily to at-slaughter and day $14(168 \mathrm{~h})$ aging points (Figure 1$)$. In the LL samples, intermediate glycolytic products, glucose 6-phosphate and fructose 6-phosphate (F-6P), increased until $24 \mathrm{~h}$ postmortem, while the downstream compounds such as fructose 1,6-bisphosphate (F-1,6P) and phosphoenolpyruvate decreased with being almost exhausted at $24 \mathrm{~h}$ postmortem, indicating the rate-determining activity of phosphofructokinase $(\mathrm{F}-6 \mathrm{P} \rightarrow \mathrm{F}-1,6 \mathrm{P})$ in postmortem LL muscle glycolysis. Additionally, in the LL muscle, most of the amino acids and identified dipeptides increased after day 1 during the aging, suggesting that protein degradation began to be dominant after day 1. Regarding ATP degradation, marked differences in compound levels were observed in the pathways of ADP $\rightarrow$ AMP $\rightarrow$ IMP $\rightarrow$ inosine $\rightarrow$ hypoxanthine between the LL and VI muscles, suggesting the intermuscular differences in enzymatic activities in the pathways [14]. These pathways in pig LL and VI muscles were overrepresented in the analysis of pathway impact by MetaboAnalyst (Figure 2), indicating a number of biologically meaningful differences in metabolic pathways between the two muscles. Such pathway analyses further afforded new insights: pathways associated with thiamine, pentose phosphate, and NADH metabolism had a high impact on postmortem aging in both muscles. In other studies analyzing bovine LL, psoas major (PM), and/or semimembranosus muscles, intermuscular metabolomic differences in energy metabolism during $24 \mathrm{~h}$ aging [16], and differences in metabolites associated with meat color and lipid oxidation during $23 \mathrm{~d}$ aging [15] were also observed by LC-MS-based approaches. Thus, the skeletal muscle type is associated with the muscle metabolome as described above, in which postmortem aging makes the intermuscular metabolome differences inherent to live animals further complicated, as shown in the complexity of the differences in the final meat quality. Nevertheless, MEATabolomics approaches have shown its advantages to discriminate meat samples by muscle type and postmortem aging conditions.

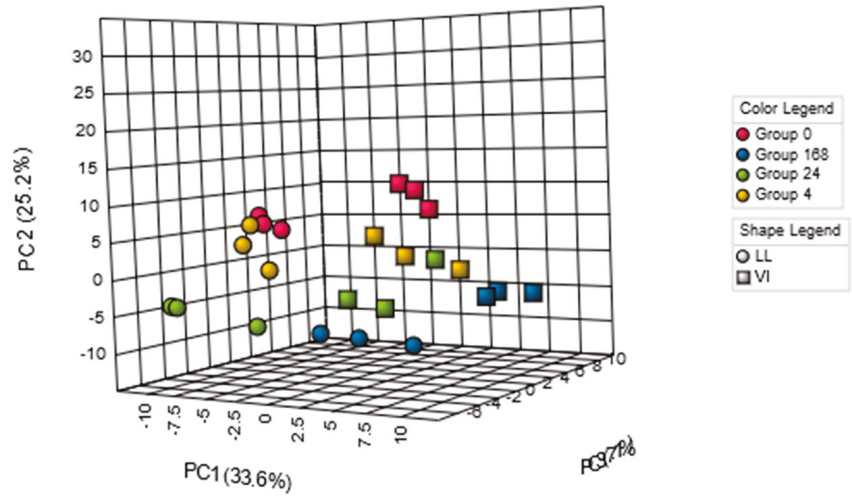

Figure 1. Classification of pig LL and VI muscle samples of different postmortem aging period by PCA. Pig LL and VI muscles were aged during $0,4,24,168 \mathrm{~h}$ postmortem. 

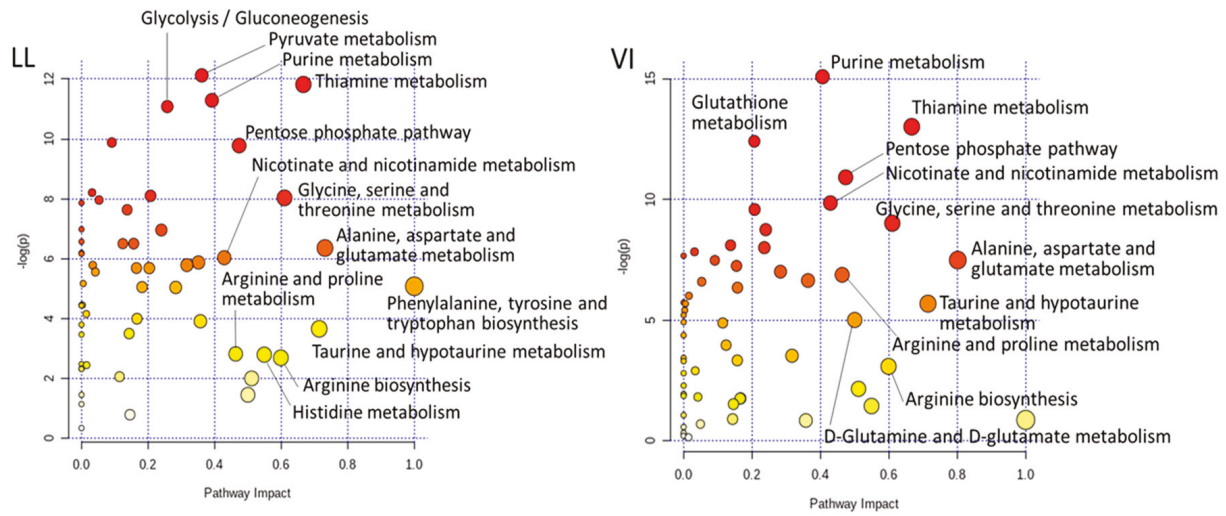

Figure 2. Metabolic pathway impact analysis of pig LL and VI muscles during postmortem aging. Pathway impact values (horizontal axis) were calculated from the pathway topology analysis in MetaboAnalyst. A pathway with small $p$ value and high impact is plotted as a red and large circle.

Regarding beef, metabolomic changes during postmortem aging was also analyzed by CE-MS [17] and NMR approaches $[18,19]$ in longissimus muscle. In the CE-MS metabolomics targeting charged metabolites, changes in nutritionally important compounds such as choline, nicotinamide, and thiamine were observed during $14 \mathrm{~d}$ aging of JB cattle beef, some of which contributed to classification of the samples by the aging period [17]. On the other hand, NMR studies were conducted for metabolomic profiling during 10 weeks [18] and 21 days [19] postmortem. The former NMR study aimed to explore excellent biomarkers of postmortem JB beef aged up to 10 weeks, and revealed that leucine and creatine are available as biomarkers for long-term aging ( $>2$ weeks) because of positive and negative correlation with aging duration, respectively [18]. The L-value, defined as the ratio of leucine to creatine in the study, was more appropriate to evaluate meat quality during long-term aging than the conventional K-value that is calculated by using the contents of ATP, ADP, AMP, and IMP. In addition, this study also showed that the NMR metabolomics was able to estimate unsaturation degree of triacylglycerol that was correlated to content ratio of unsaturated fatty acids $\left(R^{2}=0.944\right)$ and melting point of intramuscular fat $\left(R^{2}=0.871\right)$. This suggests that such an approach can evaluate not only postmortem meat aging and intramuscular fat content, but also palatability-associated quality traits such as juiciness and WHC that might be assessed through NMR-measured $\mathrm{pH}$ profile [18] in highly marbled JB beef. Besides, biomarker candidates for prolonged postmortem aging (up to 44 days) of Piedmontese LT beef was explored by the LC-MS approach [55]. The results indicated the possibility of glutamate, serine, and arginine being candidates of postmortem aging indicators due to their constant increase until day 44 . The prolonged aging period likely enhanced the accumulation of amino acids and some other metabolites. In these studies, metabolomics approaches combined with statistical analyses have enabled screening of metabolic biomarker candidates of postmortem aging from the meat metabolome dataset. Thus, metabolomics have contributed not only to the understanding of postmortem muscle metabolisms but also to the finding of better biomarkers that the conventional studies have never assessed. These MEATabolomics of postmortem aging of pork and beef re-emphasize the possibility that the amino acid generation during cold storage is primarily due to postmortem protein degradation in the muscles, as shown in previous studies of aging [31,94-101]. Besides, the elucidation of flavor-associated compound generation from muscle metabolites has been accelerated with the accumulating knowledge of the uncovered meat metabolomes through postmortem aging studies on cooked meat [43].

Other optional postmortem storage conditions are also to be investigated due to the influences on a variety of metabolites associated with meat quality traits. A dry-aging condition of $3{ }^{\circ} \mathrm{C}$ with 
$0.2 \mathrm{~m} / \mathrm{s}$ air-velocity for 3 weeks showed high improvement of beef palatability in a sensory panel evaluation compared to conditions of general wet-aging and other dry conditions, with higher contents of compounds including branched amino acids and IMP in a study by an NMR approach [56]. The air flow of this storage method might simply accelerate the up-concentration of the flavor-associated metabolites, but other undetermined factors are likely also involved. On the other hand, the chilling rate of lamb LT muscle during aging affected the muscle energy metabolism within $24 \mathrm{~h}$ postmortem, which was associated with meat tenderness in a study utilizing ${ }^{1} \mathrm{H}-,{ }^{31} \mathrm{P}-\mathrm{NMR}$, and LC-MS [57]. MEATabolomics was also conducted to investigate roles of NADH in discoloration mechanism via mitochondria activity during postmortem wet-aging of bovine LL muscle [58]. The results suggested that discoloration during postmortem aging of beef was due to increased oxidative stress, mitochondrial damages, and decreasing metabolite sources to regenerate NADH.

\subsection{Processing}

Meat processing is important to improve the microbiological safety, color, flavor, and texture for the development of favorable meat products. Product chemical information in processing is useful to monitor the processing steps for quality management and to design better products for quality development. To date, metabolomics has been used for the characterization of processed products in studies that assess effects of marinating conditions of chicken breast fillets $[7,29]$ and the marination time on pork [30], and processing conditions on dry-cured hams $[27,28]$ and fermented sausages [59], and texture defects in dry-cured ham [102]. Metabolomic profiling of hams from Japan and European countries identified a total of 203 charged metabolites by CE-MS, and revealed that redness and fat whiteness are associated with metabolomic profiles. The result of this study suggested that the metabolome of hams might be affected by country of origin and processing methods such as smoking and use of starter culture [27]. Difference between Chinese dry-cured and other hams were investigated by NMR metabolomics, in which a total of 33 charged metabolites were identified and each ham was characterized by a specific metabolite set [28]. In an application to a traditional Spanish fermented salchichón sausage, a HR-MAS NMR approach showed that the metabolome profile changed, depending primarily on the fermentation and secondarily on the drying process [59]. This result suggested that proteolysis and lipolysis attributed to microbial activities could be monitored by NMR metabolomics.

Metabolomics was also used to test the effect of marination and storage conditions on preservation and sensory quality of chicken breast fillets. By comparison between different types of marinade, temperatures, and intervals during marinating process, each marinade was characterized by a distinct organic acid profile [29]. The results of this study also showed that marinating time has influence on the indigenous microorganisms and the sensory characteristics. A subsequent study to test the effect of the processing conditions (marinade type, storage, and microbial load) showed that the profiles of organic acid and volatile compounds were discriminated between pomegranate-based marinated and control samples according to storage time, microbial load, and sensory score [7]. On the other hand, in a Chinese traditional marinated meat product, amino acids, sugars, acetate, succinate, uracil and inosine increased during marinating, while lactate, creatine, IMP and anserine decreased [30]. In this study, combined with sensory test, a negative effect on the taste of marinating meat in soy sauce was observed during the late stage of dry-ripening, accompanying decreases in most of the metabolites, which suggests that shortening the dry-ripening period could be better to improve the taste quality.

\subsection{Spoilage}

Spoilage developed by microbiological activity has been the greatest concern for consumers in terms of meat safety. Meat spoilage is developed through microbial growth during meat cutting, storage, and distribution processes after the slaughter of animals. Microbial growth causes chemical changes that result from changes in the microorganism itself and the metabolic output on meat. Therefore, chemical information acquired by metabolomics is expected to be utilized to quantify the 
degree of spoilage and predict the number or activity of microorganisms [103]. As a candidate of such compound set, volatile organic compounds (VOC) have been focused on in metabolomic studies of spoilage. In an attempt targeting microorganism-associated VOC, metabolome profiling, combined with a multivariate analysis utilizing total ion currents, was able to distinguish naturally spoiled pork samples from those artificially contaminated with Salmonella typhimurium, a food poisoning pathogen commonly recovered from pork products [62]. Levels of a total of 16 compounds, including phenylethyl alcohol and dimethyl disulfide, differed between the contaminated and non-contaminated pork samples. However, the identified compounds in such approaches to microorganism-associated VOC may depend on the multivariate analysis algorithm or other factors originating from meat samples [63]. Impact of other microorganisms (Pseudomonas spp., Brochothrix thermosphacta, lactic acid bacteria, Enterobacteriaceae, and yeasts/molds) on minced beef under the various temperature and packaging conditions was also investigated by a LC-MS approach [64].

With high sensitivity and good selectivity, HS/SPME GC-MS was applied in a study to assess spoilage of minced beef [65]. In this study, the authors focused on influences of temperature and atmosphere in packaging during storage of minced beef with indigenous microorganisms. According to the result, identified compounds such as 2-pentanone and 2-heptanone significantly contributed to unacceptable sensory scores and were associated with spoiled samples. This study showed that the GC-MS-based approach has a potential to estimate microbial counts of the different microorganisms and sensory scores of a meat samples independently of storage conditions.

\subsection{Authentication}

To address the growing consumer awareness, the meat industry has a need for systems to guarantee the authenticity of the meat and the products in order to take measures against increasing fraud/adulteration in the growing complexity of global food chains. Regarding beef, concerns about bovine spongiform encephalopathy (BSE) have increased the awareness of safety. In this field, numerous DNA and protein-based target detection techniques, including species-specific PCR, has been proved to be effective. MEATabolomics is expected to provide useful chemical information regarding authenticity as an alternative method. In this context, analysis of geographic origin of beef by metabolomics was attempted with NMR [66] and imaging MS (IMS) [67]. In the NMR approach, extracts of imported beef from 4 countries were discriminated in the results of PCA and OPLS-DA. The major compounds responsible for the discrimination contained succinate and some of amino acids, suggesting that these compounds could be potential biomarkers to discriminate the geographic origin of beef and that NMR has potential to efficiently work for this analysis. IMS-based direct analysis visualizes distribution of target biomolecules such as lipids, glycolipids, and peptides on biological tissue section samples, without complicated pretreatment procedures. In a matrix-associated laser desorption/ionization-IMS analysis of beef from 3 different Japanese regions, the three types of beef were discriminated by subsequent PCA [67]. In these analyses of geographic origin of beef, although geographic difference in metabolome changes may be due to multiple factors of animal breeds, feeds, and environment, including water and climate, the results revealed availability of metabolomics to distinguish production regions. Geographic differences may also contain the production systems of animals. A study aiming to discriminate the pre-slaughter production system was able to distinguish differences between 1-year cattle fed with barley-based concentrate indoors and with pasture feeding outdoors, using NMR metabolomics followed by PLS-DA, analyzing the longissimus dorsi (LD) muscle [68]. The results suggested that NMR approach is suitable for authentication of cattle production history.

Challenges to discriminate beef, pork, and mixture samples by metabolomics were also conducted recently for the establishment of measures against meat adulteration. To this end, different grades of minced beef and pork samples were mixed in various ratios and analyzed by GC-MS and reverse-phase LC-MS approaches [69]. The metabolite content and percentages of fat declared on meat product labeling were correlated each other, and the species of meat was identified by chemometrics using differential metabolite sets. Another volatilomic approach using HS/SPME GC-MS was also applied 
for the similar purpose [70]. In this study, multiple volatile compounds correlated not only to beef and pork but also the mixture was identified. With use of datasets divided $70 \%$ for model calibration and $30 \%$ for model prediction, the overall correct classification rate was $99 \%$ on average in both datasets. As the authors concluded, this volatilomic approach could be developed for robust and reliable off-line discrimination of meat samples. LC-MS approaches were also applied to classification of chicken meats into normally slaughtered and dead on arrival [71,72]. Such applications along with the development of discrimination analysis is expected to solve the current fraud issues related not only to chicken but also beef and pork.

On the other hand, the irradiation of meat is currently highlighted as an issue for its negative impact in cases when used out of appropriate range of strength, despite positive evaluation of the irradiation effect on meat such as disinfestation, growth inhibition, parasite control, reduction of pathogenic bacteria, and shelf-life extension. Metabolomics is expected to be a tool to monitor the negative impact of irradiation, but it needs to be a non-time-consuming, non-invasive, and reproducible method. In this regard, NMR metabolomics seems suitable for such purpose, and, accordingly, has been used in attempts to investigate the influence of irradiation on beef [73,74]. In the analysis targeting lipids, stepwise linear discriminant analysis (sLDA) following the NMR data profiling allowed the classification of $81.9 \%$ of the beef samples according to the irradiation dose $(0,2.5,4.5$, and $8 \mathrm{kGy}$ ) [73]. Moreover, the NMR analysis targeting hydrophilic compounds, combined with subsequent classification tree (CT) analysis, was able to distinguish between the irradiated and non-irradiated beef samples [74]. In addition, glycerol, lactic acid esters, and tyramine were found to be important biomarkers for the classification. Thus, MEATabolomics has been applied in a variety of research fields associated with meat production methods and the quality traits, including authentication.

\section{Conclusions}

MEATabolomics has allowed us to better understand skeletal muscle physiology in animals and molecular factors associated with meat quality. Information raised in MEATabolomics can be used as phenotypic indices of muscle properties and meat quality traits, which connects the external phenotype of meat (the quality traits) to regulatory factors in animals or conditions in the production systems. The techniques for metabolomics have been progressing, especially in the process of statistical data analyses, as shown in some examples of attempts to introduce new algorithms and to develop models for phenotype prediction. On the other hand, metabolomics targeting animal blood and meat exudate metabolome, along with use of meat metabolome data, would promote the prediction of meat quality as a non-invasive methodology by utilizing these data. Further studies on the associations of muscles and meat with animal development, stress conditions, welfare and sustainability issues, cooking methods, consumer acceptability, and sensory characteristics would be future challenges for MEATabolomics to be applied to, despite the complex biological processes during meat production and difficulties in chemical identification of secondary metabolites observed in processed and cooked meat. Nevertheless, MEATabolomics is expected to further expand comprehensive association studies with genomics or transcriptomics in an integrative fashion, being supported by the development of new detecting techniques such as REIMS and statistical analytic resources.

Author Contributions: Conceptualization, S.M.; writing—original draft preparation, S.M.; writing—review and editing, T.M., S.U., T.K., and P.E. All authors have read and agreed to the published version of the manuscript.

Funding: This research was partly supported by the Japan Society for the Promotion of Science (JSPS KAKENHI JP18K05960 to SM).

Conflicts of Interest: The authors declare no conflict of interest. 


\section{References}

1. Rubert, J.; Zachariasova, M.; Hajslova, J. Advances in high-resolution mass spectrometry based on metabolomics studies for food-A review. Food Addit. Contam. Part. A 2015, 32, 1685-1708. [CrossRef] [PubMed]

2. Consonni, R.; Cagliani, L.R. The potentiality of NMR-based metabolomics in food science and food authentication assessment. Magn. Reson. Chem. 2019, 57, 558-578. [CrossRef] [PubMed]

3. García-Cañas, V.; Simó, C.; Herrero, M.; Ibáñez, E.; Cifuentes, A. Present and Future Challenges in Food Analysis: Foodomics. Anal. Chem. 2012, 84, 10150-10159. [CrossRef] [PubMed]

4. Junot, C.; Fenaille, F.; Colsch, B.; Bécher, F. High resolution mass spectrometry based techniques at the crossroads of metabolic pathways. Mass Spectrom. Rev. 2014, 33, 471-500. [CrossRef]

5. Sugimoto, M.; Kawakami, M.; Robert, M.; Soga, T.; Tomita, M. Bioinformatics Tools for Mass Spectroscopy-Based Metabolomic Data Processing and Analysis. Curr. Bioinform. 2012, 7, 96-108. [CrossRef]

6. Straadt, I.K.; Aaslyng, M.D.; Bertram, H.C. An NMR-based metabolomics study of pork from different crossbreeds and relation to sensory perception. Meat Sci. 2014, 96, 719-728. [CrossRef]

7. Lytou, A.E.; Nychas, G.E.; Panagou, E.Z. Effect of pomegranate based marinades on the microbiological, chemical and sensory quality of chicken meat: A metabolomics approach. Int. J. Food Microbiol. 2018, 267, 42-53. [CrossRef]

8. Lee, S.M.; Kwon, G.Y.; Kim, K.O.; Kim, Y.S. Metabolomic approach for determination of key volatile compounds related to beef flavor in glutathione-Maillard reaction products. Anal. Chim. Acta 2011, 703, 204-211. [CrossRef]

9. Adamski, J.; Suhre, K. Metabolomics platforms for genome wide association studies-linking the genome to the metabolome. Curr. Opin. Biotechnol. 2013, 24, 39-47. [CrossRef]

10. Fontanesi, L. Metabolomics and livestock genomics: Insights into a phenotyping frontier and its applications in animal breeding. Anim. Front. 2016, 6, 73-79. [CrossRef]

11. Berri, C.; Picard, B.; Lebret, B.; Andueza, D.; Lefevre, F.; Le Bihan-Duval, E.; Beauclercq, S.; Chartrin, P.; Vautier, A.; Legrand, I.; et al. Predicting the Quality of Meat: Myth or Reality? Foods 2019, 8. [CrossRef] [PubMed]

12. Zampiga, M.; Flees, J.; Meluzzi, A.; Dridi, S.; Sirri, F. Application of omics technologies for a deeper insight into quali-quantitative production traits in broiler chickens: A review. J. Anim. Sci. Biotechnol. 2018, 9, 61. [CrossRef] [PubMed]

13. Picard, B.; Lebret, B.; Cassar-Malek, I.; Liaubet, L.; Berri, C.; Le Bihan-Duval, E.; Hocquette, J.F.; Renand, G. Recent advances in omic technologies for meat quality management. Meat Sci. 2015, 109, 18-26. [CrossRef] [PubMed]

14. Muroya, S.; Oe, M.; Nakajima, I.; Ojima, K.; Chikuni, K. CE-TOF MS-based metabolomic profiling revealed characteristic metabolic pathways in postmortem porcine fast and slow type muscles. Meat Sci. 2014, 98, 726-735. [CrossRef]

15. Ma, D.; Kim, Y.H.B.; Cooper, B.; Oh, J.H.; Chun, H.; Choe, J.H.; Schoonmaker, J.P.; Ajuwon, K.; Min, B. Metabolomics Profiling to Determine the Effect of Postmortem Aging on Color and Lipid Oxidative Stabilities of Different Bovine Muscles. J. Agric. Food Chem. 2017, 65, 6708-6716. [CrossRef]

16. Yu, Q.; Tian, X.; Shao, L.; Li, X.; Dai, R. Targeted metabolomics to reveal muscle-specific energy metabolism between bovine longissimus lumborum and psoas major during early postmortem periods. Meat Sci. 2019, 156, 166-173. [CrossRef]

17. Muroya, S.; Oe, M.; Ojima, K.; Watanabe, A. Metabolomic approach to key metabolites characterizing postmortem aged loin muscle of Japanese Black (Wagyu) cattle. Asian Australas. J. Anim. Sci. 2019, 32, 1172-1185. [CrossRef]

18. Kodani, Y.; Miyakawa, T.; Komatsu, T.; Tanokura, M. NMR-based metabolomics for simultaneously evaluating multiple determinants of primary beef quality in Japanese Black cattle. Sci. Rep. 2017, 7, 1297. [CrossRef]

19. Graham, S.F.; Kennedy, T.; Chevallier, O.; Gordon, A.; Farmer, L.; Elliott, C.; Moss, B. The application of NMR to study changes in polar metabolite concentrations in beef longissimus dorsi stored for different periods post mortem. Metabolomics 2010, 6, 395-404. [CrossRef] 
20. Carrillo, J.A.; He, Y.; Li, Y.; Liu, J.; Erdman, R.A.; Sonstegard, T.S.; Song, J. Integrated metabolomic and transcriptome analyses reveal finishing forage affects metabolic pathways related to beef quality and animal welfare. Sci. Rep. 2016, 6, 25948. [CrossRef]

21. Yu, K.; Matzapetakis, M.; Valent, D.; Saco, Y.; De Almeida, A.M.; Terre, M.; Bassols, A. Skeletal muscle metabolomics and blood biochemistry analysis reveal metabolic changes associated with dietary amino acid supplementation in dairy calves. Sci. Rep. 2018, 8, 13850. [CrossRef] [PubMed]

22. De Zawadzki, A.; Arrivetti, L.O.R.; Vidal, M.P.; Catai, J.R.; Nassu, R.T.; Tullio, R.R.; Berndt, A.; Oliveira, C.R.; Ferreira, A.G.; Neves-Junior, L.F.; et al. Mate extract as feed additive for improvement of beef quality. Food Res. Int. 2017, 99, 336-347. [CrossRef] [PubMed]

23. Li, G.; Fu, Y.; Han, X.; Li, X.; Li, C. Metabolomic investigation of porcine muscle and fatty tissue after Clenbuterol treatment using gas chromatography/mass spectrometry. J. Chromatogr. A 2016, 1456, 242-248. [CrossRef] [PubMed]

24. Watanabe, G.; Kobayashi, H.; Shibata, M.; Kubota, M.; Kadowaki, M.; Fujimura, S. Regulation of free glutamate content in meat by dietary lysine in broilers. Anim. Sci. J. 2015, 86, 435-442. [CrossRef]

25. Liu, C.; Pan, D.; Ye, Y.; Cao, J. ${ }^{1} \mathrm{H}$ NMR and multivariate data analysis of the relationship between the age and quality of duck meat. Food Chem. 2013, 141, 1281-1286. [CrossRef]

26. Guitton, Y.; Dervilly-Pinel, G.; Jandova, R.; Stead, S.; Takats, Z.; Le Bizec, B. Rapid evaporative ionisation mass spectrometry and chemometrics for high-throughput screening of growth promoters in meat producing animals. Food Addit. Contam. Part A 2018, 35, 900-910. [CrossRef]

27. Sugimoto, M.; Obiya, S.; Kaneko, M.; Enomoto, A.; Honma, M.; Wakayama, M.; Soga, T.; Tomita, M. Metabolomic Profiling as a Possible Reverse Engineering Tool for Estimating Processing Conditions of Dry-Cured Hams. J. Agric. Food Chem. 2017, 65, 402-410. [CrossRef]

28. Zhang, J.; Ye, Y.; Sun, Y.; Pan, D.; Ou, C.; Dang, Y.; Wang, Y.; Cao, J.; Wang, D. ${ }^{1} \mathrm{H}$ NMR and multivariate data analysis of the differences of metabolites in five types of dry-cured hams. Food Res. Int. 2018, 113, 140-148. [CrossRef]

29. Lytou, A.E.; Panagou, E.Z.; Nychas, G.E. Effect of different marinating conditions on the evolution of spoilage microbiota and metabolomic profile of chicken breast fillets. Food Microbiol. 2017, 66, 141-149. [CrossRef]

30. Yang, Y.; Ye, Y.; Pan, D.; Sun, Y.; Wang, Y.; Cao, J. Metabonomics profiling of marinated meat in soy sauce during processing. J. Sci. Food Agric. 2018, 98, 1325-1331. [CrossRef]

31. Huff-Lonergan, E.; Zhang, W.; Lonergan, S.M. Biochemistry of postmortem muscle-Lessons on mechanisms of meat tenderization. Meat Sci. 2010, 86, 184-195. [CrossRef]

32. Ramautar, R.; Somsen, G.W.; De Jong, G.J. CE-MS for metabolomics: Developments and applications in the period 2016-2018. Electrophoresis 2019, 40, 165-179. [CrossRef]

33. Stolz, A.; Jooss, K.; Hocker, O.; Romer, J.; Schlecht, J.; Neususs, C. Recent advances in capillary electrophoresis-mass spectrometry: Instrumentation, methodology and applications. Electrophoresis 2019, 40, 79-112. [CrossRef]

34. Welzenbach, J.; Neuhoff, C.; Looft, C.; Schellander, K.; Tholen, E.; Grosse-Brinkhaus, C. Different Statistical Approaches to Investigate Porcine Muscle Metabolome Profiles to Highlight New Biomarkers for Pork Quality Assessment. PLoS ONE 2016, 11, e0149758. [CrossRef] [PubMed]

35. Welzenbach, J.; Neuhoff, C.; Heidt, H.; Cinar, M.U.; Looft, C.; Schellander, K.; Tholen, E.; Grosse-Brinkhaus, C. Integrative Analysis of Metabolomic, Proteomic and Genomic Data to Reveal Functional Pathways and Candidate Genes for Drip Loss in Pigs. Int. J. Mol. Sci. 2016, 17. [CrossRef] [PubMed]

36. Gredell, D.A.; Schroeder, A.R.; Belk, K.E.; Broeckling, C.D.; Heuberger, A.L.; Kim, S.-Y.; King, D.A.; Shackelford, S.D.; Sharp, J.L.; Wheeler, T.L.; et al. Comparison of Machine Learning Algorithms for Predictive Modeling of Beef Attributes Using Rapid Evaporative Ionization Mass Spectrometry (REIMS) Data. Sci. Rep. 2019, 9, 5721. [CrossRef]

37. Cameron, S.J.S.; Alexander, J.L.; Bolt, F.; Burke, A.; Ashrafian, H.; Teare, J.; Marchesi, J.R.; Kinross, J.; Li, J.V.; Takáts, Z. Evaluation of Direct from Sample Metabolomics of Human Feces Using Rapid Evaporative Ionization Mass Spectrometry. Anal. Chem. 2019, 91, 13448-13457. [CrossRef] [PubMed]

38. Balog, J.; Sasi-Szabó, L.; Kinross, J.; Lewis, M.R.; Muirhead, L.J.; Veselkov, K.; Mirnezami, R.; Dezső, B.; Damjanovich, L.; Darzi, A.; et al. Intraoperative tissue identification using rapid evaporative ionization mass spectrometry. Sci. Transl. Med. 2013, 5, 194ra193. [CrossRef] [PubMed] 
39. Ritota, M.; Casciani, L.; Failla, S.; Valentini, M. HRMAS-NMR spectroscopy and multivariate analysis meat characterisation. Meat Sci. 2012, 92, 754-761. [CrossRef] [PubMed]

40. Subbaraj, A.K.; Kim, Y.H.; Fraser, K.; Farouk, M.M. A hydrophilic interaction liquid chromatography-mass spectrometry (HILIC-MS) based metabolomics study on colour stability of ovine meat. Meat Sci. 2016, 117, 163-172. [CrossRef]

41. Beauclercq, S.; Nadal-Desbarats, L.; Hennequet-Antier, C.; Collin, A.; Tesseraud, S.; Bourin, M.; Le Bihan-Duval, E.; Berri, C. Serum and Muscle Metabolomics for the Prediction of Ultimate pH, a Key Factor for Chicken-Meat Quality. J. Proteom. Res. 2016, 15, 1168-1178. [CrossRef] [PubMed]

42. Takakura, Y.; Sakamoto, T.; Hirai, S.; Masuzawa, T.; Wakabayashi, H.; Nishimura, T. Characterization of the key aroma compounds in beef extract using aroma extract dilution analysis. Meat Sci. 2014, 97, 27-31. [CrossRef] [PubMed]

43. Watanabe, A.; Kamada, G.; Imanari, M.; Shiba, N.; Yonai, M.; Muramoto, T. Effect of aging on volatile compounds in cooked beef. Meat Sci. 2015, 107, 12-19. [CrossRef] [PubMed]

44. Ueda, S.; Iwamoto, E.; Kato, Y.; Shinohara, M.; Shirai, Y.; Yamanoue, M. Comparative metabolomics of Japanese Black cattle beef and other meats using gas chromatography-mass spectrometry. Biosci. Biotechnol. Biochem. 2018. [CrossRef]

45. Xiao, Z.; Ge, C.; Zhou, G.; Zhang, W.; Liao, G. ${ }^{1}$ H NMR-based metabolic characterization of Chinese Wuding chicken meat. Food Chem. 2019, 274, 574-582. [CrossRef]

46. Sundekilde, U.K.; Rasmussen, M.K.; Young, J.F.; Bertram, H.C. High resolution magic angle spinning NMR spectroscopy reveals that pectoralis muscle dystrophy in chicken is associated with reduced muscle content of anserine and carnosine. Food Chem. 2017, 217, 151-154. [CrossRef]

47. Abasht, B.; Mutryn, M.F.; Michalek, R.D.; Lee, W.R. Oxidative Stress and Metabolic Perturbations in Wooden Breast Disorder in Chickens. PLoS ONE 2016, 11, e0153750. [CrossRef]

48. Wang, Y.; Yang, Y.; Pan, D.; He, J.; Cao, J.; Wang, H.; Ertbjerg, P. Metabolite profile based on ${ }^{1} \mathrm{H}$ NMR of broiler chicken breasts affected by wooden breast myodegeneration. Food Chem. 2020, 310, 125852. [CrossRef]

49. Xing, T.; Zhao, X.; Xu, X.; Li, J.; Zhang, L.; Gao, F. Physiochemical properties, protein and metabolite profiles of muscle exudate of chicken meat affected by wooden breast myopathy. Food Chem. 2020, 316, 126271. [CrossRef]

50. Boerboom, G.; Van Kempen, T.; Navarro-Villa, A.; Perez-Bonilla, A. Unraveling the cause of white striping in broilers using metabolomics. Poult. Sci. 2018, 97, 3977-3986. [CrossRef]

51. Consolo, N.R.B.; Silva, J.D.; Buarque, V.L.M.; Higuera-Padilla, A.; Barbosa, L.; Zawadzki, A.; Colnago, L.A.; Netto, A.S.; Gerrard, D.E.; Silva, S.L. Selection for Growth and Precocity Alters Muscle Metabolism in Nellore Cattle. Metabolites 2020, 10. [CrossRef] [PubMed]

52. Sakuma, H.; Saito, K.; Kohira, K.; Ohhashi, F.; Shoji, N.; Uemoto, Y. Estimates of genetic parameters for chemical traits of meat quality in Japanese black cattle. Anim. Sci. J. 2017, 88, 203-212. [CrossRef] [PubMed]

53. Komatsu, T.; Komatsu, M.; Uemoto, Y. The NT5E gene variant strongly affects the degradation rate of inosine 5'-monophosphate under postmortem conditions in Japanese Black beef. Meat Sci. 2019, 158, 107893. [CrossRef] [PubMed]

54. Muroya, S.; Oe, M.; Ojima, K. Thiamine accumulation and thiamine triphosphate decline occur in parallel with ATP exhaustion during postmortem aging of pork muscles. Meat Sci. 2018, 137, 228-234. [CrossRef]

55. Lana, A.; Longo, V.; Dalmasso, A.; D'Alessandro, A.; Bottero, M.T.; Zolla, L. Omics integrating physical techniques: Aged Piedmontese meat analysis. Food Chem. 2015, 172, 731-741. [CrossRef]

56. Kim, Y.H.B.; Kemp, R.; Samuelsson, L.M. Effects of dry-aging on meat quality attributes and metabolite profiles of beef loins. Meat Sci. 2016, 111, 168-176. [CrossRef]

57. Warner, R.D.; Jacob, R.H.; Rosenvold, K.; Rochfort, S.; Trenerry, C.; Plozza, T.; McDonagh, M.B. Altered post-mortem metabolism identified in very fast chilled lamb $\mathrm{M}$. longissimus thoracis et lumborum using metabolomic analysis. Meat Sci. 2015, 108, 155-164. [CrossRef]

58. Mitacek, R.M.; Ke, Y.; Prenni, J.E.; Jadeja, R.; VanOverbeke, D.L.; Mafi, G.G.; Ramanathan, R. Mitochondrial Degeneration, Depletion of NADH, and Oxidative Stress Decrease Color Stability of Wet-Aged Beef Longissimus Steaks. J. Food Sci. 2019, 84, 38-50. [CrossRef]

59. García-García, A.B.; Lamichhane, S.; Castejón, D.; Cambero, M.I.; Bertram, H.C. ${ }^{1}$ H HR-MAS NMR-based metabolomics analysis for dry-fermented sausage characterization. Food Chem. 2018, 240, 514-523. [CrossRef] 
60. Jiang, T.; Bratcher, C.L. Differentiation of commercial ground beef products and correlation between metabolites and sensory attributes: A metabolomic approach. Food Res. Int. 2016, 90, 298-306. [CrossRef]

61. Suzuki, K.; Shioura, H.; Yokota, S.; Katoh, K.; Roh, S.-g.; Iida, F.; Komatsu, T.; Syoji, N.; Sakuma, H.; Yamada, S. Search for an index for the taste of Japanese Black cattle beef by panel testing and chemical composition analysis. Anim. Sci. J. 2017, 88, 421-432. [CrossRef] [PubMed]

62. Xu, Y.; Cheung, W.; Winder, C.L.; Goodacre, R. VOC-based metabolic profiling for food spoilage detection with the application to detecting Salmonella typhimurium-contaminated pork. Anal. Bioanal. Chem. 2010, 397, 2439-2449. [CrossRef]

63. Xu, Y.; Cheung, W.; Winder, C.L.; Dunn, W.B.; Goodacre, R. Metabolic profiling of meat: Assessment of pork hygiene and contamination with Salmonella typhimurium. Analyst 2011, 136, 508-514. [CrossRef] [PubMed]

64. Argyri, A.A.; Doulgeraki, A.I.; Blana, V.A.; Panagou, E.Z.; Nychas, G.-J.E. Potential of a simple HPLC-based approach for the identification of the spoilage status of minced beef stored at various temperatures and packaging systems. Int. J. Food Microbiol. 2011, 150, 25-33. [CrossRef] [PubMed]

65. Argyri, A.A.; Mallouchos, A.; Panagou, E.Z.; Nychas, G.J. The dynamics of the HS/SPME-GC/MS as a tool to assess the spoilage of minced beef stored under different packaging and temperature conditions. Int. J. Food Microbiol. 2015, 193, 51-58. [CrossRef]

66. Jung, Y.; Lee, J.; Kwon, J.; Lee, K.S.; Ryu, D.H.; Hwang, G.S. Discrimination of the geographical origin of beef by ${ }^{1} \mathrm{H}$ NMR-based metabolomics. J. Agric. Food Chem. 2010, 58, 10458-10466. [CrossRef]

67. Zaima, N.; Goto-Inoue, N.; Hayasaka, T.; Enomoto, H.; Setou, M. Authenticity assessment of beef origin by principal component analysis of matrix-assisted laser desorption/ionization mass spectrometric data. Anal. Bioanal. Chem. 2011, 400, 1865-1871. [CrossRef]

68. Osorio, M.T.; Moloney, A.P.; Brennan, L.; Monahan, F.J. Authentication of beef production systems using a metabolomic-based approach. Animal 2012, 6, 167-172. [CrossRef]

69. Trivedi, D.K.; Hollywood, K.A.; Rattray, N.J.; Ward, H.; Greenwood, J.; Ellis, D.I.; Goodacre, R. Meat, the metabolites: An integrated metabolite profiling and lipidomics approach for the detection of the adulteration of beef with pork. Analyst 2016, 141, 2155-2164. [CrossRef]

70. Pavlidis, D.E.; Mallouchos, A.; Ercolini, D.; Panagou, E.Z.; Nychas, G.E. A volatilomics approach for off-line discrimination of minced beef and pork meat and their admixture using HS-SPME GC/MS in tandem with multivariate data analysis. Meat Sci. 2019, 151, 43-53. [CrossRef]

71. Sidwick, K.L.; Johnson, A.E.; Adam, C.D.; Pereira, L.; Thompson, D.F. Use of Liquid Chromatography Quadrupole Time-of-Flight Mass Spectrometry and Metabonomic Profiling To Differentiate between Normally Slaughtered and Dead on Arrival Poultry Meat. Anal. Chem. 2017, 89, 12131-12136. [CrossRef]

72. Cao, M.; Han, Q.; Zhang, J.; Zhang, R.; Wang, J.; Gu, W.; Kang, W.; Lian, K.; Ai, L. An untargeted and pseudotargeted metabolomic combination approach to identify differential markers to distinguish live from dead pork meat by liquid chromatography-mass spectrometry. J. Chromatograph. A 2020, 1610, 460553. [CrossRef] [PubMed]

73. Zanardi, E.; Caligiani, A.; Padovani, E.; Mariani, M.; Ghidini, S.; Palla, G.; Ianieri, A. Detection of irradiated beef by nuclear magnetic resonance lipid profiling combined with chemometric techniques. Meat Sci. 2013, 93, 171-177. [CrossRef]

74. Zanardi, E.; Caligiani, A.; Palla, L.; Mariani, M.; Ghidini, S.; Di Ciccio, P.A.; Palla, G.; Ianieri, A. Metabolic profiling by ${ }^{1} \mathrm{H}$ NMR of ground beef irradiated at different irradiation doses. Meat Sci. 2015, 103, 83-89. [CrossRef] [PubMed]

75. Ren, S.; Hinzman, A.A.; Kang, E.L.; Szczesniak, R.D.; Lu, L.J. Computational and statistical analysis of metabolomics data. Metabolomics 2015, 11, 1492-1513. [CrossRef]

76. Hollung, K.; Timperio, A.M.; Olivan, M.; Kemp, C.; Coto-Montes, A.; Sierra, V.; Zolla, L. Systems biology: A new tool for farm animal science. Curr. Protein Pept. Sci. 2014, 15, 100-117. [CrossRef] [PubMed]

77. Carpenter, C.E.; Cornforth, D.P.; Whittier, D. Consumer preferences for beef color and packaging did not affect eating satisfaction. Meat Sci. 2001, 57, 359-363. [CrossRef]

78. Arihara, K.; Yokoyama, I.; Ohata, M. DMHF (2,5-dimethyl-4-hydroxy-3(2H)-furanone), a volatile food component with attractive sensory properties, brings physiological functions through inhalation. Adv. Food Nutr. Res. 2019, 89, 239-258. [PubMed]

79. Nishimura, T. Mechanism Involved in the Improvement of Meat Taste during Postmortem Aging. Food Sci. Technol. Int. Tokyo 1998, 4, 241-249. [CrossRef] 
80. Mottram, D.S. Flavour formation in meat and meat products: A review. Food Chem. 1998, 62, 415-424. [CrossRef]

81. Kuttappan, V.A.; Hargis, B.M.; Owens, C.M. White striping and woody breast myopathies in the modern poultry industry: A review. Poult. Sci. 2016, 95, 2724-2733. [CrossRef] [PubMed]

82. Andersen, H.J.; Oksbjerg, N.; Young, J.F.; Therkildsen, M. Feeding and meat quality-A future approach. Meat Sci. 2005, 70, 543-554. [CrossRef] [PubMed]

83. Xing, T.; Gao, F.; Tume, R.K.; Zhou, G.; Xu, X. Stress Effects on Meat Quality: A Mechanistic Perspective. Compr. Rev. Food Sci. Food Saf. 2019, 18, 380-401. [CrossRef]

84. Hwang, Y.-H.; Kim, G.-D.; Jeong, J.-Y.; Hur, S.-J.; Joo, S.-T. The relationship between muscle fiber characteristics and meat quality traits of highly marbled Hanwoo (Korean native cattle) steers. Meat Sci. 2010, 86, 456-461. [CrossRef]

85. Chang, K.C.; Da Costa, N.; Blackley, R.; Southwood, O.; Evans, G.; Plastow, G.; Wood, J.D.; Richardson, R.I. Relationships of myosin heavy chain fibre types to meat quality traits in traditional and modern pigs. Meat Sci. 2003, 64, 93-103. [CrossRef]

86. Lee, S.H.; Choe, J.H.; Choi, Y.M.; Jung, K.C.; Rhee, M.S.; Hong, K.C.; Lee, S.K.; Ryu, Y.C.; Kim, B.C. The influence of pork quality traits and muscle fiber characteristics on the eating quality of pork from various breeds. Meat Sci. 2012, 90, 284-291. [CrossRef] [PubMed]

87. Nam, Y.J.; Choi, Y.M.; Lee, S.H.; Choe, J.H.; Jeong, D.W.; Kim, Y.Y.; Kim, B.C. Sensory evaluations of porcine longissimus dorsi muscle: Relationships with postmortem meat quality traits and muscle fiber characteristics. Meat Sci. 2009, 83, 731-736. [CrossRef]

88. Lefaucheur, L. A second look into fibre typing-relation to meat quality. Meat Sci. 2010, 84, 257-270. [CrossRef]

89. Schiaffino, S.; Reggiani, C. Fiber types in mammalian skeletal muscles. Physiol. Rev. 2011, 91, 1447-1531. [CrossRef]

90. Eggert, J.M.; Depreux, F.F.S.; Schinckel, A.P.; Grant, A.L.; Gerrard, D.E. Myosin heavy chain isoforms account for variation in pork quality. Meat Sci. 2002, 61, 117-126. [CrossRef]

91. Oe, M.; Ohnishi-Kameyama, M.; Nakajima, I.; Muroya, S.; Shibata, M.; Ojima, K.; Kushibiki, S.; Chikuni, K. Proteome analysis of whole and water-soluble proteins in masseter and semitendinosus muscles of Holstein cows. Anim. Sci. J. 2011, 82, 181-186. [CrossRef] [PubMed]

92. Hatazawa, Y.; Senoo, N.; Tadaishi, M.; Ogawa, Y.; Ezaki, O.; Kamei, Y.; Miura, S. Metabolomic Analysis of the Skeletal Muscle of Mice Overexpressing PGC-1 $\alpha$. PLoS ONE 2015, 10, e0129084. [CrossRef] [PubMed]

93. Bourdeau Julien, I.; Sephton, C.F.; Dutchak, P.A. Metabolic Networks Influencing Skeletal Muscle Fiber Composition. Front. Cell Dev. Biol. 2018, 6, 125. [CrossRef]

94. Muroya, S.; Nakajima, I.; Oe, M.; Chikuni, K. Difference in postmortem degradation pattern among troponin T isoforms expressed in bovine longissimus, diaphragm, and masseter muscles. Meat Sci. 2006, 72, 245-251. [CrossRef] [PubMed]

95. Muroya, S.; Ertbjerg, P.; Pomponio, L.; Christensen, M. Desmin and troponin T are degraded faster in type IIb muscle fibers than in type I fibers during postmortem aging of porcine muscle. Meat Sci. 2010, 86, 764-769. [CrossRef]

96. Kitamura, S.-I.; Muroya, S.; Tanabe, S.; Okumura, T.; Chikuni, K.; Nishimura, T. Mechanism of production of troponin T fragments during postmortem aging of porcine muscle. J. Agric. Food Chem. 2005, 53, 4178-4181. [CrossRef] [PubMed]

97. Muroya, S.; Kitamura, S.-I.; Tanabe, S.; Nishimura, T.; Nakajima, I.; Chikuni, K. N-terminal amino acid sequences of troponin $\mathrm{T}$ fragments, including $30 \mathrm{kDa}$ one, produced during postmortem aging of bovine longissimus muscle. Meat Sci. 2004, 67, 19-24. [CrossRef] [PubMed]

98. Muroya, S.; Ohnishi-Kameyama, M.; Oe, M.; Nakajima, I.; Chikuni, K. Postmortem changes in bovine troponin $\mathrm{T}$ isoforms on two-dimensional electrophoretic gel analyzed using mass spectrometry and western blotting: The limited fragmentation into basic polypeptides. Meat Sci. 2007, 75, 506-514. [CrossRef]

99. Lametsch, R.; Karlsson, A.; Rosenvold, K.; Andersen, H.J.; Roepstorff, P.; Bendixen, E. Postmortem proteome changes of porcine muscle related to tenderness. J. Agric. Food Chem. 2003, 51, 6992-6997. [CrossRef]

100. Marino, R.; Albenzio, M.; Della Malva, A.; Santillo, A.; Loizzo, P.; Sevi, A. Proteolytic pattern of myofibrillar protein and meat tenderness as affected by breed and aging time. Meat Sci. 2013, 95, 281-287. [CrossRef] 
101. Della Malva, A.; De Palo, P.; Lorenzo, J.M.; Maggiolino, A.; Albenzio, M.; Marino, R. Application of proteomic to investigate the post-mortem tenderization rate of different horse muscles. Meat Sci. 2019, 157, 107885. [CrossRef] [PubMed]

102. López-Pedrouso, M.; Pérez-Santaescolástica, C.; Franco, D.; Fulladosa, E.; Carballo, J.; Zapata, C.; Lorenzo, J.M. Comparative proteomic profiling of myofibrillar proteins in dry-cured ham with different proteolysis indices and adhesiveness. Food Chem. 2018, 244, 238-245. [CrossRef] [PubMed]

103. Nychas, G.-J.E.; Skandamis, P.N.; Tassou, C.C.; Koutsoumanis, K.P. Meat spoilage during distribution. Meat Sci. 2008, 78, 77-89. [CrossRef] [PubMed]

(C) 2020 by the authors. Licensee MDPI, Basel, Switzerland. This article is an open access article distributed under the terms and conditions of the Creative Commons Attribution (CC BY) license (http://creativecommons.org/licenses/by/4.0/). 

MDPI

St. Alban-Anlage 66

4052 Basel

Switzerland

Tel. +41616837734

Fax +41 613028918

www.mdpi.com

Metabolites Editorial Office

E-mail: metabolites@mdpi.com www.mdpi.com/journal/metabolites

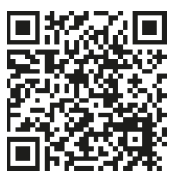



MDPI

St. Alban-Anlage 66

4052 Basel

Switzerland

Tel: +41 616837734

Fax: +41 613028918

www.mdpi.com 